\title{
Zweikernige Nickel(II)-Komplexe und dreikernige Kupfer(II)-Komplexe als \\ Baueinheiten im Molekularen Magnetismus
}

\author{
Dissertation \\ zur Erlangung des Doktorgrades \\ der Mathematisch-Naturwissenschaftlichen Fakultäten \\ der Georg-August-Universität zu Göttingen \\ vorgelegt von
}

Diplom-Chemiker

Serhiy Demeshko

aus Schostka (Ukraine)

Göttingen, den 19.09.2006 
D7

Referent: Prof. Dr. Franc Meyer

Korreferent: Prof. Dr. Dietmar Stalke

Tag der mündlichen Prüfung: 1. November 2006 
Ich erkläre hiermit an Eides statt, dass ich diese Arbeit selbständig und ohne unerlaubte Hilfsmittel angefertigt habe. 
Die vorliegende Arbeit entstand im Zeitraum von Oktober 2002 bis Juli 2006 unter Anleitung von Herrn Professor Dr. Franc Meyer am Anorganisch-Chemischen Institut der Georg-August-Universität Göttingen. 
1. Einleitung 1

2. Zielsetzung der Arbeit 5

3. Einführung in die Grundlagen des Molekularen Magnetismus $\quad 7$

3.1. Magnetisches Moment und Magnetisierung 7

3.2. Die van-Vleck-Gleichung und die isotrope Austauschwechselwirkung 9

3.3. Darstellung der magnetischen Eigenschaften 14

4. Zweikernige Nickel(II)-Komplexe mit kontrollierter Azid-Koordination als

Baueinheiten für höhernukleare Systeme

4.1. Überblick über die Chemie der Nickel(II)-Azid-Verbindungen und die magneto-strukturellen Korrelationen

4.1.1. Koordinationsvarianten sowie Austauschmechanismen des Azidliganden 16

4.1.2. $\mu$-1,3-azidverbrückte Komplexe 19

4.1.3. $\mu$-1,1-azidverbrückte Komplexe 23

4.1.4. $\mu_{3}$ - und $\mu_{4}$-azidverbrückte Komplexe 27

4.1.5. Kombinationen von EE- und EO- Azidobrücken. Topologisch ferrimagnetische Ketten $\quad 28$

4.1.6. Sprunghafte Änderung im magnetischen Verhalten, verursacht durch eine Phasenumwandlung in Azid-Komplexen $\quad 30$

4.1.7 Zusammenfassung 32

4.2. Das pyrazolbasierte Ligandsystem 33

4.2.1. Eigenschaften der Pyrazolliganden 33

4.2.2. Synthese der Liganden 34

4.3. Synthese, Molekülstrukturen und magnetische Eigenschaften der pyrazolatbasierten Nickel(II)-Azid-Komplexe 38

4.3.1. Zweikernige Nickel(II)-Komplexe 38

4.3.1.1. Synthese und grundlegende Koordinationschemie der zweikernigen $\begin{array}{ll}\text { Nickel(II)-Komplexe } & 38\end{array}$

4.3.1.2. Komplexe mit $\mu$-1,1-verbrückendem Azid 39

4.3.1.3. Komplexe mit $\mu$-1,3-verbrückendem Azid 43

4.3.2. Vierkernige Nickel(II)-Komplexe unterschiedlicher Topologie 49

4.3.2.1. Rechteckige Anordnung der Nickel(II)-Zentren mit $\mu$-1,1- und $\mu$-1,3Azidbrücken

4.3.2.2. Trapezförmige Anordnung der Nickel(II)-Zentren mit zentralen $\mu$-1,1,3-Aziden 
4.3.2.3. Rechteckige Anordnung der Nickel(II)-Zentren mit einem zentralen $\mu$-1,1,3,3-Azid

4.3.2.4. Kettenförmige Anordnung der Nickel(II)-Zentren 78

4.3.3. $J$-alternierende Ketten, bestehend aus $S=1$ Spinträgern 87

4.3.3.1. Die Steuerung des Alternanzmusters durch die Ligandgestaltung 87

4.3.3.2. Variationen an interdimeren Kopplungen in 1D-alternierenden Ketten

4.3.3.3. Topologisch ferrimagnetische Kette

4.3.4. Magnetische Hysteresebistabilität basierend auf einem molekularen Azid-Schalter: das „Chamäleon-System“ “ $\left[\mathrm{L}^{6} \mathrm{Ni}_{2}\left(\mathrm{~N}_{3}\right)_{3}\right]$

\section{Dreikernige Kupfer(II)-Triazin-Bausteine für den Aufbau polynuklearer} Spezies

5.1. Der triazinbasierte Ligand $\mathbf{L}^{\mathbf{8}}$ und seine Eigenschaften

5.2. Synthese, Molekülstrukturen und magnetische Eigenschaften der triazinbasierten Kupfer(II)-Komplexe

5.3. Anion- $\pi$ Wechselwirkung in einem karussellförmigen Kupfer(II)-Triazinkomplex

6. Experimenteller Teil

6.1. Allgemeine Arbeitstechniken und analytische Methoden

6.2. Synthese der Pyrazolatliganden $\mathbf{H L}^{\mathbf{1 - 7}}$

6.3. Synthese der Metallkomplexe

6.3.1. Vorbemerkungen

6.3.2. Synthese der zweikernigen Nickel(II)-Komplexe

6.3.3. Synthese der vierkernigen Nickel(II)-Komplexe

6.3.4. Synthese der $J$-alternierenden Ketten, bestehend aus $S=1$ Spinträgern

6.3.5. Synthese der Kupfer(II)-Triazinkomplexe

6.4. Magnetische Untersuchungen

6.4.1. Probenvorbereitung

6.4.2. Magnetische Messungen

6.4.3. Auswertung von Suszeptibilitätsmessungen

6.4.4. Anpassung an die experimentellen Daten

6.5. Kristalldaten und Angaben zur Struktur im Festkörper 
Formelübersicht

Überblick magnetischer Parameter

Danksagung

Lebenslauf 


\begin{tabular}{|c|c|}
\hline Ada & Adamantyl \\
\hline $\mathrm{AF}$ & antiferromagnetisch \\
\hline ber. & berechnet \\
\hline${ }^{t} \mathrm{Bu}$ & tert-Butyl \\
\hline CSD & Cambridge Structural Database \\
\hline DHP & Dihydropyran \\
\hline DMSO & Dimethylsulfoxid \\
\hline DSC & Dynamic Scanning Calorimetry \\
\hline $\mathrm{EE}$ & end-to-end \\
\hline EI & Elektronrnstoß-Ionisation \\
\hline EO & end-on \\
\hline EPR & Electron Paramagnetic Resonance \\
\hline ESI & Elektrospray-Ionisation \\
\hline Et & Ethyl \\
\hline $\mathrm{EtOH}$ & Ethanol \\
\hline exp. & experimentell \\
\hline $\mathrm{F}$ & ferromagnetisch \\
\hline FAB & Fast Atom Bombardment \\
\hline IR & Infrarot \\
\hline $\mathrm{L}$ & Ligand \\
\hline $\mathrm{Me}$ & Methyl \\
\hline $\mathrm{MeCN}$ & Acetonitril \\
\hline $\mathrm{MeOH}$ & Methanol \\
\hline MS & Massenspektrometrie \\
\hline NMR & Nuclear Magnetic Resonance \\
\hline Ac & Acetat \\
\hline${ }^{i} \operatorname{Pr}$ & iso-Propyl \\
\hline $\mathrm{Ph}$ & Phenyl \\
\hline $\mathrm{pz}$ & Pyrazol \\
\hline $\mathrm{R}$ & Rest \\
\hline SQUID & Superconducting Quantum Interference Device \\
\hline TIP & Temperature Independent Paramagnetism \\
\hline UV/Vis & Ultraviolett/Visible \\
\hline
\end{tabular}




\section{Einleitung}

„I have been more and more fascinated by the beauty of this (molecular magnetism) area and by the diversity it presents..."

O. Kahn, „Molecular Magnetism”

Das Naturphänomen des Magnetismus interessiert die Menschheit seit Urzeiten. Bereits im Altertum wurde die Anziehungskraft des Magnetits auf Eisen durch den griechischen Philosophen Thales von Milet im 6. Jh. v. Chr. beschrieben. ${ }^{[1,2]}$ Die Entwicklung der fundamentalen Gesetze und darauf basierender Anwendungen in neunzehnten und zwanzigsten Jahrhunderten führte dazu, dass der Magnetismus inzwischen für das moderne Leben unverzichtbar geworden ist. Heutzutage finden Magnete zahlreiche Anwendungen: von magneto-mechanischen Maschinen (magnetische Separatoren) über Datenverarbeitung (Speicher), Informationstechnik (Schalter, Sensoren) bis hin zu Elektromotoren. Derzeit werden dafür allerdings konventionelle Materialien verwendet, deren magnetische Kenngrößen sich über ein weites Spektrum anpassen lassen. Zu diesen Materialien gehören Metalle, Metalloxide oder Legierungen, die nach metallurgischen Verfahren bei hohen Temperaturen hergestellt werden.

In jüngster Zeit hat sich der Molekulare Magnetismus als ein Bereich der modernen Naturwissenschaften etabliert, der sich mit der Entwicklung neuer magnetischer Materialien auf molekularer Basis beschäftigt. ${ }^{[3]}$ Dabei ist es möglich, das erarbeitete Syntheserepertoire der Chemie für die gezielte Variation der Materialeigenschaften zum Einsatz zu bringen. Das primäre Ziel dabei ist es, die magnetischen Eigenschaften mit anderen technisch relevanten wie optischen, elektrischen oder mechanischen zu kombinieren. Somit bilden Magnete auf der Basis molekularer Bausteine ein Kernstück moderner Materialforschung. Beispielsweise können molekulare Systeme, die magnetische Bistabilität aufweisen, eine wichtige Rolle im Bereich der molekularen Elektronik spielen, z.B. in entsprechenden Speicherelementen oder in der Sensortechnologie. Die Spinübergänge in einigen Übergangsmetallkomplexen liefern dafür die besten Beispiele.

Auf der anderen Seite bleibt die Vielseitigkeit des Molekularen Magnetismus nicht nur auf die materialwissenschaftlichen Aspekte begrenzt. Hier bietet sich auch die Möglichkeit an, biologische Prozesse, wie z.B. die Wechselwirkungen in den metallhaltigen aktiven Zentren der Enzyme, aus magnetochemischer Sicht zu studieren. ${ }^{[4]}$

Das in vielerlei Hinsicht interdisziplinäre Feld des molekularen Magnetismus umfasst die synthetische und theoretische Chemie. Für die synthetische Chemie ist das Design und die Darstellung der molekularen Verbindungen mit vorhersagbaren magnetischen Eigenschaften 
eine der größten Herausforderungen. Den Hintergrund für die verwendeten Synthesestrategien und die physikalische Interpretation der magnetischen Eigenschaften bildet die theoretische Chemie. ${ }^{[5]}$

Der gezielte Aufbau von Koordinationspolymeren, welche aus maßgeschneiderten molekularen Bausteinen bestehen, wird zur Zeit intensiv erforscht. Insbesondere gibt es in den letzten Jahren eine rasante Entwicklung im Bereich des Magnetismus der niederdimensionalen Systeme. ${ }^{[6]}$ Die 1D-Ketten paramagnetischer Zentren nehmen eine Zwischenstellung auf dem Weg von isolierten, molekularen Spezies zu den unter materialwissenschaftlichen Aspekten besonders interessanten dreidimensionalen Architekturen ein. ${ }^{[7,8]}$ Unter anderem erlauben niedrigdimensionale Systeme die Überprüfung von Modellen und Konzepten zur Deutung magnetischer Phänomene, die in höherer Dimensionalität schwierig zu beschreiben sind. Eine wesentliche Voraussetzung für Fortschritte in diesem interdisziplinären Gebiet ist die Fähigkeit, neue Typen niedrigdimensionaler Systeme in kontrollierter Weise aus molekularen Einheiten aufzubauen. Von besonderem Interesse sind die $J$-alternierenden Ketten (Abb. 1.1) u.a. im Zusammenhang mit Fragen zu Spin-Peierls-Übergängen und zur Haldane-Vermutung. ${ }^{[5,6]}$

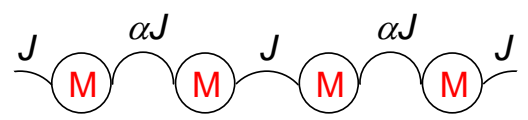

Abb. 1.1. 1D-Kette mit Alternanz der Kopplungskonstanten $J$ und $\alpha J$.

Mittlerweile ist eine ganze Reihe ungewöhnlicher 1D-Systeme mit den unterschiedlichsten Spintopologien bekannt. ${ }^{[6]}$ Hierzu gehören insbesondere homometallische Ketten mit verschiedenen Alternanzmustern bzw. Sequenzen von antiferromagnetischen und ferromagnetischen Kopplungen. ${ }^{[9,10]}$

Eine der Strategien für das Design der molekülbasierten Magnetmaterialien ist die Verknüpfung der paramagnetischen Zentren durch die Auswahl geeigneter Brückenliganden. Für diesen Zweck wird sehr oft das Azidion verwendet. Dieser vielfältige Ligand hat die unterschiedlichsten Koordinationsmöglichkeiten und vermittelt dabei sehr effektiv magnetische Austauschwechselwirkungen, deren Art und Stärke vom Koordinationsmodus und von geometrischen Parametern abhängt. ${ }^{[11,12]}$ Als Spinträger werden oft 3dÜbergangsmetallionen wie $\mathrm{Fe}^{\mathrm{II}}, \mathrm{Mn}^{\mathrm{II}}$, $\mathrm{Cu}^{\mathrm{II}}$ verwendet. Da ein Grundzustand mit hohem Spin, ${ }^{[13]}$ aber auch eine große magnetische Anisotropie ${ }^{[7,14]}$ gute Voraussetzungen für interessante magnetische Eigenschaften sind, wird besonders häufig $\mathrm{Ni}^{\mathrm{II}}$ als paramagnetisches 
Zentrum eingesetzt. Die Kombination von Nickel(II)-Ionen mit Azid als Brückenliganden hat zu einem breiten Spektrum an Molekülarchitekturen geführt. Unter anderem hat sich Azid als besonders nützliches Werkzeug für den Aufbau von 1D-Systemen mit alternierenden ferromagnetischen (durch $\mu$-1,1-Azid) und antiferromagnetischen (durch $\mu$-1,3-Azid) Wechselwirkungen etabliert. ${ }^{[15]}$ In der Regel werden diese Verbindungen jedoch in mehr oder minder zufälliger Weise durch Selbstorganisation aus Metallsalzen und mehrzähnigen Liganden gewonnen, was eine gezielte Variation individueller Parameter, wie z.B. des Alternanzparameters $\alpha$ (vgl. Abb. 1.1), kaum zulässt. Die Problematik beim Design von Nickel(II)-Azid-Systemen mit vorhersagbaren magnetischen Eigenschaften besteht in der Kontrolle der Art und Weise des Azideinbaus zwischen zwei paramagnetischen Zentren. Noch im Jahre 1999 wurde in einem Übersichtsartikel die synthetische Kontrolle der AzidKoordination als unmöglich bezeichnet. ${ }^{[12]}$

Ein anderer Weg zu neuen Klassen von Verbindungen mit magnetisch relevanten Eigenschaften basiert auf der Entwicklung bzw. Anwendung von Ligandsystemen, die ausgehend von Theorien des Magnetismus mehrere Metallzentren so koordinieren sollten, dass sie ferromagnetisch gekoppelt sind. Kompartimentliganden, abgeleitet von 1,3,5-Triazin, sind in dieser Hinsicht attraktive Gerüste, werden aber kaum in der Koordinationschemie verwendet. ${ }^{[16,17,18,19]}$ Die Besonderheit der 1,3,5-Triazine beruht auf den in meta-Position lokalisierten potenziellen N-Donoratomen, welche beispielsweise analog zu metaPhenylenbrücken (Abb. 1.2) ferromagnetische Wechselwirkung zwischen paramagnetischen Zentren dank des sogenannten Spinpolarisationsmechanismus ermöglichen sollten. ${ }^{\text {[20,21,22] }}$

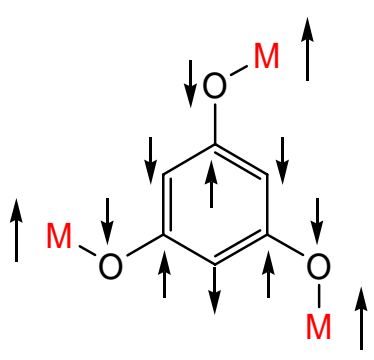

a

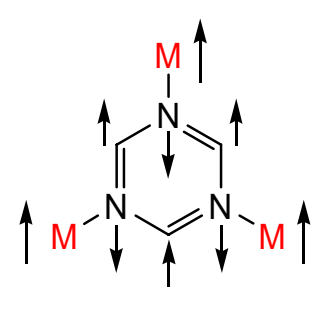

b

Abb. 1.2. Meta-phenylenverbrückte ferromagnetisch gekoppelte Komplexe auf Trihydroxybenzol- (a) und Triazinbasis (b).

Aus diesem Grund können triazinbasierende trimetallische Baueinheiten einen hohen SpinGrundzustand haben und durch Zusammenfügen $\mathrm{zu}$ neuen Typen von molekülbasierten magnetischen Materialien entwickelt werden. Es sollte angemerkt werden, dass bisher nur in 
wenigen Fällen die N-Donoratome des Triazinringes an Metallionen koordiniert werden konnten. ${ }^{[18,22,23]}$ 


\section{Zielsetzung der Arbeit}

Ziel dieser Arbeit ist, neue Klassen molekularer Verbindungen mit interessanten magnetischen Eigenschaften zu entwickeln. Die dafür ausgewählten Strategien sind die Verwendung des Azidions als Brückenligand für die Synthese von mehrkernigen Komplexen und Ketten sowie der Einsatz der Triazinliganden zum Aufbau dreikerniger Baueinheiten mit hohem Spin-Grundzustand. Strukturelle Kontrolle in polymeren Architekturen ist dabei Voraussetzung für die Darstellung von Materialien mit spezifischen physiko-chemischen Eigenschaften. In der vorliegenden Arbeit wird dies durch den Einsatz von polydentaten Liganden (Abb. 2.1) ermöglicht, die Metallionen in bestimmten Anordnungen vororganisieren können.

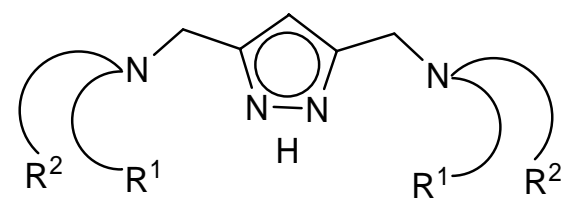

a<smiles>[R]c1nc([R])nc([R])n1</smiles>

b

Abb.2.1. Kompartimentliganden auf Pyrazol- (a) und Triazinbasis (b).

Die erste Aufgabe zu Beginn dieser Arbeit war es, das Problem der Kontrolle der AzidKoordination aus synthetischer Sicht anzugehen und zu lösen. Dafür werden mehrzähnige Kompartimentliganden auf Basis eines Pyrazolheterozyklus eingesetzt, in welchen in 3- und 5-Position Seitenarme, die unterschiedlich substituiert sein können, angebracht sind (Abb. 2.1.a). Wie in früheren Veröffentlichungen unserer Arbeitsgruppe gezeigt werden konnte, sind diese Liganden ausgezeichnet für den Aufbau zweikerniger Komplexe geeignet. In diesen kann der Abstand zwischen beiden Metallionen durch Variation der Seitenarmlänge gezielt eingestellt werden. ${ }^{[24]}$ Im Rahmen dieser Arbeit sollen Pyrazolliganden für die Synthese zweikerniger Nickel(II)-Komplexe eingesetzt werden. Die Kontrolle der AzidKoordination soll dabei durch den gezielt variierbaren Metall-Metall-Abstand gewährleistet sein. Im nächsten Schritt sollen bimetallische Einheiten im Sinne eines molekularen Baukastenprinzips in kontrollierter Weise zu höheren Aggregaten wie Clustern oder 1Dalternierenden Ketten zusammengefügt werden. Die Möglichkeit der Bildung solcher Aggregate konnte auch in ersten Vorarbeiten gezeigt werden. ${ }^{[25,26]}$ Aufgrund des modularen Syntheseansatzes sollte es dabei möglich sein, unter anderem das Verhältnis der 
alternierenden Kopplungskonstanten innerhalb der Kettenstrukturen in gewissem Rahmen zu variieren.

Im zweiten Teil der Arbeit sollen erste Versuche zur Synthese dreikerniger Komplexe der 2,4,6-substituierten Triazinliganden (Abb. 2.1.b) vorgenommen werden, in welchen die Metallzentren an die N-Triazinatome koordinieren und nach dem Spinpolarisationsmechanismus ferromagnetisch gekoppelt sein sollten. Da trimetallische Einheiten sich als geeignete Bausteine für den Aufbau von 2D-Schichten erwiesen, ${ }^{[16,27]}$ sollte es möglich sein, auf Basis solcher Komplexe interessante Materialien zu konstruieren, indem man sie zu höheren Aggregaten zusammenfügt.

Fasst man die oben erwähnten Aspekte zusammen, kann das Ziel dieser Arbeit folgendermaßen formuliert werden:

1) Verständnis und Steuerung des magnetischen Verhaltens auf molekularer Ebene durch die Entwicklung gut definierter bi- und trimetallischer molekularer Baueinheiten, deren makroskopische magnetische Eigenschaften durch Moleküldesign vorbestimmt werden sollen;

2) Aufbau von oligonuklearen, polynuklearen 1D-Koordinationsverbindungen sowie höherdimensionalen Netzwerken durch eine Verknüpfung solcher Einheiten.

Der Schwerpunkt dieser Arbeit wird jedoch auf den pyrazolatbasierten Komplexen mit kontrollierter Nickel(II)-Azid-Koordination als Baueinheiten für molekülbasierte Materialien mit interessanten magnetischen Eigenschaften liegen. 


\section{Einführung in die Grundlagen des molekularen Magnetismus}

\subsection{Magnetisches Moment und Magnetisierung}

Die magnetischen Eigenschaften von Materie können durch die Wechselwirkung mit einem äußerem Magnetfeld $(H)$ charakterisiert werden. Das Einbringen von Materie in ein Magnetfeld ruft eine Änderung der magnetischen Induktion (B) im Vergleich zum Vakuum hervor. Diese Änderung bezeichnet man als Magnetisierung $(M)$ :

$$
B=H+4 \pi M
$$

Bei geringen Feldstärken ist die Magnetisierung proportional zur Feldstärke, wobei der Proportionalitätsfaktor bezogen auf ein Mol Substanz als molare magnetische Suszeptibilität $\left(\chi_{M}\right)$ bezeichnet wird:

$$
M=\chi_{M} H
$$

Die molare magnetische Suszeptibilität setzt sich aus einem negativen diamagnetischen Beitrag $\left(\chi_{M}^{D}\right)$ und zwei positiven paramagnetischen Beiträgen $\left(\chi_{M}^{P}\right.$ und $\left.\chi_{M}^{T I P}\right)$ zusammen:

$$
\chi_{M}=\chi_{M}^{D}+\chi_{M}^{P}+\chi_{M}^{T I P}
$$

Durch die Bewegung der Elektronen wird ein magnetisches Moment induziert, das dem äußerem Magnetfeld entgegengerichtet ist. Dies führt zum diamagnetischen Beitrag. Wenn eine Substanz ungepaarte Elektronen besitzt, richtet sich das damit verbundene Spinmoment parallel zum angelegten Magnetfeld aus. Daraus resultiert der positive paramagnetische Anteil $\left(\chi_{M}^{P}\right)$. Der dritte Summand $\left(\chi_{M}^{T I P}\right)$ berücksichtigt den temperaturunabhängigen Paramagnetismus (TIP - temperature independent paramagnetism). Er entsteht dadurch, dass im Magnetfeld eine endliche, wenn auch sehr geringe Übergangswahrscheinlichkeit eines gepaarten Elektrons in einen angeregten Zustand besteht.

Für die paramagnetischen Materialien ohne Wechselwirkung zwischen Spinmomenten gilt das empirisch gefundene Curie-Gesetz: ${ }^{[28]}$ 


$$
\chi_{M}=\frac{C}{T}
$$

mit der Curie-Konstante

$$
C=\frac{N g^{2} \beta^{2}}{3 k} S(S+1)
$$

und $S=$ Spinquantenzahl, $N=$ Avagadro-Konstante, $\beta=$ Bohr'sches Magneton, $g=$ LandeFaktor, $k=$ Boltzmann-Konstante und $T=$ Temperatur.

Das effektive magnetische Moment wird nach Gleichung 3.1.6 definiert

$$
\mu_{\text {eff }}=\sqrt{\frac{3 k}{N \beta^{2}}} \sqrt{\chi_{M} T}=2.83 \sqrt{\chi_{M} T}
$$

und führt unter Anwendung des Curie-Gesetzes zur Gleichung 3.1.7,

$$
\mu_{\text {eff }}=g \sqrt{S(S+1)} \mu_{B}
$$

welche für $\mu_{\text {eff }}$ den reinen Spinparamagnetismus wiedergibt und in Einheiten des Bohr'schen Magnetons $\mu_{B} \equiv \beta=\frac{e \hbar}{2 m_{e}}$ angegeben wird. Für paramagnetische Stoffe ist $\mu_{e f f}$ temperaturunabhängig.

Das Curie-Gesetz gilt für isolierte magnetische Momente. Abweichungen vom CurieGesetz, die durch Wechselwirkung zwischen magnetischen Zentren, eventuell aber auch durch Spin-Bahn-Kopplung oder Nullfeldaufspaltung zustande kommen, können phänomenologisch durch das Curie-Weiss-Gesetz ${ }^{[29]}$ berücksichtigt werden:

$$
\chi_{M}=\frac{C}{T-\Theta}
$$

Dabei ist $\Theta$ die Weiss-Konstante, welche positiv für ferromagnetische und negativ für antiferromagnetische Wechselwirkungen ist.

Für niedrige Temperaturen und hohe Magnetfelder liegt die magnetische Energie in der Größenordnung der thermischen Energie und die Proportionalität zwischen $M$ und $H$ 
(Gleichung 3.1.2) ist nicht mehr gegeben. In diesem Fall nähert sich die Magnetisierung einem Grenzwert - der Sättigungsmagnetisierung $M_{S}$ :

$$
M_{S}=N g S \beta \quad \text { (3.1.9). }
$$

Aus der Auftragung der Sättigungsmagnetisierung (Abhängigkeit der Magnetisierung vom Feld) in Einheiten von $N \beta$ ergibt sich die Zahl der ungepaarten Elektronen. Das gilt aber nur, wenn $\left.\left.\frac{\beta H}{k T}\right\rangle\right\rangle 1$ ist.

\subsection{Die van-Vleck-Gleichung und die isotrope Austauschwechselwirkung}

Die ausführliche Darstellung der quantenmechanischen Berechnung magnetischer Suszeptibilitäten wurde von van Vleck im Jahre 1932 in seiner Monographie gegeben. ${ }^{\left[{ }^{30]}\right.}$ Für ein einzelnes paramagnetisches Molekül mit den Energiezuständen $E_{\mathrm{n}}(\mathrm{n}=1,2, \ldots)$ in einem äußerem Magnetfeld $H$ wird die mikroskopische Magnetisierung $\mu_{\mathrm{n}}$ wie folgt definiert:

$$
\mu_{n}=-\frac{\partial E_{n}}{\partial H}
$$

Durch Summation über alle Mikrozustände gemäß einer Boltzmann-Verteilung ergibt sich die makroskopische molare Magnetisierung, die sich nach einer Reihe von Umformungen und bestimmter Näherungen, worauf hier näher nicht eingegangen werden soll, in einen analytischen Ausdruck für die molare magnetische Suszeptibilität umwandeln lässt,

$$
\chi_{M}=\frac{N \sum_{n}\left\lfloor\left(E_{n}^{(1)}\right)^{2} / k T-2 E_{n}^{(2)}\right\rfloor \exp \left(-E_{n}^{(0)} / k T\right)}{\sum_{n} \exp \left(-E_{n}^{(0)} / k T\right)}
$$

in welchem $E_{n}^{(0)}$ die Energie des n-ten Zustands ohne Magnetfeld ist und $E_{n}^{(1)}$ bzw. $E_{n}^{(2)}$ Zeeman-Koeffizienten sind. Diese Gleichung wird für die Berechnung der magnetischen Suszeptibilität molekularer Substanzen zu Grunde gelegt.

Um nun das Verhalten von Materialien mit mehreren magnetischen Zentren zu beschreiben, müssen in erster Linie die Wechselwirkungen zwischen benachbarten Spinmomenten betrachtet werden. Die Spinmomente zweier benachbarter magnetischer Zentren können parallel oder antiparallel ausgerichtet sein. Im ersten Fall handelt es sich um 
eine ferromagnetische, im zweiten um eine antiferromagnetische Kopplung. Man unterscheidet zwischen antiferromagnetischen und ferromagnetischen Kopplungen einerseits und dreidimensionalen Phänomenen wie z.B. Ferromagnetismus und Antiferromagnetismus andererseits. Während es sich beim Ferromagnetismus und Antiferromagnetismus um Austauschwechselwirkungen zwischen paramagnetischen Zentren in drei Richtungen handelt, werden bei den entsprechenden Kopplungen die zwei benachbarten Spinträger betrachtet. Für den einfachsten Fall mit $S_{A}=S_{B}=1 / 2$ resultiert mit $S=1$ ein Triplett- und mit $S=0$ ein Singulett-Grundzustand (Abb. 3.2.1).

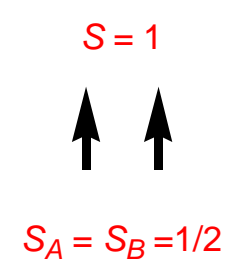

a

$$
\begin{gathered}
S=0 \\
S_{A}=S_{B}=1 / 2
\end{gathered}
$$

b

Abb. 3.2.1. Schematische Darstellung der ferromagnetischen (a) und antiferromagnetischen (b) Kopplung für zweikernige Verbindungen mit $S_{A}=S_{B}=1 / 2$.

Diese Wechselwirkungen zwischen Spinmomenten werden quantitativ auf Grundlage der Quantenmechanik durch die isotrope Austauschkopplungskonstante $J$ beschrieben. Dieser Parameter gibt den Energieabstand bei der Aufspaltung der energetischen Zustände im Magnetfeld wieder. Für $S_{A}=S_{B}=1 / 2$ ist das der energetische Unterschied vom Singulett- zum Triplett-Zustand:

$$
2 J=\Delta E=E_{(S=0)}-E_{(S=1)}
$$

Graphisch stellen sich die entsprechenden Energieniveauschemata für ferromagnetische und antiferromagnetische Kopplung wie folgt dar (Abb. 3.2.2). 


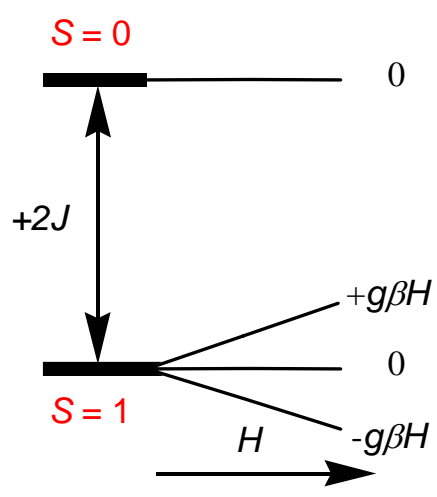

a

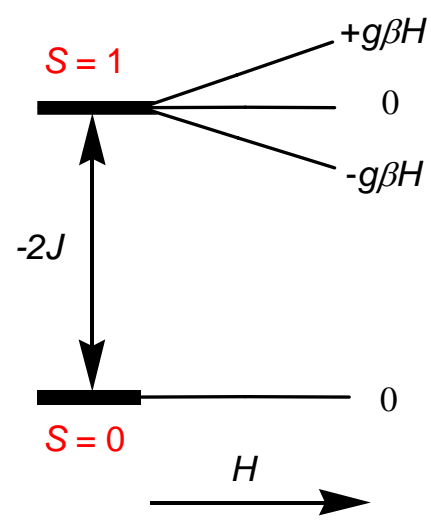

b

Abb. 3.2.2. Energiezustände von ferromagnetischer (a) und antiferromagnetischer (b) Kopplung. In einem äußeren Magnetfeld ist der Triplett-Zustand in drei Zeeman-Terme aufgespaltet.

Das Einsetzen der Energiebeträge entsprechend den Werten der Energieniveaus aus Abb. 3.3.2 in die vereinfachte Van-Vleck-Gleichung (3.2.2) führt zu folgendem Ausdruck für die molare Suszeptibilität zweikerniger Komplexe mit $S_{A}=S_{B}=1 / 2:^{[31]}$

$$
\chi_{M}=\frac{2 N g^{2} \beta^{2}}{k T\left[3+e^{-2 J / k T}\right]}
$$

Für $S_{A}=S_{B}=1$, z.B. bei zweikernigen Nickel(II)-Komplexen, gilt folgende Gleichung für die molare magnetische Suszeptibilität:

$$
\chi_{M}=\frac{2 N g^{2} \beta^{2}}{k T} \frac{e^{2(J / k T)}+5 e^{6(J / k T)}}{1+3 e^{2(J / k T)}+5 e^{6(J / k T)}}
$$

Die isotrope magnetische Austauschwechselwirkung zwischen Spinträgern wird phänomenologisch durch den Heisenberg-Dirac-van-Vleck-Hamiltonoperator beschrieben. Im Falle von zwei magnetischen Zentren gilt:

$$
\hat{H}=-2 J \hat{S}_{A} \cdot \hat{S}_{B}
$$

$\hat{S}_{A}$ und $\hat{S}_{B}$ sind lokale Spin-Operatoren, $J$ ist die Kopplungskonstante. Definitionsgemäß sind $J$-Werte für ferromagnetische Kopplungen zwischen paramagnetischen Zentren positiv und bei antiferromagnetischer Kopplung negativ. Dieser Operator betrachtet ausschließlich die Spin-Momente der an der Wechselwirkung beteiligten Zentren. Die Bahnbeiträge zum magnetischen Moment werden dabei nicht berücksichtigt. Für 3-d Übergangsmetalle ist das eine gute Näherung, da die Bahnanteile in der Regel durch das Kristallfeld unterdrückt 
werden. Mit dem Hamiltonoperator kann man auch kompliziertere Fälle isotropen magnetischen Austausches berechnen. Für eine größere molekulare Einheit oder für ausgedehnte Festkörper ergibt sich der magnetische Austausch als Summe aller paarweisen Wechselwirkungen:

$$
\hat{H}=-2 \sum J_{i j} \hat{S}_{i} \cdot \hat{S}_{j}
$$

Der tatsächliche Mechanismus der isotropen magnetischen Austauschwechselwirkung kann allerdings mit Hilfe dieses Operators nicht geklärt werden.

Die Wechselwirkung zwischen paramagnetischen Zentren kann sowohl über den Raum als auch über Bindungen zustande kommen. Magnetische Dipol-Dipol-Wechselwirkungen über den Raum sind in der Regel sehr klein und indirekt proportional dem Abstand in der dritten Potenz. Die Wechselwirkungen über Bindungen sind elektrostatischer Natur und je nachdem, ob sie direkt zwischen Spinträgern oder indirekt über verbrückende Liganden realisiert werden, unterscheidet man zwischen direktem oder indirektem Austausch.

Da sich bei dem direkten Austausch die Orbitale unmittelbar überlappen, führt dies bei der Besetzung der entstandenen Molekülorbitale nach dem Pauli-Prinzip zu einer starken antiferromagnetischen Kopplung. ${ }^{[32]}$

Der indirekte Austausch wird auch Superaustausch genannt. ${ }^{[33]}$ Der Superaustauschmechanismus dominiert bei ligandverbrückten Metallkomplexen. Für die Beschreibung dieses Phänomens werden hauptsächlich zwei Modelle verwendet, nämlich das Modell der „orthogonalisierten magnetischen Orbitale“ von P.W. Anderson ${ }^{[34]}$ und das Modell der „natürlichen magnetischen Orbitale“ von O. Kahn und B. Briat ${ }^{[35,36]}$.

Für einen zweikernigen Komplex mit den Spinzentren $\mathrm{M}(\mathrm{A})=\mathrm{M}(\mathrm{B})$ und dem Brückenliganden $\mathrm{X}$, also für den Fall $\mathrm{M}(\mathrm{A})-\mathrm{X}-\mathrm{M}(\mathrm{B})$, können magnetische Orbitale auf zwei Wegen bestimmt werden:

1) durch zwei einfach besetzte Molekülorbitale $\gamma_{1}$ und $\gamma_{2}{ }^{[34,37]}$

$$
\begin{aligned}
& \Phi_{A}^{\prime}=\frac{1}{\sqrt{2}}\left(\gamma_{1}+\gamma_{2}\right) \\
& \Phi_{B}^{\prime}=\frac{1}{\sqrt{2}}\left(\gamma_{1}-\gamma_{2}\right)
\end{aligned}
$$

wobei $\Phi_{A}^{\prime}$ und $\Phi_{B}^{\prime}$ orthogonalisiert, aber nicht streng lokalisiert sind - „orthogonalisierte magnetische Orbitale“; 
2) als HOMO's $\Phi_{A}$ und $\Phi_{B}$ für die entsprechenden Fragmente $M(\mathrm{~A})-\mathrm{X}$ und $\mathrm{X}-\mathrm{M}(\mathrm{B}),{ }^{[38]}$ an denen sie lokalisiert sind - „natürliche magnetische Orbitale“.

In beiden Modellen ergibt sich der Singulett-Triplett-Energieabstand als Summe aus einem negativen antiferromagnetischen Beitrag $J_{\text {antiferro }}$ und einem positiven ferromagnetischen Beitrag $J_{\text {ferro: }}$ :

$$
J=J_{\text {ferro }}+J_{\text {antiferro }}
$$

Im ersten Modell gilt:

$$
\begin{aligned}
& J_{\text {antiferro }}=-\frac{\Delta^{2}}{U} \\
& J_{\text {ferro }}=2 q
\end{aligned}
$$

mit $\Delta=\left|E\left(\gamma_{1}\right)-E\left(\gamma_{2}\right)\right|^{2}$, wobei $E\left(\gamma_{1}\right)$ und $E\left(\gamma_{2}\right)$ die Energien der Molekülorbitale $\gamma_{1}$ und $\gamma_{2}$ sind. $U$ ist die mit dem Transfer $\mathrm{M}(\mathrm{A})-\mathrm{X}-\mathrm{M}(\mathrm{B}) \rightarrow \mathrm{M}^{+}(\mathrm{A})-\mathrm{X}-\mathrm{M}^{-}(\mathrm{B})$ verbundene Energie, $q$ ist das zwei-Elektronen-Austauschintegral.

In zweitem Modell werden $J_{\text {antiferro }}$ und $J_{\text {ferro }}$ durch Gleichung 3.2.11 definiert, wobei S das Überlappungsintegral und $q$ das zwei-Elektronen-Austauschintegral sind.

$$
\begin{aligned}
& J_{\text {antiferro }}=-2 \Delta S \\
& J_{\text {ferro }}=2 q
\end{aligned}
$$

Wie man an Hand der Gleichungen 3.2.10 und 3.2.11 erkennen kann, wird die Kopplung zwischen Metallzentren dann ferromagnetisch, wenn das Überlappungsintegral Null ist ( $\Delta$ proportional zu S). Das ist immer dann der Fall, wenn die magnetischen Orbitale orthogonal zueinander stehen. Man unterscheidet dabei zwischen der zufälligen Orthogonalität, wenn der Winkel $\mathrm{M}(\mathrm{A})-\mathrm{X}-\mathrm{M}(\mathrm{B}) 90^{\circ}$ beträgt, ${ }^{[38,39]}$ und der strikten, durch Symmetrie bedingten Orthogonalität (unabhängig vom Winkel). ${ }^{[40]}$ Im ersten Fall kann die Art der Kopplung (ferromagnetisch oder antiferromagnetisch) schon mit geringfügigen Änderungen der strukturellen Parameter wechseln. Im zweiten Fall variiert hauptsächlich die Stärke der ferromagnetischen Kopplung.

Die beiden Modelle, „orthogonalisierte magnetische Orbitale“ und „natürliche magnetische Orbitale“, berücksichtigen bei der Klärung des Austauschphänomens ausschließlich jeweils ein ungepaartes Elektron an den Metallzentren. Da oft die zweifach besetzten Molekülorbitale 
von Brückenliganden wie Halogenen oder Hydroxid-Gruppen energetisch viel tiefer als die $d$ Orbitale der Metalle liegen, ist dies eine gute Näherung. Für den Fall, dass die HOMO’s von Liganden energetisch relativ hoch liegen, wurde von O. Kahn das Konzept der Spinpolarisation herangezogen. ${ }^{[11 \mathrm{a}]}$ Unter dem Spinpolarisationseffekt versteht man in diesem Zusammenhang die besondere Rolle eines Molekülorbitals, das von dem zweifach besetzten Orbital des Liganden und $d$-Orbitalen beider Metalle gebildet wird. An einem konkreten, für diese Arbeit relevanten Beispiel wird der Spinpolarisationseffekt an entsprechender Stelle ausführlicher betrachtet.

\subsection{Darstellung der magnetischen Eigenschaften}

Für die Charakterisierung der magnetischen Eigenschaften der Materie können unterschiedliche Messungen durchgeführt werden. Meist wird die Temperaturabhängigkeit der magnetischen Suszeptibilität gemessen. Dem entsprechend können die molare Suszeptibilität (Abb. 3.3.1.a), die reziproke molare Suszeptibilität (Abb. 3.3.1.b) und das Produkt $\chi_{M} T$ (Abb. 3.3.1.c) als Funktion der Temperatur graphisch dargestellt werden. Da $\mu_{\text {eff }}$ proportional der Wurzel von $\chi_{M} T$ ist (3.1.6), hat die Temperaturabhängigkeit des effektiven magnetischen Moments und des Produkts $\chi_{M} T$ prinzipiell den gleichen Kurvenverlauf (Abb. 3.3.1.c). Auf dem Gebiet des molekularen Magnetismus wird allerdings häufiger $\chi_{M} T$ gegen $T$ dargestellt, weil dabei die teilweise geringfügigen Änderungen in den erhaltenen Messkurven deutlicher erscheinen. 
a

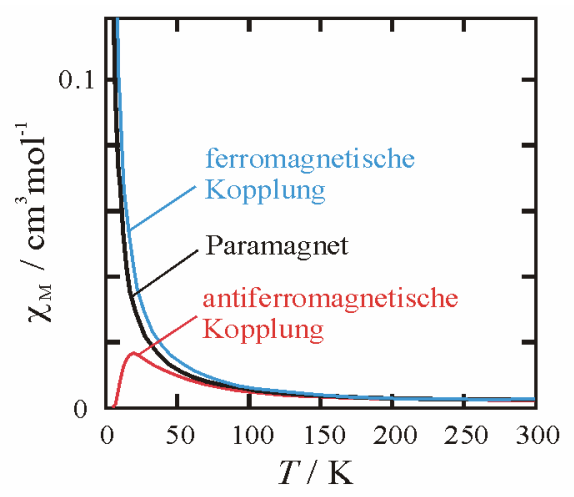

b

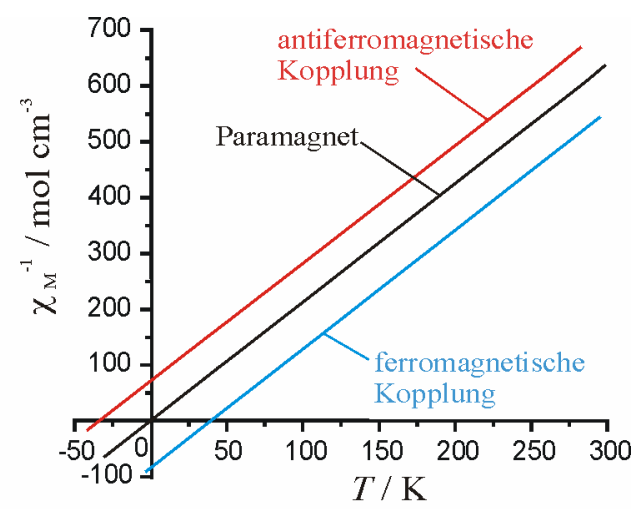

c

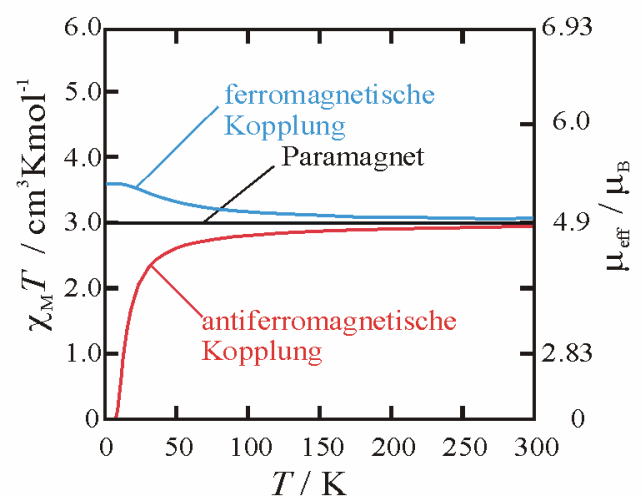

Abb. 3.3.1. Möglichkeiten für die Darstellung der temperaturabhängigen Suszeptibilitätsmessungen.

Eine andere Möglichkeit ist es, bei konstanter Temperatur die Feldabhängigkeit der Magnetisierung zu untersuchen. Entsprechende Magnetisierungskurven für die häufigsten magnetischen Phänomene sind in Abb. 3.3.2. dargestellt.

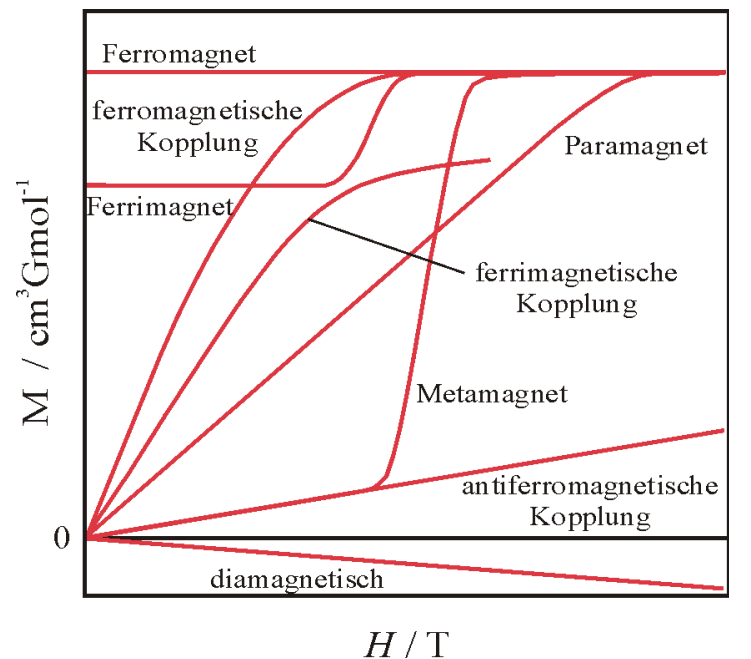

Abb. 3.3.2. Magnetisierungskurven bei verschiedenen magnetischen Verhalten.

Unterschiede zwischen ferromagnetischer Kopplung und Ferro- oder Ferrimagneten (vgl. Kapitel 3.2) zeigen sich deutlich in den Magnetisierungskurven (Abb. 3.3.2). 


\title{
4. Zweikernige Nickel(II)-Komplexe mit kontrollierter Azid-Koordination als Baueinheiten für höhernukleare Systeme
}

\author{
„The azido group, $\mathrm{N}_{3}^{-}$, is certainly one of the \\ most interesting magnetic couplers found so far \\ in molecular magnetism“ \\ O. Kahn (J. Am. Chem. Soc. 1998, 120, 5238)
}

\section{1. Überblick über die Chemie der Nickel(II)-Azid-Verbindungen und die magneto- strukturellen Korrelationen}

In diesem Kapitel sollen die für diese Arbeit relevanten Aspekte der Chemie der Nickel(II)-Azid-Verbindungen aus koordinationschemischer und magnetochemischer Sicht erläutert werden. Dies schließt in erster Linie die Koordinationsmöglichkeiten des Azids als Brückenligand und den damit verbundenen magnetischen Austausch zwischen Metallzentren, Mechanismen dieses Austausches sowie dessen Abhängigkeit von Bindungsparametern ein. Es werden auch interessante Erscheinungen in den azidhaltigen Verbindungen wie Ferrimagnetismus und magnetische Übergänge betrachtet.

\subsubsection{Koordinationsvarianten sowie Austauschmechanismen des Azidliganden}

Im Bereich des molekularen Magnetismus spielen Brückenliganden eine außergewöhnlich wichtige Rolle für die Vermittlung magnetischer Wechselwirkungen zwischen paramagnetischen Zentren. Einer der am häufigsten hierfür verwendeten Brückenliganden ist das Azidion. Es weist als Brückenligand vielfältige Möglichkeiten zur Koordination auf und vermittelt dabei den magnetischen Austausch, dessen Art und Stärke vom Koordinationsmodus und geometrischen Parametern abhängig ist, sehr effektiv. Die Verwendung von Nickel(II)-Ionen in Kombination mit Azidbrücken hat zur Entwicklung eines breiten Spektrums an Molekulararchitekturen geführt, in denen Azid unterschiedlichste Koordinationsmodi annehmen kann.

Die Nickel(II)-Azid-Verbindungen können nach ihrer Dimensionalität oder nach den Koordinationsmodi klassifiziert bzw. beschrieben werden. Es können sowohl Einzelmoleküle mit unterschiedlicher Anzahl von Nickel(II)-Ionen, als auch ein-, zwei- oder dreidimensionale Systeme sein. Klassifiziert nach Koordinationsmodi weisen diese Verbindungen $\mu-1,1$ - (end- 
on, EO), $\mu$-1,3- (end-to-end, EE), $\mu_{3}-1,1,1-, \mu_{3}-1,1,3-, \mu_{4}-1,1,1,1-$ und $\mu_{4}-1,1,3,3-A z i d b r u ̈ c k e n$ (Abb.4.1.1.1) auf. ${ }^{[12,26,41,42,43]}$ Im Falle eines Kupfer(II)-Komplexes wurde auch über eine sehr seltene $\mu_{6}-1,1,1,3,3,3-$ Koordination des Azids berichtet. ${ }^{[44]}$
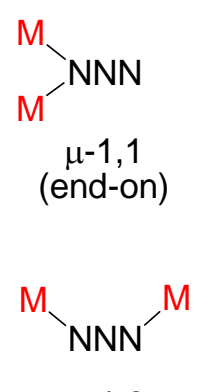

$\mu-1,3$
$($ end-to-end)

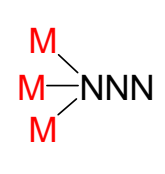

$\mu_{3}-1,1,1$

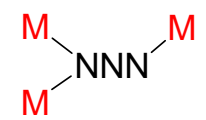

$\mu_{3}-1,1,3$

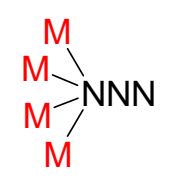

$\mu_{4}-1,1,1,1$

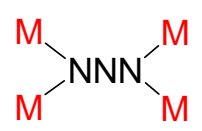

$\mu_{4}-1,1,3,3$

Abb. 4.1.1.1. Bekannte Koordinationsvarianten des Azidliganden.

Bei den Nickel(II)-Azid-Verbindungen kommen sehr oft, unabhängig von der Topologie und Dimensionalität, zweifach verbrückende (end-on oder end-to-end) Azide vor, viel seltener sind im Gegensatz dazu drei- und vierfach verbrückende. Dementsprechend wurden die magnetostrukturellen Korrelationen hauptsächlich an $\mu$-1,1- und $\mu$-1,3-azidverbrückten Systemen untersucht. Dabei wurde grundsätzlich von der Dimensionalität der Systeme ausgegangen. Im Gegensatz dazu wird hier versucht, die Vielfalt der Nickel(II)-AzidKomplexe und deren magnetische Eigenschaften einzig auf der Grundlage der Azidbindungen $\mathrm{zu}$ beschreiben. Es existieren in der Literatur exzellente und umfangreiche Übersichten, in denen Nickel(II)-Azid-Systeme unterschiedlicher Dimension beschrieben wurden. ${ }^{[12,41]}$

Beim $\mu$-1,1-Modus (EO-Koordination) der Azidbrücke tritt normalerweise ferromagnetischer und beim $\mu$-1,3-Modus (EE-Koordination) antiferromagnetischer Austausch auf. Um die Korrelationen zwischen der Art und der Stärke der Kopplung in Abhängigkeit von der Struktur zu erkennen, sollte man die geometrischen Parameter, insbesondere die Winkel, unterschiedlicher Azidbindungsmodi betrachten (Abb.4.1.1.2).
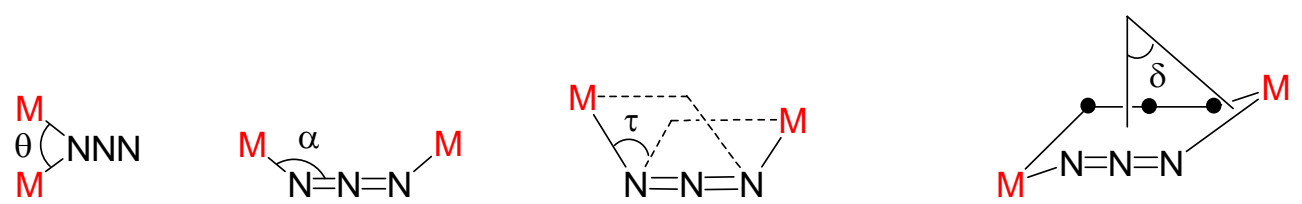

Abb. 4.1.1.2. Magnetisch relevante geometrische Parameter von Nickel(II)-Azid-Verbindungen. 
Während für die EO-Koordination der Ni-N-Ni Winkel $\theta$ ein aus magnetischer Sicht wichtiger Parameter ist, sind es für die einfache EE-Koordination der $\mathrm{Ni}-\mathrm{N}-\mathrm{N}$ Winkel $\alpha$ und der Ni-NNN-Ni Torsionswinkel $\tau$. Der Torsionswinkel $\tau$ beschreibt den Winkel zwischen den beiden Ni-N Bindungen entlang der Azid-Achse. Im Falle der doppelt EEazidverbrückten Komplexe sind auch die Diederwinkel $\delta$ für die magnetischen Eigenschaften relevant. Diese werden als Winkel zwischen den Normalen auf den zwei Ebenen, von den sechs N-Atomen sowie von den zwei N-Atomen und einem Nickel(II)-Ion, definiert.

Für die gezielte Synthese von Nickel(II)-Azid-Verbindungen mit vorhersagbaren magnetischen Eigenschaften ist es wichtig, die Frage zu klären, warum über eine $\mu-1,1-$ Azidbrücke ferromagnetischer Austausch und über eine $\mu$-1,3-Azidbrücke in der Regel antiferromagnetischer Austausch vermittelt wird. Diese Problematik wird häufig an Kupfer(II)-Systemen mittels zweier Konzepte diskutiert.

Das erste Konzept basiert auf zufälliger Orthogonalität im Rahmen des Modells der „orthogonalisierten magnetischen Orbitale“.[11d] Parallele Spinanordnung und somit eine ferromagnetische Kopplung über ein $\mu$-1,1-verbrückendes Azid wird hier durch zufällige Orthogonalität der magnetischen Orbitale erreicht. Wenn die Orbitale nicht mehr orthogonal zueinander sind, kann die Kopplung ab einem bestimmten Winkel $\theta$ (z.B. $108^{\circ}$ in Kupfer(II)Azid-Verbindungen) antiferromagnetisch werden. Übertragen auf die $\mu$-1,3-Azidverbrückung wird daher in solchen Komplexen in der Regel eine antiferromagnetische Wechselwirkung beobachtet. Diese hat ihren Grund darin, dass die strukturellen Parameter, bei denen die Bedingungen der Orthogonalität ( $\alpha$ nahe zu $180^{\circ}$ oder $\tau$ etwa $90^{\circ}$ ) erfüllt werden, normalerweise in einem anderen Bereich liegen, wenn jegliche beschränkenden Ligandgerüste fehlen.

Die zweite Erklärung von O. Kahn basiert auf dem Spinpolarisationseffekt des doppelt besetzten $\varphi_{\mathrm{A}} \mathrm{MO}$ (Abb. 4.1.1.3, unten). ${ }^{[11 \mathrm{a}]}$ Dieses MO ergibt sich aus der Kombination des $\pi_{\mathrm{g}}$-Orbitales vom $\mathrm{N}_{3}{ }^{-}$(höchstes doppelt besetztes $\mathrm{MO}^{[45]}$ ) mit den $\mathrm{d}_{\mathrm{x}-\mathrm{y}}{ }^{2}$-Orbitalen beider Metallzentren. In diesem Orbital $\left(\varphi_{\mathrm{A}}\right)$ sind beide Elektronen - ein Elektron mit $\alpha$ Spin und ein anderes mit $\beta$ Spin - auf endständigen N-Atomen lokalisiert. 


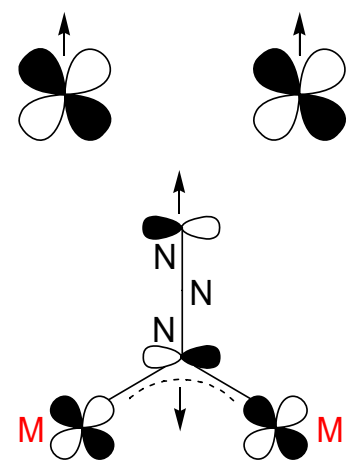

a
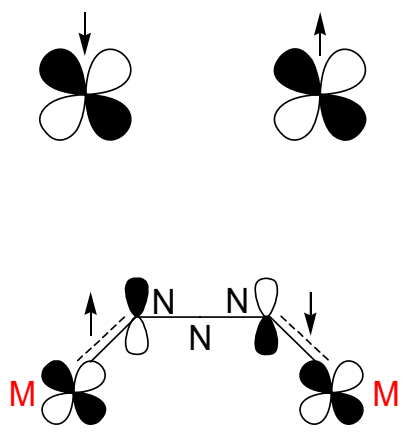

b

Abb. 4.1.1.3. Schematische Darstellung des Spinpolarisationseffektes in EO- (links) und EE- (rechts) azidverbrückten Komplexen.

Bei $\mu-1,1-$ Verbrückung ist das Elektron des verbrückenden $\mathrm{N}$-Atoms mit dem $\beta$ Spin in Richtung der $d$-Orbitale der Metallzentren delokalisiert (Abb. 4.1.1.3.a, unten). Nach der Hund'schen Regel stabilisiert das einen Zustand, in welchem Elektronen an beiden Metallzentren $\alpha$ Spin haben (ferromagnetisch gekoppelte Metallzentren, Abb. 4.1.1.3.a, oben). Da im Fall der $\mu$-1,3-Verbrückung die endständigen N-Atome unterschiedliche Spinorientierung haben, deren Delokalisation zu den $d$-Orbitalen von Metallzentren gerichtet ist (Abb. 4.1.1.3.b, unten), führt dies nach dem Pauli Prinzip zur antiparallelen Spinanordnung (antiferromagnetisch gekoppelte Metallzentren, Abb. 4.1.1.3.b, oben).

\subsection{2. $\mu$-1,3-azidverbrückte Komplexe}

Es wurden Verbindungen unterschiedlicher Architektur mit EE-Azidkoordination kristallographisch untersucht. Darunter ist eine Reihe zweikerniger Spezies mit ein bis drei Azidbrücken (Abb. 4.1.2.1a, b und c). Drei- und vierkernige Verbindungen mit jeweils $\mu$-1,3Azidverbrückung zwischen den Metallzentren (Abb. 4.1.2.1.d, e; in Abb. 4.1.2.1.e Y = verbrückende Thiophenolat und Pyridazin) sind dagegen selten.

Sehr gut untersucht sind bei den Nickel(II)-Komplexen Ketten unterschiedlicher Topologie. Hier unterscheidet man zwischen gleichartigen und alternierenden Ketten. Gleichartig sind die Ketten, wenn benachbarte Nickel(II)-Zentren mit einem (theoretisch auch mit mehreren) Azidliganden so verbrückt sind, dass das Fragment Ni-NNN-Ni immer die gleichen geometrischen Parameter für alle Glieder der Kette hat. Dementsprechend ist auch der magnetische Austausch zwischen den Nickel(II)-Zentren in jedem Kettenglied identisch. In Abhängigkeit von der Position der benachbarten Azidbrücken zueinander unterscheidet 
man zwischen 1D-cis- und 1D-trans-Ketten (Abb. 4.1.2.1.f, g). Bei alternierenden Ketten mit

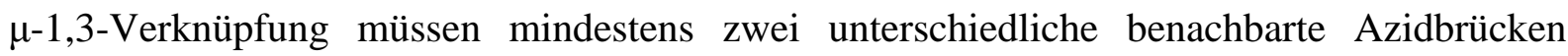
vorhanden sein. Dies kann dadurch realisiert werden, dass bei benachbarten Azidbrücken (analog zu Abb. 4.1.2.1.f, g) unterschiedliche Bindungsparameter vorliegen und sie dementsprechend magnetisch nicht äquivalent sind (Typ A). Zum Typ (B) alternierender Ketten gehören solche Verbindungen, bei denen sich ein und zwei EE-Azidbrücken abwechseln (Abb. 4.1.2.1.h).

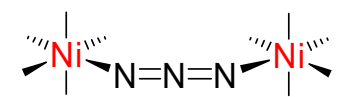

a

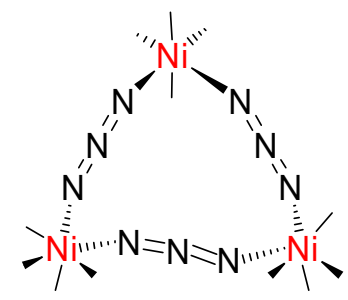

d

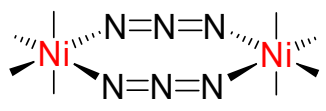

b

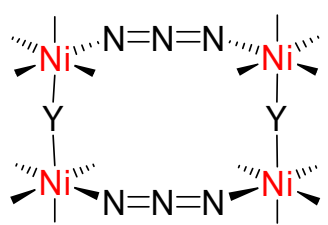

e

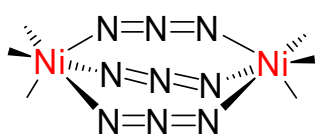

C

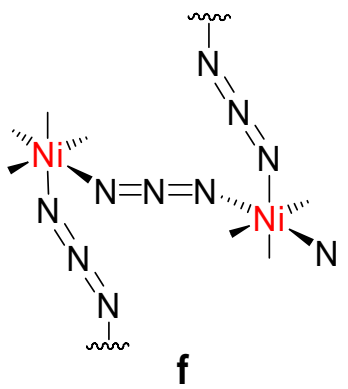

f

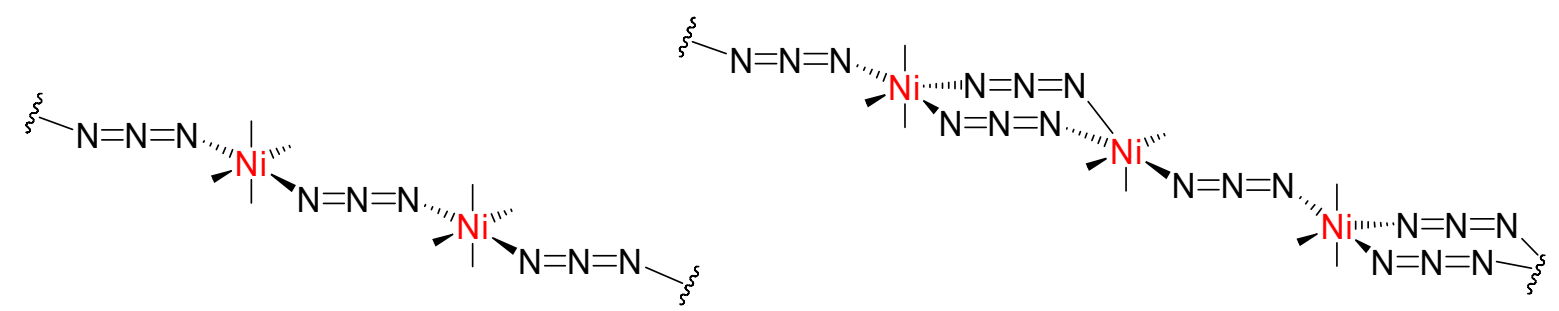

g

h

Abb. 4.1.2.1. Vielfalt der $\mu$-1,3-azidverbrückten Architekturen in Nickel(II)-Komplexen.

Wie anfangs bereits erwähnt, vermitteln $\mu$-1,3-Azidbrücken unabhängig von der Architektur der Verbindung in der Regel antiferromagnetische Kopplungen. In Tab. 4.1.2.1 sind strukturelle und magnetische Parameter verschiedener Nickel(II)-Azid-Komplexe zusammengefasst. Es ist gut erkennbar, dass die Kopplung über zwei Azidbrücken sowohl bei den zweikernigen Komplexen als auch bei den Ketten stärker als über eine einfache Azidbrücke ist. Sie hängt insbesondere von den geometrischen Parametern ab. So hat man für eine Brücke mit einem Winkel von $\alpha=110^{\circ}$ (Zweikernkomplex) bzw. $\alpha=116^{\circ}, \tau=176^{\circ}$ (1D-trans-Kette) die größte Kopplung mit $-46 \mathrm{~cm}^{-1}$ bzw. $-49 \mathrm{~cm}^{-1}$ gefunden. ${ }^{[46,47]}$ Für die zweikernigen Komplexe mit doppelten Brücken findet man einen maximalen Wert von -58 $\mathrm{cm}^{-1}$ und bei den Ketten von $-54 \mathrm{~cm}^{-1} .^{[48,49]}$ Beide Verbindungstypen weisen sehr große 
Ähnlichkeit der strukturellen Parameter auf: z.B. beträgt der Diederwinkel $\delta=3^{\circ}$ für den Zweikernkomplex und $\delta=2.7^{\circ}$ für die Kette. Auch der Winkel $\alpha$ ist sehr ähnlich: $128^{\circ}$ bzw . $139^{\circ}$ für den Zweikernkomplex und $133^{\circ}$ für die Kette. Diese Beispiele zeigen, dass nicht die Topologie der Verbindung, sondern die Geometrie der Nickel(II)-Azid-Brücke entscheidend für die Stärke der magnetischen Austauschwechselwirkung ist.

Tab. 4.1.2.1. Strukturelle und magnetische Parameter für antiferromagnetisch gekoppelte Nickel(II)-Komplexe mit $\mu$-1,3-Azidbrücken unterschiedlicher Topologie.

\begin{tabular}{|c|c|c|c|c|c|}
\hline Molekulare Architektur & $\alpha,{ }^{\circ}$ & $\tau,{ }^{\circ}$ & $\delta,^{\circ}$ & $J, \mathrm{~cm}^{-1}$ & Literatur \\
\hline $\mathrm{Ni}_{2}$-Komplexe, einfach $E E$ & $110-142$ & 180 & --- & -8 bis -46 & {$[46,50,51,52]$} \\
\hline $\mathrm{Ni}_{2}$-Komplexe, doppelt $E E$ & 119-143 & --- & $3-45$ & -2 bis -58 & {$[11 c, 48,53,54]$} \\
\hline $\mathrm{Ni}_{2}$-Komplexe, dreifach $E E$ & 116-119 & --- & --- & -34 & {$[11 c, 54 a]$} \\
\hline $\mathrm{Ni}_{3}-$ Komplex, einfach $E E$ & --- & --- & --- & -14 bis -36 & {$[54 \mathrm{~b}]$} \\
\hline 1D-trans-Ketten, einfach $E E$ & $115-141$ & $140-180$ & --- & -9 bis -49 & {$[47,55,56]$} \\
\hline 1D-cis-Ketten, einfach EE & $120-152$ & $122-147$ & --- & -1 bis -12 & {$[54 c, 57,58]$} \\
\hline 1D-altern.-Ketten, A & $123-144$ & 180 & --- & -18 bis -41 & {$[56 b, 58 b, 59]$} \\
\hline 1D-altern.-Ketten, B, $1 E E$ & 119-123 & -175 bis -180 & --- & -43 bis -44 & {$[49,60]$} \\
\hline $2 E E$ & $124-133$ & --- & 3 & -20 bis -54 & \\
\hline 2D-System, doppelt EE & $121 / 136$ & 44 & --- & -40 & {$[61]$} \\
\hline
\end{tabular}

Für ein verbrückendes Azid sind der Winkel $\alpha$ und der Torsionswinkel $\tau$ (Abb. 4.1.1.2) die zwei am häufigsten betrachteten geometrischen Parameter für magneto-strukturelle Korrelationen. ${ }^{[12,41]}$ Für einen Ni-NNN-Ni-Torsionswinkel von $\tau=180^{\circ}$ wird für die Stärke der antiferromagnetischen Kopplung ein Maximum bei $\mathrm{Ni}-\mathrm{N}-\mathrm{N}-W i n k e l n$ gegen $108^{\circ}$ vorausgesagt und sinkt bei größeren Winkeln. Die maximale Kopplung wird bei allen Ni-NN-Winkeln für eine Torsion von $180^{\circ}$ (oder $0^{\circ}$ ) erwartet. Der Einfluss des Winkels $\alpha$ auf die Stärke der Kopplung wird oft als größer betrachtet. Unter Abwesenheit von jeglichen beschränkenden Ligandgerüsten tendieren die Winkel des zentralen Nickel(II)-AzidFragments in zweikernigen Komplexen oder 1D-erweiterten Systemen mit einer $\mu-1,3-$ Azidbrücke zu Werten von $\alpha=115-145^{\circ}$ und $\tau=140-180^{\circ}$. Die resultierenden $J$-Werte werden im Bereich von $-8 \mathrm{~cm}^{-1}$ bis $-50 \mathrm{~cm}^{-1}$ gefunden. ${ }^{[12,41]}$

Für die Komplexe mit zwei EE-Azidbrücken wird der Winkel $\delta$ als der entscheidende Parameter für die Korrelation zwischen Struktur und Kopplung angesehen. So zeigen theoretische Arbeiten, dass eine maximale Kopplung bei $\delta=180^{\circ}$ (oder $0^{\circ}$ ) zu erwarten ist und dass sie bei größeren Winkeln absinkt. ${ }^{[37,48,62]}$ Diese Voraussage stimmt hervorragend mit 
experimentellen Daten überein. Die maximale Kopplung von $-58 \mathrm{~cm}^{-1}$ wurde bei $\delta=3^{\circ}$, der minimale Wert von $-2 \mathrm{~cm}^{-1}$ bei $\delta=45^{\circ}$ beobachtet.

Nur wenige Beispiele für ferromagnetisch gekoppelte $\mu$-1,3-azidverbrückte Nickel(II)Verbindungen sind bekannt. ${ }^{[63,64,65]}$ Ähnlich wie bei der Vermittlung der antiferromagnetischen Kopplung ist die $\mathrm{Ni}-\mathrm{N}_{3}-\mathrm{Ni}-$ Geometrie dabei entscheidend. Ein Beispiel ist ein zweikerniger Komplex auf Basis eines Kryptandliganden, welcher dem zentralen Ni-NNN-Ni Fragment eine ungewöhnliche, quasi-lineare Geometrie (Ni-N-N Winkel $158^{\circ} / 166^{\circ}$ ) aufzwingt. ${ }^{[63]}$ Weitere Beispiele sind ein $\mathrm{Ni}_{4}$-Komplex, ${ }^{[64]} 1 \mathrm{D}-$ Ketten $^{[65 a-c]}$ und 2D-Polymerkomplexe, ${ }^{[65 \mathrm{~d}]}$ welche passende Ni-NNN-Ni Torsionswinkel $\left(76^{\circ}, 76^{\circ}, 107^{\circ}\right.$ und $110^{\circ}$ ) aufweisen (Tab. 4.1.2.2).

Tab. 4.1.2.2. Strukturelle und magnetische Parameter für ferromagnetisch gekoppelte Nickel(II)-Komplexe mit $\mu$-1,3-Azidbrücken unterschiedlicher Topologie.

\begin{tabular}{lllll}
\hline Molekulare Architektur & $\alpha,^{\circ}$ & $\tau,^{\circ}$ & $J, \mathrm{~cm}^{-1}$ & Literatur \\
\hline $\mathrm{Ni}_{2}$-Komplex & $158 / 166$ & 177 & +6 & [63] \\
$\mathrm{Ni}_{4}$-Komplex & 124,127 & 76 & +4 & [64] \\
1D-Ketten & $124-146$ & $76 / 107$ & +3 bis +7 & [65a-c] \\
2D-System, doppelt EE & $121 / 136$ & 110 & - & [65d] \\
\hline
\end{tabular}

Im Falle des $\mathrm{Ni}_{4}$-Komplexes (Abb. 4.1.2.1.e) sowie der 1D- und 2D-Polymeren werden die positiven $J$-Werte durch eine quasi-Orthogonalität zwischen den magnetischen Orbitalen des Nickels und den p-Orbitalen des Azids erklärt. Dies führt zu einem sehr kleinen Überlappungsintegral und dementsprechend zu einer Verringerung des antiferromagnetischen Beitrags zur Kopplungskonstante (Gleichungen 3.2.10, 3.2.11), wodurch die ferromagnetische Wechselwirkung überwiegt. ${ }^{[65]}$

Wie aus Tab. 4.1.2.1 und 4.1.2.2 ersichtlich ist, liegen die Werte des Winkels $\alpha$ für antiferromagnetisch gekoppelte und für ferromagnetisch gekoppelte Systeme zwischen $110^{\circ}$ und $150^{\circ}$ (Ausnahme $\mathrm{Ni}_{2}$-Komplex, Tab. 4.1.2.2), während die Torsionswinkel im Falle der ferromagnetisch gekoppelten Verbindungen deutlich im Bereich von $90^{\circ}\left( \pm 20^{\circ}\right)$ liegen. Dies zeigt, dass der Torsionswinkel $\tau$ für den ferromagnetischen Austausch über die $\mu-1,3-$ Azidverbrückung die wichtigste Rolle spielt, obwohl dieser offensichtlich am schwierigsten zu kontrollieren ist. 


\subsection{3. $\mu$-1,1-azidverbrückte Komplexe}

Eine ganze Reihe von $\mu$-1,1-azidverbrückten Komplexen unterschiedlicher Topologie ist bekannt (Abb. 4.1.3.1). ${ }^{[12,41]}$ Die einfache $\mu-1,1$-Azidbindung kommt in „reinem“ Zustand nicht vor, es koexistiert immer die zusätzliche zweite Brücke (Abb. 4.1.3.1.a-d). Es sind zweikernige Komplexe und 1D-Ketten bekannt, sowohl mit doppelter EO-Bindung (Abb. 4.1.3.1.e) als auch mit dreifacher EO-Bindung (Abb. 4.1.3.1.f).

Die zweite Brücke im Fall einer $\mu$-1,1-Azidbindung kann von den verschiedensten Liganden gebildet werden, ein paar Beispiele sind hier gezeigt:

a) Phosphat, Wolframat (Abb. 4.1.3.1.a, zweikerniger Komplex); ${ }^{[66]}$

b) Phenolat, Oxo-diaminopropan oder Dioxo-dipyridylmethan (Abb. 4.1.3.1.b), wurden in entsprechenden zweikernigen, ${ }^{[67]}$ vierkernigen quasi-planaren $(q-p)^{[68]}$ und vierkernigen dikubanähnlichen (diq) Komplexen ${ }^{[69,70]}$ beobachtet;

c) Nicotinat (Abb. 4.1.3.1.c, 3D-Polymer); ${ }^{[71]}$

d) alternierend Acetat/Oxo-Gruppe von Carbonat oder Carbonat/Oxo-Gruppe vom Liganden (Abb. 4.1.3.1.d, $\mathrm{Ni}_{12}$-Cluster). ${ }^{[72]}$

Alle Verbindungen mit einer EO-Koordination sind unterschiedlich stark ferromagnetisch gekoppelt, von $+1.4 \mathrm{~cm}^{-1}$ bis $+26 \mathrm{~cm}^{-1}$. Dies kann sowohl durch den Einfluss des Winkels $\theta$ wie auch durch den Einfluss der zusätzlichen Brücke bedingt sein. Möglicherweise müssen sogar beide Faktoren gleichzeitig berücksichtigt werden.

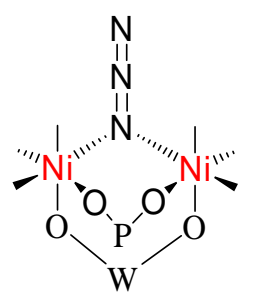

a

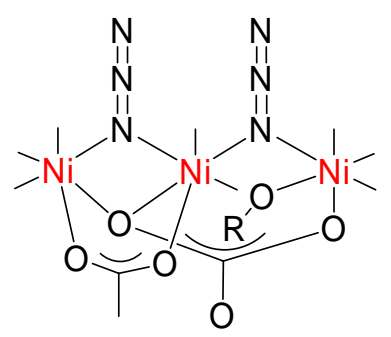

d

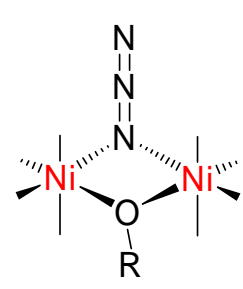

b

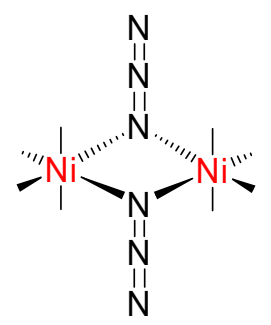

e

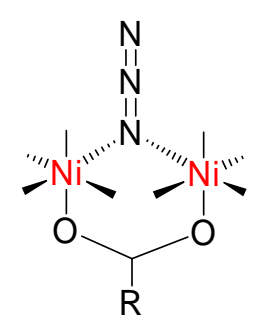

C

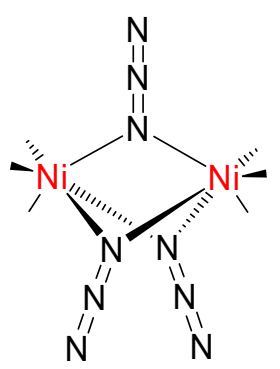

$\mathbf{f}$

Abb. 4.1.3.1. Vielfalt der Nickel(II)- $\mu-1,1-$ Azid-Architekturen. 
Zwei Aspekte sind noch interessant für ein Molekül mit zwei unterschiedlichen Brücken zwischen den Metallzentren (eine $\mu$-1,1-Azidbrücke und eine „Nichtazidbrücke“) . Erstens wurde eine ferromagnetische Kopplung $\left(+19 \mathrm{~cm}^{-1}\right)$ für die Verbindung (Abb.4.1.3.1.a) bei sehr großem Winkel $\left(\theta=129^{\circ}\right)$ beobachtet, so dass die $\mu$-1,1-Azidgruppierung als universaler Vermittler der ferromagnetischen Kopplung angesehen werden kann. ${ }^{[66]}$ Zweitens beobachtet man bei ähnlichen Winkeln (90 und ca. $\left.96^{\circ}\right)$ in einem $\mathrm{Ni}_{12}$-Cluster (Abb. 4.1.3.1.d) einen gravierenden, fast zehnfachen Unterschied in der Stärke der Kopplungskonstanten $\left(1.44 \mathrm{~cm}^{-1}\right.$ und $\left.12.7 \mathrm{~cm}^{-1}\right){ }^{[72]}$ Dies kann allein durch den Winkelunterschied nicht erklärt werden.

Die resultierenden magnetischen Eigenschaften erklären sich erst bei Berücksichtigung der zweiten Brücke, oder genauer formuliert, durch die Berücksichtigung des Zusammenspiels beider Brücken. Hier kommt der sogenannte kontra-Komplementaritätseffekt zum Tragen. Bei Anwesenheit zweier unterschiedlicher Brücken können die magnetischen Eigenschaften dieser Komplexe prinzipiell anders als bei der jeweiligen symmetrischen Verbrückung sein. Zum Beispiel kann, wenn ein $\mu$-1,1-Azid (das ferromagnetische Kopplung vermittelt) und ein Acetation (das antiferromagnetische Kopplung vermittelt) zwei Metalle verbrücken, eine Verstärkerung der ferromagnetischen Wechselwirkung beobachtet werden. Dieser Sachverhalt lässt sich mit Hilfe der MO-Theorie genauer nachvollziehen.

Nach dem Vorschlag von Hoffmann ${ }^{[37]}$ ist das Quadrat der Energieaufspaltung zwischen symmetrischen $\varphi_{\mathrm{S}}$ und antisymmetrischen $\varphi_{\mathrm{A}}$ Kombinationen von zwei magnetischen Orbitalen proportional zur antiferromagnetischen Komponente $J_{\mathrm{AF}}$ der Kopplungskonstante $J$ $\left(J=J_{\mathrm{AF}}+J_{\mathrm{F}}\right)$. Haben beide Kombinationen die gleiche Energie, ist der antiferromagnetische Beitrag gleich Null (bzw. sehr klein) und das ferromagnetische Verhalten wird beobachtet.

Für das Verständnis der Vermittlung der magnetischen Kopplung ist es sinnvoll, die HOMO’s der Brückenliganden einzubeziehen und deren Wechselwirkung mit $\varphi_{\mathrm{A}}$ und $\varphi_{\mathrm{B}} \mathrm{zu}$ betrachten. Der antibindende Charakter der einfach besetzten MO's wird durch die Überlappung mit den HOMO's der Liganden verstärkt. ${ }^{[3]}$ Haben die HOMO's beider Brücken gleiche Symmetrie, wird eine der beiden Kombinationen der magnetischen Orbitale ( $\varphi_{\mathrm{S}}$ oder $\varphi_{\mathrm{A}}$ ) verstärkt. Als Folge dessen haben $\varphi_{\mathrm{S}}$ und $\varphi_{\mathrm{A}}$ deutlich unterschiedliche Energien, der antiferromagnetischen Beitrag wird groß und dominiert. In diesem Fall (gleiche Symmetrie der Brückenliganden) spricht man von Komplementarität der Liganden.

Betrachten wir das für diese Arbeit relevante Beispiel, dass zwei Metalle über ein $\mu$-1,1Azid und einen Pyrazolatliganden verbrückt sind (Abb. 4.1.3.2.a). Die HOMO's sowohl des Azids als auch des Pyrazolats sind antisymmetrisch bezüglich der Spiegelebene, die die 
beiden Metallionen ineinander überführt. Dies führt zur Überlappung mit der antisymmetrischen $\varphi_{\mathrm{A}}$ Kombination (Abb. 4.1.3.2.a, rechts). Mit $\varphi_{\mathrm{S}}$ gibt es keine Überlappung (Abb. 4.1.3.2.a, links), somit haben $\varphi_{\mathrm{S}}$ und $\varphi_{\mathrm{A}}$ deutlich unterschiedliche Energie und man kann eine dominierende antiferromagnetische Kopplung erwarten.

Wenn das HOMO der einen Brücke symmetrisch und das der anderen antisymmetrisch ist, werden beide Kombinationen stabilisiert, so dass $\varphi_{S}$ und $\varphi_{A}$ etwa die gleiche Energie haben, der antiferromagnetische Beitrag gering ist und die Metallzentren ferromagnetisch gekoppelt sein können. In diesem Fall spricht man von kontra-Komplementarität der Liganden. ${ }^{[73,74]}$ Kombiniert man Azid (antisymmetrisches HOMO) und Acetat (symmetrisches HOMO), wird sowohl $\varphi_{\mathrm{S}}$ durch das Acetat (Abb. 4.1.3.2.b, links) als auch $\varphi_{\mathrm{A}}$ durch das Azid stabilisiert (Abb. 4.1.3.2.b, links). In diesem Fall werden recht große positive Kopplungskonstanten beobachtet, ${ }^{[75]}$ obgleich die Acetatbrücke an sich eine antiferromagnetische Kopplung vermitteln würde. ${ }^{[76]}$
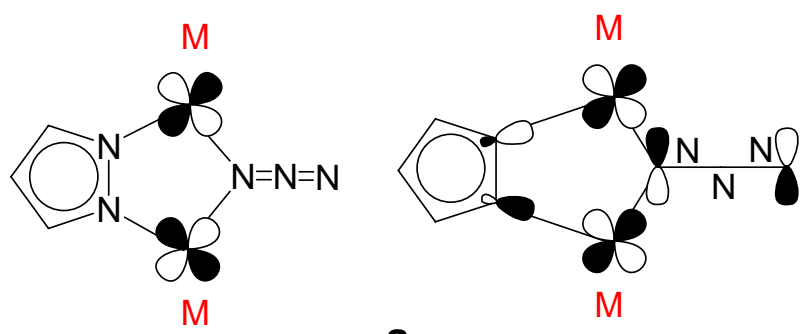

a
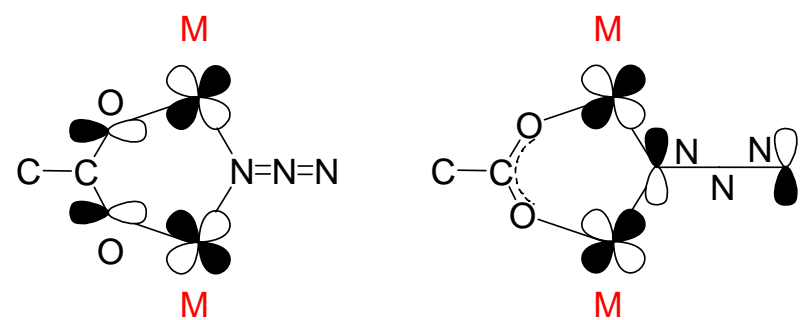

b

Abb. 4.1.3.2. Symmetrische (links) und antisymmetrische (rechts) MO Kombination für Azido-PyrazolatoBrücke (a) und Azido-Acetato-Brücke (b).

Der kontra-Komplementaritätseffekt führt zu einer verstärkten ferromagnetischen Kopplung bei Komplexen, in welchen Metalle über zwei Brücken gekoppelt sind, wovon eine zur antiferromagnetischen Kopplung führen sollte. ${ }^{[72,75]}$ Bei der Diskussion von $J$-Werten der Komplexe mit einfacher Azid-EO-Koordination sollte man jeweils prüfen, ob dieser Effekt relevant ist. Es kann daher keine klare Korrelation zwischen der Kopplungsstärke und dem 
$\mathrm{Ni}-\mathrm{N}-\mathrm{Ni}$-Winkel für einfache EO-Koordination formuliert werden, was auch zum Teil an der kleinen Zahl bekannter Verbindungen dieses Typs liegt.

Für die doppelten und dreifachen EO-Koordinationsmodi wurden DFT-Rechnungen durchgeführt, um die Natur der Austauschwechselwirkungen in solchen Systemen besser zu verstehen. ${ }^{[77]}$ Diese Rechnungen zeigen eine klare Korrelation zwischen dem Winkel $\theta$ und der Kopplungskonstanten $J$. Es wird eine ferromagnetische Wechselwirkung unabhängig vom Winkel vorausgesagt. Das Maximum wird bei $\theta \approx 104^{\circ}$ für die doppelte und bei $\theta \approx 95^{\circ}$ für die dreifache EO-Azidbindung erwartet. Auch scheint der Ni-N-Abstand im Gegensatz zu $\mu$ 1,3-azidverbrückten Komplexen einen signifikanten Einfluss zu haben, während der Winkel zwischen dem Azid und der Ni-N-Ni-Ebene für die Kopplung nicht von Bedeutung ist. Die experimentellen Daten (Tab. 4.1.3.1) zeigen, dass die Beziehung zwischen $\theta$ und den $J$ Werten nicht sehr ausgeprägt zu sein scheint, weil z.B. für bekannte $\mathrm{Ni}_{2}-$ Komplexe mit doppelter EE-Koordination die Winkel in einem sehr schmalen Bereich von $99^{\circ}$ bis $106^{\circ}$ liegen, während die Kopplungskonstanten von $+13 \mathrm{~cm}^{-1}$ bis $+39 \mathrm{~cm}^{-1}$ recht stark streuen. Dabei wurde die stärkste Kopplung bei einem Winkel von $\theta=99.5^{\circ} / 102.5^{\circ}$ gefunden. $^{[67]}$

Tab. 4.1.3.1. Strukturelle und magnetische Parameter für $\mu-1,1$-azidverbrückte Nickel(II)-Komplexe unterschiedlicher Topologie.

\begin{tabular}{llll}
\hline Molekulare Architektur & $\theta,^{\circ}$ & $\mathrm{J}, \mathrm{cm}^{-1}$ & Literatur \\
\hline $\mathrm{Ni}_{2}$-Komplex (Abb. 4.1.3.1.a) & 129 & +19 & {$[66]$} \\
$\mathrm{Ni}_{2}$-Komplex (Abb. 4.1.3.1.b) & 96 & +26 & {$[67]$} \\
$\mathrm{Ni}_{4}$-Komplex (Abb. 4.1.3.1.b), q-p & 98 & +11 & {$[68]$} \\
$\mathrm{Ni}_{4}$-Komplexe(Abb. 4.1.3.1.b), diq & $102 / 103$ & $+9 /+19$ & {$[69,70]$} \\
$3 \mathrm{D}-$-System (Abb. 4.1.3.1.c) & 125 & +11 & {$[71]$} \\
$\mathrm{Ni}_{12}$-Cluster (Abb. 4.1.3.1.d) & $90 / 96$ & $+1.4 /+12.7$ & {$[72]$} \\
$\mathrm{Ni}_{2}$-Komplexe, doppelt EO & $99-105$ & $+13-+39$ & {$[67,78]$} \\
$1 \mathrm{D}-$-Ketten, doppelt EO & $95-108$ & $+12-+29$ & {$[56 \mathrm{~b}, 79]$} \\
$\mathrm{Ni}_{2}$-Komplexe, dreifach EO & $85.9 / 86.2$ & $+15 /+9$ & {$[11 \mathrm{c}]$} \\
\hline
\end{tabular}

Auf der anderen Seite sind die gefundenen Kopplungskonstanten für $\mathrm{Ni}_{2}$-Komplexe mit dreifacher EE-Koordination relativ klein, was durch kleinere Winkel (ca. 86º) gegenüber dem für das Maximum der Kopplung notwendigen Winkel $\theta$ (95) erklärt werden kann.

Für eine exaktere Beschreibung magnetostruktureller Korrelationen von Verbindungen mit einer $\mu$-1,1-Azidbrücke müssten weitere Komplexe mit einem breiteren Bereich von $\theta$ synthetisiert und charakterisiert werden. 


\subsection{4. $\mu_{3}$ - und $\mu_{4}$-azidverbrückte Komplexe}

Drei- und vierfachverbrückende Azide sind als $\mu_{3}-1,1,1-, \quad \mu_{3}-1,1,3-, \mu_{4}-1,1,1,1-$ und $\mu_{4^{-}}$ 1,1,3,3-Koordinationsvarianten bekannt. Verbindungen dieses Typs sind allerdings selten. So wurde nur über wenige Verbindungen mit $\mu_{3}$-1,1,1-Aziden unterschiedlicher Architektur berichtet. Ein Beispiel ist ein Kuban-Cluster ${ }^{[42 a, b]}$ (Abb. 4.1.4.1.a), ein anderes ein dikubanähnlicher Komplex ${ }^{[42 f]}$ (Abb. 4.1.4.1.b). Man findet in beiden Verbindungen positive $J$-Werte von $+12 \mathrm{~cm}^{-1}$ für den Kubancluster ${ }^{[42 \mathrm{a}, \mathrm{b}]}$ und von ca. $+7.5 \mathrm{~cm}^{-1}$ für die $\mu_{3}$ - und auch die $\mu$-1,1-Azidbrücke im dikubanähnlichen $\operatorname{Komplex}^{[42 f]}$.

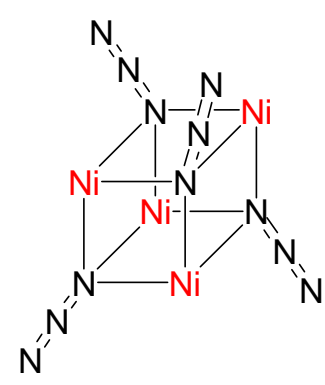

a

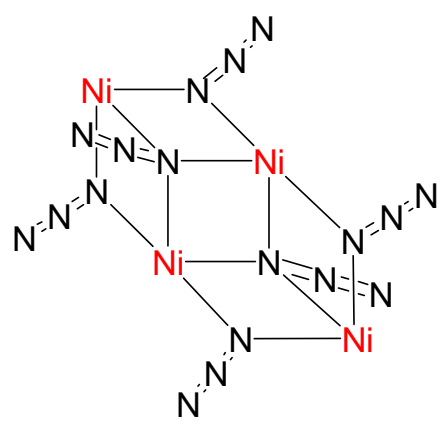

b

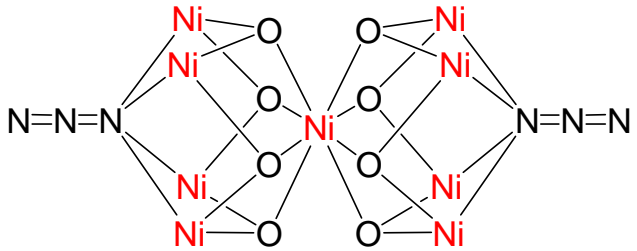

C

Abb. 4.1.4.1. Schematische Darstellung der zentralen Fragmente der Komplexe mit $\mu_{3}-1,1,1$ - und $\mu_{4}-1,1,1,1$ Azidverbrückung.

Eine ebenfalls sehr seltene $\mu_{4}$-EO-Koordination wurde in einem $\mathrm{Ni}_{9}$-Komplex (Abb. 4.1.4.1.c) beobachtet. ${ }^{[80]}$ Die Verbindung zeigt ferromagnetische Kopplungen. Interessant ist, dass auch mit Co(II) und Fe(II) ähnliche Komplexe synthetisiert und charakterisiert wurden. Während der Cog-Komplex ebenfalls ferromagnetische Kopplungen zeigt, ${ }^{[43]}$ ist der $\mathrm{Fe}_{9}$ Komplex ein SMM (single molecule magnet). ${ }^{[81]}$

Die Verbindungen mit $\mu_{3}-1,1,3-$ und $\mu_{4}-1,1,3,3$-Verbrückung, die sowohl $E O$ - als auch $E E$ Koordinationsfragmente enthalten, sind relativ lange bekannt. ${ }^{[22,83,84]}$ Man findet diese Bindungsvarianten für Polymere, oft mit Kupfer(II)-Ionen, in welchen mindestens eine der $\mathrm{Cu}-\mathrm{N}$ Bindungen sehr lang ist (semi-koordinativ). ${ }^{[83,84,]}$ Ein 2D-Nickel(II)-Komplex mit einem $\mu_{3}-1,1,3$-Azid ist auch bekannt. ${ }^{[82]}$ Aber es existieren, was für hochdimensionale Systeme aus theoretischer Sicht schwierig ist, keine magneto-strukturellen Korrelationen für diese Azid-Koordination. Molekulare Verbindungen mit „echten“ $\mu_{3}-1,1,3-$ und $\mu_{4}-1,1,3,3-$ Aziden (die Ni-N-Abstände sind alle etwa gleich lang und liegen für Nickel(II)-AzidKomplexe in einem typischen Bereich) wurden aber erst kürzlich von F. Meyer und 
Mitarbeitern veröffentlicht (siehe auch Kapitel 4.3.2.2 und 4.3.2.3). ${ }^{[26]}$ Es handelt sich dabei um $\mathrm{Ni}_{4}$-Komplexe, die ausgehend von zweikernigen pyrazolatbasierenden Baueinheiten synthetisiert wurden. Die $\mathrm{Ni}_{4}$-Komplexe mit $\mu_{3}$-1,1,3-Azidverbrückung können bei Zugabe eines Äquivalents eines Carboxylats in Komplexe mit $\mu_{4}$-1,1,3,3-Azidverbrückung überführt werden. Je nach Synthesebedingungen, z.B. Verwendung verschiedener Carboxylate, entstehen $\mathrm{Ni}_{4}$-Komplexe mit anderer räumlicher Anordnung des zentralen $\mu_{4}$-Azides. Die

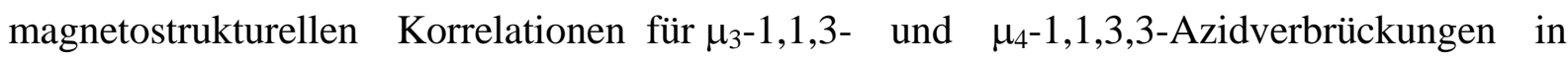
diesen und auch weiteren Komplexen diesen Typs sollen im Rahmen dieser Arbeit untersucht werden.

Über einen $\mathrm{Cu}_{4}$-Komplex mit $\mu_{4}$-1,1,3,3-Azidverbrückung ohne zusätzlichen Brückenliganden allerdings aber mit relativ langen $\mathrm{Cu}-\mathrm{N}$-Abständen von ca. $2.4 \AA$ wurde kürzlich berichtet. ${ }^{[85]}$

\subsubsection{Kombinationen von $E E$ - und $E O$ - Azidobrücken. Topologisch ferrimagnetische Ketten}

Der Azidligand kann in mehr als nur einem Koordinationsmodus auftreten. Das kommt oft bei Oligomeren und Polymeren unterschiedlicher Dimensionalität vor. ${ }^{[12,41]}$ Als besonders nützliches Werkzeug hat sich Azid daher für den Aufbau von 1D-Systemen mit alternierender ferromagnetischer (durch $\mu$-1,1-Azid) und antiferromagnetischer (durch $\mu$-1,3-Azid) Wechselwirkung etabliert. ${ }^{[15]}$ Auch für solche Verbindungen können die magnetostrukturellen Korrelationen der reinen EO- und EE-Azide prinzipiell übernommen werden. Für die oft beobachtete Topologie mit einer 2EO-2EE-Alternanz (Abb. 4.1.5.1.a) ${ }^{[86,87]}$ sinken die $J$-Werte der Ni- $\left(\mu-1,3-\mathrm{N}_{3}\right)_{2}-\mathrm{Ni}-$ Fragmente, wenn der Winkel $\delta$ größer wird. Die $\mathrm{Ni}-\mathrm{N}-\mathrm{Ni}$ Winkel liegen bei etwa $100^{\circ}$. Zwei weitere Topologien der Ketten mit abwechselnden ferro- und antiferromagnetischen Wechselwirkungen sind bekannt: mit 2EO-1EE- sowie 3EO-1EEAufeinanderfolge (Abb. 4.1.5.1.b, c). ${ }^{[88,89]}$ Interessant dabei ist, dass man für das Ni( $\mu-1,1-$ $\left.\mathrm{N}_{3}\right)_{3} \mathrm{Ni}$-Fragment sehr kleine Winkel mit $\theta$ ca. $84^{\circ}$ findet und die Kopplungskonstante einen negativen Wert von $J=-3.5 \mathrm{~cm}^{-1}$ aufweist. 


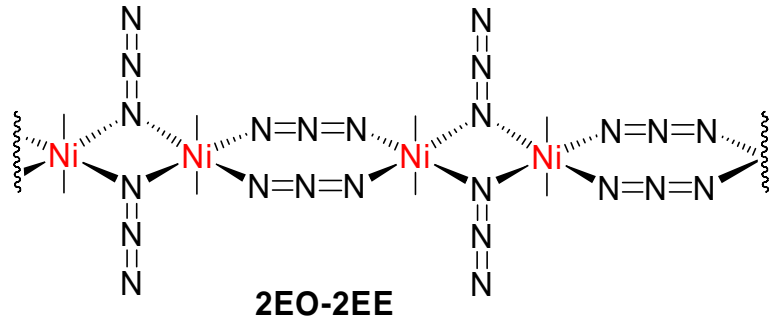

a

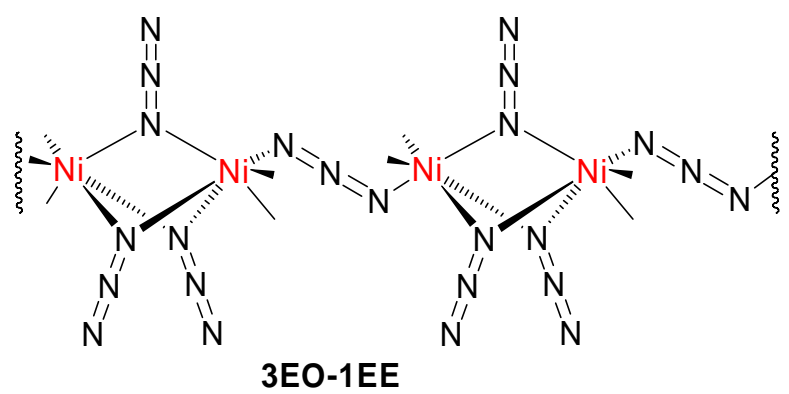

C

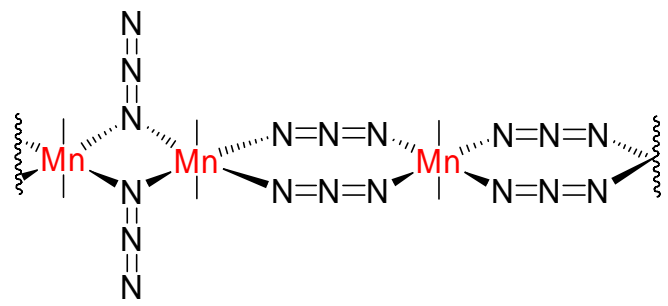

2EO-2EE-2EE

e

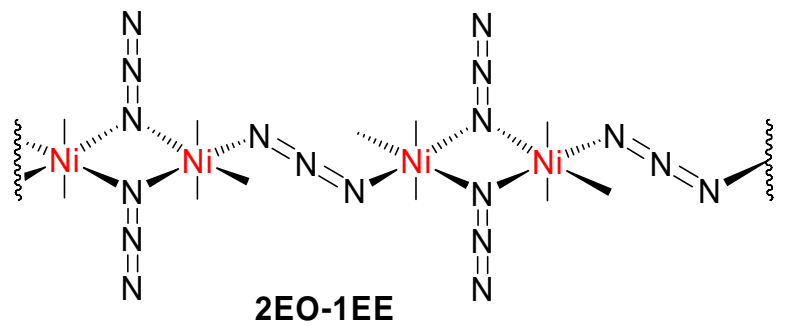

b

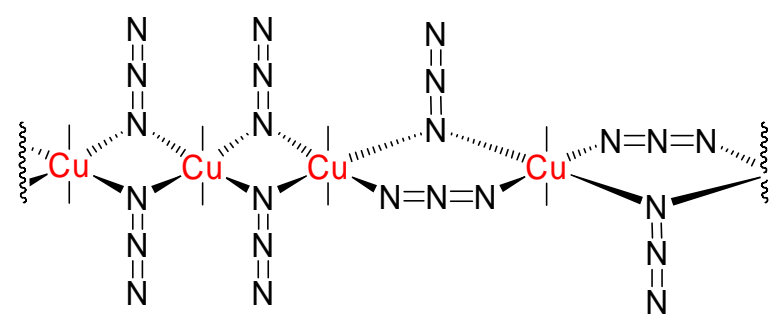

2EO-2EO-EO/EE-EO/EE

d

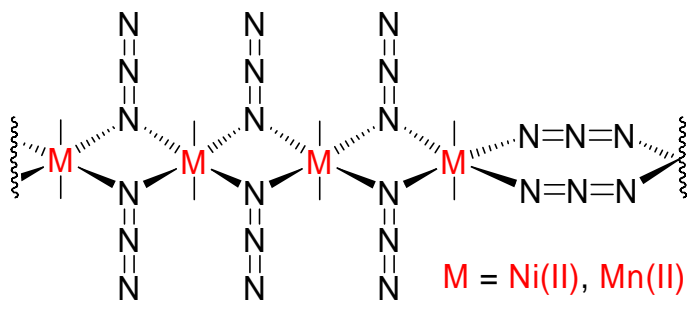

2EO-2EO-2EO-2EE

Abb.4.1.5.1. EE-EO-alternierende Ketten unterschiedlicher Topologie.

Während die ferromagnetisch-antiferromagnetische Sequenz durch die abwechselnde Aufeinanderfolge von EO-EE-Azidobrücken zum generellen antiferromagnetischen Verhalten führt, zeigen Verbindungen mit komplizierteren Alternanzmustern (Abb. 4.1.5.1.d, e) sehr ungewöhnliche magnetische Eigenschaften für derartige homometallische Verbindungen. ${ }^{[9,90,91]}$ Das magnetische Moment dieser Verbindungen nimmt beim Abkühlen zuerst ab, erreicht ein Minimum bei $10 \mathrm{~K}$ bzw. $40 \mathrm{~K}$ und steigt dann wieder an. Das ist ein typischer Kurvenverlauf für ferrimagnetischen Ketten. Nach O. Kahn ${ }^{[5]}$ wurden klassische ferrimagnetische Ketten als „systems in which two kinds of magnetic centers $A$ and $B$ regularly alternate and the interaction parameter is negative“ bezeichnet. Die genannten Verbindungen fallen zwar nicht unter diese Definition, zeigen aber trotzdem ferrimagnetisches Verhalten. Es handelt sich also um topologisch ferrimagnetische Ketten mit einer Alternanz von formal betrachtet unterschiedlichen Zentren $S_{A}$ und $S_{B}$, wobei $S_{B}$ aus zwei oder mehreren ferromagnetisch gekoppelten $S_{A}$-Zentren entsteht (Abb. 4.1.5.2). Allgemein 
wurde von A. Escuer et al. aufgrund der Analyse vorhandener topologisch ferromagnetischer Ketten folgende Regel formuliert: „Bei einer Sequenz aus einer geraden Anzahl von antiferromagnetischen Wechselwirkungen gefolgt von einer oder mehreren ferromagnetischen Wechselwirkungen tritt ein insgesamt ferrimagnetisches Verhalten auf.“[91]

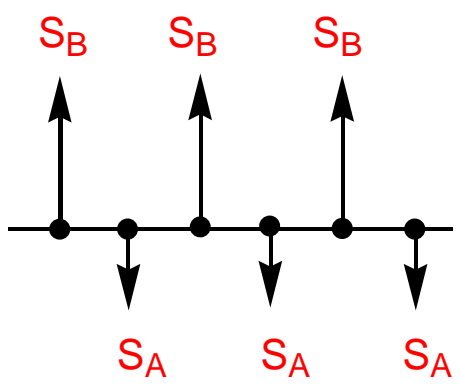

a

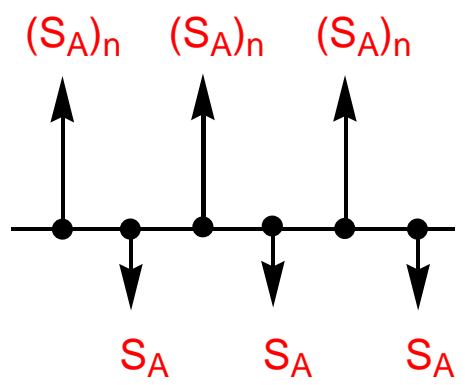

b

Abb. 4.1.5.2. Antiferromagnetische Wechselwirkung in klassischen (a) und topologischen (b) ferrimagnetischen Ketten.

Eine weitere komplexe Sequenz (Abb. 4.1.5.1.f) wurde in einem Nickel(II)-Komplex ${ }^{[92]}$ mit insgesamt antiferromagnetischem Verhalten und in einem Mn(II)-Komplex ${ }^{[91]}$, der ferrimagnetisches Verhalten zeigt, beobachtet.

Das zuvor genannte Beispiel ferrimagnetischen Verhaltens homometallischer Ketten zeigt, dass komplexe Topologien mit komplizierteren Sequenzen ein nur wenig erforschtes Gebiet des molekularen Magnetismus darstellen und dass es auch hier eine große Herausforderung sein wird, die Synthese solcher Verbindungen gezielt vornehmen zu können.

\subsubsection{Sprunghafte Änderung im magnetischen Verhalten, verursacht durch eine Phasenumwandlung in Azid-Komplexen}

Nickel(II)-Azid-Verbindungen weisen ein durchaus breites Spektrum an magnetischen Eigenschaften auf. So wurde in wenigen $\mu$-1,3-azidverbrückten Komplexen (Tab. 4.1.6.1) beispielweise eine sprunghafte Änderung in der Suszeptibilität (magnetischer Übergang) beobachtet. [55a,93,94,95,96] 
Zweikernige Nickel(II)-Azid-Komplexe als Baueinheiten für höhernukleare Systeme

Tab. 4.1.6.1. Charakteristika magnetischer Übergänge in Azid-Verbindungen unterschiedlicher Topologie.

\begin{tabular}{|c|c|c|c|c|c|}
\hline $\begin{array}{l}\text { Molekulare } \\
\text { Architektur }\end{array}$ & $T, \mathrm{~K}$ & $\begin{array}{l}\text { Kristallsystem } \\
\text { vor Übergang }\end{array}$ & $\begin{array}{l}\text { Kristallsystem } \\
\text { nach Übergang }\end{array}$ & $\begin{array}{l}J \text { (vor/nach dem } \\
\text { Übergang), } \mathrm{cm}^{-1}\end{array}$ & Literatur \\
\hline $\mathrm{Ni}_{2}-$ Komplex, $2 E E$ & 235 & orthorombisch & monoklin & $-67 /-32$ & {$[93]$} \\
\hline Ni-1D-Kette, $1 E E$ & $230-240$ & orthorombisch & monoklin & $-20 /-10$ & [55a] \\
\hline Ni-1D-Kette, $1 E E$ & $210-260$ & orthorombisch & monoklin & $-35 /-28$ & [55a] \\
\hline Ni-1D-Kette, $1 E E$ & $130-150$ & $\begin{array}{l}\text { orthorombisch } \\
\text { (Pnab) }\end{array}$ & $\begin{array}{l}\text { orthorombisch } \\
(C 221)\end{array}$ & $-21 /-20$ & [94] \\
\hline Mn-3D-Polimer, $1 E E$ & 303 & monoklin & kubisch & -2.5 & {$[95]$} \\
\hline $\mathrm{Ni}_{2}-$ Komplex, $2 E O$ & $75-175$ & triklin & triklin & +23 & {$[96]$} \\
\hline
\end{tabular}

1) in Richtung von höheren zu tieferen Temperaturen.

Solche magnetischen Übergänge weisen einen Sprung in der Suszeptibilität und einen unterschiedlich starken magnetischen Austausch vor und nach dem Übergang auf. Die röntgenographischen Untersuchungen dieser Verbindungen zeigen, dass im Temperaturbereich des magnetischen Übergangs auch ein Phasenübergang stattfindet. ${ }^{[55 a, 93,94,95]}$ Da die bisher bekannten Phasenübergänge mit einer Änderung des Kristallsystems verbunden waren, war es nicht möglich, die molekularen Strukturen beider Phasen am Einkristall zu bestimmen. Die entsprechenden kristallographischen Informationen versuchte man mittels Pulverdiffraktometrie zu erhalten. Diese magnetischen Übergänge werden über die Änderungen der M-Azid-M Bindungsparameter erklärt, was nach bekannten magnetostrukturellen Korrelationen auch die Änderung des magnetischen Verhaltens bedingen sollte. So wurde z.B. für zwei Ketten ${ }^{[55 a]}$ die Temperaturabhängigkeit der Zellparameter bestimmt. Während die $a$ - und $b$-Achse sich signifikant beim Phasenübergang ändern, bleibt die $c$-Achse fast invariant. Da die Kette entlang der $c$-Achse ausgerichtet ist, sollten die Ni-N-N-Winkel bei der Phasenumwandlung nahezu konstant bleiben. Die Zellen werden in Richtung senkrecht zu den Ketten deformiert, was vom Betrag her allein die Vergrößerung der Torsionswinkel, die vor dem magnetischen Übergang $0^{\circ}$ sind, verlangt. Diese Vergrößerung von $\tau$ sollte nach bekannten magnetostrukturellen Korrelationen zu einer schwächeren antiferromagnetischen Kopplung nach dem magnetischen Übergang führen. Dies konnte im vorliegenden Fall auch beobachtet werden: $J=-20 \mathrm{~cm}^{-1}$ bzw. $-35 \mathrm{~cm}^{-1}$ (vor dem Übergang) aufs $J=-10 \mathrm{~cm}^{-1}$ bzw. $-28 \mathrm{~cm}^{-1}$ (nach dem Übergang). Interessanterweise verlaufen einige dieser magnetischen Übergänge mit und andere ohne Hysterese.

Für einen zweikernigen Nickel(II)-Komplex mit EO-Azidobrücke wurde die beobachtete Hysterese der Suszeptibilität auf die Steigerung der Asymmetrie im zentralen nahezu 
rhombischen Ni-( $\left.\mathrm{N}_{3}\right)_{2}-\mathrm{Ni}$ Fragment zurückgeführt. ${ }^{[96]}$ Die Steigerung der Asymmetrie kann im Sinne eines Jahn-Teller-Effektes 2. Ordnung erklärt werden.

Magnetische Übergänge in Azid-Komplexen, insbesondere mit Hysterese, sind ein nur wenig erforschtes Gebiet des molekularen Magnetismus mit einem großen Potential. Es handelt sich dabei um Verbindungen, bei denen zwei relativ stabile Zustände, die sich in ihren magnetischen Eigenschaften deutlich unterscheiden und in einem Temperaturbereich koexistieren, durch Temperaturänderung umgeschaltet werden können. Dies ist für die Anwendung in der Speicher- oder Sensortechnik von großer Bedeutung. Die ersten Schritte in dieser Richtung sollten auf das Verständnis des Mechanismus und die Synthese neuer derartiger Verbindungen fokussiert werden.

\subsubsection{Zusammenfassung}

In den letzten Jahren konnte eine Vielzahl von Nickel(II)-Azid-Verbindungen unterschiedlicher Topologie hergestellt werden, die auch sehr interessant im Hinblick auf neue magnetische Materialien sind. Die Hauptschwierigkeit dabei gibt sehr gut ein Zitat von J. Ribas et al. aus dem Jahr 1999 wieder: "Synthetically it is impossible to determine, with our present stage of knowledge, which coordination mode (of the azide) will be adopted".[12] Chelatisierende Pyrazolliganden ermöglichten erste Fortschritte bei den gezielten Synthesen von Nickel(II)-Azid-Verbindungen mit kontrollierbarem Azideinbau. Die ersten diesbezüglichen Ergebnisse wurden kürzlich von F. Meyer und Mitarbeitern publiziert. ${ }^{[25,26]}$

Magnetostrukturelle Korrelationen für $\mu_{3}-1,1,3-$ und $\mu_{4}-1,1,3,3$-Azidbrücken, topologischer Ferrimagnetismus sowie magnetische Übergänge sind aktuelle und interessante Forschungsgebiete im Bereich der Nickel(II)-Azid-Chemie, die auch die Synthese neuer Verbindungen voraussetzen. 


\subsection{Das pyrazolbasierte Ligandsystem}

In diesem Kapitel werden Eigenschaften sowie die Synthese der Pyrazolliganden, die für Darstellung der Nickel(II)-Komplexe mit steuerbarer Azid-Koordination eingesetzt wurden, beschrieben.

\subsubsection{Eigenschaften der Pyrazolliganden}

Wie bei der Zielsetzung kurz erwähnt, haben die in Rahmen dieser Arbeit verwendeten Pyrazolliganden $\mathbf{H L}^{\mathbf{1 - 7}}$ (Abb.4.2.1.1) zwei wichtige Eigenschaften:

1) dank ihres Aufbaus - Substitution in 3- und 5-Position durch Seitenarme - bilden diese Liganden zwei „Taschen“, in welchen zwei Metalle, im Rahmen dieser Arbeit Nickel(II)Ionen, koordiniert werden können; der Metall-Metall-Abstand wird in bestimmtem Rahmen durch den Pyrazolring vorgegeben;

2) durch die Wahl der Seitenarmlänge kann der Abstand zwischen Metallzentren genauer eingestellt werden. Lange Seitenarme in $\mathbf{H L}^{\mathbf{1 , 2}}$ lassen einen kurzen Metall-Metall Abstand von etwa $3.6 \AA$ zu. Hingegen ziehen kurze Seitenarme in $\mathbf{H L}^{3-7}$ die Metallionen auf einen Abstand von etwa $4.4 \AA$ auseinander. ${ }^{[24]}$ Die langen Seitenarme leiten sich von 1,3Propylendiamin $\quad\left(\mathbf{H L}^{\mathbf{1 , 2}}\right)$, die kurzen von 1,2-Ethylendiamin $\left.\quad \mathbf{H L}^{\mathbf{3}}\right)$ oder von Alkylsulfanylethylaminen $\left(\mathbf{H L}^{\mathbf{4 - 7}}\right)$ ab. 
<smiles>CNCCCN(C)Cc1cc(CN(C)CCCNC)[nH]n1</smiles><smiles>CNCCN(C)Cc1cc(CN(C)CCNC)[nH]n1</smiles>

$\mathrm{HL}^{3}$<smiles>[R5]CCN(CC[R5])Cc1cc(CN(CC[R])CC[R5])[nH]n1</smiles><smiles>CNCCCN(CCCNC)Cc1cc(CN(CCCNC)CCCNC)[nH]n1</smiles>

$\mathrm{HL}^{2}$<smiles>CCSCCN(C)Cc1cc(CN(C)CCSCC)[nH]n1</smiles>

$\mathrm{HL}^{4}$

$$
\begin{aligned}
& \mathrm{HL}^{5}(\mathrm{R}=\mathrm{Me}) \\
& \mathrm{HL}^{6}(\mathrm{R}=\mathrm{Et}) \\
& \mathrm{HL}^{7}\left(\mathrm{R}={ }^{i} \mathrm{Pr}\right)
\end{aligned}
$$

Abb. 4.2.1.1. Die pyrazolbasierten Kompartimentliganden $\mathbf{H L}^{\mathbf{1 - 7}}$.

Außerdem lässt sich die Seitenarmstruktur hinsichtlich der Zahl der Donoratome sowie Substituenten leicht verändern. Durch die Variation der Anzahl der Donoratome des Liganden wird auch die Anzahl der zur weiteren Verknüpfung freien Positionen an überwiegend sechsfach koordinierten Nickel(II)-Ionen vorgegeben.

Bei Liganden $\mathbf{H L}^{4-7}$ soll die Anwesenheit „weicher“ Donoratomen wie Schwefel im Vergleich zu Stickstoff zusätzliche Flexibilität des Nickel-Nickel-Abstandes erlauben, so dass man in Komplexen dieser Liganden in gewissem Rahmen auch Selbstorganisationsphänomene erwarten könnte.

Die unterschiedlichen Reste an S-Donoratomen können bei der Deutung intermolekularer Phänomene von Nutzen sein.

\subsubsection{Synthese der Liganden}

Zur Herstellung der Liganden wurden zwei Syntheserouten verwendet. Die Herstellung erfolgte für Pyrazolliganden mit N-Donoratomen in den Seitenarmen nach bereits bekannten Verfahren. ${ }^{[24 a, 97]}$ Zuerst wurde die Pyrazol-3,5-dicarbonsäure I mit Thionylchlorid in das Pyrazol-3,5-dicarbonsäurechlorid II überführt, dieses in Gegenwart eines Triethylamin- 
überschusses mit dem sekundären Amin $\operatorname{HNR}^{1} \mathrm{R}^{2}$ (die Gruppierung $\mathrm{NR}^{1} \mathrm{R}^{2}$ enthält Stickstoff als Heteroatom, zwei oder drei je nach Ligand) im Molverhältnis 1:2 umgesetzt wurde. Anschließend wurde das entstandene Amid III mit $\mathrm{LiAlH}_{4}$ zum entsprechenden Liganden $\left(\mathbf{H L}^{1-3}\right)$ reduziert. Die dazu benötigen Amine können käuflich erworben werden. Dieser erste Syntheseweg ist in Schema 4.2.2.1 zusammengefasst.

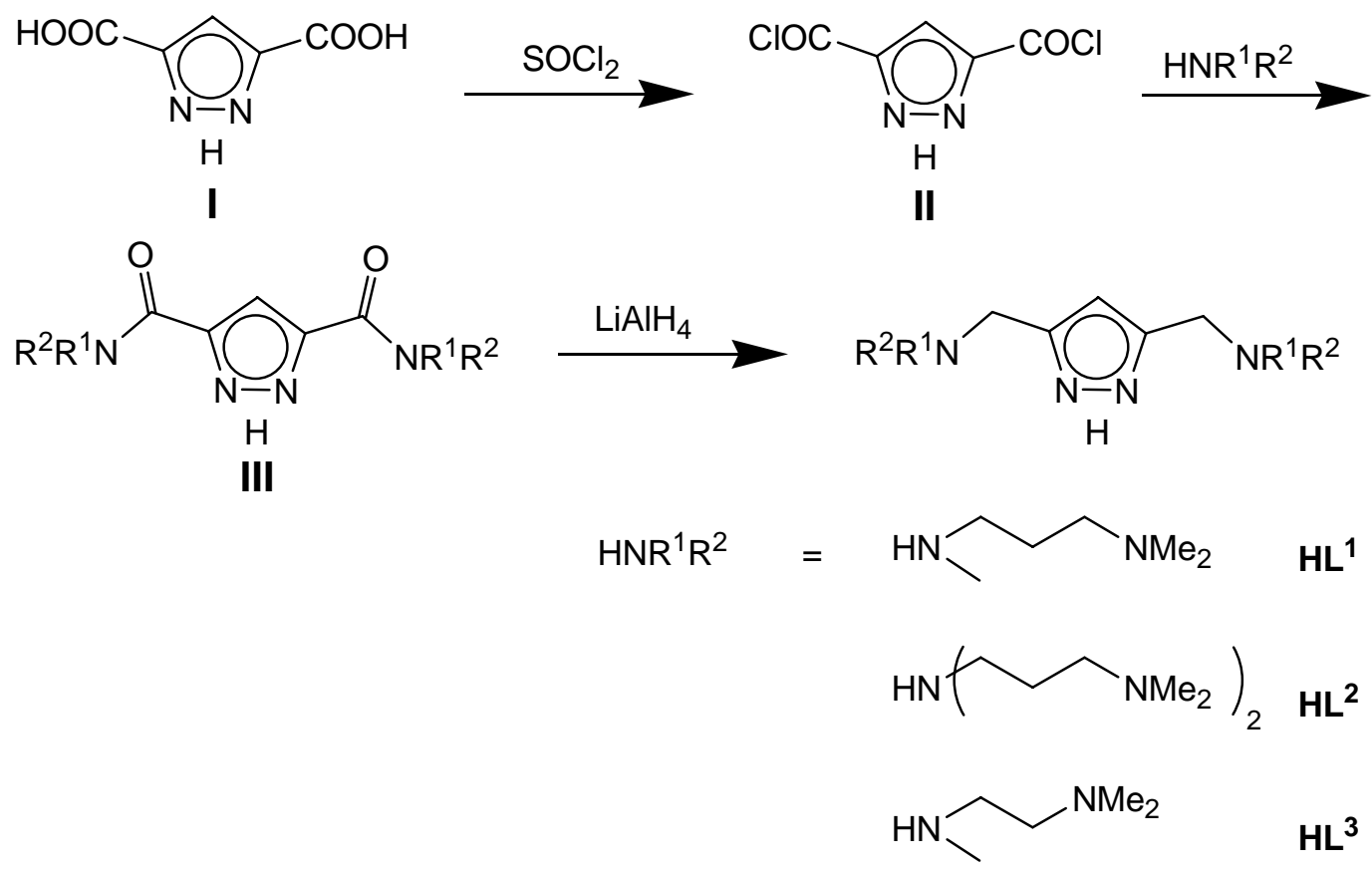

Schema 4.2.2.1. Synthese der Pyrazolliganden mit N-Donoratomen in den Seitenarmen $\left(\mathbf{H L}^{1-3}\right)$.

Dieses Verfahren erwies sich aber für die Synthese der Liganden mit S-haltigen Seitenarmen als ungünstig, da es hier verstärkt zur Spaltung der Amidbindung bei der Reduktion mit $\mathrm{LiAlH}_{4}$ kam. Bislang wurde auf diese Weise $\mathbf{H L}^{6[98]}$ erhalten.

Daher wurde für die Synthese der Liganden $\mathbf{H L}^{4-7}$ der Weg über die Schlüsselsubstanz 3,5Bis(chlormethyl)pyrazol VIII gewählt, welche in einer Mehrstufensynthese dargestellt wird. ${ }^{[99]}$ Zuerst wird das 3,5-Dimethylpyrazol IV durch $\mathrm{KMnO}_{4}$ zum Pyrazol-3,5dicarbonsäuremonokaliumsalz $\mathbf{V}$ oxidiert, welches weiter säurekatalysiert mit Methanol zu VI verestert wird. Der Ester VI wird durch $\mathrm{LiAlH}_{4}$ zum entsprechenden Alkohol VII reduziert und dieser anschließend mit $\mathrm{SOCl}_{2}$ chloriert (Schema 4.2.2.2). Das 3,5Bis(chlormethyl)pyrazol Hydrochlorid VIII wird durch die Reaktion mit 3,4-Dihydropyran in 3,5-Bis(chlormethyl)-1-(tetrahydropyran-2-yl)-1H-pyrazol IX überführt. ${ }^{[100]}$ 


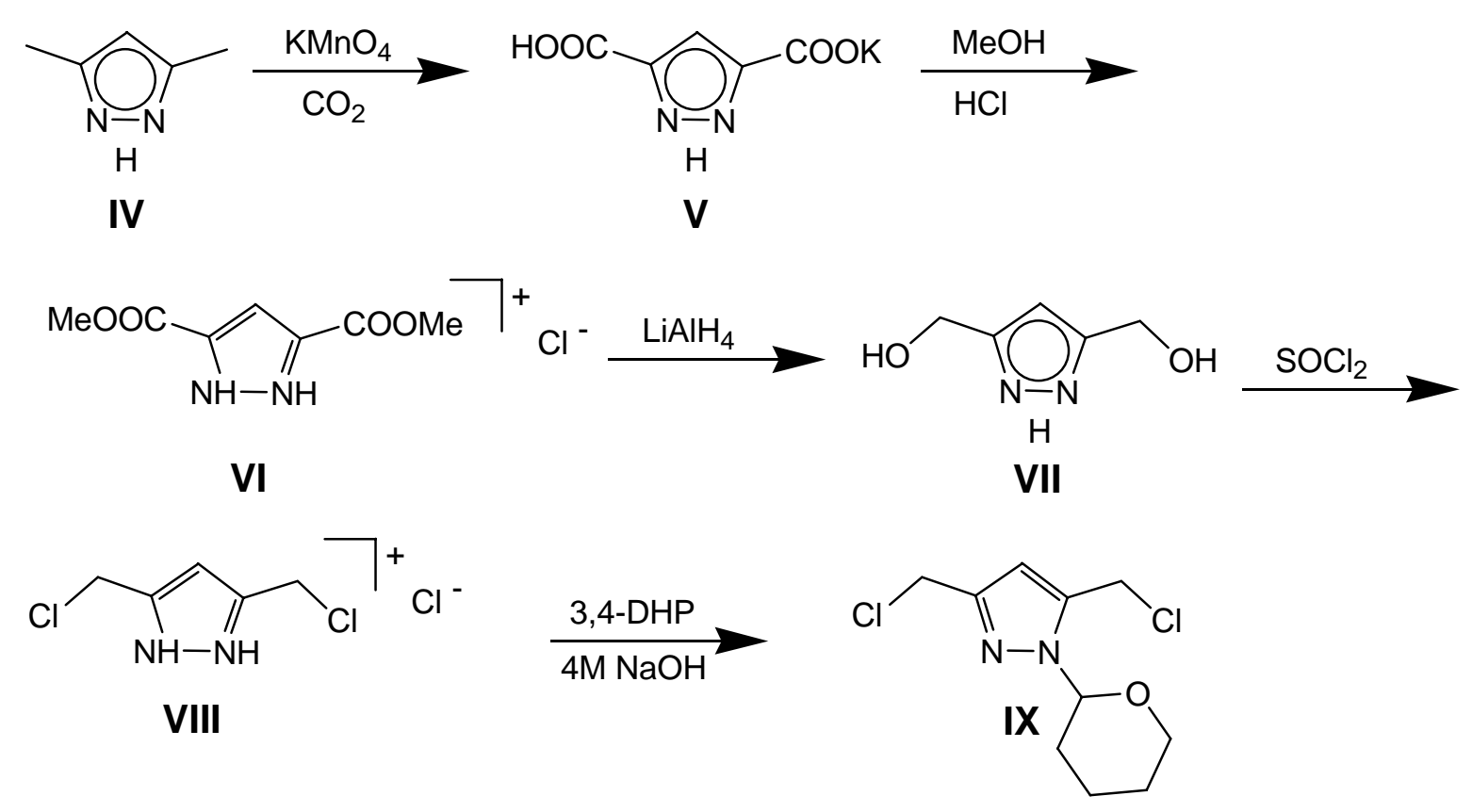

Schema 4.2.2.2. Synthese von 3,5-Bis(chlormethyl)-1-(tetrahydropyran-2-yl)-1H-pyrazol.

Durch Optimierung der Reaktion von VI zu VII konnte der $\mathrm{LiAlH}_{4}$-Verbrauch stark gegenüber der Angaben in der Literatur gesenkt werden. Zu dieser Reduktion sind pro 1 mol VI $1.25 \mathrm{~mol} \mathrm{LiAlH}_{4}$ nötig. Es wurde nur ein kleiner Überschuss eingesetzt.

Die anschließend als Seitenarme einzuführenden Alkylsulfanylethylamine wurden nach Literaturangaben synthetisiert. ${ }^{[97,101,102,103]}$ N-Methyl-[2-(ethylsulfanyl)ethyl]amin XI kann aus (2-Chlorethyl)methylamin Hydrochlorid $\mathbf{X}$ und Thioethanolat in Methanol gewonnen werden. Bis[2-(methylsulfanyl)ethyl]amin XIII, Bis[2-(ethylsulfanyl)ethyl]amin XIV und Bis[2-(1-methyl-ethylsulfanyl)ethyl]amin $\quad \mathbf{X V} \quad$ werden aus Bis(2-chlorethyl)amin Hydrochlorid XII und dem entsprechenden Thioalkoholat gewonnen (Schema 4.2.2.3).

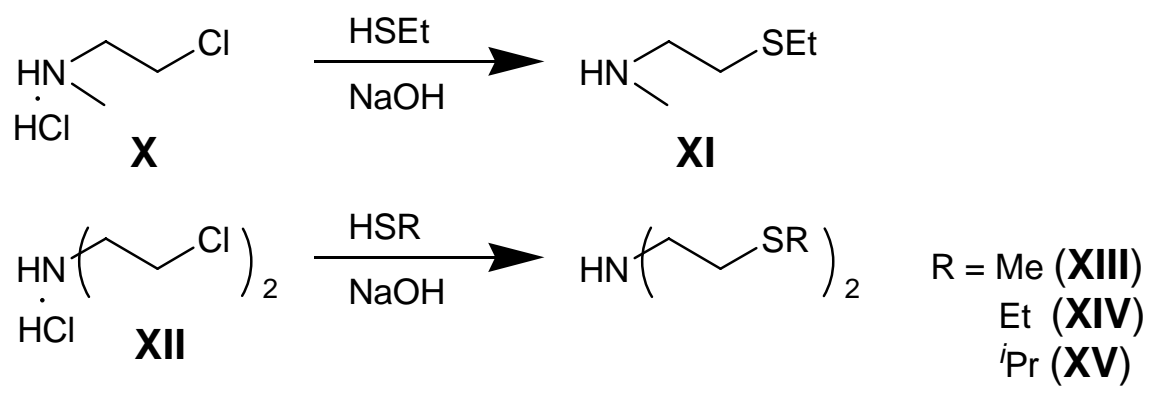

Abbildung 4.2.2.3. Synthese der Alkylsulfanylethylamine.

Zur Synthese der Liganden $\mathbf{H L}^{4-7}$ wurde das jeweilige Amin mit dem geschützten Pyrazolderivat IX im Molverhältnis 2:1 über trockenem $\mathrm{Na}_{2} \mathrm{CO}_{3}$ in Acetonitril 24 Stunden 
unter Rückfluß erhitzt. Nach Abkühlen, Filtration und Entfernen des Lösungsmittels bleibt der THP-geschützte Ligand als gelbes Öl zurück. Die Schutzgruppe wird durch EtOH/HCl abgespalten und danach das entstandene Hydrochlorid des Liganden mit wäßriger $\mathrm{NaOH}$ deprotoniert. Der Ligand wird dann durch Extraktion mit Dichlormethan und Entfernen des Lösungsmittels erhalten (Schema 4.2.2.4).

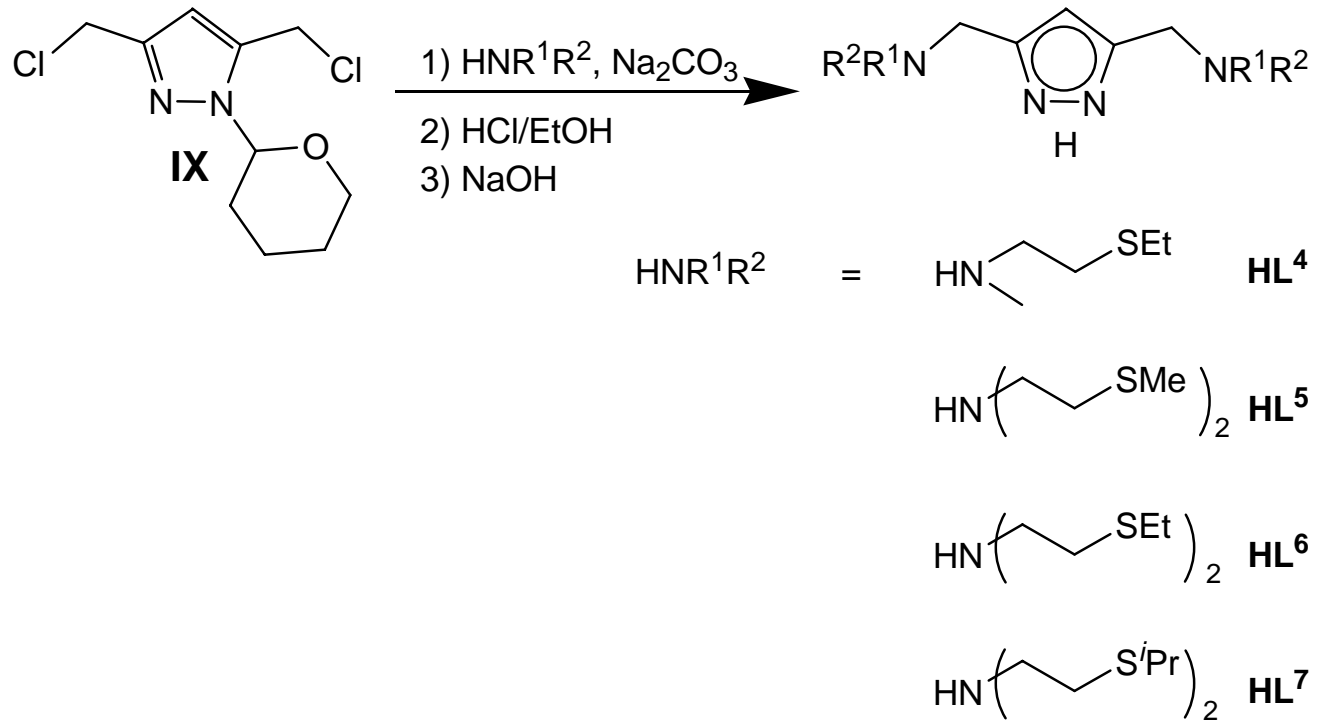

Schema 4.2.2.4. Synthese der Pyrazolliganden mit N- und S-Donoratomen in den Seitenarmen $\left(\mathbf{H L}^{4-7}\right)$.

Die Syntheseroute (Schema 4.2.2.4) kann auch für die Liganden $\mathbf{H L}^{\mathbf{1 - 3}}$ verwendet werden. Nach den Erfahrungen in unserer Arbeitsgruppe wurde versucht, $\mathbf{H L}^{1-3}$ und $\mathbf{H L}^{\mathbf{6}}$, ausgehend vom ungeschützten 3,5-Bis(chlormethyl)pyrazol zu synthetisieren. In Folge davon wurden sehr saubere Verbindungen mit der Ausbeute von über 90\% erhalten. Das Verfahren ist ansonsten völlig analog (abgesehen von der Entschützung des Liganden) der Syntheseroute, die von 3,5-Bis(chlormethyl)-1-(tetrahydropyran-2-yl)-1H-pyrazol ausgeht. 


\subsection{Synthese, Molekülstrukturen und magnetische Eigenschaften der pyrazolatbasierenden Nickel(II)-Azid-Komplexe ${ }^{1)}$}

\subsubsection{Zweikernige Nickel(II)-Azid-Komplexe}

Dieses Kapitel beschreibt die Synthese, Charakterisierung und magnetischen Eigenschaften der bimetallischen Nickel(II)-Azid-Komplexe. Es wird gezeigt, dass das Koordinationsverhalten des Azidions in der bimetallischen Umgebung der preorganizierten Dinickel(II)-Anordnung, und somit die magnetischen Eigenschaften solcher Verbindungen, durch den Einsatz von pyrazolbasierten Kompartimentliganden zielgerichtet gesteuert werden kann.

Aufgrund besonderer, in erster Linie magnetischer, Eigenschaften werden isomere zweikernige Komplexe mit der Zusammensetzung $\left[\mathrm{L}^{6} \mathrm{Ni}_{2}\left(\mathrm{~N}_{3}\right)_{3}\right]$ in einem eigenen Kapitel 4.3.4 beschrieben.

\subsubsection{Synthese und grundlegende Koordinationschemie der zweikernigen Nickel(II)-Komplexe}

Zur Darstellung von zweikernigen Nickel(II)-Azid-Komplexen werden Liganden $\mathbf{H L}^{\mathbf{1 - 4}}$ und $\mathbf{H L}^{6}$ verwendet. Die Synthese (Schema 4.3.1.1) erfolgt in Methanol durch Deprotonierung des Liganden mittels Kalium-tert-butylat und anschließender Zugabe von wasserhaltigem Nickel(II)perchlorat bzw. Nickel(II)nitrat sowie Natriumazid.

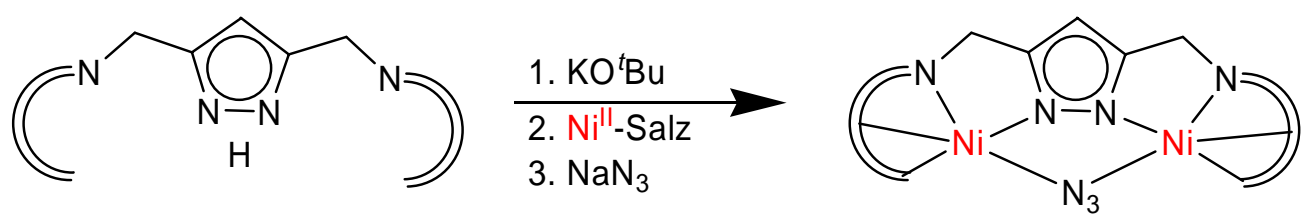

Schema 4.3.1.1. Synthese der zweikernigen Nickel(II)-Komplexe.

Die eingesetzten Liganden haben eine unterschiedliche Zahl an Donoratomen - drei in $\mathbf{H L}^{1,3,4}$ und vier in $\mathbf{H L}^{2,5-7}$ - pro Bindungstasche, welche an Metallzentren koordinieren können. Damit sind drei bzw. vier Koordinationsplätze je nach Ligand an jedem Nickel(II)Ion besetzt, noch einer wird zur Koordination des verbrückenden Azidions benötigt. Es

\footnotetext{
1) Die veröffentlichten Verbindungen werden nicht im Detail beschrieben. Ausführlichere Information ist in der entsprechenden Publikation erhältlich.
} 
bleiben noch eine oder zwei freie Koordinationsstellen bei der in diesen Systemen üblichen fünffachen bzw. sechsfachen Koordination der Nickel(II)-Ionen. Diese freien Koordinationsplätze können sowohl mit schwach koordinierenden Lösungsmitteln wie Aceton oder Acetonitril als auch mit koordinierenden Anionen wie Nitrat sowie mit terminal gebundenem Azid besetzt werden.

In allen Fällen bilden sich zweikernige Einheiten, in denen die Nickel(II)-Ionen über ein Pyrazolat und ein Azidion verbrückt sind. Die Art der Koordination des Brückenazids, $\mu-1,1-$ oder $\mu-1,3$-Verbrückung, ist streng von der Seitenarmlänge des Liganden abhängig. Bei der Verwendung der Liganden $\mathbf{H L}^{\mathbf{1}}$ und $\mathbf{H L}^{2}$ mit der Propylenbrücke zwischen den $\mathrm{N}$ Donoratomen des Seitenarms (lange Seitenarme) beobachtet man EO-Koordination, während im Fall von $\mathbf{H L}^{3-5}$ mit Ethylenbrücke zwischen den Donoratomen $\left(\{\mathrm{NN}\}\right.$ in $\mathbf{H L}^{3}$ und $\{\mathrm{NS}\}$ in $\mathbf{H L}^{4,5}$ ) des Seitenarms (kurze Seitenarme) EE-Koordination beobachtet wird. Eine Ausnahme bildet der Ligand $\mathbf{H L}^{\mathbf{6}}$, für welchen eine Lösungsmittelabhängigkeit der Azidbindung (mit bevorzugter end-to-end Koordination) beobachtet wird. Dies dürfte durch die „weichen“ SDonoren in diesem Liganden bedingt sein, so dass bei bestimmten Kristallisationsbedingungen, z.B. Kristallisation aus unterschiedlichen Lösungsmitteln, der Metall-MetallAbstand flexibel wird.

\subsubsection{Komplexe mit $\mu$-1,1-verbrückendem Azid}

Die Umsetzung des deprotonierten Liganden $\mathbf{H L}^{\mathbf{1}}$ mit Nickel(II)nitrat und Natriumazid führt zu der Verbindung 1. ${ }^{[104]}$ Einkristalle können durch Diffusion von Diethylether in eine Methanollösung des Komplexes erhalten werden. Die Verbindung kristallisiert in der Raumgruppe $P 2_{1} / c$ und enthält vier Moleküle in der monoklinen Zelle. Die Struktur der Verbindung 1 ist in Abb. 4.3.1.2.1 gezeigt.

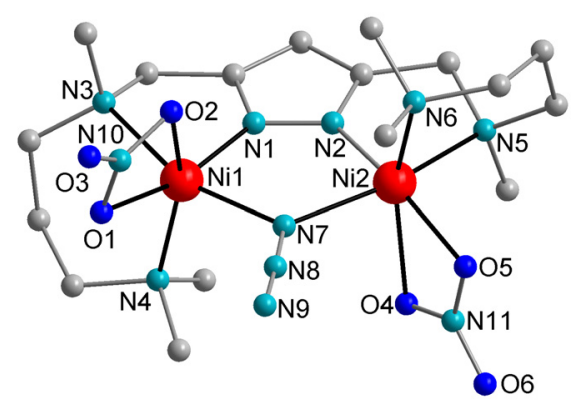

Abb. 4.3.1.2.1. Die Struktur der Verbindung 1. ${ }^{1)}$

\footnotetext{
1) Bei diesem und weiteren Komplexen wurden alle H-Atome aus Gründen der Übersichtlichkeit weggelassen
} 
Wie für die pyrazolatbasierenden Systeme zu erwarten ist, befinden sich beide Nickel(II)Ionen innerhalb ihres entsprechenden Ligandkompartiments (mit jeweils zwei Stickstoffdonoratomen vom Ligandseitenarm) und sind über das Pyrazolat verbrückt. Die $\mu$ 1,1-Azidbrücke findet sich innerhalb der bimetallischen Tasche. Die zusätzlichen Nitratliganden besetzen die restlichen freien Koordinationsstellen der Metallionen, dass jedes Nickel(II)-Ion eine pseudooktaedrische Koordinationssphäre hat. Die langen Seitenarme

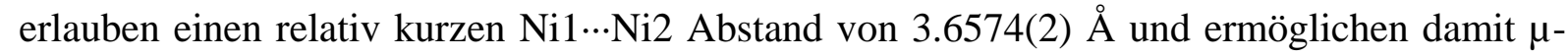
1,1-Azideinbau.

Ein ähnlicher Komplex $2^{1 \text { ), [105] }}$ bildet sich bei der Umsetzung des deprotonierten Liganden $\mathbf{H L}^{2}$, der ebenfalls lange propylenverbrückte Seitenarme besitzt, mit Nickel(II)nitrat und Natriumazid in Methanol. Dem schließt sich die Umkristallisation aus Aceton an. Die Verbindung kristallisiert wie auch 1 in der Raumgruppe $P 2_{1} / C$ und enthält vier Moleküle in der monoklinen Zelle. Die Struktur des Komplexes 2 ist in Abb. 4.3.1.2.2 gezeigt.

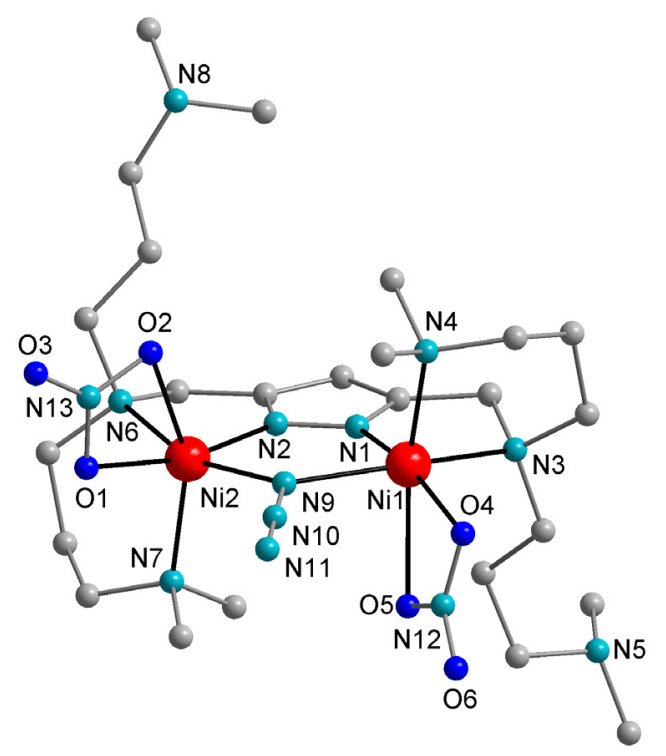

Abb. 4.3.1.2.2. Die Struktur der Verbindung 2.

2 ist ähnlich der Verbindung 1 aufgebaut, die strukturellen Parameter ändern sich nur

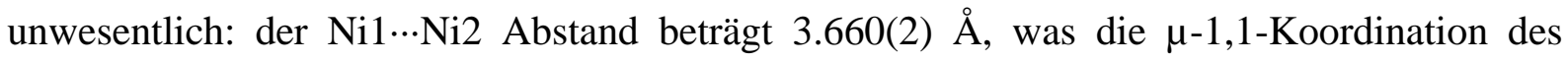
zentralen Azids ermöglicht. Interessanterweise erlaubt die vergleichsweise geringe Stabilität des Chelatsechsrings in $\mathbf{2}$ dem Nitration ein N-Donoratom des Seitenarms an jedem

\footnotetext{
${ }^{1)}$ Verbindung 2 wurde von B. Bauer-Siebenlist synthetisiert.
} 
Nickel(II)-Ion zu verdrängen. Als Folge dessen ist auf jeder Seite des Komplexes ein Seitenarm des Liganden an der Koordination nicht beteiligt.

Die temperaturabhängigen Messdaten für die magnetische Suszeptibilität $\chi_{M}$ sowie das Produkt $\chi_{M} T$ für die Komplexe 1 und 2 sind in Abb. 4.3.1.2.3 dargestellt.

Für 1 wird ein $\chi_{M} T$-Wert von $2.30 \mathrm{~cm}^{3} \mathrm{Kmol}^{-1}\left(4.32 \mu_{\mathrm{B}}\right)$ bei Raumtemperatur beobachtet. Dieser Wert liegt sehr nahe am „spin-only“ Wert für zwei ungekoppelte $S=1$ Nickel(II) Ionen (2.22 $\mathrm{cm}^{3} \mathrm{Kmol}^{-1}$ für $g=2.11$ ) und bleibt fast konstant bis zu $125 \mathrm{~K}$ (Abb. 4.3.1.2.3, links). Unterhalb dieser Temperatur steigt $\chi_{M} T$ allmählich an und erreicht ein Maximum von $3.32 \mathrm{~cm}^{3} \mathrm{Kmol}^{-1}\left(5.16 \mu_{\mathrm{B}}\right)$ bei $8 \mathrm{~K}$, was auf ferromagnetischen Austausch hindeutet. Bei weiterer Abkühlung erfolgt allerdings ein abrupter Abfall von $\chi_{M} T$, welcher durch die Nullfeldaufspaltung oder intermolekulare antiferromagnetische Wechselwirkungen verursacht werden könnte. Die Daten der Suszeptibilitätsmessungen von 1 wurden zuerst im Temperaturbereich von $295 \mathrm{~K}$ bis $10 \mathrm{~K}$ unter Verwendung des Programms ORIGIN nach Gleichung 6.4.4.2 angepasst. Als Parameter ergaben sich $g=2.10 \pm 0.01$ und $J=+5.6 \pm 0.5$ $\mathrm{cm}^{-1}\left(\rho=3 \%\right.$, TIP $\left.=1.0 \cdot 10^{-4} \mathrm{~cm}^{3} \mathrm{~mol}^{-1}\right)$. Dies bestätigt eine schwache ferromagnetische Kopplung. Um aber die magnetischen Eigenschaften von 1 im ganzen Temperaturbereich beschreiben zu können, wurde das Programm jul $X^{[106]}$ verwendet. Der zugehörige isotrope Heisenberg-Dirac-van-Vleck-Hamiltonoperator (Gleichung 4.3.1.2.1) lautet

$$
\hat{H}=-2 J \hat{S}_{1} \hat{S}_{2}+\sum_{i=1}^{2}\left(D \hat{S}_{i z}^{2}+g_{i} \mu_{B} B \hat{S}_{i z}\right)
$$

wobei die zusätzlichen Terme die Nullfeldaufspaltung $|D|$ und die Zeeman-Aufspaltung berücksichtigen. Die $g$-Werte wurden für alle Positionen als identisch angenommen. Die Berücksichtigung von $|D|$ ist für die Beschreibung ferromagnetisch gekoppelter Systeme, also für den Fall, dass der Grundzustand $S_{T}>0$ ist, physikalisch sinnvoll. Dadurch kann der abrupte Abfall von $\chi_{M} T$ im Tieftemperaturbereich erklärt und beschrieben werden. Gute Übereinstimmung wurde mit den Parametern $g=2.09 \pm 0.01, J=+5.7 \pm 0.25 \mathrm{~cm}^{-1}$ und $|D|=$ $3.5 \pm 0.2 \mathrm{~cm}^{-1}\left(\rho=1 \%\right.$, TIP $\left.=2.0 \cdot 10^{-4} \mathrm{~cm}^{3} \mathrm{~mol}^{-1}\right)$ erhalten. Die durchgezogene Linie in Abb. 4.3.1.2.3 (links) gibt die beste Anpassung für 1 wieder. Der Ni1-N7-Ni2 Winkel, der durch das Ligandgerüst vorgegeben ist, ist in 1 ungewöhnlich groß $\left(116.3(1)^{\circ}\right)$ für Nickel(II)-AzidSysteme. Trotzdem und trotz des kleinen antiferromagnetischen Beitrags der Pyrazolatbrücke ist der ferromagnetische Austausch, vermittelt durch das $\mu$-1,1-Azid, signifikant.

Im Fall von 2 bleibt der bei Raumtemperatur beobachtete $\chi_{M} T$-Wert von $2.66 \mathrm{~cm}^{3} \mathrm{Kmol}^{-1}$ (4.62 $\mu_{\mathrm{B}}$ ) bis $\mathrm{zu} 100 \mathrm{~K}$ konstant und steigt danach allmählich bis zum Maximum von 3.13 
$\mathrm{cm}^{3} \mathrm{Kmol}^{-1}$ (5.01 $\mu_{\mathrm{B}}$ ) bei $12 \mathrm{~K}$ (Abb. 4.3.1.2.3, rechts). Bei weiterer Abkühlung erfolgt ebenso wie bei 1 ein abrupter Abfall von $\chi_{M} T$. Die Daten der Suszeptibilitätsmessungen von 2 wurden analog zu 1 im Temperaturbereich von $300 \mathrm{~K}$ bis $20 \mathrm{~K}$ unter Verwendung des Programms ORIGIN nach Gleichung 6.4.4.2 und im ganzen Temperaturbereich unter Verwendung des Programms jul $X^{[106]}$ (Hamiltonoperator siehe Gleichung 4.3.1.2.1) angepasst. Als Parameter ergaben sich $g=2.25 \pm 0.01, J=+4.0 \pm 0.5 \mathrm{~cm}^{-1}, \rho=2 \%$ und TIP = $6.0 \cdot 10^{-4} \mathrm{~cm}^{3} \mathrm{~mol}^{-1}$ (ORIGIN) sowie $g=2.24 \pm 0.01, J=+2.8 \pm 0.3 \mathrm{~cm}^{-1},|D|=8.9 \pm 0.4 \mathrm{~cm}^{-1}, \rho$ $=0.4 \%$ und TIP $=3.7 \cdot 10^{-4} \mathrm{~cm}^{3} \mathrm{~mol}^{-1}$ (julX). Unter Verwendung beider Programme ergibt sich eine positive Kopplungskostante und bestätigt den ferromagnetischen Austausch. Die durchgezogene Linie in Abb. 4.3.1.2.3 (rechts) gibt die beste Anpassung für 2 unter Berücksichtigung der Nullfeldaufspaltung wieder.
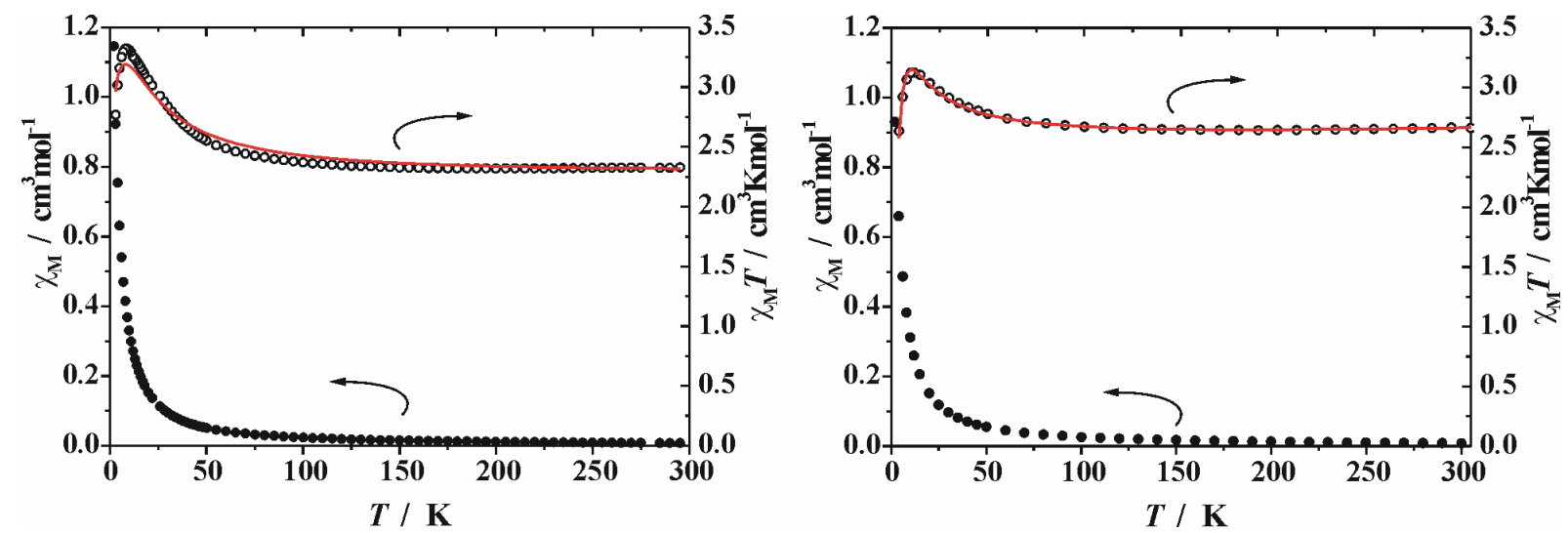

Abb. 4.3.1.2.3. Temperaturabhängigkeit von $\chi_{M}$ und $\chi_{M} T$ für 1 (links) und 2 (rechts). Die roten Linien repräsentieren die besten Anpassungen.

Unter Verwendung beider Programme ergibt sich eine positive Kopplungskostante und bestätigt den ferromagnetischen Austausch. Die durchgezogene Linie in Abb. 4.3.1.2.3 (rechts) gibt die beste Anpassung für 2 unter Berücksichtigung der Nullfeldaufspaltung wieder.

Der Vergleich von 1 und 2 zeigt, dass diese Verbindungen sowohl strukturell als auch magnetisch sehr ähnlich sind.

\section{Zusammenfassung}

Die Anwendung der Liganden $\mathbf{H L}^{\mathbf{1 , 2}}$ mit langen Seitenarmen führt zum $\mu$-1,1-Azideinbau in $\mathbf{1}$ und 2. Dabei resultierten ferromagnetische Wechselwirkungen. 
Beide Verbindungen können durch die semikoordinativ gebundenen Nitratliganden, die leicht durch einen anderen verbrückenden Liganden zu ersetzen sind, als Baueinheiten mit $S=$ 2 Spingrundzustand betrachtet werden.

\subsubsection{Komplexe mit $\mu$-1,3-verbrückendem Azid}

Die Umsetzung des deprotonierten Liganden $\mathbf{H L}^{3}$ mit Nickel(II)perchlorat und Natriumazid in Methanol und Austausch von Methanol gegen Acetonitril führt zur Verbindung 3. Einkristalle können durch Diffusion von Diethylether in eine Acetonitrillösung des Komplexes erhalten werden. Die Verbindung kristallisiert in der Raumgruppe $P \overline{1}$ und enthält je zwei kristallographisch unabhängige Moleküle in der triklinen Zelle. Die Struktur des Kations des Komplexes 3 ist in Abb. 4.3.1.3.1 gezeigt.
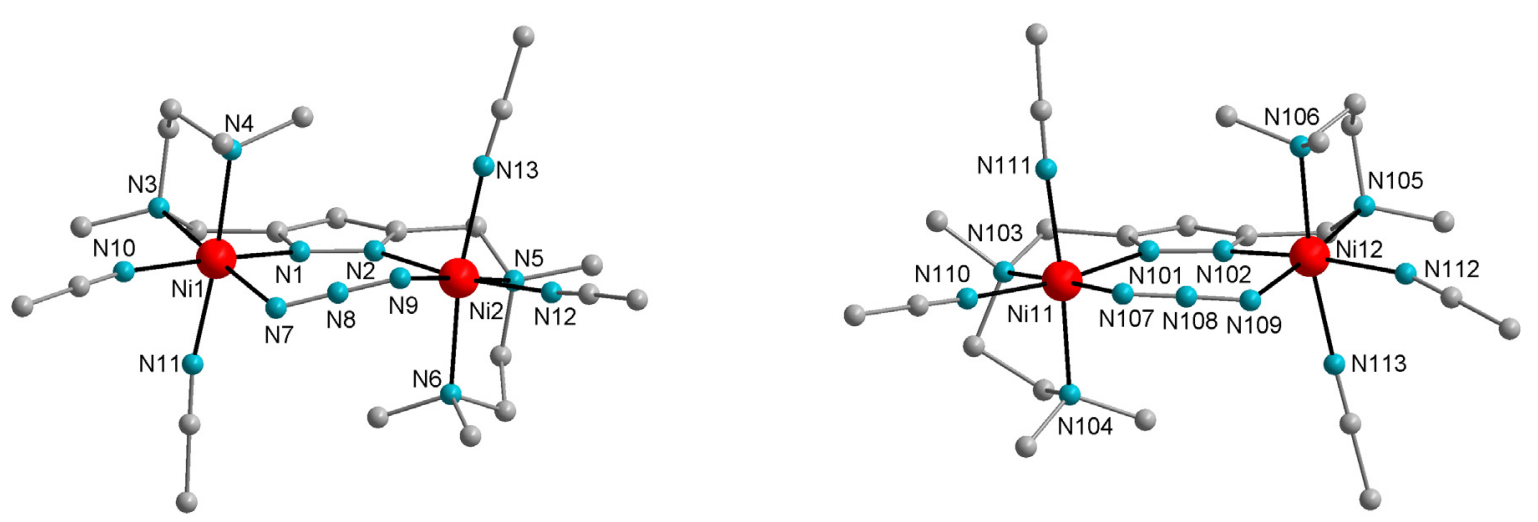

Abb. 4.3.1.3.1. Die Struktur des Kations der Verbindung 3.

Beide Moleküle von 3 sind nahezu identisch, es ändern sich allerdings geometrische Parameter des zentralen Nickel(II)-Azid-Fragmentes. Beide Nickel-Ionen befinden sich innerhalb ihres entsprechenden Ligandenkompartiments (mit jeweils zwei Stickstoffdonoratomen der Ligandseitenarme) und sind über das Pyrazolat verbrückt. Die kurze Ethylenbrücke zwischen den N-Donoratomen der Seitenarme zieht beide Metalle

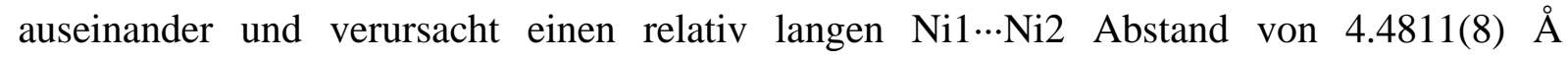
$(d(\mathrm{Ni11} \cdots \mathrm{Ni12})=4.5603(8) \AA)$, so dass das Azid im $\mu$-1,3-Modus eingebaut werden kann. Die restlichen Stellen an jedem der sechsfach koordinierten Metallionen sind durch Acetonitrilmoleküle besetzt, so dass jedes Nickel(II)-Ion eine pseudooktaedrische Koordinationssphäre hat. Der größte Unterschied zwischen beiden Molekülen wird im Torsionswinkel Ni-NNN-Ni beobachtet: für das erste Molekül (Abb. 4.3.1.3.1, links) $\tau($ Ni1- 
N7N8N9-Ni2) $=45.3(1)^{\circ}$, für das zweite (Abb. 4.3.1.3.1, rechts) $\tau($ Ni11-N107N108N109Ni12) $=23.5(1)^{\circ}$, was für die Flexibilität des zentralen Azids spricht. Der andere aus magnetischer Hinsicht wichtige Parameter, der Ni-N-N Winkel $\alpha$, ist zwar auch unterschiedlich, aber nicht so ausgeprägt: 116.1 (1) / 116.4 (1) und 120.0 (1) / 120.1 (1) (entsprechend für Molekül links und rechts in Abb. 4.3.1.3.1).

Die Temperaturabhängigkeit der magnetischen Suszeptibilität $\chi_{M}$ und des Produkts $\chi_{M} T$ für die Verbindung 3 ist in Abb. 4.3.1.3.2 dargestellt.

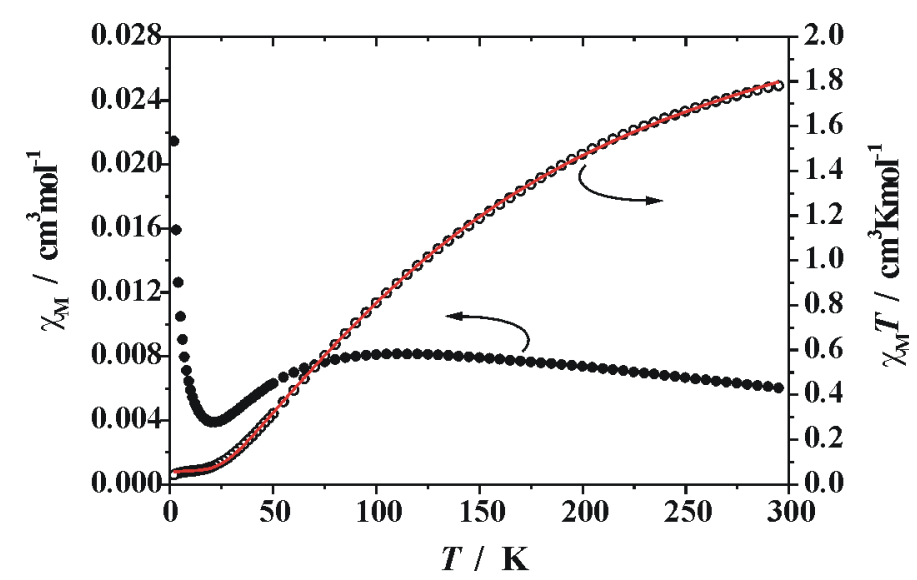

Abb. 4.3.1.3.2. Temperaturabhängigkeit von $\chi_{M}$ und $\chi_{M} T$ für 3 . Die rote Linie repräsentiert die beste Anpassung.

Der $\chi_{M} T$ Wert von $1.80 \mathrm{~cm}^{3} \mathrm{Kmol}^{-1}\left(3.80 \mu_{\mathrm{B}}\right)$ bei $295 \mathrm{~K}$ ist deutlich niedriger als der erwartete „spin-only“ Wert für zwei ungepaarte Hochspin-Nickel(II)-Ionen (2.33 $\mathrm{cm}^{3} \mathrm{Kmol}^{-1}$ für $g=2.16$ ), nimmt mit sinkender Temperatur ab und tendiert schließlich nach Null. Ein solches Verhalten ist typisch für zweikernige Komplexe mit intramolekularer antiferromagnetischer Kopplung ( $S=0$ Grundzustand) , wie für den $\mu-1,3$-azidverbrückten Komplex zu erwarten ist. Die Anwendung der Gleichung 6.4.4.2 für die Anpassung an die experimentellen Daten liefert folgende Parameter: $g=2.16 \pm 0.01, J=-42.4 \pm 0.2 \mathrm{~cm}^{-1} . \rho=4.7$ $\pm 0.1 \%$ und TIP $=5.1 \cdot 10^{-4} \pm 0.6 \cdot 10^{-4} \mathrm{~cm}^{3} \mathrm{~mol}^{-1}$. Die Kopplungskonstante $J$ liegt im typischen Bereich für die Komplexe mit der einfachen EE-Azidkoordination (siehe Kapitel 4.1.2).

Der Einzatz des Liganden $\mathbf{H L}{ }^{4}$, der sich von $\mathbf{H L}^{3}$ durch Ersatz eines N- durch ein SDonoratom in jedem Seitenarm unterscheidet, führt zu 4. Dieser Komplex kann durch die Umsetzung des deprotonierten Liganden $\mathbf{H L}^{4}$ mit Nickel(II)nitrat und Natriumazid erhalten werden, wobei die weitere Kristallisation durch Diffusion von Diethylether in eine Methanollösung des Komplexes durchgeführt wird. Die Verbindung kristallisiert in der 
Raumgruppe $C 2 / c$ und enthält vier Moleküle in der monoklinen Zelle. Die Struktur des zentrosymmetrischen Komplexes 4 ist in Abb. 4.3.1.3.3 gezeigt.

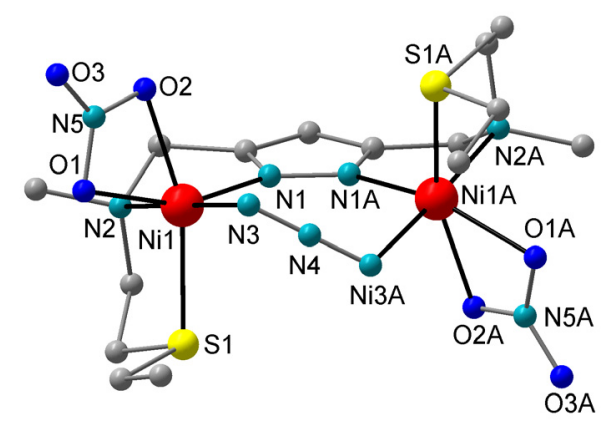

Abb. 4.3.1.3.3. Die Struktur der Verbindung 4.

Bei diesem Komplex ändert sich die durch den Liganden vorbestimmmte Koordinationsumgebung: Nickel(II)-Ionen werden durch jeweils ein $\mathrm{N}$ - und ein S-Donoratom der kurzen Seitenarme koordiniert. Die Ethylenbrücke zwischen den Seitenarmdonoratomen

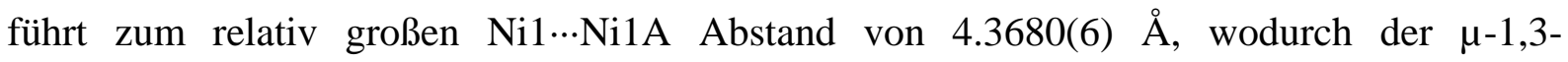
Azideinbau ermöglicht wird. Die restlichen Stellen an jedem der sechsfach koordinierten Metallionen werden durch zusätzliche Nitratliganden besetzt. Das zentrale Nickel(II)-AzidFragment ist gewinkelt: $\tau=65.4(1)^{\circ}$ und $\alpha=110.6(1)^{\circ}$.

Wie für die $\mu$-1,3-azidverbrückten Komplexe zu erwarten ist, zeigt 4 antiferromagnetisches Verhalten (Abb. 4.3.1.3.4). Der $\chi_{M} T$ Wert von $2.24 \mathrm{~cm}^{3} \mathrm{Kmol}^{-1}\left(4.26 \mu_{\mathrm{B}}\right)$ bei $300 \mathrm{~K}$ liegt nahe am „spin-only“ Wert, wie für zwei ungepaarte Hochspin-Nickel(II)-Ionen (2.31 cm $\mathrm{Kmol}^{-1}$ für $g=2.14$ ) erwartet, nimmt mit sinkender Temperatur langsamer als für $\mathbf{3}$ ab und tendiert schließlich gegen Null. Die Analyse der magnetischen Daten nach Gleichung 6.4.4.2 liefert folgende Parameter: $g=2.14 \pm 0.01, J=-15.5 \pm 0.2 \mathrm{~cm}^{-1}, \rho=8.1 \pm 0.2 \%$ und $T I P=8.7 \cdot 10^{-4}$ $\pm 0.5 \cdot 10^{-4} \mathrm{~cm}^{3} \mathrm{~mol}^{-1}$. 


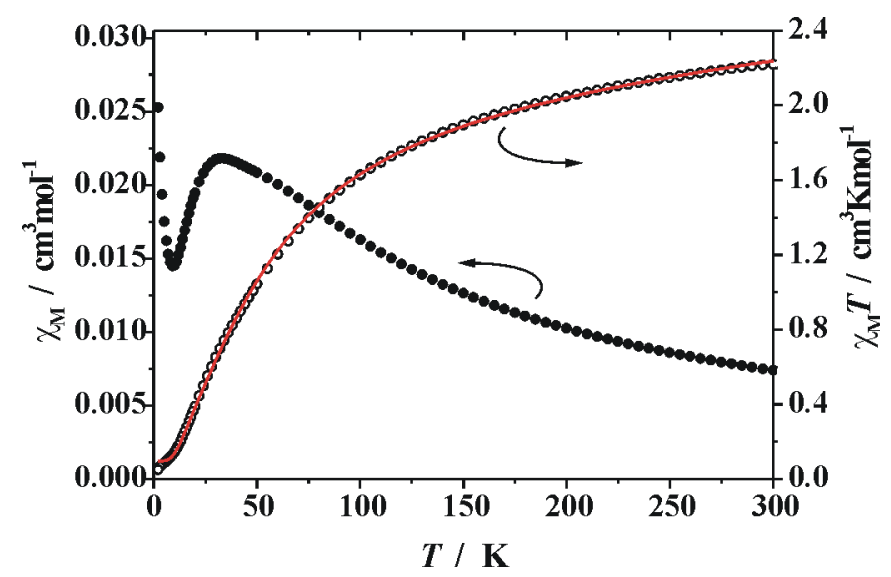

Abb. 4.3.1.3.4. Temperaturabhängigkeit von $\chi_{M}$ und $\chi_{M} T$ für 4. Die rote Linie repräsentiert die beste Anpassung.

Die Nickel(II)-Ionen in 3 sind stärker gekoppelt als in $4\left(J=-42.3 \mathrm{~cm}^{-1}\right.$ versus $J=-15.5$ $\mathrm{cm}^{-1}$ ), was durch den kleineren Torsionswinkel $\left(\tau=43.5(1)^{\circ} / 23.5(1)^{\circ}\right.$ für 3 und $\tau=65.4(1)^{\circ}$ für 4) erklärt werden kann.

Mit $\mathbf{H L}^{\mathbf{6}}$ wurden zwei weitere bimetallische Komplexe $\mathbf{5}$ und $\mathbf{6}$ mit einer $\mu$-1,3-Azidbrücke synthetisiert. $^{[107]}$ Es wurde Nickel(II)perchlorat für die Synthese beider Verbindungen eingesetzt und im Fall von 6 das Perchloration durch Zugabe von 2 Äquivqlenten $\mathrm{NaBPh}_{4}$ gegen Tetraphenylborat ausgetauscht. 5 ist hygroskopisch, was seine Darstellung aus einem wasserhaltigem Salz erschwert. Der andere Weg, diese Verbindung in sauberer Form und mit guter Ausbeute zu erhalten, verläuft über Umkristallisation des vierkernigen wasserfreien Komplexes 18 aus Aceton. Die beiden Verbindungen 5 und 6 kristallisieren in der Raumgruppe $P \overline{1}$ und enthalten jeweils zwei Moleküle in der triklinen Zelle. Die Strukturen der Kationen von 5 und $\mathbf{6}$ sind in Abb. 4.3.1.3.5 gezeigt.
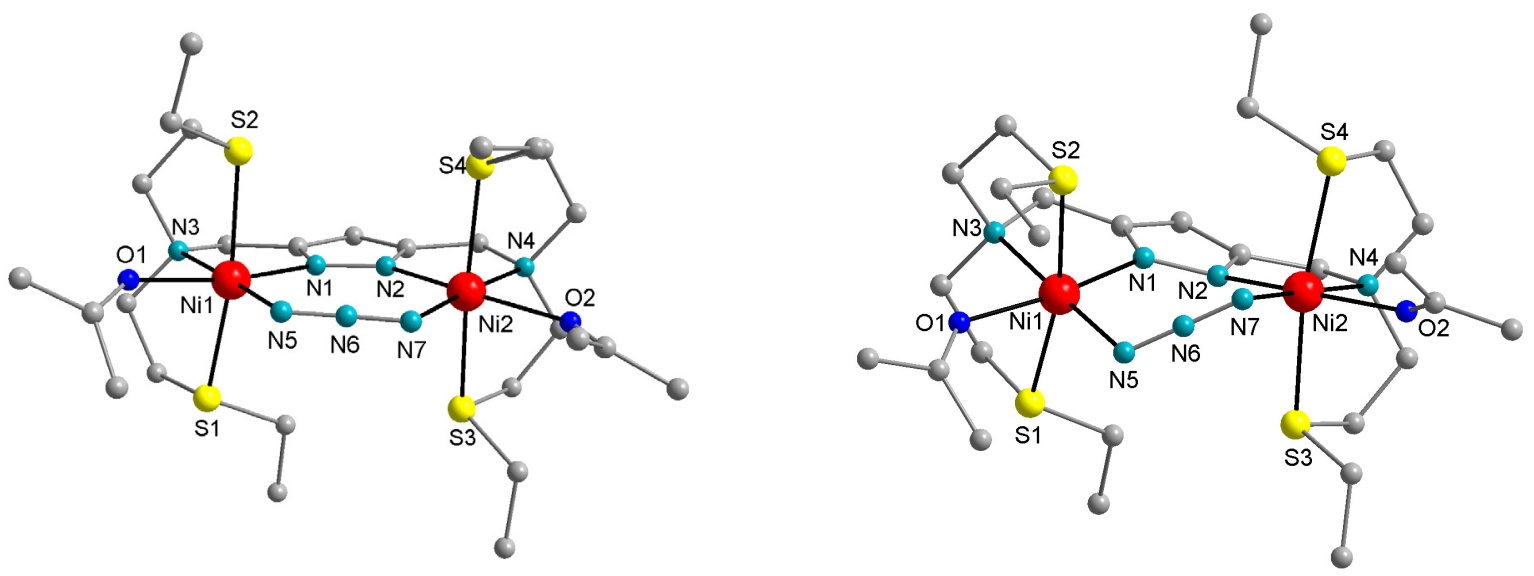

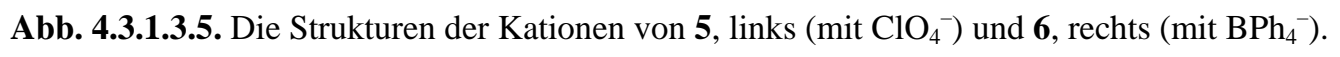


Von der Zusammensetzung her unterscheiden sich die beiden Verbindungen durch die Gegenionen: Perchlorat für 5 und Tetraphenylborat für 6. Der prinzipielle Aufbau der Kationen ist gleich: zwei Nickel(II)-Ionen sind über Pyrazolat und ein $\mu$-1,3-Azid verbrückt, drei weitere Stellen in der oktaedrischen Umgebung werden durch die zwei S- und ein NDonoratom besetzt. Die Acetonmoleküle ergänzen die Koordinationssphäre der Nickel(II)Ionen. Man findet aber sehr starke Unterschiede in den geometrischen Parametern der zentralen Fragmente (Tab. 4.3.1.3.1).

Tab. 4.3.1.3.1. Ausgewählte Abstände $(\AA)$ und Winkel $\left(^{\circ}\right)$ für 5 und 6.

\begin{tabular}{lll}
\hline Komplex & $\mathbf{5}$ & $\mathbf{6}$ \\
\hline Ni1Ni2 & $4.4409(4)$ & $4.3825(2)$ \\
Ni1-N5 & $2.0541(2)$ & $2.0635(1)$ \\
Ni2-N7 & $2.0407(2)$ & $2.0671(1)$ \\
Ni1-N5-N6 / Ni1-N7-N6 & $119.4(1) / 119.1(1)$ & $114.8(1) / 111.2(1)$ \\
Ni1-N5N6N7-Ni2 & $4.0(1)$ & $59.5(1)$ \\
Ni1-N1N2-Ni2 & $7.1(1)$ & $46.1(1)$ \\
\hline
\end{tabular}

Während $\mathrm{Ni} \cdots \mathrm{Ni}$ sowie $\mathrm{Ni}-\mathrm{N}$ Abstände sich nicht signifikant ändern, sind die Torsionswinkel, sowohl Ni-Azid-Ni als auch Ni-Pyrazolat-Ni, in beiden Molekülen sehr unterschiedlich: 4.0 (1) / 7.1 (1) in 5 gegenüber 59.5 (1) / 46.1 (1) in 6. Der Einsatz eines großen Gegenions wie Tetraphenylborat bewirkt die Verkippung des Azides in $\mathbf{6}$ und führt dagegen mit dem Perchlorat in $\mathbf{5}$ zu einer fast koplanaren Anordnung des zentralen Fragments. Dieses Beispiel zeigt, dass ein solcher aus magnetochemischer Sicht wichtiger Parameter wie der Ni-Azid-Ni Torsionswinkel sich in zweikernigen Komplexen durch die Wahl eines geeigneten Anions variieren lässt.

Nach den bekannten Korrelationen (siehe Kapitel 4.1.2) zwischen dem Winkel $\tau$ und der Kopplungskonstante $J$ sollte der Austausch in $\mathbf{5}$ mit dem kleineren Winkel viel größer als in $\mathbf{6}$ sein. Die Messungen der magnetischen Suszeptibilität und Anpassungen sind auch im Einklang mit dieser Vorhersage. Wie in Abb. 4.3.1.3.6 zu sehen ist, nimmt der bei Raumtemperatur beobachtete $\chi_{M} T$-Wert von $1.48 \mathrm{~cm}^{3} \mathrm{Kmol}^{-1}$ bei 5 viel schneller ab als bei 6 $\left(\chi_{M} T\right.$-Wert von $1.96 \mathrm{~cm}^{3} \mathrm{Kmol}^{-1}$ ), was auf die stärkere Kopplung in 5 hindeutet. 

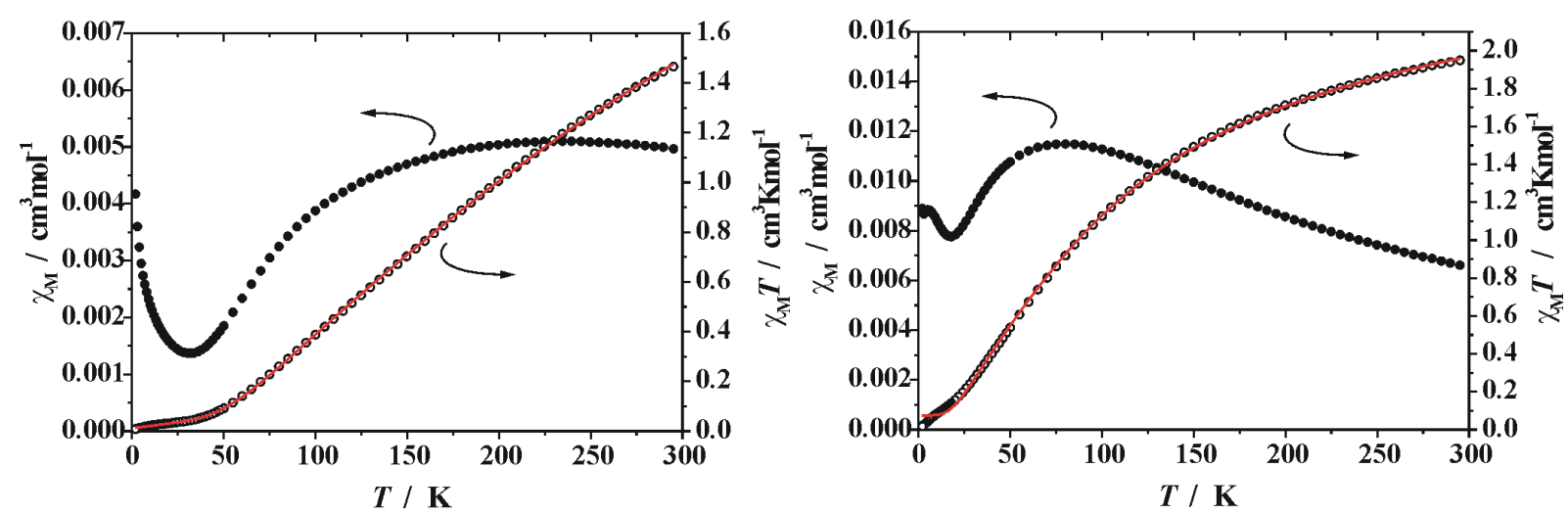

Abb. 4.3.1.3.6. Temperaturabhängigkeit von $\chi_{M}$ und $\chi_{M} T$ für 5 (links) und 6 (rechts). Die roten Linien repräsentieren die besten Anpassungen.

Die Anpassung an diese Daten nach Gleichung 6.4.4.2 liefert folgende Parameter: $g=2.22$ $\pm 0.01, J=-82.0 \pm 0.5 \mathrm{~cm}^{-1}, \rho=0.9 \pm 0.1 \%, T I P=9.7 \cdot 10^{-4} \pm 0.4 \cdot 10^{-4} \mathrm{~cm}^{3} \mathrm{~mol}^{-1}$ für 5 und $g=$ $2.13 \pm 0.02, J=-28.8 \pm 0.4 \mathrm{~cm}^{-1}, \rho=6.2 \pm 0.3 \%$, TIP $=5.8 \cdot 10^{-4} \pm 1.1 \cdot 10^{-4} \mathrm{~cm}^{3} \mathrm{~mol}^{-1}$ für 6. Die Verwendung des Programms julX ${ }^{[106]}$ für die Anpassung an die der magnetischen Daten liefert sehr ähnliche Parameter, z.B. $J=-82.0 \mathrm{~cm}^{-1}$ für 5 und $J=-28.7 \mathrm{~cm}^{-1}$ für 6. ${ }^{[107]}$

\section{Zusammenfassung}

Die Anwendung der Liganden mit kurzen Seitenarmen, sowohl des Liganden $\mathbf{H L}^{3}$ mit NDonoratomen in den Seitenarmen als auch der Liganden $\mathbf{H L}^{\mathbf{4 , 6}}$ mit einem oder zwei SDonoratomen der Ligandseitenarme führt zum $\mu$-1,3-Azideinbau mit den resultierenden antiferromagnetischen Wechselwirkungen, deren Stärke in erster Linie vom Torsionswinkel $\tau$ abhängt. Dieser Winkel kann gezielt variiert werden, indem man das komplexe Kation mit unterschiedlichen Anionen kristallisiert. Somit hat man bereits eine Möglichkeit, auch die Stärke der Kopplung zu verändern. 


\subsubsection{Vierkernige Nickel(II)-Komplexe unterschiedlicher Topologie}

In diesem Kapitel werden die Synthese und Charakterisierung vierkerniger Nickel(II)Azid-Komplexe beschrieben und deren magnetisches Verhalten vorgestellt. Es wird gezeigt, dass diese Verbindungen aus zweikernigen Einheiten, die isoliert oder in situ vorliegen, mit kontrollierter Nickel(II)-Azid-Koordination durch die Verknüpfung mit weiterem Azid (oder Azid und Carboxylat) dargestellt werden können.

\subsubsection{Rechteckige Anordnung der Nickel(II)-Zentren mit $\mu-1,1-$ und $\mu-1,3-$ Azidbrücken}

Die Verwendung von 1 und $\mathbf{2}$ als Baueinheiten für den Aufbau vierkerniger Spezies führte zur Darstellung einer Serie von $\mathrm{Ni}_{4}$-Komplexen. ${ }^{[104,105]}$ Diese Verbindungen sind über die $\mu$ 1,3-Azidverknüpfungen aus zwei $\mathrm{Ni}_{2}$-Bauelementen mit intramolekularen $\mu$-1,1-Azidbrücken erhalten worden. Während die Kopplung innerhalb der bimetallischen Einheiten durch das Design des Ligandgerüsts kontrolliert wird, ist der Spingrundzustand der $\mathrm{Ni}_{4}$-Komplexe streng vom Torsionswinkel $\mathrm{Ni}-\left(\mu-1,3-\mathrm{N}_{3}\right)-\mathrm{Ni}$ abhängig.

\section{Synthese und Charakterisierung}

Für die Vorhaben dieses Teils der Arbeit wurden die Liganden $\mathbf{H L}^{1}$ und $\mathbf{H L}^{2}$ gewählt. Durch die langen Seitenarme führen diese Liganden zum Ni $\cdots \mathrm{Ni}$-Abstand von etwa $3.6 \AA$ und somit zur $\mu$-1,1-Azidkoordination mit der resultierenden ferromagnetischen Kopplung in $\mathbf{1}$ und 2. Die Komplexe 1 und 2 können als Baueinheit für den Aufbau oligonuklearer Spezies verwendet werden, indem man ihre Nitratliganden durch Azidbrücken substituiert. Eine ähnliche Strategie wurde bereits für die Synthese vierkerniger Nickel(II)-Komplexe, ausgehend von koordinativ ungesättigten thiophenolatbasierenden Dinickel(II)-Baueinheiten oder einigen vierkernigen Nickel(II)-Komplexen auf Pyrazolatbasis verwendet. ${ }^{[26,64]}$

Die Umsetzung von 1 mit den stöchiometrischen Mengen an $\mathrm{NaN}_{3}$ und $\mathrm{NaBPh}_{4}$ in Methanol führt zu 11c (Schema 4.3.2.1.1). Allerdings können Komplexe 11a-c und 12 ${ }^{1)}$ besser direkt, ausgehend von den Liganden $\mathbf{H L}^{1}$ bzw. $\mathbf{H L}^{2}$ präpariert werden, indem man den entsprechenden deprotonierten Liganden mit zwei Äquivalenten von $\mathrm{Ni}\left(\mathrm{ClO}_{4}\right)_{2} \cdot 6 \mathrm{H}_{2} \mathrm{O}$ und zwei Äquivalenten von $\mathrm{NaN}_{3}$ in Methanol reagieren lässt.

\footnotetext{
${ }^{1)}$ Verbindung 12 wurde erstmalig von B. Bauer-Siebenlist synthetisiert.
} 


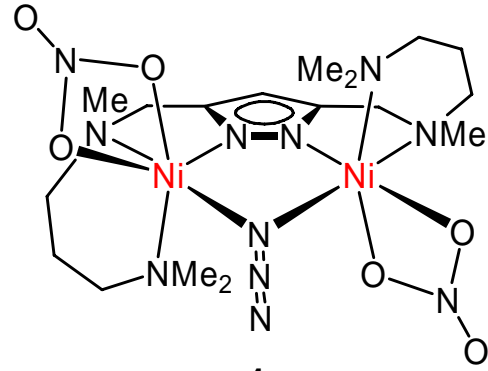

1

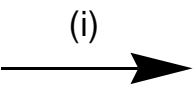

(i)

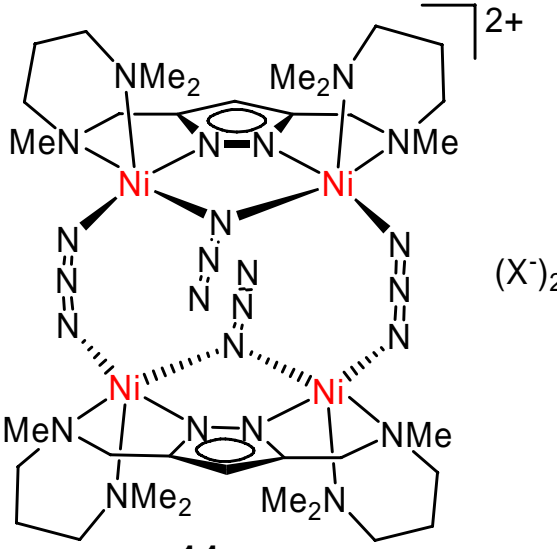

11a-c

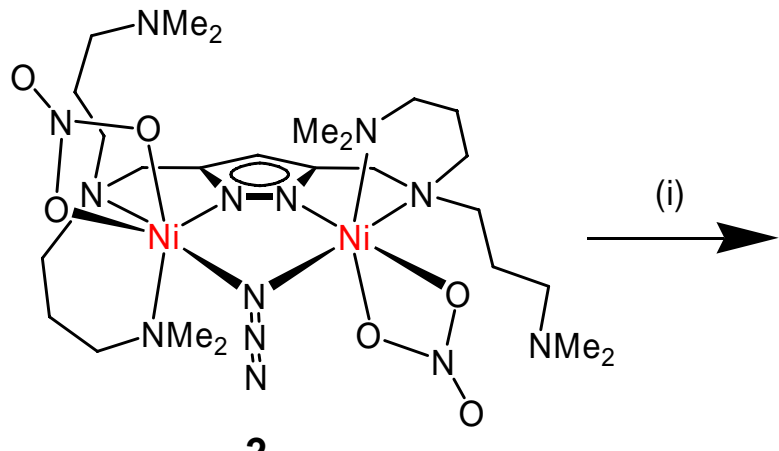

2

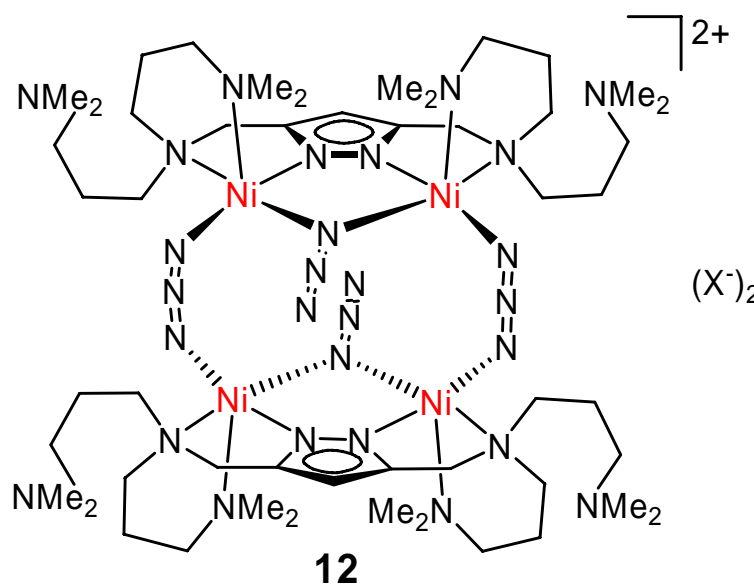

Schema 4.3.2.1.1. Synthese der $\mathrm{Ni}_{4}$-Komplexe $11\left(\mathrm{X}=\mathrm{ClO}_{4},(11\right.$ a,b $)$ oder $\left.\mathrm{BPh}_{4}(\mathbf{1 1 c})\right)$ und $12\left(\mathrm{X}=\mathrm{BPh}_{4}\right)$ aus bimetallischen Einheiten 1 und 2; (i) 1) $\mathrm{NaN}_{3} / \mathrm{MeOH}$; 2) $\mathrm{NaClO}_{4}$ (für 11a,b) oder $\mathrm{NaBPh}_{4}$ (für 11c, 12).

Auf diesem Weg wurde die kristalline Verbindung $\left[\left(\mathrm{L}^{1}\right)_{2} \mathrm{Ni}_{4}\left(\mu-1,1-\mathrm{N}_{3}\right)_{2}(\mu-1,3-\right.$ $\left.\left.\mathrm{N}_{3}\right)_{2}\right]\left(\mathrm{ClO}_{4}\right)_{2} \cdot \mathrm{CH}_{3} \mathrm{OH} \cdot 0.5 \mathrm{H}_{2} \mathrm{O}(\mathbf{1 1 a})$ isoliert. Umkristallisation aus Aceton / Petrolether führt $\mathrm{zu}$ Verbindung $\left[\left(\mathrm{L}^{1}\right)_{2} \mathrm{Ni}_{4}\left(\mu-1,1-\mathrm{N}_{3}\right)_{2}\left(\mu-1,3-\mathrm{N}_{3}\right)_{2}\right]\left(\mathrm{ClO}_{4}\right)_{2} \cdot \mathrm{C}_{3} \mathrm{H}_{6} \mathrm{O}$ (11b). In analoger Weise kann auch 12 synthetisiert werden, welches als $\mathrm{BPh}_{4}{ }^{-}$-Salz kristallisiert wurde. Die vierkernige Natur von 11a,b wird durch das Signal in FAB-MS bei $m / z=1147$, welches dem Ion $\left[\mathrm{L}_{2}^{1} \mathrm{Ni}_{4}\left(\mathrm{~N}_{3}\right)_{4}\left(\mathrm{ClO}_{4}\right)\right]^{+}$zugeordnet werden kann, bestätigt.

Die IR-Spektren von 11a-c und 12 sind im Bereich der Azidschwingungen sehr ähnlich (Tab. 4.3.2.1.1), was für einen gleichen Aufbau des zentralen Nickel(II)-Azid-Fragments in allen Verbindungen spricht. Die vier nicht koordinierten Dimethylaminogruppen in 12 üben, da sie sich im Außenbereich des Nickel(II)-Azid-Fragments befinden, keinen direkten Einfluß aus. 
Tab. 4.3.2.1.1. Ausgewählte IR-Banden für 11a-c und 12.

\begin{tabular}{ll}
\hline Komplex & $v_{\text {as }}\left(\mathrm{N}_{3}{ }^{-}\right), \mathrm{cm}^{-1}$ \\
\hline 11a & $2117(\mathrm{~s}), 2058(\mathrm{~s})$ \\
$\mathbf{1 1 b}$ & $2118(\mathrm{~s}), 2056(\mathrm{~s})$ \\
$\mathbf{1 1 c}$ & $2122(\mathrm{~s}), 2056(\mathrm{~s})$ \\
$\mathbf{1 2}$ & $2129(\mathrm{~s}), 2064(\mathrm{~s})$ \\
$\mathbf{1 2 *}$ & $2129(\mathrm{~s}), 2060(\mathrm{~s})$ \\
\hline
\end{tabular}

Die Röntgenstrukturanalyse bestätigt diese Vermutung. Als Beispiel für den Komplextyp 11 ist die Struktur des Kations von 11a in Abb. 4.3.2.1.1 abgebildet.

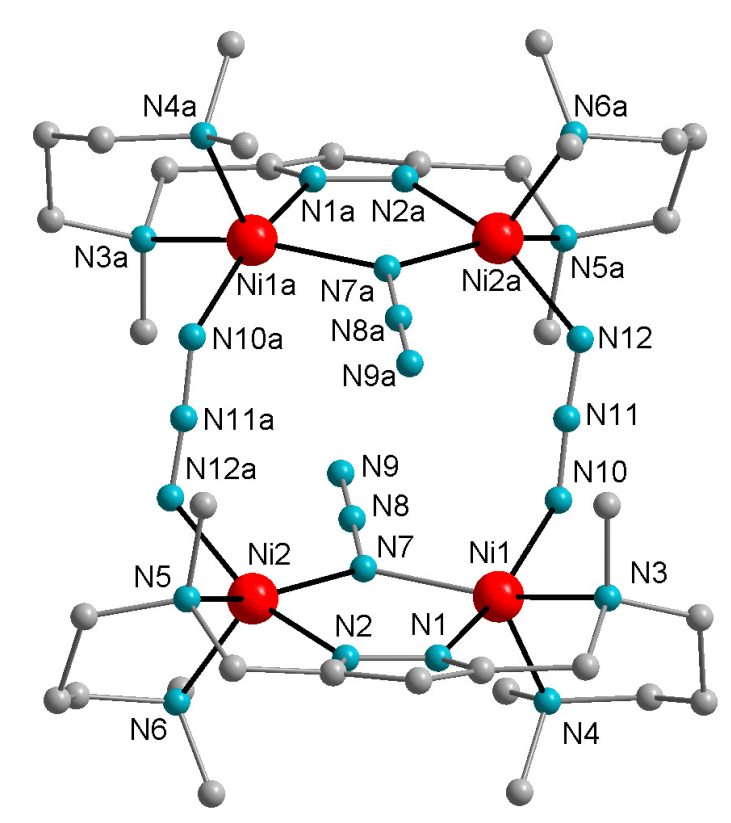

Abb. 4.3.2.1.1. Struktur des Kations von 11a.

In allen Verbindungen 11a-c und 12 sind zwei bimetallische Einheiten $\left[\left(\mathrm{L}^{1}\right)_{2} \mathrm{Ni}_{2}(\mu-1,1-\right.$ $\left.\left.\mathrm{N}_{3}\right)_{2}\right]^{2+}$ bzw. $\left[\left(\mathrm{L}^{2}\right)_{2} \mathrm{Ni}_{2}\left(\mu-1,1-\mathrm{N}_{3}\right)_{2}\right]^{2+}$ über zwei $\mu-1,3$-Azidliganden zur rechteckigen $\mathrm{Ni}_{4}-$ Anordnung mit kristallographischer $C_{\mathrm{i}}$-Symmetrie verknüpft. Die Bindungsparameter der jeweiligen bimetallischen Einheiten in den $\mathrm{Ni}_{4}$-Komplexen $11 \mathbf{a}-\mathbf{c}$ und 12 sind sehr ähnlich: $d(\mathrm{Ni} \cdots \mathrm{Ni})=3.620(1)$ (11a), 3.614(1) (11b), 3.645(1) (11c) und 3.639(2) $\AA$ (12). Die interdimeren $^{1)} \mathrm{Ni} \cdots \mathrm{Ni}$ Abstände sind 5.472(1) (11a), 5.480(1) (11b), 5.478(1) (11c) und 5.788(2) А̊ (12).

Komplex 11c kristallisiert in der Raumgruppe $P \overline{1}$ mit zwei kristallographisch unabhängigen Molekülen pro trikliner Elementarzelle. Eines von diesen Molekülen ist nahezu

\footnotetext{
1) Unter „Dimer“ wird eine zweikernige pyrazolatbasierte Einheit definiert.
} 
identisch mit 11a,b, das andere zeigt eine Fehlordnung des zentralen Nickel(II)-AzidFragments (Abb. 4.3.2.1.2). Die Fehlordnung bezieht sich auf die $\mu-1,3-A z i d-$, nicht auf die $\mu$ 1,1-Azidbrücke. Das deutet auf eine sehr hohe strukturelle Flexibilität der $\mu$-1,3-Azidbrücke hin.

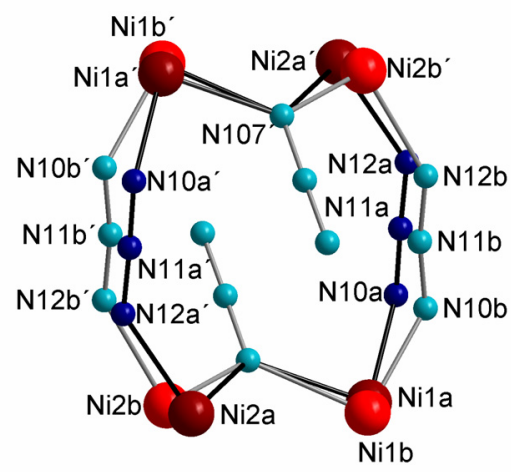

Abb. 4.3.2.1.2. Die Fehlordnung des zentralen Nickel(II)-Azid-Fragments von 11c. Ausgewählte Ni-Azid-Ni Torsionswinkel ( ${ }^{\circ}$ : Ni1a-N10a-N12a-Ni2a' 90(3), Ni1b-N10b-N12b-Ni2b' 72(7), Ni1a-N10b-N12b-Ni2a' 77(5), Ni1b-N10a-N12a-Ni2b’ 65(4).

Strukturelle Unterschiede zwischen den vier Verbindungen (11a-c und 12), verursacht durch unterschiedliche Gerüststruktur des Liganden und das variable Gegenion oder die Solvatmoleküle scheinen zwar gering zu sein, rufen aber, wie weiter gezeigt wird, drastische Unterschiede im magnetischen Verhalten hervor.

\section{Magnetische Eigenschaften}

Für 11a-c liegen die $\chi_{M} T$-Werte bei Raumtemperatur im Bereich zwischen 4.84 $\mathrm{cm}^{3} \mathrm{Kmol}^{-1}$ und $5.08 \mathrm{~cm}^{3} \mathrm{Kmol}^{-1}$. Diese Werte sind sehr nahe am „spin-only“ Wert für vier ungekoppelte $S=1$ Nickel(II)-Ionen $\left(4.83 \mathrm{~cm}^{3} \mathrm{Kmol}^{-1}\right.$ für $\left.g=2.20\right)$ und bleiben fast konstant bis zu $125 \mathrm{~K}$ für alle drei Komplexe (Abb. 4.3.2.1.3 oben für 11a und in der Mitte für 11c). Unterhalb dieser Temperatur steigt $\chi_{M} T$ allmählich und erreicht ein Maximum von 8.79 $\mathrm{cm}^{3} \mathrm{Kmol}^{-1}\left(8.06 \mu_{\mathrm{B}}\right)$ bei $8 \mathrm{~K}$ für 11a, $8.06 \mathrm{~cm}^{3} \mathrm{Kmol}^{-1}\left(8.03 \mu_{\mathrm{B}}\right)$ bei $7 \mathrm{~K}$ für $\mathbf{1 1 b}$ und 5.25 $\mathrm{cm}^{3} \mathrm{Kmol}^{-1}\left(6.48 \mu_{\mathrm{B}}\right)$ bei $30 \mathrm{~K}$ für 11c. Ein solches Verhalten deutet auf eine insgesamt ferromagnetische Wechselwirkung hin.

Für 12 ist der $\chi_{M} T$ Wert von $4.94 \mathrm{~cm}^{3} \mathrm{Kmol}^{-1}\left(6.29 \mu_{\mathrm{B}}\right)$ bei $295 \mathrm{~K}$ etwas niedriger als der „spin-only“ Wert, der für vier ungepaarte Hochspin-Nickel(II)-Ionen $\left(5.15 \mathrm{~cm}^{3} \mathrm{Kmol}^{-1}\right.$ für $g=$ 2.27) erwartet wird, nimmt mit sinkender Temperatur ab und tendiert schließlich nach Null 

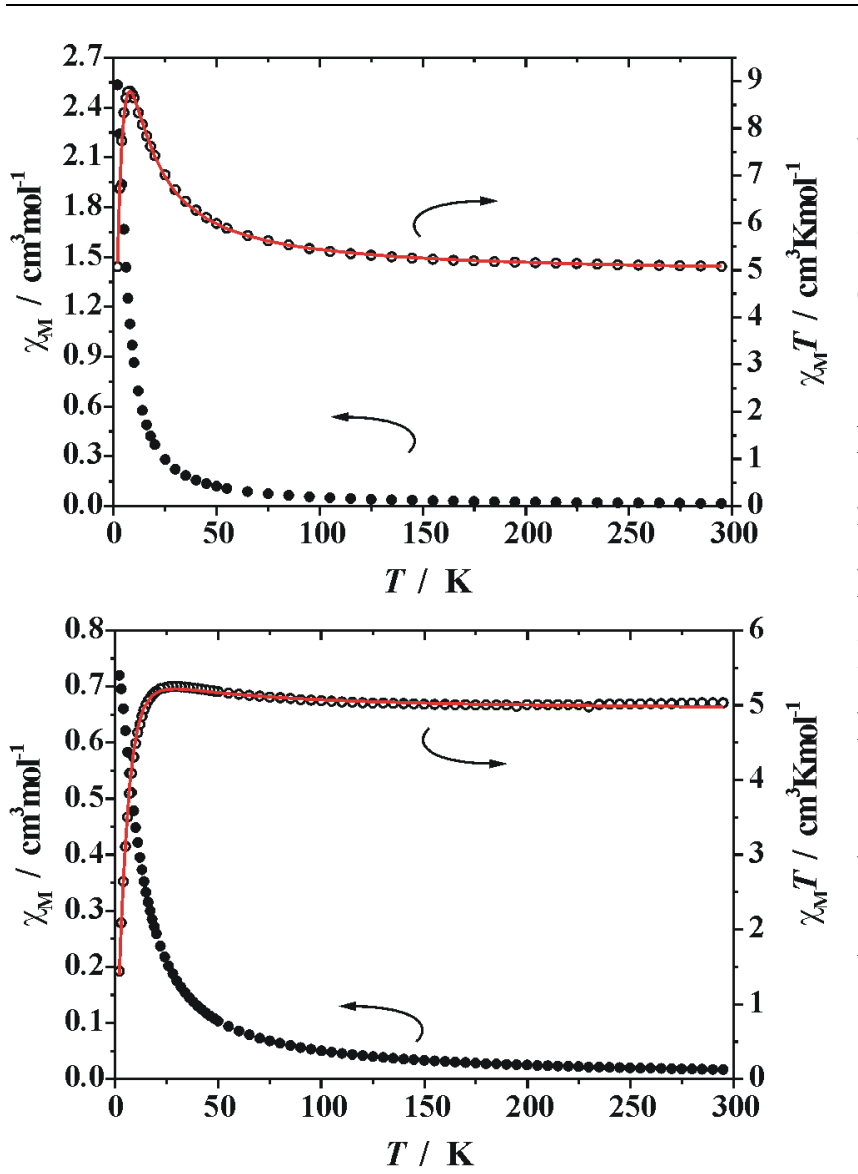

(Abb. 4.3.2.1.3 unten). Ein solches Verhalten deutet auf generelles antiferromagnetisches Verhalten $\left(S_{T}=0\right.$ Grundzustand).

Von der molekularen Struktur her können zwei prinzipielle Pfade für den magnetischen Austausch erwartet werden. Der erste Weg ist die intradimere Kopplung durch die $\mu$-1,1-Azid- und Pyrazolatbrücke, der zweite die interdimere Kopplung zwischen den Untereinheiten, vermittelt durch die $\mu-1,3-$ Azidbrücke (Schema 4.3.2.1.2).
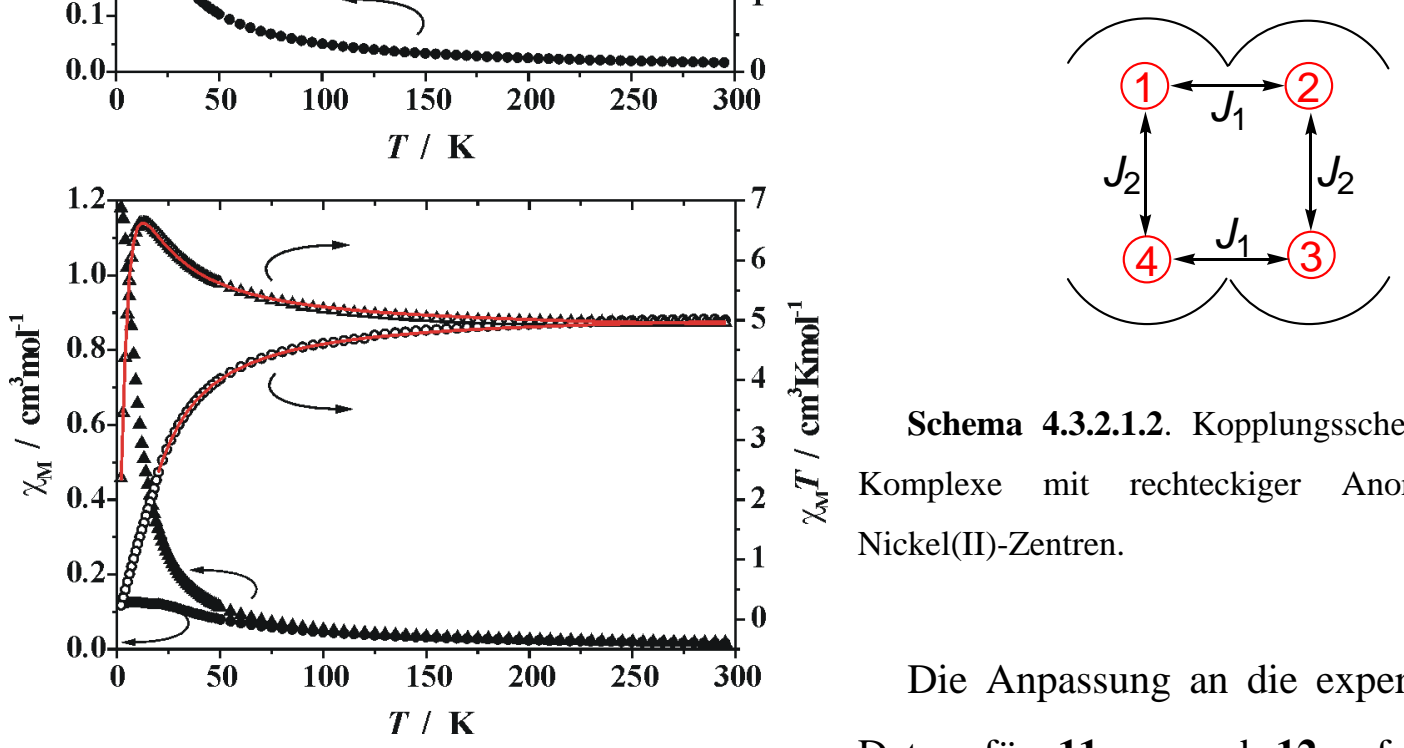

Schema 4.3.2.1.2. Kopplungsschema für $\mathrm{Ni}_{4}{ }^{-}$ Komplexe mit rechteckiger Anordnung der Nickel(II)-Zentren.

Die Anpassung an die experimentellen Daten für 11a-c und 12 erfolgte unter Abb. 4.3.2.1.3. Auftragung von $\chi_{M}$ und $\chi_{M} T$ als Funktion von $T$ für 11a (oben), 11c (in der Mitte) und 12 (unten, Kreise), 12* (unten, Dreiecke). Die roten Linien repräsentieren die besten Anpassungen.

Verwendung des Programms $j u l X,{ }^{[106]}$ das auf einem Heisenberg-Dirac-van-VleckHamiltonoperator mit zusätzlichen Termen für die Nullfeldaufspaltung und ZeemanAufspaltung basiert (Gleichung 4.3.2.1.1). Die $g$-Werte wurden für alle Positionen als identisch angenommen.

$$
\hat{H}=-2 J_{1}\left(\hat{S}_{1} \hat{S}_{2}+\hat{S}_{3} \hat{S}_{4}\right)-2 J_{2}\left(\hat{S}_{2} \hat{S}_{3}+\hat{S}_{1} \hat{S}_{4}\right)+D \sum\left(\hat{S}_{i z}^{2}-\hat{S}_{i}\left(\hat{S}_{i}+1\right) / 3\right)+g \mu_{B} B \sum \hat{S}_{i z}
$$


Die Anpassungsparameter sind in Tab.4.3.2.1.2. zusammengefasst.

Tab. 4.3.1.2. Anpassungsparameter der magnetischen Daten für 11a-c und 12. ${ }^{\mathrm{a}}$

\begin{tabular}{|c|c|c|c|c|c|}
\hline & 11a & 11b & 11c & 12 & $12^{*}$ \\
\hline$J_{1}{ }^{\mathrm{b}}, \mathrm{cm}^{-1}$ & +1.7 & +2.4 & +2.5 & +0.9 & +1.2 \\
\hline$J_{2}{ }^{\mathrm{b}}, \mathrm{cm}^{-1}$ & +4.7 & +2.6 & -0.6 & -5.9 & +4.8 \\
\hline$g$ & 2.21 & 2.16 & 2.22 & 2.28 & 2.18 \\
\hline$|D|, \mathrm{cm}^{-1}$ & 11.7 & 11.7 & 12.5 & - & 16.2 \\
\hline
\end{tabular}

Die erhaltenen $|D|$-Werte sind sehr hoch und sollten mit Vorsicht betrachtet werden. Der abrupte Abfall von $\chi_{M} T$ in diesem Typ von Komplexen ist sehr wahrscheinlich nicht nur auf Nullfeldaufspaltung, sondern auch auf intermolekulare Wechselwirkung zurückzuführen. Das Einbeziehen der Weiss-Konstante $\Theta_{W}$ für die Beschreibung der intermolekularen Wechselwirkung in die Anpassungsverfahren liefert z.B. für 11a den $|D|$-Wert von $0.93 \mathrm{~cm}^{-1}$ bei $\Theta_{W}=-2.01 \mathrm{~K}$. Dabei fällt allerdings eine starke Abhängigkeit zwischen dem $|D|$-Wert und der $\Theta_{W}$-Konstante auf. Zur Ermittlung glaubhafter $|D|$-Werte von diesen Komplexen müssen offensichtlich zusätzliche Experimente wie z.B. Hochfeld-EPR gemacht und ausgewertet werden.

Für die Interpretation der magnetischen Parameter für 11a-c und 12 scheint es sinnvoll, die einzelnen Fragmente dieser vierkernigen Komplexe separat zu betrachten. Ni1 und Ni2 (sowie Ni3 und Ni4) sind jeweils über Pyrazolat und den $\mu$-1,1-Azidliganden verbrückt. Entsprechend Schema 4.3.2.1.2. wird hierfür die Kopplung $J_{1}$, für die Wechselwirkung zwischen den bimetallischen Einheiten über das $\mu$-1,3-Azid die Kopplung $J_{2}$ zugeordnet. Wie erwartet, ist die intradimere Kopplung über die $\mu$-1,1-Azidbrücke positiv, was ferromagnetische Wechselwirkung bedeutet. Diese Werte sind allerdings etwas kleiner als in den zweikernigen Baueinheiten 1 und 2. Dies kann möglicherweise auf feine geometrische Unterschiede wie etwas größerer Ni1-N7-Ni2 Winkel in den vierkernigen (Tab. 4.3.2.1.3) im Vergleich mit den zweikernigen Verbindungen 1 und 2 (116.1 ${ }^{\circ}$ bzw. 116.3) zurückgeführt werden. 
Tab. 4.3.2.1.3. Ausgewählte Ausgewählte Winkel $\left({ }^{\circ}\right)$ von 11a,b und 12.

\begin{tabular}{llll}
\hline & $\mathbf{1 1 a}$ & $\mathbf{1 1 b}$ & $\mathbf{1 2}$ \\
\hline $\mathrm{Ni}-\mathrm{N}-\mathrm{Ni}$ & $117.5(2)$ & $116.8(1)$ & $118.3(1)$ \\
$\mathrm{Ni}-\mathrm{NNN}-\mathrm{Ni}$ & $93.0(5)$ & $91.4(3)$ & $36.1(1)$ \\
& $126.3(5) /$ & $126.0(3) /$ & $141.7(2) /$ \\
$\mathrm{Ni}-\mathrm{N}-\mathrm{N}$ & $133.5(5)$ & $134.8(3)$ & $148.1(2)$ \\
& & & \\
\hline
\end{tabular}

Es sollte angemerkt werden, dass auch die Koordinationszahlen und die Koordinationsgeometrien der zwei- und vierkernigen Komplexe unterschiedlich sind.

Der interdimere Austausch wird in erster Linie in Abhängigkeit von den zwei geometrischen Parametern, dem $\mathrm{Ni}-\mathrm{N}-\mathrm{N}$ Winkel und dem Torsionswinkel $\mathrm{Ni}-\mathrm{NNN}-\mathrm{Ni}$ entlang der $\mu$-1,3-Azidverbrückung, betrachtet (siehe Kapitel 4.1.2). Die für 11a-c und 12 gefundenen Austauschparameter sind in guter Übereinstimmung mit bekannten magnetostrukturellen Korrelationen und zeigen auch zusätzlich dazu den gravierenden Einfluss des Ni-NNN-Ni Torsionswinkels. Der merklich größere Ni-N-N Winkel von $142^{\circ} / 148^{\circ}$ in 12 im Vergleich mit 11a-c (ca. 126\%135 $)$ sollte eine stärkere ferromagnetische Kopplung verursachen. Die experimentellen Daten zeigen eine andere Tendenz. Die Kopplung ist antiferromagnetisch in 12 und ferromagnetisch in 11a,b. Dies ist offensichtlich durch den viel geringeren Ni-NNN-Ni Torsionswinkel von $36.1^{\circ}$ in 12 im Vergleich zur fast orthogonalen Situation in 11a,b (Ni-NNN-Ni nahe zu 90², Tab. 4.3.2.1.3, Abb. 4.3.2.1.4) bedingt. Der $\mathrm{Ni}-\left(\mu-1,3-\mathrm{N}_{3}\right)-\mathrm{Ni}$ Torsionswinkel ist offenbar der Hauptfaktor, der die beobachteten Unterschiede im magnetischen Verhalten in dieser Serie von vierkernigen Komplexen verursacht.

In 11c ist $\mu$-1,3-Azid partiell fehlgeordnet, und dementsprechend geben die magnetischen Daten das Mittel von verschiedenen geometrischen Orientierungen wieder. Der $J_{2}$-Wert liegt zwischen den für 11a,b und 12 gefundenen Werten. Die intradimere Kopplung wird durch die Fehlordnung nicht signifikant beeinflusst und ist etwa gleich den $J_{1}$-Weren für 11a,b und 12.
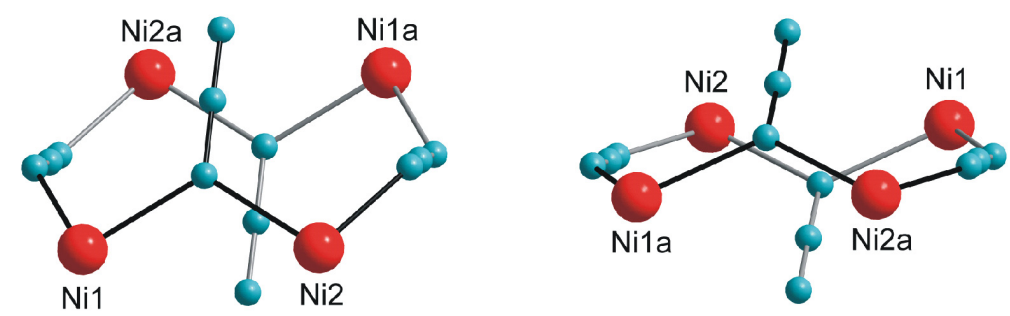

Abb.4.3.2.1.4. Ansicht der zentralen Fragmente von 11a (links) und 12 (rechts) entlang der $\mu-1,3-$ Azidbrücken. 
Interessanterweise ändern sich die magnetischen Eigenschaften von $\left.\left[\left(\mathrm{L}^{2}\right)_{2} \mathrm{Ni}_{4}\left(\mathrm{~N}_{3}\right)_{4}\right]\left(\mathrm{BPh}_{4}\right)_{2}\right] \cdot 2 \mathrm{C}_{3} \mathrm{H}_{6} \mathrm{O}$ (12) mit der Zeit, und Daten für den pulverförmigen Komplex 12*, welcher dann gebildet wird, wenn 12 entweder lange Zeit an der Luft liegt oder kurz im Vakuum gehalten worden ist, zeigen ferromagnetische Kopplung (Abb. 4.3.2.1.3 unten, Dreiecke), ähnlich zu 11a,b. Die IR-Spektren zeigen, dass die für die $\mathrm{C}=\mathrm{O}$ Schwingung von Aceton typische Bande bei $1707 \mathrm{~cm}^{-1}$ in 12 beobachtet wird (Aceton ist im Kristall eingebaut) und in 12* verschwindet. Änderungen in anderen Bereichen sind nicht signifikant, allerdings wird die Absorption in der Azid-Region beim Übergang von 12 zu 12* ähnlicher zu der von 11a,b (Tab. 4.3.2.1.1). Die Beobachtung, dass der Verlust von Lösungsmittelmolekülen das magnetische Verhalten kräftig beeinflussen kann, ist wohl bedingt durch eine damit verbundene Vergrößerung des Ni-NNN-Ni Torsionswinkels im $\mathrm{Ni}_{4}$-Fragment.

\section{Zusammenfassung}

Aus den für 11a-c und 12 erhaltenen Daten folgen drei prinzipiell wichtigen Ergebnisse:

1) $\mathrm{Ni}_{4}$-Komplexe mit der rechteckigen Anordnung der Metallzentren mit $\mu-1,1$ - und $\mu-1,3$ Azidbrücken können ausgehend von pyrazolatbasierenden zweikernigen Einheiten gezielt aufgebaut werden;

2) die Kopplung innerhalb der bimetallischen Untereinheiten ist ferromagnetisch, also gleich wie in den zweikernigen Ausgangskomplexen. Für die interdimere Kopplung zwischen solchen Einheiten ist der Ni-NNN-Ni Torsionswinkel der entscheidende Faktor, welcher auch den Grundzustand des Gesamtsystems bestimmt. 11a,b sind zwei seltene Beispiele von molekularen Komplexen, in welchen der Ni-NNN-Ni Torsionswinkel fast exakt $90^{\circ}$ ist. Das bedeutet, dass die magnetischen Orbitale der Metalle und die $p$-Orbitale des Azids orthogonal zu einander stehen. Dadurch wird die Vermittlung der ferromagnetischen Kopplung über die $\mu$-1,3-Azidbrücke möglich.

3) Die Fehlordnung der $\mu$-1,3-Azidbrücke in 11c sowie gravierende Änderungen des magnetischen Verhaltens in Abhängigkeit vom Vorhandensein von Solvatmolekülen in 12 illustriert die hohe Flexibilität der EE-Azidbrücke sogar im Festkörper. Das zeigt aber auch, dass die magnetostrukturellen Korrelationen zwischen den durch Röntgenstrukturanalyse an einem Einkristall gewonnenen geometrischen Parameter und den Daten magnetischer Messungen einer pulverförmigen Probe mit großer Vorsicht erstellt werden sollten. 


\subsubsection{Trapezförmige Anordnung der Nickel(II)-Zentren mit zentralen} $\mu-1,1,3-A z i d e n$

Es wurde jüngst über ein erstes Beispiel der bislang unbekannten Klasse von vierkernigen Nickel(II)-Komplexen berichtet, welche sich durch zwei unterschiedliche Azidbrücken auszeichnen. Bei einer dieser Azidbrücken handelt es sich um eine „echte“ (Ni-N-Abstände sind dabei etwa alle gleich) $\mu-1,1,3$-Azidverbrückung. ${ }^{[26]}$ Die Synthese neuer Verbindungen dieser Topologie sowie deren magnetischen Eigenschaften werden in diesem Kapitel beschrieben.

\section{Synthese und Charakterisierung}
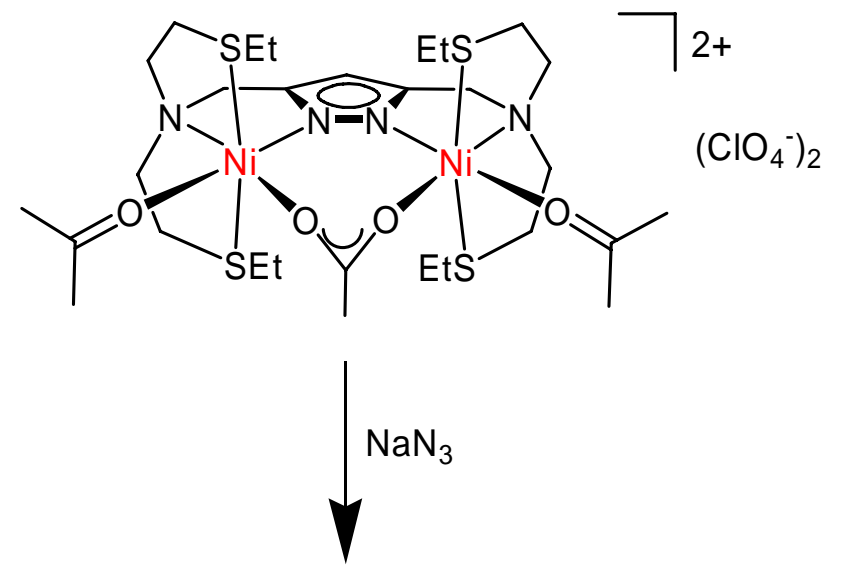

Das erste Exemplar $\left(\mathbf{1 3 a ^ { 1 ) }}\right)$ des neuen Typs 13 vierkerniger Verbindungen ist beim Versuch, ausgehend von der bimetallischen Einheit $\left[\mathrm{L}^{6} \mathrm{Ni}_{2}(\text { Ac })\right]^{2+}$ durch die Verknüpfung über Azid eine

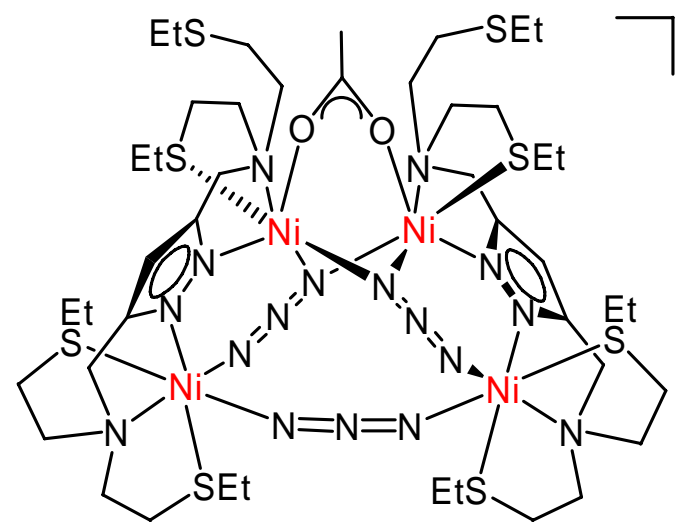

$13 a$

Schema. 4.3.2.2.1. Ursprünglicher Syntheseweg von 13a. $\mathbf{H L}^{7}: \mathrm{R}={ }^{i} \mathrm{Pr}$ ) sowie von verschiedenen
Carboxylationen sollte die Untersuchung der Flexibilität des zentralen Nickel(II)-Azid- Fragment in solchen Systemen erlauben.

\footnotetext{
${ }^{1)}$ Die Verbindung 13a wurde erstmalig von F. Meyer synthetisiert.
} 


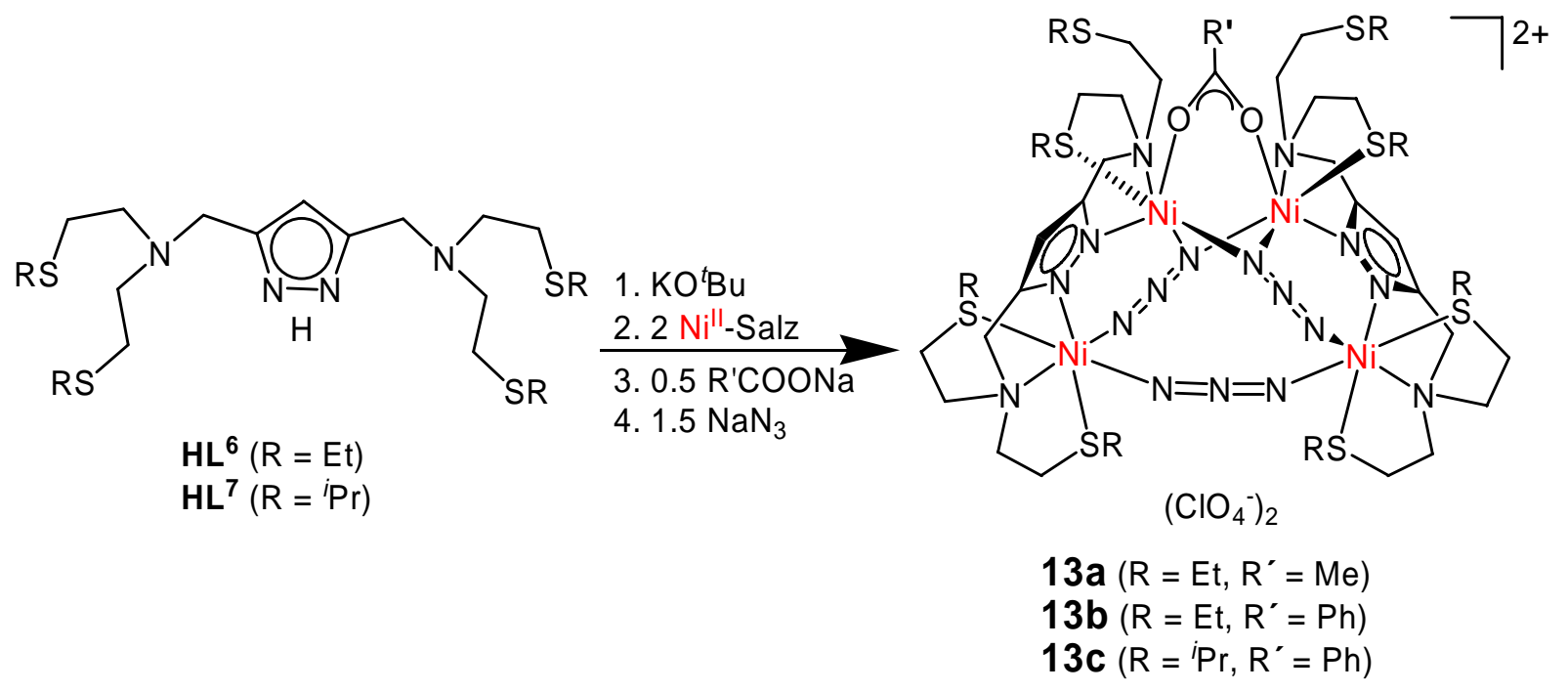

Schema. 4.3.2.2.2. Gezielte Synthese von 13a-c.

Die ersten Hinweise auf die Vierkernstruktur von 13a-b liefern die FAB-Massenspektren, in welchen das Basissignal (größte Intensität) dem Fragment $\left[\mathrm{LNi}_{2}\left(\mathrm{~N}_{3}\right)\left(\mathrm{O}_{2} \mathrm{CR}\right.\right.$ ') ${ }^{+}$zugeordnet ist, die aber auch jeweils das Fragment der vierkernigen Spezies $\left[\mathrm{L}_{2} \mathrm{Ni}_{4}\left(\mathrm{~N}_{3}\right)_{3}\left(\mathrm{O}_{2} \mathrm{CR}{ }^{\prime}\right)\left(\mathrm{ClO}_{4}\right)\right]^{+}$ zeigen. Die Übereinstimmung zwischen gefundenem und berechnetem Isotopenmuster ist am Beispiel von 13b in Abb.4.3.2.2.1. dargestellt.

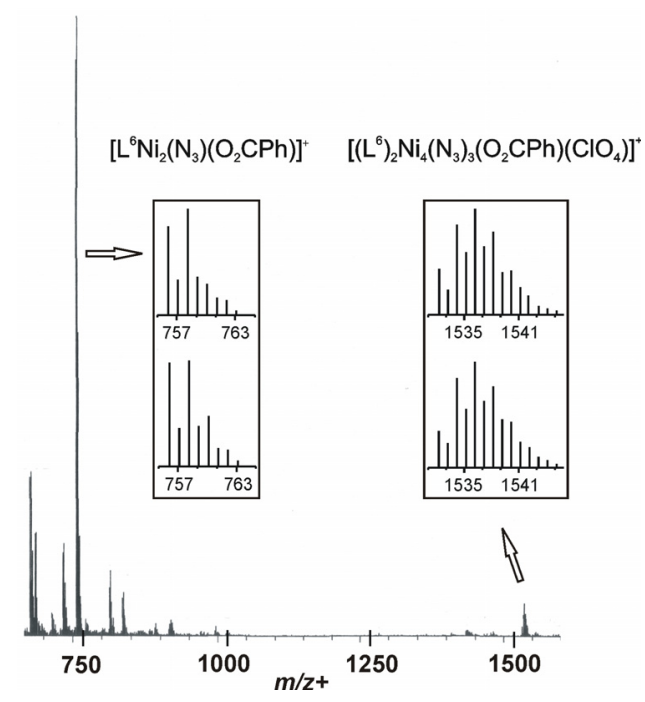

Abb. 4.3.2.2.1. FAB-Massenspektrum von 13b. Die Insets zeigen die experimentellen (unten) and theoretischen (oben) Isotopenverteilungen für zweikernige $\left[\mathrm{L}^{6} \mathrm{Ni}_{2}\left(\mathrm{~N}_{3}\right)\left(\mathrm{O}_{2} \mathrm{CPh}\right)\right]^{+}$bzw. vierkernige $\left[\left(\mathrm{L}^{6}\right)_{2} \mathrm{Ni}_{4}\left(\mathrm{~N}_{3}\right)_{3}\left(\mathrm{O}_{2} \mathrm{CPh}\right)\left(\mathrm{ClO}_{4}\right)\right]^{+}$Kationen. 
Das detaillierte Bild der molekularen Strukturen von 13a-c wurde mittels Röntgenstrukturanalyse erhalten. Die komplette Struktur des Kations sowie das in diesem Kation enthaltene ungewöhnliche zentrale Fragment von 13c sind in Abb.4.3.2.2.2 als typisches Beispiel für Komplextyp 13 dargestellt.
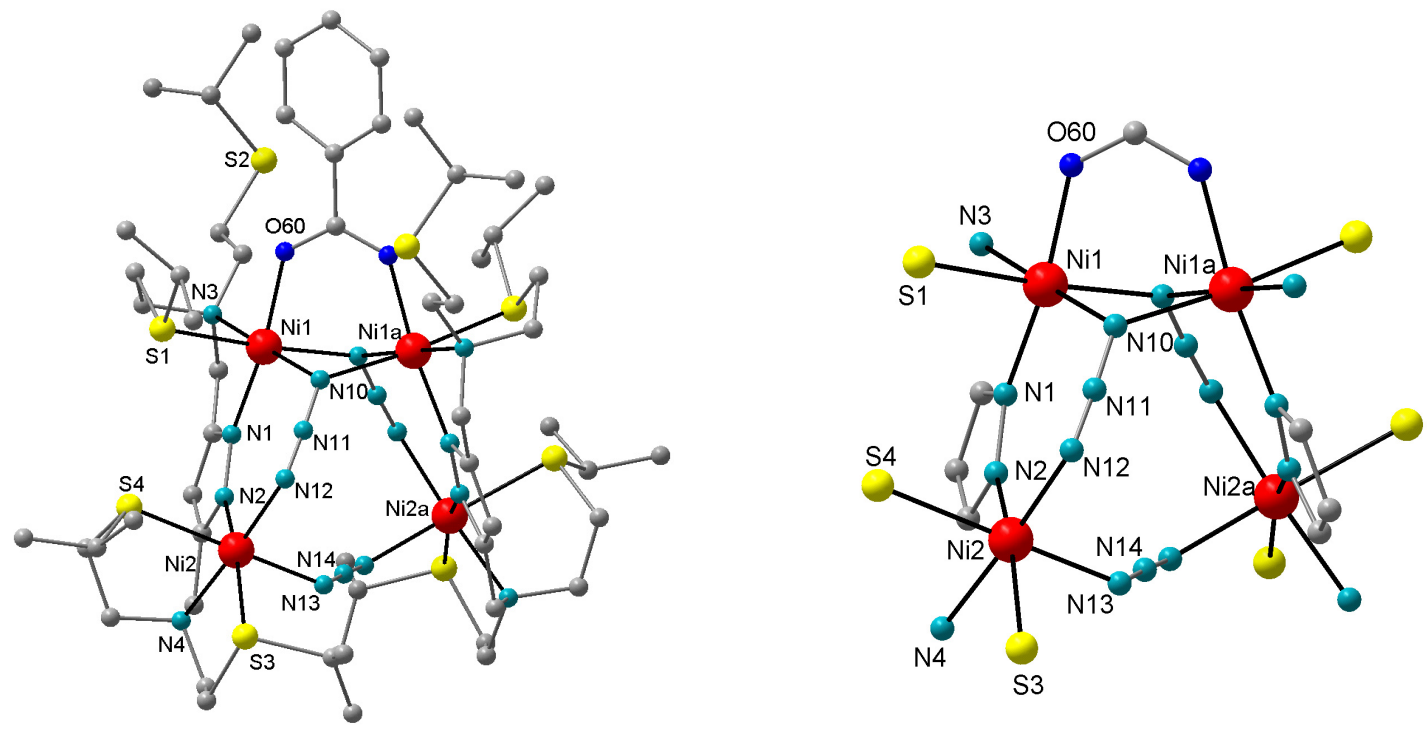

Abb. 4.3.2.2.2. Die Struktur des Kations der Verbindung 13c (links) und seines zentralen vierkernigen Fragments (rechts).

Ausgewählte Bindungsparameter und Winkel sind für alle drei Komplexe in Tab.4.3.2.2.1 zusammengefasst, wobei die Numerierung der Atome nach Abb. 4.3.2.2.3 erfolgt.

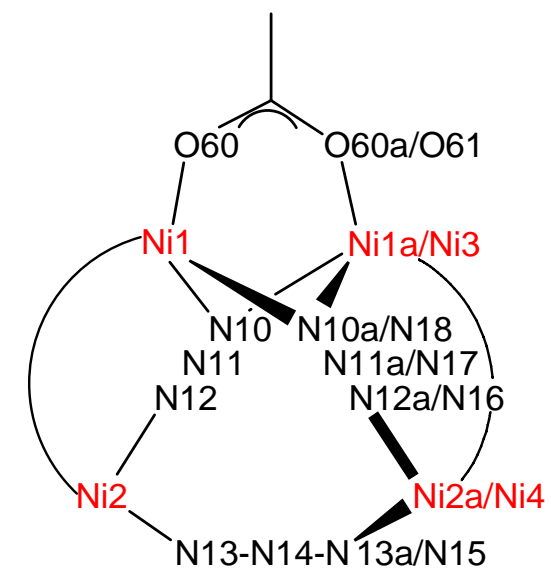

Abb. 4.3.2.2.3. Das Numerierungsschema der Atome in 13a-c. 
Zweikernige Nickel(II)-Azid-Komplexe als Baueinheiten für höhernukleare Systeme

13a konnte zwar mit zwei verschiedenen Solvatmolekülen (Aceton oder $\mathrm{CH}_{2} \mathrm{Cl}_{2}$ ) kristallisiert werden, in beiden Fällen sind die molekularen Strukturen aber nahezu identisch.

Tab. 4.3.2.2.1. Ausgewählte Bindungslängen $(\AA)$ und Winkel $\left(^{\circ}\right)$ von 13a, 13b und 13c.

\begin{tabular}{|c|c|c|c|c|}
\hline & 13a (aus Aceton) & 13a (aus $\mathrm{CH}_{2} \mathrm{Cl}_{2}$ ) & 13b & $13 c$ \\
\hline Ni1 $\cdots$ Ni1a(Ni3) & $3.151(1)$ & $3.171(1)$ & $3.175(1)$ & $3.181(2)$ \\
\hline $\mathrm{Ni} 1 \cdots \mathrm{Ni} 2 / / \mathrm{Ni} 3 \cdots \mathrm{Ni} 4$ & $4.479(1) / / 4.461(1)$ & $4.444(1) / / 4.451(1)$ & $4.466(1)$ & $4.449(2)$ \\
\hline $\mathrm{Ni} 2 \cdots \mathrm{Ni} 2 \mathrm{a}(\mathrm{Ni} 4)$ & $5.493(1)$ & $5.498(1)$ & $5.536(1)$ & $5.589(2)$ \\
\hline $\mathrm{Ni} 1 \cdots \mathrm{Ni} 2 \mathrm{a}(\mathrm{Ni} 4) / / \mathrm{Ni} 2 \cdots \mathrm{Ni} 1 \mathrm{a}(\mathrm{Ni} 3)$ & $5.499(1) / / 5.419(1)$ & $5.466(1) / / 5.427(1)$ & $5.406(1)$ & $5.494(2)$ \\
\hline Ni1-N10-Ni1a(Ni3) // Ni1-N18-Ni3 & $95.3(1) / / 94.4(1)$ & $97.0(1) / / 94.7(1)$ & $96.5(1)$ & $95.7(1)$ \\
\hline Ni1-N10-N11 // Ni3-N18-N17 & $120.2(2) / / 115.4(2)$ & $118.8(2) / / 115.2(2)$ & $118.8(2)$ & $115.1(2)$ \\
\hline $\begin{array}{l}\text { Ni1-N10a(N18)-N11a(N17) // } \\
\text { Ni3-N10-N11 }\end{array}$ & $124.0(2) / / 123.6(2)$ & $122.4(2) / / 125.7(2)$ & $123.8(2)$ & $129.8(2)$ \\
\hline Ni2-N12-N11 // Ni4-N16-N17 & $116.9(2) / / 122.3(2)$ & $117.9(2) / / 122.6(2)$ & $118.6(2)$ & $121.6(2)$ \\
\hline Ni2-N13-N14 // Ni4-N15-N14 & $128.1(2) / / 126.1(2)$ & $128.6(2) / / 122.7(2)$ & $125.6(2)$ & $126.0(2)$ \\
\hline Ni1-N10-N12-Ni2 // Ni3-N18-N16-Ni4 & $14.1(2) / / 10.4(2)$ & $20.7(2) / / 10.6(2)$ & $17.3(2)$ & $27.6(2)$ \\
\hline $\begin{array}{l}\text { Ni2-N12-N10-Ni1a(Ni3) // } \\
\text { Ni4-N16-N18-Ni1 }\end{array}$ & $108.0(2) / / 105.5(2)$ & $105.2(2) / / 104.3(2)$ & $105.4(2)$ & $95.6(2)$ \\
\hline Ni2-N13-N13a(N15)-Ni2a(Ni4) & $99.6(2)$ & 104.1(2) & $106.5(2)$ & $116.9(2)$ \\
\hline
\end{tabular}

Die zentralen Kerne aller drei Komplexe sind sehr ähnlich. Die vierkernige Einheit besteht aus zwei $\mathrm{LNi}_{2}$ Fragmenten, die über drei Azidionen und ein Carboxylation verbrückt sind (für 13b und 13c gibt es eine kristallographische $C_{2}$-Achse zwischen den zwei Einheiten). Zwei der Azidionen sind in einem sehr seltenen $\mu$-1,1,3-Koordinationsmodus, das dritte Azidion verbrückt Ni2 und Ni2a bzw. Ni4 im $\mu$-1,3-Modus. Die Ni-N $\mathrm{N}_{\text {Azid }}$-Bindungslängen für $\mu_{3}$ 1,1,3-Azidverbrückung (2.094(2)-2.160(3) $\AA$ ) sind zwar etwas länger als für $\mu$-1,3-Azid (2.058(3)-2.091(3) Å), liegen aber im üblichen Bereich für Azidbrücken. In Gegensatz dazu zeigen einige andere bekannte Systeme mit $\mu$-1,1,3-Azidbrücke normalerweise einen sehr langen M-N-Abstand (siehe Kapitel 4.1.4).

Für das Verständnis magnetischer Eigenschaften sind folgende geometrische Parameter wichtig:

1) die Ni-N-Ni Winkel des EO-verbrückenden Fragments von $\mu$-1,1,3-Azid, welche im Bereich von 94.4(1)-97.0(1) ${ }^{\circ}$ liegen;

2) die Ni-N-N Winkel (siehe Tab. 4.3.2.2.1); 
3) die Ni-NNN-Ni Torsionswinkel entlang der $\mu$-1,1,3-Azidbrücke, welche im Bereich von $10.4-27.6^{\circ}$ (Ni1/Ni2 und $\left.\mathrm{Ni} / \mathrm{Ni} 4\right)$ und 95.6-108.0 $(\mathrm{Ni} 1 / \mathrm{Ni} 2 \mathrm{a}(\mathrm{Ni} 4)$ und $\mathrm{Ni2} / \mathrm{Ni} 1 \mathrm{a}(\mathrm{Ni} 3))$ liegen;

4) die Ni-NNN-Ni-Torsionswinkel entlang der $\mu$-1,3-Azidbrücke mit Werten von 99.6$116.9^{\circ}$.

Trotz sperriger Isopropylsubstituenten an den Ligandseitenarmen in 13c unterscheiden sich die geometrischen Parameter aller dreier Verbindungen nur wenig. Diese Unterschiede könnten jedoch deutliche Einflüsse auf die magnetischen Eigenschaften von 13a-c, wie es weiter gezeigt wird, haben. Die vierkernigen Einheiten sind durch die Ligandhülle gut abgeschirmt und dadurch von benachbarten Nickel(II)-Ionen separiert: die kürzesten $\mathrm{Ni} \cdots \mathrm{Ni}$ Abstände zwischen den $\mathrm{Ni}_{4}$-Einheiten sind $7.03 \AA$ (13a, aus Aceton), $7.18 \AA$ (13a, aus $\left.\mathrm{CH}_{2} \mathrm{Cl}_{2}\right), 7.63 \AA$ (13b) und $8.60 \AA$ (13c).

Die Werte der $v_{\text {as }}\left(\mathrm{N}_{3}\right)$ Schwingungen sind in Tab. 4.3.2.2.2 zusammengefasst. Bei allen Komplexen wird eine mittelstarke Bande bei ca. $2043 \mathrm{~cm}^{-1}$ und eine sehr starke Bande bei etwa $2083 \mathrm{~cm}^{-1}$ (mit der zusätzlichen Schulter bei $2092 \mathrm{~cm}^{-1}$ im Falle von 13a) beobachtet.

Tab. 4.3.2.2.2. Ausgewählte IR-Banden für 13a, 13b und 13c.

\begin{tabular}{ll}
\hline Komplex & $\mathrm{v}_{\mathrm{as}}\left(\mathrm{N}_{3}{ }^{-}\right), \mathrm{cm}^{-1}$ \\
\hline 13a (aus Aceton) & $2042(\mathrm{~m}), 2081(\mathrm{vs}), 2092(\mathrm{sh})$ \\
13b & $2043(\mathrm{~m}), 2083(\mathrm{vs})$ \\
13c & $2043(\mathrm{~m}), 2083(\mathrm{vs})$ \\
\hline
\end{tabular}

Die Werte für $v_{\mathrm{as}}$ von 2040-2090 $\mathrm{cm}^{-1}$ liegen etwa im gleichen Bereich wie für alle ânderen zwei- und vierkernigen pyrazolatbasierten Nickel(II)-Verbindungen mit unterschiedlichen Azidkoordinationen. ${ }^{[104,105]}$

\section{Magnetische Eigenschaften}

Die $\chi_{M} T$-Werte von 13a-c nehmen mit sinkender Temperatur rapide ab. Bei Raumtemperatur liegen diese Werte im Bereich von 3.35-3.62 $\mathrm{cm}^{3} \mathrm{Kmol}^{-1}$. Das ist viel kleiner als der theoretisch erwartete „spin-only“ Wert für vier ungekoppelte $S=1$ Nickel(II)Ionen (4.83 $\mathrm{cm}^{3} \mathrm{Kmol}^{-1}$ für $\left.g=2.20\right)$ und weist auf eine resultierende sehr starke antiferromagnetische Wechselwirkung in $\mathrm{Ni}_{4}$-Fragmenten hin, welche auch durch ein breites Maximum bei $170-200 \mathrm{~K}$ im Diagramm $\chi_{M}$ gegen $T$ bestätigt wird. 


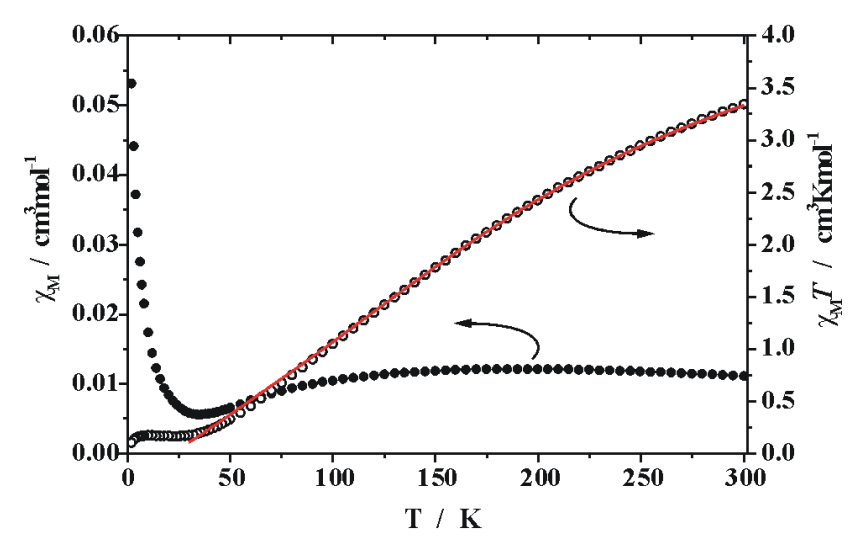

Die $\chi_{M} T$-Werte tendieren im Einklang mit dominanter antiferromagnetischer Kopplung und dem $S_{T}=0$ Grundzustand schließlich nach Null. Der Anstieg von $\chi_{M}$ bei sehr tiefen Temperaturen ist möglicherweise durch eine geringfügige Menge von paramagnetischen

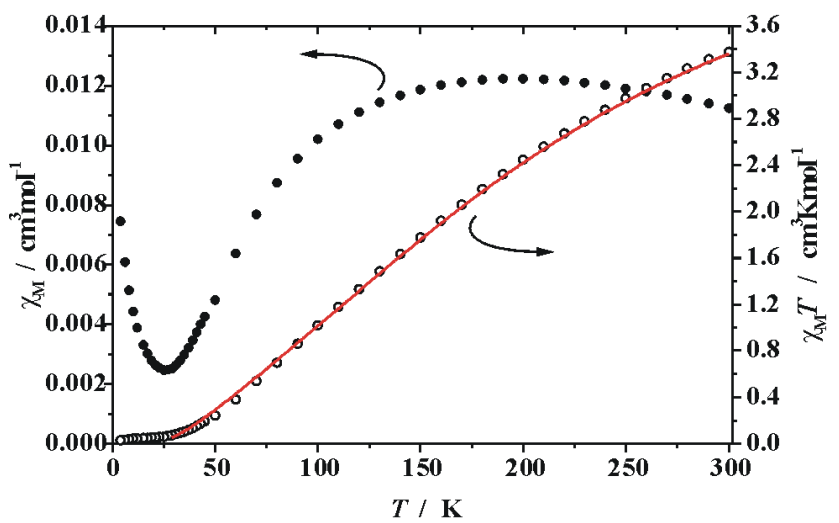

Verunreinigungen verursacht (Abb. 4.3.2.2.4).

Unter Berücksichtigung der molekularen Topologie können sechs Pfade für den magnetischen Austausch vorgeschlagen werden (Schema 4.3.2.2.3).
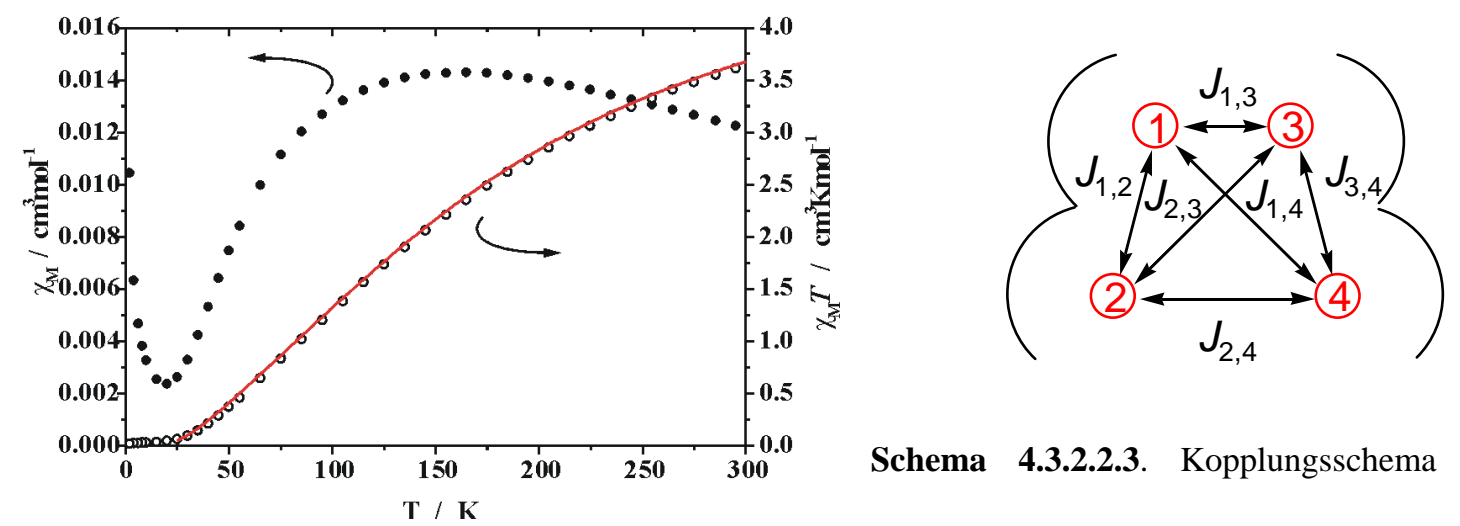

Schema 4.3.2.2.3. Kopplungsschema für $\mathrm{Ni}_{4^{-}}$ Komplexe mit der trapezförmigen Anordnung der

Abb. 4.3.2.2.4. Auftragung von $\chi_{M}$ und $\chi_{M} T$ gegen $T$ für 13a (oben), 13b (in der Mitte) und 13c (unten). Die roten Nickel(II)-Zentren.

Linien repräsentieren die besten Anpassungen.

Für die Vereinfachung der Interpretation magnetischer Daten wird angenommen, dass alle Komplexe dieses Typs zweizählige Symmetrie mit $J_{1,2}=J_{3,4}$ und $J_{1,4}=J_{2,3}$ haben. Damit enthält das Kopplungsschema eine intradimere Kopplung über Pyrazolat und den cis-Typ der end-to-end Verbrückung von $\mu_{3}-1,1,3$-Azid $\left(J_{1,2} / J_{3,4}\right)$ und drei interdimere Kopplungen: die erste erfolgt über das Carboxylat und die end-on Verbrückung der $\mu_{3}-1,1,3$-Azide $\left(J_{1,3}\right)$, die zweite ist entlang der $\mu$-1,3-Azidbrücke $\left(J_{2,4}\right)$ und die dritte entlang der Diagonalen (transTyp der end-to-end Verbrückung von $\mu_{3}$-1,1,3-Azid, $\left.J_{1,4} / J_{2,3}\right)$.

Die Anpassung an die experimentellen Daten erfolgte unter Verwendung eines Programms, ${ }^{[109]}$ basierend auf einem Heisenberg-Dirac-van-Vleck-Hamiltonoperator mit 
einem zusätzlichen Term für die Zeeman-Aufspaltung (4.3.2.2.1). Die $g$-Werte wurden für alle Positionen als identisch angenommen. Die Nullfeldaufspaltung $|D|$ wurde nicht berücksichtigt, um die Zahl der Parameter zu limitieren.

$$
\begin{aligned}
\hat{H}= & -2 J_{1,2}\left(\hat{S}_{1} \hat{S}_{2}+\hat{S}_{3} \hat{S}_{4}\right)-2 J_{1,3}\left(\hat{S}_{1} \hat{S}_{3}\right)-2 J_{2,4}\left(\hat{S}_{2} \hat{S}_{4}\right)- \\
& -2 J_{1,4}\left(\hat{S}_{1} \hat{S}_{4}+\hat{S}_{2} \hat{S}_{3}\right)+g \mu_{B} B \sum \hat{S}_{i z}
\end{aligned}
$$

Da paramagnetische Verunreinigungen und die Nullfeldaufspaltung nicht berücksichtigt werden konnten, wurden nur die Daten oberhalb 30 K (13a), 29K (13b) bzw. 25 K (13c) in die Anpassungsprozedur einbezogen. Die roten Linien in Abb. 4.3.2.2.4 repräsentieren die besten Anpassungen für 13a-c, resultierende Parameter sind in Tab. 4.3.2.2.3 zusammengefasst. Für alle Komplexe findet man zwei dominierende antiferromagnetische sowie zwei ferromagnetische Kopplungen. Ein alternatives Modell mit drei antiferromagnetischen und einer ferromagnetischen Wechselwirkungen liefert deutlich schlechtere Anpassungsergebnisse. Es sollte angemerkt werden, dass dank der Symmetrie des Hamiltonoperators die einzelnen Kopplungen $J_{1,3}, J_{1,2} / J_{3,4}, J_{1,4} / J_{2,3}$, und $J_{2,4}$ nicht eindeutig den topologischen $J$-Werten in Schema 4.3.2.2.3 zugeordnet sind. Die getroffene Zuordnung basiert auf bekannten magnetostrukturellen Korrelationen für Nickel(II)-Azid-Systeme.

Tab. 4.3.2.2.3. Anpassungsparameter der magnetischen Daten für 13a-c.

\begin{tabular}{llll}
\hline & 13a & 13b & 13c \\
\hline$J_{1,3}, \mathrm{~cm}^{-1}$ & +57 & +25 & +27 \\
$J_{1,2} / J_{3,4}, \mathrm{~cm}^{-1}$ & -51 & -61 & -53 \\
$J_{2,3} / J_{1,4}, \mathrm{~cm}^{-1}$ & -18 & -12 & -3 \\
$J_{2,4}, \mathrm{~cm}^{-1}$ & +6 & +5 & +6 \\
$g$ & 2.29 & 2.38 & 2.30 \\
\hline
\end{tabular}

Die zusätzliche Anpassung an die magnetischen Daten von 13c als Vertreter dieses Komplextyps mit dem Programm $j u l X^{[106]}$ hat gezeigt, dass bei einem System mit so vielen Parametern (vier verschiedene Kopplungskonstanten, $g$-Faktor, Anteil der paramagnetischen Verunreinigung, TIP) eine Abhängigkeit der ermittelten Werte von den Startparametern existiert. Trotzdem wird bei allen Anpassungsversuchen eine recht stark positive und eine große negative Kopplungskonstante erhalten, wie nach bekannten magneto-strukturellen Korrelationen zu erwarten. Die beiden weiteren Kopplungskonstanten können kleine positive oder kleine negative Werte annehmen, was auch durch die passenden Ni-NNN-Ni 
Torsionswinkel, die im Bereich $90 \pm 25^{\circ}$ für interdimere Brücken liegen, zu erwarten wäre. Für die weitere Diskussion werden die Werte aus der Tab. 4.3.2.2.3 verwendet.

Für die Interpretation der magnetischen Parameter für 13a-c ist es ratsam, die einzelnen Fragmente dieser vierkernigen Komplexe zu betrachten. Ni1 und Ni2 (sowie Ni3 und Ni4) sind über Pyrazolat und end-to-end Azid (als Teil des $\mu_{3}$-1,1,3-Azids verbrückt, zugeordnet zu $J_{1,2}$ und $J_{3,4}$ in Schema 4.3.2.2.3. Während das Pyrazol sicherlich zum Austausch auch beiträgt, ist es sehr wahrscheinlich, dass Azid den dominanten Austausch innerhalb der pyrazolatbasierten Einheiten vermittelt. ${ }^{[110]}$ Es gibt auch zwei interdimere end-to-end

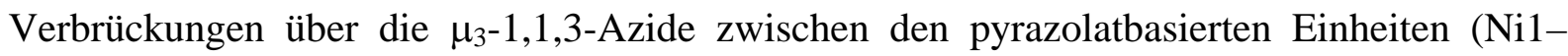
NNN-Ni2a/Ni4 und Ni2-NNN-Ni1a/Ni3, zugeordnet zu $J_{1,4}$ und $J_{2,3}$ ). Diese verschiedenen Typen von end-to-end Azidkoordination als Teil des $\mu_{3}$-1,1,3-Azidliganden unterscheiden sich sehr stark in der Größe der Ni-NNN-Ni Torsionswinkel. Diese Winkel sind klein (10.4$\left.27.6^{\circ}\right)$ innerhalb der Dimeren und sehr groß (95.6-108.0) zwischen der Dimeren. Ni2 und Ni2a (bzw. Ni4) sind über ein $\mu_{2}$-1,3-Azid verknüpft. Der Austauschpfad wird als $J_{2,4}$ gezeichnet.

Für die Interpretation der magnetischen Wechselwirkungen vermittelt über eine $\mu$-1,3Azidbrücke werden in erster Linie zwei geometrische Parameter herangezogen: der Ni-N-N Winkel und der Ni-NNN-Ni Torsionswinkel entlang der $\mu$-1,3-Azidverbrückung (die magnetostrukturellen Korrelationen dafür siehe Kapitel 4.1.2). Im Falle von 13a-c liegen die Ni-N-N Winkel für die intradimere Verbrückung über das $\mu_{3}-1,1,3-A z i d$ im Bereich von 115.6-121.7 ${ }^{\circ}$ und die Ni1-NNN-Ni2 bzw. Ni3-NNN-Ni4 Torsionswinkel weichen nicht sehr stark von Null (10.4-27.6 $)$ ab. Die größte antiferromagnetische Kopplung von etwa -50 $\mathrm{cm}^{-1}$ kann somit mit Sicherheit den intradimeren Kopplungen $J_{1,2} / J_{3,4}$ zugeordnet werden. Innerhalb der Serie von 13a-c ist der intradimere Ni-NNN-Ni Torsionswinkel mit $27.6^{\circ}$ für 13c größer als für 13a und 13b $\left(10.4-20.7^{\circ}\right)$. Man sollte bei der Erstellung von Korrelationen zwischen den magnetischen Austauschparametern mit nur kleinen Unterschieden und den geometrischen Parametern, gewonnen durch Röntgenstrukturanalyse an einem Einkristall, in gewissem Rahmen vorsichtig sein. Erstens können die Werte aus Tab. 4.3.2.2.3 nur als ungefähre Werte angesehen werden, weil solche Effekte wie z.B. Nullfeldaufspaltung nicht berücksichtigt wurden. Zweitens verwittern im Falle von 13a and 13b die Kristalle, was zu kleinen Änderungen struktureller Parameter führen könnte.

Die interdimeren Ni1-NNN-Ni2a/Ni4 und Ni2-NNN-Ni1a/Ni3 Torsionswinkel der diagonalen end-to-end Verbrückungen der $\mu_{3}$-1,1,3-Azide liegen für 13a und 13b im Bereich von $104.3-108.0^{\circ}$. Noch näher der Orthogonalität liegt mit $\tau=95.6^{\circ}$ 13c. Dementsprechend 
sollte die antiferromagnetische Kopplung $J_{2,3} / J_{1,4}$ viel kleiner als die intradimere Kopplung $J_{1,2} / J_{3,4}$ sein. Das wird auch experimentell beobachtet. Obwohl der Torsionswinkel nahe $90^{\circ}$ liegt, bleibt die Kopplung schwach antiferromagnetisch. Das kann auf relativ kleine Ni-NN Winkel (ca. $120^{\circ}$ ) zurückgeführt werden. Auf der anderen Seite weist die $\mu$-1,3-Azidbrücke zwischen Ni2 und Ni2a/Ni4 einen etwas größeren Ni-N-N Winkel (ca. 126º) auf. Zusammen mit dem entsprechenden $\mathrm{Ni}-\mathrm{NNN}-\mathrm{Ni}$ Torsionswinkel $\left(\tau=99.6-116.9^{\circ}\right)$ reicht dies offensichtlich aus, um eine schwache ferromagnetische Kopplung von $J_{2,4}=+5 \mathrm{~cm}^{-1}$ (bzw. +6 $\mathrm{cm}^{-1}$ ) für 13b (bzw. für 13a,c) zu vermitteln. Es ist interessant anzumerken, dass die Kombination der starken ferromagnetischen Kopplung im $\left\{\mathrm{Ni}\left(\mu-1,1-\mathrm{N}_{3}\right)_{2} \mathrm{Ni}\right\}$-Fragment zwischen Ni1 und Ni3/Ni1a ( $J_{1,3}$, siehe unten) und dem dominanten antiferromagnetischen intradimeren Austausch $\left(J_{1,2}, J_{3,4}\right)$ zum gewissen Grad an Frustration in der $\mathrm{Ni}_{4}$-Anordnung führen sollte, wenn die interdimere Kopplung $J_{2,4}$ nicht ferromagnetisch wäre.

Die magnetostrukturellen Korrelationen für die zweifach end-on azidverbrückten Nickel(II)-Komplexe sagen bei allen $\theta$-Werten ferromagnetische Kopplung mit einem Maximum bei $\theta=104^{\circ}$ voraus (siehe Kapitel 4.1.3). Die Ni1-N10-Ni3/Ni1a und Ni1-N18-

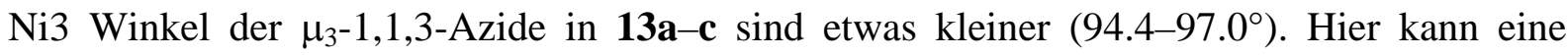
signifikante ferromagnetische Wechselwirkung zwischen Ni1 und Ni1a/Ni3 erwartet werden. Das wird durch die für $J_{1,3}$ beobachteten Werte bestätigt (Tab. 4.3.2.2.3). Der Hauptunterschied in den Strukturen zwischen 13a $\left(J_{1,3}=+57 \mathrm{~cm}^{-1}\right)$ und 13b,c $\left(J_{1,3}=+25\right.$ bzw. $+27 \mathrm{~cm}^{-1}$ ) ist das Vorhandensein der Acetatbrücke in 13a und der Benzoatbrücke in 13b,c. Diese Carboxylationen verbrücken Ni1 und Ni1a/Ni3 zusätzlich zu Azidionen. Die ungewöhnlich große Koppungskonstante in 13a kann über den kontraKomplementaritätseffekt (siehe Kapitel 4.1.3) erklärt werden. Das wirkt sich bei der Anwesenheit des Acetations zusätzlich zu zwei $\mu$-1,1-Azidbrücken als verstärkt ferromagnetische Kopplung aus. Es liegt die Vermutung nahe, dass in 13b und 13c mit einer zusätzlichen Benzoatbrücke zwischen Ni1 und Ni1a dieser Effekt weniger ins Gewicht fällt und infolge dessen etwas kleinere ferromagnetische Kopplungen von $J_{1,3}=+25 \mathrm{~cm}^{-1}$ bzw. $+27 \mathrm{~cm}^{-1}$ resultieren.

\section{Zusammenfassung}

Eine Serie von vierkernigen Nickel(II)-Verbindungen ungewöhnlicher Topologie mit sehr seltenen $\mu_{3}$-1,1,3-Azidbrücken wurde sowohl strukturell als auch magnetochemisch charakterisiert. Es wurden zwei ferromagnetische und zwei antiferromagnetische 
Wechselwirkungen festgestellt, die über verschiedene Azidpfade zustande kommen. Das Zusammenspiel dieser Wechselwirkungen führt zum $S_{T}=0$ Spingrundzustand. Die durch Anpassung der Daten der Suszeptibilitätsmessungen erhaltenen Kopplungskonstanten wurden auf der Basis der für end-to-end und end-on Azidbrücken üblichen magnetostrukturellen Korrelationen zugeordnet. Die magnetostrukturellen Korrelationen für die $\mu_{3}-1,1,3-$ Azidbrücke lassen sich also durch die gewonnenen Ergebnisse als Summe der Wechselwirkungen der einzelnen end-to-end und end-on Fragmente formulieren.

\subsubsection{Rechteckige Anordnung der Nickel(II)-Zentren mit einem zentralen $\mu$ - 1,1,3,3-Azid}

Kürzlich wurde von F. Meyer über die ersten Beispiele dieser neuen Klasse von vierkernigen Nickel(II)-Komplexen berichtet. ${ }^{[26]}$ Die Besonderheit dieser Komplexe ist das Vorhandensein von „echter“ (haben alle etwa gleich lange Ni-N-Abständen) $\mu_{4}-1,1,3,3-$ Azidkoordination. Diesen seltenen Koordinationsmodus konnte man in zwei verschiedenen Komplextypen beobachten, was auf eine hohe strukturelle Flexibilität solcher vierfach verbrückender Azide hindeutet. In diesem Kapitel wird die Synthese neuer Verbindungen mit $\mu_{4}$-1,1,3,3-Azidkoordination beschrieben und die Abhängigkeit der magnetischen Eigenschaften von der Struktur untersucht.

\section{Synthese und Charakterisierung}

Für die Synthese von 14c kann man den im vorherigen Kapitel beschriebenen Komplex 13a verwenden, indem man ein zusätzliches Äquivalent von Natriumacetat zur Lösung von 13a hinzufügt. Dabei werden die koordinativen Bindungen zweier Thioethylgruppen von 13a gelöst und unter Reorganisierung der vorhandenen Azidliganden entsteht 14c (Schema 4.3.2.3.1). ${ }^{[26]}$ 


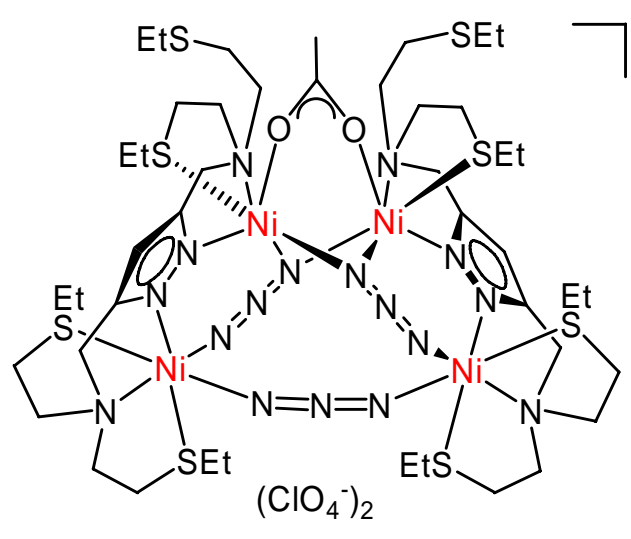

$13 a$

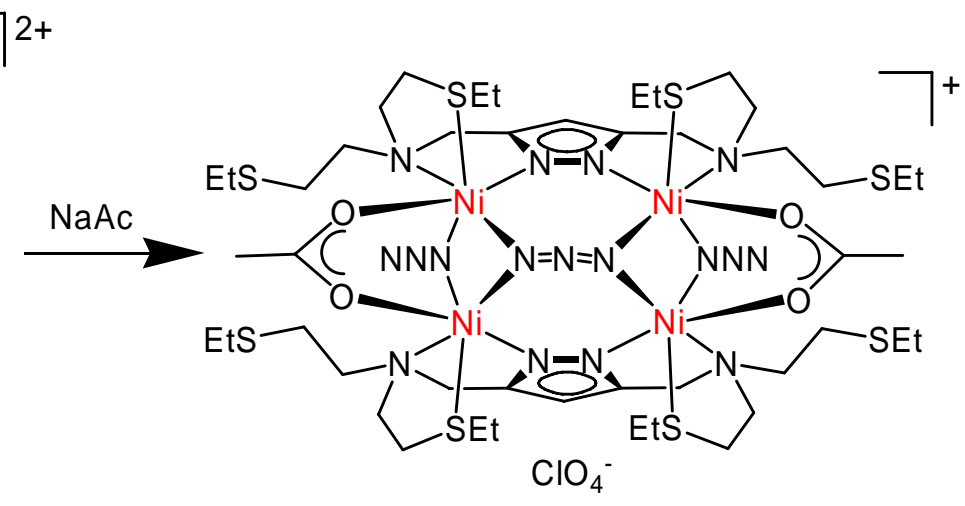

$14 c$

Schema. 4.3.2.3.1. Ursprünglicher Syntheseweg von 13a.

Die Komplexe der Typen 14 und 15 können ebenso wie des Typs 13 auch direkt aus den entsprechenden Komponenten in stöchiometrischen Mengen erhalten werden. ${ }^{1), ~}{ }^{[111]}$ In allen Komplexen 14 und 15 sind zwei $\left\{\mathrm{LNi}_{2}\right\}$ Baueinheiten über ein zentrales $\mu_{4}$-Azid unter Bildung der vierkernigen Anordnung verbrückt (Abb. 4.3.2.3.1). Die beiden $\left\{\mathrm{LNi}_{2}\right\}$ Einheiten werden im Falle von 14 zusätzlich durch jeweils zwei $\mu$-1,1-Azidbrücken und zwei Carboxylatgruppen, im Falle von 15 ausschließlich durch vier Carboxylatgruppen verknüpft. Für Komplextyp 15 konnten kristalline Substanzen in entsprechenden Mengen und mit guter Qualität nur mit dem Adamantylcarboxylat erhalten werden.

\footnotetext{
1) Die Verbindungen 14c und 15b wurden erstmalig von F. Meyer synthetisiert.
} 


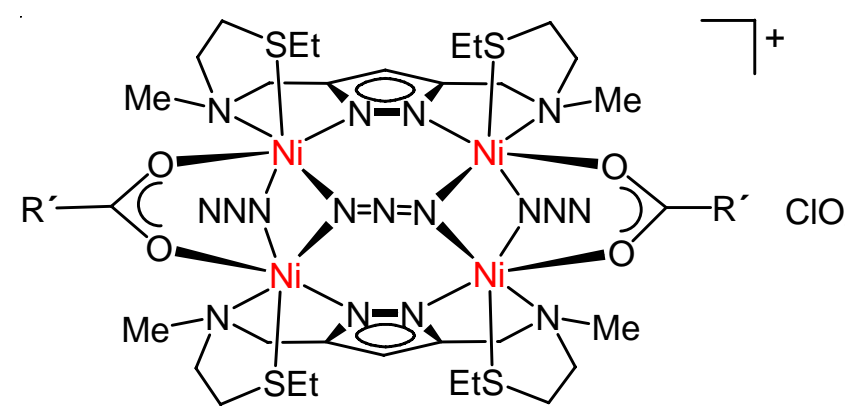

$14 a\left(R^{\prime}=M e\right)$

$14 b\left(R^{\prime}=P h\right)$

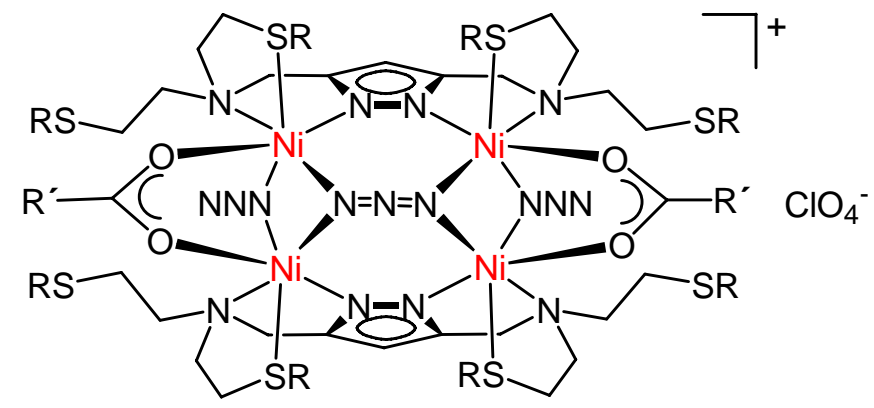

14c $\left(R=E t, R^{\prime}=M e\right)$

14d $\left(R={ }^{i} \operatorname{Pr}, R^{\prime}=M e\right)$

14e $\left(\mathrm{R}={ }^{i} \mathrm{Pr}, \mathrm{R}^{\prime}=\mathrm{Ada}\right)$

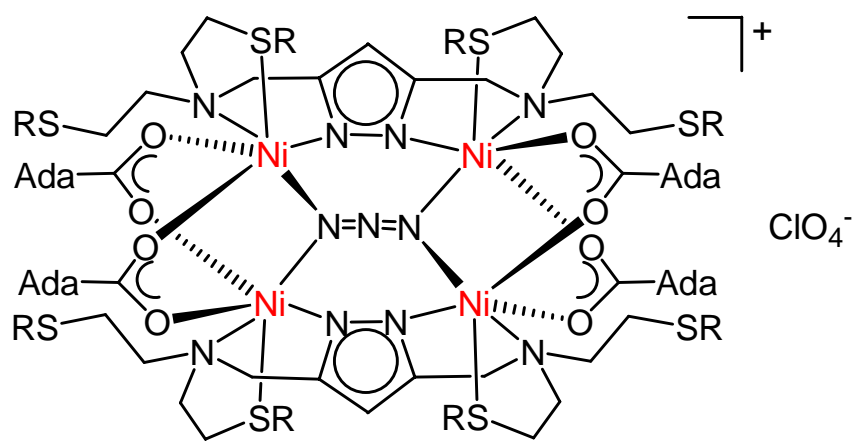

$15 \mathbf{a}(\mathrm{R}=\mathrm{Me})$

$15 b(R=E t)$

Abb. 4.3.2.3.1. Komplexe des Typs 14 und 15.

ESI- sowie FAB-Massenpektometrie sind geeignete analytische Methoden, um die vierkernige Natur dieser Verbindungen zu identifizieren, weil die entsprechenden Signale einfach geladener Kationen $\left[\mathrm{L}_{2} \mathrm{Ni}_{4}\left(\mathrm{~N}_{3}\right)_{3}\left(\mathrm{O}_{2} \mathrm{CR}\right)_{2}\right]^{+}$bzw. $\left[\mathrm{L}_{2} \mathrm{Ni}_{4}\left(\mathrm{~N}_{3}\right)\left(\mathrm{O}_{2} \mathrm{CR}\right)_{4}\right]^{+}$gut detektiert werden können. Das FAB-Massenpektrum von 14e ist als Beispiel abgebildet (Abb.4.3.2.3.2). 


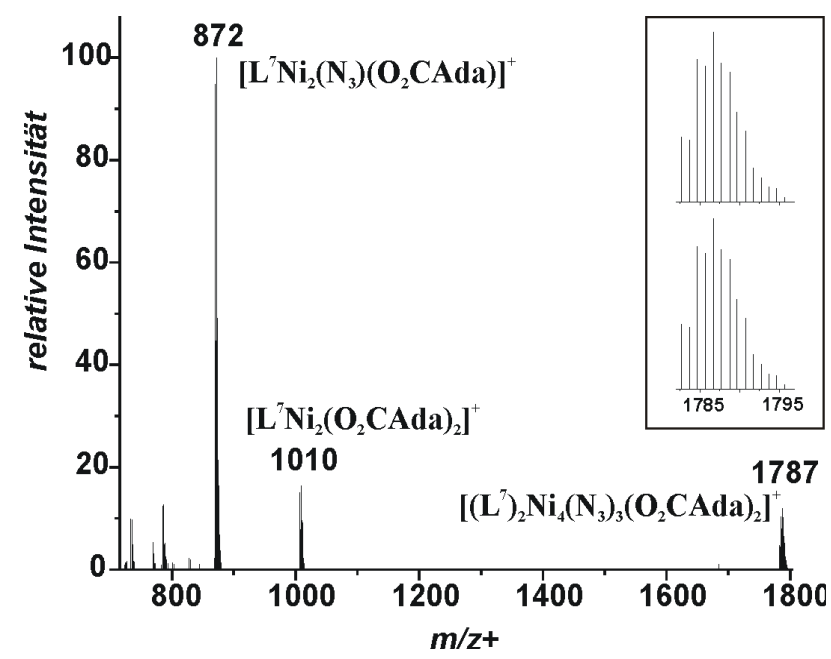

Abb. 4.3.2.3.2. FAB-Massenpektrum von 14e. Die Inset zeigt die experimentelle (unten) und theoretische (oben) Isotopenverteilung für das vierkernige Kation $\left[\left(\mathrm{L}^{7}\right)_{2} \mathrm{Ni}_{4}\left(\mathrm{~N}_{3}\right)_{3}\left(\mathrm{O}_{2} \mathrm{CAda}\right)\right]^{+}$.

Da für die Diskussion der magnetostrukturellen Korrelationen die genauen geometrischen Parameter nötig sind, wurden 14a-c,e und 15a,b röntgenographisch untersucht. Die molekularen Strukturen von 14b, 14e und 15b sowie deren zentrale vierkernige Fragmente sind in Abb. 4.3.2.3.2-4.3.2.3.4 gezeigt; ausgewählte Bindungslängen und Winkel sind in Tab.4.3.2.3.1 zusammengefasst.
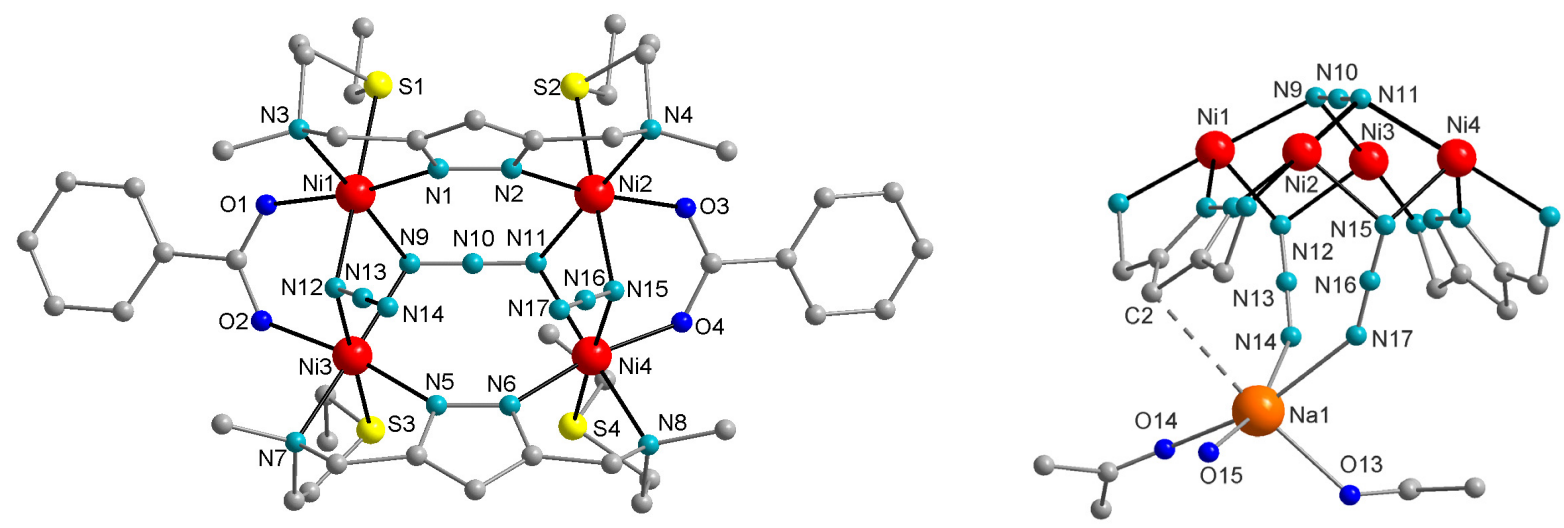

Abb. 4.3.2.3.2. Struktur des Kations der Verbindung 14b (links) und seines zentralen vierkernigen Fragments (rechts). Ausgewählte Bindungslängen ( $\AA$ ): Na1-O14 2.290(2), Na1-O15 2.335(3), Na1-O13 2.389(2), Na1-N17 2.570(3), Na1-N14 2.543(3), Na1-C2 2.994(3). 


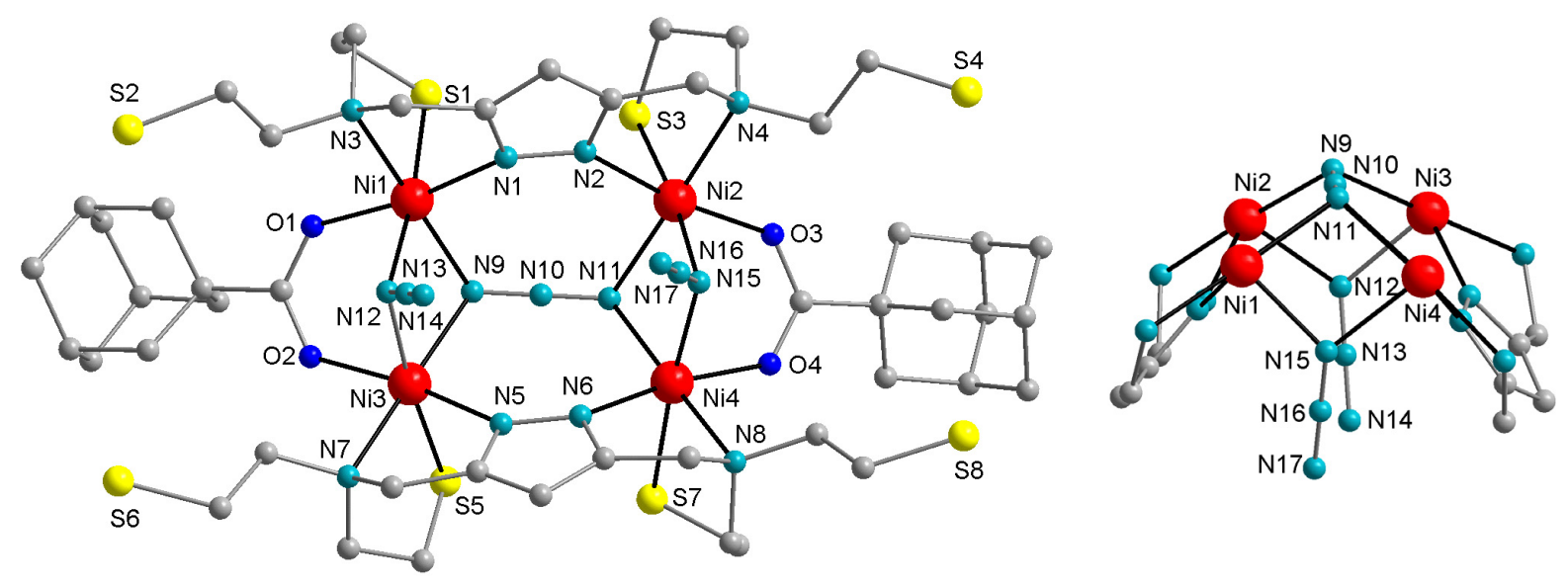

Abb. 4.3.2.3.3. Struktur des Kations der Verbindung 14e (links) und seines zentralen vierkernigen Fragments (rechts). Aus Gründen der Übersichtlichkeit wurden die am Schwefel gebundenen Isopropylgruppen weggelassen.
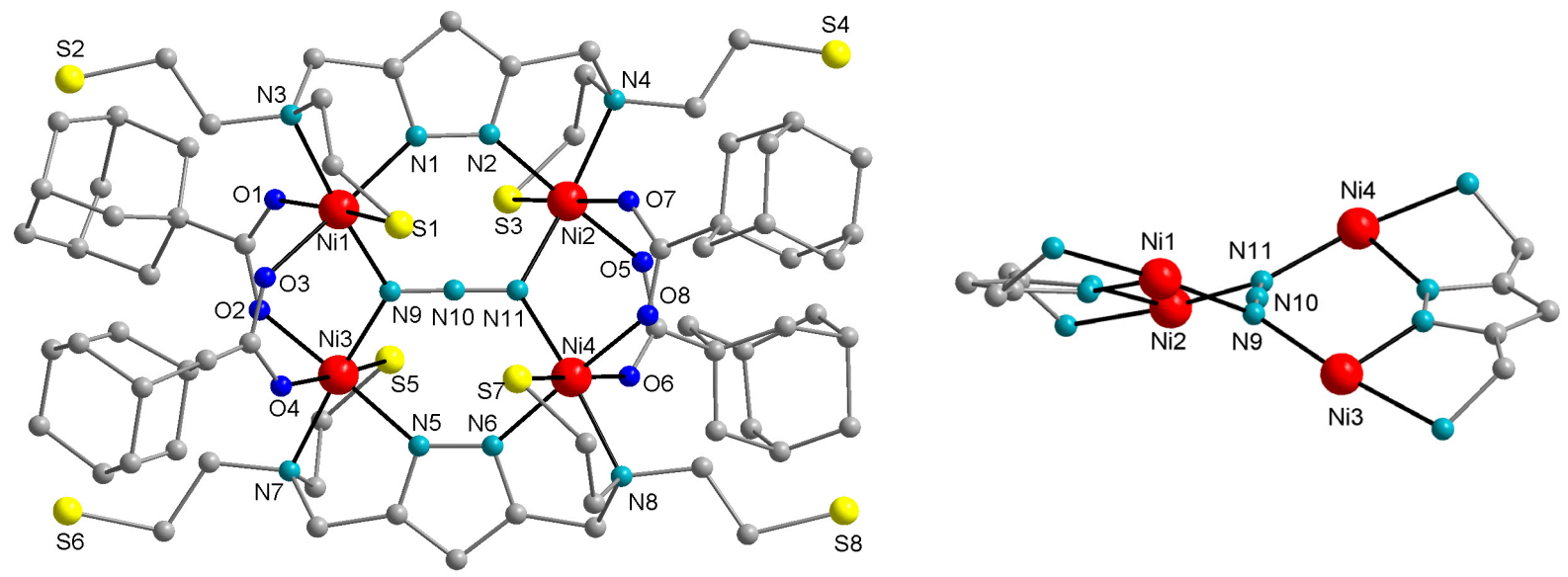

Abb. 4.3.2.3.4. Struktur des Kations der Verbindung 15b (links) und seines zentralen vierkernigen Fragments (rechts). Aus Gründen der Übersichtlichkeit wurden die am Schwefel gebundenen Ethylgruppen weggelassen.

Tab. 4.3.2.3.1. Ausgewählte Bindungslängen $(\AA)$ und Winkel $\left(^{\circ}\right)$ von 14a, 14b, 14e und $15 b$.

\begin{tabular}{lllll}
\hline & $\mathbf{1 4 a}^{\mathrm{a}}$ & $\mathbf{1 4 b}$ & $\mathbf{1 4 e}$ & $\mathbf{1 5 b}$ \\
\hline $\mathrm{Ni} 1 \cdots \mathrm{Ni} 2$ & $4.52(1)$ & $4.45(1)$ & $4.50(1)$ & $4.53(1)$ \\
$\mathrm{Ni} 1 \cdots \mathrm{Ni} 3$ & $3.15(1)$ & $3.15(1)$ & $3.16(1)$ & $3.58(1)$ \\
$\mathrm{Ni} 1 \cdots \mathrm{Ni} 4$ & $5.48(1)$ & $5.49(1)$ & $5.44(1)$ & $5.30(1)$ \\
$\mathrm{Ni} 2 \cdots \mathrm{Ni} 3$ & $5.49(1)$ & $5.41(1)$ & $5.53(1)$ & $5.31(1)$ \\
$\mathrm{Ni} 2 \cdots \mathrm{Ni} 4$ & $3.14(1)$ & $3.12(1)$ & $3.14(1)$ & $3.58(1)$ \\
$\mathrm{Ni} 3 \cdots \mathrm{Ni} 4$ & $4.48(1)$ & $4.46(1)$ & $4.49(1)$ & $4.51(1)$ \\
$\mathrm{Ni} 1-\mathrm{N} 9-\mathrm{Ni3}$ & $93.7(2)$ & $92.9(1)$ & $92.83(9)$ & $124.4(2)$ \\
\hline
\end{tabular}


Zweikernige Nickel(II)-Azid-Komplexe als Baueinheiten für höhernukleare Systeme

\begin{tabular}{lllll}
\hline Ni1-N12-Ni3 & $97.5(2)$ & $95.7(1)$ & $96.87(10)$ & -- \\
Ni2-N11-Ni4 & $93.3(2)$ & $91.0(1)$ & $91.92(9)$ & $124.1(2)$ \\
Ni2-N15-Ni4 & $97.0(2)$ & $95.6(1)$ & $96.5(1)$ & --
\end{tabular}

${ }^{a}$ Werte nur für ein von zwei unabhängigen (aber sehr ähnlichen) Molekülen sind angegeben.

Der Pyrazolatligand fungiert in allen Fällen als bis(tridentates) Ligandgerüst, weil eines der beiden S-Donoratome der Ligandseitenarme in $\mathbf{H L}^{5-7}$ unkoordiniert bleibt. Alle Nickel(II)Ionen sind sechsfach koordiniert und befinden sich in einer verzerrt oktaedrischen $\left\{\mathrm{N}_{4} \mathrm{OS}\right\}$ (Typ 14 $\}$ oder $\left\{\mathrm{N}_{3} \mathrm{O}_{2} \mathrm{~S}\right\}$ (Typ 15) Umgebung. Die Ligandgerüste geben durch das Pyrazolat

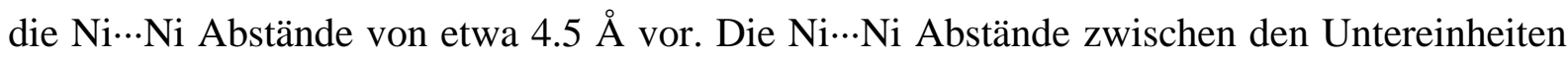
unterscheiden sich relativ stark. Für Typ 14 mit gemischten $\mu$-1,1-Azid/Carboxylatbrücken betragen sie ca. $3.15 \AA$, für Typ 15 mit zwei Carboxylatbrücken knapp 3.6 A. Diese Tatsache hat signifikante Konsequenzen für die Geometrie der vierkernigen Einheiten und für die

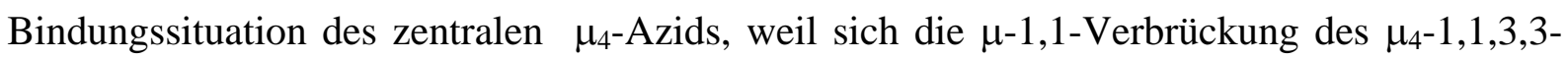
Azidliganden an diese unterschiedlichen $\mathrm{Ni} \cdots \mathrm{Ni}$ Abstände anpassen muss. In Komplexen des Typs 14 findet das $\mu_{4}$-1,1,3,3-Azid in der Ebene von vier Nickel(II)-Ionen nicht ausreichend Platz, sodass es gezwungen ist, das $\mathrm{Ni}_{4}$-Rechteck von oben zu überdecken. Die $\mathrm{Ni}-\mathrm{N}_{3}-\mathrm{Ni}$ Fragmente sind nahezu planar bezüglich der end-to-end Verbrückung, die Ni1-N9-N11-Ni2 und Ni3-N9-N11-Ni4 Torsionswinkel sind $<8.9^{\circ}$ für 14a, $<5.5^{\circ}$ für $\mathbf{1 4 b}$ und $<9^{\circ}$ für 14e. Im Gegensatz dazu liegt in Komplexen des Typs 15 das zentrale $\mu_{4}$-Azid in der Ebene der $\mathrm{Ni}_{4}$-Umgebung. Allerdings ist das Rechteck etwas verzerrt: zwischen den Ni1/N9/Ni3 und Ni2/N11/Ni4 Ebenen bildet sich ein Winkel von $\sim 53^{\circ}$. Dies stimmt mit den kleineren Ni1N9-Ni3/Ni2-N11-Ni4 Winkeln für Typ 14 (91-93) im Vergleich zu 15b (124) überein. Auch die Ni- $\mathrm{N}_{\text {Azid }}$ Bindungslängen des zentralen $\mu_{4}$-Azids unterscheiden sich relativ stark. Sie liegen im Bereich von 2.144-2.207(3) Å für Typ 14 und sind mit 2.021-2.034(5) Å kürzer für Typ 15. Die Ni-N $\mathrm{N}_{\text {Azid }}$ Abstände für das $\mu$-1,1-Azid in Typ 14 liegen in mittlerem Bereich (2.086-2.126(3) Å). Das $\mu_{4}$-Brückenazid zeigt beträchtliche strukturelle Flexibilität und kann sich an verschiedene, durch die $\mathrm{Ni}_{4}$-Umgebung vorgegebene Geometrien anpassen.

Interessanterweise bindet in $\mathbf{1 4 b}$ an die terminalen $\mathrm{N}$-Donoratome der $\mu$-1,1-Azidbrücken ein $\mathrm{Na}^{+}$-Ion von mitkristallisiertem $\mathrm{NaClO}_{4}$ (Abb. 4.3.2.3.2, rechts). $\mathrm{Na}^{+}$ist zudem koordiniert durch zwei Acetonmoleküle und ein Wassermolekül. Es existiert eine zusätzliche $\pi$ Wechselwirkung zu einem der beiden Pyrazolatringe $(d(\mathrm{Na1}-\mathrm{C} 2)=2.994(3) \AA \AA)$. Über $\pi$ Wechselwirkungen des Pyrazolatrings mit Alkalimetallen wurde kürzlich berichtet. ${ }^{[112]}$ 
Die IR-Spektroskopie ist eine sehr hilfreiche Methode für die Unterscheidung zwischen Komplexen des Typs 14 und Typs 15 (Tab. 4.3.2.3.2). Die Bindung von $\mathrm{Na}^{+}$an die $\mu-1,1-$ Azidliganden in 14b hat für $v_{\text {as }}\left(\mathrm{N}_{3}\right)$ keinen nennenswerten Einfluss.

Tab. 4.3.2.3.2. Ausgewählte IR-Banden für Komplexe Typ 14 und 15.

\begin{tabular}{ll}
\hline Komplex & $\mathrm{v}_{\mathrm{as}}\left(\mathrm{N}_{3}^{-}\right), \mathrm{cm}^{-1}$ \\
\hline 14a & $2081(\mathrm{vs}), 2070(\mathrm{vs}), 2052(\mathrm{~s})$ \\
14b & $2080(\mathrm{vs}), 2073(\mathrm{vs}), 2044(\mathrm{~s})$ \\
14c & $2079(\mathrm{vs}), 2074(\mathrm{vs}), 2043(\mathrm{~m})$ \\
14d & $2082(\mathrm{vs}), 2073(\mathrm{vs}), 2044(\mathrm{~m})$ \\
$\mathbf{1 4 e}$ & $2081(\mathrm{vs}), 2072(\mathrm{vs}), 2045(\mathrm{~m})$ \\
15a & $2128(\mathrm{~s})$ \\
15b & $2127(\mathrm{~s})$ \\
\hline
\end{tabular}

Für Komplexe des Typs 14 sind drei Banden bei $\sim 2080, \sim 2073$ and $\sim 2044 \mathrm{~cm}^{-1}$ zu sehen. Die beiden hochenergetischen Banden sind in einigen Verbindungen nicht ganz klar aufgelöst. In Typ 15 wird eine signifikante Bande bei $\sim 2127 \mathrm{~cm}^{-1}$ beobachtet. Zwei zusätzliche schwache Banden bei $\sim 2054$ und $2043 \mathrm{~cm}^{-1}$ können wahrscheinlich den Obertönen starker Perchloratschwingungen bei $\sim 1100 \mathrm{~cm}^{-1}$ zugeordnet werden. Nur für 14a ist die niederenergetische Bande im Vergleich zu anderen Komplexen dieses Typs etwas verschoben (ca $\sim 8 \mathrm{~cm}^{-1}$ ) und wird bei $2052 \mathrm{~cm}^{-1}$ beobachtet. Die Ursache dafür kann an der ungewöhnlichen Asymmetrie des zentralen $\mu_{4}$-Azids liegen. ${ }^{[111]}$

\section{Magnetische Eigenschaften}

Die Temperaturabhängigkeit von $\chi_{M}$ und $\chi_{M} T$ für $\mathbf{1 4 b , d}$ und $\mathbf{1 5 b}$ als typische Beispiele von Komplexe des Typs 14 bzw. 15 ist in Abb. 4.3.2.3.5 gezeigt. Die $\chi_{M} T$-Werte der Komplexe des Typs 14 liegen bei Raumtemperatur im Bereich von 3.3-3.9 $\mathrm{cm}^{3} \mathrm{Kmol}^{-1}$. Dies ist viel kleiner als der theoretisch erwartete „spin-only“ Wert für vier ungekoppelte $S=1$ Nickel(II)Ionen (4.62 $\mathrm{cm}^{3} \mathrm{Kmol}^{-1}$ für $g=2.15$ ), was auf eine sehr starke insgesamt antiferromagnetische Wechselwirkung in $\mathrm{Ni}_{4}$-Fragmenten hinweist. Mit sinkender Temperatur zeigt sich auch ein breites Maximum bei ca. 170-200 K in der Auftragung $\chi_{\mathrm{M}}$ gegen T, das magnetische Moment nimmt stark ab und tendiert schließlich nach Null. Diese beiden Tatsachen sind im Einklang mit dominanter antiferromagnetischer Kopplung und dem $S_{\mathrm{T}}=0$ Grundzustand. $\chi_{\mathrm{M}} T$-Werte 


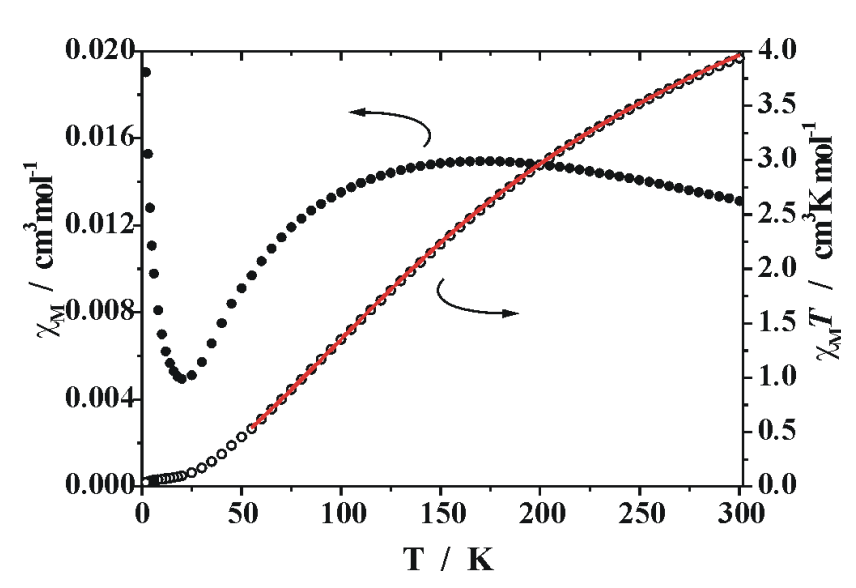

der Komplexe des Typs 15 tendieren bei sinkender Temperatur auch gegen Null, aber die antiferromagnetische Kopplung ist nicht so stark ausgeprägt wie in 14. Dafür spricht ein breites Maximum in der magnetischen Suszeptibilität erst bei viel tieferer Temperatur (ca. 50 K). Der
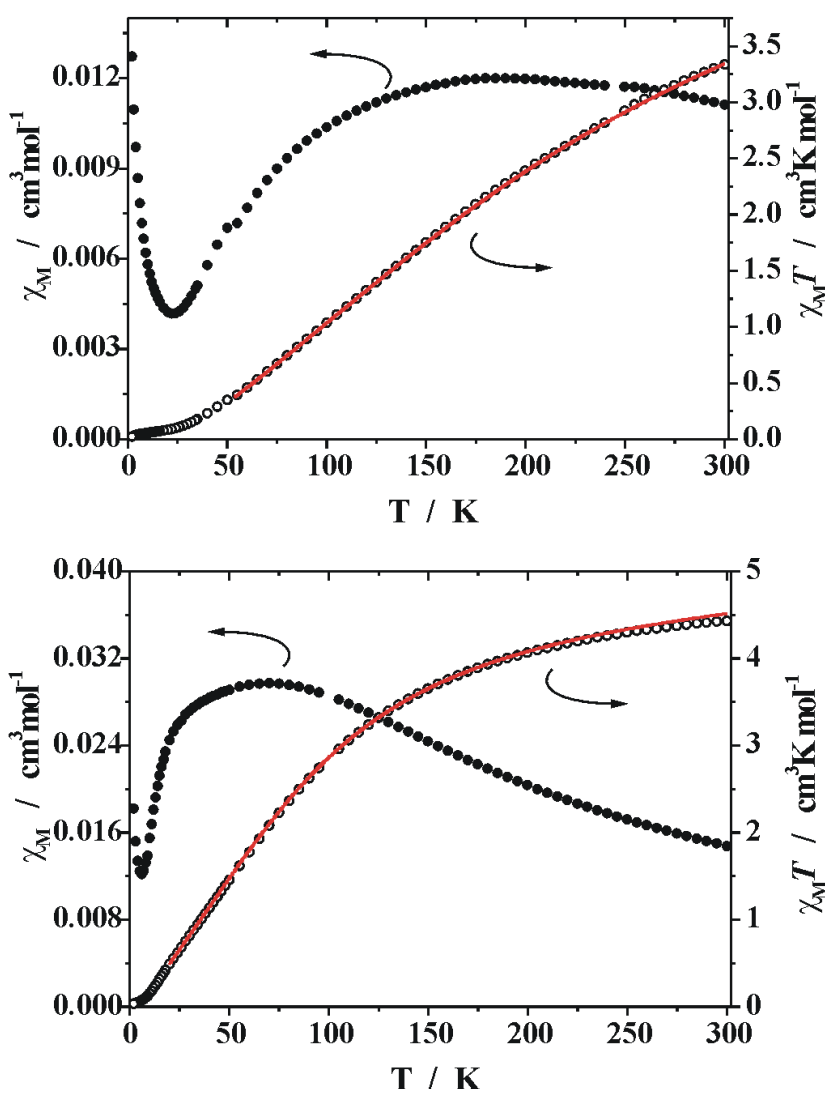

Abb. 4.3.2.3.5. Auftragung von $\chi_{M}$ und $\chi_{M} T$ gegen $T$ für 14b (oben), 14d (in der Mitte) und 15b (unten). Die roten Linien repräsentieren die besten Anpassungen.
Anstieg von $\chi_{\mathrm{M}}$ bei sehr tiefen Temperaturen ist möglicherweise durch eine Restmenge paramagnetischer Verunreinigungen bedingt.

Zur Vereinfachung der Analyse der magnetischen Daten wurde für alle $\mathrm{Ni}_{4}{ }^{-}$ Fragmente zweizählige Symmetrie angenommen. Nach der molekularen Topologie der Komplexe können drei prinzipielle Pfade des magnetischen Austauschs vorgeschlagen werden (Schema 4.3.2.3.2).

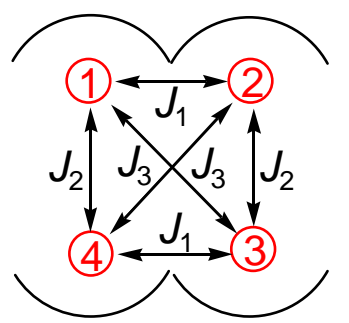

Schema 4.3.2.3.2. Kopplungsschema für $\mathrm{Ni}_{4}-$ Komplexe mit der rechteckigen Anordnung der Nickel(II)-Zentren.

Dieses Schema beinhaltet eine intradimere Kopplung über den Pyrazolatring und end-toend Verbrückung des zentralen $\mu_{4}$-1,1,3,3-Azids $\left(J_{1}\right)$ sowie zwei interdimere Kopplungen entlang der Kante $\left(J_{2}\right)$ und entlang der Diagonale $\left(J_{3}\right)$ der $\mathrm{Ni}_{4}$-Anordnung. Das diesem Schema entsprechende Modell basiert auf folgendem Heisenberg-Dirac-van-VleckHamiltonoperator: 


$$
\begin{array}{r}
\hat{H}_{J}=-2 J_{1}\left(\hat{S}_{1} \hat{S}_{2}+\hat{S}_{3} \hat{S}_{4}\right)-2 J_{2}\left(\hat{S}_{1} \hat{S}_{3}+\hat{S}_{2} \hat{S}_{4}\right)-2 J_{3}\left(\hat{S}_{1} \hat{S}_{4}+\hat{S}_{2} \hat{S}_{3}\right)+ \\
+g \mu_{B} B \sum \hat{S}_{i z}+D \sum\left(\hat{S}_{i z}^{2}-\hat{S}_{i}\left(\hat{S}_{i}+1\right) / 3\right)
\end{array}
$$

wobei die zusätzlichen Terme Zeeman-Aufspaltung und Anisotropie der einzelnen Ionen berücksichtigen.

Die experimentellen Suszeptibilitätsdaten $\chi_{M}(T)$ wurden unter Verwendung der Zweikomponentenfitfunktion analysiert. ${ }^{1)}$ Um die Parameteranzahl zu beschränken, wurde auf einen Verunreinigungsterm verzichtet. Dafür hat man die Daten erst ab einer bestimmten Temperatur (20-60 K) für die Anpassung berücksichtigt. In der magnetischen Suszeptibilität von 14c zeigt sich ein starker feldunabhängiger Anstieg ab ca. 50 K. Für dieses Verhalten konnte kein passendes Modell gefunden werden. Die nach der Analyse der magnetischen Daten erhaltenen Parameter sind in Tab. 4.3.2.3.3 zusammengefasst. Für den besseren Vergleich wurden alle $g$-Werte bei der abschließenden Anpassung auf 2.15 festgehalten.

Tab. 4.3.2.3.3. Magnetische Parameter für die Komplexe des Typs 14 und 15. ${ }^{a}$

\begin{tabular}{llllllll}
\hline Komplex & $J_{A}, \mathrm{~cm}^{-1}$ & $J_{B}, \mathrm{~cm}^{-1}$ & $J_{C}, \mathrm{~cm}^{-1}$ & $g$ (fixiert) & $\left.D, \mathrm{~cm}^{-1}\right)$ & $T I P, \mathrm{~cm}^{3} / \mathrm{mol}$ & $T_{\min }, \mathrm{K}$ \\
\hline 14a & -55 & +53 & +1 & 2.15 & -1 & 0 & 30 \\
14b & -56 & +26 & +5 & 2.15 & 1 & 0.002 (fixiert) & 50 \\
14d & -67 & +65 & +13 & 2.15 & 0 & 0 & 55 \\
14e & -56 & +43 & +3 & 2.15 & 10 & 0 & 60 \\
15a & -20 & +49 & -6 & 2.15 & -46 & 0 & 20 \\
15b & -25 & +33 & +4 & 2.15 & -35 & 0 & 20 \\
\hline
\end{tabular}

${ }^{\text {a }}$ Ungenauigkeit der $J$-Werte liegt in der Größenordnung von $3-5 \mathrm{~cm}^{-1}$.

Es ist gut zu erkennen, dass die Anpassungen für 15 deutlich kleinere antiferromagnetische Kopplungen $J_{A}$ und viel größere $D$-Werte für die Anisotropie des einzelnen Ions als für 14 liefert. Da die $D$-Werte für 15 übermäßig groß sind, sollte man die Ergebnisse von 15 mit Vorsicht interpretieren. Die starken ferromagnetischen Wechselwirkungen $J_{B}$ sind für Typ 14 und 15 fast gleich. Für $J_{C}$ wurden kleine Werte gefunden, positiv sowie negativ. Es sollte angemerkt werden, dass die einzelnen Kopplungskonstanten $J_{A}, J_{B}, J_{C}$ dank der Symmetrie

\footnotetext{
1) Die Anpassung an die Daten der Suszeptibilitätsmessungen wurden von C. Mennerich und Dr. H-H. Klauss während der Zusammenarbeit in Rahmen des DFG Schwerpunktprogramms 1137 „Molecular Magnetism“ durchgeführt. ${ }^{[111]}$
} 
des Hamiltonoperators nicht eindeutig den topologischen $J$-Werten zugeordnet werden können.

Für die Interpretation der magnetischen Parameter für 14 und $\mathbf{1 5}$ ist es sinnvoll, die einzelnen Fragmente dieser vierkernigen Komplexe separat zu betrachten. Ni1 und Ni2 (sowie Ni3 und Ni4) sind über Pyrazolat und den end-to-end Modus des zentralen $\mu_{4}$-Azids verbrückt, zugeordnet $\mathrm{zu} J_{1}$ in Schema 4.3.2.3.2. Für die Interpretation der magnetischen Wechselwirkungen, vermittelt über die $\mu$-1,3-Azidbrücke werden zwei geometrische Parameter herangezogen, nämlich der Ni-N-N Winkel und der Ni-NNN-Ni Torsionswinkel entlang der $\mu$-1,3-Azidverbrückung (die magnetostrukturellen Korrelationen dafür siehe Kapitel 4.1.2). Für 14 liegen die Ni-N-N Winkel des zentralen $\mu_{4}$-Azids im Bereich von 115.6- 121.7º Ni1-NNN-Ni2 sowie Ni3-NNN-Ni4 Torsionswinkel sind nahezu Null (0.1$8.9^{\circ}$ ). Starke antiferromagnetische intradimere Kopplung in der Größenordnung von $-50 \mathrm{~cm}^{-1}$ kann in Übereinstimmung mit den experimentellen Werten für 14a-e erwartet werden. Demgemäß ist $J_{\mathrm{A}}$ in Tab. 4.3.2.3.3 der intradimeren Kopplungskonstante $J_{1}$ in Schema 4.3.2.3.2 zugeordnet. Ein Beitrag des Pyrazolats ist sicherlich präsent, aber offensichtlich ist die Azidbrücke der dominierende Vermittler magnetischer Wechselwirkung. ${ }^{[110]}$ Interdimere Ni1-NNN-Ni4 und Ni2-NNN-Ni3 Torsionswinkel der end-to-end Verbrückung des zentralen $\mu_{4}$-Azids ( $J_{3}$ in Schema 4.3.2.3.2) liegen für alle Komplexe des Typs $14 \mathrm{im}$ Bereich von 102.7-119.7 ${ }^{\circ}$ was nahezu orthogonaler Orientierung entspricht. Der antiferromagnetische Beitrag zur Kopplungskonstante wird hier sehr klein, so dass eine ferromagnetische Wechselwirkung resultiert, welche selten für die Verbindungen mit $\mu$-1,3Verbrückung ist. Die nach der Analyse erhaltenen $J_{C}$-Werte (Tab. 4.3.2.3.3) für die ferromagnetische diagonale interdimere Wechselwirkung $\left(J_{3}\right)$ in Typ 14 liegen in der gleichen Größenordnung wie bei den aus der Literatur bekannten Werten für $\mu$-1,3-Azidbrücken mit ähnlichem Torsionswinkel (siehe Kapitel 4.1.3). Die magnetostrukturellen Korrelationen für die zweifach end-on azidverbrückten Nickel(II)-Komplexe sagen für alle $\theta$-Werten ferromagnetische Kopplung mit einem Maximum bei $\theta=104^{\circ}$ voraus (siehe Kapitel 4.1.3). Die Ni1-N9-Ni3 und Ni2-N11-Ni4 Winkel des zentralen $\mu_{4}$-Azids in Typ 14 liegen im Bereich 90.9-93.7 , sind also vergleichsweise schmal. Die Ni1-N12-Ni3 und Ni2-N15-Ni4 Winkel der $\mu$-1,1-Azidbrücke sind mit 96.5-97.9 etwas größer. Unter Berücksichtigung, dass Carboxylat dank des kontra-Komplementaritätseffekts die ferromagnetische Kopplung verstärkt (siehe Kapitel 4.1.3), kann für die interdimere Kopplung $J_{2}$ eine signifikante ferromagnetische Wechselwirkung erwartet werden. Somit wird die aus der Anpassung ermittelte große ferromagnetische Kopplung $J_{\mathrm{B}}$ der verbrückenden Kante der $\mathrm{Ni}_{4}$-Anordnung 
( $J_{2}$ in Schema 4.3.2.3.2) zugeordnet. Interessanterweise sollte diese Kombination von einer antiferromagnetischen und zwei ferromagnetischen Wechselwirkungen in Typ 14 zu einem gewissen Grad an Frustration in der $\mathrm{Ni}_{4}$-Anordnung führen, welche ihre Ursache in der ferromagnetischen diagonalen Kopplung $J_{3}$ hat.

In Gegensatz zum Typ 14 weist die molekulare Struktur von 15b große Torsionswinkel der end-to-end Verbrückung des zentralen $\mu_{4}$-Azids für Ni1-NNN-Ni2 von $52.7^{\circ}$ und für Ni3NNN-Ni4 von $52.8^{\circ}$ auf. Die Ni-N-N Winkel sind mit $117.2-118.7^{\circ}$ in derselben Größenordnung. Somit wird in Übereinstimmung mit den theoretischen Befunden für $J_{\mathrm{A}}$ eine geringe intradimere antiferromagnetische Wechselwirkung erwartet. Wegen der Anwesenheit der beiden Carboxylatbrücken sind die Ni1-N9-Ni3 $\left(124.4(2)^{\circ}\right)$ und Ni2-N11-Ni4 $\left(124.1(2)^{\circ}\right)$ Winkel viel größer für 15b als für 14. Trotzdem und trotz der Abwesenheit von zusätzlichen $\mu$-1,1-Azidbrücken ist die ferromagnetische Kopplungskonstante $J_{\mathrm{B}}$ in derselben Größenordnung sowohl für 15a, 15b als auch für Typ 14. Die Ursache dafür könnte an der Verstärkung der über die $\mu-1,1$-Verbrückung des $\mu_{4}-1,1,3,3-$ Azids vermittelten ferromagnetischen Kopplung durch die zwei Carboxylationen liegen (siehe Kapitel 4.1.3). Die Torsionswinkel entlang der diagonalen end-to-end Verbrückung sind für 15b $126.6^{\circ}$ (Ni1NNN-Ni4) und $127.9^{\circ}$ (Ni2-NNN-Ni3). Das kann bedeuten, das ferro- und antiferromagnetische Beiträge einander nahezu kompensieren. Dementsprechend beobachtet man kleine $J_{\mathrm{C}}$-Werte für $\mathbf{1 5 a}$ und $\mathbf{1 5 b}$.

Die oben diskutierten magnetostrukturellen Korrelationen für Komplextyp 15 sind von begrenztem Wert, weil die Ergebnisse der Röntgenstrukturanalyse nur einer Verbindung und die Anpassungen an die experimentellen magnetischen Daten moderater Qualität (z.B. sind die errechneten $D$-Werte unrealistisch hoch) allein noch keine sicheren Schlußfolgerungen erlauben. Ein weiterer Schritt zu genaueren magnetostrukturellen Korrelationen für diese Art des verbrückenden $\mu_{4}$-Azides wurde durch die Untersuchung von 15b mit Hilfe von Hochfeld-EPR gemacht. ${ }^{[113]}$ Dabei wurden ein $g$-Wert von 2.11 und eine Nullfeldaufspaltung des ersten angeregten Zustands mit $S=1$ von $6.7 \mathrm{~K}\left(4.7 \mathrm{~cm}^{-1}, \Delta_{1}\right.$ in Abb. 4.3.2.3.6) ermittelt, welche für eine genauere Anpassung eingesetzt wurden. Die kombinierte Analyse von $\chi_{M}(T)$ und den EPR-Daten lieferte folgende Parameter: $J_{1}=+20.2 \mathrm{~cm}^{-1}, J_{2}=-13.1 \mathrm{~cm}^{-1}, J_{3}=-13.0$ $\mathrm{cm}^{-1}$ und $D=-3.4 \mathrm{~cm}^{-1}$. Die Unterschiede in den Anpassungsergebnissen mit und ohne Einsetzen der experimentell bestimmten Parameter zeigen, wie wichtig es ist, für komplexe Systeme die Anzahl der Anpassungsparameter zu reduzieren, weil man bei sehr vielen Parametern „einen Elefanten durch ein Nadelöhr fitten kann“ (Dr. G. Leibeling, unpubliziert). 


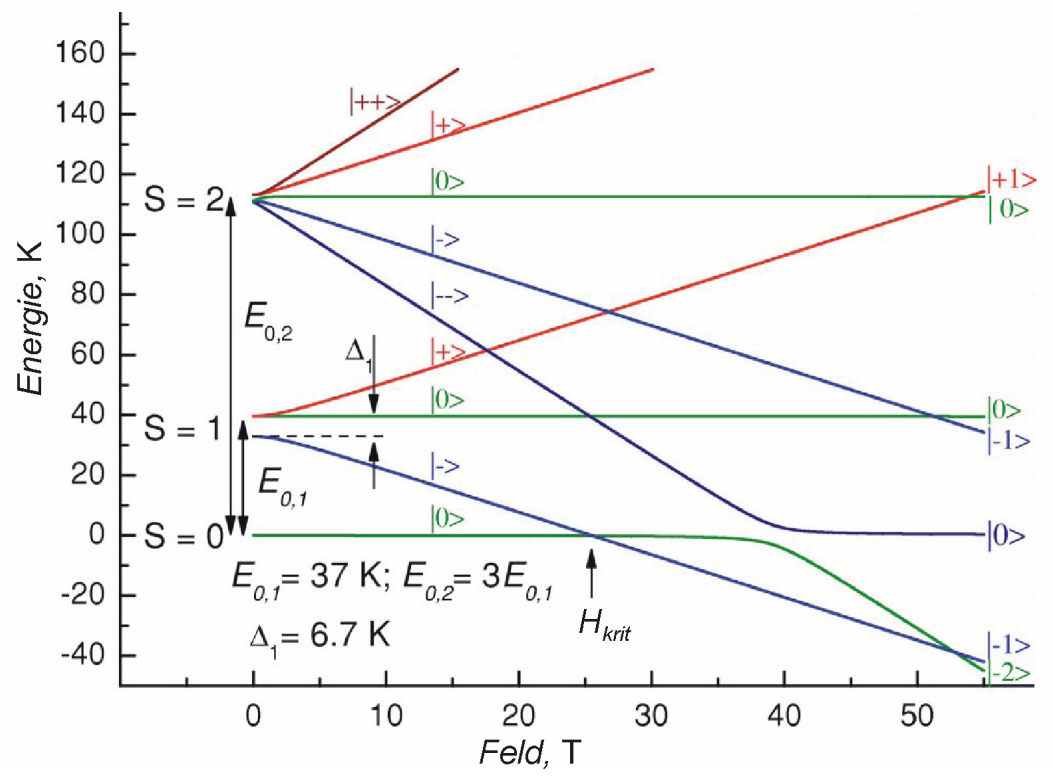

Abb. 4.3.2.3.6. Simulierte Energieniveaus von 15b und ihre Aufspaltung im magnetischen Feld. ${ }^{[13]}$

Eine auch aus Sicht des Anwendungspotentials interessante Eigenschaft von 15b wurde bei der Untersuchung der Abhängigkeit des magnetischen Moments von der angelegten Feldstärke beobachtet. ${ }^{[113]}$ Es handelt sich um die Möglichkeit, den Spingrundzustand durch das magnetische Feld von einem nichtmagnetischen bei kleineren Feldern in einen magnetischen bei einem starken Feld von $25 \mathrm{~T}$ umzuschalten. Die berechneten Energieniveaus von 15b weisen die Querung des $S_{T}=0$ Grundzustandes mit einer der Komponenten des angeregten $S=1$ Zustandes $\left(m_{S}=-1\right)$ bei einem kritischen Feld von $>25 \mathrm{~T}$ auf. Solche Quantenphänomene erregen großes Interesse wegen möglicher zukünftiger Anwendungen beispielsweise im Hinblick auf einen so genannten Quantencomputer.

\section{Zusammenfassung}

Eine Serie sehr ungewöhnlicher, aus zwei pyrazolatbasierten bimetallischen Bauelementen aufgebauten $\mathrm{Ni}_{4}$-Komplexe wurde synthetisiert und charakterisiert. Alle Komplexe beinhalten die zentrale $\mu_{4}$-1,1,3,3-Azidverbrückung. Dank offensichtlich sehr hoher Flexibilität bezüglich seiner Koordinationsgeometrie ist der Azidligand in der Lage, sich den verschiedenen Umgebungen von vier Metallzentren anzupassen. Zwei Typen von Komplexen mit deutlich unterschiedlichen $\mathrm{Ni}-\mathrm{N}_{\text {Azid }}-\mathrm{Ni}$ Winkeln und Ni-NNN-Ni Torsionswinkeln des zentralen $\mu_{4}$-Azids haben auch unterschiedliche IR-spektroskopische und magnetische Eigenschaften. Alle Verbindungen haben einen $S_{T}=0$ Grundzustand, aber individuelle intradimere und interdimere Kopplungskonstanten, die von den geometrischen Details des 
$\mathrm{Ni}_{4}$-Fragments abhängen. Die magnetostrukturellen Korrelationen der $\mu_{4}$-Azidbrücke können durch Betrachtung der Position der einzelnen $\mu$-1,1- und $\mu$-1,3-Komponenten des $\mu$-1,1,3,3Azids verstanden werden.

\subsubsection{Kettenförmige Anordnung der Nickel(II)-Zentren}

Für eine Reihe von Komplexen wurden verschiedenartige kettenförmige Anordnungen der vier Nickel(II)-Zentren beobachtet. Die zweikernigen Untereinheiten sind zueinander verschoben und in unterschiedlicher Weise über Azidionen verbrückt. Somit nehmen diese Komplexe eine Zwischenstellung auf dem Weg von zweikernigen molekularen Spezies zu 1D-polymeren Ketten ein. In diesem Kapitel werden die Synthese kettenförmiger $\mathrm{Ni}_{4}-$ Komplexe sowie deren magnetostrukturelle Korrelationen beschrieben.

\section{Synthese und Charakterisierung}

Die Komplexe 16 $^{[105]}$ und $\mathbf{1 8}^{[107]}$ (Abb. 4.3.2.4.1) sowie 17 (Schema 4.3.2.4.1) können direkt ausgehend von den Liganden $\mathbf{H L}^{1}$ bzw. $\mathbf{H L}^{\mathbf{6}}$ präpariert werden, indem man den jeweiligen Liganden mit zwei Äquivalenten von $\mathrm{Ni}\left(\mathrm{ClO}_{4}\right)_{2} \cdot 6 \mathrm{H}_{2} \mathrm{O}$ und der äquivalenten Menge an $\mathrm{NaN}_{3}$ in Methanol reagieren lässt. Für 16 wird der dabei entstandene Komplex aus Dichlormethan umkristallisiert.

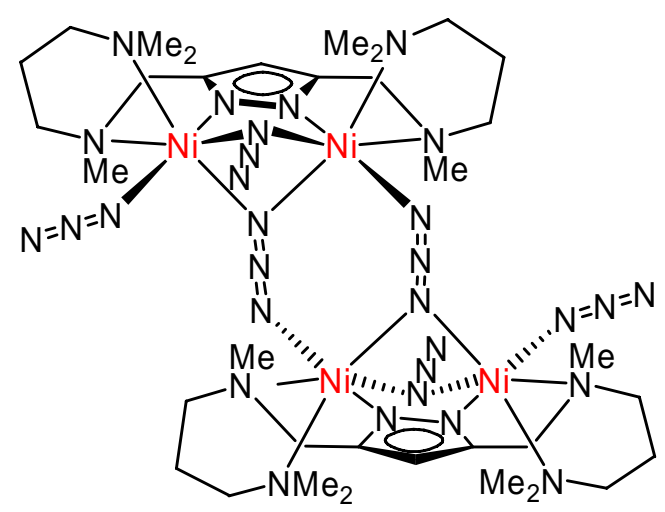

16

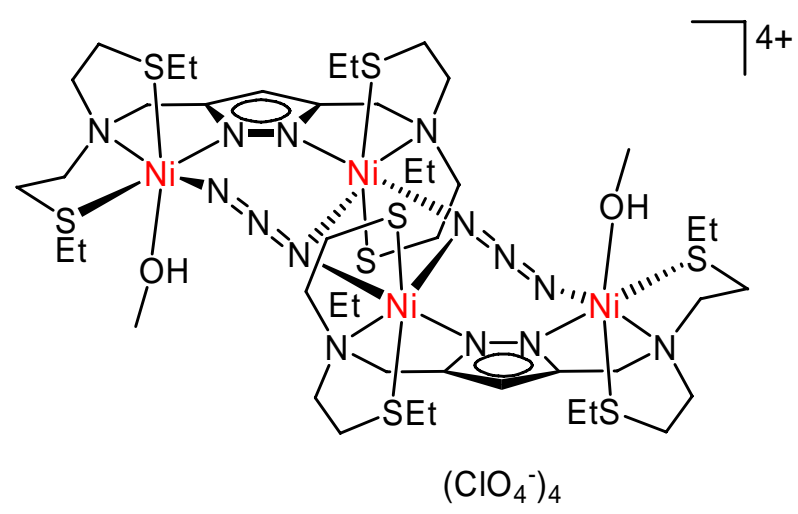

18

Abb. 4.3.2.4.1. Komplexe 16 und 18.

Während 16 und 18 nach diesem Verfahren in sauberem Zustand hergestellt werden können, erhält man im Falle von 17 Kristalle, die mit anhaftender pulvriger Substanz 
verunreinigt sind. Es ist kaum möglich, diese Verunreinigung mechanisch oder durch Umkristallisation abzutrennen. Um 17 in hohem Reinheitsgrad zu erhalten, wird kristallines 1 als Baueinheit für die Synthese von 17 verwendet, indem man zur Lösung von $\mathbf{1}$ in Methanol 2 Äquivalente $\mathrm{NaN}_{3}$ zusetzt (Schema 4.3.2.4.1). Eine ähnliche Strategie wurde für die Synthese vierkerniger Nickel(II)-Komplexe ausgehend von koordinativ ungesättigten thiophenolatbasierenden Dinickel(II)-Baueinheiten oder einigen pyrazolatbasierenden vierkernigen Nickel(II)-Komplexen verwendet. ${ }^{[26,64]}$

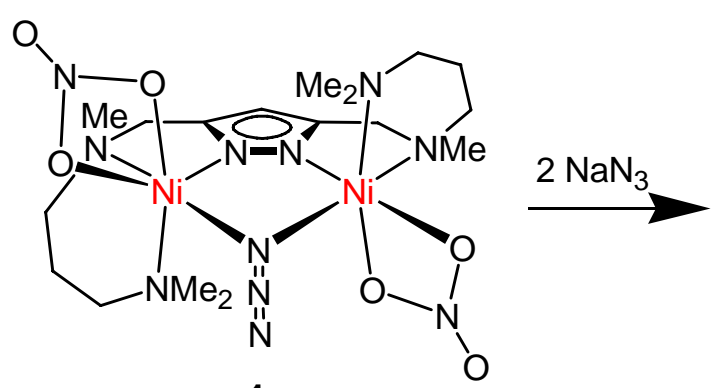

1

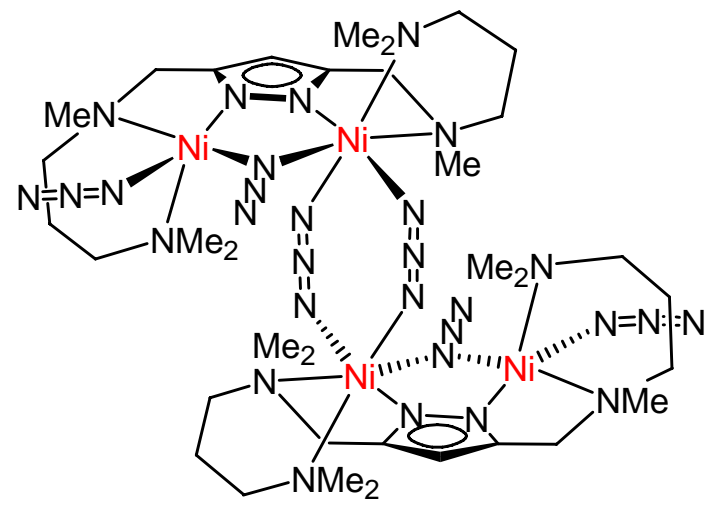

17

Schema 4.3.2.4.1. Synthese von 17 ausgehend von 1.

Die kettenförmige Natur ( $\mathrm{Ni}_{4}$-Anordnung in 16-18 als Parallelogramm) dieser Komplexe illustriert Abb. 4.3.2.4.2.

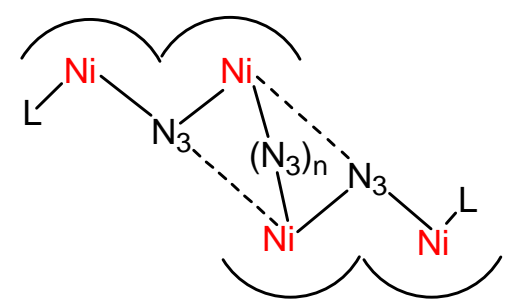

Abb. 4.3.2.4.2. Kettenförmige Anordnung der Nickel(II)-Zentren in 16-18: $n=2, \mathrm{~L}=\mathrm{N}_{3}{ }^{-}$für 16 und 17; $\mathrm{n}=$ $0, \mathrm{~L}=\mathrm{MeOH}$ für 18.

Das detaillierte Bild der molekularen Strukturen von 16-18 wurde durch Röntgenstrukturanalyse erhalten.

Die Strukturen der Kationen von 16 und 17 sind in Abb. 4.3.2.4.3 bzw. Abb. 4.3.2.4.4 gezeigt. Diese Komplexe bestehen aus zwei $\left[\mathrm{L}^{1} \mathrm{Ni}_{2}\left(\mu-1,1-\mathrm{N}_{3}\right)\right]^{2+}$ Fragmenten, die über zwei weitere Azidionen zur kettenförmigen $\mathrm{Ni}_{4}$-Anordnung mit kristallographischer $C_{\mathrm{i}}$-Symmetrie 
verknüpft sind. Die langen Seitenarme des Liganden ermöglichen die kurzen Ni1 $\cdots$ Ni2 Abstände innerhalb den bimetallischen Einheiten: 3.350(1) Å und 3.674(1) A für 16 bzw. 17. Die interdimeren Ni1 ‥Ni1a Abstände sind 5.358(1) $\AA$ und 5.123(1) $\AA$ für 16 bzw. 17. Die zweikernigen Untereinheiten in $\mathbf{1 6}$ und $\mathbf{1 7}$ sind zueinander verschoben. Da die Verschiebung im Falle von 16 kleiner als in 17 ist, beträgt der Ni2-N10 Abstand 2.443(3) Å und damit sind in 16 zwei verbrückende Azidionen zwischen den $\mathrm{Ni}_{2}$-Einheiten in einem seltenen $\mu$-1,1,3Koordinationsmodus, während sie in 17 in dem relativ häufigen $\mu$-1,3-Koordinationsmodus verknüpft sind. Als Folge dessen sind in $\mathbf{1 6}$ alle Nickel(II)-Ionen sechsfach koordiniert, in 17 hingegen sowohl fünffach (Ni2, Ni2a) als auch sechsfach (Ni1, Ni1a).

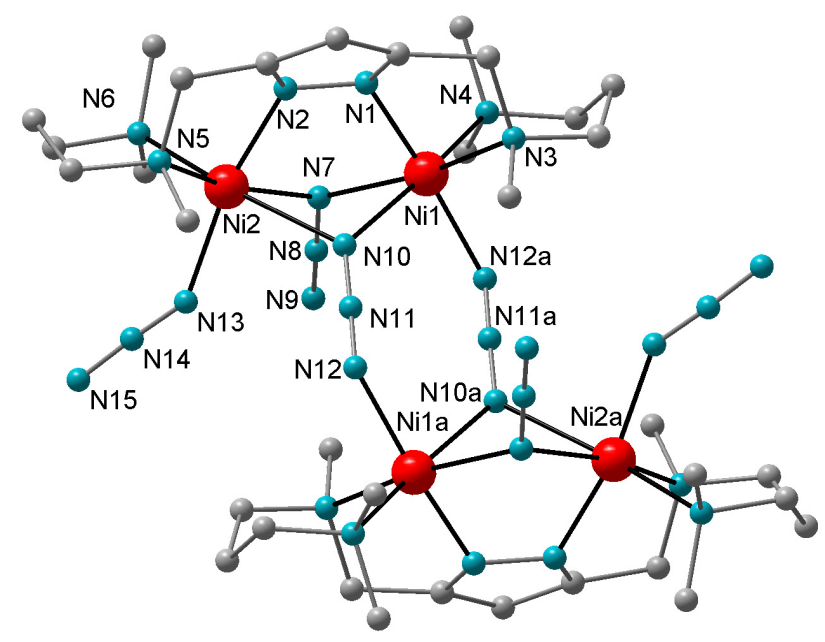

Abb. 4.3.2.4.3. Struktur Verbindung 16.

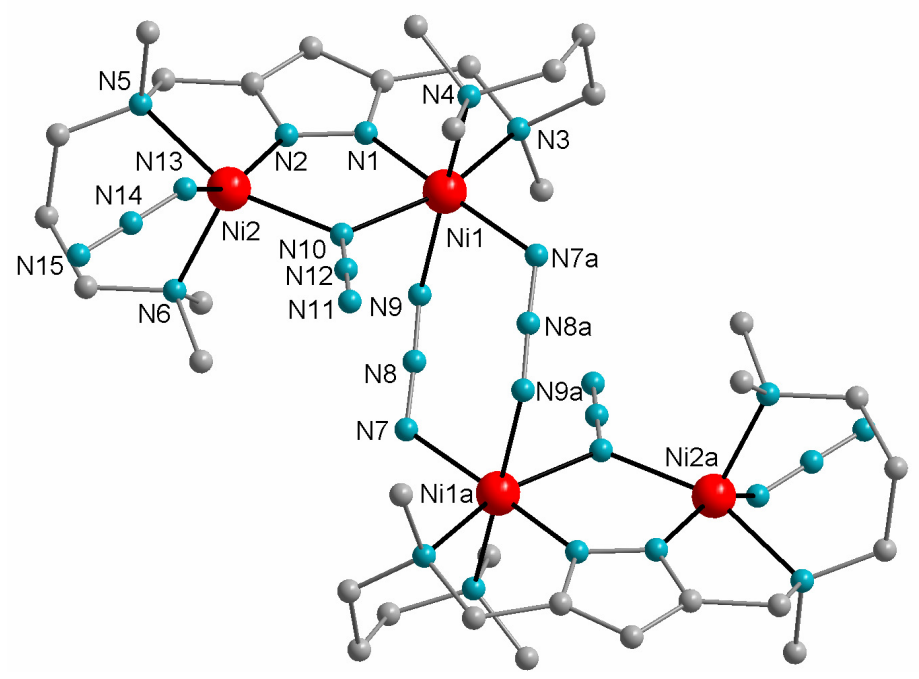

Abb. 4.3.2.4.4. Struktur der Verbindung 17. 
Dreifach verbrückende $\mu_{3}$-1,1,3-Azide sind selten und zeigen überwiegend zwei deutlich längere $\mathrm{N}_{\text {Azid }}-\mathrm{M}$ Abstände als für z.B. $\mu$-1,3-Azidbrücke. ${ }^{[83]}$ Dies belegen auch die Daten von 16. Hier liegen $d(\mathrm{Ni1}-\mathrm{N} 10)$ und $d(\mathrm{Ni2}-\mathrm{N} 10)$ bei $2.310(3) \AA$ bzw. 2.433(3) $\AA$, $d(\mathrm{Ni1}-\mathrm{N} 12 \mathrm{a})$ dagegen bei 2.066(3) $\AA$. Interessanterweise beinhaltet $\mathbf{1 6}$ drei unterschiedliche Koordinationsvarianten für den Azidliganden. Die Anwesenheit der zusätzlichen $\mu-1,1-$ Verbrückung des $\mu_{3}-1,1,3$-Azids zwischen Ni1 und Ni2 führt zu ungewöhnlich kleinen Ni-NNi Winkel (siehe Tab. 4.3.2.4.1). Normalerweise liegen die Ni-N-Ni Winkel in $\{\mathrm{Ni}-\mu-1,1-$ Ni\} pyrazolatbasierten Einheiten bei $116-118^{\circ}$. Die weiteren ebenso für die Interpretation magnetischer Eigenschaften wichtigen geometrischen Parameter sind, wie die Tab. 4.3.2.4.1 zeigt, auch unterschiedlich.

Tab. 4.3.2.4.1. Ausgewählte Winkel $\left(^{\circ}\right)$ von 16-18.

\begin{tabular}{llll}
\hline & $\mathbf{1 6}$ & $\mathbf{1 7}$ & $\mathbf{1 8}$ \\
\hline $\mathrm{Ni}-\mathrm{N}-\mathrm{N}$ (intradimer) & --- & --- & $115.3(1) / 118.8(1)$ \\
$\mathrm{Ni}-\mathrm{N}-\mathrm{N}$ (interdimer) & $127.4(2) / 133.9(2)$ & $125.7(2) / 121.2(2)$ & $118.8(1) / 135.7(1)$ \\
$\mathrm{Ni}-\mathrm{NNN}-\mathrm{Ni}$ (intradimer) & --- & --- & $42.9(1)$ \\
$\mathrm{Ni}-\mathrm{NNN}-\mathrm{Ni}$ (interdimer) & $32.7(5)$ & $63.7(5)$ & $164.5(2)$ \\
$\mathrm{Ni}-\mathrm{N}-\mathrm{Ni}$ & $102.5(1) / 89.6(1)$ & $116.1(2)$ & $101.4(2)$ \\
\hline
\end{tabular}

Die Werte für $\alpha$ sind in $\mathbf{1 6}$ nur geringfügig größer als in 17, der Torsionswinkel für $\mathbf{1 6}$ ist aber nur halb so groß wie jener von 17 (Tab. 4.3.2.4.1).

Die Struktur des Kations von 18 ist in Abb. 4.3.2.4.5 gezeigt. Dieser Komplex besteht genauso wie 16 oder 17 aus zwei bimetallischen Untereinheiten.

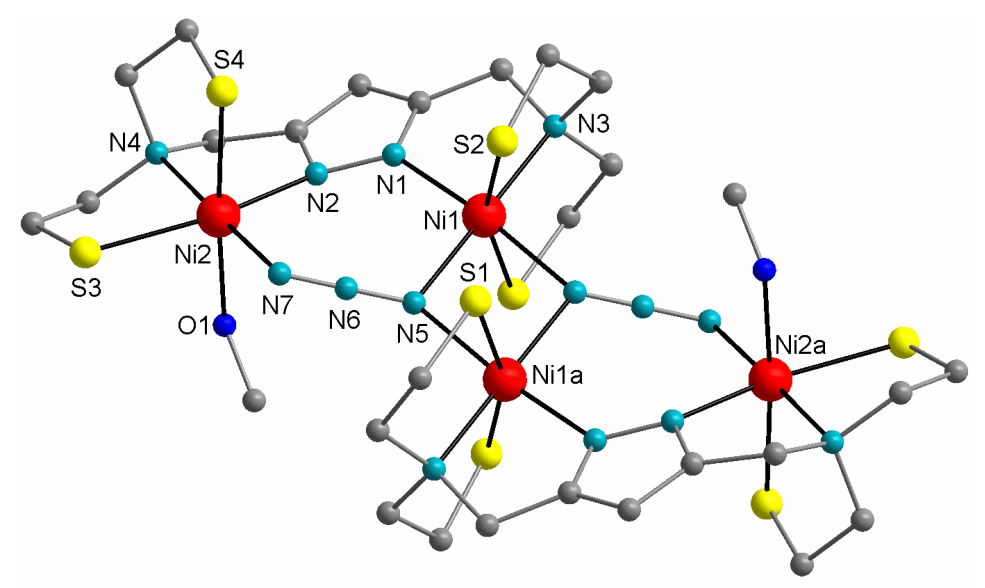

Abb. 4.3.2.4.5. Struktur des Kations der Verbindung 18. Aus Gründen der Übersichtlichkeit wurden die am Schwefel gebundene Ethylgruppe weggelassen. 
Die kurzen Seitenarme des Liganden $\mathbf{H L}^{\mathbf{6}}$ ziehen beide Nickel(II)-Ionen auseinander und

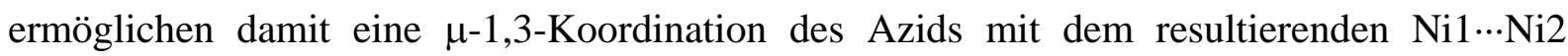
Abstand von 4.483(1) Å innerhalb der bimetallischen Untereinheiten. Allerdings sind die zweikernigen $\left[\mathrm{L}^{6} \mathrm{Ni}_{2}\left(\mu-1,1-\mathrm{N}_{3}\right)\right]^{2+}$ Fragmente noch stärker als in $\mathbf{1 6}$ bzw. 17 gegeneinander verschoben, so dass deren Verknüpfung nicht mehr über weitere Azidionen stattfinden kann. Die zweikernigen Untereinheiten werden über end-on Koordination der beiden $\mu_{3}-1,1,3$-Azide zur kettenförmigen $\mathrm{Ni}_{4}$-Anordnung mit kristallographischer $C_{\mathrm{i}}$-Symmetrie verknüpft. Der

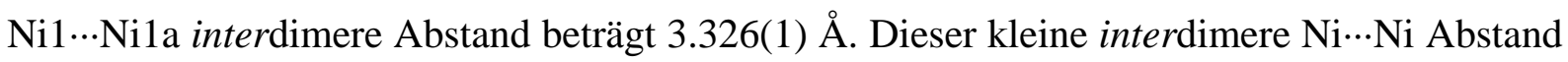
sowie die interdimere zweifache $\mu-1,1$-Verbrückung sind für pyrazolatbasierte Nickel(II)Azid-Systeme sehr ungewöhnlich. Die Ni- $\mathrm{N}_{\text {Azid }}$ Bindungslängen für das $\mu_{3}-1,1,3-A z i d$ in 16 (2.109(2)-2.189(1) Å) sind vergleichbar mit jenen für Typ 13 und liegen in üblichem Bereich für Azidbrücken. Somit ist 16 ein weiterer Vertreter des „echten“ $\mu_{3}$-1,1,3-Azidmodus, in welchem allerdings im Vergleich zum Typ 13 keine zusätzlichen Azidbrücken vorhanden sind. Der zentrale $\mu_{3}$-Azidligand verbrückt sowohl die Nickel(II)-Ionen innerhalb der bimetallischen Einheit als auch diese Einheiten untereinander. Die entsprechenden Bindungswinkel sind in Tab. 4.3.2.4.1 zusammengefasst.

Die Werte der $v_{\mathrm{as}}\left(\mathrm{N}_{3}\right)$ Schwingungen von 16-18 sind in Tab. 4.3.2.4.2 zusammengefasst. In den IR-Spektren von 16 bzw. 17 kann eine niederenergetische Bande bei $2037 \mathrm{~cm}^{-1}$ den Schwingungen der terminalen Azide zugeordnet werden, weil, erstens, diese Schwingung im Spektrum von 18 fehlt und, zweitens, die N-N-Abstände etwas kürzer sind und die Asymmetrie in den terminalen $\mathrm{N}_{3}{ }^{-}$stärker ausgeprägt ist als in den verbrückenden Aziden.

Tab. 4.3.2.4.2. Ausgewählte IR-Banden $\left(\mathrm{cm}^{-1}\right)$ für 16-18.

\begin{tabular}{ll}
\hline Komplex & $\mathrm{v}_{\mathrm{as}}\left(\mathrm{N}_{3}{ }^{-}\right)$ \\
\hline $\mathbf{1 6}$ & $2103(\mathrm{vs}), 2055(\mathrm{vs}), 2037(\mathrm{vs})$ \\
$\mathbf{1 7}$ & $2098(\mathrm{vs}), 2052(\mathrm{vs}), 2037(\mathrm{vs})$ \\
$\mathbf{1 8}$ & $2091(\mathrm{sh}), 2059(\mathrm{vs})$ \\
\hline
\end{tabular}

Die IR-Spektroskopie ist also auch für die Komplexe 16-18 eine sehr hilfreiche Methode, um die kleinsten Unterschiede im zentralen Nickel(II)-Azid-Fragment nachweisen zu können. 

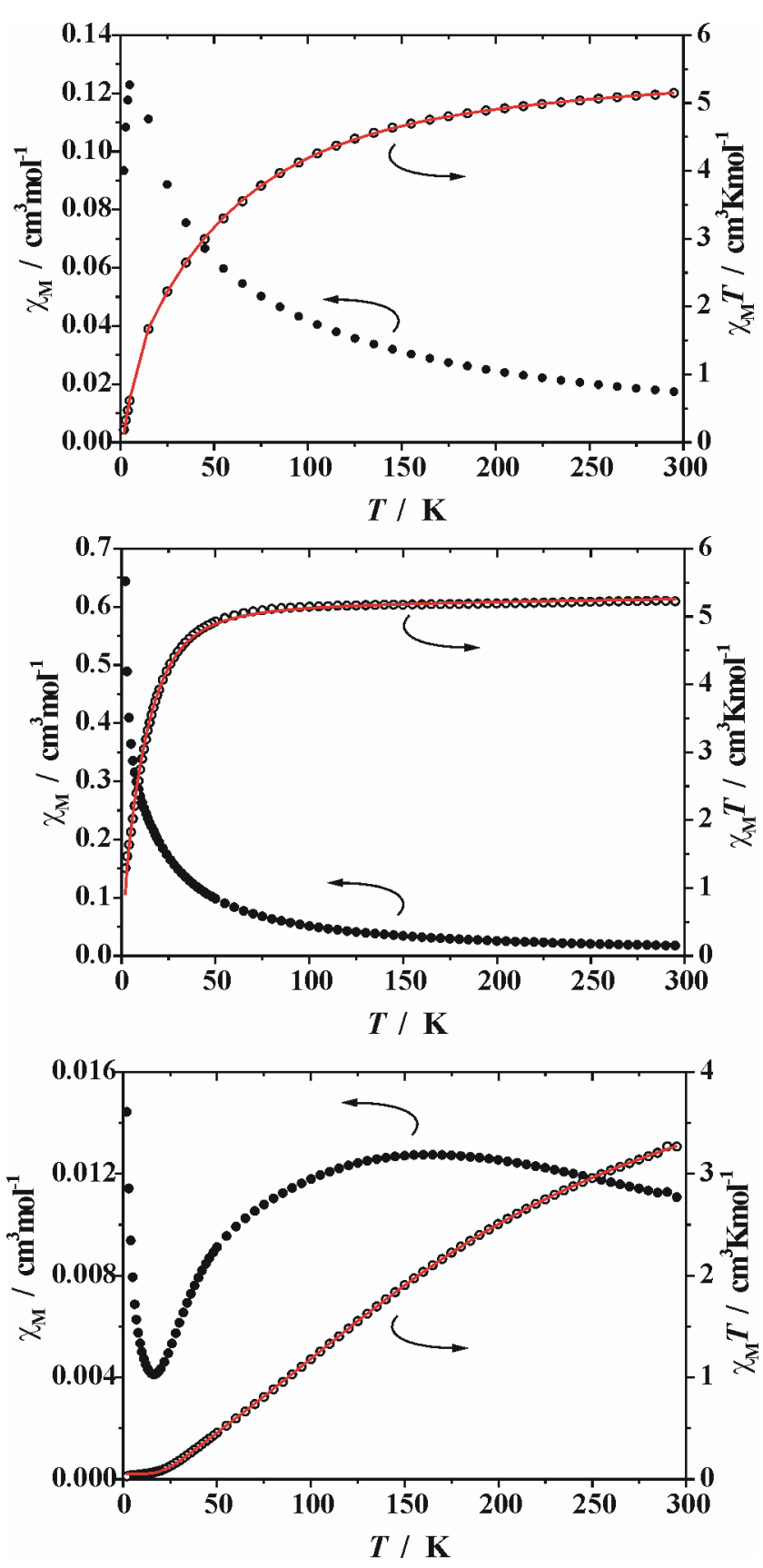

Abb. 4.3.2.4.6. Auftragung von $\chi_{M}$ und $\chi_{M} T$ gegen $T$ für 16 (oben), 17 (in der Mitte) und 18 (unten). Die roten Linien repräsentieren die besten Anpassungen.

Magnetische Eigenschaften

Die Abnahme der $\chi_{M} T$-Werte mit sinkender Temperatur im Fall von 16-18 deutet auf allgemein antiferromagnetisches Verhalten in allen drei Verbindungen hin (Abb. 4.3.2.4.6), welches allerdings unterschiedlich stark ausgeprägt ist. Während für $17 \chi_{M} T$ bis zu $50 \mathrm{~K}$ nahezu konstant bleibt und erst danach sinkt, nehmen die $\chi_{M} T$-Werte für 16 mäßig und für 18 rapide mit sinkender Raumtemperatur ab. Die $\chi_{M} T$-Werte für 16, 17 , 18 liegen bei Raumtemperatur bei 5.15 $\mathrm{cm}^{3} \mathrm{Kmol}^{-1}$, $5.27 \mathrm{~cm}^{3} \mathrm{Kmol}^{-1}$ bzw. 3.18 $\mathrm{cm}^{3} \mathrm{Kmol}^{-1}$. Für 16 und 17 sind diese Werte also etwas höher, für $\mathbf{1 8}$ niedriger als der theoretisch erwartete „spin-only“ Wert für vier ungekoppelte $S=1$ Nickel(II)-Ionen $\left(4.83 \mathrm{~cm}^{3} \mathrm{Kmol}^{-1}\right.$ für $g=$ 2.20).

Das weist auf eine viel stärkere resultierende antiferromagnetische Wechselwirkung im $\mathrm{Ni}_{4}$-Fragment von $\mathbf{1 8}$ als in 16 bzw. 17 hin. Die $\chi_{M} T$-Werte tendieren im Einklang mit dominanter antiferromagnetischer Kopplung und dem $S_{\mathrm{T}}=0$ Grundzustand in allen Komplexen schließlich nach Null.

Unter Berücksichtigung der molekularen Topologie können für den magnetischen Austausch drei Pfade in 16 bzw. $18\left(J_{1}, J_{2}\right.$ und $\left.J_{3}\right)$ und zwei in $17\left(J_{1}\right.$ und $\left.J_{2}\right)$ vorgeschlagen werden (Schema 4.3.2.4.2). 


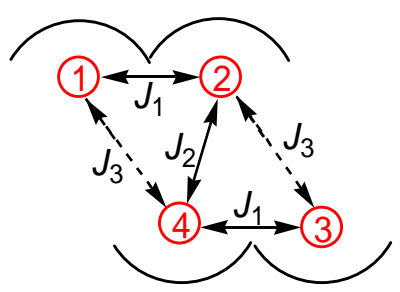

Schema 4.3.2.4.2. Kopplungsschema für $\mathrm{Ni}_{4}$-Komplexe mit der kettenförmigen Anordnung der Nickel(II)Zentren.

Da im Komplex 16 der Abstand Ni2-N10 (entspricht $J_{3}$ in Schema 4.3.2.4.2) sehr groß ist, werden für die Vereinfachung der Interpretation der magnetischen Daten nur zwei Pfade $\left(J_{1}\right.$ und $J_{2}$ ) berücksichtigt.

Die Anpassung an die experimentellen Daten für $\mathbf{1 6}$ und $\mathbf{1 7}$ erfolgte mit dem Programm julX. ${ }^{[106]}$ Es wurde ein Modell mit zwei Kopplungskonstanten, basierend auf nachstehendem Heisenberg-Dirac-van-Vleck-Hamiltonoperator (Gleichung 4.3.2.4.1), verwendet. Ein zusätzlicher Term beschreibt die Zeeman-Aufspaltung. Die $g$-Werte wurden für alle Positionen als identisch angenommen.

$$
\hat{H}=-2 J_{1}\left(\hat{S}_{1} \hat{S}_{2}+\hat{S}_{3} \hat{S}_{4}\right)-2 J_{2}\left(\hat{S}_{1} \hat{S}_{3}\right)+g \mu_{B} B \sum \hat{S}_{i z}
$$

Die dadurch erhaltenen Parameter sind: $g=2.29, J_{1}=-32.3 \mathrm{~cm}^{-1}, J_{2}=+13.7 \mathrm{~cm}^{-1} \rho=1.0 \%$, und TIP $=6.24 \times 10^{-4} \mathrm{~cm}^{3} \mathrm{~mol}^{-1}$ für 16 bzw. $g=2.20, J_{1}=-7.7 \mathrm{~cm}^{-1}, J_{2}=+4.1 \mathrm{~cm}^{-1}, \rho=1.0$ $\%$, und TIP $=3.8 \times 10^{-4} \mathrm{~cm}^{3} \mathrm{~mol}^{-1}$ für 17 .

Die Anpassung an die experimentellen Daten von 18 erfolgte ebenfalls unter Verwendung des Programms julX, ${ }^{[106]}$ welches hier auf einem Heisenberg-Dirac-van-VleckHamiltonoperator basiert, der drei Kopplungskonstanten und einen zusätzlichen Term für die Zeeman-Aufspaltung berücksichtigt (Gleichung 4.3.2.4.2). Die $g$-Werte wurden für alle Positionen als identisch angenommen.

$$
\hat{H}=-2 J_{1}\left(\hat{S}_{1} \hat{S}_{2}+\hat{S}_{3} \hat{S}_{4}\right)-2 J_{2}\left(\hat{S}_{2} \hat{S}_{4}\right)-2 J_{3}\left(\hat{S}_{1} \hat{S}_{4}+\hat{S}_{2} \hat{S}_{3}\right)+g \mu_{B} B \sum \hat{S}_{\text {iz }}
$$

Für 18 findet man eine ferromagnetische Kopplung mit $J_{2}=+32.1 \mathrm{~cm}^{-1}$ sowie zwei dominierende antiferromagnetische Kopplungen $J_{1}=-42.6 \mathrm{~cm}^{-1}$ und $J_{3}=-24.0 \mathrm{~cm}^{-1}$. Die Anpassung liefert einen $g$-Wert von 2.19. 
Für die Interpretation der magnetischen Parameter für 16-18 scheint es auch hier sinnvoll, die einzelnen Fragmente dieser vierkernigen Komplexe separat zu betrachten.

In 16 und 17 sind Ni1 und Ni2 (sowie Ni1a und Ni2a) über Pyrazolat und ein $\mu$-1,1-Azid verbrückt, zugeordnet $\mathrm{zu} J_{1}$ in Schema 4.3.2.4.2. $J_{2}$ wird der Kopplung zwischen den bimetallischen Einheiten über das $\mu$-1,3-Azid, zugeordnet. Wie erwartet, sind die intradimeren Kopplungen über die $\mu$-1,1-Azidbrücke positiv, was auf eine ferromagnetische Wechselwirkung hindeutet. Die für 16 gefundene Kopplungskonstante ist mit $13.7 \mathrm{~cm}^{-1}$ größer und die für 17 mit $4.1 \mathrm{~cm}^{-1}$ etwa gleich im Verhältnis zu der in der zweikernigen Baueinheit $1\left(5.1 \mathrm{~cm}^{-1}\right)$ oder zu denen der vierkernigen Komplexe des Typs 11 (1.7-2.5 $\mathrm{cm}^{-1}$ ). Die größere Kopplungskonstante $J_{1}$ in $\mathbf{1 6}$ kann möglicherweise auf feine geometrische Unterschiede zurückgeführt werden, da in $\mathbf{1 6}$ die Ni-N-Ni Winkel (89.6 ${ }^{\circ}$ bzw. $102.5^{\circ}$ ) kleiner sind als im Vergleich zu 1 bzw. 11a-c $\left(116^{\circ}-118^{\circ}\right)$. Dies stimmt mit den theoretischen Voraussagen überein, nach denen die maximale Kopplung bei $\theta=104^{\circ} \mathrm{zu}$ erwarten ist.

Die interdimere Kopplung $J_{2}$ ist antiferromagnetisch gemäß den bekannten magnetostrukturellen Korrelationen für die doppelten $\mu$-1,3-Azidverbrückungen. Ihre Stärke hängt von dem Diederwinkel $\delta$ ab: je größer dieser Winkel, desto schwächer ist die Kopplung (siehe Kapitel 4.1.2). Das ist auch in hervorragender Übereinstimmung mit den experimentell gefundenen Werten: für $\mathbf{1 7}$ mit einem Winkel von $\delta=38^{\circ}$ beträgt die Kopplungskonstante $J_{2}$ $=-7.7 \mathrm{~cm}^{-1}$, für 16 mit $\delta=17^{\circ}$ dagegen $J_{2}=-32.3 \mathrm{~cm}^{-1}$.

In 18 sind Ni1 und Ni2 (sowie Ni1a und Ni2a) innerhalb der zweikernigen Untereinheit über das Pyrazolat und den cis-Typ der end-to-end Koordination des $\mu_{3}-1,1,3$-Azids verbrückt. Die zwei Untereinheiten sind auf zwei Wegen miteinander gekoppelt: erstens, über doppelte end-on Verbrückung (zwischen Ni1 und Ni1a) und, zweitens, über den zweifach vorkommenden (zwischen Ni2 und Ni1a sowie Ni1 und Ni2a) trans-Typ der end-to-end Koordination der $\mu_{3}-1,1,3$-Azide. Da die ferromagnetische Kopplung bei allen $\theta$-Werten mit einem Maximum bei $\theta=104^{\circ}$ für die zweifach end-on azidverbrückten Nickel(II)-Komplexe vorausgesagt wird, kann der große ferromagnetische Wert von $+32.1 \mathrm{~cm}^{-1}$ eindeutig der Wechselwirkung $J_{2}$ zwischen Ni1 und Ni1a mit dem Winkel $\theta=101.4^{\circ}$ zugeordnet werden. Etwas schwieriger ist die Situation bei der Zuordnung von antiferromagnetischen Kopplungen $\left(J_{1}=-42.6 \mathrm{~cm}^{-1}\right.$ und $\left.J_{3}=-24.0 \mathrm{~cm}^{-1}\right)$. Während der kleinere intradimere Ni1-N5-N6 Winkel von $118.8^{\circ}$ gegenüber dem entsprechenden interdimeren Ni1a-N5-N6 Winkel von $135.7^{\circ}$ stärkere antiferromagnetische Kopplung innerhalb der bimetallischen Einheit verursachen sollte, wirken die Torsionswinkel in der entgegengesetzten Richtung. Die end-toend Azidverbrückung des trans-Typs mit dem interdimeren Winkel $\tau=164.5^{\circ}$ (nahezu 
Planarität) sollte stärkere antiferromagnetische Kopplung als das relativ stark gekippte Azid innerhalb des Dimers $\left(\tau=42.9^{\circ}\right)$ vermitteln. Da zwischen Ni1 und Ni2 zusätzlich die Pyrazolatbrücke existiert, die einen gewissen, wenn auch sehr kleinen Beitrag zur antiferromagnetischen Kopplung beisteuert, kann die größere antiferromagnetische Konstante $J_{1}$ der intradimeren und dem entsprechend die etwas kleinere Konstante $J_{3}$ der interdimeren Austauschwechselwirkung zugeordnet werden.

\section{Zusammenfassung}

$\mathrm{Ni}_{4}$-Komplexe mit kettenförmiger Anordnung der Metallzentren können ausgehend von pyrazolatbasierten zweikernigen Einheiten zusammengefügt werden.

Die Kopplungen innerhalb der bimetallischen Untereinheiten sind ferromagnetisch oder antiferromagnetisch je nach Koordinationsmodus des verbrückenden Azids, welcher durch das Design der Pyrazolliganden vorbestimmt werden kann.

Die bimetallischen Einheiten sind mehr oder weniger gegeneinander verschoben, so dass sich kettenartige Strukturen ausbilden können. Die Verschiebung der zweikernigen Baueinheiten ist unterschiedlich stark und hängt von den eingesetzten Liganden, von der Stöchiometrie sowie von den Kristallisationsbedingungen ab. Zwischen den bimetallischen Baueinheiten können sowohl ferromagnetische wie auch antiferromagnetische Wechselwirkungen auftreten. 


\subsection{3. $J$ - alternierende Ketten, bestehend aus $S=1$ Spinträgern}

Die hier verfolgte allgemeine Designstrategie für die Darstellung $J$-alternierender 1DSysteme basiert auf der Anwendung von zweikernigen Nickel(II)-Baueinheiten, die auf der Basis von Pyrazolliganden aufgebaut sind. ${ }^{[25]}$ In solchen zweikernigen Komplexen kann der Azidmodus durch die Ligandseitenarme vorgegeben werden (siehe Kapitel 4.3.1). Da Metallionen solcher zweikernigen Komplexe meistens freie Koordinationsstellen besitzen, können sie sich zu höheren Aggregaten z.B. vierkernigen Komplexen (siehe Kapitel 4.3.2) verbinden. In diesem Kapitel werden Synthesen, basierend auf oben erwähnter Strategie unter Verwendung der Liganden $\mathbf{H L}^{2,3,6}$, sowie Molekülstrukturen und Eigenschaften von $J$ alternierenden Ketten beschrieben.

\subsubsection{Die Steuerung des Alternanzmusters durch die Ligandgestaltung}

Für den gezielten Aufbau von 1D-Systemen mit alternierenden ferromagnetischen (durch $\mu-1,1-$ Azid) und antiferromagnetischen (durch $\mu$-1,3-Azid) Wechselwirkungen ist die Kontrolle der Art und Weise des Azideinbaus eine wichtige Voraussetzung. Die Pyrazolliganden $\mathbf{H L}^{2}$ und $\mathbf{H L}^{3}$ sind geeignet, zweikernige Komplexe mit vorhersagbarer Azidkoordination, end-on mit $\mathbf{H L}^{2}$ und end-to-end mit $\mathbf{H L}^{3}$, zu bilden (Kapitel 4.3.1). Es ist offensichtlich, dass aus sterischen Gründen zweikernige Einheiten über eine $\mu$-1,3Azidbrücke verknüpft werden sollten. Der Aufbau von Ketten mit ferro-antiferro- und antiferro-antiferro-Alternanzmuster wird somit, wie weiter beschrieben, durch die Wahl geeigneter Liganden vorgegeben.

\section{Synthese und Charakterisierung}

Die Komplexe 19 und 20 (Abb. 4.3.3.1.1) können direkt aus den Liganden $\mathbf{H L}^{2}$ bzw. $\mathbf{H L}^{\mathbf{3}}$ präpariert werden, indem man den jeweiligen deprotonierten Liganden mit zwei Äquivalenten $\mathrm{Ni}\left(\mathrm{ClO}_{4}\right)_{2} \cdot 6 \mathrm{H}_{2} \mathrm{O}$ in Methanol reagieren lässt. Die Lösung von 19 wird danach mit zwei Äquivalenten $\mathrm{NaN}_{3}$ umgesetzt, die Lösung von 20 nach Lösungsmittelwechsel von Methanol zu Aceton mit einem Äquivalent $\mathrm{NaN}_{3}$. Im Falle von 20 entsteht sehr viel öliges Nebenprodukt. Deswegen ist die Ausbeute an Kristallen sehr gering. Die Kristalle von 19 sowie von 20 sind geeignet, durch Röntgenstrukturanalyse ein detailliertes Bild zu liefern (Abb. 4.3.3.1.2 und Abb. 4.3.3.1.3). 


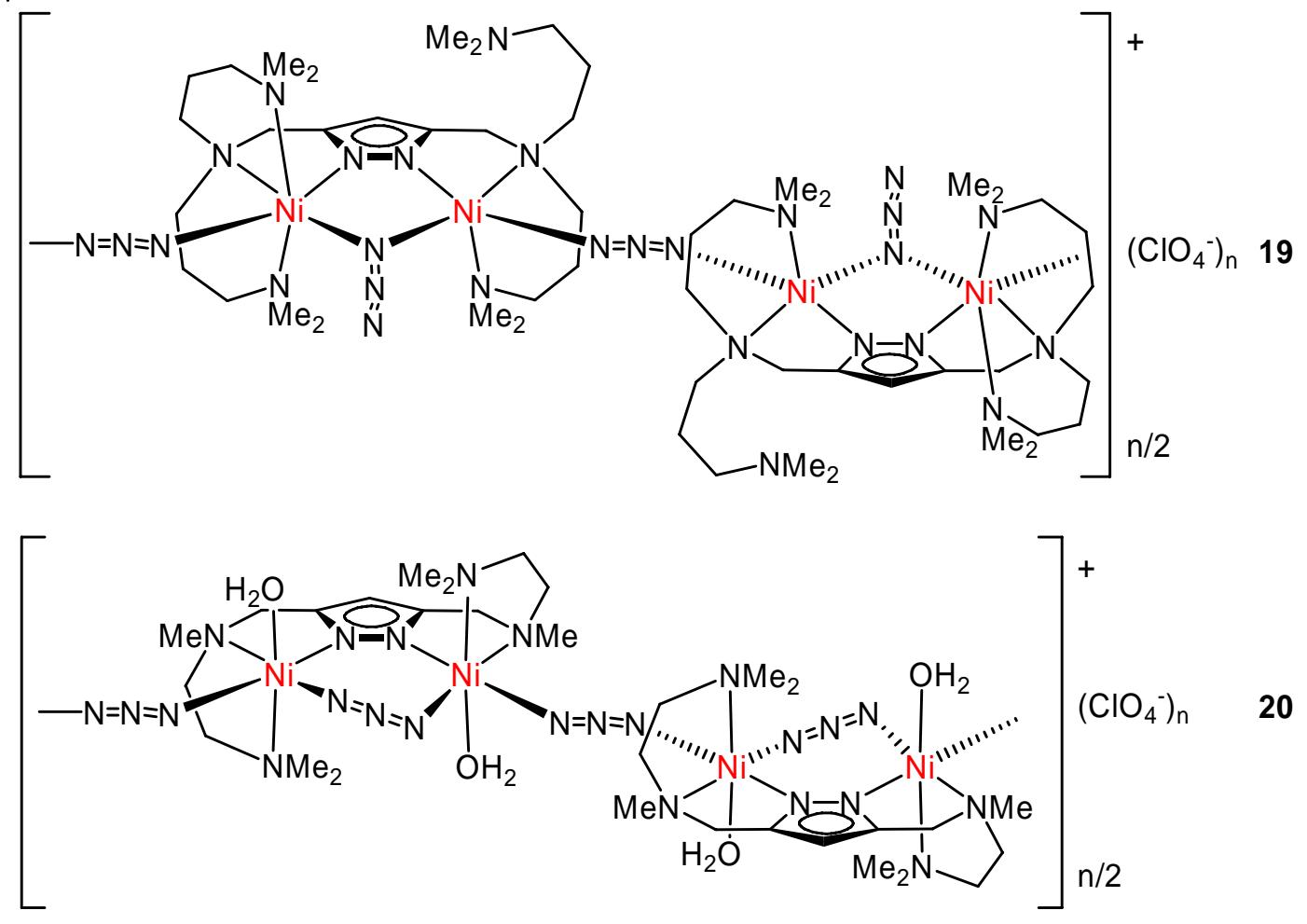

Abb. 4.3.3.1.1. Komplexe 19 und 20.

Beide Ketten bestehen aus zweikernigen pyrazolatbasierten Einheiten, in welchen die Azidkoordination durch die Liganden vorgegeben ist. Die langen Arme in $\mathbf{H L}^{2}$ erlauben den kurzen intradimeren ${ }^{1)}$ Abstand von 3.722(1) Å zwischen den Nickel(II)-Ionen in 19 und damit end-on Koordination des verbrückenden Azids innerhalb der zweikernigen Baueinheit.

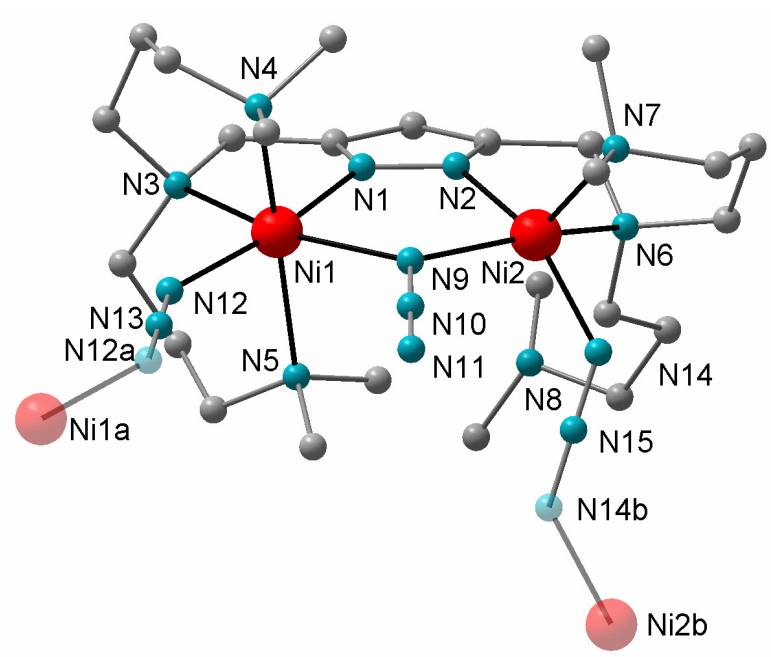

Abb. 4.3.3.1.2. Struktur der molekularen Baueinheit von 19.

\footnotetext{
${ }^{1)}$ Hier und weiter wird analog zu vierkernigen Komplexen als „Dimer“ eine zweikernige pyrazolatbasierte Einheit definiert.
} 
Interessanterweise haben die Nickel(II)-Ionen in $\mathbf{1 9}$ eine unterschiedliche Koordinationsumgebung: $\mathrm{Ni}(1)$ ist sechsfach $\left\{\mathrm{N}_{6}\right\}$, Ni(2) dagegen fünffach $\left\{\mathrm{N}_{5}\right\}$ koordiniert. Der kürzeste Abstand von Ni(2) zum potentiellen sechsten Donoratom N(8) beträgt 5.520(4) Å, während alle anderen Bindungsabstände der Nickel(II)-Ionen zu den Donoratomen in 19 unter $2.3 \AA$ Å liegen. Im Gegensatz zu 19 ziehen kurze Ligandseitenarme in 20 die Metallionen auseinander, wodurch ein größerer intradimerer $\mathrm{Ni}(1) \cdots \mathrm{Ni}(2)$-Abstand von 4.494(1) $\AA$ resultiert und sich somit eine end-to-end Koordination des diese Ionen verbrückenden Azids ergibt.

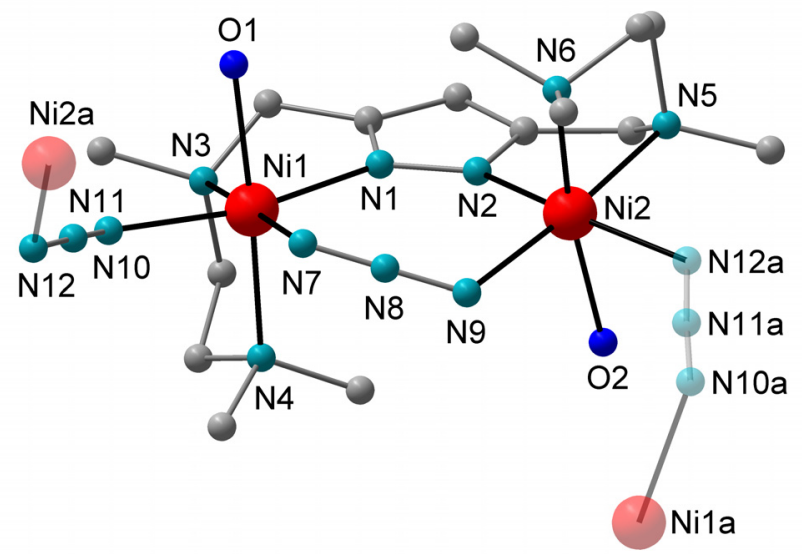

Abb. 4.3.3.1.3. Struktur der molekularen Baueinheit von 20.

Erwartungsgemäß sind die zweikernigen Baueinheiten über end-to-end Azidverbrückung miteinander verknüpft und somit weist Kette 19 ein EO-EE-Alternanzmuster und Kette 20 ein EE-EE-Alternanzmuster auf. Die interdimere Verknüpfung in $\mathbf{1 9}$ ist so gestaltet, dass fünffach bzw. sechsfach koordinierte Nickel(II)-Ionen benachbarter bimetallischer Einheiten jeweils nebeneinander stehen. Dadurch resultieren zwei interdimere end-to-end Azidbrücken mit etwas unterschiedlichen Bindungsparametern (Tab. 4.3.3.1.1).

Tab. 4.3.3.1.1. Ausgewählte Winkel $\left({ }^{\circ}\right)$ von 19 und 20.

\begin{tabular}{lll}
\hline & $\mathbf{1 9}$ & $\mathbf{2 0}$ \\
\hline $\mathrm{Ni}-\mathrm{N}-\mathrm{N}$ (intradimer) & --- & $114.6(2) / 117.9(2)$ \\
$\mathrm{Ni}-\mathrm{N}-\mathrm{N}$ (interdimer) & $133.0(2)\left\{\mathrm{N}_{5}\right\} / 137.0(2)\left\{\mathrm{N}_{6}\right\}$ & $126.9(2) / 130.3(2)$ \\
$\mathrm{Ni}-\mathrm{NNN}-\mathrm{Ni}$ (intradimer) & --- & $43.2(2)$ \\
Ni-NNN-Ni (interdimer) & $180.0(2)$ & $48.8(2)$ \\
$\mathrm{Ni}-\mathrm{N}-\mathrm{Ni}$ & $116.1(1)$ & --- \\
\hline
\end{tabular}


Die Nickel(II)-Ionen stehen in 19 in trans-Position bezüglich des interdimeren Azids und in 20 in cis-Position mit relativ ähnlichen Ni-N-N-Winkeln (Tab. 4.3.3.1.1). Dadurch sind

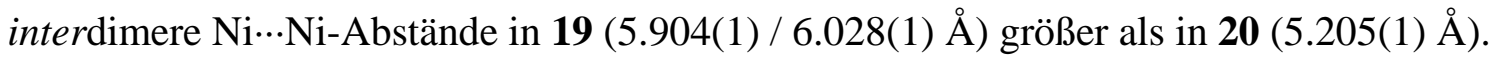

In den IR-Spektren beider Verbindungen beobachtet man eine starke hochenergetische Azidbande bei $2130 \mathrm{~cm}^{-1}$ für 19 und $2102 \mathrm{~cm}^{-1}$ für 20. Im niederenergetischen Bereich der Azidschwingungen liegen die Banden relativ nahe beieinander: eine sehr starke bei $2067 \mathrm{~cm}^{-1}$ für 19 und zwei schwach aufgelöste sehr starke bei $2067 \mathrm{~cm}^{-1}$ und $2045 \mathrm{~cm}^{-1}$ für 20.

\section{Magnetische Eigenschaften}

Die Abnahme der $\chi_{M} T$-Werte mit sinkender Temperatur von 19 sowie 20 deutet auf allgemein antiferromagnetisches Verhalten hin (Abb. 4.3.3.1.4). Allerdings ist dies unterschiedlich stark. Während in $\mathbf{1 9}$ das Produkt $\chi_{M} T$ zunächst nur schwach abnimmt bei Abkühlung auf $75 \mathrm{~K}$ und erst ab dieser Temperatur stark, zeigen die $\chi_{M} T$-Werte für 20 eine rapide Abnahme bereits ab Raumtemperatur. Dementsprechend liegen die $\chi_{M} T$-Werte bei Raumtemperatur bei $2.47 \mathrm{~cm}^{3} \mathrm{Kmol}^{-1}$ und $1.61 \mathrm{~cm}^{3} \mathrm{Kmol}^{-1}$ für $19 \mathrm{bzw}$. 20. Der Wert für 19 ist etwas höher, jener für $\mathbf{2 0}$ viel kleiner als der theoretisch erwartete „spin-only“ Wert für zwei ungekoppelte $S=1$ Nickel(II)-Ionen (2.42 $\mathrm{cm}^{3} \mathrm{Kmol}^{-1}$ für $\left.g=2.20\right)$. Das weist auf viel stärkere resultierende antiferromagnetische Wechselwirkung in 20 als in 19 hin. Die $\chi_{M} T$ Werte tendieren im Einklang mit dominanter antiferromagnetischer Kopplung und dem $S_{\mathrm{T}}=0$ Grundzustand in allen Komplexen schließlich nach Null.
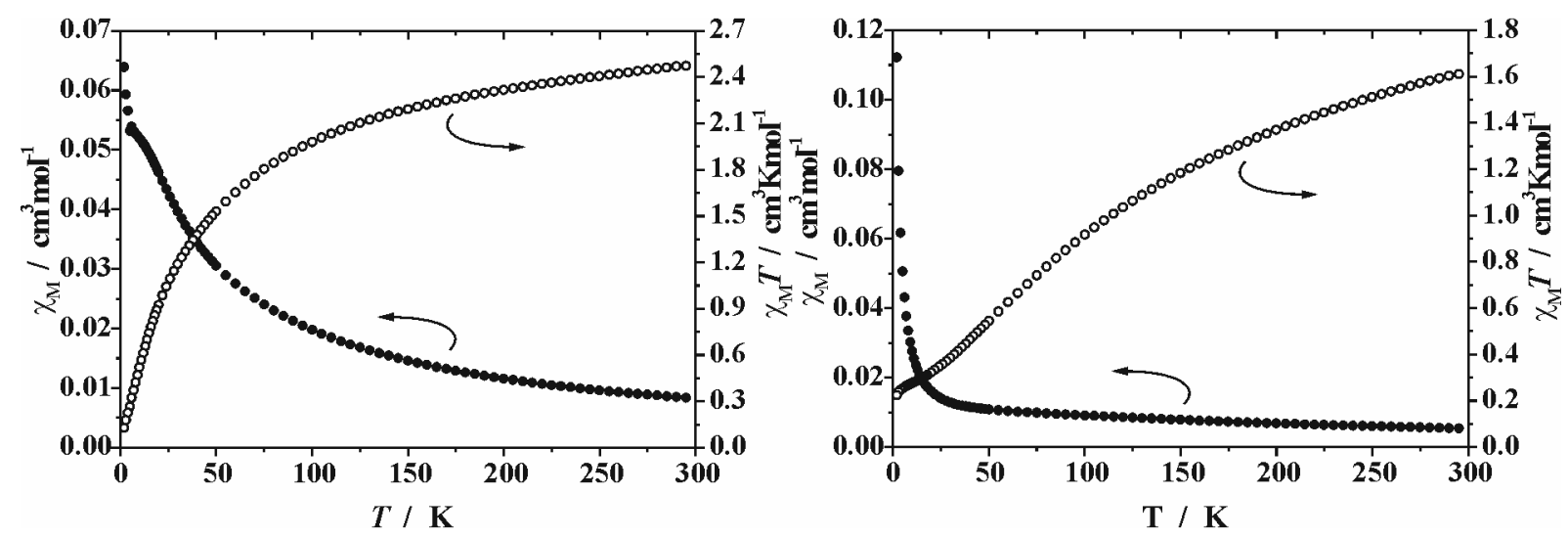

Abb. 4.3.3.1.4. Auftragung von $\chi_{M}$ und $\chi_{M} T$ gegen $T$ für 19 (links) und 20 (rechts).

Unter Berücksichtigung der magnetostrukturellen Korrelationen, sowohl den aus der Literatur (Kapitel 4.1) bekannten als auch den während dieser Arbeit festgestellten, kann man 
19 als eine Kette mit der Alternanz ferromagnetischer (durch $\mu$-1,1-Azid) und antiferromagnetischer (durch $\mu$-1,3-Azid) Kopplung betrachten. 20 stellt dann eine Kette dar, bei welcher zwei unterschiedlich starke antiferromagnetische Kopplungen alternieren, wobei die Unterschiede in der Kopplungsstärke durch die geometrischen Parameter $\alpha$ und $\tau$ verursacht werden. Nach der schematischen Darstellung der Alternanz in 19 und 20 (Abb. 4.3.3.1.5) wird ersichtlich, dass in beiden Verbindungen der $S_{T}=0$ Grundzustand erwartet werden sollte.
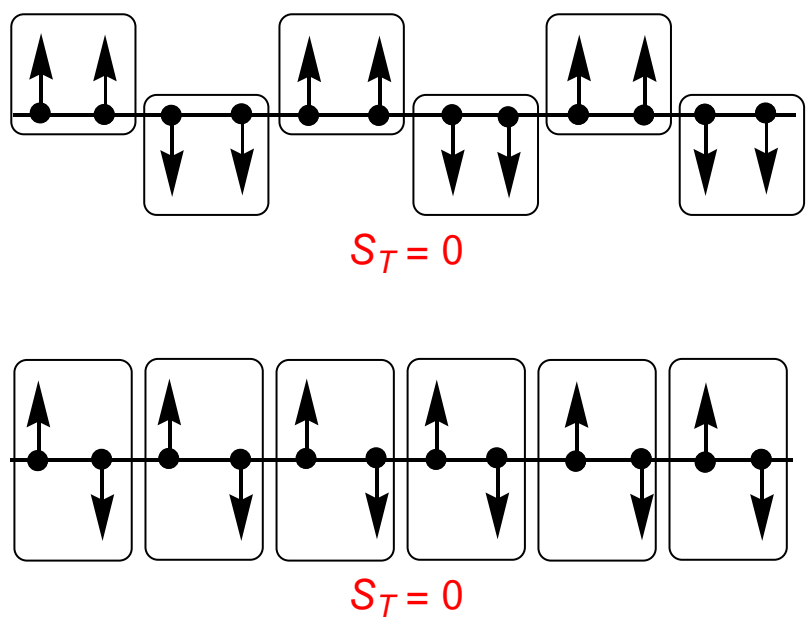

Abb. 4.3.3.1.5. Kopplungsalternanz in 19 (oben) und 20 (unten).

Die langsamere Abnahme der $\chi_{M} T$-Werte mit sinkender Temperatur in 19 kann durch das Vorhandensein ferromagnetischer Kopplung erklärt werden, auch wenn in $\mathbf{1 9}$ die antiferromagnetische Kopplungskonstante stärker sein sollte als jede der beiden antiferromagnetischen Kopplungen in 20 (Torsionswinkel von $180.0^{\circ}$ in $\mathbf{1 9}$ gegen $43.2^{\circ}$ bzw . $48.2^{\circ}$ in 20).

\section{Zusammenfassung}

Die kettenförmigen alternierenden 1D-Systeme 19 und 20 bilden sich ausgehend aus den in situ entstehenden pyrazolatbasierten zweikernigen Einheiten zusammengefügt werden.

Die Kopplungen innerhalb der bimetallischen Untereinheiten sind ferromagnetisch oder antiferromagnetisch je nach Koordinationsmodus des verbrückenden Azids, welcher durch das Design der Pyrazolliganden vorbestimmbar ist.

Die bimetallischen Untereinheiten sind über end-to-end Koordination verknüpft. Somit sind 19 und 20 alternierende Ketten, die auf kontrollierte Weise aufgebaut werden. 


\subsubsection{Variationen an interdimeren Kopplungen in 1D-alternierenden Ketten}

Im Kapitel 4.3.3.1 wurde die Möglichkeit gezeigt, Ketten mit kontrolliertem Alternanzmuster zu synthetisieren. In diesem Kapitel werden drei weitere Beispiele für die Synthese solcher Ketten ausgehend von zweikernigen Baueinheiten beschrieben. Dabei wird der schwefelhaltige Ligand $\mathbf{H L}^{\mathbf{6}}$ verwendet. Die unterschiedlichen Synthesebedingungen und die Möglichkeit, das Gegenion zu variieren, führen zu Ketten mit nahezu gleichen intradimeren aber unterschiedlichen interdimeren geometrischen Parametern. Die mit Hilfe von Quantum Monte Carlo Simulationen errechneten Kopplungskonstanten lassen sich auf der Basis der Struktur interpretieren.

\section{Synthese und Charakterisierung}

Der einfache Austausch von labilen terminal gebundenen Lösungsmittelmolekülen in 5, 6 und 18 sowie das leichte Aufbrechen der vierkernigen Struktur von 18 erlauben es, diese Systeme als geeignete Baueinheiten für den kontrollierten Aufbau (Schema 4.3.3.2.1) der alternierenden 1D-Ketten zu verwenden. Die Zugabe eines zusätzlichen Azidäquivalents führt zu polymeren Komplexen 21-23. ${ }^{[107]}$ Ausgehend von 5 oder 18 in Methanol fällt nach der Zugabe von einem Äquivalent des Azids ein grünes Pulver aus, welches in größeren Mengen auch in situ durch Umsetzung des deprotonierten Liganden $\mathbf{H L}^{6}$ mit 2 Äquivalenten $\mathrm{Ni}\left(\mathrm{ClO}_{4}\right)_{2} \cdot 6 \mathrm{H}_{2} \mathrm{O}$ und 2 Äquivalenten $\mathrm{NaN}_{3}$ in Methanol dargestellt werden kann. Die Umkristallisation dieses Pulvers aus Dichlormethan liefert in Abhängigkeit von der verwendeten Lösungsmittelmenge die Ketten 21 (Raumgruppe $P 2{ }_{1} / n$ ) bzw. 22 (Raumgruppe $P 2_{1} / c$ ), die gleiche Zusammensetzung haben. Die Kristalle der Kette 22 verwittern nach ihrer Isolierung aufgrund relativ schneller Abgabe (wenige Minuten) von Dichlormethanmolekülen aus dem Kristallgitter. Nach der Umsetzung von 6 mit 1 Äquivalent $\mathrm{NaN}_{3}$ in Aceton fällt 23 als Pulver aus. 23 kann auch direkt aus $\left[\mathbf{L}^{6}\right]^{-}, \mathrm{Ni}\left(\mathrm{ClO}_{4}\right)_{2} \cdot 6 \mathrm{H}_{2} 0, \mathrm{NaN}_{3}$ und $\mathrm{NaBPh}_{4}$ dargestellt werden. Man verwendet allerdings nur die Hälfte der stöchiometrischen Menge an $\mathrm{Ni}\left(\mathrm{ClO}_{4}\right)_{2} \cdot 6 \mathrm{H}_{2} 0$, was die Gewinnung des Komplexes in Lösung vor der Zugabe von 2 Äquivalenten $\mathrm{NaBPh}_{4}$ ermöglicht. Die Kristalle von 23 können aus der Lösung in Aceton durch Überschichtung mit Petrolether erhalten werden. 


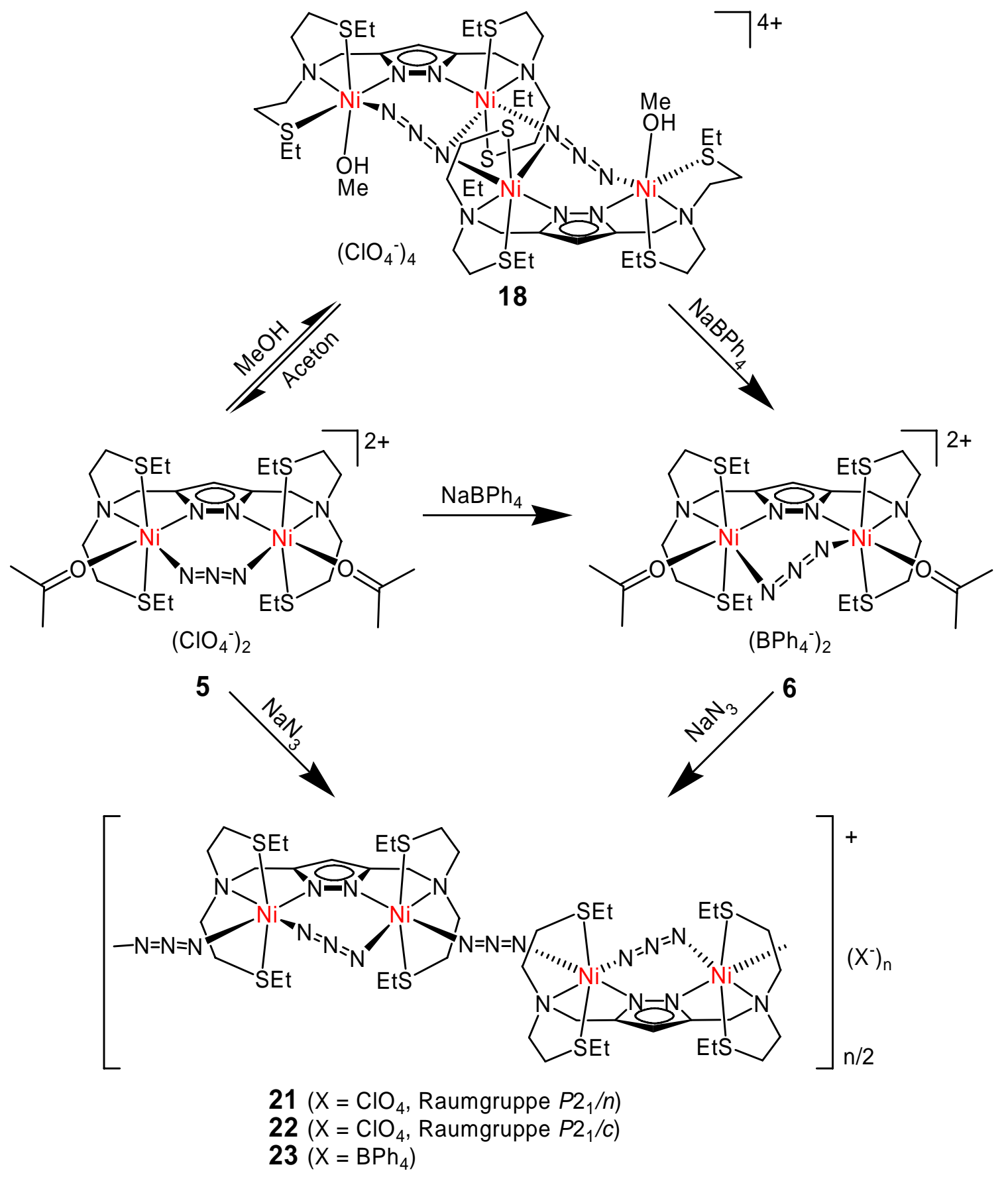

Schema 4.3.3.2.1. Synthetische Zusammenhänge zwischen den Komplexen 5, 6, 18 sowie 21-23.

Die 1D-Kettenstrukturen von 21-23 wurden durch Röntgenstrukturanalyse identifiziert (Abb. 4.3.3.2.1). Die Details der pyrazolatbasierten bimetallischen Untereinheiten sind in Abb. 4.3.3.2.2-4.3.3.2.4 gezeigt. 

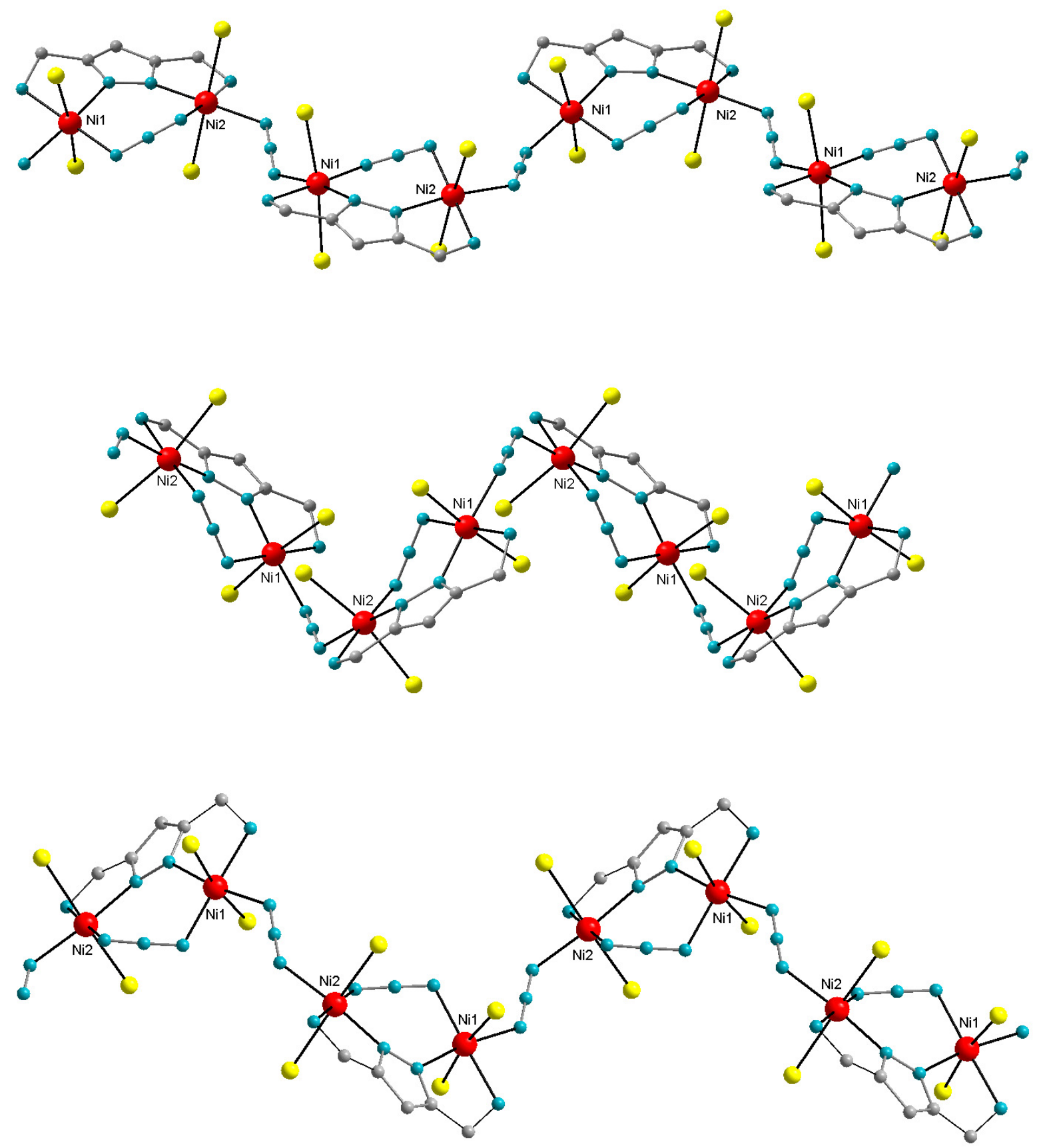

Abb. 4.3.3.2.1. 1D-Ketten 21 (oben), 22 (in der Mitte) und 23 (unten). 


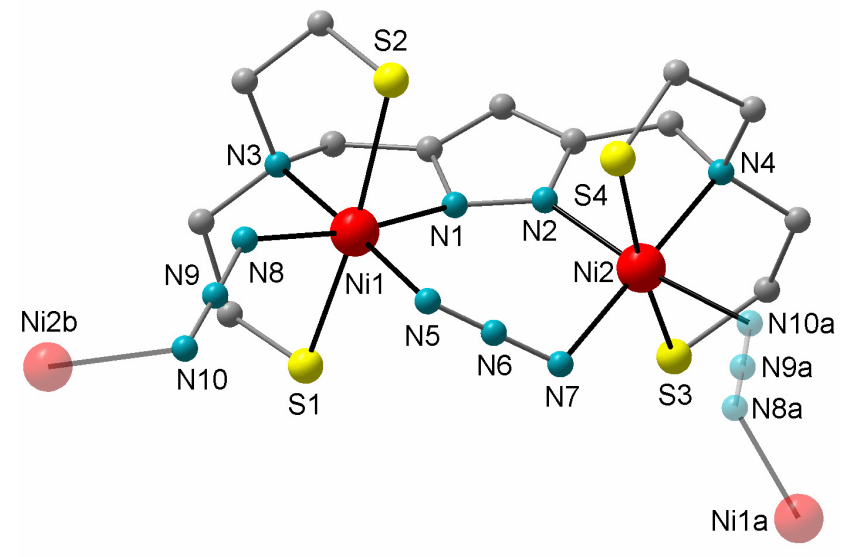

Abb. 4.3.3.2.2. Struktur der molekularen Baueinheit von 21.

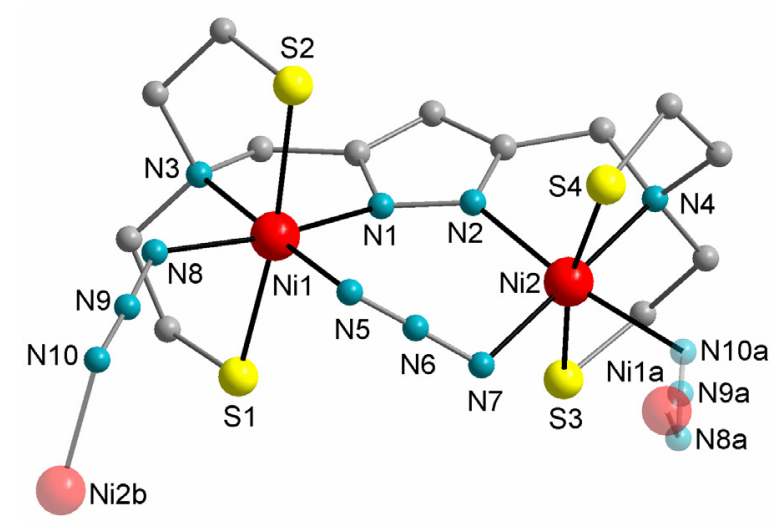

Abb. 4.3.3.2.3. Struktur der molekularen Baueinheit von 22.

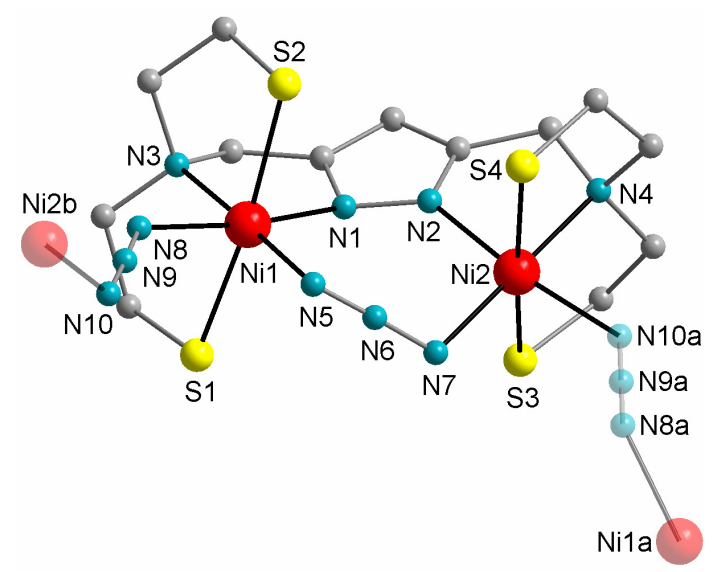

Abb. 4.3.3.2.4. Struktur der molekularen Baueinheit von 23.

In alle drei Verbindungen 21-23 ziehen kurze Ligandseitenarme die Metallionen auseinander, wodurch ein größerer intradimerer $\mathrm{Ni}(1) \cdots \mathrm{Ni}(2)$-Abstand von ca. $4.4 \AA$ (Tab. 4.3.3.2.1) resultiert und sich eine end-to-end Koordination des diese Ionen verbrückenden Azids ergibt. 
Strukturelle Parameter für die pyrazolatbasierten Untereinheiten (Abb. 4.3.3.2.2-4.3.3.2.4) sind sehr ähnlich für alle drei Systeme. Beispielweise liegen die intradimeren Ni-NNN-NiTorsionswinkel in einem sehr engen Bereich von 56-59 (Tab. 4.3.3.2.1).

Tab. 4.3.3.2.1. Ausgewählte Bindungslänge $(\AA)$ und Winkel $\left(^{\circ}\right)$ von 21, 22 und 23.

\begin{tabular}{llll}
\hline & $\mathbf{2 1}$ & $\mathbf{2 2}$ & $\mathbf{2 3}$ \\
\hline $\mathrm{Ni} \cdot \mathrm{Ni}$ (intradimer) & $4.383(1)$ & $4.440(1)$ & $4.348(1)$ \\
$\mathrm{Ni} \cdot \mathrm{Ni}$ (interdimer) & $5.864(1)$ & $5.328(1)$ & 5.824 \\
$\mathrm{Ni}-\mathrm{N}-\mathrm{N}$ (intradimer) & $111.1(2) / 115.6(2)$ & $112.4(2) / 113.2(2)$ & $112.3(4) / 112.8(4)$ \\
$\mathrm{Ni}-\mathrm{N}-\mathrm{N}($ interdimer) & $123.4(2) / 134.3(2)$ & $121.0(2) / 134.3(2)$ & $128.2(4) / 131.4(4)$ \\
$\mathrm{Ni}-\mathrm{NNN}-\mathrm{Ni}$ (intradimer) & $56.1(2)$ & $58.6(2)$ & $56.3(2)$ \\
$\mathrm{Ni}-\mathrm{NNN}-\mathrm{Ni}$ (interdimer) & $144.0(2)$ & $69.6(2)$ & $136.3(2)$ \\
\hline
\end{tabular}

Die pyrazolatbasierten Untereinheiten sind über den zusätzlichen end-to-end Azidliganden verbrückt. Die Nickel(II)-Ionen in 21 und 23 stehen in trans-Position ( $\tau=144.0(2)^{\circ}$ bzw. 136.3(2) $\left.{ }^{\circ}\right)$ bezüglich des interdimeren Azids und in 22 in cis-Position $\left(\tau=69.6(2)^{\circ}\right)$ mit relativ ähnlichen $\mathrm{Ni}-\mathrm{N}-\mathrm{N}-W i n k e l n$ (Tab. 4.3.3.2.1). Dadurch sind interdimere $\mathrm{Ni} \cdots \mathrm{Ni}$ Abstände in 21 und 23 um ca. $0.5 \AA$ größer als in 22 (Tab. 4.3.3.2.1). Die kompaktere Packung der pyrazolatbasierten Einheiten in 22 durch die cis-Position bezüglich des interdimeren Azids im Vergleich zu 21 könnte dadurch zustande kommen, dass die Kristalle von 22 aus der zweifach konzentrierteren Lösung als im Falle 21 entstehen, so dass die bimetallischen Einheiten „gezwungen“ sind, sich kompakt zu lagern. 21-23 beinhalten zwar gleiche end-to-end Azidkoordination entlang der aus Nickel(II)-Ionen bestehenden Kette, allerdings liegen zwei Arten vor, die sich in ihren geometrischen Parametern unterscheiden. In Folge dessen stellen 21-23 1D-Strukturen mit einem EE-EE-Alternanzmuster vor.

IR-Spektren der drei Verbindungen sind ähnlich. (Tab. 4.3.3.2.2). Man beobachtet zwei starke Banden bei $2080-2092 \mathrm{~cm}^{-1}$ und $2040 \mathrm{~cm}^{-1}$.

Tab. 4.3.3.2.2. Ausgewählte IR-Banden $\left(\mathrm{cm}^{-1}\right)$ für $21-23$..

\begin{tabular}{ll}
\hline Komplex & $v_{\mathrm{as}}\left(\mathrm{N}_{3}^{-}\right)$ \\
\hline $\mathbf{2 1}$ & $2120(\mathrm{sh}), 2084(\mathrm{~s}), 2039(\mathrm{vs}), 2008(\mathrm{sh})$ \\
$\mathbf{2 2}$ & $2102(\mathrm{sh}), 2080(\mathrm{vs}), 2041(\mathrm{vs})$ \\
$\mathbf{2 3}$ & $2092(\mathrm{~s}), 2041(\mathrm{vs})$ \\
\hline
\end{tabular}


Interessanterweise kann man der Verlust des Dichlormethans in 22 mit Hilfe der IRSpektroskopie verfolgen. Die Bande im IR-Spektrum von 21 bei $3058 \mathrm{~cm}^{-1}$, zugeordnet zu

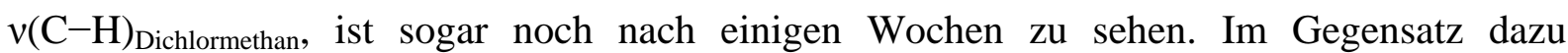
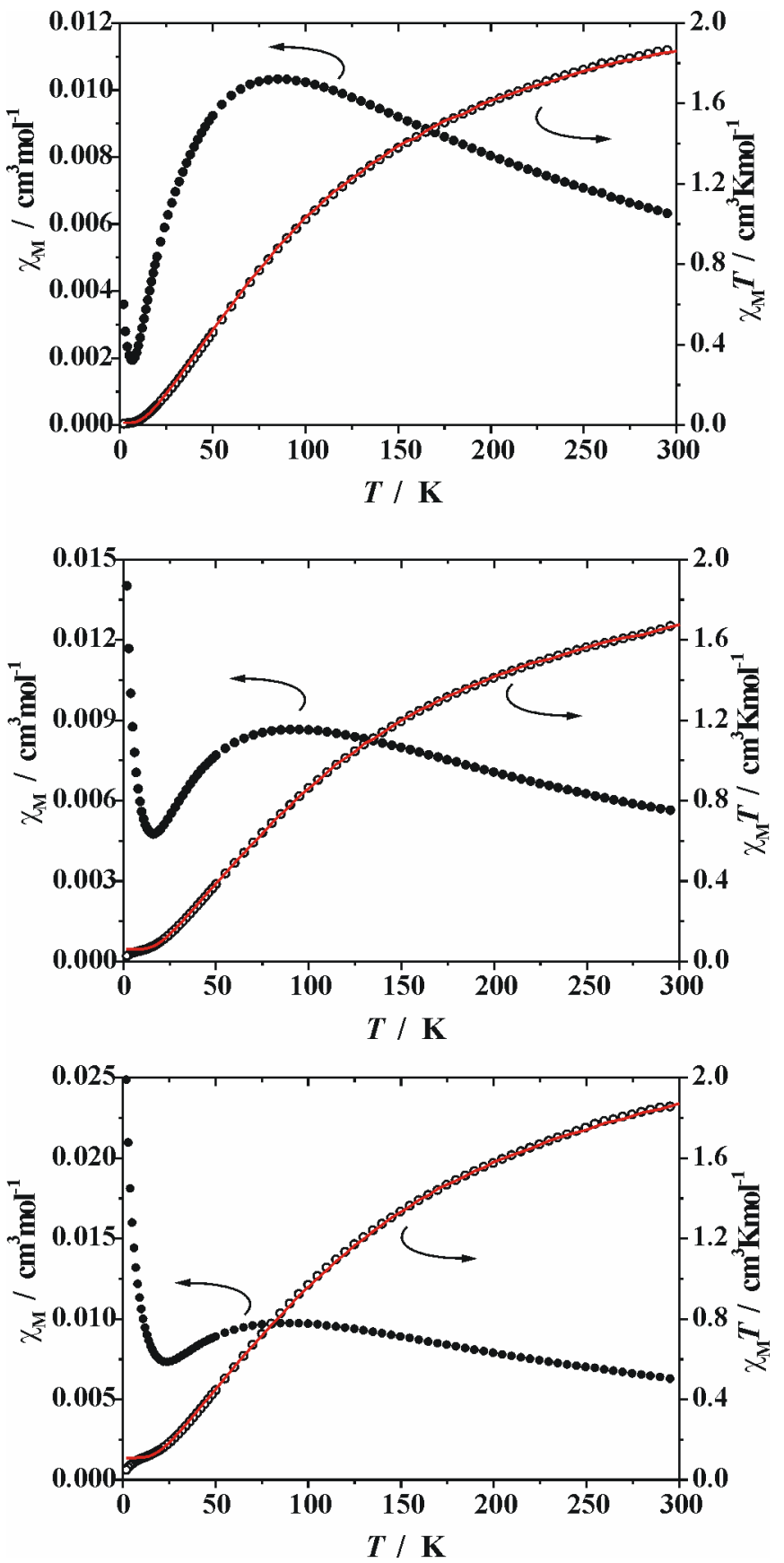

Abb. 4.3.3.2.5. Auftragung von $\chi_{M}$ und $\chi_{M} T$ gegen $T$ für 21 (oben), 22 (in der Mitte) und 23 (unten). Die roten Linien repräsentieren die besten Anpassungen. verwittern Kristalle von 22 unter Abgabe der Lösungsmittelmoleküle schon nach 2-3 Minuten, so dass im IR-Spektrum die Bande von Dichlormethan fehlt. Die frisch isolierten Kristalle von 22, die auch nur sehr kurz gemörsert sind, zeigen jedoch eine Bande bei $3058 \mathrm{~cm}^{-1}$, die im Vergleich zu 21 schwächer ausgeprägt ist.

\section{Magnetische Eigenschaften}

Die Abnahme der $\chi_{M}$ T-Werte mit sinkender Temperatur und das Maximum in den $\chi_{M}$-Kurven deutet auf allgemein antiferromagnetisches Verhalten und den $S_{\mathrm{T}}=0$ Grundzustand in allen Komplexen hin (Abb. 4.3.3.2.5), was für Systeme mit ausschließlich end-to-end verbrückenden Aziden im Einklang mit der in der Regel zu erwartenden Kopplung steht.

Die Anpassung an die experimentellen Daten erfolgte durch Quanten Monte Carlo (QMC) Simulationen. ${ }^{1)}$ Gemäß der Topologie (Schema 4.3.3.2.2) wurden 21-23 durch ein eindimensionales $S=1$ HeisenbergModell beschrieben. Somit lautet der

\footnotetext{
1) Die Anpassungen an die magnetische Suszeptibilität wurden von S. Fuchs und Prof. T. Pruschke während der Zusammenarbeit in Rahmen des SFB 602 „Komplexe Strukturen in kondensierter Materie von atomarer bis mesoskopischer Skala“ durchgeführt.
} 
Operator für den isotropen Austausch wie folgt:

$$
\hat{H}=-2 J_{1} \sum_{i} \hat{S}_{2 i} \hat{S}_{2 i+1}-2 J_{2} \sum_{i} \hat{S}_{2 i+1} \hat{S}_{2 i+2}
$$

Dabei beschreibt eine Kopplungkonstante $J_{1}$ die intradimeren Wechselwirkungen (innerhalb der pyrazolatbasierten zweikernigen Baueinheit), die andere $J_{2}$ die interdimeren (zwischen pyrazolatbasierten Baueinheiten) Wechselwirkungen in allen drei Verbindungen. Die Simulationen wurden für die Systeme von 100 Spinträgern ausgeführt. Dies ist ausreichend, um die Effekte des Kettenabbruchs in den Suszeptibilitätsrechnungen auszuschließen.

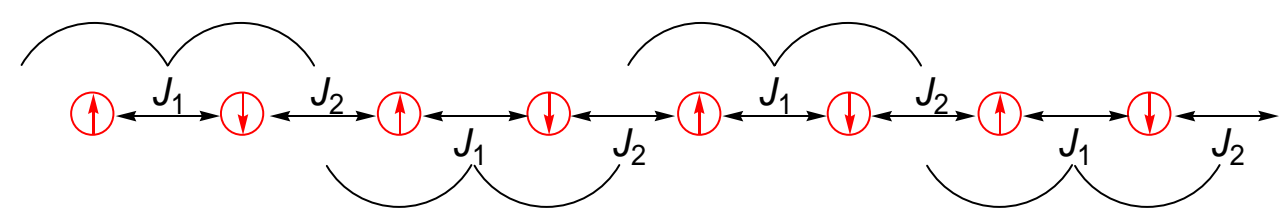

Schema 4.3.3.2.2. Kopplungsschema für Nickel(II)-Ketten 21-23 mit alternierenden Kopplungskonstanten $J_{1}$ und $J_{2}$.

Weitere Parameter, die dieses Modell optimiert, sind der $g$-Wert und die Konzentration von paramagnetischen „Verunreinigungen“ $\rho$, die für die Erklärung des beobachteten Anstiegs von $\chi_{M}$ bei tiefen Temperaturen herangezogen werden. Es wurde vorausgesetzt, dass diese „Verunreinigungen“ Curie-Verhalten zeigen. Die roten Linien in Abb. 4.3.3.2.5 repräsentieren die besten Anpassungen für 21-23, die resultierenden Parameter sind in Tab. 4.3.3.2.3 zusammengefasst.

Tab. 4.3.3.2.3. Anpassungsparameter an die Daten der Suszeptibilitätsmessungen für 21-23.

\begin{tabular}{cccc}
\hline Komplex & $g$ & $J_{1}, \mathrm{~cm}^{-1}$ & $J_{2}, \mathrm{~cm}^{-1}$ \\
\hline $\mathbf{2 1}$ & 2.20 & -29.5 & -9.7 \\
$\mathbf{2 2}$ & 2.09 & -34.7 & -8.3 \\
$\mathbf{2 3}$ & 2.20 & -36.1 & -9.0 \\
\hline
\end{tabular}

Die $g$-Werte haben die für Nickel(II)-Ionen typischen Größe von 2.09 bis 2.20 (siehe Tab. 4.3.3.2.3), die Konzentration von „Verunreinigungen“ liegt auch im akzeptablen Bereich (0.5 \%, 2.8 \% und 4.6 \% für entsprechend 21, 22 und 23). Für alle Komplexe findet man zwei negative Werte, die antiferromagnetische Kopplungen beschreiben. Eine liegt im Bereich von -8 bis $-10 \mathrm{~cm}^{-1}$, die andere ist etwa dreifach größer $\left(-29\right.$ bis $\left.-36 \mathrm{~cm}^{-1}\right)$. 
Die Zuordnung der berechneten Kopplungskonstanten kann auf Basis der Strukturparameter unter Berücksichtigung der bekannten magnetostrukturellen Korrelationen für end-to-end verbrückende Azidliganden (siehe Kapitel 4.1.2) sowie durch Vergleich mit den Eigenschaften der zweikernigen Baueinheit 6 getroffen werden. Die Ni-N-N-Winkel für die intradimeren Verbrückungen sind in allen drei Verbindungen kleiner (111-116 $)$ als für die interdimeren $\left(121-135^{\circ}\right)$. Daraus sollte eine stärkere Kopplung innerhalb der pyrazolatbasierten Baueinheiten resultieren. Die Torsionswinkel für die intradimeren Verbrückungen liegen in einem sehr schmalen Bereich (56-59 ) und sind sehr unterschiedlich für die interdimeren Verbrückungen $\left(144.0^{\circ}, 69.6^{\circ}\right.$ und $136.3^{\circ}$ für 21, 22 und 23). Das interdimere Nickel(II)-Azid-Fragment in 22 mit dem Torsionswinkel von $69.6^{\circ}$ ist am nächsten zur Orthogonalität, was die kleine interdimere Kopplungskonstante verursachen sollte. Schließlich beobachtet man für die zweikernige Baueinheit 6, die sehr ähnliche Bindungsparameter von $\theta=59.5^{\circ}$ und $\tau=111-114^{\circ}$ bezüglich der pyrazolatbasierten Fragmente in 21-23 aufweist, eine Kopplungskonstante von $-28.8 \mathrm{~cm}^{-1}$. Somit werden die Kopplungskonstanten $J_{1}$ den intradimeren und $J_{2}$ den interdimeren Austauschwechselwirkungen zugeordnet.

\section{Zusammenfassung}

Die J-alternierenden 1D-Systeme 21-23 können sowohl ausgehend von den in situ entstehenden pyrazolatbasierten zweikernigen Einheiten als auch aus den vorher isolierten Baueinheiten 5 bzw. 6 gebildet werden.

Die zweikernigen Einheiten in 21-23 haben ähnliche geometrische Parameter und sind über einen zusätzlichen end-to-end Azidliganden verbrückt. Die interdimeren Nickel(II)Azid-Fragmente unterscheiden sich relativ stark, vor allem im Torsionswinkel. Diese Unterschiede können durch Wahl des Gegenions sowie durch die Kristallisationsbedingungen beeinflusst werden.

Die Messungen der magnetischen Suszeptibilität weisen auf allgemein antiferromagnetisches Verhalten aller drei Verbindungen hin. Die qualitative Berechnung der magnetischen Parameter von 21-23 wurde unter Verwendung von Quanten Monte Carlo Simulationen durchgeführt. Für jede Kette wurden zwei alternierende antiferromagnetische Kopplungen gefunden, wobei die intradimeren deutlich stärker als die interdimeren sind. 


\subsubsection{Topologisch ferrimagnetische Kette}

Die Variation der Kristallisationsbedingungen erlaubte bei Einsatz des Pyrazolliganden $\mathbf{H L}^{6}$ die Darstellung einer Kette 24, die in der Zusammensetzung (abgesehen von den im Kristallgitter eingebauten Lösungsmittelmolekülen) identisch zu 21 bzw. 22 ist, aber ein anderes Alternanzmuster besitzt. Es handelt sich um ein 1D System, bei dem innerhalb der pyrazolatbasierten Baueinheiten alternierend end-on bzw. end-to-end Azidverbrückungen auftreten. 24 zeigt den für ferrimagnetische Ketten typischen Kurvenverlauf des magnetischen Moments als Funktion der Temperatur. Da 24 ausschließlich $S=1$ Spinträger besitzt, kann das ferrimagnetische Verhalten nur durch die Topologie zustande kommen. Dadurch wird 24 als topologisch ferrimagnetische Kette bezeichnet.

\section{Synthese und Charakterisierung}

24 wird direkt aus $\left[\mathbf{L}^{6}\right]^{-}, \mathrm{Ni}\left(\mathrm{ClO}_{4}\right)_{2} \cdot 6 \mathrm{H}_{2} \mathrm{O}$ und $\mathrm{NaN}_{3}$ in Methanol dargestellt. Man verwendet allerdings nur 1.2 Äquivalenten an $\mathrm{NaN}_{3}$ und erhält dabei den Komplex in Lösung. Die Kristalle von 24 können aus der methanolischen Lösung durch Überschichtung mit Diethylether erhalten werden. Alternativ können auch 2 Äquivalente von $\mathrm{NaN}_{3}$ verwendet werden. Man filtriert dabei ausgefallenes Pulver ab und lässt die Lösung einige Wochen stehen.

Die 1D-Kettenstruktur von 24 wurde durch die Röntgenstrukturanalyse aufgeklärt (Abb. 4.3.3.3.1). Im Detail ist das elementare Bauelement in Abb. 4.3.3.3.2 gezeigt.

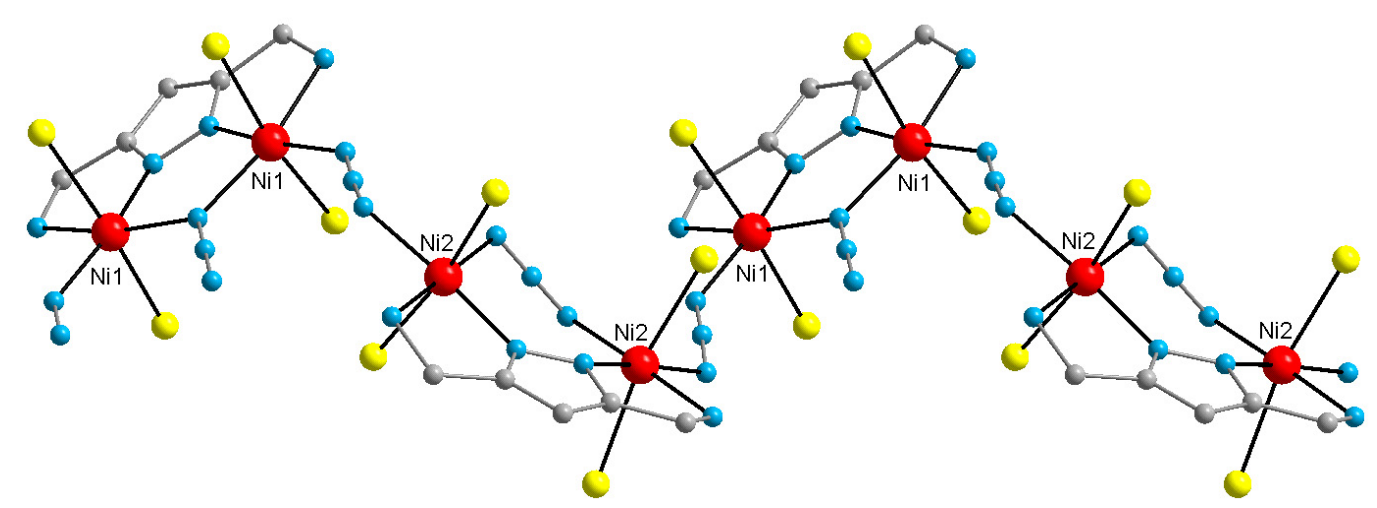

Abb. 4.3.3.3.1. 1D-kettenartige Struktur des Kations von 24. 


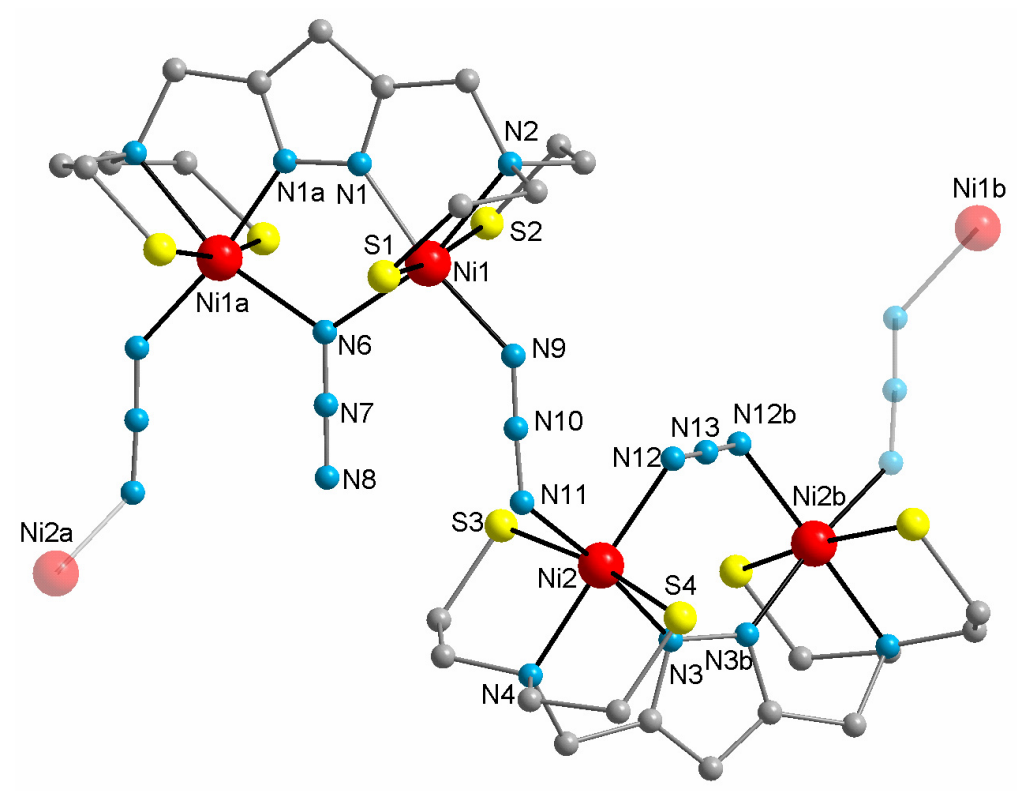

Abb. 4.3.3.3.2. Struktur des elementaren Baufragments von 24. Die am Schwefel gebundenen Ethylgruppen wurden aus Übersichtlichkeitsgründen weggelassen.

Die einzelnen Kettenmoleküle von $\mathbf{2 4}$ stapeln sich entlang der $\boldsymbol{a}$-Achse und bilden Kanäle, die mit Lösungsmittelmolekülen (Wasser und Methanol) gefüllt sind. Da die Lösungsmittelmoleküle nicht gebunden sind, verwittern Kristalle sofort nach der Isolierung aus der Mutterlauge.

Die Kette besteht aus alternierenden zweikernigen pyrazolatbasierten Einheiten: eine Hälfte davon besitzt end-on, die andere Hälfte end-to-end verbrückende Azidliganden. Diese Pyrazolateinheiten sind über die zusätzlichen end-to-end Azidliganden verbrückt. Somit besteht das elementare Fragment aus zwei bimetallischen pyrazolatbasierten Einheiten mit der alternierenden intradimeren end-on und end-to-end sowie interdimeren end-to-end Verbrückungen. Diese Fragmente sind über ein end-to-end Azid verbrückt, so dass eine Kette mit EO-EE-EE-EE Alternanz resultiert. Die ausgewählten strukturellen Parameter sind in Tab. 4.3.3.3.1 zusammengefasst.

Tab. 4.3.3.3.1. Ausgewählte Bindungslängen $(\AA)$ und Winkel $\left(^{\circ}\right)$ von 24.

\begin{tabular}{|c|c|c|c|}
\hline Parameter & Wert & Parameter & Wert \\
\hline Ni1 $\cdots$ Ni1a (intradimer) & $3.598(1)$ & Ni1-N9-N10 (interdimer) & $138.3(3)$ \\
\hline Ni2 $\cdots \mathrm{Ni} 2 \mathrm{~b}$ (intradimer) & $4.442(1)$ & Ni2-N11-N10 (interdimer) & 122.7(3) \\
\hline Ni1 $\cdots$ Ni2 (interdimer) & $5.717(1)$ & Ni2-N12-N13 (intradimer) & $113.8(3)$ \\
\hline \multirow[t]{2}{*}{ Ni1-N6-Ni1a (intradimer) } & $114.7(3)$ & Ni1-N9-N11-Ni2 (interdimer) & $121.7(3)$ \\
\hline & & Ni2-N12-N12b-Ni2b (intradimer) & $56.0(3)$ \\
\hline
\end{tabular}


Die bimetallische Pyrazolateinheit mit $\mu$-1,3-verbrückendem Azid hat strukturelle Parameter, die sehr ähnlich zu den entsprechenden in den Ketten 21-23 sind (Tab. 4.3.3.2.1, Tab. 4.3.3.3.1). Das Zusammenwirken des Liganden $\mathbf{H L}^{\mathbf{6}}$, der weiche S-Donoratome besitzt, mit geeigneten Kristallisationsbedingungen (in erster Linie bestimmt durch das Lösungsmittel), erlaubt neben $\mu$-1,3- auch $\mu$-1,1-Verbrückung des Azidliganden innerhalb der pyrazolatbasierten Baueinheit in einer Verbindung. Der $\mathrm{Ni} \cdots \mathrm{Ni}$-Abstand für des end-on verbrückende Fragment wird dabei ca. $0.85 \AA$ Aürzer und beträgt 3.598(1) Å. Der Ni-N-NiWinkel ist 114.7 (3) o, was sogar etwas kleiner ist als die entsprechenden Winkel in Komplexen mit den langen Ligandseitenarmen (116.1-118.3 ${ }^{\circ}$ ). Die interdimeren Ni-N-NWinkel sind vergleichbar mit den entsprechenden Winkeln in 21-23 (Tab. 4.3.3.2.1, Tab. 4.3.3.3.1). Der interdimere Torsionswinkel in 24 (121.7(3) ${ }^{\circ}$ ) ist dagegen größer als in 22 $\left(69.6(2)^{\circ}\right)$ und kleiner als in 21 bzw. 23 (144.0(2) ${ }^{\circ}$ und $\left(136.3(2)^{\circ}\right)$.

In IR-Spektrum von 24 beobachtet man zwei sehr starke Banden bei $2122 \mathrm{~cm}^{-1}$ und 2043 $\mathrm{cm}^{-1}$. Im Vergleich zu 21-23 ist die höherenergetische Bande um ca. 30-40 $\mathrm{cm}^{-1} \mathrm{zu}$ kürzeren Wellenlängen verschoben.

\section{Magnetische Eigenschaften}

Die magnetischen Eigenschaften wie auch die Struktur von $\mathbf{2 4}$ sind für diese Verbindungsklasse sehr ungewöhnlich. Die Temperaturabhängigkeit von $\chi_{M}$ und $\chi_{M} T$ für 24 ist in Abb. 4.3.3.3.1 gezeigt.

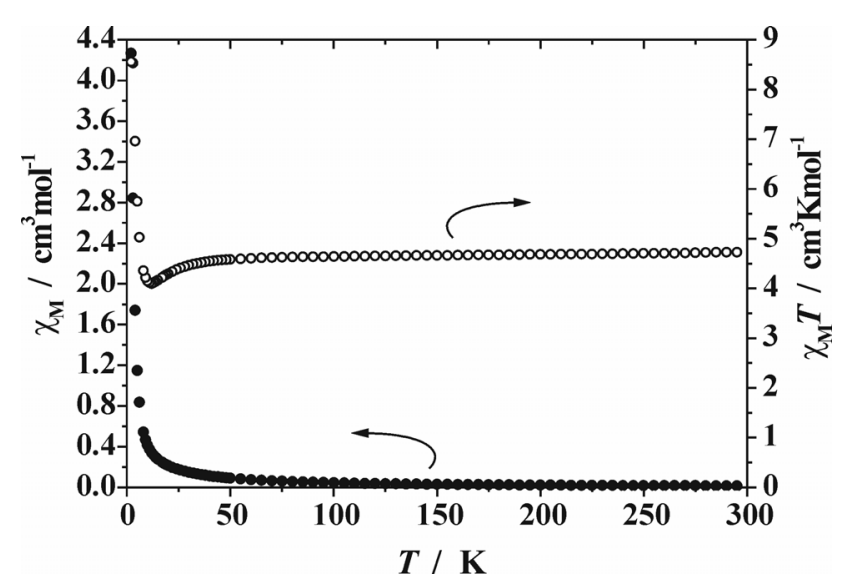

Abb. 4.3.3.3.3. Auftragung von $\chi_{M}$ und $\chi_{M} T$ gegen $T$ für 24 . 
Der $\chi_{M} T$-Wert des Komplexes liegt bei Raumtemperatur bei $4.73 \mathrm{~cm}^{3} \mathrm{Kmol}^{-1}$. Dies ist sehr nahe am theoretisch erwarteten „spin-only“ Wert für vier ungekoppelte $S=1$ Nickel(II)-Ionen (4.83 $\mathrm{cm}^{3} \mathrm{Kmol}^{-1}$ für $\left.g=2.20\right)$. Mit sinkender Temperatur zeigt sich zuerst ein Minimum bei ca. $12 \mathrm{~K}$ in der Auftragung $\chi_{M} T$ gegen $T$, danach steigt das magnetische Moment rapide an $\left(8.53 \mathrm{~cm}^{3} \mathrm{Kmol}^{-1}\right.$ bei $2 \mathrm{~K}$ ). Ein solcher Kurvenverlauf des magnetischen Moments bzw. von $\chi_{M} T$ ist typisch für ferrimagnetische Ketten. 24 ist aber keine heterometallische Verbindung, für welche das ferrimagnetische Verhalten durch die unterschiedliche Größe der Spinquantenzahlen der paramagnetischen Zentren erklärt werden kann. Dadurch ist der Ferrimagnetismus in 24 offensichtlich topologischer Natur (siehe Kapitel 4.1.5). Andererseits sollte ein insgesamt ferrimagnetisches Verhalten erst dann auftreten, wenn eine Sequenz aus einer geraden Zahl von antiferromagnetischen Wechselwirkungen einer oder mehreren ferromagnetischen Wechselwirkungen folgt. ${ }^{[91]}$ Wenn aber 24 nach bekannten magnetostrukturellen Korrelationen analysiert wird, ergibt sich eine Sequenz aus einer ungeraden Zahl von antiferromagnetischen Wechselwirkungen (zwei mal intradimere und ein mal interdimere end-to-end Verbrückungen) gefolgt von ferromagnetischen Wechselwirkungen (ein mal intradimere end-on Verbrückungen). Dies sollte zu einem insgesamt antiferromagnetischen Verhalten mit dem $S_{T}=0$ Grundzustand führen (Abb. 4.3.3.3.4 a).

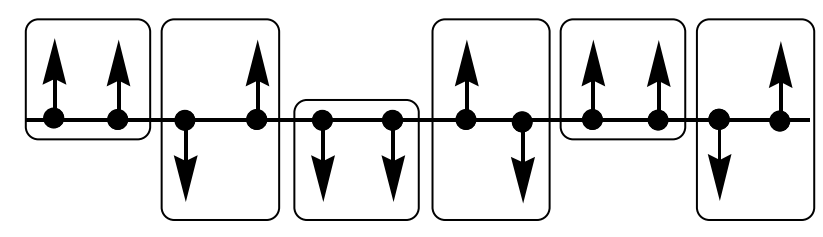

a $\quad S_{T}=0$

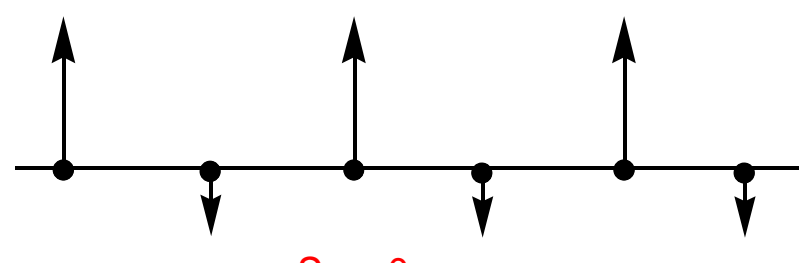

b $\quad S_{T}>0$

Abb. 4.3.3.3.4.Varianten der Kopplungsalternanz in 24: a) mit einem insgesamt antiferromagnetischen Verhalten; b) mit einem insgesamt ferrimagnetischen Verhalten.

Für die Erklärung des beobachteten ferrimagnetischen Verhaltens können einige Hypothesen vorgeschlagen werden. Wenn die bimetallischen Pyrazolateinheiten als einzelne Spinträger betrachtet werden, hat man dann in zweikernigen Einheiten mit einer $\mu$-1,1- 
Azidbrücke einen $S_{T}=2$ Grundzustand und in zweikernigen Einheiten mit einer $\mu$-1,3Azidbrücke einen $S_{T}=0$ Grundzustand. Durch die Alternanz der Einheiten mit unterschiedlichem Spin ergibt sich ein ferrimagnetisches Verhalten (Abb. 4.3.3.3.4 b). Nach einer anderen Hypothese kann man sich vorstellen, dass sich durch die Abgabe des Lösungsmittels und damit verbundene Verwitterung der Kristalle die Parameter und dementsprechend die dadurch vermittelte Kopplung ändern. Dies wäre nicht ungewöhnlich, wenn man die Flexibilität insbesondere von end-to-end verbrückendem Azid berücksichtigt. Nach diesem Modell sollte allerdings in jedem zweiten interdimeren Nickel(II)-AzidFragment der Torsionswinkel sich in Richtung $90^{\circ}$ ändern, so dass eine zweite ferromagnetische Kopplung vermittelt wird und sich die F-F-AF-AF-Sequenz ergibt. Es kann nicht ausgeschlossen werden, dass bei sehr tiefen Temperaturen ein Phasenübergang stattfindet, der die Ursache dieses Verhaltens ist.

\section{Zusammenfassung}

Das 1D System mit EO-EE-EE-EE Alternanzmuster 24 wurde synthetisiert. Im Unterschied zu den Ketten 21 und 22, die dieselbe Zusammensetzung haben, beobachtet man den ständigen Wechsel zwischen $\mu$-1,1- und $\mu$-1,3-Azidverbrückung aufeinander folgender Pyrazolateinheiten. Eine entscheidende Rolle bei der Bildung der Kristalle von 24 kommt dem Lösungsmittel zu. Das insgesamt ferrimagnetische Verhalten von $\mathbf{2 4}$ ist topologisch bedingt. 


\subsubsection{Magnetische Hysteresebistabilität basierend auf einem molekularen Azid- Schalter: das „Chamäleon-System“ $\left[\mathrm{L}^{6} \mathrm{Ni}_{2}\left(\mathrm{~N}_{3}\right)_{3}\right]$}

In diesem Kapitel werden zweikernige Komplexe mit der Zusammensetzung $\left[\mathrm{L}^{6} \mathrm{Ni}_{2}\left(\mathrm{~N}_{3}\right)_{3}\right]$ beschrieben. Es handelt sich dabei um ein System, das auf kleine Änderungen der Kristallisationsbedingungen sehr flexibel reagiert. Dementsprechend führt das zur Kristallisation koordinationsisomerer ${ }^{1)}$ Verbindungen. Das magnetische Verhalten isomerer Verbindungen des „Chamäleon-Systems“ ist unterschiedlich. Zwei Verbindungen aus dieser Gruppe weisen eine sehr ungewöhnliche magnetische Hysteresebistabilität auf.

Materialien, die Hysteresebistabilität bereits bei Temperaturen nahe der Raumtemperatur aufweisen, können große Bedeutung für zukünftige Speicher- oder Sensoranwendungen haben. ${ }^{[114]}$ Die thermisch-magnetische Hysterese mit einer abrupten Eigenschaftenänderung bei Raumtemperatur ist besonders selten für Materialien auf molekularer Basis. ${ }^{[115]}$ Spincrossover-Komplexe liefern dafür mit Sicherheit die besten Beispiele. ${ }^{[116]}$ Kürzlich wurde magnetische Hysterese auch für einige molekulare organische Radikale ${ }^{[117]}$ und für Übergangsmetallkomplexe beobachtet. ${ }^{[118,119]}$ Hier kommt die Umwandlung zwischen zwei Zuständen durch den thermischen Phasenübergang mit Orientierungsänderungen im 3DKristallgitter zustande.

In diesem Kapitel wird unter anderem die thermische Hysterese der magnetischen Suszeptibilität für Einkristalle des molekularen Dinickel(II)-Komplexes $\left[\mathrm{L}^{6} \mathrm{Ni}_{2}\left(\mathrm{~N}_{3}\right)_{3}\right] \mathbf{9}^{[120]}$ beschrieben, wobei der $\mu_{1,3}$-verbrückende Azidligand als Ein-/Ausschalter für die intramolekulare antiferromagnetische Kopplung zwischen zwei Metallionen fungiert.

\section{Synthese und Charakterisierung}

Die isomeren Verbindungen 7-10 ${ }^{2}$ (Abb. 4.3.4.1) werden wie folgt synthetisiert. Die Umsetzung des deprotonierten Liganden $\mathbf{H L}^{6}$ mit 1 Äquivalent Nickel(II)perchlorat und 3.5 Äquivalenten Natriumazid in Methanol führt zu 7. Beim Ansatz in DMSO mit veränderter Stöchiometrie (2 Äquivalente Nickel(II)perchlorat und 2 Äquivalente Natriumazid) wird der Komplex 8 erhalten, welcher in $\mathbf{7}$ umgewandelt werden kann, indem man die Kristalle von 8 in Methanol auflöst und mit Diethylether aussalzt. Für die Synthese von 9 erhöht man die Azidmenge im Vergleich zu 8 auf 3.5 Äquivalente (die Mende von Nickel(II)perchlorat bleibt

\footnotetext{
1) Abgesehen von im Kristallgitter eingebauten Lösungsmittelmolekülen in $\mathbf{7}$ und $\mathbf{8}$.

2) Die Verbindung 9 wurde erstmalig von G. Leibeling synthetisiert.
} 
dieselbe) und tauscht dann Methanol gegen Aceton aus. Nach der Überschichtung der Lösung des Komplexes mit n-Hexan erhält man 9 (Raumgruppe Pbcn).

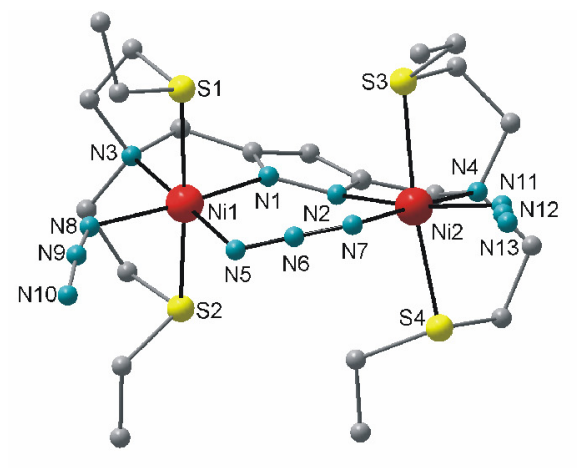

7 aus Methanol

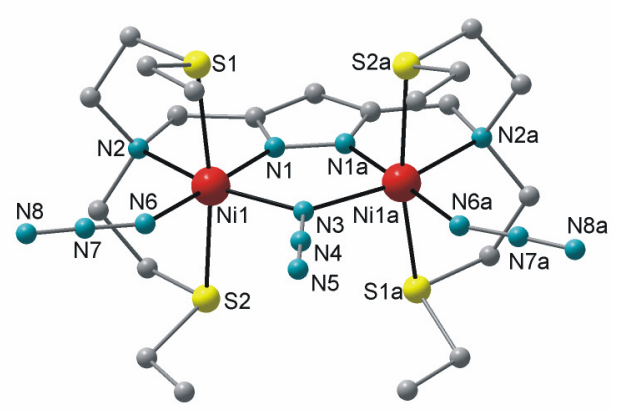

8

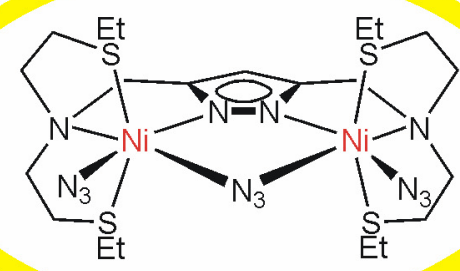

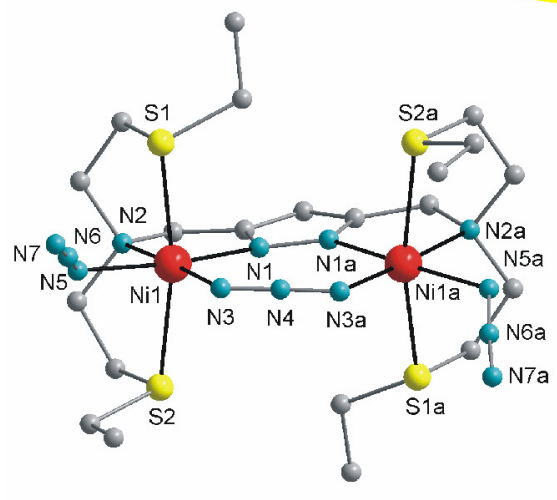

9 aus Aceton (Raumgruppe $\mathrm{Pbcn}$ )

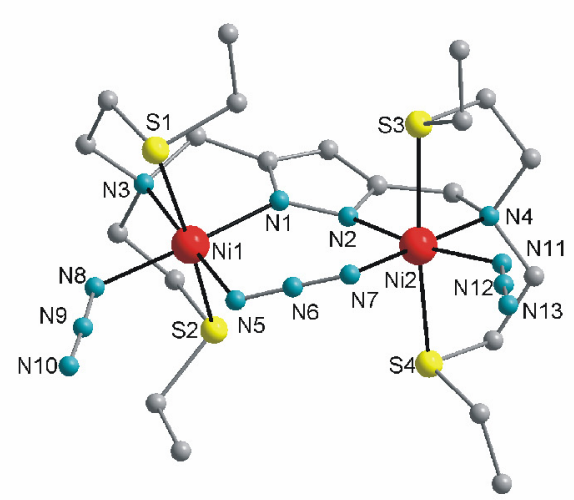

10 aus Aceton (Raumgruppe $P \overline{1}$ )

Abb.4.3.4.1. Die Strukturen der isomeren Verbindungen 7-10 des „Chamäleon-Systems“ bei 133K. In der Mitte ist die allgemeine schematische Formel dargestellt.

Verbindung 10 wird analog zu 9 hergestellt. Man überschichtet allerdings die Lösung nicht, sondern lässt sie zwei Tage stehen, wobei die Kristalle von 10 (Raumgruppe $P \overline{1}$ ) entstehen. Die abdekantierte und mit n-Hexan überschichtete Lösung ergibt wieder 9. Die Verbindung 9 ist auch aus $\mathbf{8}$ darstellbar, indem $\mathbf{8}$ in Aceton aufgelöst und mit n-Hexan überschichtet wird. Löst man 9 und 10 in Methanol auf und überschichtet mit Diethylether, entsteht der Komplex 7. 
Das detaillierte Bild der molekularen Strukturen von 7-10 wurde durch Röntgenstrukturanalyse erhalten (Abb. 4.3.4.1). Wie erwartet, sitzen beide Nickel(II)-Ionen innerhalb ihres entsprechenden Ligandkompartiments von drei Donoratomen (zwei Schwefel, ein Stickstoff) des Ligandseitenarms (im Falle von 8 und 9 in der $C_{2}$-symmetrischen Anordnung) und sind über das Pyrazolat verbrückt. Die Azidbrücke findet sich innerhalb der zweikernigen Tasche, und ein zusätzlicher terminaler Azidligand füllt die letzte Koordinationsstelle der oktaedrisch koordinierten Metallionen. Die kurzen Seitenarme des Liganden $\mathbf{H L}^{6}$ ziehen beide Nickel(II)-Ionen auseinander und ermöglichen damit $\mu$-1,3Koordination des Azids in 7,9 und $\mathbf{1 0}$ mit dem resultierenden Ni1 $\cdots$ Ni2(Ni1a) Abstand von ca. $4.5 \AA$ (Tab. 4.3.4.1, der kürzere Ni1 ‥Ni1a Abstand für 8 wird weiter unten diskutiert).

Tab. 4.3.4.1. Ausgewählte Bindungslängen $(\AA)$ und Winkel $\left(^{\circ}\right)$ von 7-10 bei $133 \mathrm{~K}$.

\begin{tabular}{lllll}
\hline & $\mathbf{7}$ & $\mathbf{8}$ & $\mathbf{9}$ & $\mathbf{1 0}$ \\
\hline $\mathrm{Ni} \cdots \mathrm{Ni}$ & $4.4703(6)$ & $3.6463(2)$ & $4.5538(8)$ & $4.4757(4)$ \\
$\mathrm{Ni}-\mathrm{N}-\mathrm{N}$ & $117.1(2) /$ & -- & $120.7(3)$ & $117.5(2) /$ \\
& $118.1(2)$ & & & $118.1(2)$ \\
& $34.2(2)$ & --- & $4.3(4)$ & $34.5(2)$ \\
$\mathrm{Ni}-\mathrm{NNN}_{\text {Azid }}-\mathrm{Ni}$ & $46.6(2)$ & $5.0(1)$ & $23.1(6)$ & $47.1(2)$ \\
$\mathrm{Ni}-\mathrm{NN}$ & & $117.8(1)$ & --- & --- \\
$\mathrm{Ni}-\mathrm{N}-\mathrm{Ni}$ & --- & & & \\
\hline
\end{tabular}

Die Verbindungen $\mathbf{7}$ und $\mathbf{1 0}$ sind in ihrer Struktur sehr ähnlich und beinhalten beide ein gekipptes Azid mit dem Torsionswinkel von 34 ${ }^{\circ} .7$ besitzt im Vergleich zu 10 ein im Kristallgitter eingebautes Methanolmolekül pro zwei Komplexmoleküle. Im Gegensatz zu 7 und 10 beträgt in 9 der $\mathrm{Ni}-\mathrm{NNN}-\mathrm{Ni}$ Torsionswinkel $4.3^{\circ}$ bei $133 \mathrm{~K}$, so dass beinahe Planaritär erreicht wird. Während das verbrückende Azid in 7, 9 und 10 den end-to-end Modus einnimmt, findet man in 8 das $\mu$-1,1-verbrückende Azid und den relativ kurzen Ni1 ‥Ni1a Abstand von $3.6463 \AA$ A. Die end-on Koordination des Azids sowie der kurze Metall-Metall Abstand in Komplexen, basierend auf Liganden mit kurzen Ligandseitenarmen, ist höchst ungewöhnlich. Die Ursache dafür könnte an den weichen SDonoren in $\mathbf{H L}^{\mathbf{6}}$ liegen, welche zusammen mit den passenden Kristallisationsbedingungen und damit verbundenen günstigen Kristallpackungseffeken eine Anpassung an die end-on Koordination des Azids ermöglichen. 8 besitzt zwei im Kristallgitter eingebaute Moleküle DMSO. Der Ni-N-Ni Winkel in 8 (Tab.4.3.4.1) liegt für die pyrazolatbasierenden Komplexe mit einer $\mu$-1,1-Azidbrücke in üblichem Bereich (116-118º). 
Die IR-Spektren aller Verbindungen sind sehr ähnlich (Tab.4.3.4.2). Für die Azidschwingungen findet man für 7, 9 sowie 10 eine sehr starke Bande bei ca. $2040 \mathrm{~cm}^{-1}$ und für 8 bei ca. $2050 \mathrm{~cm}^{-1}$.

Tab. 4.3.4.2. Ausgewählte IR-Banden für 7-10.

\begin{tabular}{ll}
\hline Komplex & $v_{\text {as }}\left(\mathrm{N}_{3}^{-}\right), \mathrm{cm}^{-1}$ \\
\hline $\mathbf{7}$ & $2039(\mathrm{vs})$ \\
$\mathbf{8}$ & $2048(\mathrm{vs})$ \\
$\mathbf{9}$ & $2043(\mathrm{vs})$ \\
$\mathbf{1 0}$ & $2040(\mathrm{vs})$ \\
\hline
\end{tabular}

Die IR-spektroskopischen Eigenschaften dieses „Chamäleon-Systems“ sind zwar ähnlich, aber magnetische Messungen zeigen für einige Verbindungen sehr interessante Phänomene.

\section{Magnetische und kalorimetrische Eigenschaften}

Die Temperaturabhängigkeit von $\chi_{M}$ und $\chi_{M} T$ für 7 und 8 ist in Abb. 4.3.4.2. gezeigt. Der $\chi_{M} T$-Wert von 7 beträgt bei Raumtemperatur $1.7 \mathrm{~cm}^{3} \mathrm{Kmol}^{-1}$. Dies ist viel kleiner als der theoretisch erwartete „spin-only“ Wert für zwei ungekoppelte $S=1$ Nickel(II)-Ionen (2.42 $\mathrm{cm}^{3} \mathrm{Kmol}^{-1}$ für $\left.g=2.20\right)$. Das weist zusammen mit einer starken Abnahme von $\chi_{\mathrm{M}} T$ und einem breiten Maximum bei ca. $175 \mathrm{~K}$ in der Auftragung $\chi_{M}$ gegen $T$ auf eine sehr starke antiferromagnetische Wechselwirkung und einem $S_{T}=0$ Grundzustand in diesem Komplex hin.
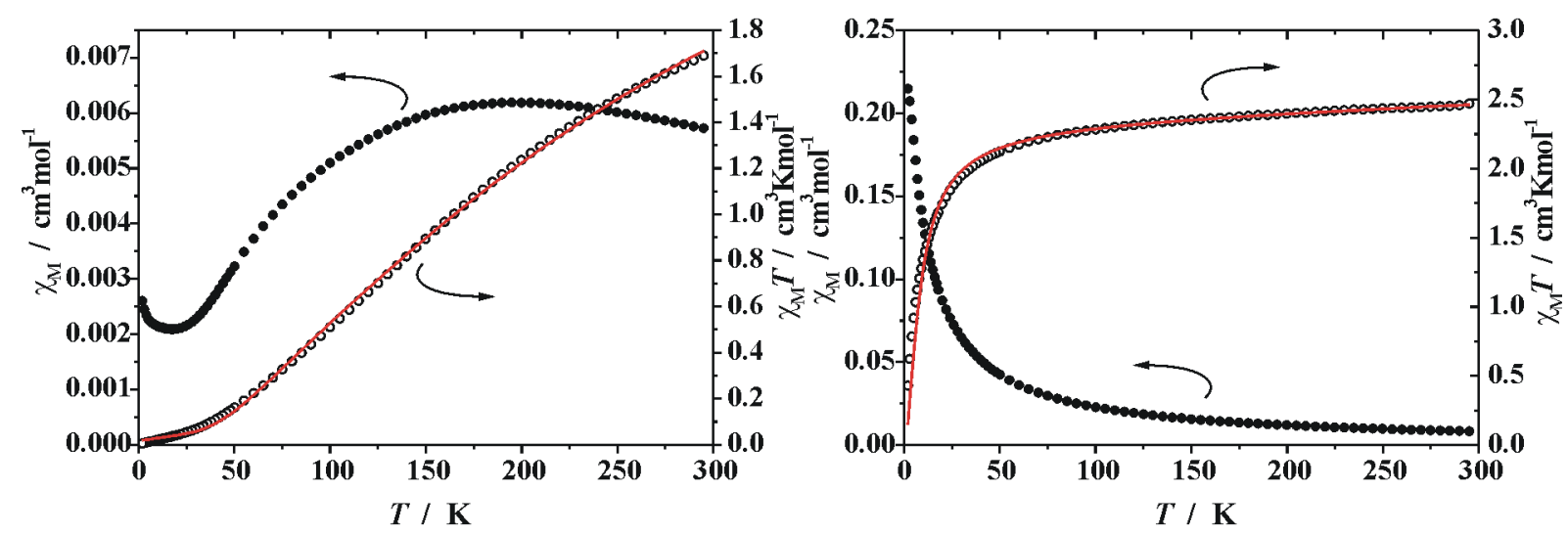

Abb. 4.3.4.2. Temperaturabhängigkeit von $\chi_{M}$ und $\chi_{M} T$ für 7 (links) und 8 (rechts). Die roten Linien repräsentieren die besten Anpassungen. 
Der $\chi_{M} T$-Wert von 8 ist bei Raumtemperatur $2.46 \mathrm{~cm}^{3} \mathrm{Kmol}^{-1}$ und liegt somit etwas höher als der „spin-only“ Wert. Mit sinkender Temperatur bleibt das Produkt $\chi_{M} T$ bis zu $50 \mathrm{~K}$ nahezu konstant und nimmt dann ab (Abb. 4.3.4.2, rechts). Dies könnte für eine sehr schwache antiferromagnetische Kopplung bzw. starke Nullfeldaufspaltung sprechen.

Die Anpassung an die experimentellen Daten von 7 und 8 nach Gleichung 6.4.4.2 liefert folgende Parameter: $g=2.20 \pm 0.01, J=-67.8 \pm 0.5 \mathrm{~cm}^{-1} \cdot \rho=0.7 \pm 0.1 \%$, TIP $=12.0 \cdot 10^{-4}$ $\mathrm{cm}^{3} \mathrm{~mol}^{-1}$ für 7 und $g=2.19 \pm 0.01, J=-1.3 \mathrm{~cm}^{-1} \cdot \rho=5.0 \%$, TIP $=5.1 \cdot 10^{-4} \pm 1.1 \cdot 10^{-4}$ $\mathrm{cm}^{3} \mathrm{~mol}^{-1}$ für 8. Während die große antiferromagnetische Kopplungskonstante $J$ für 7 im typischen Bereich für die Komplexe mit der einfachen EE-Azidkoordination (siehe Kapitel 4.1.2) liegt, ist die in 8 gefundene schwache antiferromagnetische Kopplung für $\mu-1,1$ azidverbrückte Komplexe sehr ungewöhnlich. Über Kupfer(II)-Komplexe mit einer end-on Azidverbrückung und einer zweiten „Nichtazidbrücke“, bei welcher die antiferromagnetische Kopplung auf Komplementaritätseffekt zurückzuführen war, wurde berichtet. ${ }^{[121]}$ Offensichtlich liegt in $\mathbf{8}$ auch die Situation vor, dass der antiferromagnetische Beitrag $J_{\mathrm{AF}} \mathrm{zu}$ der Kopplungskonstante $J\left(J=J_{\mathrm{AF}}+J_{\mathrm{F}}\right)$ sehr groß ist. Dieser Beitrag ist umso größer je stärker die Überlappung zwischen den Orbitalen der beiden Liganden gleicher Symmetrie mit den Orbitalen der Metalle (siehe Kapitel 4.1.3) ausgeprägt ist. In 8 hat man im Vergleich zu anderen pyrazolatbasierten end-on verbrückten Komplexen mit ferromagnetischer Kopplung die besondere Situation, dass der „out-of-plane“ Winkel (Winkel zwischen Ni-N-Ni-Ebene und der Geraden, die durch das Azid gelegt wird) exakt $0^{\circ}$ ist. Dieser Winkel liegt im Bereich von $7-12^{\circ}$ für die ferromagnetischen zweikernigen Komplexe $\mathbf{1}$ und $\mathbf{2}$ sowie für die ferromagnetisch gekoppelten Nickel(II)- $\mu$-1,1-Azid-Fragmente in den vierkernigen Komplexen 11a und 12. Die planare Anordnung des Nickel(II)-Azid-Fragments in $\mathbf{8}$ bewirkt das maximal mögliche Überlappungsintegral, dessen quadrierter Wert zum antiferromagnetischen Teil der Kopplung beiträgt (Gleichungen 3.2.10, 3.2.11). Der dadurch stark ausgeprägte Komplementaritätseffekt spielt offensichtlich die entscheidende Rolle für die Art des magnetischen Austausches in diesem Komplex.

Die Daten der Suszeptibilitätsmessungrn von 8 wurden auch unter Verwendung des Programms jul $X,{ }^{[106]}$ basierend auf nachfolgendemem isotropen Heisenberg-Dirac-van-VleckHamiltonoperator (Gleichung 4.3.4.1), analysiert,

$$
\hat{H}=-2 J \hat{S}_{1} \hat{S}_{2}+\sum_{i=1}^{2} g \mu_{B} B \hat{S}_{i z}+D \sum_{i=1}^{2}\left[\hat{S}_{i z}^{2}-\hat{S}_{i}\left(\hat{S}_{i}+1\right) / 3+E_{i} / D_{i}\left(\hat{S}_{x, i}^{2}+\hat{S}_{y, i}^{2}\right)\right]
$$


wobei die zusätzlichen Terme Zeeman-Aufspaltung und Nullfeldaufspaltung ( $D_{i}$ und $E_{i}$ sind entsprechende lokale axiale bzw. rombische Parameter) berücksichtigen. Die $g$-Werte wurden für alle Positionen als identisch angenommen. Eine gute Übereinstimmung wurde mit den Parametern $g=2.13, J=+1.8 \mathrm{~cm}^{-1},|D|=22.9 \mathrm{~cm}^{-1}$, und $|E|=1.66 \mathrm{~cm}^{-1}, \rho=2.0 \%$ und TIP $=8.0 \cdot 10^{-4} \mathrm{~cm}^{3} \mathrm{~mol}^{-1}$ erhalten. Unter Verwendung des Hamiltonoperators 4.3.4.1. wird die Kopplung in 8 aufgrund des hohen Wertes des $|D|$ trotz der planaren Anordnung des Nickel(II)- $\mu$-1,1-Azid-Fragments als schwach ferromagnetisch beschrieben. Ein solch ungewöhnlich hoher Wert von $|D|$ müsste jedoch durch zusätzliche Experimente (z.B. Hochfeld-EPR) bestätigt werden.

Da die Röntgenstrukturanalyse von 9 bei 133 K keine ungewöhnlichen Strukturmerkmale aufwies, war der Verlauf der Temperaturabhängigkeit der magnetischen Suszeptibilität höchst unerwartet. Das Produkt $\chi_{M} T$ für eine polykristalline Probe von 9 ist in Abb. 4.3.4.3 sowohl für steigende als auch für sinkende Temperatur dargestellt. Der Wert von $1.82 \mathrm{~cm}^{3} \mathrm{Kmol}^{-1}$ bei $300 \mathrm{~K}$ ist etwas niedriger als der erwartete „spin-only“ Wert für zwei ungekoppelte $S=1$ Nickel(II)-Ionen (2.14 $\mathrm{cm}^{3} \mathrm{Kmol}^{-1}$ für $\left.g=2.07\right)$ und verringert sich nur leicht nach der Temperatursenkung auf $220 \mathrm{~K}$. Bei $215 \mathrm{~K}$ erfolgt allerdings ein abrupter Abfall von $\chi_{M} T$ (zentriert bei $T_{\downarrow}=212 \mathrm{~K}$ ), gefolgt von einem viel rapiderem Abfall bei tieferen Temperaturen. Unterhalb $50 \mathrm{~K}$ geht $\chi_{M} T$ gegen Null. Die Tieftemperaturdaten beweisen eine starke antiferromagnetische Kopplung und einen Grundzustand $S=0$. Die Kopplung bei einer Temperatur über $220 \mathrm{~K}$ ist anscheinend nur noch schwach. Bei der Messung bei steigendem Temperaturverlauf zeigt $\chi_{M} T$ ein analoges Verhalten, weist allerdings thermische Hysterese mit $\Delta T \sim 13 \mathrm{~K}$ auf, $T \uparrow$ ist zentriert bei $223 \mathrm{~K}$. Eine Feldabhängigkeit der magnetischen Daten wurde nicht beobachtet. Komplex 9 erlebt offensichtlich eine reversible und feldunabhängige Umwandlung mit Hysterese in einem Bereich nicht allzuweit von Raumtemperatur. Diese Umwandlung hat etwa die gleiche Größenordnung wie die magnetische Austauschwechselwirkung. 

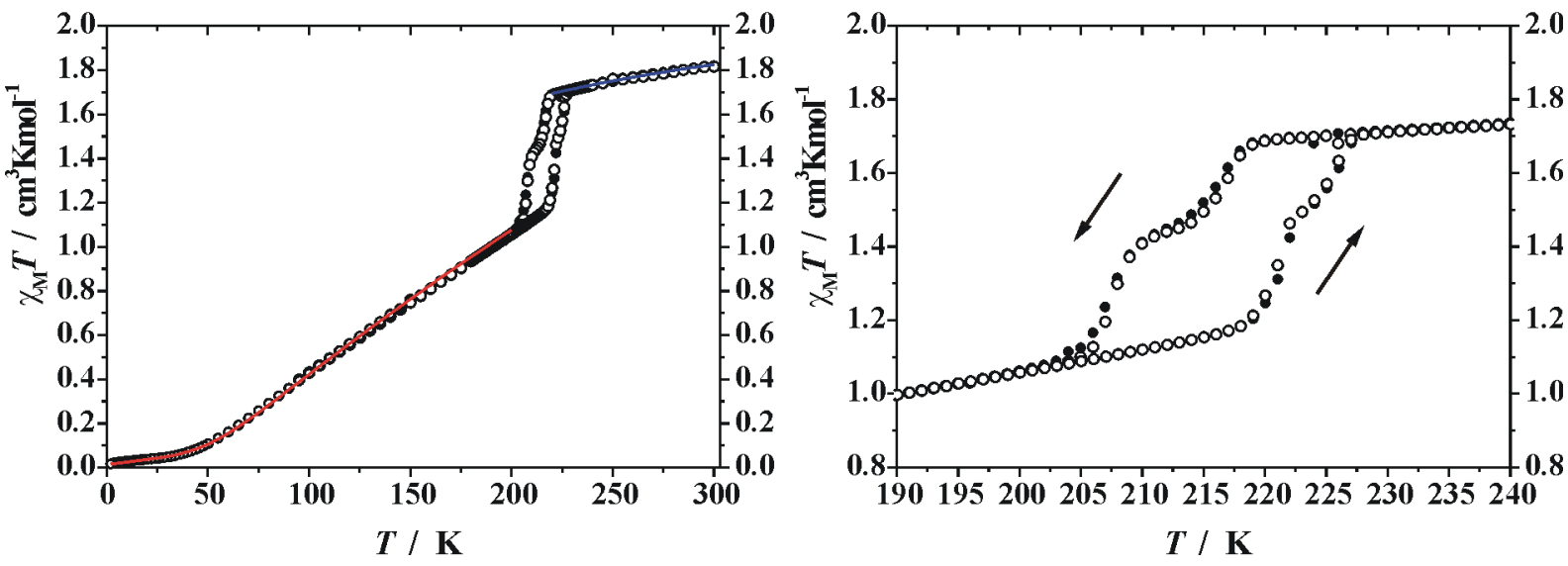

Abb. 4.3.4.3. $\chi_{M} T$ gegen $T$ für 9 über dem gesamten Temperaturbereich (links) und der vergrößerte Hysteresebereich (rechts) bei zwei verschiedenen magnetischen Feldern (gefüllte Kreise: 5000 G, offene Kreise: 2000 G) für sinkende und steigende Temperatur. Die durchgezogenen Linien geben die besten Anpassungen bei hoher (blau) und tiefer (rot) Temperatur wieder (siehe Text).

Magnetische Experimentalwerte wurden in den Bereichen von 200 bis $5 \mathrm{~K}$ und über $230 \mathrm{~K}$ unter Verwendung der Gleichung 6.4.4.2 getrennt angepasst. Gute Anpassungen wurden erhalten mit den Parametern $g=2.24 \pm 0.01$ und $J=-81 \pm 1.5 \mathrm{~cm}^{-1}(\rho=0.6 \pm 0.1 \%$; TIP $=$ $\left.12 \cdot 10^{-4}\right)$ im Tieftemperaturbereich, und $g=2.07 \pm 0.01, J=-24 \pm 1.0 \mathrm{~cm}^{-1}(\rho=0.8 \pm 0.1 \%$; $T I P=1 \cdot 10^{-4}$ ) über $230 \mathrm{~K}^{1}{ }^{1)}$ Das bestätigt drastische Unterschiede in der Stärke der antiferromagnetischen Kopplung für die zwei Temperaturbereiche, mit einer starken bzw. schwachen antiferromagnetischen Kopplung unter bzw. über der Hysteresetemperatur.

Um die molekulare Ursache für das unerwartete magnetische Verhalten zu erläutern und den magnetischen Phasenübergang zu charakterisieren, wurde der Einkristall von 9 kristallographisch bei verschiedenen Temperaturen untersucht. Der Einkristall wurde zunächst bei Raumtemperatur angesetzt und danach die Datensätze bei den Temperaturen 253 K, 173 K, $223 \mathrm{~K}$ und $195 \mathrm{~K}$ (in dieser Reihenfolge) gesammelt. In jedem Fall hat man vor Beginn der Datensammlung eine einstündige Temperaturangleichung stattfinden lassen. Aus den kristallographischen Daten wird ersichtlich, dass der Kristall unter der Temperaturänderung und bei wiederholtem Hin- und Herfahren durch den Hysteresebereich intakt bleibt; das Kristallsystem bleibt orthorhombisch bei allen Temperaturen. Die Untersuchung der molekularen Strukturen oberhalb und unterhalb des Hysteresebereiches weist allerdings signifikante Unterschiede des zweikernigen Gerüsts auf. Diese Unterschiede werden durch

\footnotetext{
1) Die für Daten oberhalb 230 K erhaltenen Parameter sollten mit Vorsicht interpretiert werden, weil nur ein relativ kleiner Temperaturbereich für die Anpassung berücksichtigt wurde.
} 
einen Vergleich der bei $133 \mathrm{~K}$ und $253 \mathrm{~K}$ erhaltenen molekularen Strukturen des zentralen Nickel(II)-Azid-Fragments hervorgehoben (Abb. 4.3.4.4).
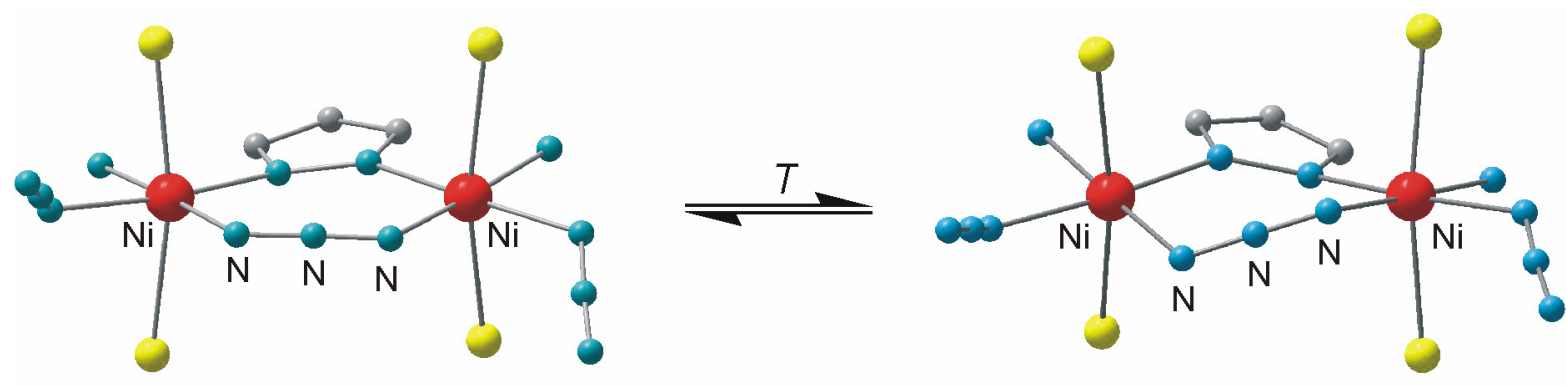

Abb. 4.3.4.4. Schalteffekt in 9: molekulare Strukturen des zentralen Fragments bei $133 \mathrm{~K}$ (links) und $253 \mathrm{~K}$ (rechts) von 9.

Strukturelle Änderungen kommen im wesentlichen durch Änderungen des Torsionswinkels, der von zwei Metallzentren und den äußeren Stickstoffatomen des verbrückenden Azids (N3 und N3a) gebildet wird, sowie des Torsionswinkels zwischen den Metallionen und Pyrazolatstickstoffatomen (N1 und N1a), zustande (Tab. 4.3.4.3). Die wesentlichste Änderung wird zwischen $195 \mathrm{~K}$ und $223 \mathrm{~K}$ beobachtet, was mit dem abrupten Abfall der magnetischen Suszeptibilität in diesem Bereich im Einklang steht. Zwischen $133 \mathrm{~K}$ und $195 \mathrm{~K}$ oder zwischen $223 \mathrm{~K}$ und $253 \mathrm{~K}$ weist der Torsionswinkel keine bedeutende Veränderung auf. Auch das ist in Übereinstimmung mit den magnetischen Daten.

Tab. 4.3.4.3. Zellparameter und ausgewählte Torsionswinkel von 9 bei unterschiedlichen Temperaturen.

\begin{tabular}{ccccccc}
\hline$T, \mathrm{~K}$ & $a, \AA$ & $b, \AA$ & $c, \AA$ & $V, \AA^{3}$ & Ni1-N1N1a-Ni1a, $^{\circ}$ & Ni1-N3N3a-Ni1a, $^{\circ}$ \\
\hline 133 & $15.9739(12)$ & $8.6167(8)$ & $22.8983(17)$ & $3151.8(4)$ & $23.1(6)$ & $4.3(4)$ \\
195 & $16.0886(12)$ & $8.6241(8)$ & $22.9437(16)$ & $3183.4(4)$ & $20.9(7)$ & $4.8(5)$ \\
223 & $16.3424(12)$ & $8.6933(8)$ & $22.9634(15)$ & $3262.4(4)$ & $-39.8(7)$ & $48.7(4)$ \\
253 & $16.3677(10)$ & $8.7169(7)$ & $22.9876(13)$ & $3279.8(4)$ & $-38.8(6)$ & $46.6(4)$ \\
\hline
\end{tabular}

Die ungewöhnlichen magnetischen Eigenschaften von 9 können durch die kristallographischen Befunde erklärt werden. Während ein gewisser Beitrag zur magnetischen Kopplung seitens des Pyrazolats mit Sicherheit vorhanden ist, scheint die Azidbrücke den dominierenden Einfluß bei der intramolekularen Wechselwirkung zu haben. ${ }^{[10]}$ Magnetostrukturelle Korrelationen für die $\mu$-1,3-azidverbrückten zweikernigen Nickel(II)-Komplexe haben aufgezeigt, dass zwei geometrische Parameter, die Winkel $\mathrm{Ni}-\mathrm{N}-\mathrm{N}$ und der 
Torsionswinkel $\tau$ entlang der Azidliganden, von größter Bedeutung sind (siehe Kapitel 4.1.2). Im Falle von 9 sind die Winkel Ni1-N3-N4 ziemlich klein und ähnlich sowohl bei $133 \mathrm{~K}$ $\left(120.7(3)^{\circ}\right)$, als auch bei $253 \mathrm{~K}\left(116.7(3)^{\circ}\right)$; aber der Torsionswinkel Ni-NNN-Ni unterscheidet sich stark $\left(4.3(4)^{\circ}\right.$ gegen $\left.46.6(4)^{\circ}\right)$. Die bekannten magnetostrukturellen Korrelationen vorausgesetzt kann somit eine große antiferromagnetische Kopplung in der Größenordnung von beinahe $-100 \mathrm{~cm}^{-1}$ für die Tieftemperaturphase (wo der Torsionswinkel nahe Null ist) erwartet werden, während die bedeutend verminderte Kopplung in der Hochtemperaturspezies durch die stark gekippte Azidbrücke bedingt sein sollte. Dies steht im Einklang mit den experimentellen Befunden $\left(-81 \pm 1.5 \mathrm{~cm}^{-1}\right.$ gegen $\left.-24 \pm 1.0 \mathrm{~cm}^{-1}\right)$. Somit kann das Brückenazid innerhalb der bimetallischen Tasche des Dinickel(II)-Gerüstes von 9 als ein molekularer Ein-/Ausschalter für die antiferromagnetische Kopplung zwischen zwei Metallionen angesehen werden.

Die eingehende Untersuchung der kristallographischen Änderungen, die den Phasenübergang begleiten, zeigt auf, dass oberhalb des Hysteresebereichs (bei $223 \mathrm{~K}$ und 253 K) die schwefelgebundenen Ethylgruppen der Ligandseitenarme von $\left(\mathbf{L}^{6}\right)^{-}$bei zwei Positionen mit temperaturabhängigen Besetzungsfaktoren für S2-Et fehlgeordnet sind. Diese Fehlordnung ist unterhalb des Hysteresebereichs verschwunden. Die kristallographische $a$ Achse schrumpft abrupt nach der Abkühlung unter den Hysteresebereich. Änderungen der $b$ und $c$-Achsen sind dagegen nur geringfügig (Tab. 4.3.4.3). Wie aus dem Bild der Elementarzelle (Abb. 4.3.4.5) ersichtlich, beeinflußt die fehlgeordnete, an S2 gebundene Ethylgruppe die Stapelung der Moleküle entlang der $a$-Achse. Das abrupte Schrumpfen dieser Achse kann somit mit dem Verschwinden des Fehlordnungsphänomens in Verbindung gebracht werden. Offensichtlich wird dadurch eine effizientere Packung der Moleküle bei niedrigen Temperaturen möglich. Es ist allerdings noch unklar, wie das Erscheinen oder Verschwinden der Fehlordnung mit den Änderungen des Ni-NNN-Ni-Torsionswinkels innerhalb der zweikernigen Moleküle zusammenhängt. 

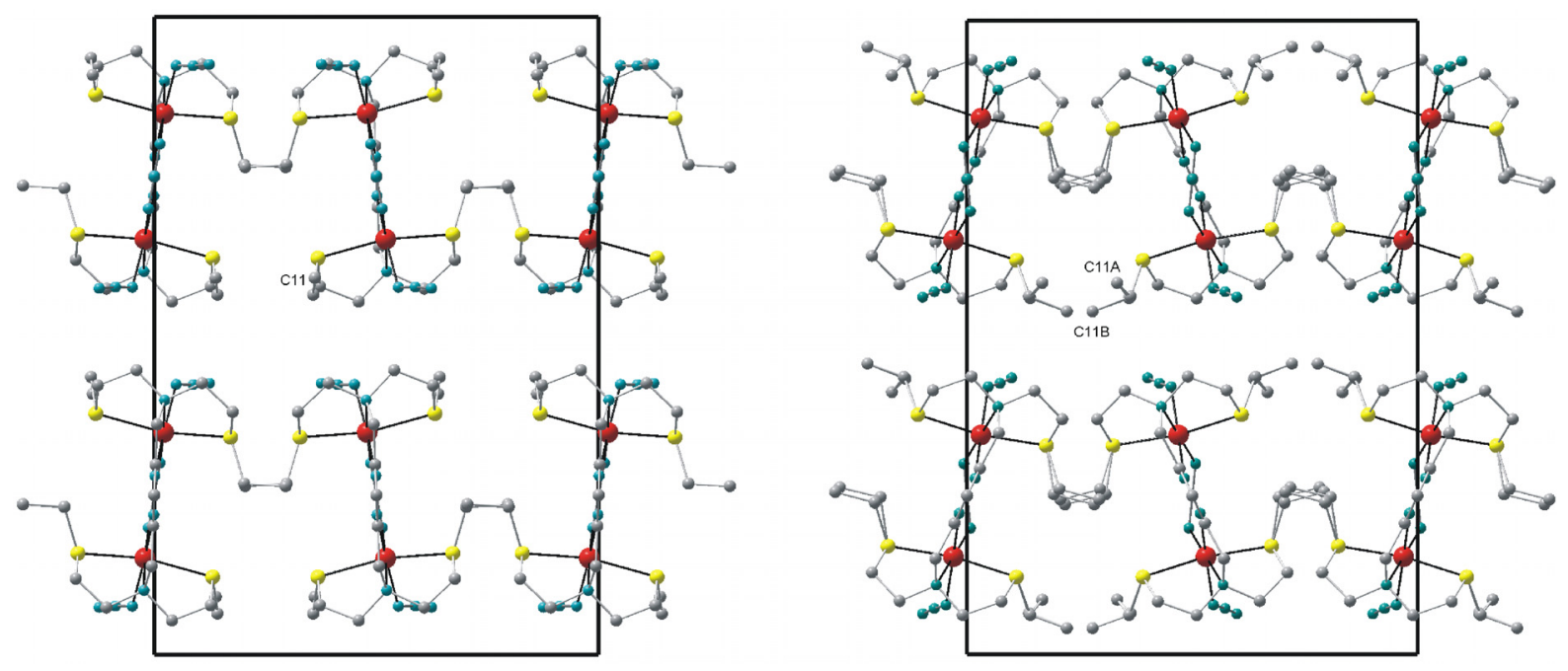

Abb. 4.3.4.5. Die Elementarzellen von 9 bei 193 K (links) und 223 K (rechts).

Es ist auch von Interesse, dass eine Zweistufigkeit des Phasenübergangs aus den magnetischen Daten erkennbar ist (Abb. 4.3.4.3). Eine DSC-Kurve ${ }^{1)}$ zeigt nicht nur die $20 \mathrm{~K}$ breite Hysterese, sondern auch den in zwei Stufen ablaufenden Phasenübergang (Abb.4.3.4.6).

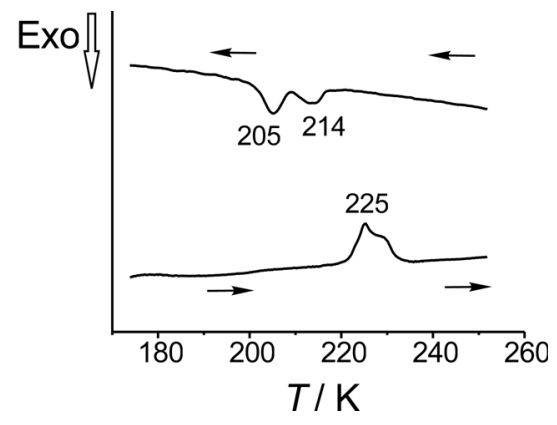

Abb. 4.3.4.6. DSC-Kurve für 9, darstellend die zweistufige thermische Hysterese zwischen 205 und $230 \mathrm{~K}$.

Obwohl das Phänomen eines Sprung von $\chi_{M} T$ (hier mit Hysterese) an ein SpincrossoverVerhalten erinnert, ist der Ursprung der thermischen magnetischen Bistabilität von 9 jedoch grundsätzlich anders: beide Metallionen behalten ihren individuellen Hochspin-Zustand ( $S=$ 1), das Brückenazid fungiert allerdings als Kippschalter, der die antiferromagnetische Kopplung zwischen den Nickel(II)-Ionen ein- oder ausschaltet. Phasenübergänge, verbunden mit kleinen Änderungen in den magnetischen Eigenschaften, wurden für polymere MetallAzid-Systeme beobachtet (siehe Kapitel 4.1.6). Die für 9 so deutlich beobachtete Hysterese ist präzedenzlos und ist nach unserem Wissen der erste Fall, wo sowohl die Tieftemperatur- als

1) DSC - Dynamic Scanning Calorimetry (Dynamische Differenz Kalorimetrie). 
auch die Hochtemperatur-Phase kristallographisch charakterisiert wurden, um das Azid zu identifizieren, das als ein gut definierter molekularer Schalter funktioniert.

Bei der weiteren Untersuchung von 9 hat sich herausgestellt, dass die Form und die Lage der Hysterese sich leicht variieren lässt (Abb. 4.3.4.7).
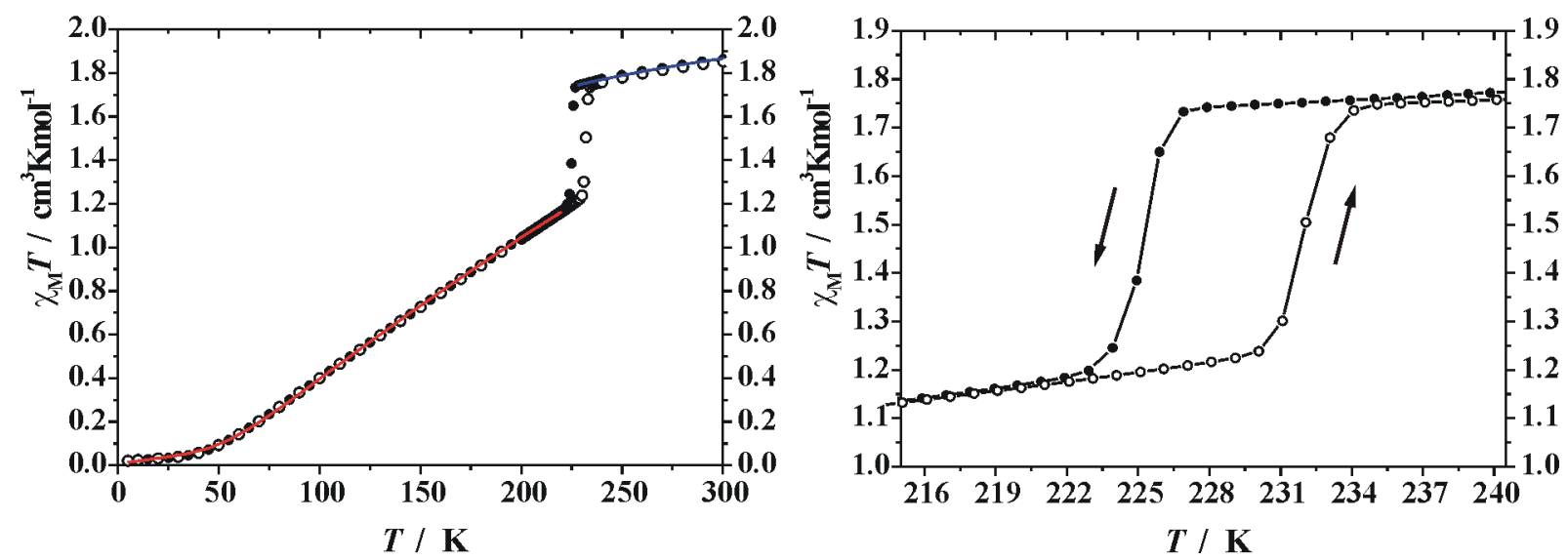

Abb. 4.3.4.7. $\chi_{M} T$ gegen $T$ für 9 über dem gesamten Temperaturbereich (links) und der vergrößerte Hysteresebereich (rechts) (schraffierte Kreise: sinkende Temperatur, nichtschraffierte Kreise: steigende Temperatur) ohne Stufe. Die durchgezogenen Linien geben die besten Anpassungen bei hoher (blau) und tiefer (rot) Temperatur wieder (sieh Text).

In verschiedenen Chargen von 9, die allerdings auf gleichem Weg erhalten wurden, kann die Hysterese bei den temperaturabhängigen Messungen sowohl mit als auch ohne Stufe auftreten. Wenn keine Stufe beobachtet wird, ist der Abfall abrupter, die Hysterese ist schmaler (7 K gegen $13 \mathrm{~K}$ mit der Stufe) und zu etwas höherer Temperatur (zentriert bei 229 K gegen 223 mit der Stufe) verschoben (Abb. 4.3.4.7). Die Anpassung an die experimentellen Daten wurde auch für eine Charge ohne Zweistufigkeit in den Bereichen von 200 bis $5 \mathrm{~K}$ und oberhalb $230 \mathrm{~K}$ getrennt unter Verwendung der Gleichung 6.4.4.2 durchgeführt. Gute Anpassungen wurden erhalten mit den Parametern $g=2.27 \pm 0.01$ und $J=-86 \pm 1.5 \mathrm{~cm}^{-1}(\rho=$ $0.6 \pm 0.2 \%$; TIP $=12 \cdot 10^{-4}$ ) im Tieftemperaturbereich, und $g=2.09 \pm 0.01, J=-24.2 \pm 1.0$ $\mathrm{cm}^{-1}\left(\rho=1.0 \%\right.$;IP $\left.=1.6 \cdot 10^{-4}\right)$ über $230 \mathrm{~K}$ erhalten. ${ }^{1)}$ Diese Werte sind sehr ähnlich den Werten der Charge mit zweistufiger Hysterese.

Die röntgenographischen Untersuchungen einer Charge ohne Stufe in der Hysterese oberhalb und unterhalb der Temperatur des magnetischen Übergangs zeigen keine Unterschiede im Vergleich zu Daten, die für die Charge mit der Stufe diskutiert wurden (siehe

\footnotetext{
1) Die für Daten oberhalb 230 K erhalten Parameter sollten mit der Vorsicht interpritiert werden, weil nur ein relativ kleiner Temperaturbereich für die Anpassung berücksichtigt wurde.
} 
oben). Auch die IR-Spektren beider Chargen von 9 sind nahezu identisch. Allerdings verschiebt sich die Azidbande geringfügig zu niedrigen Frequenzen in der Charge ohne Stufe im Vergleich zur Charge mit Stufe $\left(v_{\mathrm{as}}\left(\mathrm{N}_{3}{ }^{-}\right) 2038 \mathrm{~cm}^{-1}\right.$ gegen $\left.2043 \mathrm{~cm}^{-1}\right)$.

Interessanterweise sind damit auch die DSC-Messung und die magnetische Messung im Einklang. Die kalorimetrische Methode bestätigt die Anwesenheit (Abb. 4.3.4.6) oder Abwesenheit (Abb.4.3.4.8) des zweistufigen Charakters des magnetisches Übergangs je nach Komplexcharge.

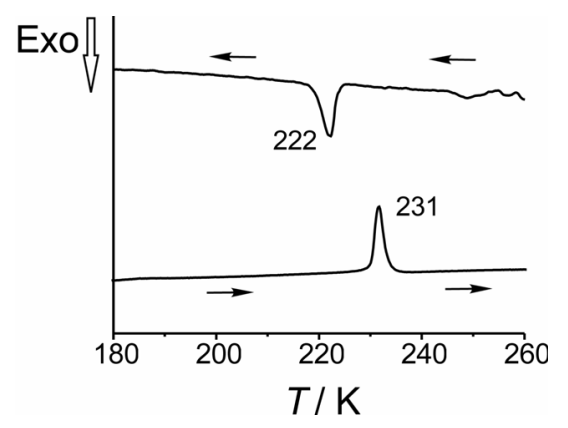

Abb. 4.3.4.8. DSC-Kurve für 9, darstellend die thermische Hysterese zwischen 222 und 231 K ohne Stufe.

Die beobachtete Abhängigkeit der magnetischen und kalorimetrischen Eigenschaften von der Synthesecharge in 9 kann auf minimale Änderungen in der Synthese oder auf unmerklich veränderte Kristallisationsbedingungen zurückgeführt werden. Es könnten Faktoren wie z.B. die Charge des verwendeten Liganden, die Raumtemperatur und damit verbundene Geschwindigkeit des Kristallwachstums, die Form des Gefäßes, Beschaffenheit der Oberfläche des Gefäßes, der Anteil an Verunreinigungen usw., die bis jetzt nicht erfasst werden konnten, die entscheidende Rolle spielen.

Die Versuche, die Synthese von 9 durch Wahl verschiedener Kristallisationsmethoden (siehe oben) zu optimieren, führten zu 10. Die ersten Analysen zeigten dieselbe Zusammensetzung und die gleichen IR-spektroskopischen Eigenschaften (Tab. 4.3.4.2) wie für 9. Der $\chi_{M} T$-Wert von $1.98 \mathrm{~cm}^{3} \mathrm{Kmol}^{-1}$ bei $300 \mathrm{~K}$ in $\mathbf{1 0}$ ist etwas niedriger als der erwartete „spin-only“ Wert für zwei ungekoppelte $S=1$ Nickel(II)-Ionen $\left(2.14 \mathrm{~cm}^{3} \mathrm{Kmol}^{-1}\right.$ für $g=$ 2.07), nimmt langsam bei Temperatursenkung ab und tendiert schließlich nach Null. Überraschenderweise wurde bei 10 aber kein magnetischer Übergang im Bereich $220 \mathrm{~K}$ festgestellt. Die magnetischen Eigenschaften von 10 oberhalb Raumtemperatur weisen eine „Verschiebung“ der Hysterese um $150 \mathrm{~K}$ nach höheren Temperaturen (Abb. 4.3.4.9) gegenüber 9 auf (Abb. 4.3.4.7). 

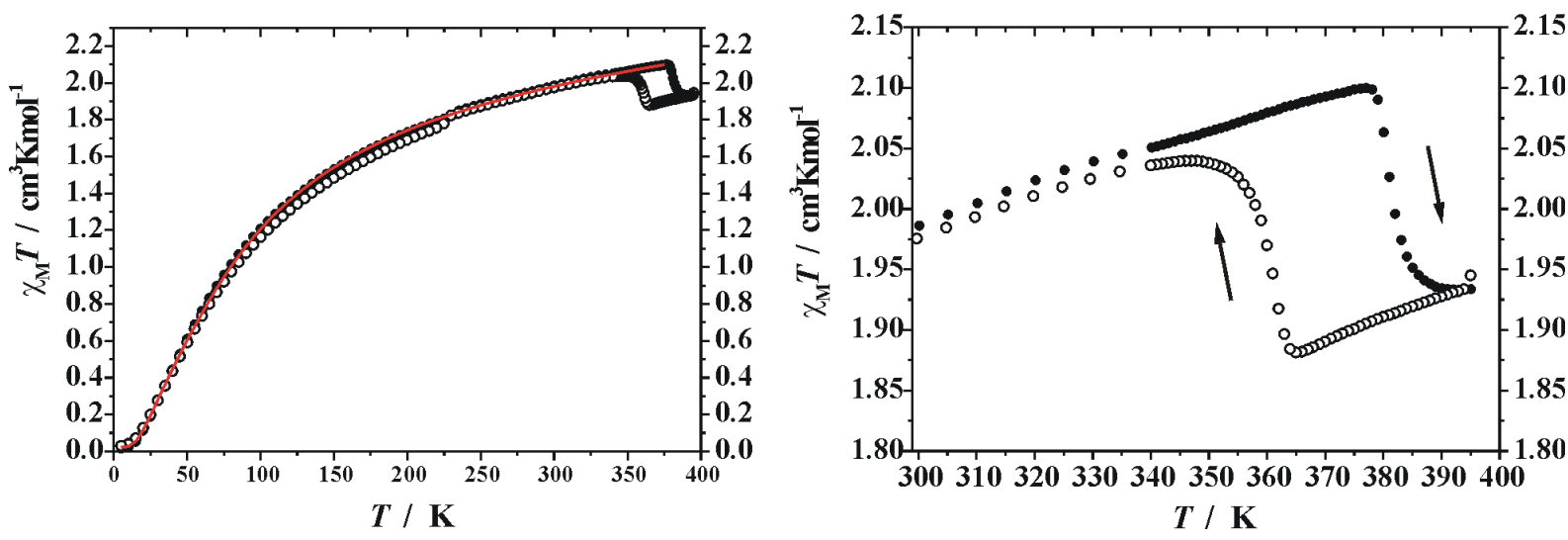

Abb. 4.3.4.9. $\chi_{M} T$ als Funktion von $T$ für 10 über dem gesamten Temperaturbereich (links), der vergrößerte Hysteresebereich (rechts) (schraffierte Kreise: steigende Temperatur, nichtschraffierte Kreise: sinkende Temperatur). Die durchgezogene rote Linie gibt die beste Anpassung wieder.

Der Wert von $1.98 \mathrm{~cm}^{3} \mathrm{Kmol}^{-1}$ bei $300 \mathrm{~K}$ vergrößert sich leicht nach der Temperatursteigerung auf $377 \mathrm{~K}$. Dann erfolgt allerdings ein abrupter Abfall von $\chi_{M} T$ (zentriert bei $T \uparrow=381 \mathrm{~K}$ ). Danach erreicht $\chi_{M} T$-Kurve ein Plateau mit der Tendenz zur weiteren Steigung. $\chi_{M} T$ zeigt als Funktion der sinkenden Temperatur von $395 \mathrm{~K}$ zu 2 K ein analoges Verhalten, weist allerdings thermische Hysterese mit $\Delta T \sim 20 \mathrm{~K}$ auf; $T \downarrow$ ist zentriert bei 360 K. Eine Feldabhängigkeit der magnetischen Daten wurde nicht beobachtet. Komplex 10 erlebt offensichtlich eine reversible und feldunabhängige Umwandlung in der Größenordnung der magnetischen Austauschwechselwirkung mit Hysterese, die im Bereich weit oberhalb von Raumtemperatur auftritt. Die magnetischen Experimentalwerte wurden in den Bereichen von 375 bis $5 \mathrm{~K}$ unter Verwendung der Gleichung 6.4.4.2 analysiert. Gute Anpassung wurde mit den Parametern $g=2.07 \pm 0.01, J=-24.7 \pm 0.2 \mathrm{~cm}^{-1}, \rho=0.8 \pm 0.14 \%$ und $T I P=6.3 \cdot 10^{-4}$ erhalten. Die Daten oberhalb der Hysterese sind wegen des kleinen Temperaturbereichs von 375-400 K nicht ausreichend für die zuverlässige Anpassung und Interpretation.

Es ist von Interesse, dass der magnetische Übergang in $\mathbf{1 0}$ genau spiegelverkehrt zum magnetischen Übergang in $\mathbf{9}$ ist. Der Abfall der $\chi_{M} T$-Kurve erfolgt für $\mathbf{9}$ in die Tieftemperaturrichtung und für $\mathbf{1 0}$ in die Hochtemperaturrichtung (ausgehend von Raumtemperatur). Während bei 9 die Phase mit zwei fehlgeordneten Ethylgruppen, gebunden an S2 und S2a, oberhalb der Hysterese liegt, beobachtet man die Fehlordnung nur einer Ethylgruppe an S1 von $\mathbf{1 0}$ in der Phase unterhalb der Hysterese. Man findet in $\mathbf{9}$ oberhalb des magnetischen Übergangs und in $\mathbf{1 0}$ unterhalb des magnetischen Übergangs magnetische sowie strukturelle Ähnlichkeiten: das verbrückende end-to-end Azid liegt in beiden 
Verbindungen gekippt vor (Torsionswinkel $\tau=46.6^{\circ}$ in 9 bzw. $34.5^{\circ}$ in 10) und vermittelt dadurch relativ schwache antiferromagnetische Kopplung $\left(24 \mathrm{~cm}^{-1}\right.$ in 9 und $25 \mathrm{~cm}^{-1}$ in 10).

Auch in $\mathbf{1 0}$ stimmen die DSC- und die magnetische Messung überein. Die kalorimetrische Methode bestätigt den Phasenübergang mit der Breite von 20 K (Abb. 4.3.4.10).

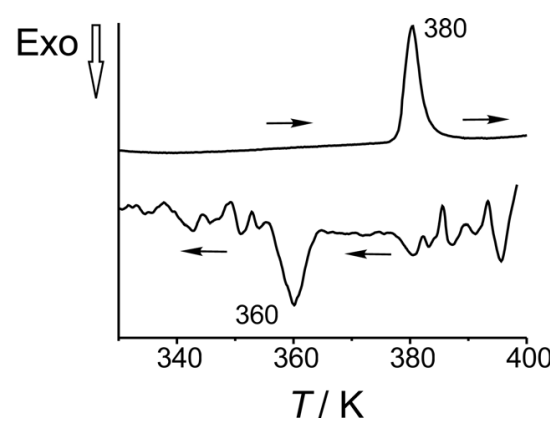

Abb. 4.3.4.10. DSC-Kurve für 10: thermische Hysterese zwischen 360 und $380 \mathrm{~K}$ ohne Stufe.

Da der Hysteresebereich in $\mathbf{1 0}$ sehr hoch liegt, ist es nicht möglich, die molekulare Ursache für dieses sehr spannende magnetische Verhalten durch Röntgenstrukturanalyse am Einkristall zu untersuchen. Man kann aber vermuten, dass z.B. das verbrückende Azid in $\mathbf{1 0}$ nach dem Übergang anders, z.B. planar (mit einem kleineren $\mathrm{Ni}-\mathrm{NN}_{\text {Azid }}-\mathrm{Ni}$ Torsionswinkel), angeordnet ist und dadurch die stärkere antiferromagnetische Kopplung eingeschaltet wird. Damit hätte man einen molekularen Komplex entdeckt, in welchem Azid als Schalter für die intramolekulare Kopplung oberhalb von Raumtemperatur fungiert. Eine der möglichen Erklärungen basiert auf dem Zusammenhang zwischen der Änderung der Azidanordnung mit der Fehlordnung S-ständiger Ethylgruppen. Da vor der Hysterese $(<360 \mathrm{~K})$ nur die Ethylgruppe an S1, fehlgeordnet ist, könnte man sich vorstellen, dass die zweite Ethylgruppe nach der Hysterese (>380 K) auch fehlgeordnet wird. Das könnte die Änderung in der Azidstellung analog wie in $\mathbf{9}$ verursachen. Der Unterschied zwischen $\mathbf{9}$ und $\mathbf{1 0}$ ist nur, dass in 9 vor der Hysterese $(<220$ K) keine Fehlordnung beobachtet wird. Der Vergleich der absoluten Änderungen der $\chi_{M} T$-Werte vor und nach der Hysterese von $\mathbf{9}$ und $\mathbf{1 0}$ spricht auch für den Zusammenhang von fehlgeordneten Ethylgruppen und Erscheinen des magnetischen Übergangs in 10. Diese Änderung $\Delta \chi_{M} T$ beträgt im Falle von 9 ca. $0.6 \mathrm{~cm}^{3} \mathrm{Kmol}^{-1}$, während für 10 dieser Wert nur halb so groß ist (ca. $0.25 \mathrm{~cm}^{3} \mathrm{Kmol}^{-1}$ ). Das ist erklärbar, wenn man berücksichtigt, dass im Falle von 9 zwei Ethylgruppen beteiligt sind und im Falle von 10 nur eine. Um diese Hypothese weiter zu untermauern, ist ein Vergleich der Strukturen von $\mathbf{7}$ und 10 hilfsreich. Der Vergleich zeigt nämlich, dass die beiden Verbindungen nahezu identisch sind und beide auch eine am S1 gebundene fehlgeordnete Ethylgruppe besitzen. Der Komplex 
7 schaltet die Kopplung im untersuchten Temperaturbereich jedoch nicht. Der einzige große Unterschied ist die Anwesenheit eines im Kristallgitter eingebauten Methanolmoleküls je zwei Komplexmoleküle in 7. Somit könnten die Methanolmoleküle als blockierende Puffer für das Entstehen der Fehlordnung der zweiten Ethylgruppe in 7 interpretiert werden, während die Abwesenheit dieses begrenzenden Faktors in 10 die Bistabilität mit der Hysterese bei 380 K beobachten lässt.

\section{Zusammenfassung}

Die Anwendung des Liganden $\mathbf{H L}^{6}$ mit weichen S-Donoratomen, die nicht mehr ganz so streng wie z.B. N-Donoratome den Metall-Metall-Abstand vorgeben können, erlaubt die Synthese isomerer zweikerniger Verbindungen 7-10 des „Chamäleon-Systems“ $\left[\mathrm{L}^{6} \mathrm{Ni}_{2}\left(\mathrm{~N}_{3}\right)_{3}\right]$. Die Variation der Synthese- und Kristallisationsbedingungen führt zu Komplexen mit dem end-on oder end-to-end verbrückenden Azid. Die außergewöhnliche Flexibilität des $\mu$-1,3Azids auch im Festkörper verursacht die magnetische Bistabilität mit Hysterese in $\mathbf{9}$ und 10, wobei dieses Phänomen bei unterschiedlichen Temperaturen auftritt. Im Falle von 9 konnte man mit Hilfe der Röntgenstrukturanalyse feststellen, dass das verbrückende Azid als wohldefinierter molekularer Schalter fungiert. Für 10 kann durch einen Vergleich der Komplexe des „Chamäleon-Systems“ miteinander das Auftreten der magnetischen Bistabilität erklärt werden. Diese Erklärung basiert, ähnlich wie in 9, auf dem Erscheinen bzw. Verschwinden der Fehlordnung der am Schwefel gebundenen Ethylgruppen bei Temperaturänderung. Ob jedoch die Änderungen in der Struktur des Nickel(II)-AzidFragments die Änderung der elektronischen Struktur und den damit verbundenen magnetischen Übergang verursachen oder umgekehrt, kann hier nicht abschließend beantwortet werden. 


\section{Dreikernige Kupfer(II)-Triazin-Bausteine für den Aufbau polynuklearer Spezies}

\subsection{Der triazinbasierte Ligand $L^{8}$ und seine Eigenschaften}

Das Konzept der Anwendung 2,4,6-trisubstituierter 1,3,5-Triazinderivate zur Darstellung neuer Klassen molekülbasierter magnetischer Materialien beruht auf dem Spinpolarisationsmechanismus. ${ }^{[122]}$ Dieser Mechanismus beschreibt, wie ein ungepaartes Elektron an einem Atom die Elektronenwolke eines Nachbaratoms in umgekehrter Weise polarisiert. ${ }^{1)}$ Bei Atomen, die Teil einer verbrückenden Einheit sind, bewirkt dies eine alternierende Spindichte und dementsprechend einen Wechsel des Vorzeichens des magnetischen Kopplungsparameters $J$ an jedem Atom der Brücke. Eine ferromagnetische Austauschwechselwirkung wäre also mit einer ungeraden Anzahl von Atomen zwischen den paramagnetischen Zentren möglich. In Abb. 5.1.1 sind einige bekannte Beispiele der verschiedenen meta-phenylenverbrückten ferromagnetischen Systeme gezeigt, die nach dieser Vorstellung entworfen und synthetisiert wurden.

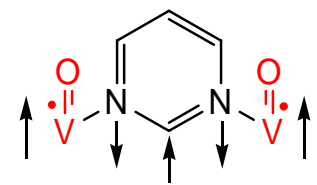

a

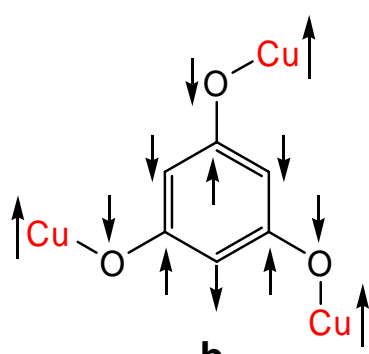

b

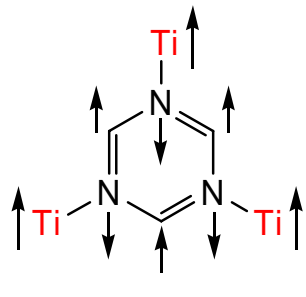

C

Abb. 5.1.1. Spinpolarisationsmechanismus zur Erklärung der ferromagnetischen Kopplung in zwei- (a) und dreikernigen (b, c) Komplexen.

So konnte gezeigt werden, dass sich zwei Vanadylzentren über eine Pyrimidinbrücke ferromagnetisch koppeln lassen (Abb. 5.1.1.a). ${ }^{[123]}$ Die Anwendung eines modifizierten 1,3,5Trihydroxibenzolliganden $^{[21 a]}$ oder von Trithiocyanurat $^{[124]}$ führte $\mathrm{zu}$ dreikernigen Verbindungen mit ferromagnetischer Kopplung zwischen den Metallionen (Abb. 5.1.1.b, c).

1) Streng betrachtet, dominiert für das Metall-Donoratom-Fragment in der Regel der Spindelokalisationsmechanismus mit resultierender gleicher Ausrichtung der Spindichte am Donoratom sowie am Metallzentrum, was allerdings keine Konsequenz auf das Vorzeichen der Metall-Metall-Kopplungsparameter hat. $^{[122 c]}$ 
Einer der prominentesten triazinbasierten Liganden ist 2,4,6-Tris(di-2-pyridylamino)-1,3,5triazin, das in einem Schritt in hoher Ausbeute dargestellt werden kann (Schema. 5.1.1). ${ }^{\text {[125] }}$<smiles>C/C=C\C=C(c1nc(N(c2ccccn2)c2ccccn2)nc(N(c2ccccn2)c2ccccn2)n1)N(C(/C=C\C)=C/C)[C@H](C)Cl</smiles>

Schema. 5.1.1. Synthese des Liganden $\mathbf{L}^{\mathbf{8}}$.

Diese Verbindung wurde wegen ihrer folgenden Eigenschaften auch in Rahmen dieser Arbeit als Ligand $\left(\mathbf{L}^{\mathbf{8}}\right)$ verwendet:

1) der polydentate Ligand $\mathbf{L}^{\mathbf{8}}$ hat dreizählige Symmetrie mit zwei- bzw. drei NDonoratomen je Bindungstasche, in Abhängigkeit davon, ob N-Donoren in meta-Positionen des Triazinrings beteiligt sind (Abb. 5.1.2). Dies kann die Synthese dreikerniger Komplexe ermöglichen;

2) der Triazinring mit seinen N-Donoren in meta-Positionen sollte, falls es gelingt die Metalle an diese Atome $\mathrm{zu}$ binden, nach dem Spinpolarisationsmechanismus ferromagnetische Kopplung vermitteln; somit hätte man dreikernige ferromagnetische Spezies mit dem höchst möglichen Spin-Grundzustand;

3) da der Ligand zwei- bzw. drei N-Donoren pro Bindungstasche besitzt, welche an ein Metall koordinieren können (Abb. 5.1.2), bleiben an oft fünf- bzw. sechsfach koordinierten Kupfer(II)-Ionen freie Koordinationsstellen übrig, über welche solche dreikernige Einheiten zu höheren Aggregaten agglomerieren könnten. 
<smiles></smiles>

a

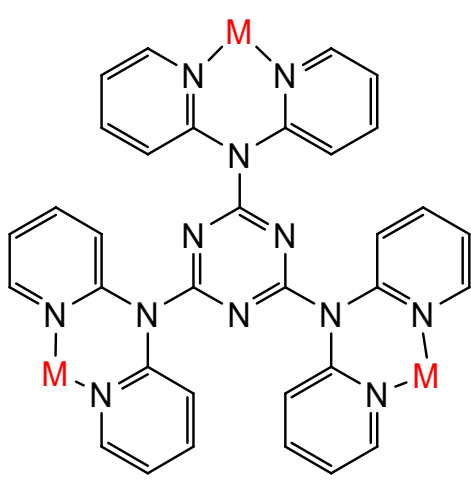

b

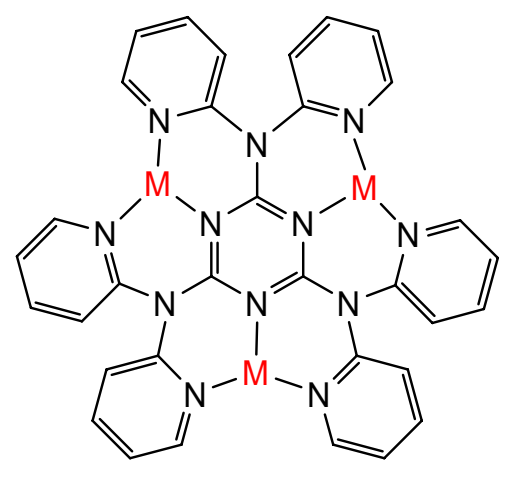

C

Abb. 5.1.2. Mögliche Bindungsmodi des Liganden $\mathbf{L}^{\mathbf{8}}$ an drei Metallionen: a) terminal, b) bidentat chelatisiert; c) tridentat chelatisiert.

Es soll angemerkt werden, dass sich auch andere Forschungsgruppen mit Komplexen auf der Basis dieses Liganden in unterschiedlichen Hinsichten (Magnetismus, Katalyse, supramolekulare sowie organometallische Chemie) erfolgreich beschäftigen. ${ }^{[19,126]}$

\subsection{Synthese, Molekülstrukturen und magnetische Eigenschaften der triazinbasierten Kupfer(II)-Komplexe}

Zwei neue dreikernige Komplexe 25 und 26, ausgehend von dem Liganden $\mathbf{L}^{\mathbf{8}}$ und Kupfer(II)chlorid sowie ein 1D-polymeres zickzack-System 27 wurden dargestellt und strukturell sowie magnetisch charakterisiert. ${ }^{[127,128]}$ Die magnetischen Eigenschaften dieser Komplexe können auf der Basis der Struktur interpretiert werden.

\section{Synthese und Charakterisierung}

Für die Synthese der neuen Komplexe wurde $\mathbf{L}^{\mathbf{8}}$ mit $\mathrm{CuCl}_{2} \cdot 2 \mathrm{H}_{2} \mathrm{O}$ umgesetzt. In Abhängigkeit von der Stöchiometrie der Reaktanten, der verwendeten Lösungsmittel sowie der Kristallisationsbedingungen wurden drei unterschiedliche Komplexe erhalten. Dunkelgrüne Kristalle von 25 (Raumgruppe $P 2_{1} 2_{1} 2_{1}$ ) wurden aus dem Lösungsmittelgemisch $\mathrm{CH}_{3} \mathrm{OH} / \mathrm{CH}_{2} \mathrm{Cl}_{2}$ isoliert. Wenn die Reaktion zwischen $\mathbf{L}^{\mathbf{8}}$ und $\mathrm{CuCl}_{2} \cdot 2 \mathrm{H}_{2} \mathrm{O}$ in $\mathrm{CH}_{2} \mathrm{Cl}_{2}$ verläuft, entsteht ein grünes Pulver. Dies kann in DMSO gelöst werden. Die entstandene Lösung wurde mit $\mathrm{CH}_{2} \mathrm{Cl}_{2}$ vorsichtig unterschichtet, was zu Kristallen von 26 führte (Raumgruppe $P 2_{1} / c$ ). Schließlich erhielt man nach direkter Unterschichtung der Lösung von $\mathbf{L}^{\mathbf{8}}$ und $\mathrm{CuCl}_{2} \cdot 2 \mathrm{H}_{2} \mathrm{O}$ in DMSO durch $\mathrm{CH}_{2} \mathrm{Cl}_{2} 27$ (Raumgruppe $P \overline{1}$ ). 
Die Struktur des Kations von 25 weist eine karussellförmige Anordnung auf. Diese besteht aus zwei Triazinliganden und drei Kupfer(II)-Ionen, welche an je vier N-Pyridyldonoratome und an ein apikales Chloridion koordiniert sind (Abb. 5.2.1). Die Ladung ist durch das zusätzliche Chloridion und das $\left[\mathrm{CuCl}_{4}\right]^{2-}$-Ion ausgeglichen. Die besonders interessante Eigenschaft von 25 betrifft die Lage der Anionen: das Chloridion befindet sich oberhalb eines Triazinrings, und die gegenüberstehende Seite des $\left[\mathrm{L}_{2}^{8}(\mathrm{CuCl})_{3}\right]^{3+}$-Gerüsts ist mit dem ClAtom von $\left[\mathrm{CuCl}_{4}\right]^{2-}$ überkappt, was auf die Existenz einer nicht kovalenten elektrostatischen Bindung zwischen den Anionen und den $\pi$-elektronenarmen Triazinringen hindeutet. ${ }^{[127]}$ Dieser ungewöhnliche Aspekt und die strukturellen Feinheiten von 25 werden im nächsten Kapitel beschrieben.

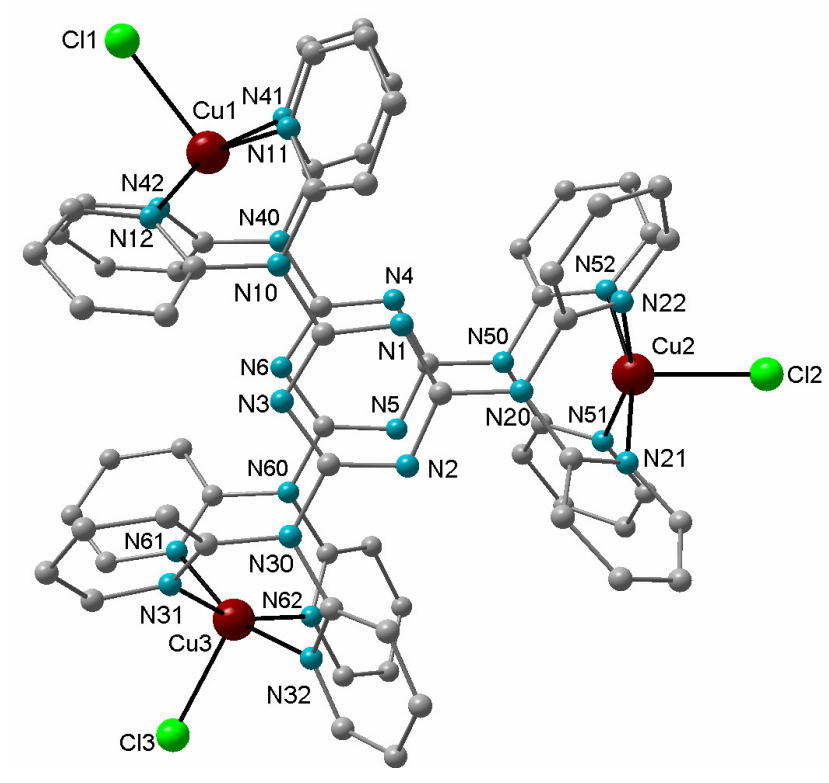

Abb. 5.2.1. Molekulare Struktur des Kations von 25.

Im Gegensatz zu 25 ist das Metall:Triazin-Verhältnis in Komplexen 26 und 27 gleich 3:1. Jedes Kupfer(II)-Ion in $\mathbf{2 6}$ sowie $\mathbf{2 7}$ ist an zwei N-Pyridyldonoratome der Ligandseitenarme des Liganden $\mathbf{L}^{\mathbf{8}}$ koordiniert (Abb. 5.2.2 und Abb. 5.2.3). 


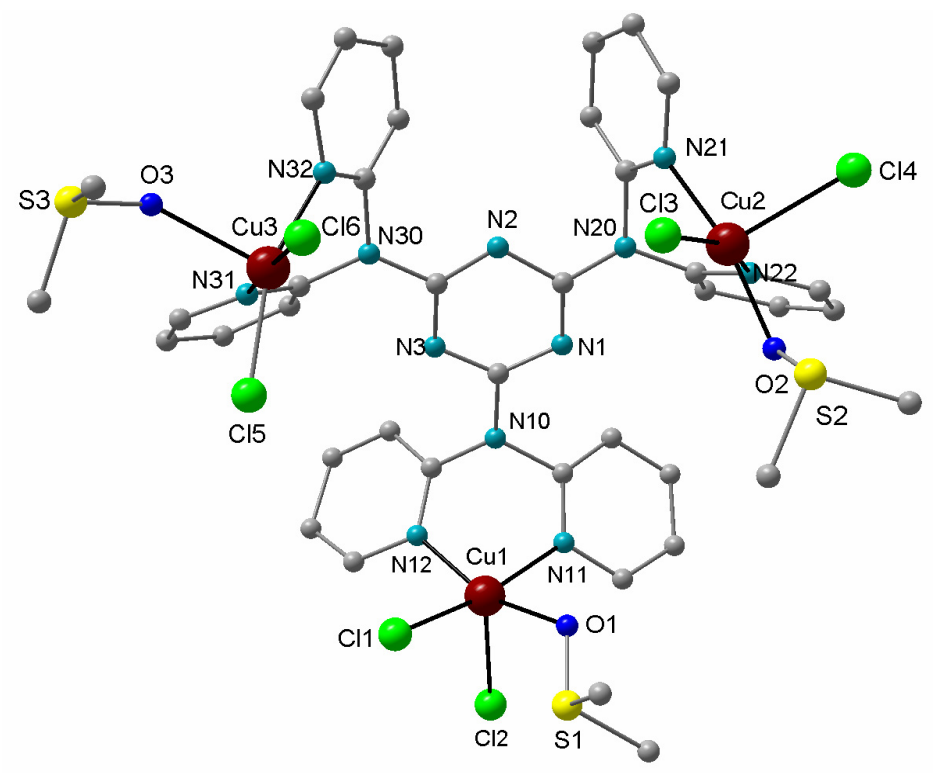

Abb. 5.2.2. Molekulare Struktur von 26.

Im Falle von 26 ist die Koordinationssphäre bei jedem Kupfer(II)-Ion durch zwei Chloridionen sowie durch das O-Donoratom von DMSO ergänzt. Damit sind alle Metallionen fünffach koordiniert. Die Koordinationsgeometrie kann mit Hilfe des Parameters $\tau$ quantitativ beschrieben werden. Dieser Parameter ist gleich Null im Falle einer idealen tetragonalen Pyramide und gleich eins für die ideale trigonal bipyramidale Geometrie. ${ }^{[129]}$ Diese Werte sind 0.09 für $\mathrm{Cu}(1), 0.03$ für $\mathrm{Cu}(2)$ und 0.31 für $\mathrm{Cu}(3)$ in 26 und lassen somit die Geometrie der Koordinationspolyeder als verzerrt quadratisch pyramidal beschreiben. Die Winkel in der basalen Ebene um die Kupfer(II)-Ionen herum variieren von 84.4(5) bis 93.0(4) ${ }^{\circ}$ für $\mathrm{Cu}(1)$, 82.2(5) bis $95.0(4)^{\circ}$ für $\mathrm{Cu}(2)$ und 85.2(5) bis $93.9(4)^{\circ}$ für $\mathrm{Cu}(3)$. Interessanterweise kann jeder der beiden Coliganden (Chlorid $[\mathrm{Cu}(1)$ und $\mathrm{Cu}(2)]$ oder DMSO [Cu(3)]) die axiale Position besetzen, was auf vergleichbare Ligandstärke hindeutet. Die $\mathrm{Cu}-\mathrm{Cl}$ und $\mathrm{Cu}-\mathrm{O}$ Bindungslängen für die entsprechenden apikalen Liganden sind in besonderem Maße länger (um ca. $0.2 \AA$ ) als die basalen Kupfer-Ligand-Bindungen (Tab. 5.2.1).

Im Falle von 27 ist DMSO nur an einem von drei Kupfer(II)-Ionen je trimetallischer Einheit gebunden (in der axialen Position von Cu(1), Abb. 5.2.3). 


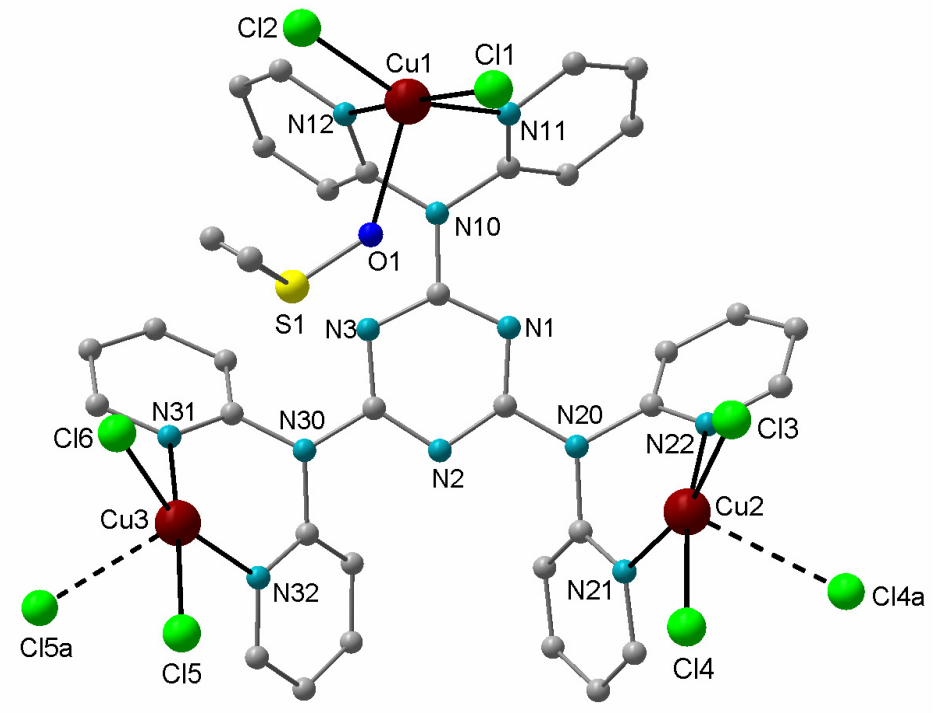

Abb. 5.2.3. Molekulare Struktur von 27.

Die Koordinationssphäre der zwei anderen Kupfer(II)-Ionen ist durch die langen axialen Bindungen zu den Chloridionen von benachbarten Molekülen und vice versa gefüllt. In Folge dessen resultiert eine eindimensionale zickzack-Kette, welche aus sich an einander gereihten trimetallischen Einheiten besteht (Abb. 5.2.4).

Die $\mathrm{Cu}-\mathrm{Cl}$ Abstände von den apikalen, kettenformgebenden Bindungen sind deutlich größer (0.6 bis $0.9 \AA$ ) als die basalen $\mathrm{Cu}-\mathrm{Cl}$ Bindungen (Tab. 5.2.1).

Tab. 5.2.1. Ausgewählte Bindungslängen $(\AA)$ und Winkel $\left(^{\circ}\right)$ von 25, 26 und 27.

\begin{tabular}{|c|c|c|c|c|c|c|c|}
\hline & 25 & 26 & 27 & & 25 & 26 & 27 \\
\hline $\mathrm{Cu}(1)-\mathrm{Cl}(1)$ & $2.407(1)$ & $2.294(5)$ & $2.279(1)$ & $\mathrm{Cu}(3)-\mathrm{N}(31)$ & $2.027(4)$ & $2.04(1)$ & $2.019(4)$ \\
\hline $\mathrm{Cu}(1)-\mathrm{Cl}(2)$ & - & $2.464(5)$ & $2.233(1)$ & $\mathrm{Cu}(3)-\mathrm{N}(32)$ & $2.028(4)$ & $2.07(1)$ & $2.054(4)$ \\
\hline $\mathrm{Cu}(2)-\mathrm{Cl}(3)$ & $2.382(1)^{\mathrm{a}}$ & $2.271(5)$ & $2.246(1)$ & $\mathrm{Cu}(1)-\mathrm{O}(1)$ & - & $1.99(1)$ & $2.256(3)$ \\
\hline \multirow[t]{2}{*}{$\mathrm{Cu}(2)-\mathrm{Cl}(4)$} & - & $2.496(4)$ & $2.255(1)$ & $\mathrm{Cu}(2)-\mathrm{O}(2)$ & - & $1.98(1)$ & - \\
\hline & & & $3.134(2)^{c}$ & & & & \\
\hline \multirow[t]{2}{*}{$\mathrm{Cu}(3)-\mathrm{Cl}(5)$} & $2.399(1)^{\mathrm{b}}$ & $2.238(5)$ & $2.261(1)$ & $\mathrm{Cu}(3)-\mathrm{O}(3)$ & - & $2.25(1)$ & - \\
\hline & & & $2.898(2)^{\mathrm{d}}$ & & & & \\
\hline $\mathrm{Cu}(3)-\mathrm{Cl}(6)$ & - & $2.297(5)$ & $2.274(1)$ & $\mathrm{Cu}(1) \cdots \mathrm{Cu}(2)$ & $8.4146(7)$ & 8.309(3) & $7.964(1)$ \\
\hline $\mathrm{Cu}(1)-\mathrm{N}(11)$ & $2.044(4)$ & $2.04(1)$ & $2.035(4)$ & $\mathrm{Cu}(1) \cdots \mathrm{Cu}(3)$ & $8.2916(7)$ & $7.878(3)$ & $7.787(1)$ \\
\hline $\mathrm{Cu}(1)-\mathrm{N}(12)$ & $2.040(4)$ & $1.96(1)$ & $2.088(4)$ & $\mathrm{Cu}(2) \cdots \mathrm{Cu}(3)$ & $8.2706(8)$ & 7.831(3) & $8.224(1)$ \\
\hline $\mathrm{Cu}(2)-\mathrm{N}(21)$ & $2.037(4)$ & $2.03(2)$ & $2.031(4)$ & $\mathrm{Cu}(2) \cdots \mathrm{Cu}(2 \mathrm{a})$ & - & - & $3.986(1)$ \\
\hline $\mathrm{Cu}(2)-\mathrm{N}(22)$ & $2.033(4)$ & $2.05(1)$ & $2.012(4)$ & $\mathrm{Cu}(3) \cdots \mathrm{Cu}(3 \mathrm{a})$ & - & - & $3.838(1)$ \\
\hline $\mathrm{Cl}(1)-\mathrm{Cu}(1)-\mathrm{Cl}(2)$ & - & $98.7(2)$ & $92.40(6)$ & & & & \\
\hline $\mathrm{Cl}(3)-\mathrm{Cu}(2)-\mathrm{Cl}(4)$ & - & $101.5(2)$ & $93.09(5)$ & & & & \\
\hline $\mathrm{Cl}(5)-\mathrm{Cu}(3)-\mathrm{Cl}(6)$ & - & $90.6(2)$ & $93.54(5)$ & & & & \\
\hline
\end{tabular}




$\begin{array}{llll}\mathrm{Cu}(2)-\mathrm{Cl}(4)-\mathrm{Cu}(2 \mathrm{a}) & - & - & 93.97(5) \\ \mathrm{Cu}(3)-\mathrm{Cl}(5)-\mathrm{Cu}(3 \mathrm{a}) & - & - & 95.34(5)\end{array}$

${ }^{\mathrm{a}} \mathrm{Cl}(3)=\mathrm{Cl}(2),{ }^{\mathrm{b}} \mathrm{Cl}(5)=\mathrm{Cl}(3),{ }^{\mathrm{c}} \mathrm{Cl}(4 \mathrm{a}),{ }^{\mathrm{d}} \mathrm{Cl}(5 \mathrm{a})$.

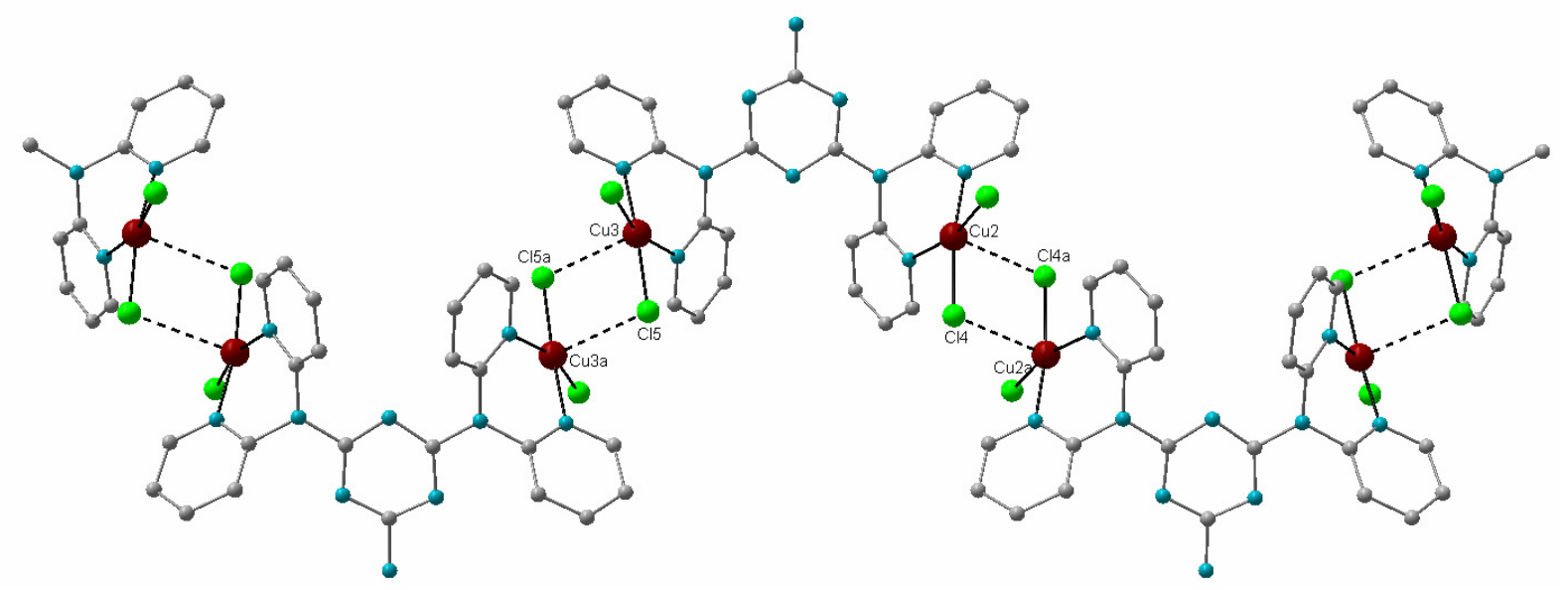

Abb. 5.2.4. Kettenförmige Struktur von 27 mit den pseudodimeren rhombischen $\left\{\mathrm{Cu}_{2} \mathrm{Cl}_{2}\right\}$-Untereinheiten.

Dies deutet auf relativ lockere Assoziation der trimetallischen Baueinheiten hin. Die zwei $\mathrm{Cu}_{2}(\mu-\mathrm{Cl})_{2}$ Rhomben sind in unabhängigen Inversionszentren der Raumgruppe $P \overline{1}$ angeordnet. Die Winkel in der basalen Ebene in 27 liegen im Bereich von 85.1(2) bis 92.5(1) für $\mathrm{Cu}(1)$, 86.7(1) bis 93.5(1) ${ }^{\circ}$ für $\mathrm{Cu}(2)$ und 86.7(1) bis 93.54(5) ${ }^{\circ}$ für $\mathrm{Cu}(3)$. ). Die $\tau$ Werte betragen 0.34 für $\mathrm{Cu}(1), 0.06$ für $\mathrm{Cu}(2)$ und 0.21 für $\mathrm{Cu}(3)$. Dies zeigt analog zu 26, dass die Koordinationsgeometrie von Kupfer(II)-Ionen zwischen der für $\mathrm{Cu}(2)$ fast idealen und der für $\mathrm{Cu}(1)$ sowie $\mathrm{Cu}(3)$ verzerrten tetragonalen Pyramide liegt. Über einen Kupfer(II)-Komplex von $\mathrm{N}, \mathrm{N}^{\prime}-\{2,4-\mathrm{Di}[($ di-pyridin-2-yl)amine]-1,3,5-triazine\}ethylenediamin (opytrizediam) mit ähnlichen molekularen Parametern wurde kürzlich berichtet. ${ }^{[130]}$ In diesem Komplex weisen zwei von drei unterschiedlichen Kupfer(II)-Ionen eine verzerrt quadratisch planare Umgebung ( $\tau=0.38$ und 0.39 ) auf, während das dritte Kupfer(II)-Ion sechsfach koordiniert ist. Wie im Falle von 27 stellt der Opytrizediamkomplex ein eindimensionales zickzack-System durch die Bis( $\mu$-chloro)verbrückung zwischen benachbarten Molekülen dar. Allerdings sind die intermolekularen $\mathrm{Cu} \cdots \mathrm{Cu}$ Abstände $(d(\mathrm{Cu} \cdots \mathrm{Cu})=3.6635(4)$ und 3.6769(3) $\AA$ ) innerhalb des $\mathrm{Cu}_{2} \mathrm{Cl}_{4}$ Rhombus bis zu $0.3 \AA$ kürzer als in 27. Wie weiter unten diskutiert wird, hat dies drastische Konsequenzen für die magnetischen Eigenschaften. 
EPR-Messungen der gefrorenen Methanollösungen von 25-27 weisen Spektren axialen Typs mit $g_{\perp} \approx 2.10$ und mit der im $g \|$ Bereich erkennbaren Hyperfeinaufspaltung von $124 \cdot 10^{-4}-130 \cdot 10^{-4} \mathrm{~cm}^{-1}$ auf (Abb. 5.2.5, [128]).

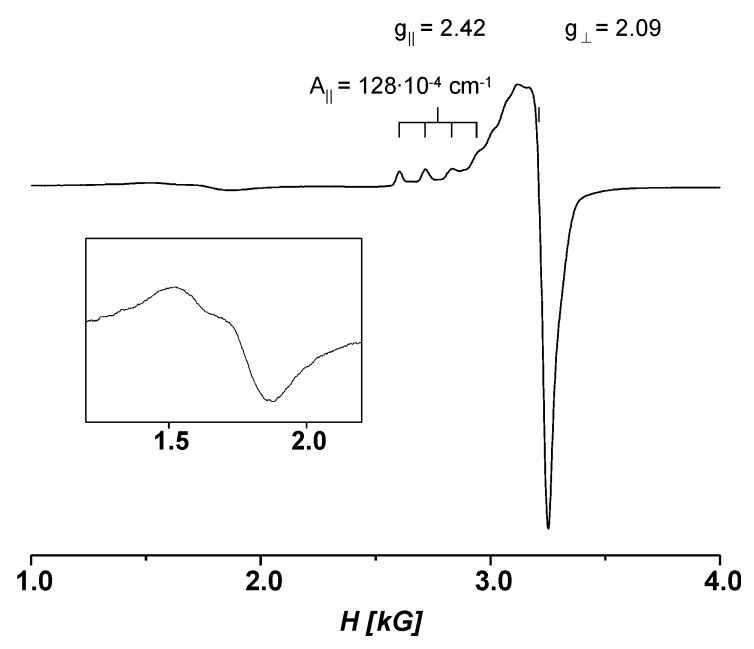

Abb. 5.2.5. EPR-Spektrum der gefrorenen Methanollösungen von 27 bei $130 \mathrm{~K}$.

Nur im Falle von 27 ist ein Halbfeldsignal dank des Übergangs mit $\Delta M_{\mathrm{S}}=2$ erkennbar (Abb. 5.2.5), was auf Vorhandensein der $\mu$-Chlorverbrückung in der pseudodimeren Einheit auch in der Lösung hindeutet. Dies steht in Übereinstimmung mit der ESImassenspektrometrischen Untersuchung von 27, die Signale von Spezies wie $\left[\mathrm{Cu}_{5}\left(\mathrm{~L}^{8}\right)_{2} \mathrm{Cl}_{9}\right]^{+}$ $(m / z=1806)$ und $\left[\mathrm{Cu}_{4}\left(\mathrm{~L}^{8}\right)_{2} \mathrm{Cl}_{7}\right]^{+}(m / z=1673)$ ebenso wie die Präsenz höhernuklearer Aggregate in der Lösung bestätigt. UV/Vis-Spektren von 27 sind lösungsmittelabhängig. Dies bestätigt die Anwesenheit von Spezies mit der unterschiedlichen Kupfer(II)koordination in $\mathrm{MeOH}\left(\lambda_{\max }=692 \mathrm{~nm}\right)$ und DMSO $\left(\lambda_{\max }=855 \mathrm{~nm}\right)$, was nicht zulässt, eindeutige Korrelationen zwischen den spektroskopischen Daten in Lösung und den strukturellen sowie magnetischen Eigenschaften im Festkörper zu formulieren.

\section{Magnetische Eigenschaften}

Die $\chi_{M} T$-Werte von 26 und 27 liegen bei Raumtemperatur bei ca. $1.21 \mathrm{~cm}^{3} \mathrm{Kmol}^{-1}$. Dieser Wert ist sehr nahe am „spin-only“ Wert für drei ungekoppelte $S=1 / 2$ Kupfer(II)-Ionen (1.18 $\mathrm{cm}^{3} \mathrm{Kmol}^{-1}$ für $g=2.05$ ) und weist auf keine signifikante magnetische Wechselwirkungen zwischen Metallionen hin. Der $\chi_{M} T$-Wert von 25 beträgt bei Raumtemperatur 1.67 $\mathrm{cm}^{3} \mathrm{Kmol}^{-1}$, was dem „spin-only“ Wert für vier ungekoppelte $S=1 / 2 \mathrm{Kupfer(II)-Ionen} \mathrm{(1.57}$ 

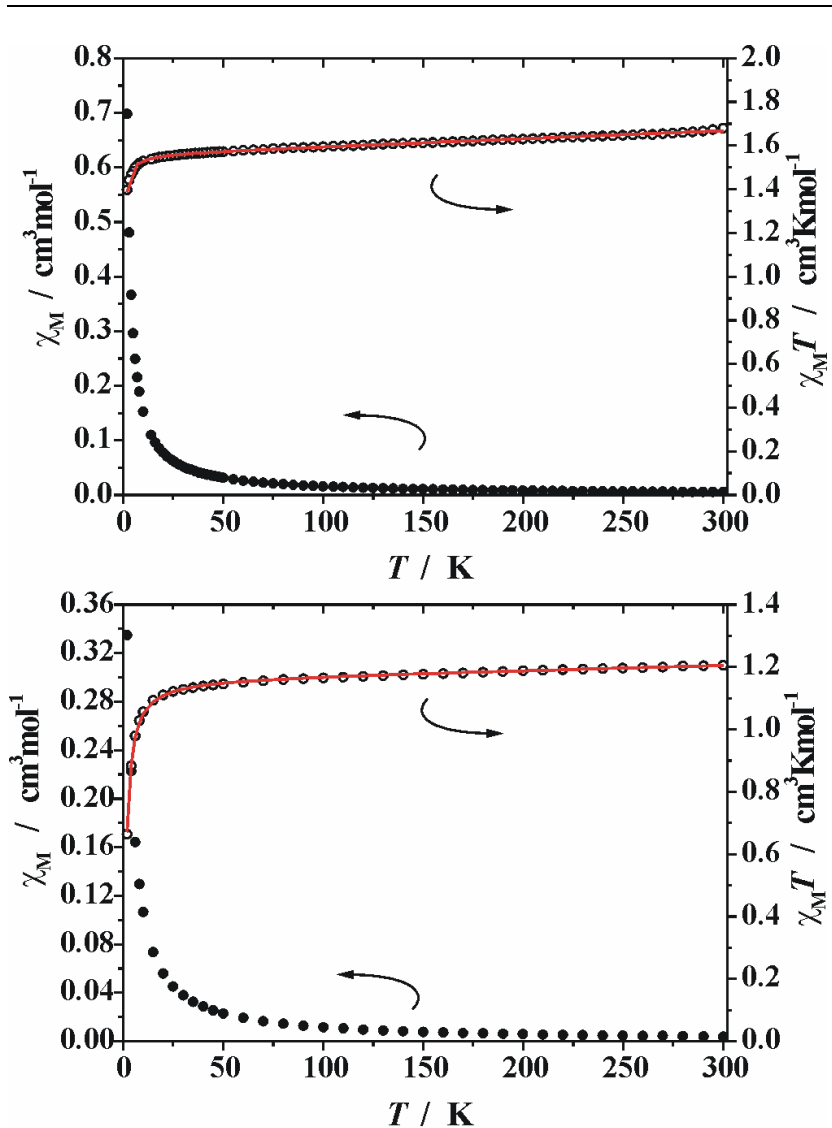

$\mathrm{cm}^{3} \mathrm{Kmol}^{-1}$ für $\left.g=2.05\right)$ entspricht. Dies ist auch sinnvoll, weil auch das Tetrachlorocupratanion bei der Rechnung berücksichtigt werden muss.

Das Produkt $\chi_{M} T$ von 25 nimmt bei Abkühlung zuerst sehr wenig ab: von 1.67 $\mathrm{cm}^{3} \mathrm{Kmol}^{-1}\left(3.66 \mu_{\mathrm{B}}\right)$ bei $295 \mathrm{~K}$ bis 1.55 $\mathrm{cm}^{3} \mathrm{Kmol}^{-1}\left(3.53 \mu_{\mathrm{B}}\right)$ bei $20 \mathrm{~K}$. Unterhalb von $20 \mathrm{~K}$ wird die Abnahme stärker, das Produkt $\chi_{M} T$ erreicht einen Wert von 1.40 $\mathrm{cm}^{3} \mathrm{Kmol}^{-1}\left(3.35 \mu_{\mathrm{B}}\right)$ bei 2 K. Die Temperaturabhängigkeiten von $\chi_{M} T$ für 26 und 27 sind ähnlich. Die Abnahme erfolgt zuerst sehr mäßig von $1.21 \mathrm{~cm}^{3} \mathrm{Kmol}^{-1}$ $\left(3.12 \mu_{\mathrm{B}}\right)$ bei $295 \mathrm{~K}$ bis $1.15 \mathrm{~cm}^{3} \mathrm{Kmol}^{-1}$

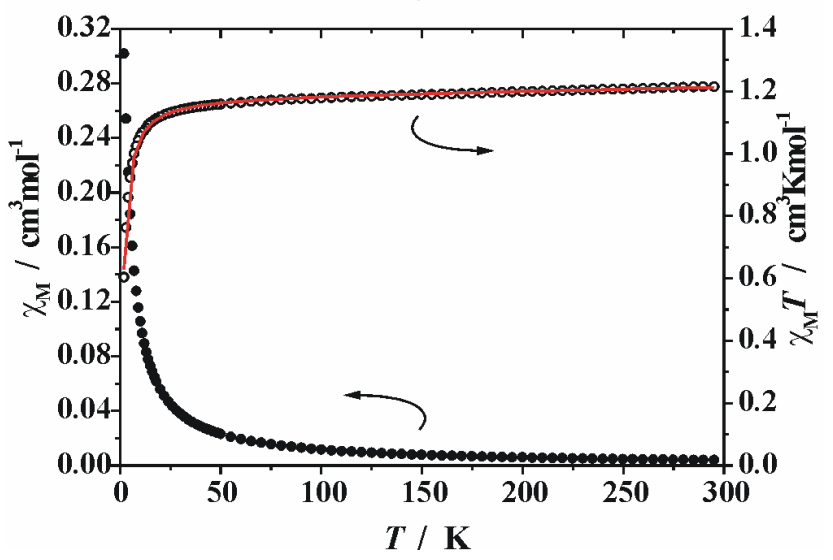

Abb. 5.2.6. Auftragung von $\chi_{M}$ und $\chi_{M} T$ gegen $T$ für 25 (oben), 26 (in der Mitte) und 27b (unten). Die roten Linien repräsentieren die besten Anpassungen. $\left(3.04 \mu_{\mathrm{B}}\right)$ bei $50 \mathrm{~K}$. Unterhalb von $50 \mathrm{~K}$ nehmen die $\chi_{M} T$-Werte schneller ab: bis $0.66 \mathrm{~cm}^{3} \mathrm{Kmol}^{-1}\left(2.31 \mu_{\mathrm{B}}\right)$ und 0.60 $\mathrm{cm}^{3} \mathrm{Kmol}^{-1}\left(2.20 \mu_{\mathrm{B}}\right)$ bei $2 \mathrm{~K}$ für 26 und 27. Offensichtlich ist das magnetische Verhalten aller drei Kupfer(II)-Komplexe ähnlich, alle drei weisen schwache antiferromagnetische Kopplung innerhalb der trimetallischen Einheit und die zusätzlich bei tieferen Temperaturen wichtigen intermolekularen antiferromagnetischen Wechselwirkungen auf.

Die Anpassung an die experimentellen Daten für 25-27 erfolgte mit dem Programm ORIGIN unter Verwendung des Heisenberg-Dirac-Van-Vleck-Hamiltonoperators mit den zusätzlichen Termen für die paramagnetische Verunreinigung $(\rho)$ und den temperaturunabhängigen Paramagnetismus (TIP). Es wurde ein Modell für die dreikernige Anordnung der Metallzentren in der Form eines gleichseitigen Dreiecks angewendet. ${ }^{[5,131]}$ In dieser Situation wird der erwartete Hamiltonoperator (Gleichung 5.2.1) 


$$
\hat{H}=-2 J_{12} \hat{S}_{1} \hat{S}_{2}--2 J_{23} \hat{S}_{2} \hat{S}_{3}--2 J_{13} \hat{S}_{1} \hat{S}_{3}
$$

vereinfacht und enthält nur eine Kopplungskonstante $J\left(J_{12}=J_{23}=J_{13}=J\right.$, Gleichung 5.2.2). Für Komplex 25 wurde ein Curie-Term für das Kupfer(II)-Ion in $\left[\mathrm{CuCl}_{4}\right]^{2-}$ zusätzlich addiert. Wenn man die Wechselwirkung innerhalb der bis( $\mu$-chloro)verbrückten Untereinheit von 27 als dominant betrachtet, können experimentelle magnetische Daten für diesen Komplex alternativ behandelt werden, indem man ein Modell mit den gekoppelten isolierten Kupfer(II)-Dimeren und einem zusätzlichen Curie-Term für das monomere dritte Kupfer(II)Ion (Gleichung 5.2.3) verwendet.

$$
\begin{gathered}
\chi_{M}=\frac{N g^{2} \beta^{2}}{4 k T} \frac{1+5 e^{(3 J / k T)}}{1+e^{(3 J / k T)}}(1-\rho)+\frac{3 N g^{2} \beta^{2}}{4 k T} \rho+T I P \\
\chi_{M}=\left(\frac{2 N g_{1}^{2} \beta^{2}}{k T\left(3+e^{(-2 J / k T)}\right)}+\frac{N g^{2} \beta^{2}}{4 k T}\right)(1-\rho)+\frac{3 N g^{2} \beta^{2}}{4 k T} \rho+T I P
\end{gathered}
$$

Nach Analyse der magnetischen Daten unter Verwendung der Gleichung 5.2.2 erhält man die Parameter: $g_{1}=2.04 \pm 0.01, g_{2}=2.07 \pm 0.01$ ( $\left[\mathrm{CuCl}_{4}\right]^{2-}$-Ion), $J=-0.21 \pm 0.01 \mathrm{~cm}^{-1}, \rho=1.0$ $\%$ und TIP $=3.6 \times 10^{-4} \mathrm{~cm}^{3} \mathrm{~mol}^{-1}$ für 25, $g=2.04 \pm 0.01, J=-0.71 \pm 0.01 \mathrm{~cm}^{-1}, \rho=1.5 \%$ und $T I P=1.5 \times 10^{-4} \mathrm{~cm}^{3} \mathrm{~mol}^{-1}$ für 26 und $g=2.05 \pm 0.01, J=-0.82 \pm 0.01 \mathrm{~cm}^{-1}, \rho=0.8 \%$ und TIP $=1.1 \times 10^{-4} \mathrm{~cm}^{3} \mathrm{~mol}^{-1}$ für 27. Im Falle von 27 liefert die Anpassung nach Gleichung 5.2 .3 mit einer vergleichbar guten Qualität folgende Parameter: $g_{1}=2.05 \pm 0.01, g_{2}=2.03 \pm 0.01$ (Einzelnion), $J=-1.83 \pm 0.02 \mathrm{~cm}^{-1}, \rho=3.2 \%$ und $T I P=1.7 \times 10^{-4} \mathrm{~cm}^{3} \mathrm{~mol}^{-1}$. Alle $g$-Werte sind in vernünftigem Bereich und liegen nahe zu Werten, die durch EPR-spektroskopische Untersuchungen an den gefrorenen Methanollösungen gefunden wurden (siehe oben). Die $J$ Werte bestätigen, dass die magnetische Austauschwechselwirkung zwischen den Metallionen in allen drei Komplexen sehr schwach ist. Das nach der Verwendung des dimeren Modells erhaltene Ergebnis deutet darauf hin, dass die beobachtete Abnahme des magnetischen Moments auch auf den schwachen intermolekularen Austausch zwischen den trimetallischen Baueinheiten zurückzuführen ist.

Im Hinblick auf die Schwäche der intramolekularen Austauschwechselwirkung in $\mathbf{2 5}$ und 26 sollte angemerkt werden, dass sich die basalen Koordinationsebenen von den Kupfer(II)- 
Ionen annähernd senkrecht zu dem zentralen Triazinring befinden und die Delokalisierung der magnetischen $d_{x-y}^{2}{ }^{2}$-Orbitale in die Richtung der N-Pyridindonoren $\sigma$-Charakter hat. Die Pyridinringe sind stark gekippt zueinander sowie zum zentralen Triazinring und vermitteln dadurch keine effiziente magnetische Kopplung durch die Bindungen.

Interessant ist, dass in $\mathbf{2 7}$ die schwache antiferromagnetische Kopplung in der dimeren bis( $\mu$-chloro)verbrückten Kupfer(II)-Untereinheit gefunden wurde, aber die Wechselwirkung in der ähnlichen opytrizediambasierten zickzack Kette ferromagnetischer Natur ist. ${ }^{[130]}$ Dieser Unterschied kann durch die detaillierte Betrachtung der pseudodimeren $\mathrm{Cu}_{2}(\mu-\mathrm{Cl})_{2}$ - $\mathrm{Rhomben}$ beleuchtet werden. ${ }^{[132]}$ In beiden Verbindungen resultiert der $\mathrm{Cu}_{2}(\mu-\mathrm{Cl})_{2}$-Rhombus durch den Zusammenbau von zwei qudratisch pyramidalen Kupfer(II)-Untereinheiten. Dabei gilt die Spitze einer Pyramide gleichzeitig als der Punkt des in der basalen Ebene liegenden Vierecks der anderen Pyramide (Abb. 5.2.4). Da die Spindichte in der basalen Ebene liegt, ist die Überlappung der magnetischen Orbitale sehr klein, was im Einklang mit der sehr schwachen intradimeren Wechselwirkung steht. Allerdings wurde eine verfeinerte Korrelation für diesen

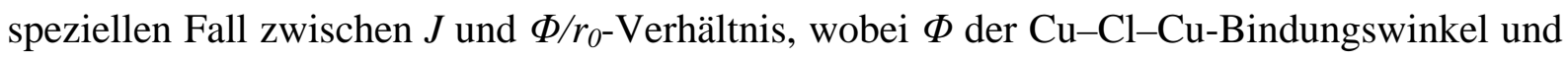
$r_{0}$ der längste $\mathrm{Cu}-\mathrm{Cl}$ Abstand ist, veröffentlicht. ${ }^{[133]}$ Das $\Phi / r_{0}$-Verhältnis für 27 beträgt $30.0^{\circ} \cdot \AA^{-1}[\mathrm{Cu}(2) / \mathrm{Cl}(4)]$ und $32.9^{\circ} \cdot \AA^{-1}[\mathrm{Cu}(3) / \mathrm{Cl}(5)]$. Dies liegt im Bereich, für den antiferromagnetische Kopplung vorausgesagt und beobachtet wird. Ferromagnetisches Verhalten erwartet man für ein größeres $\Phi / r_{0}$-Verhältnis im Bereich zwischen $33^{\circ} \cdot \AA^{-1}$ und $35^{\circ} \cdot \AA^{-1}$. Die Werte von $34.8^{\circ} \cdot \AA^{-1}$ und $35.6^{\circ} \cdot \AA^{-1}$ wurden in der Tat im opytrizediambasierten Kupfer(II)-Komplex beobachtet. Die magnetischen Eigenschaften der zwei $\mathrm{Cu}_{2}(\mu-\mathrm{Cl})_{2}$ Rhomben von sehr ähnlichen zickzack-Ketten unterscheiden sich merklich, obwohl die $\mathrm{Cu}-$ $\mathrm{Cl}-\mathrm{Cu}$ Winkel $\left(94.0-95.3^{\circ}\right)$ fast gleich sind. Die relativ kleine Größe der $\mathrm{Cu}_{2}(\mu-\mathrm{Cl})_{2}$ intradimeren Kopplung in $\mathbf{2 7}$ ist sehr wahrscheinlich auf die ineffiziente Überlappung wegen der langen axialen $\mathrm{Cu}-\mathrm{Cl}$-Abstände (2.898(2) und 3.134(2) in 27 versus 2.695(1) und 2.670(1) für den opytrizediambasierten Komplex) zurückzuführen.

\section{Zusammenfassung}

Drei Kupfer(II)-Komplexe verschiedener Topologie wurden auf der Basis des Triazinliganden $\mathbf{L}^{\mathbf{8}}$ synthetisiert und strukturell sowie magnetisch charakterisiert. Im Falle aller Verbindungen binden sich drei Di(2-pyridyl)amineinheiten jeweils an ein Kupfer(II)-Ion durch zwei N-Pyridyldonoratome. Die leichten Modifikationen in den Synthese- oder Kristallisationsbedingungen können zu unterschiedlichen Typen der Aggregation sowie 
Dimensionalität im Festkörper führen (molekulare dreikernige (25, 26) oder 1D-polymere (27) Spezies). Wenn man die Anwesenheit von freien, durch Lösungsmittelmoleküle besetzten Koordinationsstellen von Kupfer(II)-Ionen in $\mathbf{2 6}$ berücksichtigt, kann dieser Komplex als ein geeigneter Baustein für den kontrollierten Aufbau von bienenwabenähnlichen 2D-Netzwerken betrachtet werden. In allen drei Komplexen nehmen die N-Triazindonoratome bei der Koordination an Kupfer(II)-Ionen nicht teil. In Folge dessen resultiert kein effizienter Pfad für den magnetischen Austausch und somit nur sehr schwache magnetische Wechselwirkung innerhalb der Dreikernkomplexe von 25-27. 


\subsection{Anion- $\pi$ Wechselwirkung in einem karussellförmigen Kupfer(II)- Triazinkomplex}

Supramolekulare Chemie - die Chemie nichtkovalenter Wechselwirkungen - ist neben dem molekularen Magnetismus ein sehr attraktives Forschungsgebiet der modernen Naturwissenschaft. Nichtkovalente supramolekulare Wechselwirkungen, insbesondere unter Beteiligung aromatischer $\pi$-Systeme, spielen eine wichtige Rolle in chemischen sowie biologischen Erkennungsprozessen. ${ }^{[134]}$ Das Design und die Synthese von supramolekularen Gast-Wirt-Verbindungen mit nichtkovalenten Kation- $\pi$ Wechselwirkungen hat in den letzten zwanzig Jahren dank ihrer entscheidenden Bedeutung für viele biologische Systeme eine rasante Entwicklung erlebt. ${ }^{[134,135]}$ Im Gegensatz dazu ist die Chemie von nichtkovalenten Anion- $\pi$ Wechselwirkungen viel weniger untersucht und erarbeitet. ${ }^{[136,137]}$ Der Grund dafür liegt offensichtlich darin, dass intuitiv zwischen Anionen (Elektronendonoren) und aromatischen $\pi$-Systemen eine Abstoßung erwartet wird. Trotz der Tatsache, dass die Koordinationschemie von Anionen ${ }^{[136,138]}$ viel weniger erforscht ist als die Koordinationschemie von Kationen, ist sie eine der relevantesten und attraktivsten Gebiete der supramolekularen Chemie. ${ }^{[139]}$ In den letzten vier Jahren stieg die Intensität der Forschung an aromatischen Wirtsystemen als molekulare Rezeptoren für die Erkennung von anionischen Gästen. Mehr als 70\% der Enzymsubstrate und Cofaktoren sind anionischer Natur, ${ }^{[138]}$ und arylbasierte Wirte könnten potenzielle medizinische und biologische Anwendungen haben. Andere wichtige Anwendungsbereiche der molekularen Erkennung sind Katalyse, analytische Chemie sowie Templatsynthese an Anionen.

Auf die Assoziation zwischen elektronenarmen neutralen $\pi$-Systemen und Anionen wiesen zuerst einige NMR-spektroskopische Untersuchungen hin. ${ }^{[135 a, 140,141]}$ Der Begriff „Anion- $\pi$ Wechselwirkung“ wurde erstmals in 2002 in einer theoretischen Arbeit verwendet, in welcher die Autoren durch Rechnungen zeigen konnten, dass die Wechselwirkung zwischen solchen aromatischen $\pi$-Systemen wie Hexafluorobenzol oder Trinitrobenzol und verschiedenen Anionen energetisch günstig ist. ${ }^{[142]}$ Die gleichen Ergebnisse wurden auch für die synthetisch vielseitigeren $\pi$-elektronenarmen heterocyclischen 1,3,5-Triazine und 1,2,4,5-Tetrazine erhalten. ${ }^{[143,144,145]}$ Mascal et al. haben durch die Rechnung einen Abstand von 3.2 A zwischen Chlorid und Arylzentroid für einen $\mathrm{Cl}^{-}$...Triazinkomplex, in welchem sich das Anion auf der $C_{3}$-Achse befindet (Abb. 5.3.1), vorhergesagt. ${ }^{[143]}$ Während eindeutige kristallographische Nachweise für solche Anion- $\pi$ Wechselwirkungen mit Halogeniden fehlten, führten die theoretischen Voraussagen von interessanten Anion- $\pi$ Wechselwirkungen zum Vorschlag, 
Hetorocyclen wie Triazin oder Tetrazin als Module von neuen Rezeptoren für molekulare Anionerkennung zu betrachten. ${ }^{[143]}$

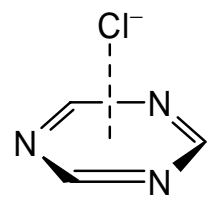

Abb. 5.3.1. Anion- $\pi$ Wechselwirkung im berechneten $\mathrm{Cl}^{-} \cdots$ Triazinkomplex.

Bei unseren Untersuchungen an strukturellen und magnetischen Eigenschaften von Kupfer(II)-Komplexen mit $\mathbf{L}^{\mathbf{8}}$ wurde ein Kupfer(II)-Triazinkomplex $\mathbf{2 5}^{[127]}$ erhalten. In diesem Komplex sind die metrischen Chlorid-Triazin Parameter nahezu identisch zu den für dieses Fragment zwei Jahre früher theoretisch vorhergesagten(Abb.5.3.1). ${ }^{[143]}$

\section{Strukturelle und MS-spektrometrische Charakterisierung}

25 wurde aus Kupfer(II)-Chlorid und $\mathbf{L}^{\mathbf{8}}$ synthetisiert (siehe Kapitel 5.2). Der kationische Teil besteht aus zwei Liganden $\mathbf{L}^{\mathbf{8}}$, welche in paralleler Anordnung aufgeschichtet und durch drei Kupfer(II)-Ionen zu einer karussellförmigen $\left[\left(\mathrm{L}^{8}\right)_{2}(\mathrm{CuCl})_{3}\right]^{3+}$ Struktur zusammengefügt sind (Abb. 5.2.1, 5.3.2).

Interessanterweise bilden beide Triazinringe in $\mathbf{2 5}$ eine fast perfekte face-to-face Anordnung, in welcher die $\mathrm{N}$-Atome eines Triazinringes über $\mathrm{N}$-Atomen des anderen Triazinringes liegen (Abb. 5.3.2). Der Abstand zwischen beiden Triazinebenen ist mit $3.78 \AA$ etwas größer als der übliche Abstand für aromatisches Stacking, der bei $3.5 \AA$ liegt. Eine solche Abweichung wird aber häufig bei aromatischen, Stickstoff enthaltenden Heterocyclen, welche als Liganden eingesetzt werden, beobachtet. ${ }^{[146]}$ Allerdings ist dabei ein Ring typischerweise um $1.30 \AA$ in horizontaler Richtung verschoben, was in $\mathbf{2 5}$ nicht der Fall ist (0.163 $\AA$ ). Eine ähnliche face-to-face Anordnung von zwei Triazinringen wurde vor kurzem in $\left[\left\{(\mathrm{CO})_{3} \operatorname{Re}\left(\mu-\mathrm{OC}_{4} \mathrm{H}_{9}\right)_{2} \mathrm{Re}(\mathrm{CO})_{3}\right\}_{3}(2,4,6 \text {-tri(pyridin-3-yl)-1,3,5-triazin })_{2}\right]$ beobachtet. Hier beträgt der Abstand zweier Ringzentroide $3.55 \AA .^{[147]}$ Im Gegensatz zu 25 beinhaltet ein $\mathrm{Cu}(\mathrm{I})$-Triazin-Komplex $\left[(\mathrm{CuCl})_{6}\left(\mathrm{P}(\mathrm{OPh})_{3}\right)_{6}(1,3,5 \text {-triazin })_{2}\right]$ eine face-to-face Anordnung, bei der die Triazinringe um $60^{\circ}$ zueinander verschoben sind, d.h. das N-Atom eines Triazinrings steht über dem C-Atom des anderen Triazinrings. ${ }^{[148]}$ Diese zweite Stapelungsvariante scheint hier elektrostatisch günstiger zu sein und dementsprechend wurden kürzere Abstände von 3.41 / $3.45 \AA$ in diesem Fall beobachtet. 


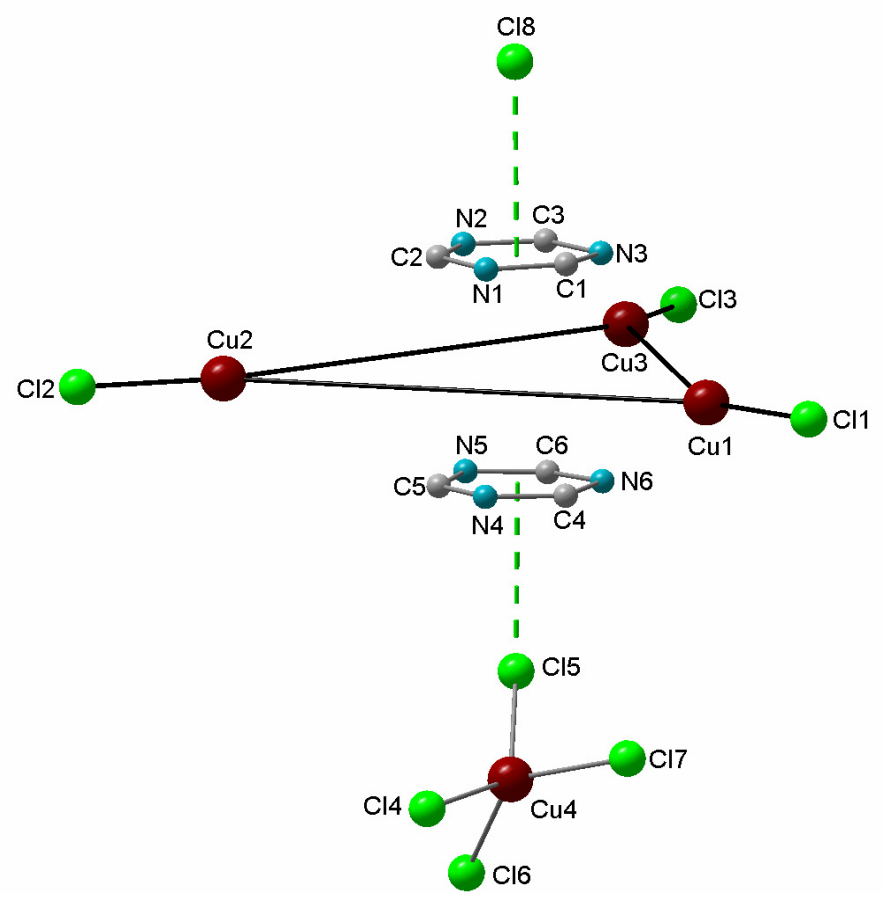

Abb. 5.3.2. Die Position der Chloridionen $\mathrm{Cl}(5)$ und $\mathrm{Cl}(8)$ des Kations von 25 weist auf die Anion- $\pi$ Wechselwirkung hin. Von $\mathbf{L}^{\mathbf{8}}$ ist jeweils nur der Triazinring dargestellt..

Außer der einzigartigen Karussellstruktur von $\left[\left(\mathrm{L}^{8}\right)_{2}(\mathrm{CuCl})_{3}\right]^{3+}$ ist die interessanteste Eigenschaft von 25 die Position der ladungsausgleichenden Chlorid- und [ $\left.\mathrm{CuCl}_{4}\right]^{2-}$-Ionen. $\mathrm{Cl}(8)$ befindet sich über einem von beiden Triazinringen (Abb. 5.3.2), der Abstand zwischen dem Zentroid vom Ring und dem Chlorid beträgt 3.17 Å. Der Winkel zwischen der $\mathrm{Cl}^{-}$ -.ZZentroidachse und der Ringebene $\left(8^{\circ}\right)$ erlaubt die Aussage, dass das Chloridion fast perfekt auf der $\mathrm{C}_{3}$-Achse über dem Triazinring lokalisiert ist.

Die beiden Werte (Abstand und Winkel) sind in hervorragender Übereinstimmung mit den Werten (3.2 $\AA$ and $90^{\circ}$ ), die durch $a b$ initio MO-Rechnungen für den Chlorid-1,3,5-TriazinKomplex (Abb. 5.3.1) erhalten wurden. ${ }^{[143]}$ Infolgedessen ist die Aussage möglich, dass in 25 ein $\mathrm{Cl}^{-} \cdots$ Triazin-Komplex mit elektrostatischer Anion- $\pi$ Wechselwirkung vorliegt. Der kürzeste Abstand von $\mathrm{Cl}(8)$ zu Nicht-Wasserstoffatomen vom benachbarten $\left[\left(\mathrm{L}^{8}\right)_{2}(\mathrm{CuCl})_{3}\right]^{3+}$ Fragment beträgt etwa $3.6 \AA$.

In der gleichen Weise ist der zweite Triazinring des $\left[\left(\mathrm{L}^{8}\right)_{2}(\mathrm{CuCl})_{3}\right]^{3+}$-Karussells mit dem zweiten Gegenion überkappt. Zwischen dem Chloratom $\mathrm{Cl}(5)$ vom $\left[\mathrm{CuCl}_{4}\right]^{2-}$-Ion und dem Zentroid des [C(4)-N(6)]-Triazinrings wurde mit $3.11 \AA$ sogar ein kleinerer Abstand beobachtet (Abb. 5.3.2). Auch hier ist das Chloratom fast ideal über dem Triazinring zentriert, der Winkel zwischen der Triazin-Ringebene und der $\mathrm{Cl} \cdots$ Zentroid-Achse beträgt $88^{\circ}$. 
Die Wechselwirkungen zwischen den Chloridionen und $\left[\left(\mathrm{L}^{8}\right)_{2}(\mathrm{CuCl})_{3} \mathrm{Cl}_{2}\right]^{+}$scheinen auch in Lösung sowie in der Gasphase vorhanden zu sein. Das stabile $\left[\left(\mathrm{L}^{8}\right)_{2}(\mathrm{CuCl})_{3}\right]^{3+}$-Ion kann als deutliches Signal im ESI-Massenspektrum von $\left[\left[\left(\mathrm{L}^{8}\right)_{2}(\mathrm{CuCl})_{3}\right]\left[\mathrm{CuCl}{ }_{4}\right] \mathrm{Cl}\right.$ in Methanol detektiert werden (Abb. 5.3.3).

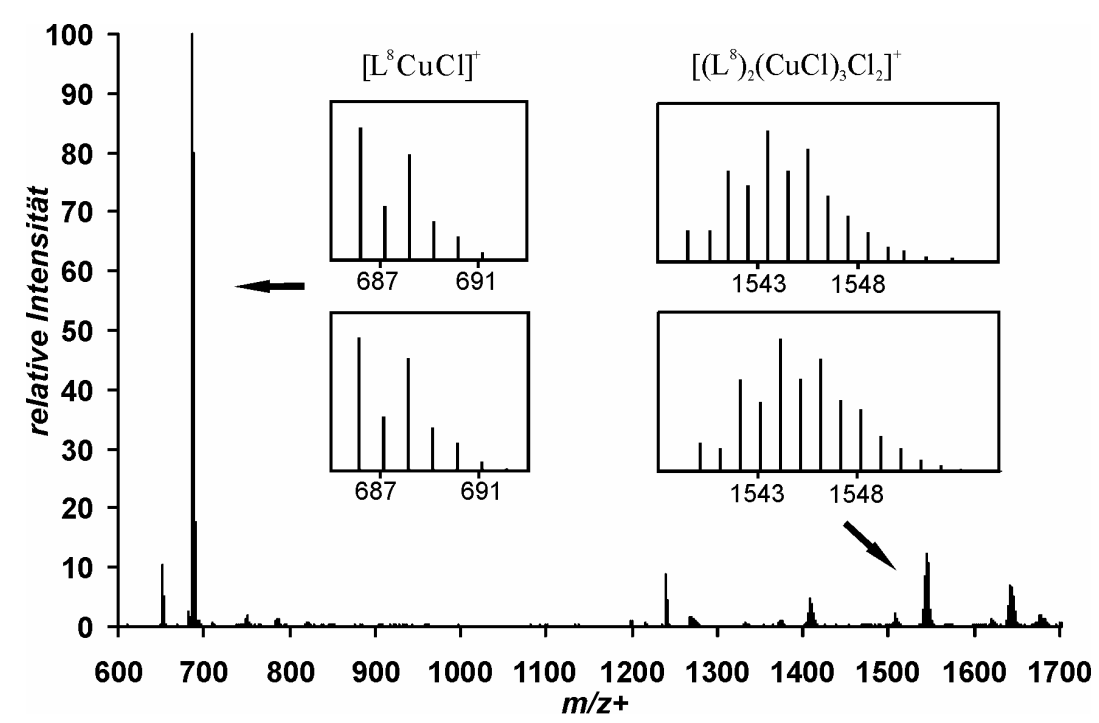

Abb. 5.3.3. ESI-Massenpektrum von 25 in Methanol. Die Insets zeigen die experimentellen (unten) and theoretischen (oben) Isotopenverteilungen der charakteristischen Fragmente.

\section{Zusammenfassung}

Der Vergleich der Ergebnisse von kürzlich berichteten theoretischen Arbeiten ${ }^{[143,144,145]}$ und den oben beschriebenen kristallographischen Daten bestätigt offensichtlich das Vorhandensein einer Anion- $\pi$ Wechselwirkung in $\left[\left(\mathrm{L}^{8}\right)_{2}(\mathrm{CuCl})_{3}\right]\left[\mathrm{CuCl}_{4}\right] \mathrm{Cl}$. Die Überprüfung auf Anwesenheit von intra- oder intermolekularen Kontakten zwischen Halogeniden und 1,3,5-Triazinringen in der CSD (Cambridge Structural Database) zeigt, dass die Abstände zwischen Halogeniden und Triazinringzentroiden kleiner als $3.4 \AA$ sind und auch in einigen Melaminsalzen von $\mathrm{Cu}_{2} \mathrm{X}_{6}{ }^{2-}$-Dimeren auftreten. ${ }^{[149]}$ Eine ähnliche Untersuchung ${ }^{[142]}$ der in der CSD vorhandenen Strukturen lieferte knapp zweitausend Fragmente, in welchen anhand struktureller Parameter nichtkovalente $\pi$-Wechselwirkungen zwischen elektronegativen Atomen wie $\mathrm{F}, \mathrm{Cl}, \mathrm{Br}, \mathrm{O}, \mathrm{S}$ sowie $\mathrm{N}$ und perfluorierten Benzolderivaten formuliert werden können. Ohne als solches zuvor erkannt zu sein, ist die Anion- $\pi$ Wechselwirkung sicherlich ein strukturbestimmendes Motiv in vielen Komplexen mit elektronenarmen aromatischen Heterocyclen und scheint allgemeine Wichtigkeit für die Festkörperstrukturen sowie den supramolekularen Aufbau zu haben; außerdem kann sie in die Strategie der Synthese 
molekularer Rezeptoren für Anionerkennung einbezogen werden. Dies bestätigt auch eine ganze Reihe experimenteller und theoretischer Arbeiten in den letzten zwei Jahren. Beispielweise konnten sowohl $\mathrm{Cl}^{-}$als auch ein großes Anion wie $\mathrm{SbF}_{6}^{-}$wegen der stabilisierenden Wirkung der Anion- $\pi$ Wechselwirkungen in geeignete Container eingebaut werden. ${ }^{[150,151]}$ Durch UV/Vis- und NMR-spektroskopische Untersuchungen sowie Berechnungen wurde gezeigt, dass elektronenarme $\pi$-Systeme als Komponente in der Designstrategie für gezielte Anionerkennung verwendet werden können. ${ }^{[152,153,154]}$ Über Anion $-\pi$ Wechselwirkungen als kontrollierendes Element in der Selbstorganisation wurde neulich ebenso berichtet. ${ }^{[155,156]}$ Unter Berücksichtigung, dass nicht nur Halogenide bzw. halogenhaltige Anionen, sondern auch andere Atome wie beispielweise das O-Atom in Nitrat ${ }^{[157]}$ oder Triflat ${ }^{[158]}$ mit den $\pi$-Systemen in Wechselwirkung treten können, sind Anion$\pi$ Wechselwirkungen sehr wahrscheinlich auch in der Natur von großer Bedeutung. 


\section{Experimenteller Teil}

\subsection{Allgemeine Arbeitstechniken und analytische Methoden}

Apparaturen. Es wurden konventionelle Glasapparaturen oder Schlenktechnik ${ }^{1)}$ verwendet. Sofern nötig, wurden die Apparaturen unter Verwendung eines Heißluftföns ausgeheizt, mehrmals im Ölpumpenvakuum evakuiert und mit Stickstoff gespült.

Absolute Lösungsmittel: Lösungsmittel wurden nach herkömmlichen Methoden getrocknet: ${ }^{[159]}$

\begin{tabular}{ll||ll} 
Aceton & Calziumoxid & n-Hexan & Natrium \\
Acetonitril & Molsieb $\AA 3$ & Ethanol & Calziumoxid \\
Dichlormethan & Phosphorpentoxid & Methanol & Magnesium \\
Diethylether & Natrium & Petrolether 40/60 & Natrium \\
Dimethylsulfoxid & Molsieb $\AA 3$ & Tetrahydrofuran & Natrium/Kalium
\end{tabular}

Reagentien: Handelsübliche Chemikalien wurden in der Qualität „Reinheit zur Synthese“ eingesetzt. Die folgende Reagentien wurden nach bekannten Vorschriften dargestellt:

N-Methyl-[2-(ethylsulfanyl)ethyl]amin ${ }^{\text {[101] }}$

Bis[2-(methylsulfanyl)ethyl]amin ${ }^{[102]}$

Bis[2-(ethylsulfanyl)ethyl]amin ${ }^{[97]}$

Bis[2-(1-methyl-ethylsulfanyl)ethyl]amin ${ }^{[103]}$

5-Bis(chlormethyl)-1-(tetrahydropyran-2-yl)-1H-pyrazol ${ }^{[100]}$

Natriumcarbonat: zur Entfernung von Feuchtigkeitsspuren wurde Natriumcarbonat bei $100{ }^{\circ} \mathrm{C}$ und 0.002 mbar getrocknet.

Elementaranalysen: Die C,H,N-Analysen wurden vom Analytischen Labor des Anorganisch-Chemischen Instituts der Universität Göttingen an einem CHN-O-Rapid Gerät von HERAEUS durchgeführt.

Infrarotspektren: Die IR-Spektren wurden als KBr-Preßling an einem Digilab Excalibur IR-Spektrometer gemessen. Die Intensitäten und Formen der Banden sind wie folgt abgekürzt:

${ }^{1}$ ) Bei einigen Stufen der Ligandsynthese sowie bei der Synthese des hygroskopischen Komplexes 5. 
$(\mathrm{v}) \mathrm{s}=(\mathrm{sehr})$ stark $\quad \mathrm{m}=$ mittel $\quad \mathrm{w}=$ schwach $\quad$ br $=$ breit $\quad \mathrm{sh}=$ Schulter

Massenspektren: Die Massenspektren wurden an folgenden Geräten aufgenommen: Finnigan MAT 8200 (EI-MS), Finnigan MAT 95 (FAB-MS, 3-Nitrobenzylalkohol-Matrix) und Finnigan MAT LCQ (ESI-MS). Isotopensimulationen wurden mit dem Programm Molecular Weight Calculator durchgeführt.

Kernresonanzspektren: Die ${ }^{1} \mathrm{H}$ - und ${ }^{13} \mathrm{C}-\mathrm{NMR}-$ Spektren wurden an einem Bruker Avance 500 Spektrometer bei $500.13 \mathrm{MHz}\left({ }^{1} \mathrm{H}\right)$ und $125.77 \mathrm{MHz}\left({ }^{13} \mathrm{C}\right)$ in deuteriertem Chloroform bei einer Temperatur von 300 K gemessen. Zur Standardisierung der chemischen Verschiebung $(\delta$ in $\mathrm{ppm})$ wurden die Werte des $\mathrm{CDCl}_{3}\left(\delta_{H}=7.27, \delta_{C}=77.0\right)$ als interner Standard verwendet. Die Signalmultiplizitäten werden wie folgt abgekürzt:

$\mathrm{s}=$ Singulett $\quad \mathrm{d}=$ Dublett $\quad \mathrm{t}=$ Triplett $\quad \mathrm{q}=$ Quartett $\quad \mathrm{m}=$ Multiplett sept $=$ Septett $\quad$ br $=$ breit

UV/Vis-Spektren: Die UV/Vis-Spektren wurden an einem Varian Cary-5000 Spektrometer aufgenommen.

EPR-Spektren: Die EPR-Spektren wurden an einem Bruker ELEXSYS E500 Spektrometer vermessen.

Röntgenstrukturanalysen: Die Bestimmung der Elementarzellen und die Sammlung der Messdaten erfolgte an einem STOE/IPDS II - Gerät mit graphitmonochromatisierter Mo-K $\alpha_{\alpha^{-}}$ Strahlung ( $\lambda=0.711 \AA$ ). Die Messwerte wurden bezüglich Lorentz- und Polarisationseffekten korrigiert. Zusätzlich erfolgten Absorptionskorrekturen. Lösung und Verfeinerung wurden mit den Programmen SHELXS-97 ${ }^{[160]}$ und SHELXL-97 ${ }^{[161]}$ durchgeführt.

\subsection{Synthese der Pyrazolatliganden $\mathrm{HL}^{1-7}$}

Die Herstellung der Liganden $\mathbf{H L}^{\mathbf{1 - 3}[24 a, 97]}$ und $\mathbf{L}^{\mathbf{8}[125]}$ erfolgte nach bekannten Verfahren.

Allgemeine Syntheseroute der Liganden $\mathbf{H L}^{1-3}$ : Die Reaktionsmischung von vorgetrocknetem $\mathrm{Na}_{2} \mathrm{CO}_{3}$ (42 g, $\left.400 \mathrm{mmol}\right)$, trockenem Acetonitril (400 ml), 3,5Bis(chlormethyl)-1-(tetrahydropyran-2-yl)-1H-pyrazol (10 g, $40 \mathrm{mmol}) \quad$ und dem 
entsprechenden Amin (90 mmol) wird unter $\mathrm{N}_{2}$-Schutzgasatmosphäre 24 h unter Rückfluss erhitzt, anschließend filtriert und alle flüchtigen Komponenten werden im Vakuum entfernt. Der Rückstand wird in $50 \mathrm{ml}$ Ethanol aufgenommen, mit ethanolischer Salzsäure versetzt und über Nacht gerührt. Die Zugabe von Diethylether führt zum Ausfallen des entsprechenden Hydrochlorides des Liganden. Man filtriert das ausgefallene Salz ab und gibt wässrige $\mathrm{NaOH}-\mathrm{Lösung}(4 \mathrm{M})$ zu. Die wässrige Phase wird mehrmals mit Dichlormethan extrahiert. Die vereinigten organischen Phasen werden mit $\mathrm{MgSO}_{4}$ getrocknet und filtriert. Nach Entfernen des Lösungsmittels im Vakuum bleibt das jeweilige Produkt als gelbes Öl zurück.

\section{Analytische Daten:}

\section{3,5-Bis[N-methyl-N-(2-ethylsulfanyl)ethyl]aminomethyl-1H-pyrazol $\left(\mathrm{HL}^{4}\right)$}

Ausbeute

Summenformel

Molmasse, g/mol

${ }^{1} \mathrm{H}-\mathrm{NMR}$

${ }^{13} \mathrm{C}-\mathrm{NMR}$

MS (EI+), $m / z(\%)$

Elementaranalyse, \%
7.9 g, $60 \%$

$\mathrm{C}_{15} \mathrm{H}_{30} \mathrm{~N}_{4} \mathrm{~S}_{2}$

330.6

$\delta=1.12\left(\mathrm{t}, J(\mathrm{HH})=7.4 \mathrm{~Hz}, \mathrm{CH}_{2} \mathrm{CH}_{3}, 3 \mathrm{H}\right), 2.19\left(\mathrm{~s}, \mathrm{NCH}_{3}, 3 \mathrm{H}\right), 2.40$

$\left(\mathrm{q}, J(\mathrm{HH})=7.4 \mathrm{~Hz}, \mathrm{CH}_{2} \mathrm{CH}_{3}, 2 \mathrm{H}\right), 2.52\left(\mathrm{~s}, \mathrm{NCH}_{2} \mathrm{CH}_{2} \mathrm{~S}, 4 \mathrm{H}\right), 3.52$

(s, $\left.\mathrm{pzCH}_{2}, 4 \mathrm{H}\right), 6.01\left(\mathrm{~s}, \mathrm{pz}-\mathrm{H}^{4}, 1 \mathrm{H}\right)$

$\delta=14.5\left(\mathrm{CH}_{2} \mathrm{CH}_{3}\right), 25.7\left(\mathrm{NCH}_{3}\right), 28.8\left(\mathrm{SCH}_{2}\right), 41.9\left(\mathrm{SCH}_{2}\right), 53.4$

$\left(\mathrm{pzCH}_{2}\right), 56.2\left(\mathrm{CH}_{2} \mathrm{CH}_{3}\right), 103.5\left(\mathrm{pz}-\mathrm{C}^{4}\right), 145.0\left(\mathrm{pz}-\mathrm{C}^{3,5}\right)$

330 (2), [M] ; 329 (4), [M-H] ; 269 (10), [M-SC $\left.{ }_{2} \mathrm{H}_{5}\right]^{+} ; 255$ (100),

$\left[\mathrm{M}-\mathrm{CH}_{2} \mathrm{SC}_{2} \mathrm{H}_{5}\right]^{+} ; 212$ (60), [M- $\left.\mathrm{CH}_{3} \mathrm{~N}\left(\mathrm{CH}_{2}\right)_{2} \mathrm{SC}_{2} \mathrm{H}_{5}\right]^{+}$; 89 (45),

$\left[\left(\mathrm{CH}_{2}\right)_{2} \mathrm{SC}_{2} \mathrm{H}_{5}\right]^{+}$

ber.: C 54.50, H 9.15, N 16.95; gef.: C 54.19, H 8.97, N 17.64

\section{3,5-Bis $\left\{\mathrm{N}, \mathrm{N}-\mathrm{bis}\left[2-\left(\right.\right.\right.$ methylsulfanyl)ethyl]aminomethyl\}-1H-pyrazol $\left(\mathrm{HL}^{5}\right)$}

Ausbeute

Summenformel

Molmasse, g/mol

${ }^{1} \mathrm{H}-\mathrm{NMR}$

$$
12.1 \mathrm{~g}, 72 \%
$$

$\mathrm{C}_{17} \mathrm{H}_{34} \mathrm{~N}_{4} \mathrm{~S}_{4}$

422.7

$\delta=1.85\left(\mathrm{~s}, \mathrm{SCH}_{3}, 12 \mathrm{H}\right), 2.39\left(\mathrm{t}, J(\mathrm{HH})=6.5 \mathrm{~Hz}, \mathrm{SCH}_{2}, 8 \mathrm{H}\right), 2.53$

$\left(\mathrm{t}, \mathrm{NCH}_{2}, J(\mathrm{HH})=6.5 \mathrm{~Hz}, 8 \mathrm{H}\right), 3.52\left(\mathrm{~s}, \mathrm{pzCH}_{2}, 4 \mathrm{H}\right), 5.89\left(\mathrm{~s}, \mathrm{pz}-\mathrm{H}^{4}\right.$, 
1H), 10.65 (br, NH, 1H)

${ }^{13} \mathrm{C}-\mathrm{NMR}$

$\delta=15.1\left(\mathrm{SCH}_{3}\right), 31.4\left(\mathrm{SCH}_{2}\right), 49.7\left(\mathrm{NCH}_{2}\right), 52.6\left(\mathrm{pzCH}_{2}\right), 103.5$ $\left(\mathrm{pz}-C^{4}\right), 149.0\left(\mathrm{br}, \mathrm{pz}-C^{3,5}\right)$

MS (EI+), m/z (\%) 361 (100), $\left[\mathrm{M}-\mathrm{CH}_{2} \mathrm{SCH}_{3}\right]^{+} ; 258$ (25), [M-N(C $\left.\left.\mathrm{C}_{2} \mathrm{H}_{4} \mathrm{SCH}_{3}\right)_{2}\right]^{+} ; 75$ (60), $\left[\mathrm{CH}_{3}\left(\mathrm{CH}_{2}\right)_{2}\right]^{+}$

Elementaranalyse, \% $\quad$ ber.: C 48.30, H 8.11, N 13.25; gef.: C 48.48, H 8.07, N 13.62

\title{
3,5-Bis $\left\{\mathrm{N}, \mathrm{N}-\mathrm{bis}\left[2-(\right.\right.$ ethylsulfanyl)ethyl]aminomethyl $\}-1 \mathrm{H}$-pyrazol $\left(\mathrm{HL}^{6}\right)$
}

\author{
Ausbeute \\ $14.5 \mathrm{~g}, 76 \%$ \\ Summenformel \\ $\mathrm{C}_{21} \mathrm{H}_{42} \mathrm{~N}_{4} \mathrm{~S}_{4}$ \\ Molmasse, g/mol \\ 478.9 \\ ${ }^{1} \mathrm{H}-\mathrm{NMR}$ \\ $\delta=1.10\left(\mathrm{t}, J(\mathrm{HH})=7.3 \mathrm{~Hz}, \mathrm{CH}_{2} \mathrm{CH}_{3}, 12 \mathrm{H}\right), 2.35\left(\mathrm{q},{ }^{3} J(\mathrm{HH})=7.3\right.$ \\ $\mathrm{Hz}, \mathrm{CH}_{2} \mathrm{CH}_{3}, 8 \mathrm{H}$ ), 2.50-2.75 (m, $\mathrm{NCH}_{2} \mathrm{CH}_{2} \mathrm{~S}, 16 \mathrm{H}$ ), 3.56 (s, $\mathrm{pzCH}_{2}$, \\ 4H), $5.92\left(\mathrm{~s}, \mathrm{pz}-\mathrm{H}^{4}, 1 \mathrm{H}\right)$ \\ ${ }^{13} \mathrm{C}-\mathrm{NMR}$ \\ $\delta=14.7\left(\mathrm{CH}_{2} \mathrm{CH}_{3}\right), 26.0\left(\mathrm{SCH}_{2}\right), 29.4\left(\mathrm{NCH}_{2}\right), 53.65\left(\mathrm{pzCH}_{2}\right)$, \\ $103.3\left(\mathrm{pz}-C^{4}\right), 145.5\left(\mathrm{pz}-C^{3,5}\right)$ \\ MS (EI+), $m / z(\%)$ \\ 403 (100), [M- $\left.\mathrm{CH}_{2} \mathrm{SC}_{2} \mathrm{H}_{5}\right]^{+} ; 286$ (25), [M-N $\left.\left(\left(\mathrm{CH}_{2}\right)_{2} \mathrm{SC}_{2} \mathrm{H}_{5}\right)_{2}\right]^{+} ; 89$ \\ (70), $\left[\mathrm{C}_{2} \mathrm{H}_{5} \mathrm{~S}\left(\mathrm{CH}_{2}\right)_{2}\right]^{+}$
}

Elementaranalyse, \% ～ber.: C 52.67, H 8.84, N 11.70; gef.: C 52.95, H 8.72, N 11.97

\section{3,5-Bis $\left\{\mathrm{N}, \mathrm{N}-\mathrm{bis}\left[2-(1-\right.\right.$ methylethylsulfanyl)ethyl]aminomethyl $\}-1 \mathrm{H}$-pyrazol $\left(\mathrm{HL}^{7}\right)$}

Ausbeute

Summenformel

Molmasse, g/mol

${ }^{1} \mathrm{H}-\mathrm{NMR}$

${ }^{13} \mathrm{C}-\mathrm{NMR}$
14.9 g, $70 \%$

$\mathrm{C}_{25} \mathrm{H}_{50} \mathrm{~N}_{4} \mathrm{~S}_{4}$

535.0

$\delta=1.20\left(\mathrm{~d}, J(\mathrm{HH})=6.7 \mathrm{~Hz}, \mathrm{CHCH}_{3}, 24 \mathrm{H}\right), 2.51-2.65(\mathrm{~m}$, $\mathrm{NCH}_{2} \mathrm{CH}_{2} \mathrm{~S}, 16 \mathrm{H}$ ), 2.80 (sept, $J(\mathrm{HH})=6.7 \mathrm{~Hz}, \mathrm{CHCH}_{3}, 4 \mathrm{H}$ ), 3.62 (s, pz- $\left.\mathrm{CH}_{2}, 4 \mathrm{H}\right), 5.95$ (s, pz- $\left.\mathrm{H}^{4}, 1 \mathrm{H}\right)$

$\delta=23.3\left(\mathrm{CHCH}_{3}\right), 28.27\left(\mathrm{SCH}_{2}\right), 34.7\left(\mathrm{NCH}_{2}\right), 50.2\left(\mathrm{CHCH}_{3}\right), 53.7$ $(\mathrm{pzCH}), 103.3\left(\mathrm{pz}-\mathrm{C}^{4}\right), 145.7\left(\mathrm{br}, \mathrm{pz}-\mathrm{C}^{3,5}\right)$ 


\section{MS (EI+), $m / z(\%) \quad 403$ (100), $\left[\mathrm{M}-\mathrm{CH}_{2} \mathrm{SC}_{2} \mathrm{H}_{5}\right]^{+} ; 286(25),\left[\mathrm{M}-\mathrm{N}\left(\left(\mathrm{CH}_{2}\right)_{2} \mathrm{SC}_{2} \mathrm{H}_{5}\right)_{2}\right]^{+} ; 89$ (70) $\left[\mathrm{C}_{2} \mathrm{H}_{5} \mathrm{~S}\left(\mathrm{CH}_{2}\right)_{2}\right]^{+}$}

Elementaranalyse, \% ～ber.: C 56.13, H 9.42, N 10.47; gef.: C 55.96, H 9.38, N 10.86

\subsection{Synthese der Metallkomplexe}

Vorsicht! Azid- und Perchloratkomlexe der Übergangsmetalle sind potentiell explosionsgefährlich und sollten mit entsprechenden Sicherheitsmaßnahmen in kleinen Mengen gehandhabt werden.

\subsubsection{Vorbemerkungen}

Die Metallkomplexe wurden durchwegs nur in kristalliner Form verwendet, weil die kristallinen Substanzen meist einen höheren Reinheitsgrad als amorphe Pulver aufweisen. Dies ist für die magnetischen Untersuchungen von großer Bedeutung. Um geeignete Kristalle $\mathrm{zu}$ erhalten, wurden unterschiedliche Kristallisationsmethoden aus den entsprechenden Lösungen bei Raumtemperatur eingesetzt.

Methode A: Unmittelbar nach Beendigung der Reaktion wurden die filtrierten Lösungen zur Kristallisation gestellt.

Methode B: Nach diesem Verfahren wurden die Kristallisationsversuche durch Überschichtung (bzw. Unterschichtung) einer Lösung der zu kristallisierenden Substanz mit einem 3- bis 4-fachen Überschuß an Fällungsmittel durchgeführt. Die meisten Metallkomplexe konnten nach diesem Verfahren in kristalliner Form erhalten werden.

Methode C (Dreischichtverfahren): Dieses Verfahren ist eine Komplexbildungsmethode durch Diffusion, bei der unmittelbar während der Reaktion Kristalle erhalten werden können. Es werden drei Schichten aufgebaut, wobei die mittlere Schicht ein Gemisch aus den reinen Lösungsmitteln ist. Die untere und obere Schicht enthalten jeweils eine die beteiligten Reaktionskomponenten. 
Isolierung der Metallkomplexe: Nach Abtrennung der Komplexe aus der Mutterlauge wurden diese kurzzeitig an der Luft getrocknet. Einige Verbindungen enthalten im Gitter eingebaute Lösungsmittelmoleküle, die bei längerer Lagerung abgegeben werden können. Dies hat zur Folge, dass die Kristalle verwittern. Aus diesem Grund wurden die elementaranalytischen, spektroskopischen sowie magnetischen Untersuchungen, soweit möglich, unmittelbar nach der Isolierung der Komplexe aus der Mutterlauge durchgeführt. Bei einigen Verbindungen läuft die Verwitterung sehr schnell ab, so dass die durch Elementaranalyse bestimmte Zusammensetzung von der per Röntgenstrukturanalyse ermittelten abweicht. Da für die Auswertung magnetischer Messungen die reale Zusammensetzung wichtig ist, wird nachfolgend die auf der Elementaranalyse basierende Molmasse angegeben.

\subsubsection{Synthese der zweikernigen Nickel(II)-Komplexe}

\section{$\left[\mathrm{L}^{1} \mathrm{Ni}_{2}\left(\mathrm{~N}_{3}\right)\left(\mathrm{NO}_{3}\right)_{2}\right](\mathbf{1})$}

Zur einer Lösung des Liganden $\mathbf{H L}^{1}$ (324 mg, $1.0 \mathrm{mmol}$ ) in $25 \mathrm{ml}$ Methanol werden nacheinander 1 Äquivalent $\mathrm{KO}^{t} \mathrm{Bu}(112 \mathrm{mg}, 1.0 \mathrm{mmol})$ und 2 Äquivalente $\mathrm{Ni}\left(\mathrm{NO}_{3}\right)_{2} \cdot 6 \mathrm{H}_{2} \mathrm{O}$ (581 mg, $2.0 \mathrm{mmol}$ ) zugegeben. Nach einer Rührdauer von $1 \mathrm{~h}$ wird die Reaktionsmischung filtriert, dem Filtrat 1 Äquivalent $\mathrm{NaN}_{3}(65 \mathrm{mg}, 1.0 \mathrm{mmol}$ ) zugeführt und über Nacht weiter gerührt. Nach Überschichtung der Komplexlösung mit Diethylether bilden sich durch Diffusion grüne Kristalle, welche nach dem Isolieren langsam verwittern.

Ausbeute $\quad 226 \mathrm{mg}, 33 \%$

Summenformel $\quad \mathrm{C}_{17} \mathrm{H}_{35} \mathrm{~N}_{11} \mathrm{Ni}_{2} \mathrm{O}_{6}$

Molmasse, g/mol $\quad 606.9$

IR $(\mathrm{KBr}), \mathrm{cm}^{-1} \quad 2062(\mathrm{~s}),\left[v\left(\mathrm{~N}_{3}{ }^{-}\right)\right] ; 1384(\mathrm{~s})\left[\mathrm{v}\left(\mathrm{NO}_{3}{ }^{-}\right)\right]$

Elementaranalyse, \% ～ber.: C 33.64, H 5.81, N 25.37; gef.: C 33.00, H 5.91, N 25.71

$\left[\mathbf{L}^{2} \mathbf{N i}_{\mathbf{2}}\left(\mathbf{N}_{3}\right)\left(\mathbf{N O}_{3}\right)_{2}\right]^{[105]}$ (2) (diese von B. Bauer-Siebenlist synthetisierte Verbindung wurde für den Vergleich magnetischer Eigenschaften in Rahmen dieser Arbeit untersucht).

\section{$\left[\mathrm{L}^{3} \mathrm{Ni}_{2}\left(\mathrm{~N}_{3}\right)(\mathrm{MeCN})_{4}\right]\left(\mathrm{ClO}_{4}\right)_{2}(3)$}

$\mathrm{Zu}$ einer Lösung des Liganden $\mathbf{H L}^{3}$ (89 mg, $0.3 \mathrm{mmol}$ ) in $25 \mathrm{~mL}$ Methanol werden 1 Äquivalent $\mathrm{KO}^{t} \mathrm{Bu}(34 \mathrm{mg}, 0.3 \mathrm{mmol})$ und 2 Äquivalente $\mathrm{Ni}\left(\mathrm{ClO}_{4}\right)_{2} \cdot 6 \mathrm{H}_{2} \mathrm{O}(219 \mathrm{~g}, 0.6 \mathrm{mmol})$ 
zugegeben. Nach 1 Stunde Rühren filtriert man Kaliumperchlorat ab und zieht das Filtrat im Vakuum trocken. Die zurückbleibende grüne Substanz wird in $15 \mathrm{ml}$ Aceton aufgenommen und mit 1 Äquivalent $\mathrm{NaN}_{3}$ (20 mg, $0.3 \mathrm{mmol}$ ) versetzt. Die Reaktionsmischung wird über Nacht weiter gerührt und anschließend filtriert. Das im Vakuum trocken gezogene Filtrat wird in $15 \mathrm{ml}$ Acetonitril aufgenommen und mit Diethylether überschichtet. Dies führt zur Bildung blauer Kristalle.
Ausbeute
$150 \mathrm{mg}, 61 \%$
Summenformel
$\mathrm{C}_{23} \mathrm{H}_{43} \mathrm{Cl}_{2} \mathrm{~N}_{13} \mathrm{Ni}_{2} \mathrm{O}_{8}$
Molmasse, g/mol
818.02
IR $(\mathrm{KBr}), \mathrm{cm}^{-1}$
$2313(\mathrm{~m}), 2282(\mathrm{~m})\left[\mathrm{v}(\mathrm{C} \equiv \mathrm{N})_{\text {Acetonitril }] ;} 2062(\mathrm{vs})\left[\mathrm{v}\left(\mathrm{N}_{3}{ }^{-}\right)\right] ; 1469(\mathrm{w})\right.$; $1419(\mathrm{w}) ; 1091(\mathrm{vs})\left[\mathrm{v}\left(\mathrm{ClO}_{4}{ }^{-}\right)\right]$

Elementaranalyse, \% ～ber.: C 33.77, H 5.30, N 22.26; gef.: C 33.57, H 5.41, N 21.68

\section{$\left[\mathrm{L}^{4} \mathrm{Ni}_{2}\left(\mathrm{~N}_{3}\right)\left(\mathrm{NO}_{3}\right)_{2}\right]$ (4)}

Zur einer Lösung des Liganden $\mathbf{H L}^{4}$ (341 mg, $1.03 \mathrm{mmol}$ ) in $40 \mathrm{ml}$ Methanol werden nacheinander 1 Äquivalent $\mathrm{KO}^{t} \mathrm{Bu}(115 \mathrm{mg}, 1.0 \mathrm{mmol})$ und 2 Äquivalente $\mathrm{Ni}\left(\mathrm{NO}_{3}\right)_{2} \cdot 6 \mathrm{H}_{2} \mathrm{O}$ (598 mg, 2.06 mmol) zugegeben. Nach einstündigem Rühren der Reaktionsmischung wird filtriert, dem Filtrat 1 Äquivalent $\mathrm{NaN}_{3}$ (67 mg, $1.03 \mathrm{mmol}$ ) zugesetzt, über Nacht gerührt und dann mit Diethylether überschichtet. Es scheiden sich grüne Kristalle ab.

Ausbeute $374 \mathrm{mg}, 59 \%$

Summenformel

$$
\mathrm{C}_{15} \mathrm{H}_{29} \mathrm{~N}_{9} \mathrm{Ni}_{2} \mathrm{O}_{6} \mathrm{~S}_{2}
$$

Molmasse, g/mol

613.0

IR $(\mathrm{KBr}), \mathrm{cm}^{-1}$ 2047 (s), $\left[v\left(\mathrm{~N}_{3}{ }^{-}\right)\right] ; 1498(\mathrm{~m}), 1490(\mathrm{~m}), 1384(\mathrm{~s})\left[\mathrm{v}\left(\mathrm{NO}_{3}{ }^{-}\right)\right]$

Elementaranalyse, \% ber.: C 29.39, H 4.77, N 20.57; gef.: C 28.47, H 4.66, N 20.17

\section{$\left[\mathrm{L}^{6} \mathrm{Ni}_{2}\left(\mathrm{~N}_{3}\right)\left(\mathrm{C}_{3} \mathrm{H}_{6} \mathrm{O}\right)_{2}\right]\left(\mathrm{ClO}_{4}\right)_{2} \cdot \mathrm{C}_{3} \mathrm{H}_{6} \mathrm{O}(5)$}

$\mathrm{Zu}$ einer Lösung des Liganden $\mathbf{H L}^{6}$ (240 mg, $0.5 \mathrm{mmol}$ ) in $25 \mathrm{~mL}$ Methanol werden 1 Äquivalent $\mathrm{KO}^{t} \mathrm{Bu}(67 \mathrm{mg}, 0.5 \mathrm{mmol})$ und 2 Äquivalente $\mathrm{Ni}\left(\mathrm{ClO}_{4}\right)_{2} \cdot 6 \mathrm{H}_{2} \mathrm{O}(365 \mathrm{mg}, 1.0$ mmol) zugegeben. $1 \mathrm{~h}$ wird die Reaktionsmischung gerührt, dann ausgefallenes Kaliumperchlorat abfiltriert und das Filtrat trocken gezogen. Die erhaltene grüne Substanz wird in $25 \mathrm{ml}$ Aceton aufgenommen und mit 0.92 Äquivalente $\mathrm{NaN}_{3}$ (30 mg, $0.46 \mathrm{mmol}$ ) versetzt. Dann rührt man über Nacht, filtriert und überschichtet anschließend mit Petrolether. 
Die dabei erhaltenen grünen Kristalle (29 mg, 7 \%) sind hygroskopisch und geben partiell Lösungsmittel ab.

Alternativ kann 5 ausgehend von 18 in größerer Menge dargestellt werden. Dafür löst man 225 mg 18 in 25 ml Aceton und überschichtet mit Petrolether. Durch die Diffusion entstehen grüne Kristalle (216 mg, 82 \%).

Summenformel $\quad \mathrm{C}_{30} \mathrm{H}_{59} \mathrm{Cl}_{2} \mathrm{~N}_{7} \mathrm{Ni}_{2} \mathrm{O}_{11} \mathrm{~S}_{4}$

Molmasse, g/mol $\quad 1010.4$

IR (KBr), $\mathrm{cm}^{-1} \quad 2967(\mathrm{w}) ; 2930(\mathrm{w}) ; 2872(\mathrm{w}) ; 2097(\mathrm{vs}), 2061$ (s) [v( $\left.\left.2 \mathrm{~N}_{3}{ }^{-}\right)\right] ; 1702$

(w) $\left[v(\mathrm{C}=\mathrm{O})_{\text {Aceton }}\right] ; 1459(\mathrm{w}) ; 1425(\mathrm{w}) ; 1098(\mathrm{vs})\left[\mathrm{v}\left(\mathrm{ClO}_{4}{ }^{-}\right)\right]$

MS (FAB+), m/z (\%) $793(8),\left[\mathrm{L}^{6} \mathrm{Ni}_{2}\left(\mathrm{~N}_{3}\right)\left(\mathrm{C}_{3} \mathrm{H}_{6} \mathrm{O}\right)\left(\mathrm{ClO}_{4}\right)\right]^{+} ; 736(100),\left[\mathrm{L}^{6} \mathrm{Ni}_{2}\left(\mathrm{~N}_{3}\right)\left(\mathrm{ClO}_{4}\right)\right]^{+}$

Elementaranalyse, \% ～ber.: C 35.66, H 5.89, N 9.70; gef.: C 35.60, H 5.75, N 9.35

\section{$\left[\mathbf{L}^{6} \mathrm{Ni}_{2}\left(\mathrm{~N}_{3}\right)\left(\mathrm{C}_{3} \mathrm{H}_{6} \mathrm{O}\right)_{2}\right]\left(\mathrm{BPh}_{4}\right)_{2} \cdot \mathrm{C}_{3} \mathrm{H}_{6} \mathrm{O}(\mathbf{6})$}

$\mathrm{Zu}$ einer Lösung des Liganden $\mathbf{H L}^{6}$ (240 mg, $0.5 \mathrm{mmol}$ ) in $25 \mathrm{~mL}$ Methanol werden 1 Äquivalent $\mathrm{KO}^{t} \mathrm{Bu}(56 \mathrm{mg}, 0.5 \mathrm{mmol})$ und 2 Äquivalente $\mathrm{Ni}\left(\mathrm{ClO}_{4}\right)_{2} \cdot 6 \mathrm{H}_{2} \mathrm{O}(365 \mathrm{mg}, 1.0$ mmol) zugegeben. Nach einstündigem Rühren wird Kaliumperchlorat abfiltriert, dem Filtrat 1 Äquivalent $\mathrm{NaN}_{3}$ (33 mg, $0.5 \mathrm{mmol}$ ) zugefügt, nochmals eine Stunde gerührt und dann zu der grünen Lösung 2 Äquivalente $\mathrm{NaBPh}_{4}$ (342 mg, 1.0 mmol) gegeben. Nach weiterem einstündigem Rühren wird der hellgrüne Komplex abfiltriert, in 25 ml Aceton gelöst und mit Petrolether überschichtet. Dadurch werden grüne Kristalle erhalten.

Ausbeute

Summenformel

Molmasse, g/mol

IR $(\mathrm{KBr}), \mathrm{cm}^{-1}$

MS (FAB+), $m / z(\%)$

Elementaranalyse, \%
$260 \mathrm{mg}, 36 \%$

$\mathrm{C}_{78} \mathrm{H}_{99} \mathrm{~B}_{2} \mathrm{~N}_{7} \mathrm{Ni}_{2} \mathrm{O}_{3} \mathrm{~S}_{4}$

1449.9

3053 (m); 2980 (w); 2930 (w); 2093 (w), 2056 (vs) [v( $\left.\left.\mathrm{N}_{3}{ }^{-}\right)\right] ; 1713$

$(\mathrm{w})\left[v(\mathrm{C}=\mathrm{O})_{\text {Aceton }}\right] ; 1456(\mathrm{w}) ; 1425(\mathrm{w})$

954 (8), $\left[\mathrm{L}^{6} \mathrm{Ni}_{2}\left(\mathrm{~N}_{3}\right)\left(\mathrm{BPh}_{4}\right)\right]^{+} ; 637(100)\left[\mathrm{L}^{6} \mathrm{Ni}_{2}\left(\mathrm{~N}_{3}\right)\right]^{+}$

ber.: C 64.61, H 6.88, N 6.76; gef.: C 63.22, H 6.84, N 6.92 


\section{$\left[\mathrm{L}^{6} \mathrm{Ni}_{2}\left(\mathrm{~N}_{3}\right)_{3}\right] \cdot 0.5 \mathrm{CH}_{3} \mathrm{OH}(7)$}

7 (119 mg, $0.136 \mathrm{mmol}$ ) wird in $30 \mathrm{ml}$ Methanol aufgenommen, nach einstündigem Rühren abfiltriert und mit Diethylether überschichtet. Das langsame Eindiffundieren von Diethylether in eine Lösung des Komplexes liefert grüne Kristalle.

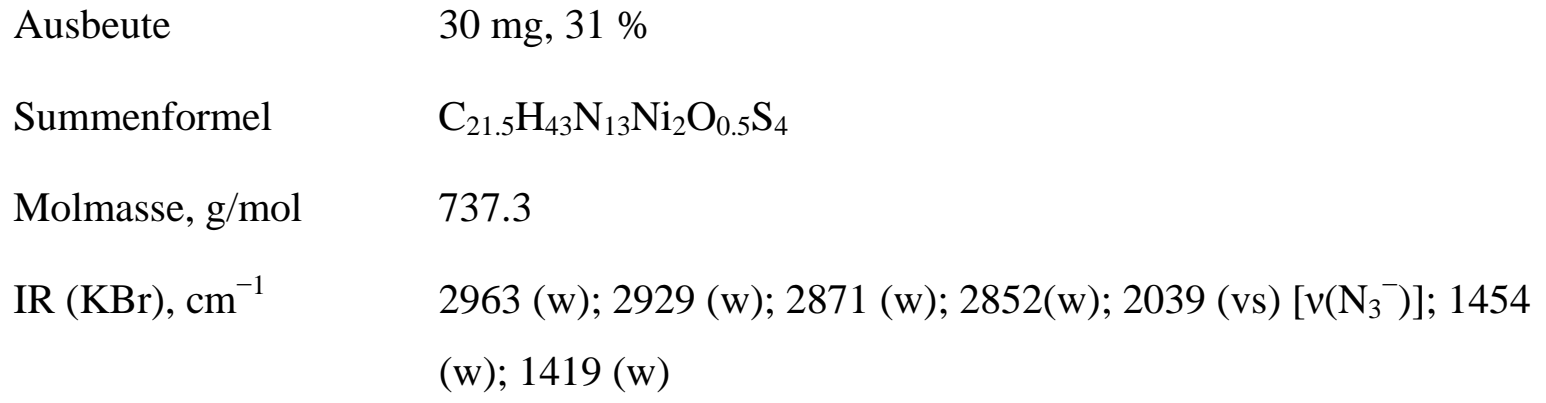

\section{$\left[\mathrm{L}^{6} \mathrm{Ni}_{2}\left(\mathrm{~N}_{3}\right)_{3}\right] \cdot 2 \mathrm{C}_{2} \mathrm{H}_{6} \mathrm{SO}(8)$}

Zu einer Lösung des Liganden $\mathbf{H L}^{6}$ (1.024 g, $2.14 \mathrm{mmol}$ ) in $125 \mathrm{~mL}$ Methanol werden 1 Äquivalent $\mathrm{KO}^{t} \mathrm{Bu}(307 \mathrm{mg}, 2.74 \mathrm{mmol})$ und 2 Äquivalente $\mathrm{Ni}\left(\mathrm{ClO}_{4}\right)_{2} \cdot 6 \mathrm{H}_{2} \mathrm{O}(1.565 \mathrm{~g}, 4.28$ mmol) zugegeben. Nach 1 h Rühren filtriert man das Kaliumperchlorat ab, fügt zu der grünen Lösung 2 Äquivalente $\mathrm{NaN}_{3}$ (278 mg, $4.28 \mathrm{mmol}$ ) hinzu und lässt weitere 2 h rühren. Der entstandene grüne Niederschlag wird abfiltriert und erst mit Aceton und danach mit Dichlormethan so lange gewaschen, bis diese Lösungsmittel farblos ablaufen. Der Rückstand wird in $10 \mathrm{~mL}$ DMSO aufgenommen und zur Kristallisation gestellt. Über Nacht entstehen grüne Kristalle.
Ausbeute $\quad 148 \mathrm{mg}, 8 \%$
Summenformel $\quad \mathrm{C}_{25} \mathrm{H}_{53} \mathrm{~N}_{13} \mathrm{Ni}_{2} \mathrm{O}_{2} \mathrm{~S}_{6}$
Molmasse, g/mol $\quad 877.6$
IR (KBr), $\mathrm{cm}^{-1} \quad 2966(\mathrm{w}) ; 2930(\mathrm{w}) ; 2870(\mathrm{w}) ; 2844(\mathrm{w}) ; 2048(\mathrm{vs})\left[\mathrm{v}\left(\mathrm{N}_{3}{ }^{-}\right)\right] ; 1456$
(w); $1418(\mathrm{w})$

Elementaranalyse, \% ber.: C 34.23, H 6.09, N 20.75, S 21.92; gef.: C 34.26, H 6.11, N 20.77, S 22.25

\section{$\left[\mathbf{L}^{6} \mathrm{Ni}_{2}\left(\mathbf{N}_{3}\right)_{3}\right](\mathbf{9})$}

Zu einer Lösung des Liganden $\mathbf{H L}^{6}$ (512 mg, $1.07 \mathrm{mmol}$ ) in $65 \mathrm{~mL}$ Methanol wird 1 Äquivalent $\mathrm{KO}^{t} \mathrm{Bu}(120 \mathrm{mg}, 1.07 \mathrm{mmol})$ zugegeben und nach einstündigem Rühren bei Raumtemperatur werden 2 Äquivalente $\mathrm{Ni}\left(\mathrm{ClO}_{4}\right)_{2} \cdot 6 \mathrm{H}_{2} \mathrm{O}(783 \mathrm{mg}, 2.14 \mathrm{mmol})$ zugefügt. Die 
Reaktionsmischung wird über Nacht gerührt, dann das ausgefallene Kaliumperchlorat abfiltriert, die resultierende grüne Lösung mit 3.5 Äquivalenten $\mathrm{NaN}_{3}$ (244 mg, 3.75 mmol) versetzt und erneut über Nacht gerührt. Der entstandene hellgrüne Niederschlag wird abfiltriert und das Filtrat im Vakuum trocken gezogen. Die zurückbleibende grüne Substanz wird in $15 \mathrm{ml}$ Aceton aufgenommen. Aus dieser Lösung scheiden sich dann nach Überschichtung mit n-Hexan grüne Kristalle ab (Raumgruppe Pbcn).

Ausbeute $275 \mathrm{mg}, 36 \%$

Summenformel $\mathrm{C}_{21} \mathrm{H}_{41} \mathrm{~N}_{13} \mathrm{Ni}_{2} \mathrm{~S}_{4}$

Molmasse, g/mol 721.3

IR (KBr), $\mathrm{cm}^{-1}$ 2965 (w); 2924 (w); $2870(\mathrm{w}) ; 2854(\mathrm{w}) ; 2043(\mathrm{vs})\left[\mathrm{v}\left(\mathrm{N}_{3}{ }^{-}\right)\right] ; 1454$ (w); $1418(\mathrm{w})$

MS (FAB+), m/z (\%) $677(100),\left[\mathrm{L}^{6} \mathrm{Ni2}\left(\mathrm{N}_{3}\right)_{2}\right]^{+} ; 635(15),\left[\mathrm{L}^{6} \mathrm{Ni}_{2}\left(\mathrm{~N}_{3}\right)\right]^{+}$

Elementaranalyse, \% ber.: C 34.97, H 5.73, N 25.24; gef.: C 35.03, H 5.75, N 24.60

\section{$\left[\mathrm{L}^{6} \mathrm{Ni}_{2}\left(\mathbf{N}_{3}\right)_{3}\right](\mathbf{1 0})$}

Zu einer Lösung des Liganden $\mathbf{H L}^{\mathbf{6}}$ (1.312 g, $\left.2.74 \mathrm{mmol}\right)$ in $250 \mathrm{~mL}$ Methanol werden 1 Äquivalent $\mathrm{KO}^{t} \mathrm{Bu}(307 \mathrm{mg}, 2.74 \mathrm{mmol})$ und 2 Äquivalente $\mathrm{Ni}\left(\mathrm{ClO}_{4}\right)_{2} \cdot 6 \mathrm{H}_{2} \mathrm{O}(2.00 \mathrm{~g}, 5.48$ mmol) zugegeben und $1 \mathrm{~h}$ gerührt. Nach Filtration des Kaliumperchlorats zieht man das Filtrat im Vakuum trocken, nimmt die zurückbleibende grüne Substanz in $100 \mathrm{ml}$ Aceton auf und fügt zu der resultierenden grünen Lösung 3.5 Äquivalente $\mathrm{NaN}_{3}$ (623 mg, 9.59 mmol) hinzu. Nach 1 h Rühren wird die Lösung filtriert und zum Kristallisieren gestellt. Über Nacht entstehen grüne Kristalle (Raumgruppe $P \overline{1}$ ).

Ausbeute $\quad 686 \mathrm{mg}, 35 \%$

Summenformel $\quad \mathrm{C}_{21} \mathrm{H}_{41} \mathrm{~N}_{13} \mathrm{Ni}_{2} \mathrm{~S}_{4}$

Molmasse, g/mol $\quad 721.3$

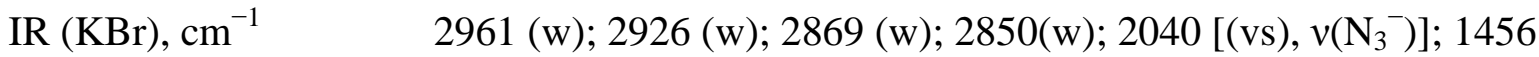
(w); $1418(\mathrm{w})$

Elementaranalyse, \% ber.: C 34.97, H 5.73, N 25.24; gef.: C 35.05, H 5.70, N 25.02 


\subsubsection{Synthese der vierkernigen Nickel(II)-Komplexe}

\section{$\left[\left(\mathrm{L}^{1}\right)_{2} \mathrm{Ni}_{4}\left(\mathrm{~N}_{3}\right)_{4}\right]\left(\mathrm{ClO}_{4}\right)_{2} \cdot \mathrm{CH}_{3} \mathrm{OH} \cdot 0.5 \mathrm{H}_{2} \mathrm{O}(11 \mathrm{a})$}

Zu einer Lösung des Liganden $\mathbf{H L}^{\mathbf{1}}$ (259 mg, $0.80 \mathrm{mmol}$ ) in $30 \mathrm{~mL}$ Methanol werden nacheinander 1 Äquivalent $\mathrm{KO}^{t} \mathrm{Bu}(90 \mathrm{mg}, 0.80 \mathrm{mmol})$ und 2 Äquivalente $\mathrm{Ni}\left(\mathrm{ClO}_{4}\right)_{2} \cdot 6 \mathrm{H}_{2} \mathrm{O}$ (585 mg, 1.60 mmol) zugegeben. Nach 1 h Rühren wird die Reaktionsmischung filtriert und zum Filtrat 2 Äquivalente $\mathrm{NaN}_{3}$ (104 mg, $1.60 \mathrm{mmol}$ ) hinzugefügt. Die Reaktionsmischung wird weiter über Nacht gerührt und anschließend zur Kristallisation gestellt. Dadurch enthält man grüne Kristalle, die wegen des partiellen Verlustes von Methanol nach der Abtrennung von der Mutterlauge verwittern.

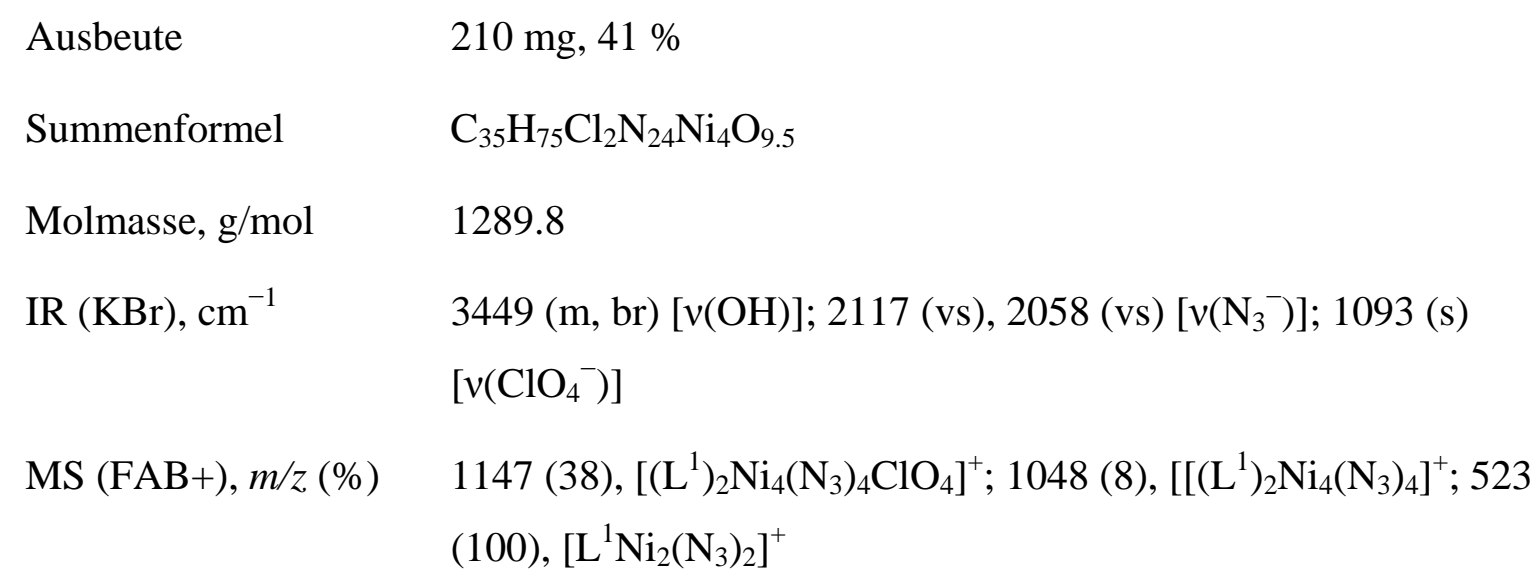

Elementaranalyse, \% ～ber.: C 32.59, H 5.86, N 26.06; gef.: C 31.70, H 5.67, N 26.12

\section{$\left[\left(\mathrm{L}^{1}\right)_{2} \mathrm{Ni}_{4}\left(\mathrm{~N}_{3}\right)_{4}\right]\left(\mathrm{ClO}_{4}\right)_{2} \cdot \mathrm{C}_{3} \mathrm{H}_{6} \mathrm{O}(11 b)$}

11a wird in Aceton aufgenommen und mit Petrolether überschichtet. Das langsame Eindiffundieren von Petrolether in die Lösung des Komplexes liefert grüne Kristalle. Aufgrund des partiellen Verlustes von Lösungsmittelmolekülen nach der Abtrennung von der Mutterlauge verwittern die Kristalle.

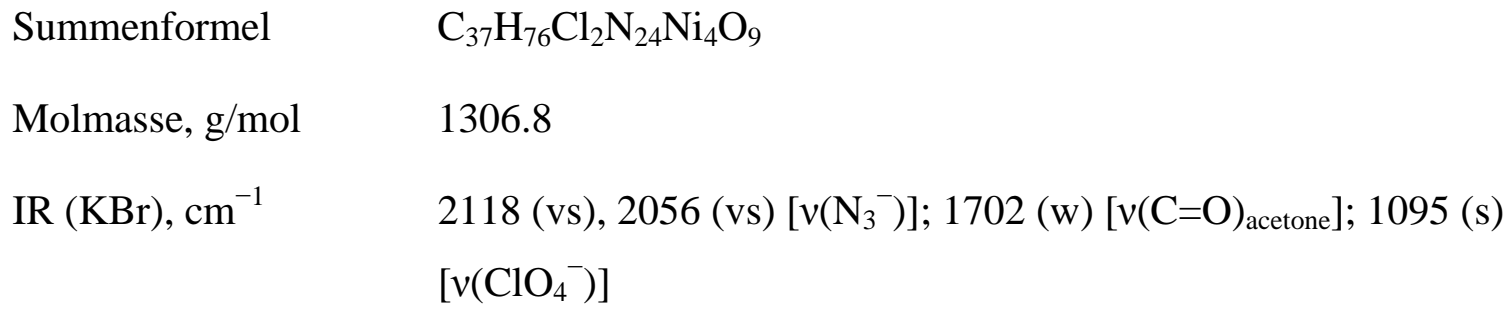

Elementaranalyse, \% ～ber.: C 34.01, H 5.86, N 25.72; gef.: C 33.80, H 6.05, N 23.40 


\section{$\left[\left(\mathrm{L}^{1}\right)_{2} \mathrm{Ni}_{4}\left(\mathrm{~N}_{3}\right)_{4}\right]\left(\mathrm{BPh}_{4}\right)_{2} \cdot \mathrm{C}_{3} \mathrm{H}_{6} \mathrm{O}(\mathbf{1 1 c})$}

Die Verbindung 1 (112 mg, $0.185 \mathrm{mmol}$ ) wird in $25 \mathrm{ml}$ Methanol aufgenommen. Zu dieser Lösung wird zuerst $\mathrm{NaN}_{3}$ (10.6 mg, $\left.0.163 \mathrm{mmol}\right)$ und nach 1h Rühren $\mathrm{NaBPh}_{4}$ (55,7 mg, $0.163 \mathrm{mmol}$ ) zugegeben. Das dabei entstehende grüne Pulver wird abfiltriert und in $20 \mathrm{~mL}$ Aceton gelöst. Die Komplexlösung wird mit Petrolether überschichtet. Durch langsame Diffusion bilden sich grüne Kristalle, die wegen der Abgabe von Lösungsmittelmolekülen verwittern.

Ausbeute $63 \mathrm{mg}, 44 \%$

Summenformel

$$
\mathrm{C}_{85} \mathrm{H}_{116} \mathrm{~B}_{2} \mathrm{~N}_{24} \mathrm{Ni}_{4} \mathrm{O}
$$

Molmasse, g/mol

1746.4

IR $(\mathrm{KBr}), \mathrm{cm}^{-1}$ 2122 (s), $2056(\mathrm{~s})\left[v\left(\mathrm{~N}_{3}{ }^{-}\right)\right]$

MS (FAB+), $m / z(\%)$

1370 (2), $\left[\left(\mathrm{L}^{1}\right)_{2} \mathrm{Ni}_{4}\left(\mathrm{~N}_{3}\right)_{4} \mathrm{BPh}_{4}\right]^{+} ; 523(18),\left[\mathrm{L}^{1} \mathrm{Ni}_{2}\left(\mathrm{~N}_{3}\right)_{2}\right]^{+}$

Elementaranalyse, \% ber.: C 58.46, H 6.70, N 19.25; gef.: C 59.20, H 6.94, N 18.86

\section{$\left.\left[\left(\mathrm{L}^{2}\right)_{2} \mathrm{Ni}_{4}\left(\mathrm{~N}_{3}\right)_{4}\right]\left(\mathrm{BPh}_{4}\right)_{2}\right] \cdot 2 \mathrm{C}_{3} \mathrm{H}_{6} \mathrm{O}(\mathbf{1 2})$}

Die Verbindung 12 wurde nach einer Literaturvorschrift synthetisiert. ${ }^{[105]}$ Die erhaltene Substanz wird erst mit Wasser, danach mit Methanol gewaschen und anschließend an der Luft getrocknet.

Summenformel $\quad \mathrm{C}_{104} \mathrm{H}_{158} \mathrm{~B}_{2} \mathrm{~N}_{28} \mathrm{Ni}_{4} \mathrm{O}_{2}$

Molmasse, g/mol $\quad 2089.0$

IR (KBr), $\mathrm{cm}^{-1} \quad 2129(\mathrm{~s}), 2064(\mathrm{~s})\left[v\left(\mathrm{~N}_{3}{ }^{-}\right)\right] ; 1707(\mathrm{w})\left[\mathrm{v}(\mathrm{C}=\mathrm{O})_{\text {Aceton }}\right]$

Elementaranalyse, \% ～ber.: C 59.80, H 7.62, N 18.77; gef.: C 59.83, H 7.68, N 18.37

Wegen der Abgabe von im Gitter eingebauten Acetonmolekülen zerfallen die Kristalle in das Pulver 12*.

IR (KBr), $\mathrm{cm}^{-1} \quad 2129(\mathrm{~s}), 2060(\mathrm{~s})\left[\mathrm{v}\left(\mathrm{N}_{3}{ }^{-}\right)\right]$ 


\section{3a-c: $\left.\left[\left(\mathrm{L}^{6}\right)_{2} \mathrm{Ni}_{4}\left(\mathrm{~N}_{3}\right)_{3}\left(\mathrm{O}_{2} \mathrm{CMe}\right)\right]\left(\mathrm{ClO}_{4}\right)_{2}\right) \cdot 0.75 \mathrm{C}_{3} \mathrm{H}_{6} \mathrm{O} \quad$ (13a) \\ $\left.\left[\left(\mathrm{L}^{6}\right)_{2} \mathrm{Ni}_{4}\left(\mathrm{~N}_{3}\right)_{3}\left(\mathrm{O}_{2} \mathrm{CMe}\right)\right]\left(\mathrm{ClO}_{4}\right)_{2}\right) \cdot 0.5 \mathrm{CH}_{2} \mathrm{Cl}_{2} \quad\left(13 \mathrm{a}^{*}\right)$ \\ $\left.\left[\left(\mathrm{L}^{6}\right)_{2} \mathrm{Ni}_{4}\left(\mathrm{~N}_{3}\right)_{3}\left(\mathrm{O}_{2} \mathrm{CPh}\right)\right]\left(\mathrm{ClO}_{4}\right)_{2}\right)$ \\ (13b) \\ $\left[\left(\mathrm{L}^{7}\right)_{2} \mathrm{Ni}_{4}\left(\mathrm{~N}_{3}\right)_{3}\left(\mathrm{O}_{2} \mathrm{CPh}\right)\right]\left(\mathrm{ClO}_{4}\right)_{2}$

Für 13a: zu einer Lösung des Liganden $\mathbf{H L}^{6}$ (256 mg, $0.535 \mathrm{mmol}$ ) in $30 \mathrm{ml}$ Methanol werden nacheinander 1 Äquivalent $\mathrm{KO}^{t} \mathrm{Bu}(63 \mathrm{mg}, 0.535 \mathrm{mmol}), 1.75$ Äquivalente $\mathrm{Ni}\left(\mathrm{ClO}_{4}\right)_{2} \cdot 6 \mathrm{H}_{2} \mathrm{O}$ (294 mg, $\left.0.803 \mathrm{mmol}\right)$ und 0.25 Äquivalente $\mathrm{Ni}\left(\mathrm{O}_{2} \mathrm{CMe}\right)_{2} \cdot 4 \mathrm{H}_{2} \mathrm{O}(33 \mathrm{mg}$, 0.134 mmol) zugegeben. Für 13b und 13c: zu einer Lösung des Liganden $\mathbf{H L}^{\mathbf{6}}$ (256 mg, 0.535 mmol) für 13b oder entsprechend $\mathbf{H L}^{7}$ (286 mg, $0.535 \mathrm{mmol}$ ) für 13c in $30 \mathrm{ml}$ Methanol werden nacheinander 1 Äquivalent $\mathrm{KO}^{t} \mathrm{Bu}(63 \mathrm{mg}, 0.535 \mathrm{mmol}), 2$ Äquivalente $\mathrm{Ni}\left(\mathrm{ClO}_{4}\right)_{2} \cdot 6 \mathrm{H}_{2} \mathrm{O}$ (391 mg, $1.07 \mathrm{mmol}$ ) und 0.5 Äquivalente von $\mathrm{NaO}_{2} \mathrm{CPh}$ (39 mg, 0.268 mmol) zugegeben. Nach 2h Rühren wird die Reaktionsmischung im Vakuum trockengezogen. Die zurückbleibende grüne Substanz wird in $25 \mathrm{ml}$ Aceton aufgenommen, die resultierende grüne Lösung mit 1.5 Äquivalenten $\mathrm{NaN}_{3}$ (52 mg, $0.803 \mathrm{mmol}$ ) unter Rühren über Nacht umgesetzt, dann filtriert und mit Petrolether überschichtet. Ein Teil der so erhaltenen Substanz wird nochmals aus Dichlormethan/Petrolether umkristallisiert. Die aus Dichlormethan erhaltenen grünen Kristalle von 13a* und 13b verwittern bei längerer Aufbewahrung.

\section{3a}

Ausbeute $187 \mathrm{mg}, 43 \%$

Summenformel $\mathrm{C}_{46.25} \mathrm{H}_{89.5} \mathrm{Cl}_{2} \mathrm{~N}_{17} \mathrm{Ni}_{4} \mathrm{O}_{10.75} \mathrm{~S}_{8}$

Molmasse, g/mol 1618.0

IR (KBr), $\mathrm{cm}^{-1}$ 2092 (sh), 2081 (vs), 2042 (m) [v( $\left.\left.\mathrm{N}_{3}{ }^{-}\right)\right] ; 1707$ (w) [v(C=O) $\left.)_{\text {Aceton }}\right]$; 1584(m) $\left[v(\mathrm{C}=\mathrm{O})_{\text {Acetat }}\right] ; 1090(\mathrm{vs})\left[v\left(\mathrm{ClO}_{4}{ }^{-}\right)\right]$ MS (FAB+), $m / z(\%)$ 1474 (6), $\left[\left(\mathrm{L}^{6}\right)_{2} \mathrm{Ni}_{4}\left(\mathrm{~N}_{3}\right)_{3}\left(\mathrm{O}_{2} \mathrm{CMe}\right)\left(\mathrm{ClO}_{4}\right)\right]^{+} ; 753$ (12), $\left[\mathrm{L}^{6} \mathrm{Ni}_{2}\left(\mathrm{O}_{2} \mathrm{CMe}\right)\left(\mathrm{ClO}_{4}\right)\right]^{+} ; 736(25),\left[\mathrm{L}^{6} \mathrm{Ni}_{2}\left(\mathrm{~N}_{3}\right)\left(\mathrm{ClO}_{4}\right)\right]^{+} ; 696$ (100), $\left[\mathrm{L}^{6} \mathrm{Ni}_{2}\left(\mathrm{~N}_{3}\right)\left(\mathrm{O}_{2} \mathrm{CMe}\right)\right]^{+} ; 677$ (35), [ $\left.\mathrm{L}^{6} \mathrm{Ni}_{2}\left(\mathrm{~N}_{3}\right)_{2}\right]^{+}$

Elementaranalyse, \% $\quad$ ber.: C, 34.33; H, 5.57; N, 14.72; gef.: C, 34.20; H, 5.58; N, 14.87 


\section{3a*}

Summenformel

Molmasse, g/mol

Elementaranalyse, \%
$\mathrm{C}_{44.5} \mathrm{H}_{86} \mathrm{Cl}_{3} \mathrm{~N}_{17} \mathrm{Ni}_{4} \mathrm{O}_{10} \mathrm{~S}_{8}$

1616.9

ber.: C, 33.06, H, 5.36, N, 14.73, Cl 6.58; gef.: C, 33.21, H, 5.15, N, 14.08, Cl 6.06.

13b

Ausbeute

Summenformel

Molmasse, g/mol

IR (KBr), $\mathrm{cm}^{-1}$

MS (FAB+), m/z (\%)

$13 c$

Ausbeute

Summenformel

Molmasse, g/mol

IR (KBr), $\mathrm{cm}^{-1}$

MS (FAB +$), m / z(\%)$
$202 \mathrm{mg}, 46 \%$

$\mathrm{C}_{49} \mathrm{H}_{87} \mathrm{Cl}_{2} \mathrm{~N}_{17} \mathrm{Ni}_{4} \mathrm{O}_{10} \mathrm{~S}_{8}$

1636.5

2083 (vs), 2043 (m) [v( $\left.\left.\mathrm{N}_{3}{ }^{-}\right)\right] ; 1598$ (m), 1563 (m) [v(C=O) $\left.)_{\text {Benzoat }}\right]$; 1097 (vs) $\left[v\left(\mathrm{ClO}_{4}{ }^{-}\right)\right]$

1536 (7), $\left[\left(\mathrm{L}^{6}\right)_{2} \mathrm{Ni}_{4}\left(\mathrm{~N}_{3}\right)_{3}\left(\mathrm{O}_{2} \mathrm{CPh}\right)\left(\mathrm{ClO}_{4}\right)\right]^{+} ; 758$ (100),

$\left[\mathrm{L}^{6} \mathrm{Ni}_{2}\left(\mathrm{~N}_{3}\right)\left(\mathrm{O}_{2} \mathrm{CPh}\right)_{2}\right]^{+} ; 677$ (33), [ $\left.\mathrm{L}^{6} \mathrm{Ni}_{2}\left(\mathrm{~N}_{3}\right)_{2}\right]^{+} ; 635$ (18),

$\left[\mathrm{L}^{6} \mathrm{Ni}_{2}\left(\mathrm{~N}_{3}\right)\right]^{+}$

ber.: C, 35.96, H, 5.36, N, 14.55; ber.: C, 36.12, H, 5.19, N, 14.59
$270 \mathrm{mg}, 58 \%$

$\mathrm{C}_{57} \mathrm{H}_{103} \mathrm{~N}_{17} \mathrm{Ni}_{4} \mathrm{O}_{10} \mathrm{~S}_{8} \mathrm{Cl}_{2}$

1748.7

2083 (vs), $2043(\mathrm{~m})\left[v\left(\mathrm{~N}_{3}{ }^{-}\right)\right] ; 1600(\mathrm{~m}), 1564$ (m) [v(C=O) $\left.)_{\text {Benzoat }}\right]$;

$1098(\mathrm{vs})\left[\mathrm{v}\left(\mathrm{ClO}_{4}{ }^{-}\right)\right]$

1648 (8), $\left[\left(\mathrm{L}^{7}\right)_{2} \mathrm{Ni}_{4}\left(\mathrm{~N}_{3}\right)_{3}\left(\mathrm{O}_{2} \mathrm{CPh}\right)\left(\mathrm{ClO}_{4}\right)\right]^{+} ; 814$ (100),

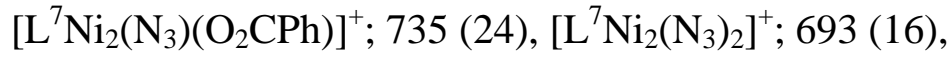

$\left[\mathrm{L}^{7} \mathrm{Ni}_{2}\left(\mathrm{~N}_{3}\right)\right]^{+}$

Elementaranalyse, \% $\quad$ ber.: C, 39.15, H, 5.94, N, 13.62; gef.: C, 39.27, H, 6.02, N, 13.70 


\section{$\left[\left(\mathrm{L}^{4}\right)_{2} \mathrm{Ni}_{4}\left(\mathrm{~N}_{3}\right)_{3}\left(\mathrm{O}_{2} \mathrm{CMe}\right)_{2}\right]\left(\mathrm{ClO}_{4}\right) \cdot \mathrm{C}_{3} \mathrm{H}_{6} \mathrm{O}(\mathbf{1 4 a})$}

$\mathrm{Zu}$ einer Lösung des Liganden $\mathbf{H L}^{4}$ (198 mg, $0.60 \mathrm{mmol}$ ) in $30 \mathrm{ml}$ Methanol werden nacheinander 1 Äquivalent $\mathrm{KO}^{t} \mathrm{Bu}\left(71 \mathrm{mg}, 0.60 \mathrm{mmol}\right.$ ), 1.5 Äquivalente $\mathrm{Ni}\left(\mathrm{ClO}_{4}\right)_{2} \cdot 6 \mathrm{H}_{2} \mathrm{O}$ (329 mg, $0.90 \mathrm{mmol}$ ) und 0.5 Äquivalente $\mathrm{Ni}\left(\mathrm{O}_{2} \mathrm{CMe}\right)_{2} \cdot 4 \mathrm{H}_{2} \mathrm{O}(75 \mathrm{mg}, 0.30 \mathrm{mmol}$ ) zugegeben. Nach 1h Rühren wird die Reaktionsmischung im Vakuum trocken gezogen. Die zurückbleibende grüne Substanz wird in $25 \mathrm{ml}$ Aceton aufgenommen und die grüne Lösung wird mit 1.5 Äquivalenten $\mathrm{NaN}_{3}$ (59 mg, $0.90 \mathrm{mmol}$ ) versetzt. Die Reaktionsmischung wird weitere $12 \mathrm{~h}$ gerührt und anschließend filtriert. Aus dem Filtrat scheiden sich dann nach Überschichtung mit Petrolether grüne Kristalle ab.

Ausbeute

Summenformel

Molmasse, g/mol

IR $(\mathrm{KBr}), \mathrm{cm}^{-1}$

MS (FAB+), $m / z(\%)$

$$
\begin{aligned}
& 1136(74),\left[\left(\mathrm{L}^{4}\right)_{2} \mathrm{Ni}_{4}\left(\mathrm{~N}_{3}\right)_{3}\left(\mathrm{O}_{2} \mathrm{CMe}\right)_{2}\right]^{+} ; 563(72),\left[\mathrm{L}^{4} \mathrm{Ni}_{2}\left(\mathrm{O}_{2} \mathrm{CMe}\right)_{2}\right]^{+} \text {; } \\
& 546(100),\left[\mathrm{L}^{4} \mathrm{Ni}_{2}\left(\mathrm{~N}_{3}\right)\left(\mathrm{O}_{2} \mathrm{CMe}\right)\right]^{+} ; 504(5)\left[\mathrm{L}^{4} \mathrm{Ni}_{2}\left(\mathrm{O}_{2} \mathrm{CMe}\right)\right]^{+}, 445 \\
& \text { (12) }\left[\mathrm{L}^{4} \mathrm{Ni}_{2}\right]^{+}
\end{aligned}
$$

Elementaranalyse, \% ～ber.: C 34.30, H 5.47, N 18.40; gef.: C 34.30, H 5.40, N 18.15

\section{$\left[\left(\mathrm{L}^{4}\right)_{2} \mathrm{Ni}_{4}\left(\mathrm{~N}_{3}\right)_{3}\left(\mathrm{O}_{2} \mathrm{CPh}\right)_{2}\right]\left(\mathrm{ClO}_{4}\right) \cdot \mathrm{NaClO}_{4} \cdot \mathrm{C}_{3} \mathrm{H}_{6} \mathrm{O} \cdot \mathrm{H}_{2} \mathrm{O}(\mathbf{1 4 b})$}

$\mathrm{Zu}$ einer Lösung des Liganden $\mathbf{H L}^{4}$ (198 mg, $0.60 \mathrm{mmol}$ ) in $30 \mathrm{ml}$ Methanol werden nacheinander 1 Äquivalent $\mathrm{KO}^{t} \mathrm{Bu}(71 \mathrm{mg}, 0.60 \mathrm{mmol})$ und 2 Äquivalente $\mathrm{Ni}\left(\mathrm{ClO}_{4}\right)_{2} \cdot 6 \mathrm{H}_{2} \mathrm{O}$ (439 mg, $1.20 \mathrm{mmol}$ ) zugegeben. Nach 1h Rühren wird die Reaktionsmischung trocken gezogen, der erhaltene grüne Rückstand in $25 \mathrm{ml}$ Aceton aufgenommen, es werden 1 Äquivalent $\mathrm{Na}\left(\mathrm{O}_{2} \mathrm{CPh}\right.$ ) (86 mg, $0.60 \mathrm{mmol}$ ) sowie 1.5 Äquivalente $\mathrm{NaN}_{3}$ (59 mg, $0.90 \mathrm{mmol}$ ) zugesetzt, dann über Nacht gerührt und anschließend filtriert. Durch das Überschichten des Filtrats mit Petrolether werden grüne Kristalle gebildet, welche nach Abtrennung von der Mutterlauge durch partielle Abgabe des enthaltenen Lösungsmittels verwittern.

Ausbeute

Summenformel

Molmasse, g/mol
$117 \mathrm{mg}, 24 \%$

$\mathrm{C}_{47} \mathrm{H}_{76} \mathrm{~N}_{17} \mathrm{NaNi}_{4} \mathrm{O}_{14} \mathrm{~S}_{4} \mathrm{Cl}_{2}$

1560.1 
IR $(\mathrm{KBr}), \mathrm{cm}^{-1}$

3425 (m, br) [v(OH)]; 2080 (vs), 2073 (vs), 2044 (s) [v( $\left.\left.\mathrm{N}_{3}{ }^{-}\right)\right] ; 1708$

(w) $\left[v(\mathrm{C}=\mathrm{O})_{\text {Aceton }}\right] ; 1601$ (s), 1567 (s) $\left[v(\mathrm{C}=\mathrm{O})_{\text {Benzoat }}\right]$; 1121 (s), 1107

(s), $1091(\mathrm{~s})\left[\mathrm{v}\left(\mathrm{ClO}_{4}{ }^{-}\right)\right]$

MS (FAB+), m/z (\%) 1260 (92), $\left[\left(\mathrm{L}^{4}\right)_{2} \mathrm{Ni}_{4}\left(\mathrm{~N}_{3}\right)_{3}\left(\mathrm{O}_{2} \mathrm{CPh}\right)_{2}\right]^{+} ; 687(68),\left[\mathrm{L}^{4} \mathrm{Ni}_{2}\left(\mathrm{O}_{2} \mathrm{CPh}\right)_{2}\right]^{+}$;

608 (100), $\left[\mathrm{L}^{4} \mathrm{Ni}_{2}\left(\mathrm{~N}_{3}\right)\left(\mathrm{O}_{2} \mathrm{CPh}\right)\right]^{+} ; 507$ (15), [L $\left.{ }^{4} \mathrm{Ni}\left(\mathrm{O}_{2} \mathrm{CPh}\right)\right]^{+}$

Elementaranalyse, \% ～ber.: C 36.18, H 4.91, N 15.26; gef.: C 36.37, H 4.87, N 14.82

14c-e: $\left[\left(\mathrm{L}^{6}\right)_{2} \mathrm{Ni}_{4}\left(\mathrm{~N}_{3}\right)_{3}\left(\mathrm{O}_{2} \mathrm{CMe}\right)_{2}\right]\left(\mathrm{ClO}_{4}\right)$

$\left.\left[\left(\mathrm{L}^{7}\right)_{2} \mathrm{Ni}_{4}\left(\mathrm{~N}_{3}\right)_{3}\left(\mathrm{O}_{2} \mathrm{CMe}\right)_{2}\right]\left(\mathrm{ClO}_{4}\right)\right) \cdot 2 \mathrm{H}_{2} \mathrm{O}$

$\left[\left(\mathrm{L}^{7}\right)_{2} \mathrm{Ni}_{4}\left(\mathrm{~N}_{3}\right)_{3}\left(\mathrm{O}_{2} \mathrm{CAda}\right)_{2}\right]\left(\mathrm{ClO}_{4}\right) \cdot \mathrm{C}_{3} \mathrm{H}_{6} \mathrm{O}$

(14c)

(14d)

(14e)

Die Synthese von 14c bzw. 14d erfolgt analog zur Darstellung des Komplexes 14a, ausgehend von $\mathbf{H L}^{6}$ (287 mg, $0.60 \mathrm{mmol}$ ) bzw. von $\mathbf{H L}^{7}$ (321 mg, $0.60 \mathrm{mmol}$ ). Die Synthese von 14e wird analog zur Darstellung des Komplexes 14b, ausgehend von $\mathbf{H L}^{7}$ (321 mg, 0.60 mmol) durchgeführt. Das hier benötigte $\mathrm{K}\left(\mathrm{O}_{2} \mathrm{CAda}\right.$ ) wird aus $\mathrm{HO}_{2} \mathrm{CAda}$ (108 mg, $0.60 \mathrm{mmol}$ ) und $\mathrm{KO}^{t} \mathrm{Bu}(71 \mathrm{mg}, 0.60 \mathrm{mmol})$ in situ hergestellt.

\section{$14 c$}

Ausbeute $175 \mathrm{mg}, 38 \%$

Summenformel

$$
\mathrm{C}_{46} \mathrm{H}_{88} \mathrm{ClN}_{17} \mathrm{Ni}_{4} \mathrm{O}_{8} \mathrm{~S}_{8}
$$

Molmasse, g/mol 1534.1

IR $(\mathrm{KBr}), \mathrm{cm}^{-1}$ 2079 (vs), 2074 (vs), 2043 (m) [( $\left.\left.\mathrm{N}_{3}^{-}\right)\right] ; 1587$ (s) [v(C=O) $\left.)_{\text {Acetat }}\right] ; 1122$ (m), $1096(\mathrm{~s})\left[v\left(\mathrm{ClO}_{4}^{-}\right)\right]$

MS (FAB +$), m / z(\%) 1434(4),\left[\left(\mathrm{L}^{6}\right)_{2} \mathrm{Ni}_{4}\left(\mathrm{~N}_{3}\right)_{3}\left(\mathrm{O}_{2} \mathrm{CMe}\right)_{2}\right]^{+} ; 1375$ (5), $\left[\left(\mathrm{L}^{6}\right)_{2} \mathrm{Ni}_{4}\left(\mathrm{~N}_{3}\right)_{3}\left(\mathrm{O}_{2} \mathrm{CMe}\right)\right]^{+} ; 696(100),\left[\mathrm{L}^{6} \mathrm{Ni}_{2}\left(\mathrm{~N}_{3}\right)\left(\mathrm{O}_{2} \mathrm{CMe}\right)\right]^{+} ; 635$ (20), $\left[\mathrm{L}^{6} \mathrm{Ni}_{2}\left(\mathrm{~N}_{3}\right)\right]^{+} ; 536(12),\left[\mathrm{L}^{6} \mathrm{Ni}\right]^{+}$

Elementaranalyse, \% ～ber.: C 36.02, H 5.78, N 15.52; gef.: C 35.42, H 5.73, N 15.07 
14d

Ausbeute

Summenformel

Molmasse, g/mol

IR $(\mathrm{KBr}), \mathrm{cm}^{-1}$

MS (FAB+), m/z (\%)

Elementaranalyse, \%
$112 \mathrm{mg}, 22 \%$

$$
\mathrm{C}_{54} \mathrm{H}_{108} \mathrm{Cl} \mathrm{N}{ }_{17} \mathrm{Ni}_{4} \mathrm{O}_{10} \mathrm{~S}_{8}
$$

1682.3

3425 (m, br) [v(OH)]; 2082 (vs), 2073 (vs), 2044 (m) [v( $\left.\left.\mathrm{N}_{3}{ }^{-}\right)\right] ; 1587$

(s) $\left[v(\mathrm{C}=\mathrm{O})_{\text {Acetat }}\right] ; 1122(\mathrm{~m}), 1095(\mathrm{~s})\left[\mathrm{v}\left(\mathrm{ClO}_{4}{ }^{-}\right)\right]$

1546 (10), $\left[\left(\mathrm{L}^{7}\right)_{2} \mathrm{Ni}_{4}\left(\mathrm{~N}_{3}\right)_{3}\left(\mathrm{O}_{2} \mathrm{CMe}\right)_{2}\right]^{+} ; 769$ (24), $\left[\mathrm{L}^{7} \mathrm{Ni}_{2}\left(\mathrm{O}_{2} \mathrm{CMe}\right)_{2}\right]^{+}$; 750 (100), [ $\left.\mathrm{L}^{7} \mathrm{Ni}_{2}\left(\mathrm{~N}_{3}\right)\left(\mathrm{O}_{2} \mathrm{CMe}\right)\right]^{+} ; 735$ (20), $\left[\mathrm{L}^{7} \mathrm{Ni}_{2}\left(\mathrm{~N}_{3}\right)_{2}\right]^{+} ; 693$ (7), $\left[\mathrm{L}^{7} \mathrm{Ni} \mathrm{N}\right]^{+} ; 650(12),\left[\mathrm{L}^{7} \mathrm{Ni}\right]^{+}$

ber.: C 38.55, H 6.47, N 14.15; gef.: C 38.05, H 6.13, N 13.58
$14 e$

Ausbeute

Summenformel

Molmasse, g/mol

IR $(\mathrm{KBr}), \mathrm{cm}^{-1}$

MS (FAB+), $m / z(\%)$
$135 \mathrm{mg}, 23 \%$

$\mathrm{C}_{75} \mathrm{H}_{134} \mathrm{Cl} \mathrm{N}_{17} \mathrm{Ni}_{4} \mathrm{O}_{9} \mathrm{~S}_{8}$

1944.7

2081 (vs), 2072 (vs), 2045 (m) [v( $\left.\left.\mathrm{N}_{3}^{-}\right)\right] ; 1705$ [w, v(C=O) $\left.)_{\text {Aceton }}\right]$; 1567 (s) [v(C=O) $\left.)_{\text {Adamantat }}\right]$; 1121 (m), 1096 (s) [v( $\left.\left.\mathrm{ClO}_{4}{ }^{\top}\right)\right]$

$1787(12)\left[\left(\mathrm{L}^{7}\right)_{2} \mathrm{Ni}_{4}\left(\mathrm{~N}_{3}\right)_{3}\left(\mathrm{O}_{2} \mathrm{Ada}\right)_{2}\right]^{+} ; 1010(16)\left[\mathrm{L}^{7} \mathrm{Ni}_{2}\left(\mathrm{O}_{2} \mathrm{CAda}\right)_{2}\right]^{+}$, $872(100)\left[\mathrm{L}^{7} \mathrm{Ni}_{2}\left(\mathrm{~N}_{3}\right)\left(\mathrm{O}_{2} \mathrm{CAda}\right)\right]^{+}, 735(12)\left[\mathrm{L}^{7} \mathrm{Ni}\left(\mathrm{N}_{3}\right)_{2}\right]^{+}, 693(8)$ $\left[\mathrm{L}^{7} \mathrm{Ni}\left(\mathrm{N}_{3}\right)\right]^{+}$

ber.: C 46.32, H 6.95, N 12.24; gef.: C 46.19, H 6.93, N 11.96

\section{$\left[\left(\mathrm{L}^{5}\right)_{2} \mathrm{Ni}_{4}\left(\mathrm{~N}_{3}\right)\left(\mathrm{O}_{2} \mathrm{CAda}\right)_{4}\right]\left(\mathrm{ClO}_{4}\right)(15 \mathrm{a})$}

Zu einer Lösung des Liganden $\mathbf{H L}^{\mathbf{5}}$ (254 mg, $0.60 \mathrm{mmol}$ ) in $20 \mathrm{ml}$ Methanol werden nacheinander 1 Äquivalent $\mathrm{KO}^{t} \mathrm{Bu}(71 \mathrm{mg}, 0.60 \mathrm{mmol})$ und 2 Äquivalente $\mathrm{Ni}\left(\mathrm{ClO}_{4}\right)_{2} \cdot 6 \mathrm{H}_{2} \mathrm{O}$ (439 mg, $1.20 \mathrm{mmol}$ ) zugegeben. Nach 1h Rühren fügt man 1.5 Äquivalente $\mathrm{NaN}_{3}$ (59 mg, $0.90 \mathrm{mmol}$ ) hinzu, wobei die Farbe der Lösung von blau auf grün wechselt. Man überschichtet diese grüne Lösung zuerst mit $20 \mathrm{ml}$ Methanol und danach wird mit der in situ hergestellten Lösung von $\mathrm{KO}_{2} \mathrm{CAda}$ (131 mg, $0.60 \mathrm{mmol}$ ) in 20ml Methanol die dritte Schicht aufgebaut. Durch langsam verlaufende Diffusionsprozesse zwischen den Schichten, wobei 
Moleküle aus der ersten und dritten Schicht zu einander diffundieren, werden grüne Kristalle erhalten.

Ausbeute

Summenformel

Molmasse, g/mol

IR (KBr), $\mathrm{cm}^{-1}$

$\mathrm{MS}(\mathrm{FAB}+), m / z(\%)$

MS (ESI+, MeCN),

$m / z(\%)$
$27 \mathrm{mg}, 5 \%$

$\mathrm{C}_{78} \mathrm{H}_{126} \mathrm{~N}_{11} \mathrm{Ni}_{4} \mathrm{~S}_{8} \mathrm{O}_{12} \mathrm{Cl}$

1936.7

2128 (s) $\left[v\left(\mathrm{~N}_{3}{ }^{-}\right)\right] ; 2054$ (m); 1585 (vs) [v(C=O) $\left.)_{\text {Adamantat }}\right] ; 1142$ (vs), 1107 (vs), 1089 (vs) $\left[v\left(\mathrm{ClO}_{4}^{-}\right)\right]$

1835 (12), $\left[\left(\mathrm{L}^{5}\right)_{2} \mathrm{Ni}_{4}\left(\mathrm{~N}_{3}\right)\left(\mathrm{O}_{2} \mathrm{CAda}\right)_{4}\right]^{+} ; 897$ (45), $\left[\mathrm{L}^{5} \mathrm{Ni}_{2}\left(\mathrm{O}_{2} \mathrm{CAda}\right)_{2}\right]^{+}$; 758 (15), $\left[\mathrm{L}^{5} \mathrm{Ni}_{2}\left(\mathrm{O}_{2} \mathrm{CAda}\right)\left(\mathrm{N}_{3}\right)\right]^{+}$

$1835(100)\left[\left(\mathrm{L}^{5}\right)_{2} \mathrm{Ni}_{4}\left(\mathrm{~N}_{3}\right)\left(\mathrm{O}_{2} \mathrm{CAda}\right)_{4}\right]^{+}$

ber.: C 48.37, H 6.58, N 7.96; gef.: C 48.09, H 6.72, N 7.68

\section{$\left[\left(\mathrm{L}^{6}\right)_{2} \mathrm{Ni}_{4}\left(\mathrm{~N}_{3}\right)\left(\mathrm{O}_{2} \mathrm{CAda}\right)_{4}\right]\left(\mathrm{ClO}_{4}\right)(15 b)$}

Zu einer Lösung des Liganden $\mathbf{H L}^{6}$ (287 mg, $0.60 \mathrm{mmol}$ ) in $20 \mathrm{ml}$ Dichlormethan werden nacheinander 1 Äquivalent $\mathrm{KO}^{t} \mathrm{Bu}(71 \mathrm{mg}, 0.60 \mathrm{mmol})$ und 2 Äquivalente $\mathrm{Ni}\left(\mathrm{ClO}_{4}\right)_{2} \cdot 6 \mathrm{H}_{2} \mathrm{O}$ (439 mg, 1.20 mmol) zugegeben. Nach 1 h Rühren fügt man 2 Äquivalente $\mathrm{HO}_{2} \mathrm{CAda}$ (216 $\mathrm{mg}, 1.20 \mathrm{mmol}$ ), 2 Äquivalente $\mathrm{KO}^{t} \mathrm{Bu}\left(142 \mathrm{mg}, 1.20 \mathrm{mmol}\right.$ ) und 0.67 Äquivalent $\mathrm{NaN}_{3}$ (30 mg, $0.45 \mathrm{mmol}$ ) hinzu. Die Reaktionsmischung wird über Nacht gerührt und anschließend filtriert. Das Filtrat wird mit Petrolether überschichtet und man erhält grüne Kristalle.

Ausbeute

Summenformel

Molmasse, g/mol

IR (KBr), $\mathrm{cm}^{-1}$

MS (FAB+), m/z (\%)

Elementaranalyse, \% $107 \mathrm{mg}, 17 \%$

$\mathrm{C}_{86} \mathrm{H}_{142} \mathrm{~N}_{11} \mathrm{Ni}_{4} \mathrm{~S}_{8} \mathrm{O}_{12} \mathrm{Cl}$

2048.9

2127 (s) $\left[v\left(\mathrm{~N}_{3}{ }^{-}\right)\right] ; 2043(\mathrm{~m}) ; 1582$ (vs) [v(C=O) $\left.)_{\text {Adamantat }}\right] ; 1144$ (s), 1103 (vs), 1088 (vs) $\left[v\left(\mathrm{ClO}_{4}^{-}\right)\right]$

1949 (5), [( $\left.\left.\mathrm{L}^{6}\right)_{2} \mathrm{Ni}_{4}\left(\mathrm{~N}_{3}\right)\left(\mathrm{O}_{2} \mathrm{CAda}\right)_{4}\right]^{+}$; 953(100), [ $\left.\mathrm{L}^{6} \mathrm{Ni}_{2}\left(\mathrm{O}_{2} \mathrm{CAda}\right)_{2}\right]^{+}$; 816 (12), [ $\left.\mathrm{L}^{6} \mathrm{Ni}_{2}\left(\mathrm{~N}_{3}\right)\left(\mathrm{O}_{2} \mathrm{CAda}\right)\right]^{+}$

ber.: C 50.41, H 6.99, N 7.52; gef.: C 50.13, H 6.94, N 7.57 


\section{$\left[\left(\mathrm{L}^{1}\right)_{2} \mathrm{Ni}_{4}\left(\mathrm{~N}_{3}\right)_{6}\right] \cdot 2 \mathrm{CH}_{2} \mathrm{Cl}_{2}(16)$}

$\mathrm{Zu}$ einer Lösung des Liganden $\mathbf{H L}^{\mathbf{1}}$ (255 mg, $0.786 \mathrm{mmol}$ ) in $20 \mathrm{ml}$ Methanol werden nacheinander 1 Äquivalent $\mathrm{KO}^{t} \mathrm{Bu}(92.7 \mathrm{mg}, 0.786 \mathrm{mmol})$ und 2 Äquivalente $\mathrm{Ni}\left(\mathrm{ClO}_{4}\right)_{2} \cdot 6 \mathrm{H}_{2} \mathrm{O}$ (580.7 mg, $1.572 \mathrm{mmol}$ ) zugegeben. Nach $30 \mathrm{~min}$. Rühren wird die Reaktionsmischung filtriert und das Filtrat im Vakuum trocken gezogen. Die zurückbleibende grüne Substanz wird in 25 ml Aceton aufgenommen, mit 3 Äquivalenten $\mathrm{NaN}_{3}$ (154.8 mg, 2.358 mmol) versetzt und über Nacht gerührt. Das dabei entstehende grüne Pulver wird abfiltriert, der Filtrationsrückstand in $25 \mathrm{ml}$ Dichlormethan gelöst und mit Petrolether überschichtet. Nach einigen Tagen werden grüne Kristalle erhalten.

$\begin{array}{ll}\text { Ausbeute } & 148 \mathrm{mg}, 29 \% \\ \text { Summenformel } & \mathrm{C}_{36} \mathrm{H}_{74} \mathrm{~N}_{30} \mathrm{Ni}_{4} \mathrm{Cl}_{4} \\ \text { Molmasse, g/mol } & 1303.8 \\ \text { IR (KBr), cm } & \\ \text { MS (FAB+), } \mathrm{m} / \mathrm{z}(\%) & 1092(16),\left[\left(\mathrm{L}^{1}\right)_{2} \mathrm{Ni}_{4}\left(\mathrm{~N}_{3}\right)_{5}\right]^{+} ; 525\left(100,\left[\mathrm{~L}^{1} \mathrm{Ni}_{2}\left(\mathrm{~N}_{3}\right)_{2}\right]^{+}\right) ; 424(12), \\ & {\left[\mathrm{L}^{1} \mathrm{Ni}\left(\mathrm{N}_{3}\right)\right]^{+}}\end{array}$

Elementaranalyse, \% ～ber.: C 33.17, H 5.72, N 32.23; gef.: C 33.59, H 5.78, N 32.50

\section{$\left[\left(\mathbf{L}^{1}\right)_{2} \mathrm{Ni}_{4}\left(\mathbf{N}_{3}\right)_{6}\right](\mathbf{1 7})$}

Zu einer Lösung des Liganden $\mathbf{H L}^{1}$ (162 mg, $0.5 \mathrm{mmol}$ ) in $40 \mathrm{ml}$ Methanol werden nacheinander 1 Äquivalent $\mathrm{KO}^{t} \mathrm{Bu}(56 \mathrm{mg}, 0.5 \mathrm{mmol})$ und 2 Äquivalente $\mathrm{Ni}\left(\mathrm{ClO}_{4}\right)_{2} \cdot 6 \mathrm{H}_{2} \mathrm{O}$ (365.7 mg, $1.0 \mathrm{mmol}$ ) zugegeben. Nach $30 \mathrm{~min}$. Rühren wird die Reaktionsmischung filtriert, dem Filtrat werden 4 Äquivalente $\mathrm{NaN}_{3}(130 \mathrm{mg}, 2.0 \mathrm{mmol}$ ) zugesetzt und dann wird weitere 2 h gerührt. Der dabei entstandene Niederschlag wird abfiltriert und das Filtrat zur Kristallisation gestellt. Nach zwei Tagen wird die Methanollösung filtriert und das Filtrat wieder zur Kristallisation gestellt. Aus der Methanollösung erhält man grüne Kristalle, die aber mit einem hellgrünen Pulver, das sich auch absetzt, behaftet sind (Ausbeute insgesamt 236 mg, 83 \%). Das erschwert die Gewinnung der Verbindung in einem Reinheitsgrad, der für die magnetischen Untersuchungen notwendig ist.

17 kann aber in ausreichender Reinheit durch Reaktion von kristallinem 1 mit Natriumazid in Methanol dargestellt werden. Dafür wird 1 (50 mg, $0.0824 \mathrm{mmol}$ ) in $20 \mathrm{ml}$ MeOH gelöst, mit 2 Äquivalenten $\mathrm{NaN}_{3}$ (10.7 mg, $0.1648 \mathrm{mmol}$ ) versetzt, eine halbe Stunde gerührt und mit Diethylether überschichtet. Dadurch enthält man 17 in reiner kristalliner Form (13 mg, 28 \%), frei von anhaftender pulvriger Substanz. 
Summenformel $\quad \mathrm{C}_{34} \mathrm{H}_{70} \mathrm{~N}_{30} \mathrm{Ni}_{4}$

Molmasse, g/mol $\quad 1134.0$

IR (KBr), $\mathrm{cm}^{-1} \quad 2098(\mathrm{vs}), 2052(\mathrm{vs}), 2037$ (vs) $\left[\mathrm{v}\left(\mathrm{N}_{3}{ }^{-}\right)\right]$

Elementaranalyse, \% ～ber.: C 36.01, H 6.22, N 37.06; gef.: C 36.19, H 6.34, N 35.22

\section{$\left[\left(\mathrm{L}^{6}\right)_{2} \mathrm{Ni}_{4}\left(\mathrm{~N}_{3}\right)_{2}(\mathrm{MeOH})_{2}\right]\left(\mathrm{ClO}_{4}\right)_{4}(18)$}

Zur einer Lösung des Liganden $\mathbf{H L}^{6}$ (240 mg, $0.5 \mathrm{mmol}$ ) in $25 \mathrm{ml}$ Methanol werden nacheinander 1 Äquivalent $\mathrm{KO}^{t} \mathrm{Bu}(56 \mathrm{mg}, 0.5 \mathrm{mmol})$ und 2 Äquivalente $\mathrm{Ni}\left(\mathrm{ClO}_{4}\right)_{2} \cdot 6 \mathrm{H}_{2} \mathrm{O}$ (365 mg, $1.0 \mathrm{mmol}$ ) zugegeben. Nach einer Rührdauer von $1 \mathrm{~h}$ wird die Reaktionsmischung filtriert, dem Filtrat 1 Äquivalent $\mathrm{NaN}_{3}$ (33 mg, $0.5 \mathrm{mmol}$ ) zugesetzt und dann weitere $2 \mathrm{~h}$ gerührt. Nach Überschichtung der Komplexlösung mit Diethylether bilden sich durch Diffusion grüne Kristalle.

Ausbeute $305 \mathrm{mg}, 35 \%$

Summenformel

$$
\mathrm{C}_{44} \mathrm{H}_{90} \mathrm{Cl}_{4} \mathrm{~N}_{14} \mathrm{Ni}_{4} \mathrm{O}_{18} \mathrm{~S}_{8}
$$

Molmasse, g/mol

1736.4

IR $(\mathrm{KBr}), \mathrm{cm}^{-1}$ 2091 (sh), 2059 (vs) [v( $\left.\left.\mathrm{N}_{3}^{-}\right)\right], 1454$ (w); 1418 (w); 1093 (vs) $\left[v\left(\mathrm{ClO}_{4}^{-}\right)\right]$

Elementaranalyse, \% ～ber.: C 30.44, H 5.22, N 11.29; gef.: C 29.13, H 5.05, N 11.27

\subsubsection{Synthese der $J$-alternierenden Ketten, bestehend aus $S=1$ Spinträgern $^{1)}$}

\section{$\left[\mathrm{L}^{2} \mathrm{Ni}_{2}\left(\mathrm{~N}_{3}\right)_{2}\right]_{\mathbf{n}}\left(\mathrm{ClO}_{4}\right)_{\mathbf{n}} \cdot \mathbf{0 . 5 n C H} \mathrm{n}_{3} \mathrm{OH}(19)$}

Zur einer Lösung des Liganden $\mathbf{H L}^{2}$ (234 mg, $0.25 \mathrm{mmol}$ ) in $20 \mathrm{ml}$ Methanol werden nacheinander 1 Äquivalent $\mathrm{KO}^{t} \mathrm{Bu}(28 \mathrm{mg}, 0.25 \mathrm{mmol})$ und 2 Äquivalente $\mathrm{Ni}\left(\mathrm{ClO}_{4}\right)_{2} \cdot 6 \mathrm{H}_{2} \mathrm{O}$ (183 mg, $0.5 \mathrm{mmol}$ ) zugegeben. Nach einer Rührdauer von $1 \mathrm{~h}$ wird die Reaktionsmischung filtriert, dem Filtrat werden 2 Äquivalente $\mathrm{NaN}_{3}$ (33 mg, $0.5 \mathrm{mmol}$ ) zugesetzt. Es wird eine weitere Stunde gerührt und anschließend filtriert. Nach Überschichtung der Komplexlösung mit Diethylether wachsen, bedingt durch Diffusion, grüne Kristalle.

Ausbeute

$39 \mathrm{mg}, 20 \%$

\footnotetext{
1) Summenformel und Molmasse beziehen sich bei den Ketten auf die kristallographisch kleinste Einheit.
} 
Summenformel $\quad \mathrm{C}_{25.5} \mathrm{H}_{55} \mathrm{ClN}_{14} \mathrm{Ni}_{2} \mathrm{O}_{4.5}$

Molmasse, g/mol $\quad 782.6$

IR (KBr), $\mathrm{cm}^{-1} \quad 2130$ (s), 2067 (vs) $\left[\mathrm{v}\left(\mathrm{N}_{3}{ }^{-}\right)\right] ; 1464(\mathrm{w}) ; 1095$ (vs) $\left[\mathrm{v}\left(\mathrm{ClO}_{4}{ }^{-}\right)\right]$

Elementaranalyse, \% ～ber.: C 39.13, H 7.08, N 25.06; gef.: C 37.96, H 6.59, N 24.91

\section{$\left[\mathrm{L}^{3} \mathrm{Ni}_{2}\left(\mathrm{~N}_{3}\right)_{2}\left(\mathrm{H}_{2} \mathrm{O}\right)_{2}\right]_{\mathbf{n}}\left(\mathrm{ClO}_{4}\right)_{\mathbf{n}} \cdot \mathbf{n C}_{3} \mathrm{H}_{6} \mathrm{O}(20)$}

$\mathrm{Zu}$ einer Lösung des Liganden $\mathbf{H L}^{3}$ (148 $\mathrm{mg}, 0.5 \mathrm{mmol}$ ) in $20 \mathrm{~mL}$ Methanol werden 1 Äquivalent $\mathrm{KO}^{t} \mathrm{Bu}(56 \mathrm{mg}, 0.5 \mathrm{mmol})$ und 2 Äquivalente $\mathrm{Ni}\left(\mathrm{ClO}_{4}\right)_{2} \cdot 6 \mathrm{H}_{2} \mathrm{O}(365 \mathrm{mg}, 1.0$ mmol) zugegeben. Eine Stunde wird die Reaktionsmischung gerührt, dann ausgefallenes Kaliumperchlorat abfiltriert und das Filtrat trockengezogen. Die zurückbleibende grüne Substanz wird in $25 \mathrm{ml}$ Aceton aufgenommen und mit 1 Äquivalent $\mathrm{NaN}_{3}$ (33 mg, 0.5 mmol) versetzt, über Nacht gerührt, dann filtriert und das Filtrat anschließend mit Petrolether überschichtet. Es werden grün-blaue Kristalle erhalten.

$\begin{array}{ll}\text { Ausbeute } & 9 \mathrm{mg}, 5 \% \\ \text { Summenformel } & \mathrm{C}_{18} \mathrm{H}_{41} \mathrm{ClN}_{12} \mathrm{Ni}_{2} \mathrm{O}_{7} \\ \text { Molmasse, g/mol } & 690.4 \\ \text { IR (KBr), } \mathrm{cm}^{-1} & 2102(\mathrm{~s}), 2067(\mathrm{vs}), 2045(\mathrm{vs})\left[\mathrm{v}\left(\mathrm{N}_{3}{ }^{-}\right)\right] ; 1738(\mathrm{w})\left[\mathrm{v}(\mathrm{C}=\mathrm{O})_{\text {Aceton }}\right] ; \\ & 1463(\mathrm{w}) ; 1120(\mathrm{~s}), 1109(\mathrm{~s}), 1092(\mathrm{~s})\left[\mathrm{v}\left(\mathrm{ClO}_{4}{ }^{-}\right)\right]\end{array}$

\section{$\left[\mathrm{L}^{6} \mathrm{Ni}_{2}\left(\mathrm{~N}_{3}\right)_{2}\right]_{\mathbf{n}}\left(\mathrm{ClO}_{4}\right)_{\mathbf{n}} \cdot 2 \mathrm{nCH}_{2} \mathrm{Cl}_{2}(\mathbf{2 1})$}

Zu einer Lösung des Liganden HL $^{6}$ (480 mg, $1.0 \mathrm{mmol}$ ) in $50 \mathrm{~mL}$ Methanol werden 1 Äquivalent $\mathrm{KO}^{t} \mathrm{Bu}(112 \mathrm{mg}, 1.0 \mathrm{mmol})$ und 2 Äquivalente $\mathrm{Ni}\left(\mathrm{ClO}_{4}\right)_{2} \cdot 6 \mathrm{H}_{2} \mathrm{O}(731 \mathrm{mg}, 2.0$ mmol) zugegeben. Nach 1 h Rühren filtriert man das Kaliumperchlorat ab, fügt zu der grünen Lösung 2 Äquivalente $\mathrm{NaN}_{3}$ (130 mg, $2.0 \mathrm{mmol}$ ) hinzu und lässt weitere zwei Stunden rühren. Der entstandene grüne Niederschlag wird abfiltriert und erst mit Aceton und danach mit Dichlormethan gewaschen. Der Rückstand wird nun in $40 \mathrm{~mL}$ Dichlormethan aufgenommen und mit Petrolether überschichtet. Nach einigen Tagen bilden sich grüne Kristalle (Raumgruppe $P 2_{1} / n$ ).

Ausbeute

$398 \mathrm{mg}, 42 \%$

Summenformel

$\mathrm{C}_{23} \mathrm{H}_{45} \mathrm{Cl}_{5} \mathrm{~N}_{10} \mathrm{Ni}_{2} \mathrm{O}_{4} \mathrm{~S}_{4}$

Molmasse, g/mol

948.6 
IR (KBr), $\mathrm{cm}^{-1} \quad 3058(\mathrm{w})\left[\mathrm{v}(\mathrm{C}-\mathrm{H})_{\text {Dichlormethan }}\right] 2963(\mathrm{w}) ; 2929(\mathrm{w}) ; 2869$ (w); 2120 (sh), 2084 (s), 2039 (vs), 2008 (sh), [v( $\left.\left.\mathrm{N}_{3}{ }^{-}\right)\right] ; 1456$ (w); 1418 (w); 1091 (vs) $\left[\mathrm{v}\left(\mathrm{ClO}_{4}{ }^{-}\right)\right]$

Elementaranalyse, \% ber.: C 29.12, H 4.78, N 14.77; gef.: C 28.81, H 4.71, N 15.13

\section{$\left[\mathrm{L}^{6} \mathrm{Ni}_{2}\left(\mathrm{~N}_{3}\right)_{2}\right]_{\mathbf{n}}\left(\mathrm{ClO}_{4}\right)_{\mathrm{n}}(22)$}

22 wird analog zu 21 mit einer auf 20 ml reduzierten Dichlormethanmenge dargestellt. Die Konzentration des Komplexes in der Lösung ist demnach doppelt so groß. 22 kristallisiert in der Raumgruppe $P 2_{1} / c$. Durch relativ schnelle Abgabe von Dichlormethanmolekülen verwittern die Kristalle nach ihrer Isolierung.

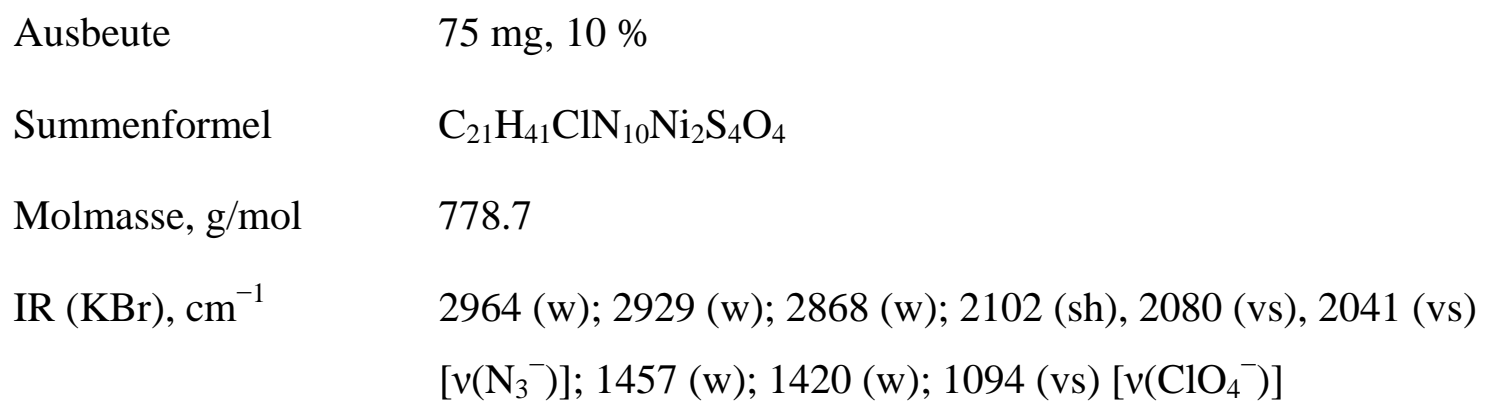

Elementaranalyse, \% ～ber.: C 32.39, H 5.31, N 17.99; gef.: C 32.27, H 5.37, N 17.62

\section{$\left[\mathrm{L}^{6} \mathrm{Ni}_{2}\left(\mathrm{~N}_{3}\right)_{2}\right]_{\mathbf{n}}\left(\mathrm{BPh}_{4}\right)_{\mathbf{n}} \cdot 0.58 \mathrm{nC}_{3} \mathrm{H}_{6} \mathrm{O}(23)$}

Zur einer Lösung des Liganden $\mathbf{H L}^{6}$ (240 mg, $0.5 \mathrm{mmol}$ ) in $30 \mathrm{ml}$ Methanol werden nacheinander 1 Äquivalent $\mathrm{KO}^{t} \mathrm{Bu}(56 \mathrm{mg}, 0.5 \mathrm{mmol})$ und 1 Äquivalent $\mathrm{Ni}\left(\mathrm{ClO}_{4}\right)_{2} \cdot 6 \mathrm{H}_{2} \mathrm{O}(183$ mg, $0.5 \mathrm{mmol}$ ) zugegeben. Nach einer Rührzeit von $1 \mathrm{~h}$ filtriert man das Kaliumperchlorat ab, fügt zu der grünen Lösung 2 Äquivalente $\mathrm{NaN}_{3}$ (65 mg, $1.0 \mathrm{mmol}$ ) hinzu, lässt weitere zwei Stunden rühren und gibt danach 1 Äquivalent $\mathrm{NaBPh}_{4}$ (171 mg, $\left.0.5 \mathrm{mmol}\right) \mathrm{zu}$. Nach weiterer einstündiger Rührdauer wird der hellgrüne Komplex abfiltriert, in $30 \mathrm{ml}$ Aceton gelöst und mit Petrolether überschichtet. Dadurch werden grüne Kristalle erhalten, welche nach Abtrennung aus der Mutterlauge durch partielle Abgabe von Lösungsmittelmolekülen verwittern.

Ausbeute

Summenformel

Molmasse, g/mol

IR $(\mathrm{KBr}), \mathrm{cm}^{-1}$
$70 \mathrm{mg}, 27 \%$

$$
\mathrm{C}_{46.74} \mathrm{H}_{64.48} \mathrm{BN}_{10} \mathrm{Ni}_{2} \mathrm{O}_{0.58} \mathrm{~S}_{4}
$$

1032.2

2092 (s), 2041 (vs), $\left[v\left(\mathrm{~N}_{3}{ }^{-}\right)\right] ; 1456(\mathrm{w}) ; 1423(\mathrm{w})$ 
Elementaranalyse, \% ～ber.: C 54.39, H 6.30, N 13.57; gef.: C 53.90, H 5.96, N 13.62

\section{$\left[\left(\mathrm{L}^{6}\right)_{2} \mathrm{Ni}_{4}\left(\mathrm{~N}_{3}\right)_{4}\right]_{\mathbf{n}}\left(\mathrm{ClO}_{4}\right)_{2 \mathrm{n}} \cdot \mathrm{nH}_{2} \mathrm{O} \cdot \mathrm{nCH}_{3} \mathrm{OH}(\mathbf{2 4})$}

Zur einer Lösung des Liganden $\mathbf{H L}^{6}$ (240 mg, $0.5 \mathrm{mmol}$ ) in $35 \mathrm{ml}$ Methanol werden nacheinander 1 Äquivalent $\mathrm{KO}^{t} \mathrm{Bu}(56 \mathrm{mg}, 0.5 \mathrm{mmol})$ und 2 Äquivalente $\mathrm{Ni}\left(\mathrm{ClO}_{4}\right)_{2} \cdot 6 \mathrm{H}_{2} \mathrm{O}$ (365 mg, $1.0 \mathrm{mmol}$ ) zugegeben. Nach einer Rührdauer von $1 \mathrm{~h}$ wird die Reaktionsmischung filtriert, dem Filtrat 1.2 Äquivalente $\mathrm{NaN}_{3}$ (39 mg, $0.6 \mathrm{mmol}$ ) zugesetzt und es wird weiter $2 \mathrm{~h}$ gerührt. Nach Überschichtung der Komplexlösung mit Diethylether entstehen nach einiger Zeit grüne Kristalle, die nach Isolierung aus der Mutterlauge sehr schnell verwittern.

Ausbeute $92 \mathrm{mg}, 38 \%$

Summenformel

$$
\mathrm{C}_{43} \mathrm{H}_{88} \mathrm{Cl}_{2} \mathrm{~N}_{20} \mathrm{Ni}_{4} \mathrm{O}_{10} \mathrm{~S}_{8}
$$

Molmasse, g/mol

1607.5

IR $(\mathrm{KBr}), \mathrm{cm}^{-1}$

2122(vs), 2043 (vs) [v( $\left.\left.\mathrm{N}_{3}^{-}\right)\right] ; 1461$ (w); 1422 (w); 1089 (vs)

$\left[v\left(\mathrm{ClO}_{4}^{-}\right)\right]$

Elementaranalyse, \% ～ber.: C 32.13, H 5.22, N 17.43; gef.: C 31.93, H 5.20, N 17.30

\subsubsection{Synthese der Kupfer(II)-Triazinkomplexe}

\section{$\left[\left(\mathrm{L}^{8}\right)_{2}(\mathrm{CuCl})_{3}\right]\left[\mathrm{CuCl}_{4}\right] \mathrm{Cl} \cdot \mathrm{CH}_{3} \mathrm{OH} \cdot 2 \mathrm{CH}_{2} \mathrm{Cl}_{2}(25)$}

Eine Lösung des Liganden $\mathbf{L}^{\mathbf{8}}$ (294.3 mg, $0.5 \mathrm{mmol}$ ) in $20 \mathrm{ml}$ Dichlormethan wird mit 20 ml einer 1:1 Mischung von Methanol und Dichlormethan und dann noch mit einer Lösung von $\mathrm{CuCl}_{2} \cdot 2 \mathrm{H}_{2} \mathrm{O}$ (255.8 mg, $1.5 \mathrm{mmol}$ ) in $20 \mathrm{~mL}$ Methanol überschichtet. Durch langsame Diffusion der in der ersten und dritten Schicht enthaltenen Komponenten zueinander ergeben sich grüne Kristalle. Diese verwittern sehr schnell nach der Isolierung des Komplexes aus der Mutterlauge, weil der Komplex Moleküle der Lösungsmittels abgibt.
Ausbeute
$422.0 \mathrm{mg}, 88 \%$
Summenformel
$\mathrm{C}_{69.5} \mathrm{H}_{57} \mathrm{~N}_{24} \mathrm{Cu}_{4} \mathrm{Cl}_{13} \mathrm{O}$
Molmasse, g/mol
1959.4
IR $(\mathrm{KBr}), \mathrm{cm}^{-1}$
1606 (m); 1579 (w); 1553 (m); 1482 (m); 1467 (m); 1320 (m);
1300(m); 1276 (m); 1252 (m); 1252 (m); 1160 (m)

$\mathrm{UV} / \mathrm{Vis} \lambda$, $\mathrm{nm}$

in DMSO: 835 (338); in MeOH: 681 (390) 


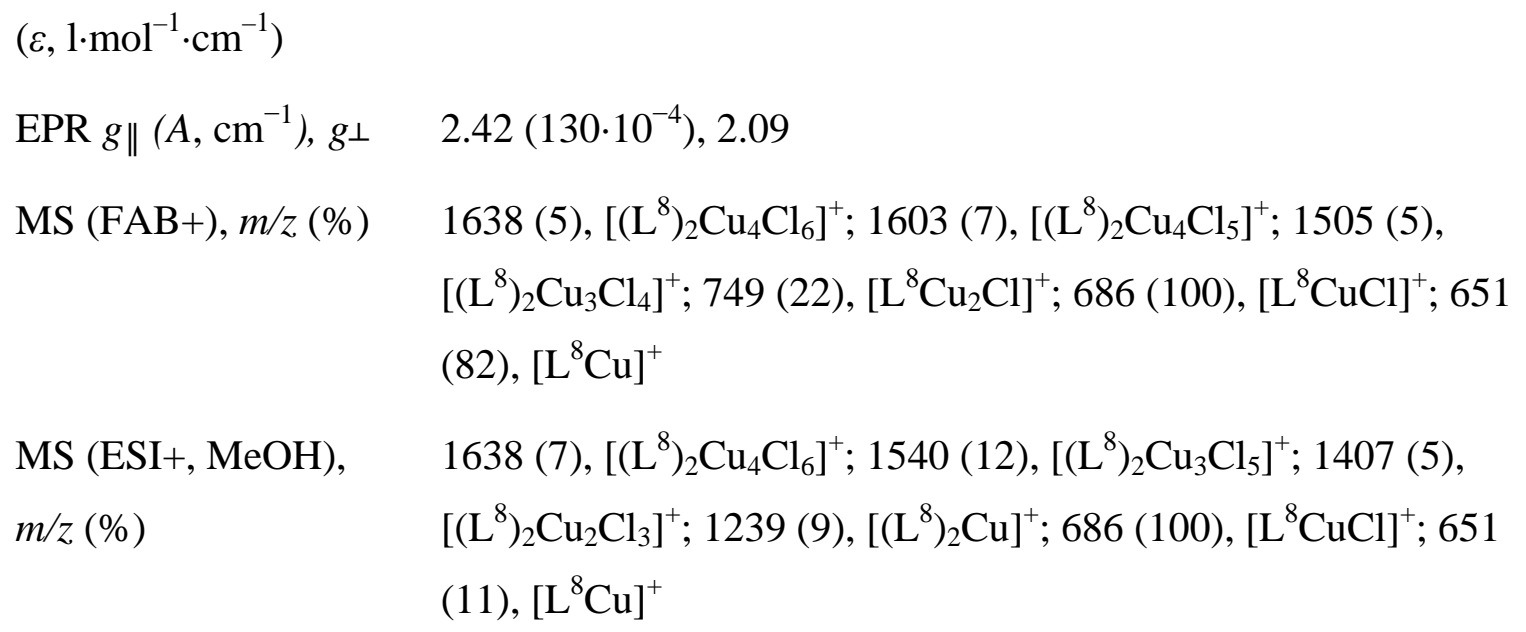

Elementaranalyse, \% ～ber.: C 42.60, H 2.93, N 17.16; gef.: C 42.58, H 2.97, N 17.49

\section{$\left[\mathrm{L}^{8}\left(\mathrm{CuCl}_{2}\right)_{3}(\mathrm{DMSO})_{3}\right](\mathbf{2 6})$}

Zu einer Lösung des Liganden $\mathbf{L}^{8}$ (294 mg, $0.5 \mathrm{mmol}$ ) in $40 \mathrm{ml}$ Dichlormethan werden 3 Äquivalente $\mathrm{CuCl}_{2} \cdot 2 \mathrm{H}_{2} \mathrm{O}$ (256 mg, $1.5 \mathrm{mmol}$ ) zugegeben. Nach $12 \mathrm{~h}$ Rühren filtriert man den ausgefallenen grünen Niederschlag ab und löst ihn unter Erwärmen in 25 ml DMSO. Nach Abkühlen auf Raumtemperatur wird diese Lösung vorsichtig mit $80 \mathrm{ml}$ Dichlormethan unterschichtet. Es bilden sich grüne Kristalle.

Ausbeute

Summenformel

Molmasse, g/mol

IR (KBr), $\mathrm{cm}^{-1}$

$\mathrm{UV} / \mathrm{Vis} \lambda$, $\mathrm{nm}$

$\left(\varepsilon, \mathrm{l} \cdot \mathrm{mol}^{-1} \cdot \mathrm{cm}^{-1}\right)$

EPR $g \|\left(A, \mathrm{~cm}^{-1}\right), g \perp \quad 2.42\left(124 \cdot 10^{-4}\right), 2.10$

MS (FAB+), m/z (\%) $1239(100),\left[\left(\mathrm{L}^{8}\right)_{2} \mathrm{Cu}\right]^{+} ; 847$ (18), $\left[\mathrm{L}^{8} \mathrm{Cu}_{3} \mathrm{Cl}_{2}\right]^{+} ; 749$ (35), $\left[\mathrm{L}^{8} \mathrm{Cu}_{2} \mathrm{Cl}\right]^{+}$; 686 (100), $\left[\mathrm{L}^{8} \mathrm{CuCl}\right]^{+} ; 651(78),\left[\mathrm{L}^{8} \mathrm{Cu}\right]^{+}$

Elementaranalyse, \% ～ber.: C 38.20, H 3.45, N 13.71; gef.: C 37.67, H 3.45, N, 13.73 (m); $1276(\mathrm{~m}) ; 1257$ (m); 1160 (m)

in DMSO: 864 (198)

\section{$\left[\mathrm{L}^{8}\left(\mathrm{CuCl}_{2}\right)_{2} \mathrm{CuCl}_{2}(\mathrm{DMSO})\right]_{\mathbf{n}} \cdot \mathrm{nDMSO} \cdot \mathrm{nCH}_{2} \mathrm{Cl}_{2}(27)$}

Zu einer Lösung des Liganden $\mathbf{L}^{\mathbf{8}}$ (100 mg, $0.17 \mathrm{mmol}$ ) in $20 \mathrm{ml}$ heißem DMSO ( ca. $150^{\circ}$ ) werden 4 Äquivalente $\mathrm{CuCl}_{2} \cdot 2 \mathrm{H}_{2} \mathrm{O}$ (119 mg, $\left.0.70 \mathrm{mmol}\right)$ zugegeben. Nach Abkühlen 
auf Raumtemperatur wird die Lösung mit $80 \mathrm{ml}$ Dichlormethan unterschichtet. Durch langsame Diffusion erhält man grüne Kristalle, die nach Isolierung langsam verwittern.

Ausbeute $93 \mathrm{mg}, 44 \%$

Summenformel

$\mathrm{C}_{38} \mathrm{H}_{38} \mathrm{Cl}_{8} \mathrm{Cu}_{3} \mathrm{~N}_{12} \mathrm{O}_{2} \mathrm{~S}_{2}$

Molmasse, g/mol

1233.2

IR $(\mathrm{KBr}), \mathrm{cm}^{-1}$ 1608 (m); 1580 (w); 1552 (m); 1479 (m); 1467 (m); 1324 (m); 1299 (m); 1276 (m); 1257 (m); 1157 (m)

$\mathrm{UV} / \mathrm{Vis} \lambda$, $\mathrm{nm}$ in DMSO: 855 (237); in MeOH): 692 ( 223) $\left(\varepsilon, \mathrm{l} \cdot \mathrm{mol}^{-1} \cdot \mathrm{cm}^{-1}\right)$ EPR $g \|\left(A, \mathrm{~cm}^{-1}\right), g \perp \quad 2.42\left(128 \cdot 10^{-4}\right), 2.09$; (bei ca. $g=4$ breites Halbfeldsignal) MS (FAB+), m/z (\%) 847 (24), $\left[\mathrm{Cu}_{3} \mathrm{~L}^{8} \mathrm{Cl}_{2}\right]^{+} ; 814$ (52), $\left[\mathrm{Cu}_{3} \mathrm{~L}^{8} \mathrm{Cl}\right]^{+} ; 749$ (48), $\left[\mathrm{Cu}_{2} \mathrm{~L}^{8} \mathrm{Cl}\right]^{+}$; 686 (45), $\left[\mathrm{CuL}^{8} \mathrm{Cl}\right]^{+} ; 651$ (100), $\left[\mathrm{CuL}^{8}\right]^{+}$

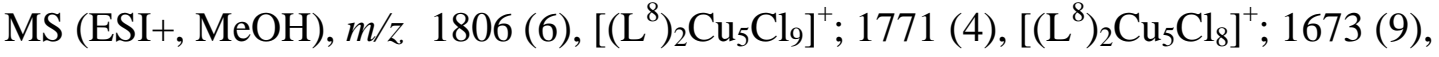
$\left[\left(\mathrm{L}^{8}\right)_{2} \mathrm{Cu}_{4} \mathrm{Cl}_{7}\right]^{+} ; 1638(5),\left[\left(\mathrm{L}^{8}\right)_{2} \mathrm{Cu}_{4} \mathrm{Cl}_{6}\right]^{+} ; 1540(11),\left[\left(\mathrm{L}^{8}\right)_{2} \mathrm{Cu}_{3} \mathrm{Cl}_{5}\right]^{+}$; 686 (100), $\left[\mathrm{L}^{8} \mathrm{CuCl}\right]^{+}$

Elementaranalyse, \% ～ber.: C 37.01, H 3.11, N 13.63; gef.: C 36.71, H 3.15, N 13.23

\subsection{Magnetische Untersuchungen}

Für die Untersuchung der magnetischen Eigenschaften wurde ein MPMS-5S-SQUIDMagnetometer (Superconducting Quantum Interference Device) der Firma Quantum-Design verwendet. SQUID's sind die empfindlichsten, heute verfügbaren, Detektoren für magnetische Felder. Ihre Funktion beruht auf dem physikalischen Effekt der Supraleitung und der Verdrängung von Magnetfeldern aus einem Supraleiter. Dabei wird der magnetische Fluß in eine Spannung umgewandelt. ${ }^{[162]}$ Das im Rahmen dieser Arbeit verwendete Gerät erlaubt Temperaturen zwischen $1.7 \mathrm{~K}$ und $400 \mathrm{~K}$ und homogene Magnetfelder bis zu $5 \mathrm{~T}$.

\subsubsection{Probenvorbereitung}

Für die magnetischen Messungen wurden die kristallinen Komplexe pulverisiert. Die optimale Einwaage der zu untersuchenden Verbindungen liegt bei 10-20 mg Substanz. Einige Verbindungen wurden aber auch mit nur 3-4 mg Probenmasse gemessen. Die pulverförmigen 
Proben wurden in handelsüblichen Gelatinekapseln (Größe 4; 5 mm Durchmesser) präpariert (siehe Bild). Der obere Teil (kleinerer Durchmesser) wurde gekürzt, um den diamagnetischen Beitrag der Kapsel zu verringern. ${ }^{[163]}$ Die Gelatinekapsel wird in einem Kunststoffröhrchen (Strohalm) fixiert und dieses am Probenhalter des SQUIDMagnetometers befestigt. Bei luftempfindlichen Substanzen wurde die Probenvorbereitung in einer Glove-Bag unter $\mathrm{N}_{2}$-Schutzgasatmosphäre durchgeführt. Die Proben wurden in einem Schlenkgefäß zum SQUID-Magnetometer transportiert.

\subsubsection{Magnetische Messungen}

Bei allen Verbindungen wurden grundsätzlich folgenden Messungen durchgeführt: die magnetische Suszeptibilität wurde bei zwei Feldern, nämlich 5000 Oe bzw. 2000 Oe, im Temperaturbereich zwischen 300 K bzw. 295 K und 2 K, die Sättigungsmagnetisierung bei 2 $\mathrm{K}$ und $5 \mathrm{~K}$ im Bereich von $0 \mathrm{~T}$ bis $5 \mathrm{~T}$ gemessen.

Die Suszeptibilitätmessung bei zwei Feldern dient neben der Möglichkeit wissenschaftlich relevanten Phänomene (z.B. Ferromagnetismus) abzucheken auch der zusätzlichen Kontrolle der Reinheit der Probe, weil bei molekülbasierenden Verbindungen sowie bei niederdimensionalen Systemen in der Regel keine signifikante Feldabhängigkeit beobachtet werden sollte. Das trifft auf die in dieser Arbeit untersuchten Verbindungen zu. Die manchmal beobachtete Feldabhängigkeit der Suszeptibilität war verursacht durch Verunreinigungen und konnte durch die Umkristallisation beseitigt werden.

Die Messung von Raumtemperatur abwärts zu tieferen Temperaturen ermöglicht den Verbleib der im Gitter eingebauten Lösungsmittelmoleküle. Bei einigen Verbindungen des „Chamäleon-Systems“ wurden die Messungen bis $400 \mathrm{~K}$ durchgeführt. Das erlaubt die Beobachtung interessanter magnetischer Effekte und gestattet die Untersuchung des Einflusses eingebauter Lösungsmittelmoleküle.

\subsubsection{Auswertung von Suszeptibilitätsmessungen}

Für die Auswertung der temperaturabhängigen magnetischen Messungen müssen die erhaltenen Rohdaten zuerst um die Werte des Probenhalters korrigiert werden. Für die Ermittlung der molaren Suszeptibilität benötigt man die genaue Einwaage, das Molekulargewicht und die angelegte Feldstärke. Dann wird die molare Suszeptibilität um den diamagnetischen Beitrag der untersuchten Probe korrigiert, der sowohl aus experimentellen 
Daten entsprechender diamagnetischer Verbindungen als auch aus den Inkrementen ${ }^{[164]}$ bestimmt werden kann (Verwendung der Pascal-Konstanten bzw. der Inkrementmethode nach Haberditzl).

\subsubsection{Anpassung an die experimentellen Daten}

Die erhaltene Suszeptibilität kann nach Gleichung 3.1.6 in das effektive magnetische Moment umgerechnet werden. Der dabei aus dem Experiment erhaltene Wert wird mit einem theoretisch erwarteten, dem so genannten „spin-only“ Wert verglichen. Der „spin-only“ Wert für Verbindungen mit Wechselwirkungen zwischen paramagnetischen Zentren wird nach Gleichung (3.1.7) berechnet, in welcher $S$ der Gesamtspin ist.

Für den Fall von beispielsweise zwei paramagnetischen Zentren ohne Wechselwirkungen ergibt sich der „spin-only“ Wert nach folgender Gleichung:

$$
\mu_{\text {eff }}=g \sqrt{S_{A}\left(S_{A}+1\right)+S_{B}\left(S_{B}+1\right)} \mu_{\beta}
$$

Die Anpassung an die Daten der magnetischen Messungen der zweikernigen Nickel(II)Komplexe wurde mit dem Programm ORIGIN durchgeführt. Die nichtlineare Regression basiert auf der Methode der kleinsten Quadrate und wurde unter Verwendung des LEVENBERG-MARQUARDT-Algoritmus nach Gleichung 6.4.4.2 durchgeführt.

$$
\chi_{M}=\frac{2 N g^{2} \beta^{2}}{k T} \frac{e^{2(J / k T)}+5 e^{6(J / k T)}}{1+3 e^{2(J / k T)}+5 e^{6(J / k T)}}(1-\rho)+\frac{2 N g^{2} \beta^{2}}{3 k T} \rho+T I P
$$

Als Ergebnis werden dabei der Austauschparameter $J$ sowie der $g$-Faktor, der Molanteil der Verunreinigungen $\rho$ und der Beitrag des temperaturunabhängigen Paramagnetismus TIP ermittelt. Unter Verunreinigung versteht man in diesem Zusammenhang einen sehr kleinen Anteil paramagnetischer Spezies in der Probe. Es wird vorausgesetzt, dass die Verunreinigung Curie-Verhalten zeigt, dieselbe molare Masse und denselben $g$-Faktor wie die untersuchte Verbindung hat. Um den TIP zu berücksichtigen, wird ein additives Glied hinzugefügt.

Die Analyse der magnetischen Daten für einige zwei- und vierkernige Nickel(II)Komplexe und Ketten wurde mit Hilfe der verschiedenen, an den entsprechenden Stellen erwähnten Programme durchgeführt. Sehr oft erfolgten dabei die Anpassungen mit dem 
Programm julX. Dieses Programm wurde von Dr. E. Bill, Max-Planck-Institut für Bioanorganische Chemie, Mülheim an der Ruhr, zur Verfügung gestellt.

julX simuliert auf der Basis des üblichen Spin-Hamiltonoperator-Formalismus molare magnetische Suszeptibilitäten für paramagnetische Systeme von bis $\mathrm{zu}$ vier Spins mit isotropen Austauschwechselwirkungen und lokalen axialen und rhombischen Nullfeldwechselwirkungen. Die verwendeten Heisenberg-Dirac-van-Vleck-Operatoren für die Austauschwechselwirkung sind an entsprechenden Stellen im Text angegeben. Die ZeemanWechelwirkung wird richtungsabhängig berücksichtigt und für Pulverproben entweder über die drei kanonischen Hauptachsen oder über ein 16-Punkte Lebedev-Gitter gemittelt. Die Diagonalisierung der Hamiltonmatrix erfolgt numerisch mit der LAPACK Routine ZHEEV. Magnetische Momente werden durch numerische Ableitung der Energien nach dem Feld berechnet. Einzelne oder mehrere Parameter der Hamiltonoperatoren können mit der Simplexroutine AMOEBA von NUMERICAL RRECIPIES automatisch optimiert werden, wobei paarweise Korrelation von beliebigen Variablen möglich sind. Für Visualisierungszwecke können Energiediagramme geplottet werden und für systematische Fehlerbetrachtungen können Fehler-Kontourplots oder Fehlerflächen für beliebige VariablenPaare gerechnet werden.

Die Anpassung an die Daten der magnetischen Messungen für die Kupfer(II)-TriazinKomplexe wurde mit dem Programm ORIGIN durchgeführt. Die dazu verwendeten Gleichungen sind an den entsprechenden Stellen angegeben. 


\subsection{Kristalldaten und Angaben zur Struktur im Festkörper}

1, 11a, 11b, 11c; $;^{[104]} 2,12,17 ; ;^{[105]} 5,6,18,21,22,23 ;{ }^{[107]} 9,{ }^{[120]} 13 a, 13 b, 13 c ;{ }^{[108]} 14 a$, 14b, 14e, 15b; ${ }^{[111]} 14 c,{ }^{[26]} 25,{ }^{[127]} 26,27 .^{[128]}$

\begin{tabular}{|c|c|c|c|}
\hline & 3 & 4 & 7 \\
\hline Formel & $\mathrm{C}_{23} \mathrm{H}_{43} \mathrm{Cl}_{2} \mathrm{~N}_{13} \mathrm{Ni}_{2} \mathrm{O}_{8}$ & $\mathrm{C}_{15} \mathrm{H}_{29} \mathrm{~N}_{9} \mathrm{Ni}_{2} \mathrm{O}_{6} \mathrm{~S}_{2}$ & $\mathrm{C}_{21.50} \mathrm{H}_{43} \mathrm{~N}_{13} \mathrm{Ni}_{2} \mathrm{O}_{0.50} \mathrm{~S}_{4}$ \\
\hline$M, \mathrm{~g} / \mathrm{mol}$ & 818.02 & 613.01 & 737.35 \\
\hline Kristallgröße, mm & $0.39 \times 0.30 \times 0.21$ & $0.39 \times 0.34 \times 0.22$ & $0.18 \times 0.15 \times 0.13$ \\
\hline Kristallsystem & Triklin & Monoklin & Triklin \\
\hline Raumgruppe & $P \overline{1}$ & $C 2 / c$ & $P \overline{1}$ \\
\hline$a, \AA$ & $12.1031(5)$ & $12.7751(8)$ & $8.6530(6)$ \\
\hline$b, \AA$ & $13.1282(6)$ & $9.4749(9)$ & $11.9457(9)$ \\
\hline$c, \AA$ & $22.7377(10)$ & $20.4706(13)$ & $16.0004(12)$ \\
\hline$\alpha,{ }^{\circ}$ & $85.251(4)$ & 90 & $96.065(6)$ \\
\hline$\beta,{ }^{\circ}$ & $86.010(4)$ & $103.589(5)$ & $100.102(6)$ \\
\hline$\gamma,{ }^{\circ}$ & 87.899(4) & 90 & $100.591(6)$ \\
\hline$V, \mathrm{~cm}^{3}$ & $3590.0(3)$ & 2408.5(3) & $1584.2(2)$ \\
\hline$Z$ & 4 & 4 & 2 \\
\hline$\rho_{\text {ber., }} \mathrm{g} / \mathrm{cm}^{3}$ & 1.514 & 1.6913 & 1.546 \\
\hline$T, \mathrm{~K}$ & $133(2)$ & $133(2)$ & $133(2)$ \\
\hline$h k l$-Bereich & $\pm 14, \pm 15, \pm 26$ & -15 bis $14, \pm 11,-22$ bis 24 & $\pm 10,-14$ bis $13, \pm 18$ \\
\hline$\theta$-Bereich, ${ }^{\circ}$ & 1.69 bis 24.71 & 2.05 bis 24.80 & 1.75 bis 24.84 \\
\hline Gemessene Reflexe & 63825 & 11111 & 17578 \\
\hline Unabhängige Refl. $\left(R_{\text {int }}\right)$ & $12164(0.0522)$ & $2061(0.0325)$ & $5433(0.0544)$ \\
\hline Verfeinerte Parameter & 856 & 157 & 413 \\
\hline Goodness-of-fit & 1.034 & 1.032 & 1.004 \\
\hline Restelektronendichte, e $/ \AA^{3}$ & $1.164 /-0.775$ & $0.330 /-0.243$ & $0.288 /-0.533$ \\
\hline$R 1(I>2 \sigma(I))$ & 0.0507 & 0.0247 & 0.0316 \\
\hline$w R 2$ (alle Daten) & 0.1397 & 0.0597 & 0.0717 \\
\hline
\end{tabular}


Experimenteller Teil

\begin{tabular}{|c|c|c|c|}
\hline & 8 & 10 & 16 \\
\hline Formel & $\mathrm{C}_{25} \mathrm{H}_{53} \mathrm{~N}_{13} \mathrm{Ni}_{2} \mathrm{O}_{2} \mathrm{~S}_{6}$ & $\mathrm{C}_{21} \mathrm{H}_{41} \mathrm{~N}_{13} \mathrm{Ni}_{2} \mathrm{~S}_{4}$ & $\mathrm{C}_{34} \mathrm{H}_{70} \mathrm{~N}_{30} \mathrm{Ni}_{4}$ \\
\hline$M, \mathrm{~g} / \mathrm{mol}$ & 877.58 & 721.33 & 1134.04 \\
\hline Kristallgröße, mm & $0.20 \times 0.12 \times 0.09$ & $0.50 \times 0.46 \times 0.43$ & $0.36 \times 0.32 \times 0.19$ \\
\hline Kristallsystem & Monoklin & Triklin & Monoklin \\
\hline Raumgruppe & $C 2 / c$ & $P \overline{1}$ & $C 2 / c$ \\
\hline$a, \AA$ & $25.4129(15)$ & $8.6294(5)$ & $25.113(2)$ \\
\hline$b, \AA$ & $12.2651(7)$ & $11.8874(7)$ & $10.0778(5)$ \\
\hline$c, \AA$ & 13.8943(8) & $15.8256(10)$ & $21.0736(14)$ \\
\hline$\alpha,^{\circ}$ & 90 & $96.129(5)$ & 90 \\
\hline$\beta, \circ$ & $116.8370(10)$ & $99.241(5)$ & $113.518(5)$ \\
\hline$\gamma, \circ$ & 90 & $100.496(5)$ & 90 \\
\hline$V, \mathrm{~cm}^{3}$ & $3864.3(4)$ & 1559.98(16) & $4890.3(6)$ \\
\hline$Z$ & 4 & 2 & 4 \\
\hline$\rho_{\text {ber. }}, \mathrm{g} / \mathrm{cm}^{3}$ & 1.508 & 1.536 & 1.540 \\
\hline$T, \mathrm{~K}$ & 293(2) & $133(2)$ & $133(2)$ \\
\hline$h k l$-Bereich & -33 bis 29,0 bis 15,0 bis18 & $\pm 11,-13$ bis $15, \pm 20$ & $\pm 29, \pm 11, \pm 24$ \\
\hline$\theta$-Bereich, ${ }^{\circ}$ & 1.80bis 28.28 & 1.76 bis 27.28 & 1.77 bis 24.72 \\
\hline Gemessene Reflexe & 13239 & 30699 & 12846 \\
\hline Unabhängige Refl. ( $\left.R_{\text {int }}\right)$ & $4585(0.032)$ & $6943(0.0361)$ & $4130(0.0368)$ \\
\hline Verfeinerte Parameter & 325 & 385 & 313 \\
\hline Goodness-of-fit & 0.967 & 1.060 & 1.018 \\
\hline Restelektronendichte, e/ $\AA^{3}$ & $0.555 /-0.254$ & $0.883 /-0.424$ & $0.303 /-0.202$ \\
\hline$R 1(I>2 \sigma(I))$ & 0.0307 & 0.0266 & 0.0272 \\
\hline$w R 2$ (alle Daten) & 0.0735 & 0.0731 & 0.0622 \\
\hline
\end{tabular}


Experimenteller Teil

\begin{tabular}{|c|c|c|c|}
\hline & 19 & 20 & 24 \\
\hline Formel & $\mathrm{C}_{25.50} \mathrm{H}_{55} \mathrm{ClN}_{14} \mathrm{Ni}_{2} \mathrm{O}_{4.50}$ & $\mathrm{C}_{18} \mathrm{H}_{41} \mathrm{ClN}_{12} \mathrm{Ni}_{2} \mathrm{O}_{7}$ & $\mathrm{C}_{42} \mathrm{H}_{82} \mathrm{Cl}_{2} \mathrm{~N}_{20} \mathrm{Ni}_{4} \mathrm{O}_{8} \mathrm{~S}_{8}$ \\
\hline$M, \mathrm{~g} / \mathrm{mol}$ & 782.71 & 690.50 & 1557.50 \\
\hline Kristallgröße, mm & $0.24 \times 0.22 \times 0.18$ & $0.43 \times 0.28 \times 0.26$ & $0.41 \times 0.33 \times 0.25$ \\
\hline Kristallsystem & Monoklin & Monoklin & Monoklin \\
\hline Raumgruppe & $C 2 / c$ & $P 2_{1} / n$ & $P 2 / C$ \\
\hline$a, \AA$ & $20.7829(10)$ & $12.9789(5)$ & $12.5218(8)$ \\
\hline$b, \AA$ & $14.1370(6)$ & 11.1834(6) & $20.6000(9)$ \\
\hline$c, \AA$ & $25.7571(14)$ & $20.7165(10)$ & $13.9506(10)$ \\
\hline$\alpha,^{\circ}$ & 90 & 90 & 90 \\
\hline$\beta,^{\circ}$ & $108.214(4)$ & $98.125(4)$ & $95.436(6)$ \\
\hline$\gamma^{\circ}{ }^{\circ}$ & 90 & 90 & 90 \\
\hline$V, \mathrm{~cm}^{3}$ & $7188.5(6)$ & $2976.8(2)$ & $3582.4(4)$ \\
\hline$Z$ & 8 & 4 & 2 \\
\hline$\rho_{\text {ber. }}, \mathrm{g} / \mathrm{cm}^{3}$ & 1.446 & 1.541 & 1.444 \\
\hline$T, \mathrm{~K}$ & 133(2) & 133(2) & 133(2) \\
\hline hkl-Bereich & $\pm 24, \pm 16, \pm 30$ & -15 bis $14, \pm 13, \pm 24$ & $\pm 11,-13$ bis $15, \pm 20$ \\
\hline$\theta$-Bereich, ${ }^{\circ}$ & 1.66 bis 24.65 & 1.75 to 24.65 & 1.63 bis 24.70 \\
\hline Gemessene Reflexe & 21540 & 29408 & 18545 \\
\hline Unabhängige Refl. ( $\left.R_{\text {int }}\right)$ & $6051(0.0435)$ & $5031(0.0527)$ & $6079(0.0449)$ \\
\hline Verfeinerte Parameter & 432 & 385 & 350 \\
\hline Goodness-of-fit & 1.054 & 1.042 & 1.019 \\
\hline Restelektronendichte, e/ $\AA^{3}$ & $0.984 /-0.782$ & $0.650 /-0.378$ & $0.695 /-0.673$ \\
\hline$R 1(I>2 \sigma(I))$ & 0.0419 & 0.0347 & 0.0443 \\
\hline wR2 (alle Daten) & 0.1106 & 0.0761 & 0.1042 \\
\hline
\end{tabular}




\section{Zusammenfassung}

Die vorliegende Arbeit befasst sich mit dem Aufbau zweikerniger Nickel(II)-Komplexe und dreikerniger Kupfer(II)-Komplexe sowie mit der Anwendung solcher Bausteine im Bereich des molekularen Magnetismus für die Synthese neuer Klassen von Verbindungen mit interessanten magnetischen Eigenschaften.

Der erste Teil der Arbeit betrifft Nickel(II)-Azid-Komplexe und deren magnetische Eigenschaften. Die wichtigste Aufgabenstellung in der Nickel(II)-Azid-Chemie besteht im rationalen Design der Verbindungen mit vorhersagbaren, also steuerbaren magnetischen Eigenschaften. Dazu ist die synthetische Kontrolle des Azidkoordinationsmodus, der für die magnetischen Eigenschaften bestimmend ist, notwendig. Erst dann ist der gezielte Aufbau der oligo- und polymeren Komplexe unterschiedlicher Topologie möglich. Da die zweikernigen Komplexe die kleinsten verbrückten Einheiten sind, ist es wichtig, die Koordination des Azids in solchen Komplexen vorbestimmen zu können.

Für die Synthese zweikerniger Nickel(II)-Komplexe wurden die PyrazolDikompartimentliganden $\mathbf{H L}^{\mathbf{1 - 7}}$ (siehe z.B. Formelübersicht) dargestellt. $\mathbf{H L}^{\mathbf{1 - 3 , 6}}$ sind bereits bekannt, ${ }^{[24 a, 97,98]} \mathbf{H L}^{4,5,7}$ wurden erstmals synthetisiert. Für die magnetischen Eigenschaften, die von der Struktur abhängig sind, ist entscheidend, das Ligandgerüst so zu variieren, dass dabei, je nach Wunsch, der Azidligand zwischen den beiden Nickel(II)-Ionen entweder $\mu_{1,1}$ oder $\mu_{1,3}$ verbrückt wird. Der Koordinationsmodus des Azidliganden innerhalb der bimetallischen Tasche der präorganisierten Dinickel(II)-Anordnung - und somit auch die magnetischen Eigenschaften - ist, wie gezeigt werden konnte, in erster Linie abhängig von der Länge der Ligandseitenarme. Während die Liganden mit langen Seitenarmen die zweikernigen Komplexe mit $\mu_{1,1}$-Azidverbrückung $(\mathbf{1}, \mathbf{2})$ ergeben, führen die Liganden mit kurzen Seitenarmen zu Verbindungen mit $\mu_{1,3}$-Azidverbrückung (3-7, 9 und 10). 


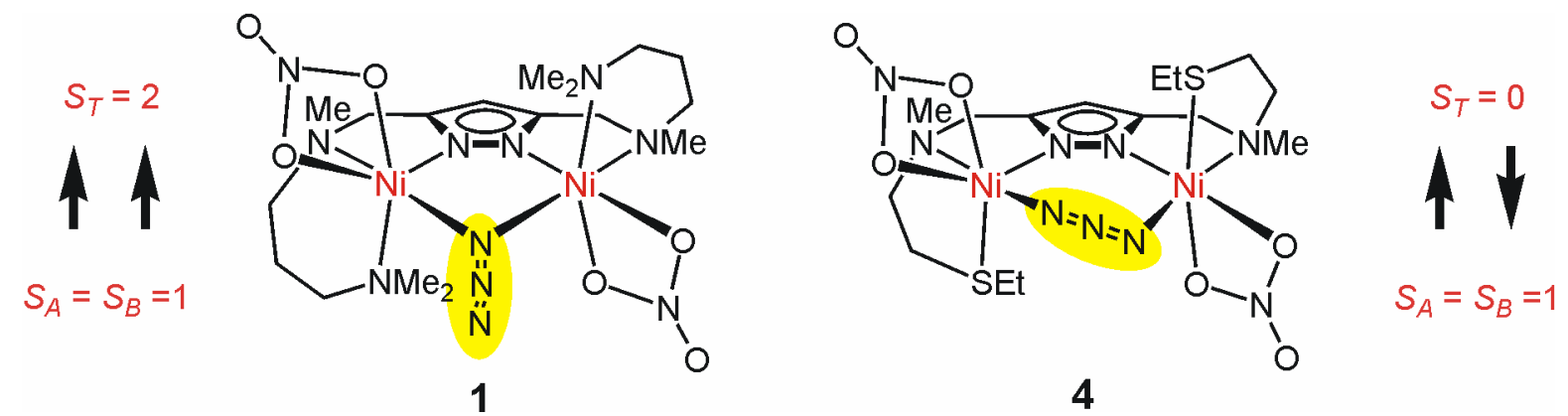

Lange Seitenarme bedingen einen kurzen Abstand zwischen den Nickel(II)-Ionen, kurze dagegen einen größeren. Mit $\mathbf{H L}^{\mathbf{6}}$ wurden die zweikernigen Komplexe 5-10 erhalten. Vergleicht man $\mathbf{7}$ und $\mathbf{8}$, so stellt man bei gleicher Zusammensetzung und Länge der Seitenarme für $7 \mu_{1,3^{-}}$, für $8 \mu_{1,1}$-Koordination fest. Für diesen Ausnahmefall (sowie auch für die Kette 24) sind offensichtlich auch die Reaktions- und Kristallisationsbedingungen von großer Bedeutung, weil die weichen S-Donoratome der Seitenarme sehr flexibel sind und den Abstand zwischen den Metallionen nicht mehr so streng wie Liganden mit ausschließlich NDonoratomen vorgeben können.

Es hat sich gezeigt, dass die hohe Flexibilität der Azidbrücke für die magnetischen Eigenschaften eine wesentliche Rolle spielt. Dadurch ist die Herstellung von Materialien mit magnetischer Bistabilität mit Hysterese möglich. Die Temperaturen, bei denen dieses Phänomen auftritt, sind unterschiedlich. Für 9 liegt dafür die Temperatur bei 220 K, für 10 bei 380 K. Im Falle von 9 konnte mit Hilfe der Röntgenstrukturanalyse festgestellt werden, dass das verbrückende Azid als wohldefinierter molekularer Schalter fungiert.

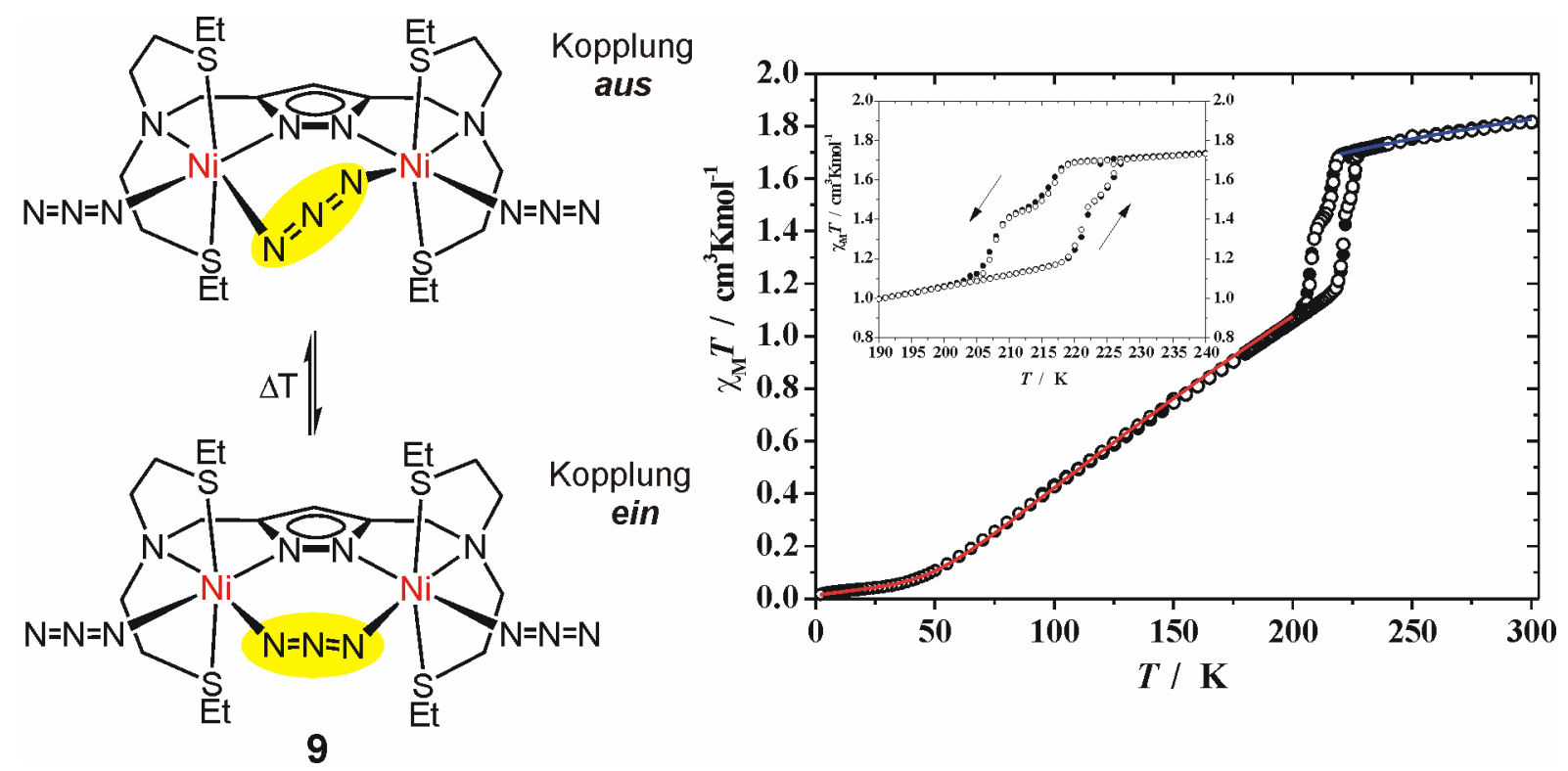


Die pyrazolatbasierten bimetallischen Einheiten, die terminale schwach gebundene Lösungsmittelmoleküle oder Gegenionen besitzen, können als Bausteine für die Darstellung von tetranuklearen Spezies verschiedener Topologie eingesetzt werden. Beispielweise konnte 11c ausschließlich ausgehend von 1 in sauberer Form synthetisiert werden.

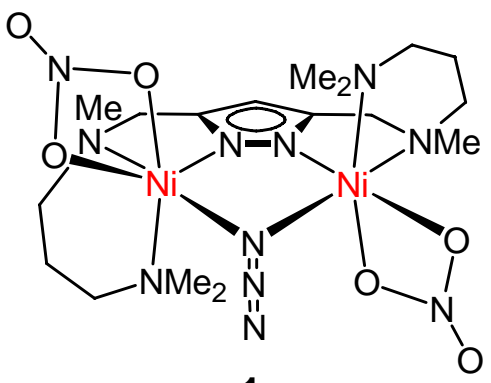

1
1) $\mathrm{NaN}_{3}$

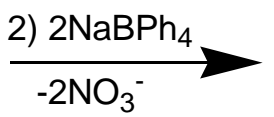

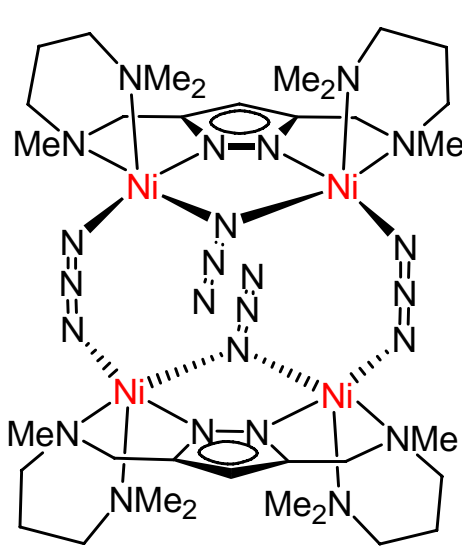

$2+$

$\left.\left(\mathrm{BPh}_{4}\right)_{2}\right)_{2}$ 11c

Es wurde festgestellt, dass die interdimere Kopplung zwischen den pyrazolatbasierten Einheiten streng von den geometrischen Parametern der interdimeren Verbrückung, in erster Linie vom Ni-NNN-Ni Torsionswinkel, abhängt. Dieser ist allerdings nicht vorhersagbar. Für vierkernige Komplexe 11a-c und 12 steuert der interdimere Ni-NNN-Ni Torsionswinkel den Spingrundzustand. Komplexe 11a,b sind zwei seltene Beispiele für molekulare Komplexe, in welchen der Ni-NNN-Ni Torsionswinkel fast exakt $90^{\circ}$ ist. In 12 kann der Spingrundzustand durch die Entfernung von den im Kristallgitter eingebauten Lösungsmittelmolekülen von $S_{T}=0$ zu $S_{T}=4$ geändert werden (siehe Abbildung). Dabei ändert sich offensichtlich der interdimere Ni-NNN-Ni Torsionswinkel von $36^{\circ}$ auf ca. $90^{\circ}$. 


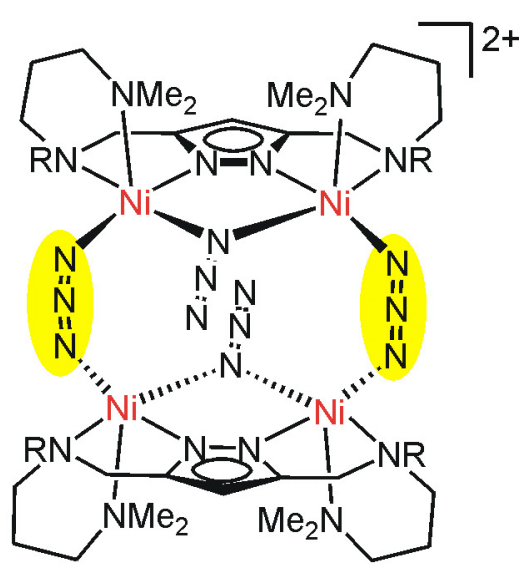

11a-c, 12

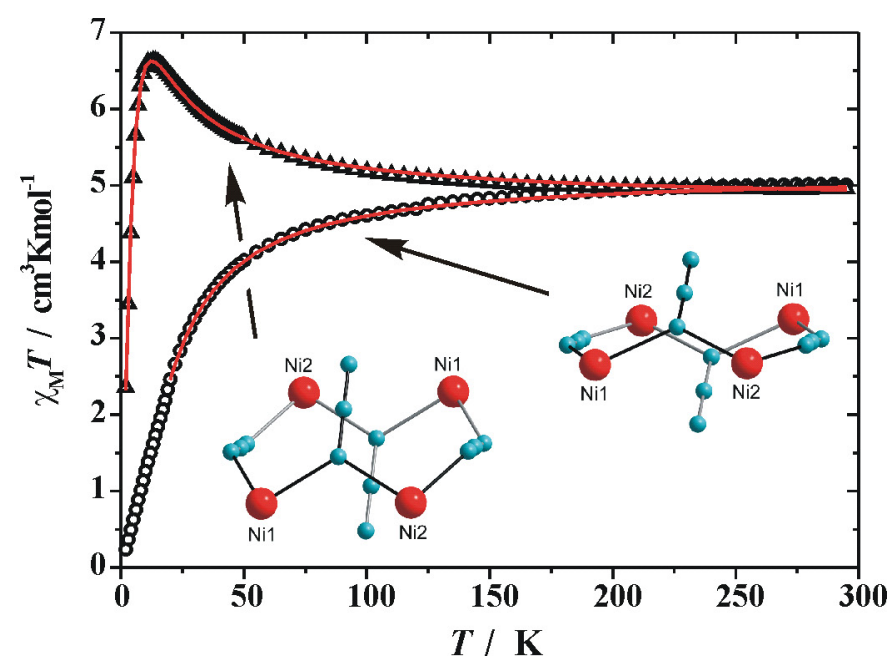

Neben den vierkernigen Komplexen mit rechteckiger Anordnung der Nickel(II)-Ionen mit $\mu$-1,1- und $\mu$-1,3-Azidbrücken (11a-c, 12) gelang auch die Synthese einer ganzen Reihe von vierkernigen Nickel(II)-Verbindungen ungewöhnlicher Topologie mit sehr seltenen $\mu_{3}-1,1,3-$ Azidbrücken. Es sind die Verbindungen 13a-c, welche auch magnetochemisch untersucht wurden. Neben 13 wurden auch für 16 und $18 \mu_{3}$-1,1,3-Azidbrücken gefunden. Die magnetostrukturellen Korrelationen für die $\mu_{3}$-1,1,3-Azidbrücke können als Summe der Wechselwirkungen der einzelnen end-to-end und end-on Fragmente formuliert werden und sind für 13b in folgendem Kopplungsschema zusammengefasst. 


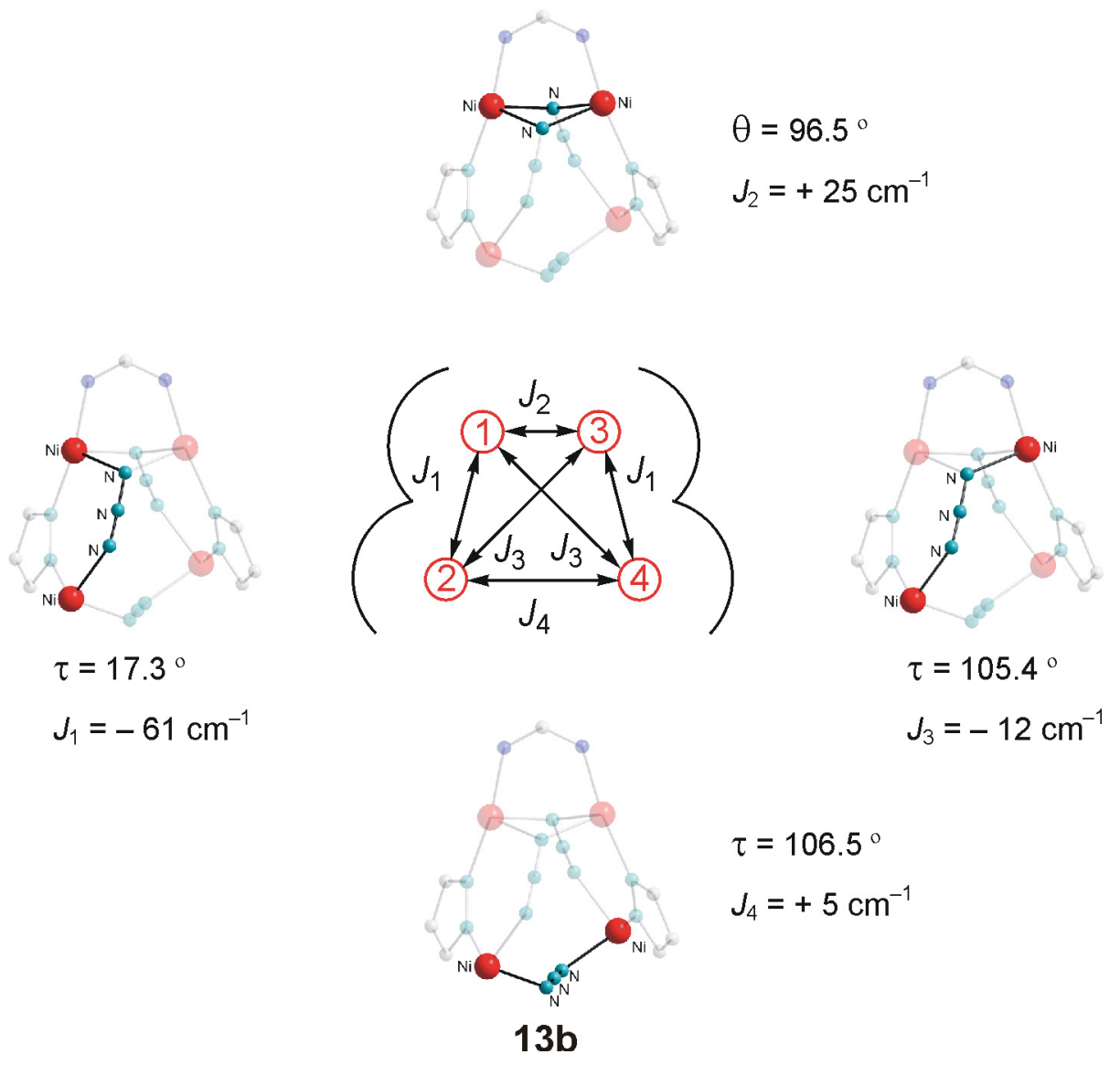

Weiters wurde eine Serie sehr interessanter $\mathrm{Ni}_{4}$-Komplexe, welche die zuvor unbekannte, in diesem Falle zentrale $\mu_{4}-1,1,3,3$-Azidverbrückung beinhalten, synthetisiert und charakterisiert. Dank offensichtlich sehr hoher Flexibilität bezüglich seiner Koordinationsgeometrie ist der $\mu_{4}$-Azidligand in der Lage, sich verschiedenen Umgebungen von vier Metallzentren anzupassen. Dadurch entstehen zwei Typen (14 und 15) von Komplexen mit deutlich unterschiedlicher Koordinationsgeometrie des zentralen $\mu_{4^{-}}$ Azidliganden. Die Folge sind unterschiedliche IR-spektroskopische und magnetische

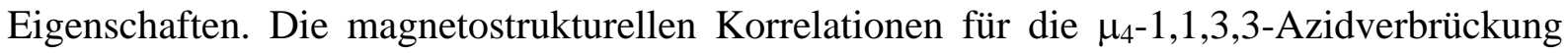
von vier Nickel(II)-Ionen können, wie die Darstellung des Kopplungsschemas von 14b und 15b verdeutlicht, durch die Betrachtung der einzelnen $\mu$-1,1- und $\mu$-1,3-Komponenten verstanden werden. 


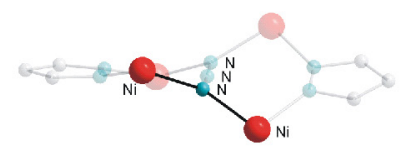

$14 b$

$$
\begin{aligned}
& \theta=92.9^{\circ} \\
& J_{1}=+26 \mathrm{~cm}^{-1}
\end{aligned}
$$

$$
J_{2}=-56 \mathrm{~cm}^{-1}
$$$$
\tau=107.8^{\circ}
$$$$
J_{3}=+5 \mathrm{~cm}^{-1}
$$
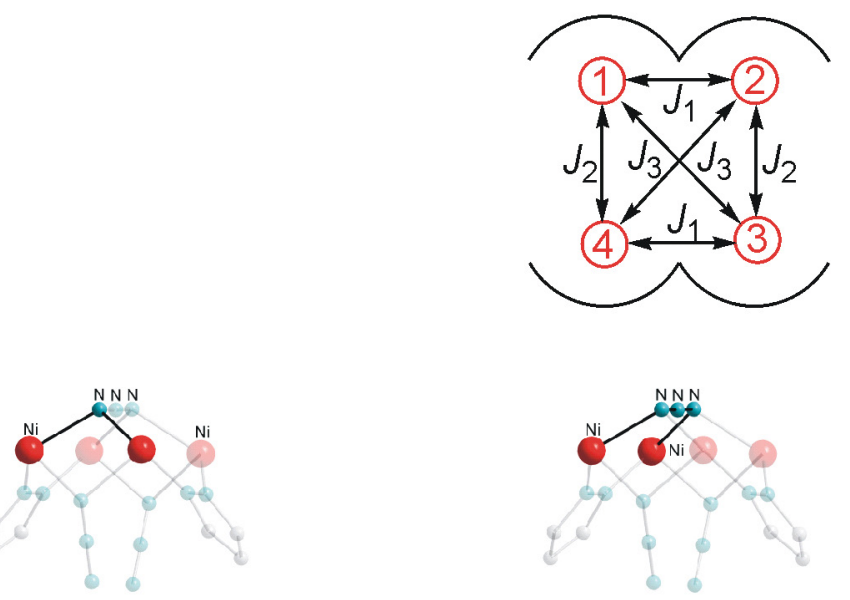

$15 \mathbf{b} \begin{aligned} \theta & =124.4^{\circ} \\ J_{1} & =+33 \mathrm{~cm}^{-1}\end{aligned}$

$$
\begin{aligned}
& \tau=52.6^{\circ} \\
& J_{?}=-25 \mathrm{~cm}^{-1}
\end{aligned}
$$

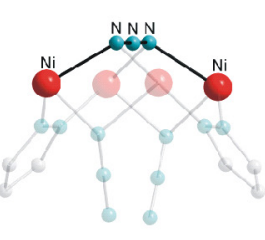

$\tau=126.6^{\circ}$

$J_{3}=+4 \mathrm{~cm}^{-1}$

Der nichtmagnetische Spingrundzustand $S_{T}=0$ in 15b kann durch ein sehr hohes Magnetfeld (25 T) in den magnetischen Spinzustand $S=1$ überführt werden. Die Wechsel im Spinzustand sind generell von großem Interesse wegen möglicher Anwendung in der Quantentechnologie.

Die Anwendung der pyrazolatbasierten bimetallischen Einheiten ermöglichte nicht nur die gezielte Synthese der tetranuklearen Nickel(II)-Komplexe, sondern eröffnete auch den Zugang zu den $J$-alternierenden 1D-Systemen 19-24 mit unterschiedlichen Alternanzmustern. So führt die Umsetzung von 5 mit Natriumazid zu 21 mit EE-EE-Alternanz. 


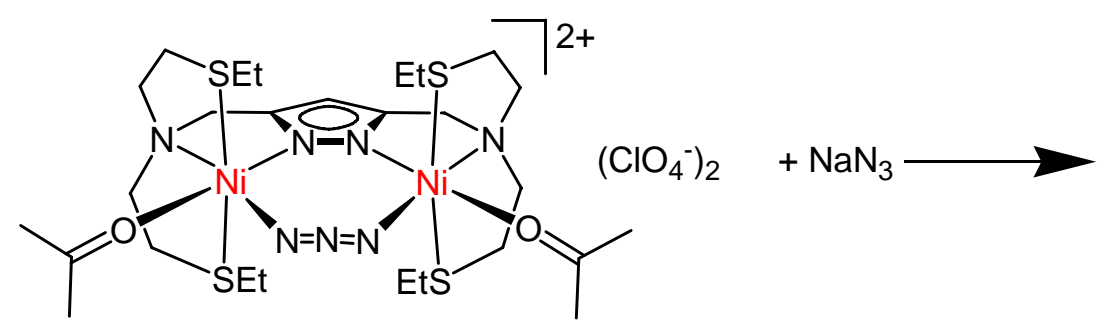

5

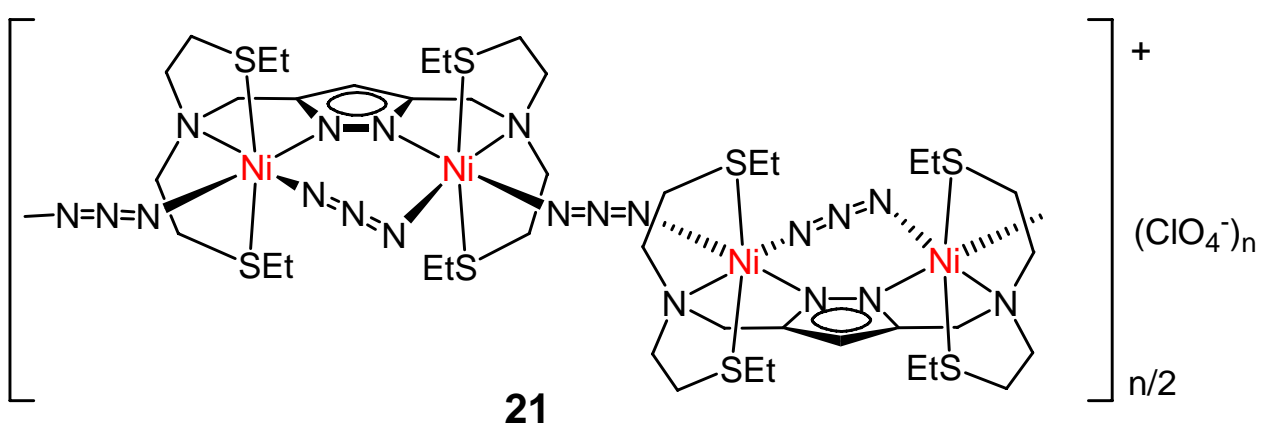

Die Variation der Reaktionsbedingungen sowie der Einsatz anderer bimetallischer Einheiten ergibt die 1D-Ketten 19, 20, 22-24. Auch die direkte Synthese, bei der die bimetallischen Einheiten nicht isoliert werden, ist möglich.

Da die interdimere Azidverbrückung aus sterischen Gründen immer end-to-end ist und die intradimere Verbrückung durch den Liganden vorbestimmbar ist, wird auch das gesamte Alternanzmuster durch das Ligandgerüst vorgegeben: Alternanz von aufeinander folgenden intradimeren end-on und interdimeren end-to-end Verbrückungen (EO-EE-Alternanz) in 19 (Ligand mit langen Seitenarmen) und Alternanz zwei geometrisch und dadurch auch magnetisch unterschiedlicher end-to-end Azidverbrückungen (EE-EE-Alternanz) in 20-23 (Liganden mit kurzen Seitenarmen). Bei der intradimeren Azid-Verbrückung in $\mathbf{2 4}$ wechseln end-on und end-to-end Verknüpfung einander ab und führen so zum EO-EE-EE-EEAlternanzmuster. 24 zeigt wegen dieser komplexen Alternanz das sehr ungewöhnliche Verhalten einer ferrimagnetischen Kette. Die Tatsache, dass mit $\mathbf{H L}^{6}$ sowohl $\mu-1,1-$ als auch $\mu$-1,3-Verbrückung der Azidionen festgestellt wurde, ist von besonderem Interesse. Dies zeigt die Flexibilität des Liganden mit S-Donoratomen bezüglich der Einstellung des MetallMetall-Abstandes, was zu Verbindungen mit interessanten magnetischen Eigenschaften führt (siehe auch $\mathbf{9}, 10$ oben).

Die magnetischen Eigenschaften der Ketten 21-23 wurden in Zusammenarbeit mit theoretischen Physikern mittels Quanten-Monte-Carlo-Methoden analysiert.

Im zweiten Teil der Arbeit wurde versucht, basierend auf dem Spinpolarisationsmechanismus unter Anwendung des Triazinliganden $\mathbf{L}^{\mathbf{8}}$ molekulare dreikernige Kupfer(II)-Komplexe mit ferromagnetischer Kopplung zu generieren, um diese 
weiter zu polymeren magnetischen Materialien zusammenzufügen. Während der Durchführung dieses Teils der Arbeit wurden drei Kupfer(II)-Triazin-Komplexe verschiedener Topologie, nämlich die zwei dreikernigen 25 und 26 sowie die 1D-Kette 27, synthetisiert und charakterisiert. Die 1D-polymere Spezies 27 besteht formal aus dreikernigen Baueinheiten 26, in welchen zwei von drei Kupfer(II)-Ionen an der Bildung der kettenförmigen Struktur beteiligt sind. Dies zeigt die Möglichkeit, die molekularen dreikernigen Komplexe $\mathbf{2 5}$ und $\mathbf{2 6}$ als geeignete Bausteine für den kontrollierten Aufbau von 1D-Ketten sowie von bienenwabenähnlichen 2D-Netzwerken zu betrachten. Voraussetzung für ferromagnetischen Austausch wäre die Koordination der Kupfer(II)-Ionen an die NDonoratome in Position 1,3,5 des Triazinrings. Da die Koordination von Kupfer(II)-Ionen aber nur an den N-Atomen der 2,2'-Dipyridylaminosubstituenten stattfindet, ist dies nicht der Fall und es kommt nur zu sehr schwacher magnetischer Wechselwirkung innerhalb der Kupfer(II)-Triazin-Komplexe 25-27.

In der dreikernigen Kupfer(II)-Verbindung 25 mit zwei Triazinliganden und karussellartiger Struktur konnte erstmals kristallographisch eine Anion- $\pi$ Wechselwirkung bestätigt werden, in exzellenter Übereinstimmung mit theoretischen Vorhersagen.
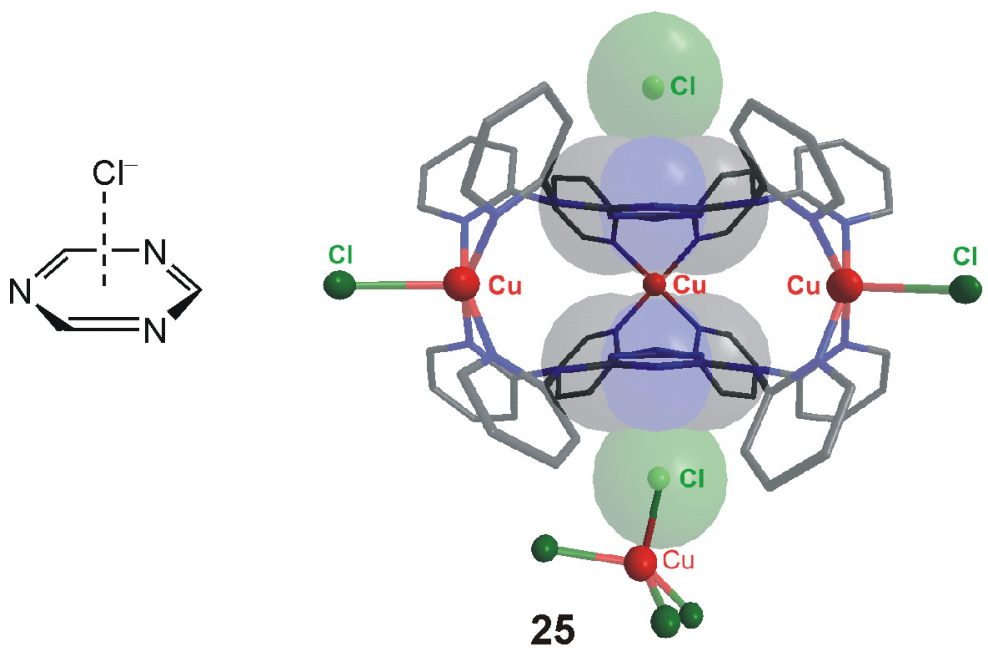

Solche Wechselwirkungen sind von großer Bedeutung in der Supramolekularen Chemie und auch sehr wahrscheinlich in biologischen Prozessen.

Zusammengefasst für beide Teile der Arbeit kann also festgestellt werden, dass es gelungen ist, voll definierte zwei- und dreikernige molekulare Baueinheiten zu synthetisieren und diese strukturell, spektroskopisch und magnetisch $\mathrm{zu}$ charakterisieren. Solche Baueinheiten konnten erfolgreich bei der Synthesestrategie für die Darstellung oligo- und polynuklearer Koordinationsverbindungen, unter anderem auch für $J$-alternierende 1D-Ketten 
eingesetzt werden. Durch die hier gewonnenen Erkenntnisse kann die Natur und Steuerung des magnetischen Verhaltens auf molekularer Ebene besser verstanden werden. 


\section{Literaturverzeichnis}

[1] A. Kloss, „Geschichte des Magnetismus”, VDE-Verlag Berlin und Offenbach, 1994.

[2] W. Plass, Chem. Unserer Zeit 1998, 32, 323 - 333.

[3] M. Verdaguer, Polyhedron 2001, 20, 1115-1128.

[4] J. Müller, T. Weyhermüller, E. Bill, P. Hildebrandt, L. Ould-Moussa, T. Glaser, K. Wieghardt, Angew. Chem. 1998, 110, 637-640; Angew. Chem. Int. Ed. 1998, 37, 616619.

[5] O. Kahn, „Molecular Magnetism“, VCH Publishers, Weinheim, 1993.

[6] J.S. Miller, M. Drillon, „Magnetism: Molecules to Materials - Models and Experiments”, WILEY-VCH Weinheim, 2001.

[7] O. Kahn, „Metal-Organic and Organic Molecular Magnets”, Spec. Publ. 252, Royal Society of Chemistry 2000, 150-168.

[8] P. Delhaes, M. Drillon, „Organic and Inorganic Low Dimensional Crystalline Material”, NATO ASI Series, Series B: Physics Vol. 168, Plenum Press, New York, 1987.

[9] M.A.M. Abu-Youssef, M. Drillon, A. Escuer, M.A.S. Goher, F.A. Mautner, R. Vicente, Inorg. Chem. 2000, 39, 5022-5027.

[10] R. Wang, E. Gao, M. Hong, S. Gao, J. Luo, Z. Han, R. Cao, Inorg. Chem. 2003, 42, 5486-5488.

[11] a) M.F. Charlot, O. Kahn, M. Chaillet, C. Larrieu, J. Am. Chem. Soc. 1986, 108, 25742581; b) P. Chauduri, T. Weyhermüller, E. Bill, K. Wieghardt, Inorg. Chim. Acta 1996, 252, 195-202; c) L.K. Thompson, S.S. Tandon, Comments Inorg. Chem. 1996, 18, 125 144.

[12] J. Ribas, A. Escuer, M. Monfort, R. Vicente, R. Cortés, L. Lezama, T. Rojo, Coord. Chem. Rev. 1999, 193-195, 1027-1068; und dort zitierte Literatur.

[13] a) R. Sessoli, H.-L. Tsai, A.N. Schake, S. Wang, J.B. Vincent, K. Folting, D. Gatteschi, G. Christou, D.N. Hendrickson, J. Am. Chem. Soc. 1993, 115, 1804-1816; b) A.K. Powell, S.L. Heath, D. Gatteschi, L. Pardi, R. Sessoli, G. Spina, F. Del Giallo, F. Pieralli, J. Am. Chem. Soc. 1995, 117, 2491-2502; c) A. Müller, F. Peters, M.T. Pope, D. Gatteschi, Chem. Rev. 1998, 98, 239-271; d) D. Gatteschi, R. Sessoli, A. Cornia, Chem. Commun. 2000, 725-732; e) J. Larionova, M. Gross, M. Pilkington, H. Andres, H. Stoeckli-Evans, H.U. Güdel, S. Decurtins, Angew. Chem. 2000, 112, 1667-1672; Angew. Chem. Int. Ed. 2000, 39, 1605-1609. 
[14] a) O. Kahn, J. Larionova, L. Ouahab, Chem. Commun. 1999, 945-952; b) A. Caneschi, D. Gatteschi, C. Sangregorio, R. Sessoli, L. Sorace, A. Cornia, M.A. Novak, C. Paulsen, W. Wernsdorfer, J. Magn. Magn. Mater. 1999, 200, 182-201.

[15] G. Viau, M.G. Lombardi, G. De Munno, M. Julve, F. Lloret, J. Faus, A. Caneschi, J.M. Clemente-Juan, Chem. Commun. 1997, 1195-1196.

[16] a) P.N.W. Baxter, in Comprehensive Supramolecular Chemistry, ed. J.-M. Lehn, Pergamon, Oxford, 1996, 9, 165; b) B.F. Abrahams, S.R. Batten, M.J. Grannas, H. Hamit, B.F. Hoskins, R. Robson, Angew. Chem. 1999, 111, 1538-1540; Angew. Chem. Int. Ed. 1999, 38, 1475-1477; c) S.-Y. Wan, Y.-Z. Li, T.-A. Okamura, J. Fan, W.-Y. Sun, N. Ueyama, Eur. J. Inorg. Chem. 2003, 3783-3789.

[17] a) S.R. Batten, B.F. Hoskins, B. Moubaraki, K.S. Murray, R. Robson, Chem. Commun. 2000, 1095-1096; b) M. Fujita, N. Fujita, K. Ogura, K. Yamaguchi, Nature 1999, 400, 52-55; c) E. Bosch, C.L. Barnes, Inorg. Chem. 2002, 41, 2543-2547.

[18] a) A.M. Garcia, D.M. Bassani, J.-M. Lehn, G. Baum, D. Fenske, Chem. Eur. J. 1999, 5, 1234-1238; b) C. Metcalfe, S. Spey, H. Adams, J.A. Thomas, Dalton Trans. 2002, 47324739; c) J.R. Galán-Mascarós, J.-M. Clemente-Juan, K.R. Dunbar, Dalton Trans. 2002, 2710-2713; d) C.R. de Silva, J. Wang, M.D. Carducci, S.A. Rajapakshe, Z. Zheng, Inorg. Chim. Acta 2004, 357, 630-634.

[19] a) P. Gamez, P. de Hoog, O. Roubeau, M. Lutz, W.L. Driessen, A.L. Spek, J. Reedijk, Chem. Commun. 2002, 1488-1489; b) P. Gamez, P. de Hoog, M. Lutz, W.L. Driessen, A.L. Spek, J. Reedijk, Polyhedron 2003, 22, 205-210; c) P. Gamez, P. de Hoog, M. Lutz, A.L. Spek, J. Reedijk, Inorg. Chim. Acta 2003, 351, 319-325.

[20] H. Iwamura, Adv. Phys. Org. Chem. 1990, 26, 179-253.

[21] a) T. Glaser, M. Gerenkamp, R. Fröhlich, Angew. Chem. 2002, 114, 3984-3986; Angew. Chem. Int. Ed. 2002, 41, 3823-3825; b) T. Glaser, M. Heidemeier, T. Lügger, Dalton Trans. 2003, 2381-2383.

[22] T. Glaser, T. Lügger, R. Fröhlich, Eur. J. Inorg. Chem. 2004, 394-400.

[23] E.I. Lerner, S.J. Lippard, Inorg. Chem. 1977, 16, 1537-1546.

[24] a) F. Meyer, S. Beyreuther, K. Heinze, L. Zsolnai, Chem. Ber./Recueil 1997, 130, 605613; b) F. Meyer, K. Heinze, B. Nuber, L. Zsolnai, J. Chem. Soc., Dalton Trans. 1998, 207-213; c) F. Meyer, P. Rutsch, Chem. Commun. 1998, 1037-1038; d) F. Meyer, E. Kaifer, P. Kircher, K. Heinze, H. Pritzkow, Chem. Eur. J. 1999, 5, 1617-1630; e) J. Ackermann, F. Meyer, E. Kaifer, H. Pritzkow, Chem. Eur. J. 2002, 8, 247-258.

[25] F. Meyer, H. Pritzkow, Inorg. Chem. Commun. 2001, 4, 305-307. 
[26] F. Meyer, P. Kircher, H. Pritzkow, Chem. Commun. 2003, 774-775.

[27] a) S.R. Batten, R. Robson, Angew. Chem. 1998, 110, 1558-1595; Angew. Chem. Int. Ed. 1998, 37, 1460-1494; b) M. Bertelli, L. Carlucci, G. Ciani, D.M. Proserpio, M. Davide, A. Sironi, J. Mater. Chem. 1997, 7, 1271-1276.

[28] P. Curie, Ann. Chim. Phys. 1895, 5, 289.

[29] P. Weiss, J. Phys. 1907, 6, 667.

[30] J.H. van Vleck, „The Theory of Electric and Magnetic Susceptibilities”, Oxford University Press, Oxford 1932.

[31] B. Bleaney, K.D. Bowers, Proc. Roy. Soc. (London) Ser. A 1952, 214, 451-465.

[32] R. Werner, Dissertation 2000, Fachbereich Chemie der TU Darmstadt.

[33] H.A. Kramers, Physica 1 1934, 182-192.

[34] a) P.W. Anderson, Phys. Rev. 1959, 115, 2-13; b) P.W. Anderson, Solid State Phys. 1963, 14, 99 - 214.

[35] O. Kahn, Angew. Chem. 1985, 97, 837-853; Angew. Chem. Int. Ed. 1985, 24, 834-850.

[36] a) O. Kahn, B. Briat, J. Chem. Soc., Faraday Trans. 1976, 72, 268-281; b) O. Kahn, B. Briat, J. Chem. Soc., Faraday Trans. 1976, 72, 1441-1446.

[37] P.J. Hay, J.C. Thibeault, R.J. Hoffmann, J. Am. Chem. Soc. 1975, 97, 4884-4899.

[38] 0. Kahn, M.F. Charlot, Now. J. Chim. 1980, 4, 567-576.

[39] M.D. Glick, R.L. Lintvedt, D.P. Gavel, B. Tomlonovic, Inorg. Chem. 1976, 15, 16541660.

[40] O. Kahn, J. Galy, Y. Journaux, J. Jaud, I. Morgenstern-Badarau, J. Am. Chem. Soc. 1982, 104, 2165-2176.

[41] F. Meyer, H. Kozlowski in Comprehensive Coordination Chemistry II (Eds.: J.A. McCleverty, T.J. Meyer), Pergamon, 2004, vol. 6, 247-554.

[42] a) M.A. Halcrow, J.C. Huffman, G. Christou, Angew. Chem. 1995, 107, 971-973; Angew. Chem. Int. Ed. 1995, 34, 889-891; b) M.A. Halcrow, J.-S. Sun, J.C. Huffman, G. Christou, Inorg. Chem. 1995, 34, 4167-4177; c) M.W. Wemple, D.M. Adams, K.S. Hagen, K. Folting, D.N. Hendrickson, G. Christou, Chem. Commun. 1995, 1591-1593; d) D. Ma, S. Hikichi, M. Akita, Y. Moro-oka, J. Chem. Soc., Dalton Trans. 2000, 11231134; e) M.A.S. Goher, J. Cano, Y. Journaux, M.A.M. Abu-Youssef, F.A. Mautner, A. Escuer, R. Vicente, Chem. Eur. J. 2000, 6, 778-784; f) T.K. Karmakar, S.K. Chandra, J. Ribas, G. Mostafa, T.H. Luc, B.K. Ghosh, Chem. Commun. 2002, 2364-2365.

[43] G.S. Papaefstathiou, S.P. Perlepes, A. Escuer, R. Vicente, M. Font-Bardia, X. Solans, Angew. Chem. 2001, 113, 908-910; Angew. Chem. Int. Ed. 2001, 40, 884-886. 
[44] P. Mialane, A. Dolbecq, J. Marrot, E. Rivière, F. Sécheresse, Chem. Eur. J. 2005, 11, 1771-1778.

[45] A.R. Rossi, R.H. Bartram, J. Chem. Phys. 1979, 70, 532-537.

[46] J. Hausmann, M.H. Klingele, V. Lozan, G. Steinfeld, D. Siebert, Y. Journaux, J.J. Girerd, B. Kersting, Chem. Eur. J. 2004, 10, 1716-1728.

[47] R. Vicente, A. Escuer, J. Ribas, M.S. El Fallah, X. Solans, M. Font-Bardia, Inorg. Chem. 1995, 34, 1278-1281

[48] J. Ribas, M. Monfort, C. Diaz, C. Bastos, X. Solans, Inorg. Chem. 1993, 32, 3557-3561.

[49] S.C. Manna, S. Konar, E. Zangrando, M.G.B. Drew, J. Ribas, N.R. Chaudhuri, Eur. J. Inorg. Chem. 2005, 1751-1758

[50] C.G. Pierpont, D.N. Hendrickson, D.M. Duggan, F. Wagner, E.K. Barefield, Inorg. Chem. 1975, 14, 604-610.

[51] G.A. McLachlan, G.D. Fallon, R.L. Martin, B. Moubaraki, K.S. Murray, L. Spiccia, Inorg. Chem. 1994, 33, 4663-4668.

[52] Z.-H. Zhang, X.-H. Bu, Z.-H. Ma, W.-M. Bu, Y. Tang, Q.-H. Zaho, Polyhedron 2000, 19, 1559-1566.

[53] E.-Q. Gao, D.-Z. Liao, Z.-H. Jiang, S.-P. Yan, S.-F. Zhang, Нuaxue Xuebao 2001, 59, 1294-1298

[54] a) P. Chaudhuri, M. Guttmann, D. Ventur, K. Wieghardt, B. Nuber, J. Weiss, J. Chem. Soc., Chem. Commun. 1985, 1618-1620; b) A. Escuer, I. Castro, F.A. Mautner, M.S. El Fallah, R. Vicente, Inorg. Chem. 1997, 36, 4633-4640 ; c) J. Ribas, M. Monfort, B.K. Gosh, R. Cortés, X. Solans, M. Font-Bardia, Inorg. Chem. 1996, 35, 864-868 ; d) A.M. Escuer, R. Vicente, M.S. El Fallah, X. Solans, M. Font-Bardia, Inorg. Chim. Acta 1998, 278, 43-50.

[55] a) M. Monfort, J. Ribas, X. Solans, M. Font-Bardia, Inorg. Chem. 1996, 35, 7633-7638; b) A. Escuer, R. Vicente, J. Ribas, M.S. El Fallah, X. Solans, Inorg. Chem. 1993, 32, 1033-1035; c) Y. Yu, Z. Honda, K. Katsumata, T. Ohishi, T. Manabe, M. Yamashita, Mol. Cryst. Liq. Cryst. 1996, 286, 121-126; d) A. Escuer, R. Vicente, J. Ribas, M.S. El Fallah, X. Solans, M. Font-Bardia, Inorg. Chem. 1993, 32, 3727-3732.

[56] a) A. Escuer, R. Vicente, M.S. El Fallah, J. Ribas, X. Solans, M. Font-Bardia, J. Chem. Soc., Dalton Trans. 1993, 2975-2976; b) M. Monfort, I. Resino, M.S. El Fallah, J. Ribas, X. Solans, M. Font-Bardia, H. Stoeckli-Evans, Chem. Eur. J. 2001, 7, 280-287.

[57] J. Ribas, M. Monfort, C. Diaz, C. Bastos, C. Mer, X. Solans, Inorg. Chem. 1995, 34, 4986-4990. 
[58] a) R. Cortés, K.; Urtiaga, L. Lezama, J.L. Pizarro, A. Goñi, M.I. Arriortua, T. Rojo, Inorg. Chem. 1994, 33, 4009-4015; b) A. Escuer, R. Vicente, J. Ribas, M.S. El Fallah, X. Solans, M. Font-Bardia, Inorg. Chem. 1994, 33, 1842-1847.

[59] M. Hagiwara, Y. Narumi, K. Kindo, M. Kohno, H. Nakano, R. Sato, M. Takahashi, Phys. Rev. Lett. 1998, 80, 1312-1315

[60] a) R. Vicente, A. Escuer, J. Ribas, X. Solans, Inorg. Chem. 1992, 31, 1726-1728; b) R. Vicente, A. Escuer, Polyhedron, 1995, 14, 2133-2138.

[61] M.L. Hernández, M.G. Barandika, M.K. Urtiaga, R. Cortés, L. Lezama, M.I. Arriortua, J. Chem. Soc., Dalton Trans. 2000, 79-84.

[62] F.F. de Biani, E. Ruiz, J. Cano, J.J. Novoa, S. Alvarez, Inorg. Chem. 2000, 39, 32213229.

[63] A. Escuer, C.J. Harding, Y. Dussart, J. Nelson, V. McKee, R. Vicente, J. Chem. Soc., Dalton Trans. 1999, 223-227.

[64] B. Kersting, G. Steinfeld, D. Siebert, Chem. Eur. J. 2001, 7, 4253-4258.

[65] a) C.S. Hong, Y. Do, Angew. Chem. 1999, 111, 153-155; Angew. Chem. Int. Ed. 1999, 38, 193-195; b) C.S. Hong, J. Koo, S.-K. Son, Y.S. Lee, Y.-S. Kim, Y. Do, Chem.-Eur. J. 2001, 7, 4243-4252; c) P.S. Mukherjee, S. Dalai, E. Zangrando, F. Lloret, N.R. Chaudhuri, Chem. Commun. 2001, 1444-1445; d) M. Montfort, I. Resino, J. Ribas, H. Stoeckli-Evans, Angew. Chem. 2000, 112, 197-199; Angew. Chem. Int. Ed. 2000, 39, 191-193.

[66] P. Mialane, A. Dolbecq, E. Rivière, J. Marrot, F. Sécheresse, Angew. Chem. 2004, 116, 2324-2327; Angew. Chem. Int. Ed . 2004, 43, 2274-2277.

[67] S.K. Dey, N. Mondal, M.S. El Fallah, R. Vicente, A. Escuer, X. Solans, M. Font-Bardía, T. Matsushita, V. Gramlich, S. Mitra, Inorg. Chem. 2004, 43, 2427-2434.

[68] R. Ribas, M. Monfort, R. Costa, X. Solans, Inorg. Chem. 1993, 32, 695-699.

[69] Z.E. Serna, L. Lezama, M.K. Urtiaga, M.I. Arriortua, M.G. Barandika, R. Cortés, T. Rojo, Angew. Chem. 2000, 112, 352-355; Angew. Chem. Int. Ed. 2000, 39, 344-347.

[70] Z.E. Serna, M.G. Barandika, R. Cortés, M.K. Urtiaga, G.E. Barberis, T. Rojo, J. Chem. Soc., Dalton Trans. 2000, 29-34.

[71] F.-C. Liu, Y.-F. Zeng, J.-R. Li, X.-H. Bu, H.-J. Zhang, J. Ribas, Inorg. Chem. 2005, 44, 7298-7300.

[72] M.-L. Tong, M. Monfort, J.M.C. Juan, X-M. Chen, X.-H. Bu, M. Ohbad, S. Kitagawa, Chem. Commun. 2005, 233-235

[73] V. McKee, M. Zvagulis, C.A. Reed, Inorg. Chem. 1985, 24, 2914-2919. 
[74] L.K. Thompson, S.S. Tandon, F. Lloret, J. Cano, M. Julve, Inorg. Chem. 1997, 36, 33013306.

[75] A. Escuer, R. Vicente, F.A. Mautner, M.A.S. Goher, Inorg. Chem. 1997, 36, 1233-1236.

[76] K.-S. Bürger, P. Chaudhuri, K. Wieghardt, Inorg. Chem. 1996, 35, 2704-2707.

[77] E. Ruiz, J. Cano, S. Alvarez, P. Alemany, J. Am. Chem. Soc. 1998, 120, 11122-11129.

[78] X.-J. Lin, Z. Shen, Y. Song, H.-J. Xu, Y.-Z. Li, X.-Z. You, Inorg. Chim. Acta 2005, 358, 1963-1969.

[79] J. Ribas, M. Monfort, C. Diaz, C. Bastos, X. Solans, Inorg. Chem. 1994, 33, 484-489.

[80] G.S. Papaefstathiou, A. Escuer, R. Vicente, M. Font-Bardia, X. Solans, S.P. Perlepes, Chem. Commun. 2001, 2414-2415.

[81] A.K. Boudalis, B. Donnadieu, V. Nastopoulos, J.M. Clemente-Juan, A. Mari, Y. Sanakis, J.-P. Tuchagues, S.P. Perlepes, Angew. Chem. 2004, 116, 2316-2320; Angew. Chem. Int. Ed. 2004, 43, 2266-2270.

[82] a) M. Montfort, J. Ribas, X. Solans, J. Chem. Soc., Chem. Commun. 1993, 350-351; b) J. Ribas, M. Montfort, X. Solans, M. Drillon, Inorg. Chem., 1994, 33, 742-745.

[83] a) M.A.S. Goher, A. Escuer, M.A.M. Abu-Youssef, F.A. Mautner, Polyhedron, 1998, 17, 4265-4273; b) T.K. Maji, P.S. Mukherjee, S. Koner, G. Mostafa, J.-P.Tuchagues, N.R. Chaudhuri, Inorg, Chim. Acta, 2001, 314, 111-116; c) L.Zhang, L.-F. Tang, Z.-H. Wang, M. Du, M. Julve, F. Lloret, J.-T.Wang, Inorg. Chem., 2001, 40, 3619-3622.

[84] M.A.S. Goher, F.A. Mautner, Polyhedron, 1995, 14, 1439-1446.

[85] P.K. Nanda, G. Aromí, D. Ray, Chem. Commun. 2006, 3181-3183.

[86] Borrás-Almenar, J.J.; Clemente-Juan, J.M.; Coronado, E.; Llorte, E. Chem. Phys. Lett. 1997, 275, 79-84.

[87] a) L. Li, D. Liao, Z. Jiang, S. Yan, Polyhedron, 2000, 19, 1575-1578; b) J. Ribas, M. Monfort, K.B. Gosh, X. Solans, M. Font-Bardia, J. Chem. Soc. Chem. Commun. 1995, 2375-2376 ; c) M. Monfort, I. Resino, J. Ribas, P. Rabu, M. Drillon, Inorg. Chem. 2000, 39, 2572-2576 ; d) M. Monfort, I. Resino, J. Ribas, X. Solans, M. Font-Bardia, New. J. Chem. 2001, 25, 1577-1582.

[88] A. Escuer, R. Vicente, M.S. El Fallah, S.B. Kumar, F.A. Mautner, D. Gatteschi, J. Chem. Soc., Dalton Trans. 1998, 3905-3910.

[89] J. Ribas, M. Monfort, K.B. Ghosh, X. Solans, Angew. Chem. 1994, 106, 2177-2179; Angew. Chem. Int. Ed. Engl. 1994, 33, 2087-2089.

[90] A. Escuer, R. Vicente, M.S. El Fallah, M.A.S. Goher, F.A. Mautner, Inorg. Chem. 1998, 37, 4466-4469. 
[91] M.A.M. Abu-Youssef, A. Escuer, M.A.S. Goher, F.A. Mautner, G.J. Reiß, R. Vicente, Angew. Chem. 2000, 112, 1681-1683; Angew. Chem. Int. Ed. 2000, 39, 1624-1626.

[92] J. Ribas, M. Monfort, I. Resino, X. Solans, P. Rabu, F. Maingot, M. Drillon, Angew. Chem. 1996, 108, 2671-2673; Angew. Chem. Int. Ed. 1996, 35, 2520-2522.

[93] A. Escuer, R. Vicente, M.S. El Fallah, X. Solans, M. Font-Bardia, Inorg. Chim. Acta 1998, 278, 43-50.

[94] M.S. El Fallah, A. Escuer, R. Vicente, X. Solans, M. Font-Bardia, M. Verdaguer, Inorg. Chim. Acta 2003, 344, 133-142.

[95] F.A. Mautner, R. Cortés, L. Lezama, T. Rojo, Angew. Chem. 1996, 108, 96-98; Angew. Chem. Int. Ed. 1996, 35, 78-80.

[96] A. Escuer, R. Vicente, J. Ribas, X. Solans, Inorg. Chem. 1995, 34, 1793-1798.

[97] F. Meyer, U. Ruschewitz, P. Schober, B. Antelmann, L. Zsolnai, J. Chem. Soc., Dalton Trans. 1998, 199-205.

[98] M. Konrad, F. Meyer, K. Heinze, L. Zsolnai, J. Chem. Soc., Dalton Trans. 1998, 11811186.

[99] T.G. Schenck, J.M. Downes, C.R.C. Milne, P.B. Mackenzie, T.G. Boucher, J. Whelan, B. Bosnich, Inorg. Chem. 1985, 24, 2334-2337.

[100] J.C. Röder, F. Meyer, H. Pritzkow, Organometallics 2001, 20, 811-817.

[101] a) R.A.B. Bannard, J.H. Parkkari, I.W. Coleman, Can. J. Chem. 1962, 40, 1909-1916; b) J.H. Parkkari, R.A.B. Bannard, I.W. Coleman, Can. J. Chem. 1965, 43, 3119-3128.

[102] D.S. McGuinness, P. Wasserscheid, W. Keim, D. Morgan, J.T. Dixon, A. Bollmann, H. Maumela, F. Hess, U. Englert, J. Am. Chem. Soc. 2003, 125, 5272-5273.

[103] X.-Y. Le, J.-E. Shi, Wuji Huaxue Xuebao 1999, 15, 128-131.

[104] S. Demeshko, G. Leibeling, S. Dechert, F. Meyer, Dalton Trans. 2006, 3458-3465.

[105] G. Leibeling, S. Demeshko, B. Bauer-Siebenlist, F. Meyer, H. Pritzkow, Eur. J. Inorg. Chem. 2004, 2413-2420.

[106] Das hier verwendete Programm julX wurde von Dr. E. Bill, Max-Planck-Institut für Bioanorganische Chemie, Mülheim an der Ruhr, zur Verfügung gestellt.

julX simuliert auf der Basis des üblichen Spin-Hamiltonoperator-Formalismus molare magnetische Suszeptibilitäten für paramagnetische Systeme von bis zu vier Spins mit isotropen Austauschwechselwirkungen und lokalen axialen und rhombischen Nullfeldwechselwirkungen. Die verwendeten Heisenberg-Dirac-van-Vleck-Operatoren für die Austauschwechselwirkung sind an entsprechenden Stellen im Text angegeben. Die Zeeman-Wechelwirkung wird richtungsabhängig berücksichtigt und für 
Pulverproben entweder über die drei kanonischen Hauptachsen oder über ein 16-Punkte Lebedev-Gitter gemittelt. Die Diagonalisierung der Hamiltonmatrix erfolgt numerisch mit der LAPACK Routine ZHEEV. Magnetische Momente werden durch numerische Ableitung der Energien nach dem Feld berechnet. Einzelne oder mehrere Parameter der Hamiltonoperatoren können mit der Simplexroutine AMOEBA von NUMERICAL RRECIPIES automatisch optimiert werden, wobei paarweise Korrelation von beliebigen Variablen möglich ist. Für Visualisierungszwecke können Energiediagramme geplottet werden und für systematische Fehlerbetrachtungen können Fehler-Kontourplots oder Fehlerflächen für beliebige Variablen-Paare gerechnet werden.

[107] S. Demeshko, G. Leibeling, S. Dechert, S. Fuchs, T. Pruschke, F. Meyer, ChemPhysChem 2007, im Druck.

[108] F. Meyer, S. Demeshko, G. Leibeling, E. Kaifer, H. Pritzkow, Chem. Eur. J. 2005, 11, 1518-1526.

[109] Das hier verwendete Programm ist eine modifizierte Version des in [64] beschriebenen. Die Diagonalisierung der Hamiltonmatrix erfolgte numerisch, um die Energiewerte der einzehlnen Spinzustände zu erhalten. Das Einsetzen dieser Parameter in die Van-VleckGleichung ergibt die theoretischen Werte für die magnetische Suszeptibilität. Das Programm vergleicht die berechneten und experimentell beobachteten Werte unter Verwendung der Methode der kleinsten Quadrate miteinander und variiert die Parameter, um die beste Anpassung zu erhalten.

[110] M. Konrad, F. Meyer, A. Jacobi, P. Kircher, P. Rutsch, L. Zsolnai, Inorg. Chem. 1999, 38, 4559-4566.

[111] S. Demeshko, G. Leibeling, W. Maringgele, F. Meyer, C. Mennerich, H.-H. Klauss, H. Pritzkow, Inorg. Chem. 2005, 44, 519-528.

[112] a) Z. Hu, S.M. Gorun, Inorg. Chem. 2001, 40, 667-671; b) J.C. Röder, F. Meyer, E. Kaifer, Angew. Chem. 2002, 114, 2414-2417; Angew. Chem. Int. Ed. 2002, 41, 23042306.

[113] C. Golze, A. Alfonsov, R. Klingeler, B. Büchner, V. Kataev, C. Mennerich, H.-H. Klauss, M. Goiran, J.-M. Broto, H. Rakoto, S. Demeshko, G. Leibeling, F. Meyer, Phys. Rev. B 2006, 73, 224403.

[114] O. Kahn, J. P. Launay, Chemtronics 1988, 3, 140-151.

[115] J.S. Miller, Angew. Chem. 2003, 115, 27-29; Angew. Chem. Int. Ed. Engl. 2003, 42, 2729. 
[116] a) P. Gütlich, A. Hauser, H. Spiering, Angew. Chem. 1994, 106, 2109-2141; Angew. Chem. Int. Ed. Engl. 1994, 33, 2024-2054; b) O. Kahn, C.J. Martinez, Science 1998, 279, 44-48; c) O. Kahn, J. Kröber, C. Jay, Adv. Mater. 1992, 4, 718-728; d) A. Bousseksou, G. Molnár, G. Matouzenko, Eur. J. Inorg. Chem. 2004, 4353-4369.

[117] a) W. Fujita, K. Awaga, Science 1999, 286, 261-262; b) W. Fujita, K. Awaga, J. Solid State Chem. 2001, 159, 451-454; c) M.E. Itkis, X. Chi, A.W. Cordes, R.C. Haddon, Science 2002, 296, 1443-1445; d) J.L. Brussom, O.P. Clements, R.C. Haddon, M.E. Itkis, A.A. Leitch, R.T. Oakley, R.W. Reed, J.F. Richardson, J. Am. Chem. Soc. 2004, 126, 14692-14693.

[118] F. L. De Panthou, D. Luneau, R. Musin, L. Öhrström, A. Grand, P. Turek, P. Rey, Inorg. Chem. 1996, 35, 3484-3491.

[119] a) J.M. Migliori, W.M. Reiff, A.M. Arif, J.S. Miller, Inorg. Chem. 2004, 43, 68756877; b) S.M. Humphrey, P.T. Wood, J. Am. Chem. Soc. 2004, 126, 13236-13237.

[120] G. Leibeling, S. Demeshko, S. Dechert, F. Meyer, Angew. Chem., 2005, 117, 72737276; Angew. Chem. Int. Ed., 2005, 44, 7111-7114.

[121] a) S.S. Tandon, L.K. Thompson, M.E. Manuel, J.N. Bridson, Inorg. Chem. 1994, 33, 5555-5570 ; b) A. Escuer, R. Vicente, F.A. Mautner, M.A.S. Goher, Inorg. Chem. 1997, $36,1233-1236$.

[122] a) H.M. McConnell, J. Chem. Phys. 1963, 39, 1910; b) D.A. Dougherty, Acc. Chem. Res. 1991, 24, 88-94; c) J. Cano, E. Ruiz, S. Alvarez, M. Verdaguer, Comments Inorg. Chem. 1998, 20, 27-56.

[123] S. Mitsubori, T. Ishida, T. Nogami, H. Iwamura, Chem. Lett. 1994, 94, 285-288.

[124] G. Glatz, Diplomarbeit, Göttingen, 2005.

[125] a) J. Pang, Y. Tao, S. Freiberg, X.-P. Yang, M. D'Iorio, S. Wang, J. Mater. Chem. 2002, 12, 206-212; b) P. de Hoog, P. Gamez, W.L. Driessen, J. Reedijk, Tetrahedron Letters 2002, 43, 6783-6786.

[126] C. Seward, S. Wang, Comments Inorg. Chem. 2005, 26, 103-125.

[127] S. Demeshko, S. Dechert, F. Meyer, J. Am. Chem. Soc. 2004, 126, 4508-4509.

[128] S. Demeshko, G. Leibeling, S. Dechert, F. Meyer, Dalton. Trans. 2004, 3782-3787.

[129] A.W. Addison, T.N. Rao, J. Reedijk, J. van Rijn, G.C. Verschoor, J. Chem. Soc., Dalton Trans. 1984, 1349-1356.

[130] P. de Hoog, P. Gamez, O. Roubeau, M. Lutz, W.L. Driessen, A. L. Spek, J. Reedijk, New J. Chem. 2003, 27, 18-21.

[131] C. J. O’Connor, Prog. Inorg. Chem. 1982, 29, 203-283. 
[132] A. Rodriguez-Fortea, P. Alemany, S. Alvarez, E. Ruiz, Inorg. Chem. 2002, 41, 37693778.

[133] a) W.E. Marsh, W.E. Hatfield, D.J. Hodgson, Inorg. Chem. 1982, 2, 2679-2684.

b) T. Rojo, M. I. Arriortua, J. Ruiz, J. Darriet, G. Villeneuve, D. Beltran-Porter, J. Chem. Soc., Dalton Trans. 1987, 285-291.

[134] R.K. Castellano, F. Diederich, E.A. Meyer, Angew. Chem. 2003, 115, 1244-1287; Angew. Chem. Int. Ed. 2003, 42, 1210-1250.

[135] a) H.-J. Schneider, Angew. Chem. 1991, 103, 1419-1439; Angew. Chem. Int. Ed. Engl. 1991, 30, 1417-1436; b) H.-J. Schneider, A. Yatsimirsky, Principles and Methods in Supramolecular Chemistry, Wiley \& Sons, Chichester, 2000.

[136] P.D. Beer, P.A. Gale, Angew. Chem. 2001, 113, 502-532; Angew. Chem. Int. Ed. 2001, 40, 486-516.

[137] R. Vilar, Angew. Chem. 2003, 115, 1498-1516; Angew. Chem. Int. Ed. 2003, 42, $1460-$ 1477.

[138] A. Bianchi, K. Bowman-James, E. García-España, Supramolecular Chemistry of Anions, Wiley-VCH, New York, 1997.

[139] J.W. Steed, J.L. Atwood, Supramolecular Chemistry, Wiley \& Sons, Chichester, 2000.

[140] a) H.-J. Schneider, T. Blatter, B. Palm, U. Pfingstag, V. Ruediger, I. Theis, J. Am. Chem. Soc. 1992, 114, 7704-7708.

[141] H.-J. Schneider, F. Werner, T. Blatter, J. Phys. Org. Chem. 1993, 9, 590-594.

[142] D. Quiñonero, C. Garau, C. Rotger, A. Frontera, P. Ballester, A. Costa, P.M. Deyà, Angew. Chem. 2002, 114, 3539-3542; Angew. Chem. Int. Ed. 2002, 41, 3389-3392.

[143] M. Mascal, A. Armstrong, M.D. Bartberger, J. Am. Chem. Soc. 2002, 124, 6274-6276.

[144] C. Garau, D. Quiñonera, A. Frontera, A. Costa, P. Ballester, P.M. Deyà, Chem. Phys. Lett. 2003, 370, 7-13.

[145] C. Garau, A.Frontera, D. Quiñonero, P. Ballester, A. Costa, P.M. Deyà, ChemPhysChem 2003, 4, 1344-1348.

[146] C. Janiak, J. Chem. Soc., Dalton. Trans. 2000, 3885-3896.

[147] B. Manimaran, T. Rajendran, Y.-L. Lu, G.-H. Lee, S.-M. Peng, K.-L. Lu, Eur. J. Inorg. Chem. 2001, 633-636.

[148] R.D. Pike, B.D. Borne, J.T. Maeyer, A.L. Rheingold, Inorg. Chem. 2002, 41, 631-633.

[149] a) A. Colombo, L. Mewabue, A. Motori, G.C. Pellacani, W. Porzio, F. Sandrolini, R.D. Willett, Inorg. Chem. 1985, 24, 2900-2905; b) B. Scott, U. Geiser, R.D. Willett, B. 
Patyal, C.P. Landee, R.E. Greeney, T. Manfredini, G.C. Pellacani, A.B. Corradi, L.P. Battaglia, Inorg. Chem. 1988, 27, 2454-2460.

[150] P. de Hoog, P. Gamez, I. Mutikainen, U. Turpeiner, J. Reedijk, Angew. Chem. 2004, 116, 5939-5941; Angew. Chem. Int. Ed. 2004, 43, 5815-5817.

[151] R.M. Fairchild, K.T. Holman, J. Am. Chem. Soc. 2005, 127, 16364-16365.

[152] Y.S. Rosokha, S.Y. Lindeman, S.V. Rosokha, J.K. Kochi, Angew. Chem. 2004, 116, 4750-4752; Angew. Chem. Int. Ed. 2004, 43, 4650-4652.

[153] A. Frontera, F. Saczewski, M. Gdaniec, E. Dziemidowicz-Borys, A. Kurland, P.M. Deyà, D. Quiñonero, C. Garau, Chem. Eur. J. 2005, 11, 6560-6567.

[154] O.B. Berryman, F. Hof, M.J. Hynes, D.W. Johnson, Chem. Commun. 2006, 506-508.

[155] B.L. Schottel, J. Bacsa, K.R. Dunbar, Chem. Commun. 2005, 46-47.

[156] B.L. Schottel, H.T. Chifotides, M. Shatruk, A. Chouai, L.M. Pérez, J. Bacsa, K.R. Dunbar, J. Am. Chem. Soc. 2006, 128, 5895-5912.

[157] H. Casellas, C. Massera, P. Gamez, A.M. Manotti Lanfredi, J. Reedijk, Eur. J. Inorg. Chem. 2005, 2902-2908.

[158] P. Gamez, G.A. van Albada, I. Mutikainen, U. Turpeinen, J. Reedijk, Inorg. Chim. Acta 2005, 358, 1975-1980.

[159] a) F. Hein, E. Petzschner, K. Wagner, F. A. Segnitz, Z. anorg. Allg. Chem. 1924, 141, 161-227; b) B. Heyn, B. Hipler, G. Kreisel, H. Schreer, D. Walther, „Anorganische Synthesechemie - Ein integriertes Praktikum“, Springer Verlag Heidelberg, 1990, 2. Auflage.

[160] G.M. Sheldrick, SHELXS-97, „Program for Crystal Structure Solution“, Universität Göttingen 1997.

[161] G.M. Sheldrick, SHELXL-97, „Program for Crystal Structure Refinement“, Universität Göttingen 1997.

[162] S. J. Swithenby, Contemp. Phys. 1974, 15, 249-267.

[163] G. Leibeling, Dissertation, Universität Jena 2003.

[164] a) W. Haberditzl, Angew. Chem. 1966, 78, 277-288; Angew. Chem. Int. Ed. Engl. 1966, 5, 288-298 ; b) W. Haberditzl, „Magnetochemie”, Akademie-Verlag, 1968; d) LandoltBörnstein, „Zahlenwerte und Funktionen aus Naturwissenschaften und Technik”; Neue Serie, Gruppe II: Atom- und Molekularphysik, Bd. 2: „Magnetische Eigenschaften der Koordinations- und metallorganischen Verbindungen der Übergangselemente”, Springerverlag Berlin, 1966; c) L. N. Mulay, E. A. Boudreaux, „Theory and Applications of Molecular Diamagnetism”, Wiley New York, 1976. 


\section{Formelübersicht}
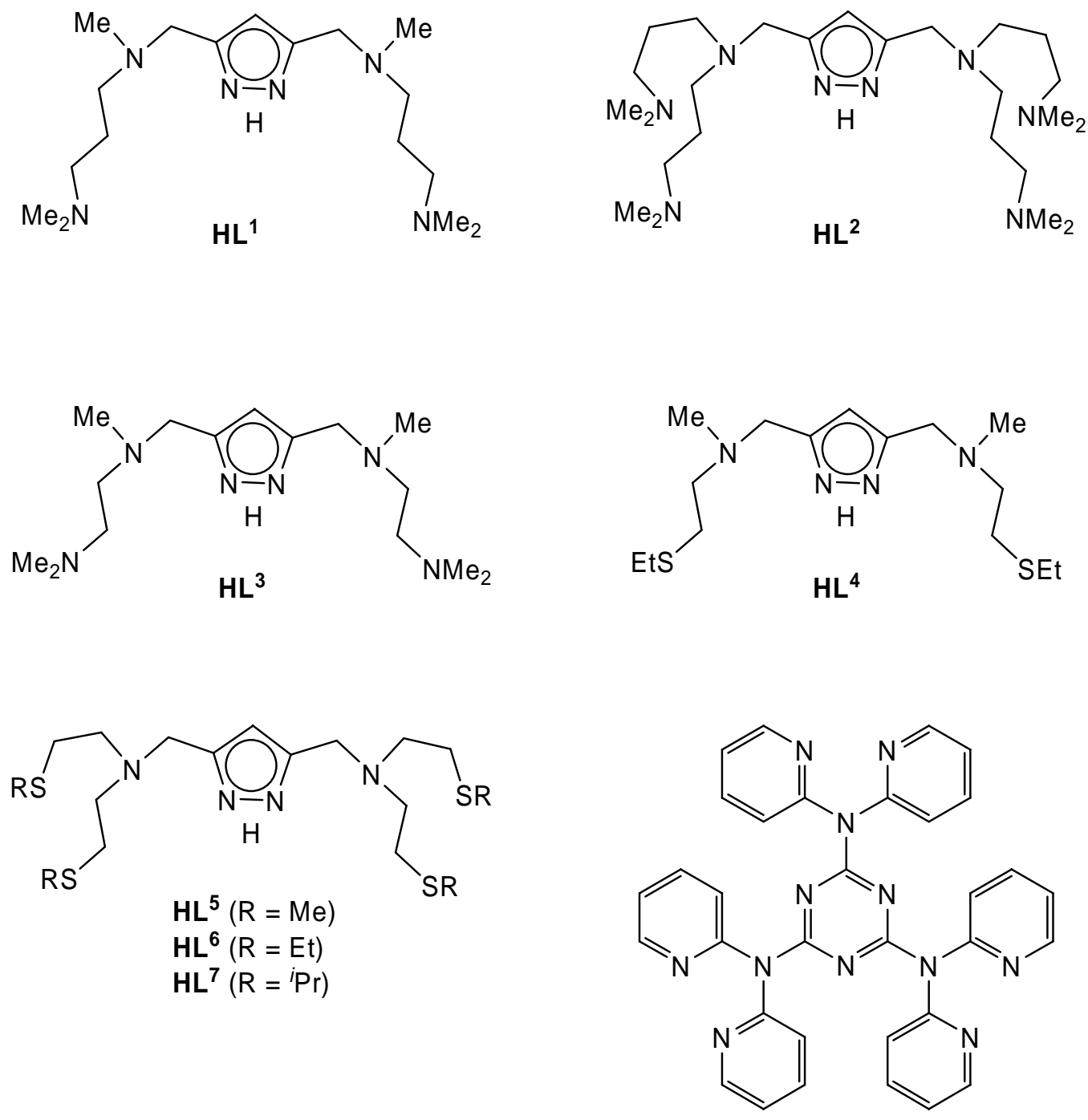

$\mathbf{L}^{8}$ 


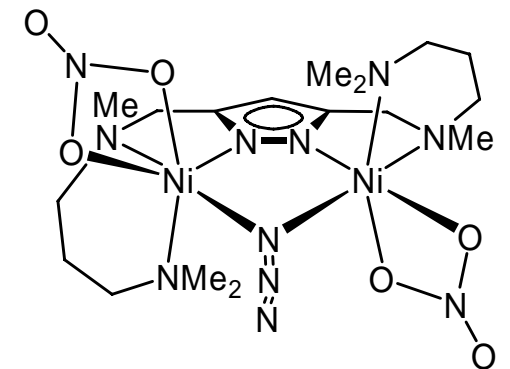

1

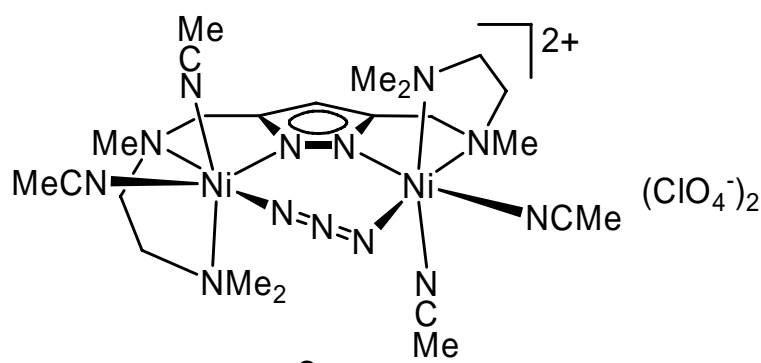

3

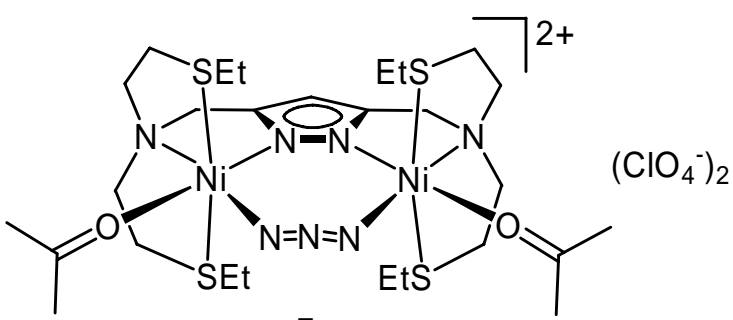

5

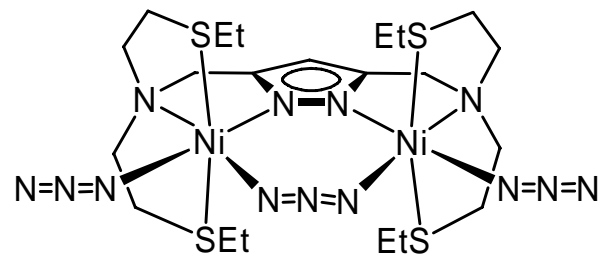

7
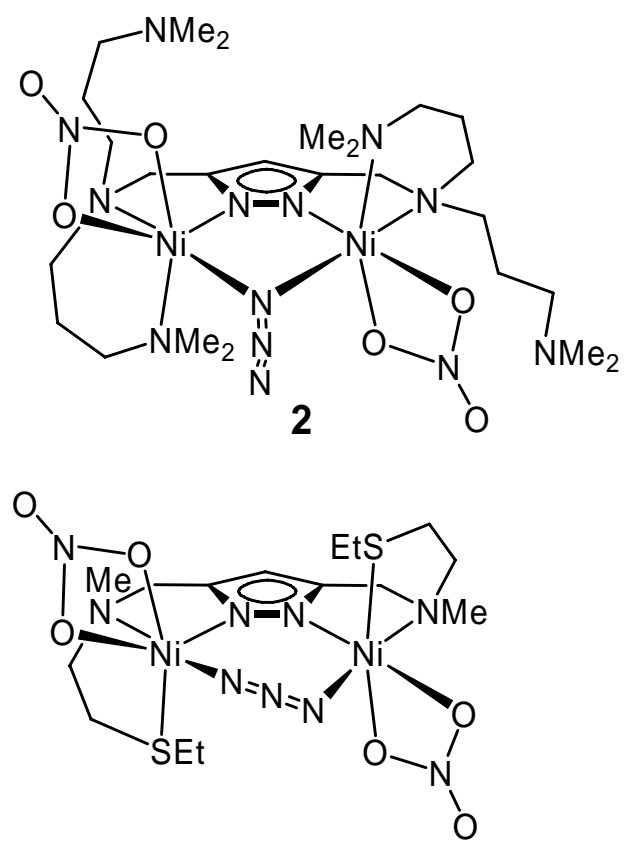

4
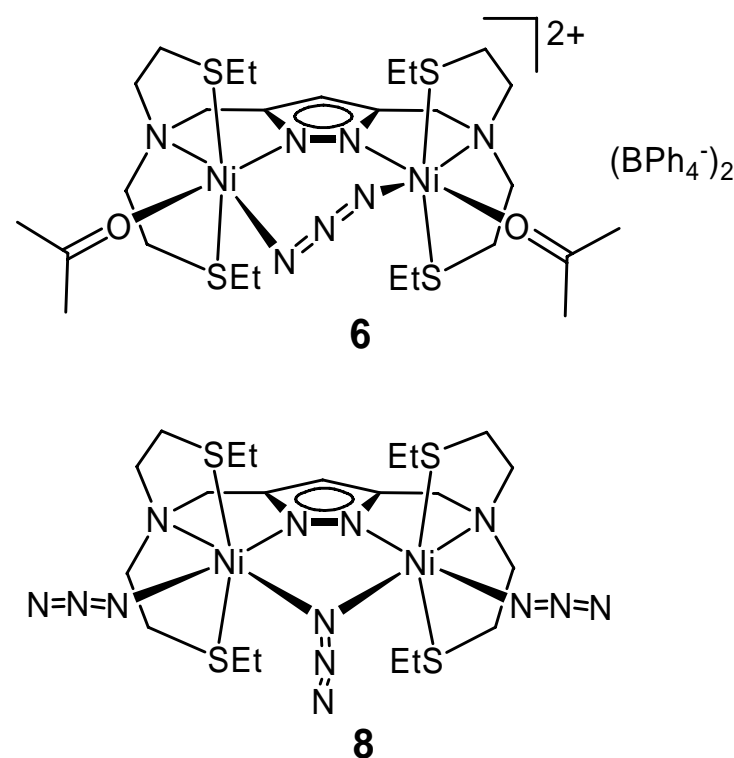

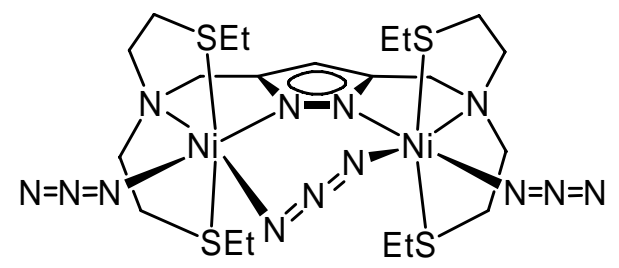

9 (Raumgruppe $P b c n$ )

10 (Raumgruppe $P \overline{1}$ ) 


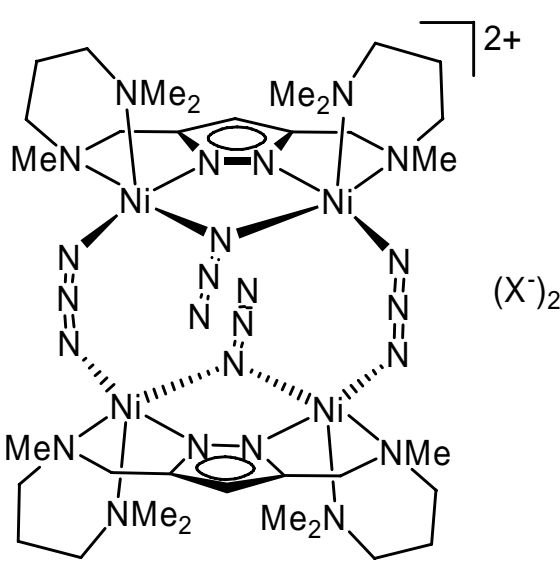

$11 \mathrm{a}\left(\mathrm{X}=\mathrm{ClO}_{4}\right.$, aus Methanol)

11b $\left(X=\mathrm{ClO}_{4}\right.$, aus Aceton $)$

11c $\left(\mathrm{X}=\mathrm{BPh}_{4}\right)$

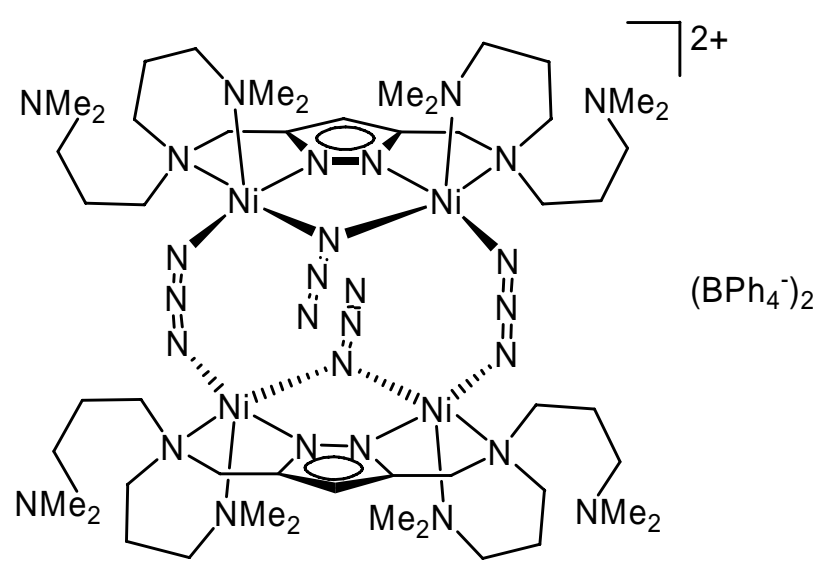

12

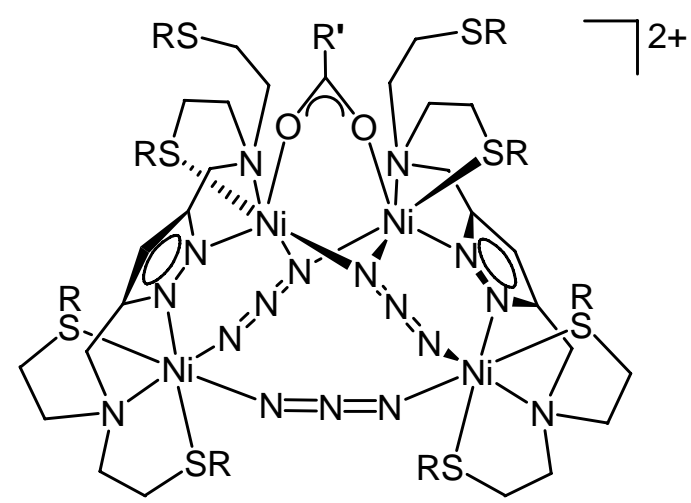

$$
\begin{array}{ll} 
& 13 \mathbf{a}\left(\mathrm{R}=\mathrm{Et}, \mathrm{R}^{\prime}=\mathrm{Me}\right) \\
\left(\mathrm{ClO}_{4}{ }^{-}\right)_{2} & \text { 13b }\left(\mathrm{R}=\mathrm{Et}, \mathrm{R}^{\prime}=\mathrm{Ph}\right) \\
& 13 \mathbf{c}\left(\mathrm{R}={ }^{i} \mathrm{Pr}, \mathrm{R}^{\prime}=\mathrm{Ph}\right)
\end{array}
$$

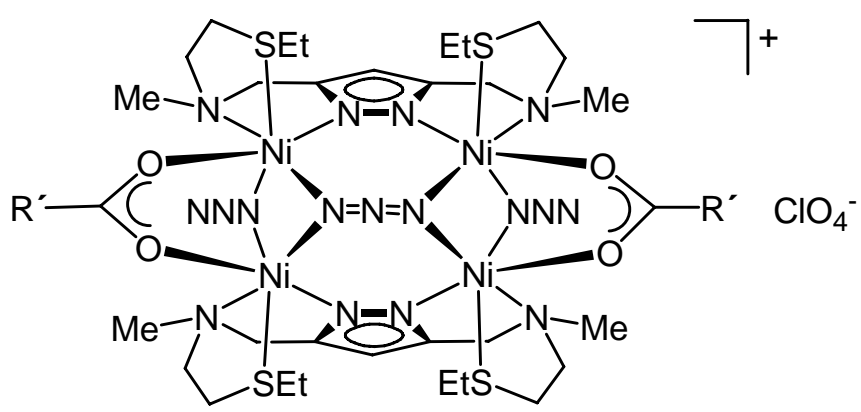

$14 a\left(R^{\prime}=M e\right)$

$14 b\left(R^{\prime}=P h\right)$

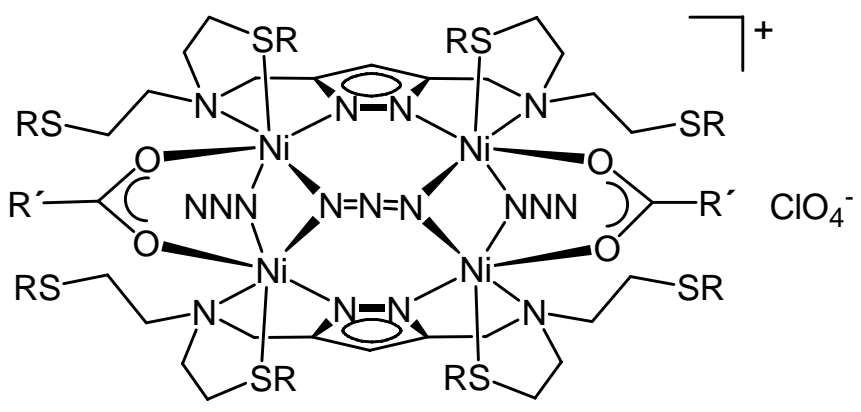

14c $\left(R=E t, R^{\prime}=M e\right)$

14d $\left(\mathrm{R}={ }^{i} \operatorname{Pr}, \mathrm{R}^{\prime}=\mathrm{Me}\right)$

$14 \mathrm{e}\left(\mathrm{R}={ }^{i} \mathrm{Pr}, \mathrm{R}^{\prime}=\mathrm{Ada}\right)$ 


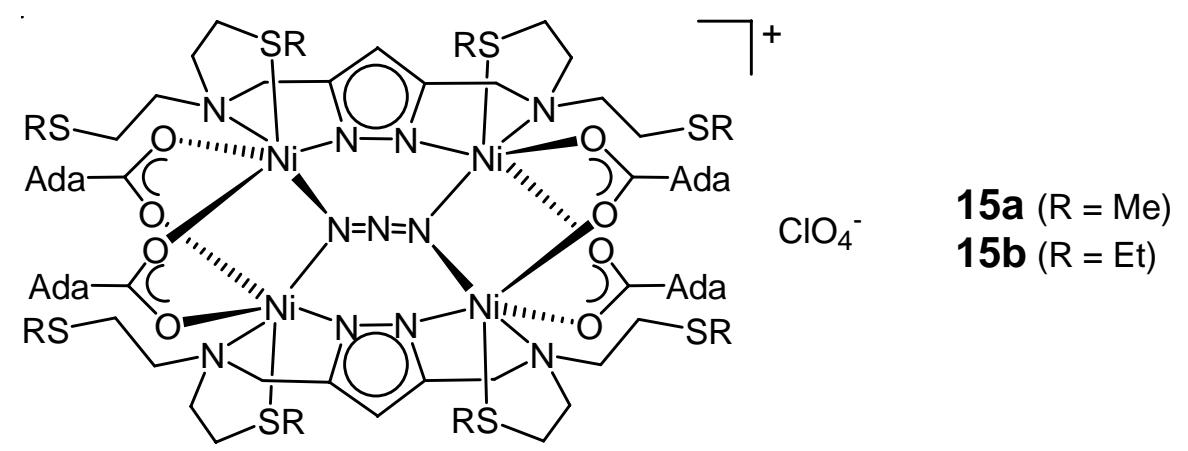

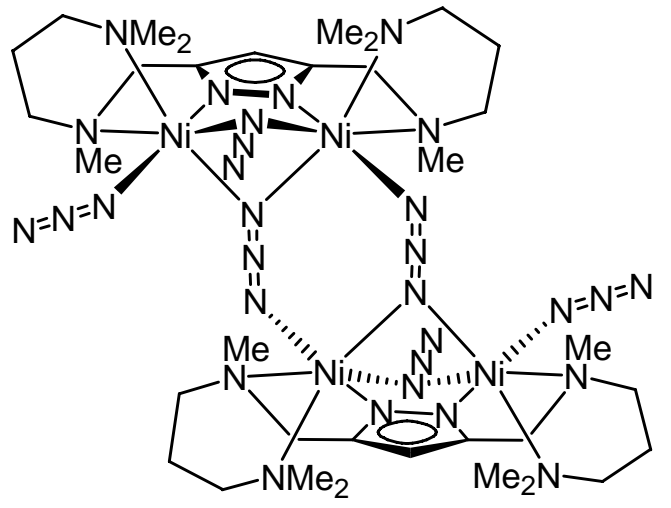

16

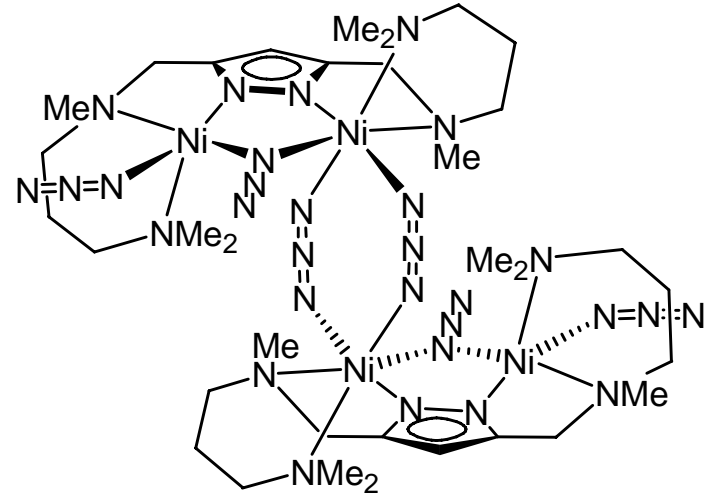

17

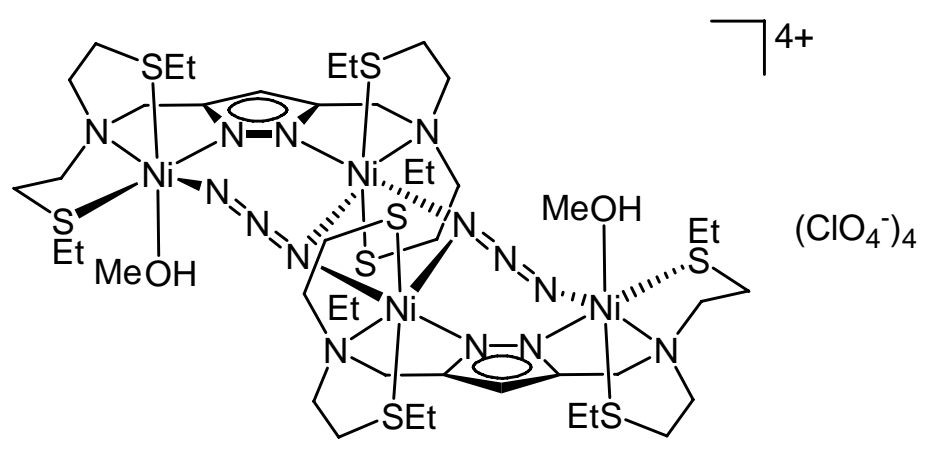

18

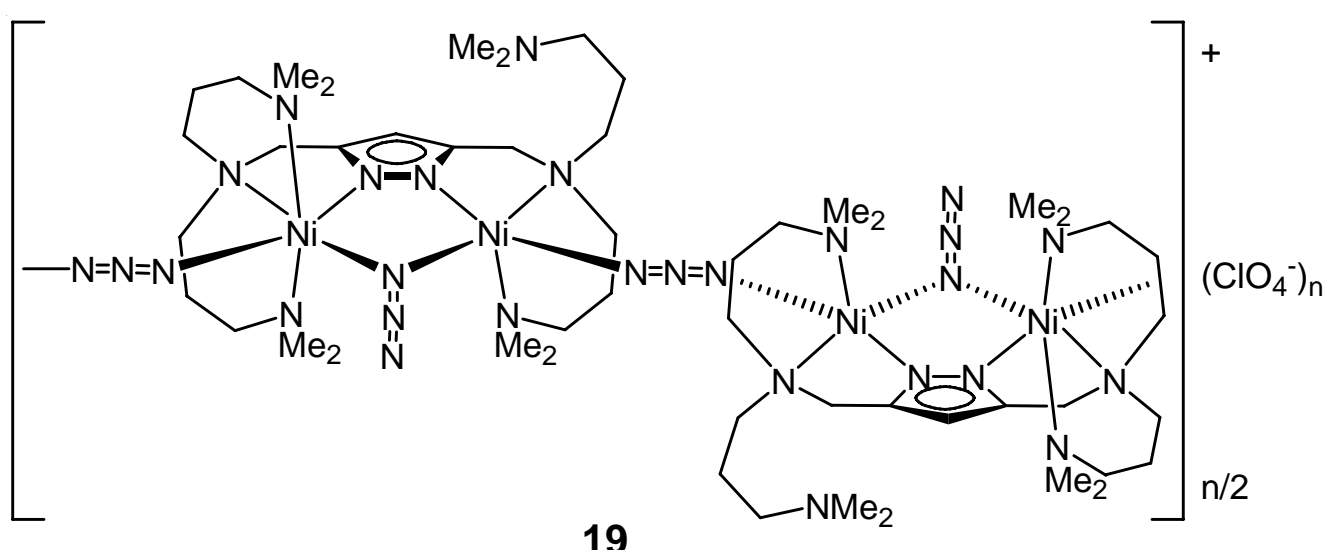



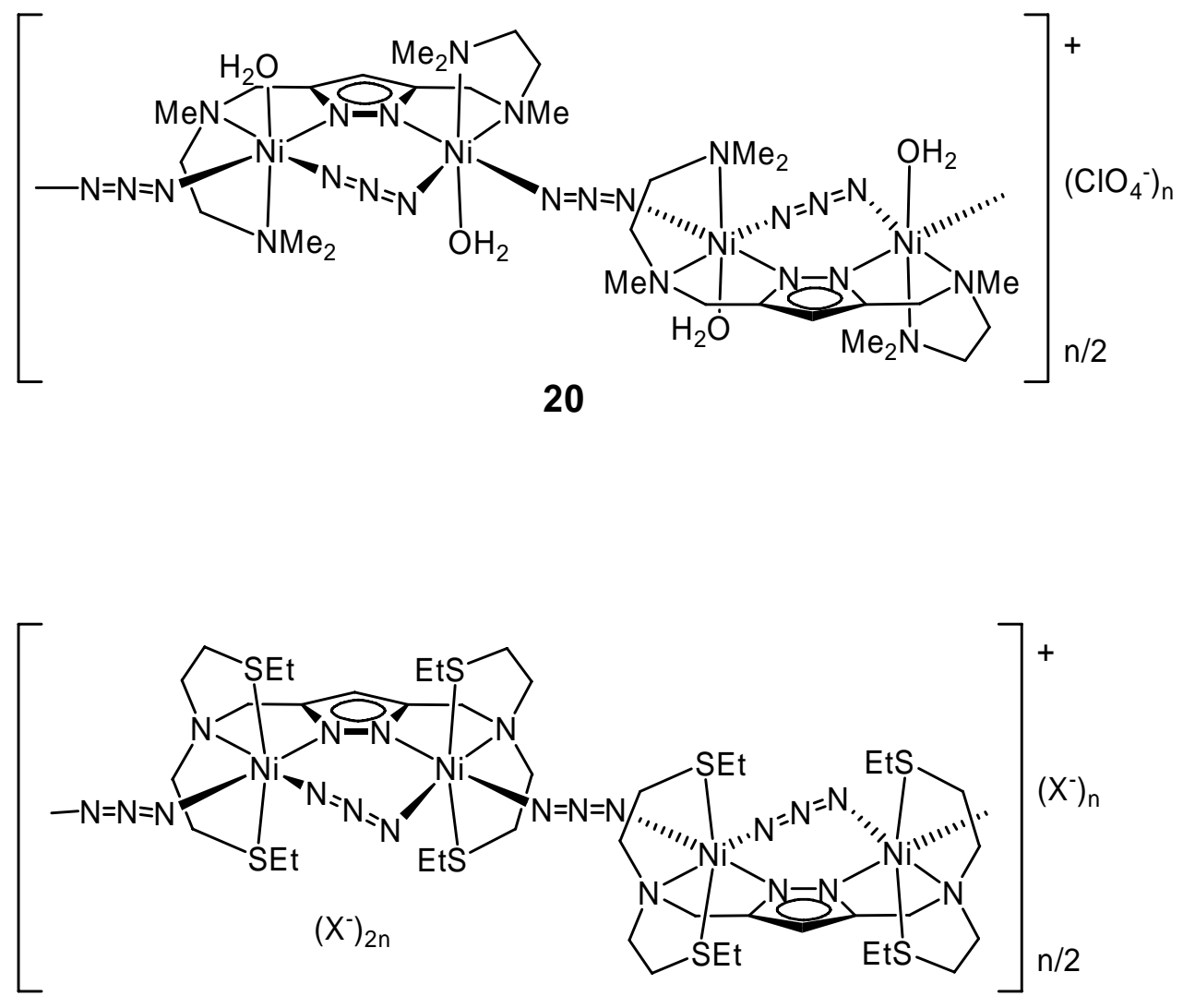

$21\left(\mathrm{X}=\mathrm{ClO}_{4}\right.$, Raumgruppe $\left.P 2_{1} / n\right)$

$22\left(\mathrm{X}=\mathrm{ClO}_{4}\right.$, Raumgruppe $\left.P 2_{1} / \mathrm{C}\right)$

$23\left(\mathrm{X}=\mathrm{BPh}_{4}\right)$

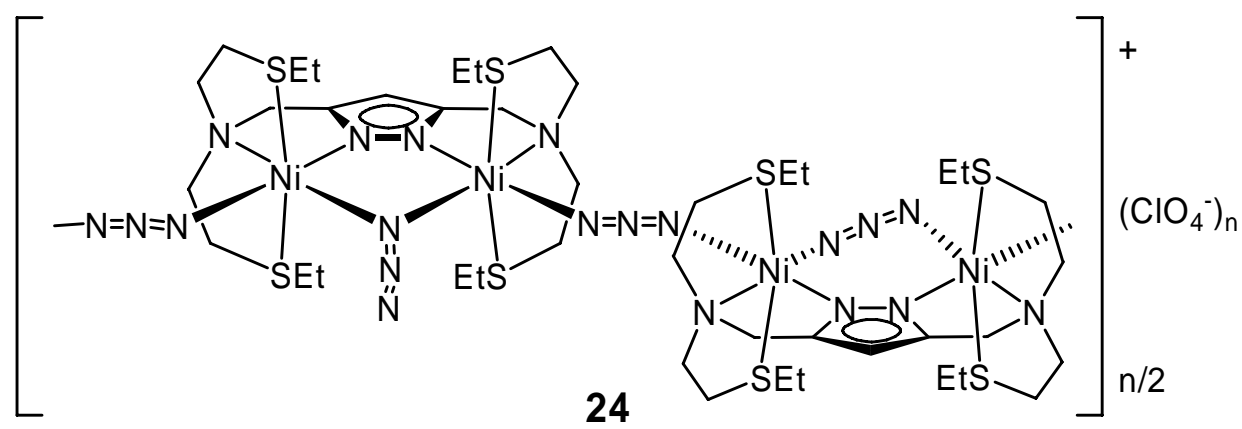




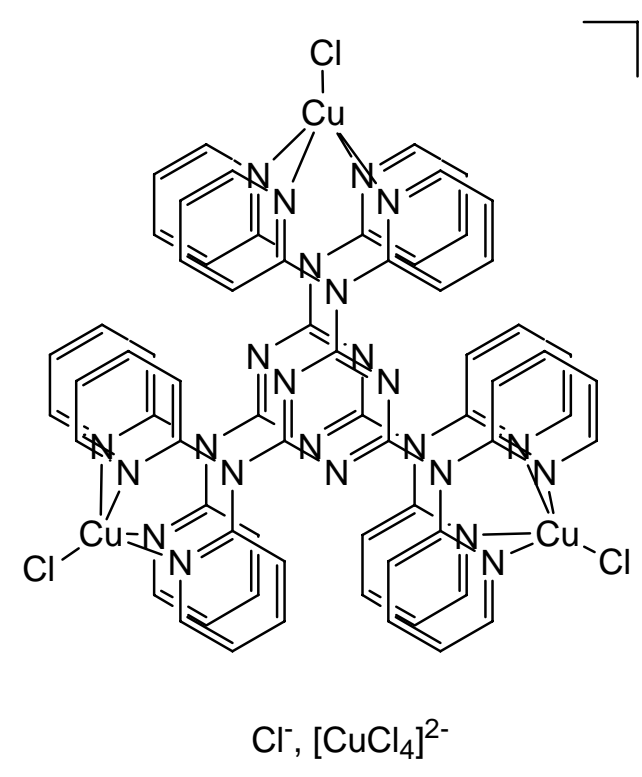

25

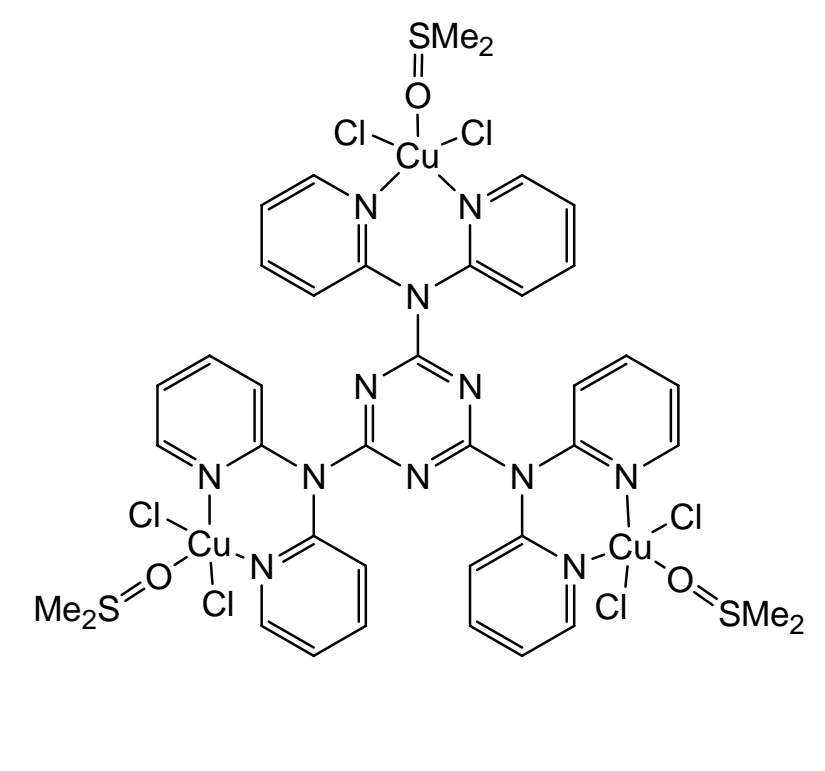

26

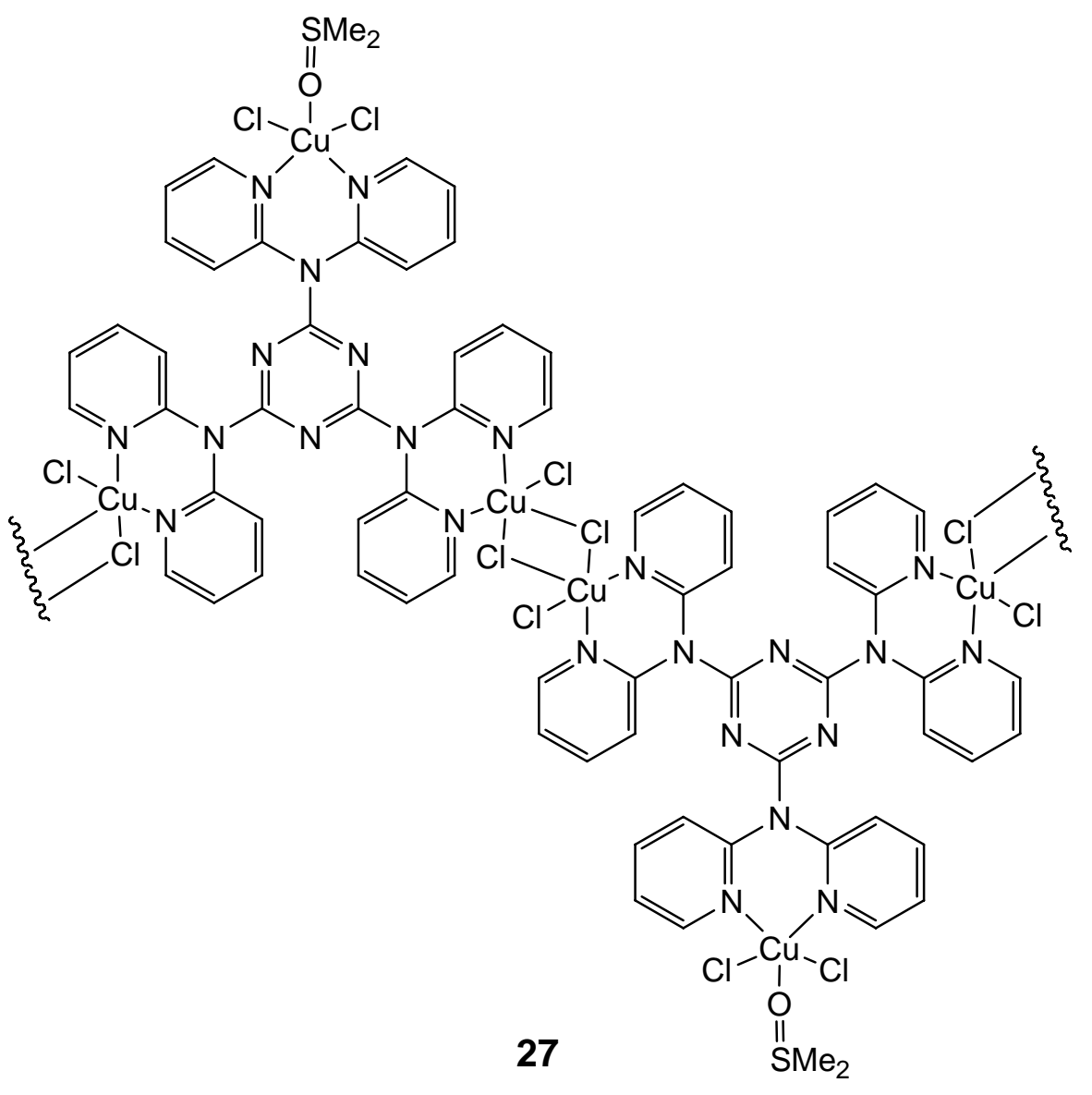




\section{Überblick magnetischer Parameter}

\section{Nickel(II)-Komplexe}

\begin{tabular}{|c|c|c|c|c|c|c|c|c|}
\hline Komplex & $g$ & $J_{1}, \mathrm{~cm}^{-1}$ & $J_{2}, \mathrm{~cm}^{-1}$ & $J_{3}, \mathrm{~cm}^{-1}$ & $J_{4}, \mathrm{~cm}^{-1}$ & $|D|, \mathrm{cm}^{-1}$ & $\begin{array}{l}\text { TIP, } \\
\mathrm{cm}^{3} / \mathrm{mol}\end{array}$ & Programm \\
\hline 1 & 2.10 & +5.6 & - & - & - & - & $1.0 \cdot 10^{-4}$ & ORIGIN \\
\hline 1 & 2.09 & +5.7 & - & - & - & 3.5 & $2.0 \cdot 10^{-4}$ & julX \\
\hline 2 & 2.25 & +4.0 & - & - & - & - & $6.0 \cdot 10^{-4}$ & ORIGIN \\
\hline 2 & 2.24 & +2.8 & - & - & - & 8.9 & $3.7 \cdot 10^{-4}$ & julX \\
\hline 3 & 2.16 & -42.4 & - & - & - & - & $5.1 \cdot 10^{-4}$ & ORIGIN \\
\hline 4 & 2.14 & -15.5 & - & - & - & - & $8.7 \cdot 10^{-4}$ & ORIGIN \\
\hline 5 & 2.22 & -82.0 & - & - & - & - & $9.7 \cdot 10^{-4}$ & ORIGIN \\
\hline 5 & 2.22 & -82.0 & - & - & - & - & $9.7 \cdot 10^{-4}$ & julX \\
\hline 6 & 2.13 & -28.8 & - & - & - & - & $5.8 \cdot 10^{-4}$ & ORIGIN \\
\hline 6 & 2.11 & -28.7 & - & - & - & - & $8.0 \cdot 10^{-4}$ & julX \\
\hline 7 & 2.20 & -67.8 & - & - & - & - & $12.0 \cdot 10^{-4}$ & ORIGIN \\
\hline 8 & 2.19 & -1.3 & - & - & - & - & $5.1 \cdot 10^{-4}$ & ORIGIN \\
\hline 8 & 2.13 & +1.8 & - & - & - & 22.9 & $8 \cdot 10^{-4}$ & julX \\
\hline $9(>230 \mathrm{~K})$ & 2.07 & -24.0 & - & - & - & - & $1 \cdot 10^{-4}$ & ORIGIN \\
\hline $9(<200 \mathrm{~K})$ & 2.24 & -81.0 & - & - & - & - & $12.0 \cdot 10^{-4}$ & ORIGIN \\
\hline 10 & 2.07 & -24.7 & - & - & - & - & $6.3 \cdot 10^{-4}$ & ORIGIN \\
\hline $11 a$ & 2.21 & +1.7 & +4.7 & - & - & 11.7 & - & julX \\
\hline $11 b$ & 2.16 & +2.4 & +2.6 & - & - & 11.7 & - & julX \\
\hline $11 \mathrm{c}$ & 2.22 & +2.5 & -0.6 & - & - & 12.5 & - & julX \\
\hline 12 & 2.28 & +0.9 & -5.9 & - & - & - & - & julX \\
\hline $12 *$ & 2.18 & +1.2 & +4.8 & - & - & 16.2 & - & julX \\
\hline 13a & 2.29 & -57 & -18 & +57 & +6 & - & - & [108] \\
\hline $13 b$ & 2.38 & -61 & -12 & +25 & +5 & - & - & [108] \\
\hline $13 c$ & 2.30 & -53 & -3 & +27 & +6 & - & - & [108] \\
\hline $14 a$ & 2.15 & -55 & +53 & +1 & - & 1 & - & [111] \\
\hline $14 b$ & 2.15 & -56 & +26 & +5 & - & 1 & $20 \cdot 10^{-4}$ & [111] \\
\hline 14d & 2.15 & -67 & +65 & +13 & - & 0 & - & [111] \\
\hline $14 e$ & 2.15 & -56 & +43 & +3 & - & 10 & - & [111] \\
\hline $15 a$ & 2.15 & -20 & +49 & -6 & - & 46 & - & [111] \\
\hline 15b & 2.11 & -25 & +33 & +4 & - & 35 & - & [111] \\
\hline $15 b$ & 2.15 & -13.1 & +20.2 & -13 & - & 3.4 & - & [113] \\
\hline 16 & 2.29 & +13.7 & -32.3 & - & - & - & $6.2 \cdot 10^{-4}$ & julX \\
\hline 17 & 2.20 & +4.1 & -7.7 & - & - & - & $3.8 \cdot 10^{-4}$ & julX \\
\hline 18 & 2.17 & -42.6 & +32.1 & -24.0 & - & - & $8.9 \cdot 10^{-4}$ & julX \\
\hline 21 & 2.20 & -29.5 & -9.7 & - & - & - & - & QMS \\
\hline
\end{tabular}




\begin{tabular}{lllllllll}
\hline 22 & 2.09 & -34.7 & -8.3 & - & - & - & - & QMS \\
23 & 2.20 & -36.1 & -9.0 & - & - & - & QMS \\
\hline
\end{tabular}

\section{Kupfer(II)-Komplexe}

\begin{tabular}{llllll}
\hline Komplex & $g_{1}$ & $g_{2}$ & $J_{1}, \mathrm{~cm}^{-1}$ & $\begin{array}{l}\text { TIP, } \\
\mathrm{cm}^{3} / \mathrm{mol}\end{array}$ & Programm \\
\hline $\mathbf{2 5}$ & 2.04 & 2.07 & -0.21 & $3.6 .0 \cdot 10^{-4}$ & ORIGIN \\
$\mathbf{2 6}$ & 2.04 & - & -0.71 & $1.5 \cdot 10^{-4}$ & ORIGIN \\
$\mathbf{2 7}$ & 2.05 & - & -0.82 & $1.1 \cdot 10^{-4}$ & ORIGIN \\
$\mathbf{2 7}^{1)}$ & 2.05 & 2.03 & -1.83 & $1.7 \cdot 10^{-4}$ & ORIGIN \\
\hline
\end{tabular}

${ }^{1)}$ Nach der Anwendung des dimeren Modells. 


\section{Publikationen}

Teile dieser Arbeit wurden bereits veröffentlicht:

1) G. Leibeling, S. Demeshko, B. Bauer-Siebenlist, F. Meyer, H. Pritzkow, Eur. J. Inorg. Chem. 2004, 2413-2420.

„Polynuclear and extended coordination compounds from preorganized bimetallic components: tuning the magnetic properties of dinickel(II) building blocks“"

2) S. Demeshko, S. Dechert, F. Meyer, J. Am. Chem. Soc. 2004, 126, 4508-4509. „Anion- $\pi$ Interactions in a Carousel Copper(II)-Triazine Complex“

3) S. Demeshko, G. Leibeling, S. Dechert, F. Meyer, Dalton. Trans. 2004, 3782-3787. „1,3,5-Triazine-based tricopper(II) complexes: structure and magnetic properties of threefold-symmetric building blocks“

4) S. Demeshko, G. Leibeling, W. Maringgele, F. Meyer, C. Mennerich, H.-H. Klauss, H. Pritzkow, Inorg. Chem. 2005, 44, 519-528.

„Structural Variety and Magnetic Properties of Tetranuclear Nickel(II)Complexes with a Central $\mu_{4}$-Azide“

5) F. Meyer, S. Demeshko, G. Leibeling, E. Kaifer, H. Pritzkow, Chem. Eur. J. 2005, 11, 1518-1526.

„Structures and Magnetic Properties of Tetranuclear Nickel(II) Complexes with Unusual $\mu_{3}-1,1,3$ Azido Bridges“

6) G. Leibeling, S. Demeshko, S. Dechert, F. Meyer, Angew. Chem., 2005, 117, 7273-7276; Angew. Chem. Int. Ed., 2005, 44, 7111-7114.

„Hysteretic Magnetic Bistability Based on a Molecular Azide Switch“

7) S. Demeshko, G. Leibeling, S. Dechert, F. Meyer, Dalton Trans. 2006, 3458-3465. „Targeted construction of azido-bridged $\mathrm{Ni}_{4}$ complexes with decisive effect of $\mu$-1,3-azide torsion on the spin ground state“

8) C. Golze, A. Alfonsov, R. Klingeler, B. Büchner, V. Kataev, C. Mennerich, H.-H. Klauss, M. Goiran, J.-M. Broto, H. Rakoto, S. Demeshko, G. Leibeling, F. Meyer, Phys. Rev. B 2006, 73, 224403.

„Tuning the magnetic ground state of a tetranuclear nickel(II) molecular complex by high magnetic fields“

9) S. Demeshko, G. Leibeling, S. Dechert, S. Fuchs, T. Pruschke, F. Meyer, ChemPhysChem 2007, im Druck.

„S = 1 alternating $1 D$ spin chains: Controlled assembly from bimetallic building blocks and Quantum Monte Carlo simulation of spin correlation“ 


\section{Danksagung}

Mein großer Dank gilt meinem Betreuer Prof. Dr. Franc Meyer für die freundliche Aufnahme in den Arbeitskreis, die interessante Themenstellung, die hervorragende Betreuung meiner Arbeit, die gewährte wissenschaftliche Freiheit und die sehr guten Arbeitsbedingungen.

Danken möchte ich auch allen, die zum Gelingen dieser Arbeit beigetragen haben:

den Kristallographen Dr. Hans Pritzkow und insbesondere Dr. Sebastian Dechert für die Mühe mit den Kristallen sowie für die hilfreichen Diskussionen;

den Mitarbeitern der Spektroskopie- und Serviceabteilungen sowie allen anderen Institutsangehörigen für ihre Unterstützung und Hilfsbereitschaft;

Dr. Jens Ackermann und Dr. Sebastian Dechert für die Hilfe und Unterstützung an den Rechnern;

Dr. Walter Maringgele für die Unterstützung bei den synthetischen Arbeiten;

meinen Laborkollegen Dr. Guido Leibeling, Germund Glatz, Andreas Schwarz und Dr. Gilles Noël für das angenehme Arbeitsklima im Labor;

allen Kollegen des Arbeitskreises für das freundliche Arbeitsklima, die Diskussions- und Hilfsbereitschaft sowie für die vielen interessanten Gespräche. Mein besonderer Dank gilt hier Dr. Jens Ackermann, Dr. Rosana Ferrari, Petra Unger, Angelina Prokofieva, Dr. Jarl Ivar van der Vlugt und Dr. Oleksandr Prykhodko;

Christopher Mennerich und Dr. Hans-Henning Klauss (TU Braunschweig) sowie Sebastian Fuchs und Prof. Dr. Thomas Pruschke (Fakultät für Physik, Universität Göttingen) für die gute Zusammenarbeit;

Dr. Ekhard Bill (Max-Planck-Institut für Bioanorganische Chemie, Mülheim an der Ruhr) für das zur Verfügung gestellte Programm julX und die hilfreichen Diskussionen.

Bei Dr. Guido Leibeling möchte ich mich herzlich für die Einführung in die interessante Welt des Molekularen Magnetismus sowie für die vielfältige Unterstützung bedanken.

Für die hervorragende Leistung beim Korrekturlesen dieser Arbeit und die zahlreichen Tipps danke ich Dr. Guido Leibeling und insbesondere Dr. Walter Maringgele.

Ganz besonders bedanke ich mich bei meiner Familie für die Unterstützung und das Verständnis. 


\section{Lebenslauf}

Name

Geboren

Staatsangehörigkeit

Familienstand

Kinder

Schulausbildung

Hochschulstudium

(Bachelor), absolviert mit Auszeichnung

09/2001-06/2002 Taras-Schewtschenko-Universität Kiev, Magisterdiplom im Fach Chemie, Lehrer; absolviert mit Auszeichnung Magisterarbeit am Pisargevski-Institut für Physikalische Chemie der Akademie der Wissenschaft der Ukraine mit dem Thema: „Synthese, Zusammensetzung und Aufbau heterokerniger Komplexe von $\mathrm{Cu}(\mathrm{II})$, Zn(II) und Ni(II) mit verschiedenen Aminopolykarbonsäuren und Oxysäuren“

Wehrdienst 09/1999-05/2001 Militärausbildung an der Taras-SchewtschenkoUniversität Kiev 05/2002 Unterleutnant (Reserveoffizier)

Promotion

Serhiy Demeshko

am 11.12.1979, Schostka (Ukraine)

ukrainisch

verheiratet

Yuliya (12/2000) und Pavlo (11/2002)

09/1986-06/1997, Allgemeinbildende Schule Nr. 8, Schostka, absolviert mit Auszeichnung

09/1997-06/2001 Taras-Schewtschenko-Universität Kiev, Chemie

seit 10/2002 Georg-August-Universität Göttingen, unter Anleitung von Prof. Dr. Franc Meyer 